\title{
THIRTEENTH MEETING OF THE UJNR PANEL ON FIRE RESEARCH AND SAFETY, MARCH 13-20, 1996
}

\section{VOLUME 2}

Kellie Ann Beall, Editor

QC

100

.456

No.6030

V.2

1997

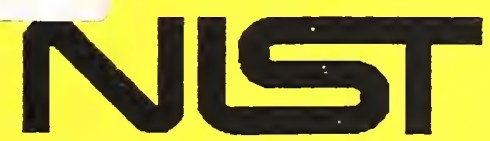

United States Department of Commerce

Technology Administration

National Institute of Standards and Technology 



\section{THIRTEENTH MEETING OF THE UJNR PANEL ON FIRE RESEARCH AND SAFETY, MARCH 13-20, 1996}

\section{VOLUME 2}

Kellie Ann Beall, Editor

June 1997

Building and Fire Research Laboratory

National Institute of Standards and Technology

Gaithersburg, MD 20899

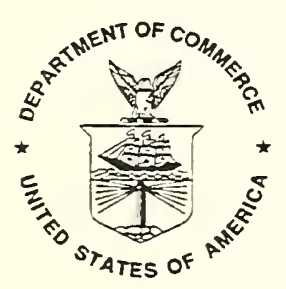

U.S. Department of Commerce

William M. Daley, Secretary

Technology Administration

Gary R. Buchula, Acting Under Secretary for Technology

National Institute of Standards and Technology

Robert E. Hebner, Acting Director 


\section{VOLUME 2}

\section{CONTENTS}

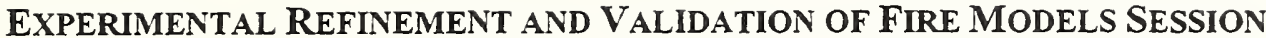

Progress Report on Fire Modeling -- Numerical Simulation of Variable Density Flow with High

Buoyancy . . . . . . . . . . . . . . . . . . . 3

Progress Report on Fire Modeling and Validation $\ldots \ldots \ldots \ldots \ldots \ldots \ldots \ldots \ldots \ldots \ldots \ldots$

Computations of Enhanced Soot Production in Flickering Diffusion Flames . . . . . . . . . 15

Experimental Study of the Exchange Flow through a Horizontal Ceiling Vent in Atrium Fires . .... 21

Modeling on Temperature and Ventilation Induced by a Model Fire in a Tall and

Narrow Atrium Space . . . . . . . . . . . . . . . . . . . . . . . . . . 31

Radiation Properties and Flame Structure of Large Hydrocarbon Pool Fires . . . . . . . . . . . 41

Carbon Monoxide Formation Algorithm . . . . . . . . . . . . . . . . . . . 51

The Fire Hazard Assessment Methodology . . . . . . . . . . . . . . . . . . . . . 61

A Room Fire Model in View of Predicting Fire Spread by External Flames . . . . . . . . . . 69

Evaluation of Complex Fire Models . . . . . . . . . . . . . . . . . . . . . . 81

Experiments on Smoke Behavior in Cavity Spaces $\ldots \ldots \ldots \ldots \ldots \ldots \ldots \ldots \ldots \ldots \ldots \ldots$

A Prototype FDMS Database for Model Verification . . . . . . . . . . . . . . . . 105

\section{SUPPRESSION SESSION}

Progress Report on Fire Suppression Research in the U.S. . . . . . . . . . . . . . . 111

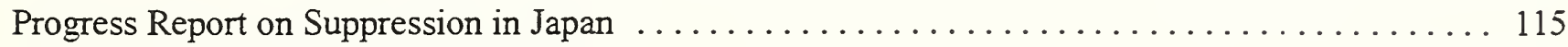

Flame Suppression by Halon Alternatives . . . . . . . . . . . . . . . . . . . . . . 119

Evaluation of Fire Suppression Efficiency of Halon Replacements in Japan . . . . . . . . . . . . 129

Status Report on Water Mist Fire Suppression Systems -- 1996 . . . . . . . . . . . . . . . 137

Experimental Study on Fire Hazard of Residential Fires Before and After Sprinklers Activation . . 145

\section{Materials AND TESTING SESSiON}

Progress Report on Materials and Test Methods . . . . . . . . . . . . . . . . . 161

Progress Report on U.S. Research on Test Methods and Materials . . . . . . . . . . . . . 167

Asia-Oceania ISO5660 Cone Calorimeter Inter-Laboratory Trials $\ldots \ldots \ldots \ldots \ldots \ldots \ldots \ldots \ldots$

Flammability of Upholstered Furniture . . . . . . . . . . . . . . . . . . . . 215

Heat Release Rates Measured by Cone Calorimeter and Intermediate Scale

Electrical Radiant Panels . . . . . . . . . . . . . . . . . . . . . . . . . 225

A Flammability Test for Granular Synthetic Resins Using a Modified Oxygen Index Method . . . . . 235

Advanced Fire safe Materials for Aircraft Interiors . . . . . . . . . . . . . . . . . . . . . . . . 249

Fire Retardant Additives for Polymeric Materials - I. Char Formation from Silica Gel--Potassium

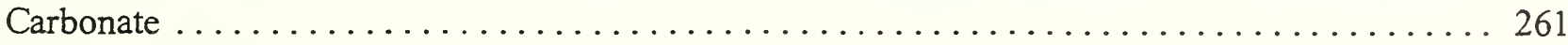




\section{FIRES AFTER EARTHQUAKES SESSION}

Progress Report on Fires Following the Northridge Earthquake . . . . . . . . . . . . 273

Progress Report on Fires Following the 1995 Great Hanshin-Awaji Earthquake . . . . . . . . . . . 283

Post-Earthquake Fires and Firefighting Activities in the Early Stage in the 1995 Great Hanshin

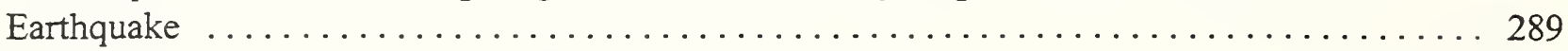

The 1994 Northridge Earthquake and the Fires That Followed .................. 303

The Performance of Fire Protection of Buildings Against the Fires Following the

Great Hanshin-Awaji Earthquake . . . . . . . . . . . . . . . 313

Fires Following the Northridge and Kobe Earthquakes . . . . . . . . . . . . . 325

Causes of the Seismic Fires Following the Great Hanshin-Awaji Earthquake-Survey . . . . . . 337

Durable Agents for Exposure Protection in Wildland/Urban Interface Conflagrations . . . . . . . 345

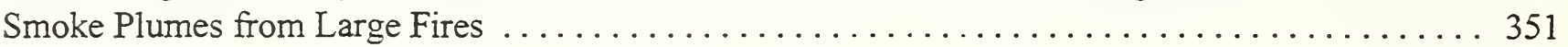

\section{DETECTION SESSION}

Progress Report on Fire Detection Research in the United States. . . . . . . . . . . . . . . 363

Progress Report on Detection Research in Japan . . . . . . . . . . . . . . . . 371

Test Fire Signatures and the Fire-Emulator/Detector-Evaluator $\ldots \ldots \ldots \ldots \ldots \ldots \ldots \ldots \ldots \ldots$

Industry Advances in Fire Detection Technology . . . . . . . . . . . . . . . . . . . 391

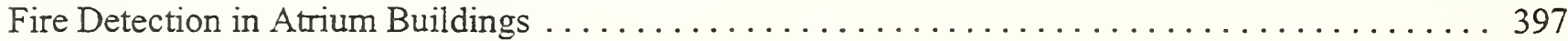

Multivariate Methods for Fire Detection . . . . . . . . . . . . . . . . . . 411

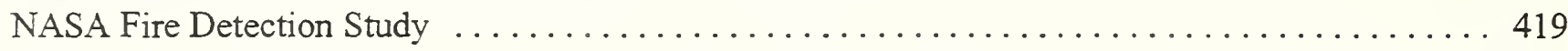

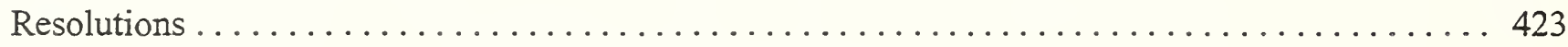

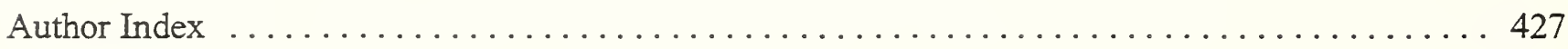


EXPERIMENTAL REFINEMENT AND VALIDATION OF FIRE MODELS 


\title{
PROGRESS REPORT ON FIRE MODELING \\ NUMERICAL SIMULATION OF \\ VARIABLE DENSITY FLOW WITH HIGH BUOYANCY
}

\author{
Yoshihiko HAYASHI, Building Research Institute, Tsukuba, Japan \\ Shuzo MURAKAMI, Tokyo University, Tokyo, Japan \\ Akashi MOCHIDA, Niigata Institute Technology, Niigata, Japan
}

\begin{abstract}
First, I am sorry that it cannot be said that this paper introduces you to the progress report on fire modeling. I will show you the validation of turbulence models applied to the flowfield around a building. In expectation of further developments in fire modeling, now 1 am entering the main subject.

Flowfields around bluff bodies can be analyzed using various turbulence models, $k^{-} \varepsilon$ two equation model ( $k \cdot \varepsilon$ model) which is the most widely used for engineering applications, Algebraic Second-moment closure Model (ASM) and Large Eddy Simulation (LES).

Next, a flowfield inside a room on fire, which is characterized by variable density distributions with high buoyance, is analyzed with the $\mathrm{k}^{-} \varepsilon$ model. This flowfield was predicted by the coupled simulation of convective and radiative heat transfer (case 1) and the result was compared with that of the non-coupled simulation (case 2).
\end{abstract}

\section{COMPARISON OF VARIOÚS TURBULENCE MODELS APPLIED TO A BLUFF BODY}

\section{1-1.INTRODUCTION}

The engineering applications of CFD (Computational Fluid Dynamics) have advanced with the development of various turbulence models, e.g. the $\mathrm{k}^{-} \varepsilon$ two equation model, the Algebraic Second-moment closure Model (ASM), the Differential Second-moment closure Model (DSM) and the Large Eddy Simulation (LES) etc. The high efficiency and accuracy of these models have been clearly confirmed when they are applied to such simple flowfields as the channel or pipe flows. However their performance is not well established for complex turbulent flowfields.

Here, three turbulence models, $k-\varepsilon$ model, ASM and LES, are applied to the flowfield around a bluff body, and thus their relative performances are examined.

\section{1-2.NUMERICAL METHODS AND BOUNDARY CONDITIONS}

The standard formulation for $k-\varepsilon$ model $[1,2]$ was adopted. The commonly adopted form for ASM was used following the methods of Rodi [3], Gibson and Launder [4] except for the treatment of the wall reflection term [5]. The values of the numerical constants in ASM follow those proposed by Launder, Reece and Rodi [6] and Launder [7]. In LES, the Smagorinsky subgrid model $[8,9]$ was applied and the value of 0.12 was selected for the Smagorinsky constant.

A staggered grid was adopted. A second-order upwind scheme was applied for the convective terms in the cases of $\mathrm{k}-\varepsilon$ model and ASM. A second-order centered difference scheme was adopted for the other spatial derivatives. The Adams-Bashforth scheme was used for time marching. Numerical methods and boundary conditions used for the three simulations are made as identical as possible [10].

\section{1-3.COMPARISON OF MEAN VELOCITY VECTOR FIELD}

The mean flowfields around a cube given by wind tunnel testing, $\mathrm{k} \cdot \approx$ model, ASM and LES are compared in terms of mean velocity vectors in Fig. 1. The vertical distributions of velocity behind the model are also shown in Fig.2. As shown in Figs.1 and 2, small differences are observed in some areas, e.g., in the separation region on the frontal comer. Although each rurbulence model seems to give good results for the mean velocity vector field, some discrepancies are often observed in the results of simulations when we compare the distributions of surface pressure and turbulence statistics. 


\section{1-4.DISCREPANCY IN SURFACE PRESSURE DISTRIBUTION}

The surface pressure distribution for a cube is compared in Fig.3. The most noticeable position of the difference is the frontal roof corner where the largest negative value appears. The result of $k \cdot \varepsilon$ model deviates greatly at this area. The large negative peak at the corner decreases rapidly in a distribution peculiar to $k \cdot \varepsilon$ model. We of ten get such inaccurate pressure distribution when $\mathrm{k}-\mathrm{z}$ model is applied to a bluff body. This is caused by the failure to reproduce the small separation here, as is shown in Fig.4, where the area around the frontal corner is magnified. We can observe a small separation in the results of the experiment, ASM and LES, but not in the result of $k-\varepsilon$ model. it should be noted again that the conditions of the numerical methods including boundary conditions are made as identical as possible for the simulations of all three turbulence models.

The discrepancy of surface pressure peculiar to $k \cdot \varepsilon$ model is very closely related to the turbulence statistics around the frontal corner. As shown in Fig. 5 , turbulence energy $\mathrm{k}$ is overestimated in the case of $\mathrm{k} \cdot \varepsilon$ model, which gives rise to a large eddy viscosity ${ }_{2}\left(\infty k^{2} / \varepsilon\right)$. Hence, the excessive mixing effect produced by this large $\nu_{t}$ eliminates the reverse flow on the roof, as is shown in Fig.4.

\section{1-5.CONCLUSION}

The flowfields around a cube predicted by LES, ASM and $\mathrm{k} \cdot \varepsilon$ model are compared with the experimental data, and the accuracy of each model is examined.

\section{COUPLED SIMULATION OF VARIABLE DENSITY FLOW WITH HIGH BUOYANCY}

\section{2-1.NUMERICAL METHODS AND BOUNDARY CONDITIONS [12]}

The standard formulation for $k \cdot \varepsilon$ model was adopted. Since the flowfield is characterized by variable densities, pseudo compressible model [11] was applied. In case of the coupled simulation of convective and radiative heat transfer, convective heat transfer at the wall was calculated by solving heat balance among conduction, convection and radiation. Emissivity at the wall was 0.95 .

The main purpose of this study is to see the validity of the numerical methods applied for the flowfield with high temperature, so chemical production during the combustion was not considered. The heater in the fire room was set to be $500^{\circ} \mathrm{C}$ higher than the ambient air. The computational domain covered an area of $1.8 \mathrm{~m} \times 1.2 \mathrm{~m} \times 1.2 \mathrm{~m}$ as shown in Fig.6. This domain was discretized into $30 \times 30 \times 30$ meshes. Hybrid scheme was applied for convective terms.

The view factor was calculated using Monte Carlo method and the numerical simulation was conducted.

\section{2-2.RESULTS OF SIMULATION}

The mean flowfields given by the simulation are compared in terms of mean velocity vectors in Fig.7. Some differences are observed in some areas, e.g., wall A-B and ceiling A.C. In these areas the mean velocity component of the coupled simulation is larger than that of the non-coupled one. Various discrepancies are also observed even in the result of the coupled simulation, e.g., the floor D-B. In this part the condition of wind vector is different from that of the experiment conducted recently. It seems that the treatment of the constant value of convective heat transfer rate should be modified in the near future.

The temperature distributions are examined in Fig.8. In case of the coupled simulation, boundary layer is observed at each wall. This is due to the higher temperature at the wall in comparison with the non-coupled simulation. However, the temperature gradient along the vertical direction is large only at the vicinity area of the wall. This point is different from the experiment in which the gradient area around the upper part is wider. Therefore another model applied for a large space was proposed [13].

\section{2-3.CONCLUSION}

The flowfield inside a room on fire was calculated by the convective-radiative coupled simulation. The result was compared with that of the non-coupled one. There is a large difference between them, so it seems to be important to apply the heat balance on the wall to such flowfields with high buoyancy. 


\section{REFERENCES}

[1] B.E.Launder and D.B.Spalding, Mathematical Models of Turbulence, Academic Press (1972)

[2] B.E.Launder and D.B.Spalding, The Numerical Computation of Turbulent Flows, Comput. Methods Appl. Mech. Eng., 3 (1974) 269-289

[3] W.Rodi, ZAMM, 58 (1976) T219-T221

[4] M.M.Gibson and B.E.Launder, Ground Effects on Pressure Fluctuations in the Atmospheric Boundary Layer, J. Fluid Mech., 86 (1978) 491-511

[5] S.Murakami, A.Mochida and Y.Hayashi, Scrutinizing k- $=$ EVM and ASM by means of LES and Wind Tunnel for Flowfield around Cube, 8th Symp. on Turbulent Shear Flows (1991)

[6] B.E.Launder, G.J.Reece and W.Rodi, Progress in the Development of a Reynolds Stress Turbulence Closure, J. Fluid Mech., 68 (1975) 537-566

[7] B.E.Launder, University of Manchester, Institute of Science and Technology, Report No.TFD/82/4 (1983)

[8] J.Smagorinsky, General Circulation Experiments with the Primitive Equations, Part 1, Basic Experiments, Mon. Weather Rev., 91 (1963) 99-164

[9] J.W.Deardorff, ANumerical Study of Three-dimensional Turbulent Channel Flow at Large Reynolds Numbers, J. Fluid Mech., 41 (1970) 453-480

[10] S.Murakami, Comparison of Various Turbulence Models Applied to a Bluff Body, J. Wind Eng. Ind. Aero., 46/47 (1993) 21-36

[11] S.Kato et al., Numerical Simulation of Variable Density Flow with High Buoyancy, Part 5, Summaries of Technical Papers of Annual Meeting Architectural Institute of Japan, D (1992) 521-522, written in Japanese

[12] Y.Hayashi et al., Numerical Simulation of Variable Density Flow with High Buoyancy, Part 15, Summaries of Technical Papers of Annual Meeting Architectural Institute of Japan, D (1994) 39-40, written in Japanese

[13] T.Chikamoto et al., New Low-Reynolds Number $k \cdot \varepsilon$ Model Applied to Stratified Flowfield, Part 1, 2 , Summaries of Technical Papers of Annual Meeting Architectural Institute of Japan, D (1994) 17-20, written in Japanese 

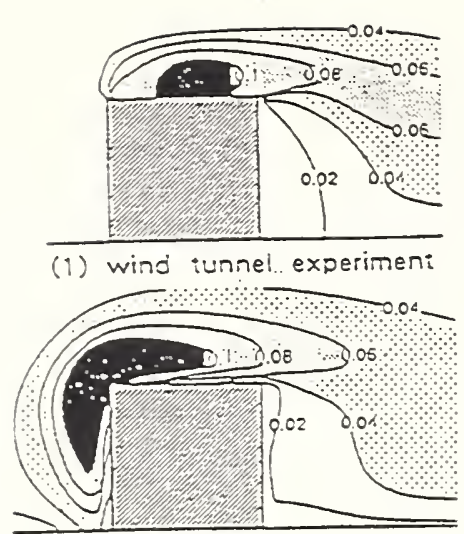

(2) $\Sigma-\varepsilon E V M$

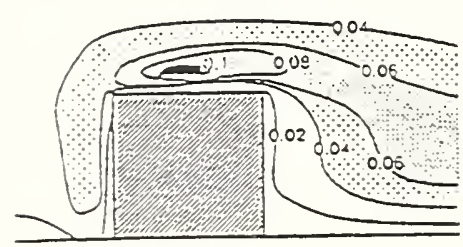

(3) ASM

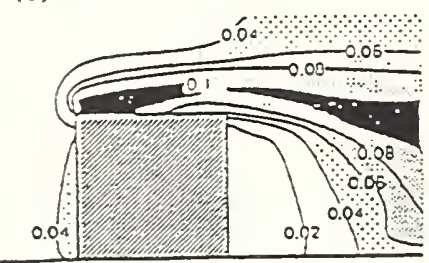

(4) LES

Fig.5 Distzibutions of $k$ at center sec.

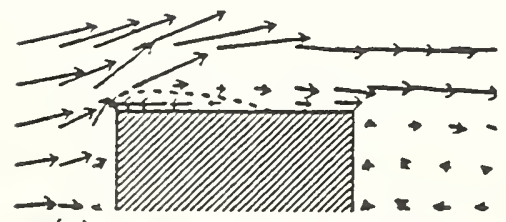

(1) wind tunnel experiment

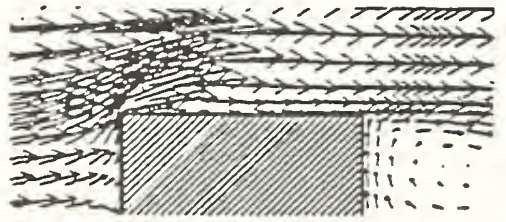

(2) $k-\varepsilon$ EVM

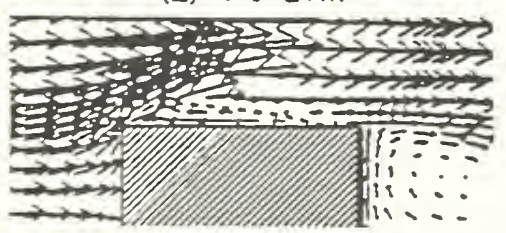

(3) ASM

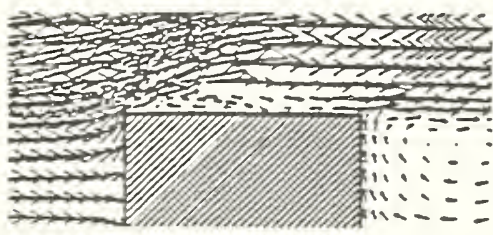

(4) LES

Fig.4 Same with Fig. 1 but magnified around frontal corner

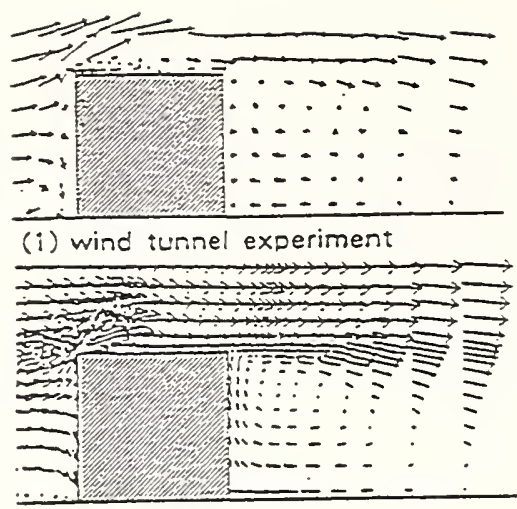

(2) $k-\varepsilon E V M$
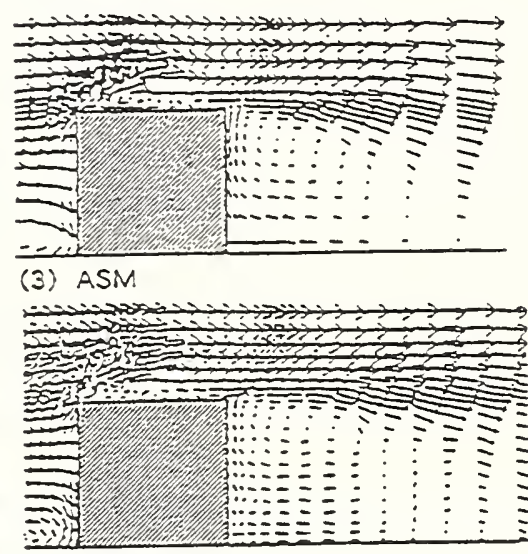

(4) LES

Fig.1 Distこi一 butions of velocity vecters

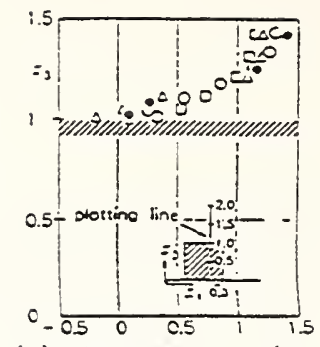

(1) above the root

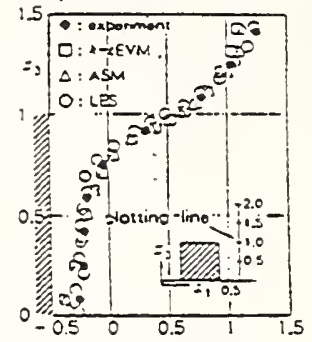

(2) benind the cube

Fig.2 Vertical profiles of $\left\langle u_{1}\right\rangle$ at center sec.

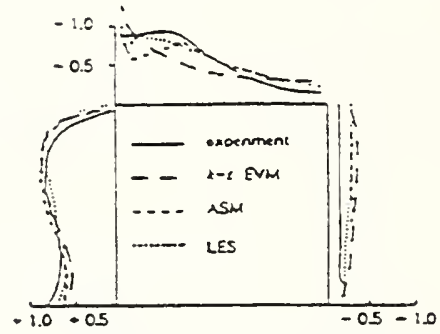

Fig.3 Dist=i-

butions of $\langle C$, $\rangle$

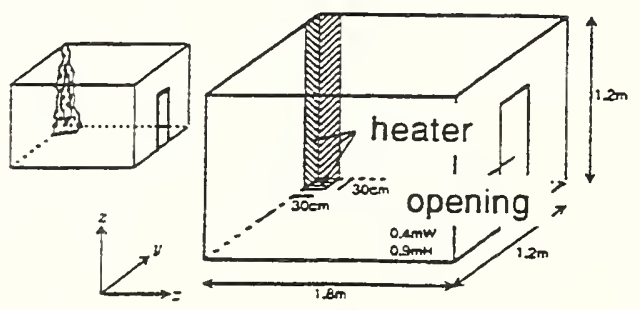

Fig.6-1 Fire room model

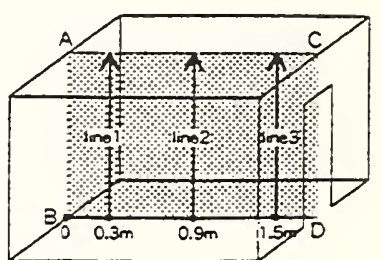

Fig.6-2 Plotting line

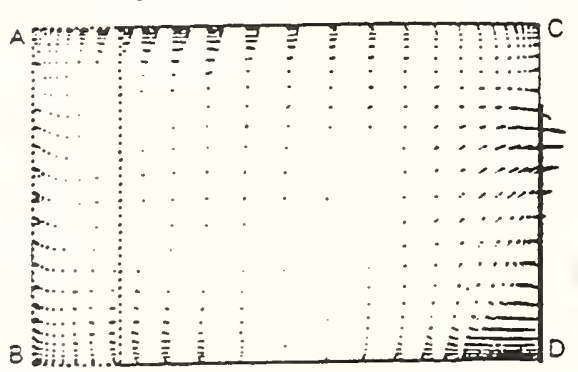

(1) Non-coupled

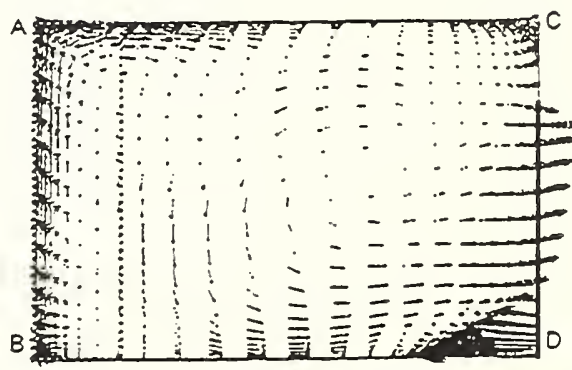

(2) Coupled $\overrightarrow{\mathrm{im} / \mathrm{s}}$

Fig.7 Distributions of velocity vectors

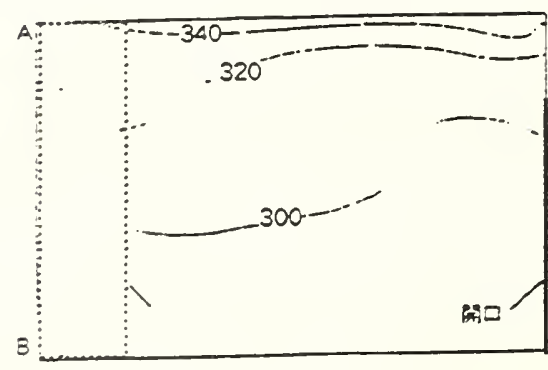

(1) Non-coupled

Fig. 8 Distributions of temperature

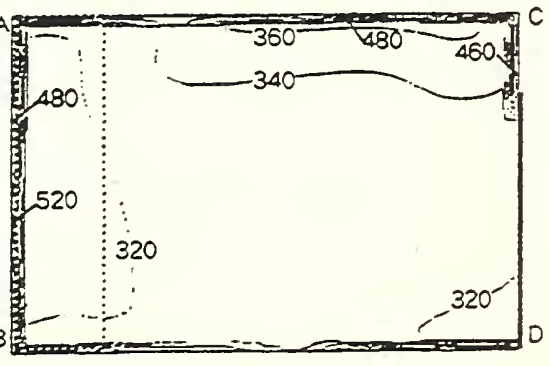

(2) Coupled 


\title{
Progress Report on Fire Modeling and Validation
}

\author{
Walter W. Jones \\ Building and Fire Research Laboratory, NIST, Gaithersburg, MD 20899, USA
}

\begin{abstract}
The nations of the world are moving toward performance based building code standards which will establish a level of safety or risk rather then the traditional prescriptive codes which specify the performance of components. Performance evaluations can then use trade offs between many factors to provide the required level of safety. Computer models are the means to ascertain the performance of buildings built with new materials and new contents. As these models progress and become more entrenched in the regulatory system, it is paramount there be a continual effort to insure their validity. The accuracy of the models of individual phenomena is, and should be, addressed during development However, the interaction of various parts of the system are not always well understood. This paper is part of the continuing effort to test the complete system model with full scale and real scale tests and experiments. ISO, together with the $\mathrm{CIB}$, is establishing a framework for deciding on the appropriateness of a model to meet the requirements of those who wish to use them in predicting the environment in a building. This paper discusses the status of this work and lays out the time table for the completion of this effort, leading to a proposal for an ISO standard.
\end{abstract}

Computer modeling of physical phenomena is becoming an increasingly important tool for understanding the physical world. The driving forces are the increased speed and availability of computers and an increased knowledge base of the physical world. These factors have led to improvements such that model predictions are being used for actual design decisions. Zone models are the present means of choice for understanding and predicting fire growth and smoke spread in compartmented structures. In the past, such models have been used largely to determine, after the fact, what occurred in specific fires. As confidence in the capability of grows, many nations are moving toward replacing much of their benchscale testing of components with computer modeling of whole systems ${ }^{1}$.

Several attempts have been made to answer partially the question of how close the models are to reality. As noted in Peacock, Jones, and Bukowski ${ }^{2}$, to date the method has been to examine plots of experimental data and model predictions. Then some qualitative and often vague statement on the "goodness" of the prediction is made. No method exists for making quantitative estimates of error in predictions and there are many difficulties in developing such a method ${ }^{3}$. The problem concerns comparing curves of variables plotted against time. A method of comparing two curves and giving a simple report on how they fit each other doesn't exist. Until some method exists, quantitative comparisons can not be done. Although we cannot yet provide such a quantitative comparison, it is possible to provide graphically a qualitative analysis. As is the practice, predictions have been plotted against measured values. This gives a visual sense of errors and any trends. The graphs used are included to allow examination of the data that leads to these conclusions.

\section{Overall Project Objective}

Our objective in the project is rather broad: To develop and promote an international protocol to assess the predictive capabilities and usability of computer-based fire models that includes the experimental, statistical, and analytical techniques to implement it. There are an number of areas, however, where significant technical issues must be resolved before such a process is workable.

\section{Issues Which Must be Addressed}

It is critical that the process of model evaluation address areas of primary interest to the users of the models. With this in mind, a number of areas of interest to model users can have an impact on future efforts in fire model evaluation: 
- Classes and uses of models - What types of models are available (zone models, field models, special purpose models) and how are they being used (design, fire recreation, litigation)? Also included is the intended domain of models (and typical use which may be different). Several summaries exist, which list, but do not evaluate these models.

- Scenarios for use in model evaluation - What fire/building scenarios are important to the users of the models? The answer to this should include sufficient information to allow appropriate experiments or simulations to be conducted (building description, design, openings and connections, materials; contents, ignition scenario, environmental conditions). Any process of model evaluation should concentrate on areas which tests the limits of the models rather than areas which most models can be expected to successfully predict.

- Developing and gaining acceptance of an evaluation method - How can we insure that whatever process is used to evaluate a model is supported by the users and developers of the models?

- Technical issues - Although we should build on extensive experimental data available, it is likely that there are areas which are yet to have been studied in detail. These include non-residential fire scenarios, statistical and analytical techniques for comparing model and experimental data, uncertainty in model and experimental results, and model sensitivity.

\section{Important Elements of a Protocol for Model Evaluation}

Once the most important models and scenarios are defined, there are multiple approaches to model evaluation: evaluation of the model theoretical basis by peer review, evaluation of the implementation and usability by model users, and evaluation of the overall predictive capability of the resulting model by comparison of the model with experimental results. A summary of these may provide a starting point for discussions towards a widely accepted protocol:

- Peer review of theoretical basis of models

- Usability and practicality of models

- Comparison of model predictions with experimental results

Sources and quality of data for comparisons

Measurement and measurement system requirements

Uncertainty in experimental results

Peer review of experimental results

Methodology for transforming measured data into values which may be compared with model predictions

Sensitivity analysis of the experimentally-based "model predictions"

Blind runs of the models

Statistical and analytical comparisons

Peer review of theoretical basis - The theoretical basis of the model should be reviewed by a group of recognized experts fully conversant with the chemistry and physics of fire phenomena. The reviewers should judge whether there is sufficient scientific evidence in the open scientific literature to justify the approaches and assumptions being used. Primarily, this group would be comprised of researchers in the field.

Issues to be resolved include who should participate in the groups, who is willing to pay for the effort, what organization should be responsible for the group, and how can the group's independence be ensured with a group composed of both model developers and users. Key to the success of such an effort, however, is some agreement on a process and criteria for submission and evaluation of models that is acceptable to the majority of the model developers and users. Such a process is critical to avoid continual emotional and political arguments of the "best" model phenomena.

Practicality and usability - In addition to peer review of the physics of a model, the ease with which data can be input to the model and the outputs of the model examined can have an impact on the appropriate and correct use of a model. Like the peer review of the theoretical 
basis of models, a group of model users could review the documentation and use to judge the level of expertise necessary to use different models.

Comparison of model predictions with experimental results - User acceptance of predictive fire models is enhanced when the predictions of the model matches the user's experience. This may come from successful recreation of actual fire incidents or through comparison of the predictions of a model with data gathered experimentally. In addition, verifying that the entire model satisfactorily predicts the course of a fire by comparing its output against the actual full-scale fire, ensures that the inevitable assumptions and errors in the individual submodels do not combine to produce incorrect predictions. Program predictions should be made without reference to the experimental data to be used for the comparison. Of course, this restriction does not include required input data that may have been obtained by bench or larger scale tests. No attempt to adjust a fit between the measurements and the predictions should be made.

The quality of experimental data must also be assured. Any experimental data used for model evaluation must be sufficiently documented and reviewed to insure that the experiments were conducted appropriately, that the facilities, instrumentation, and experimental techniques were appropriate to the scenario investigated, that the experimental data is consistent, and that the experiments have been sufficiently characterized to allow simulation by the models without actual reference to the experimental data. Physical measurements of compartments, connections, leakage, and construction materials and techniques must be adequately described to allow accurate simulation. Uncertainties in the measurements and measurement techniques themselves should be determined in a systematic and logical manner.

Model documentation - In order to evaluate the predictive capability, practicality, and usability of any model, sufficient documentation must be provided by the model developer to enable an independent review of the theoretical assumptions and mathematical techniques used in the model. Such documentation of a computer model is the primary means for the model developer to communicate the underlying physics, chemistry, and assumptions of the model.

Sensitivity analysis - Nearly all fire models are deterministic. However, uncertainties in model inputs lead to often overlooked uncertainties in the model results. Model predictions may be sensitive to uncertainties in input data, to the level of rigor employed in modeling the relevant physics and chemistry, and to use of numerical treatments. In general, we need to develop a procedure which identifies the important steps and minimum requirements for evaluation of the sensitivity of a range of fire models for consideration by national and international consensus standards organizations, and to identify and review methods for sensitivity analysis which may be appropriate for evaluation of computer based fire models.

- Organize a working group to develop a consensus on a set of fire/building scenarios of interest to model users.

- Test a range of available fire models with these scenarios (once the scenarios are adequately characterized) to refine the range over which the models should be evaluated.

- Develop a consensus document on a protocol for model evaluation. The existing ASTM guide can provide a starting point. We are currently working to expand on this guide and would welcome cooperation. ISO CD 13389 is now being circulated.

\section{The CIB W14 Project on Model Verification}

An assessment procedure for the evaluation of deterministic fire development simulation methods has been proposed. Detailed planning is made for the first phase consisting of blind, 
semiblind and open numerical simulations followed by a common comparison of simulation data with experimental data. An open comparison report is produced for every scenario of the round robin.

This proposal has been prepared within a CIB W14 sub-group 'Assessment and verification of computer codes for predicting fire development and smoke movement'. The members of the group are: Michel Curtat, Centre Scientifique et Technique du Bâtiment, France, Reinhold Dobbernack, IBMB der Technischen Universität Braunschweig, Germany, Walter W. Jones, Building and Fire Research Laboratory, NIST, USA, and Olavi Keski-Rahkonen (chair); VTT Building Technology, Finland. The proposal was discussed January 12, 1995 at the CIB W14 plenary meeting at Espoo and accepted as rules for the first rounds of the code assessment round robin.

Although numerous efforts to compare fire models with fire experiments have been published, systematic validation of the plethora of existing fire codes ${ }^{4}$ is insufficient. This deficiency has become critical by the introduction of performance based codes in several countries which place heavy emphasis on numerical fire simulations. Therefore, designers, authorities, and the end users of buildings, who may not be knowledgeable about fire simulation, should be given guidance on which codes to use and on the limits of the models. There is anecdotal information that the codes could predict whatever you want. Currently it is left up to the skills of the applicant to ensure the range of validity of a computer code applied to a particular problem.

Following several initiatives CIB W14 has set up a prenormative task group ${ }^{5}$ to plan and organize a validation program, which is intended to provide unbiased evaluations of different deterministic fire development codes and a means to test the usability of these models. Primarily, this evaluation is intended to include zone model based codes due to existing experimental information, but other types of codes, if offered to participate in the round robin, are not excluded.

\section{PROJECT OBJECTIVE}

The objectives of this group are

(1) to increase confidence on the use of fire models as tool for fire safety engineering,

(2) to support ISO/TC92/SC4 in its effort to produce a document on assessment and verification of calculation models (WD13389),

(3) to consider all aspects of code evaluation, including physics, numerics, documentation, use of the codes, and availability of appropriate data for the selected scenarios, and

(4) to carry out a round robin project on deterministic numerical fire simulation computer codes and experiments for model evaluation.

These definitions are slightly modified from those given in [10] due to opinions presented during discussion within the group.

The objectives of the evaluation are so wide, that it will require several years to reach them. It is feasible to plan in a detailed way only a project for the first phase consisting of two simple scenarios. Altogether it is foreseen that at least ten different scenarios should be considered to assess a code to the extend required for fire safety engineering. Preliminarily, these scenarios could be:

\footnotetext{
A: $\quad$ Single plume under a hood

B: $\quad$ Single room with a door opening

C: $\quad$ Single room with a door opening into a corridor

$\mathrm{D}:$ - A floor in a hotel and/or in a health care facility

E: Atrium and a room opening into an atrium
} 


$\begin{array}{ll}\text { F: } & \text { Shopping mall } \\ \text { G: } & \text { Staircase in a multifloor building } \\ \text { H: } & \text { Very large room } \\ \text { I: } & \text { Underground space, room ventilated only from above }\end{array}$

Compartment scenarios include as subscenarios different fire load configurations and boundary conditions (natural versus mechanical ventilation). Scenarios with burning walls, complicated fire loads as well as inclusion of active extinction are left to the later part of the program because the physical basis is still under development.

\section{PROJECT SCHEDULE}

Assessment consisting of different tasks is carried out as field work at different participating institutions, and as expert work within the CIB W14 task group. These are indicated below by a letter after the task number. Calculations relative to experimental data are carried out as blind, semiblind or open. The final report is intended to provide a measure of the validity of the data used for comparison, and the process used for the evaluation. For the first phase of the project, the proposed schedule is as follows:

Project program accepted (January 13, 1995).

Identification of potential participants into the first round of the round robin (March 1995).

Data for the first fire scenarios (standard problems) (March 1995)

(A) smoke filling of a hood with an analytic solution to test numerics, and

(B) three sizes of compartments with a vertical opening

(C) worked out into a standard FDMS format [3], and design reports written.

Invitations to participation into the first round of the robin sent out (April 1995)

Announcement of acceptance fulfilling preconditions (August 1995)

Filing in the blind calculation report on one-compartment fire (B). In addition to illustrating the comparability of models in actual end-use conditions, this will test the ability of the model users to develop appropriate input data for the models (ultimate deadline December 31, 1995)

Invitation to calculation of the one-compartment fire with given rate of the heat release (B), (January 1996). As a follow-on to the blind calculation, this test provides a more careful comparison of the underlying physics in the models with a more completely specified scenario.

Deadline for filing in the first simulation (March 31, 1996)

Invitation to open calculation of problem B, (April 1996). This problem provides the model user with the most complete information about the scenario, including the results of experimental tests of the scenario. Deficiencies in available input (used for the blind calculation) should become most apparent with comparison of the open and blind calculation.

Deadline to file in problem B (June 31, 1996)

Deadline for peer reviews of the codes (December 1995)

Final report on A, and B rounds \{see above for a description\} (September 1996).

\section{ASSESSMENT RULES}

The simulation code assessment includes five major components, which occur either explicitly or implicitly during the process $s^{6}$ documentation, verification, performance validation, sensitivity study, and usability. Prerequisites for participation into the round robin are:

Full written description of the code 
For documentation full description of the physical basis of the code, the main structure of the program, required input data, produced output data, and user instructions should be written. The physical basis should include all mathematical equations used in the code either fully written or referenced to in the open literature in a unique way. Other instructions should be sufficiently detailed, that an educated practitioner in the field should be able to use the code properly following instructions, and without other knowledge of the code (usability).

\section{Availability of the source code}

The source code should be available for inspection at least in one place agreeable to peer reviewers outside the institution which has developed the code (verification).

Multiple participation into the round robin.

For a code to participate into the round robin at least two different users of the code are needed, one outside the organization where code is developed. These users should simulate the given problems independently (usability).

\section{THE FIRST TWO STANDARD PROBLEMS}

SINGLE PLUME UNDER A HOOD: For a rough testing of numerics smoke filling of a hood is calculated using optional plume models, and compared with analytical solutions. For testing the sensitivity on the initial values several input data sets differing from each other only marginally are supplied. For scaling a small, medium, and large hood is to be calculated.

SINGLE ROOM WITH A DOOR: The fire scenario consists of a single room with a door, and a fire load of wood cribs. Three different sizes of rooms of approximately similar geometry are used.

\section{COMPARISON OF SIMULATIONS}

For verification a slight peer review listing models used will be carried out. It is felt at the moment, a conclusive review prior to comparisons of calculations would be practically impossible.

During this first phase the comparison between the simulated and experimental results is made by inspection. Qualitative judgement is given for the goodness of fit. More formal statistical methods may be tested, and used if practical.

A workshop on simulations has been arranged for September 1996, in conjunction with the CIB/SFPE workshop in Ottawa, Canada to discuss the results of the round robin before the comparison report is published in a final form. That workshop will plan the continuation of the round robin exercise for the next scenarios and prepare the design reports for the precalculations of those scenarios.

\section{RELATED ACTIVITIES}

The assessment of the fire simulation codes will be carried out in cooperation with CIB W14 task group of Engineering Evaluation of Building Fire Safety as well as with

ISO/TC92/SC4/WG1 and other organizations related to the work. Since the data to carry out detailed evaluations is either insufficient or nonexistent strong interaction is needed with organizations and laboratories carrying out experiments. The following activities should be discussed to find proper ways of carrying them out outside of the present program and project.

Use accumulating experience of round robins to develop rules for assessment and identify areas which need additional research.

Designing and carrying out all scale experiments of different scenarios to assess numerical fire codes. There 
exists only a limited number of relevant experimental data. Design of an experiment should be based on a precalculated scenario to define measured quantities and measurement positions in a proper way.

Use descriptions of the codes to write a handbook on simulation codes.

\section{CONCLUSION}

The development of performance-based building regulations will reduce barriers to international trade in building materials, products, design, and construction by providing objective criteria and means to establish compliance with these criteria that are not proprietary. This CIB effort is central with regard to fire regulations, and provides key support and prestandardization research to the International Standards Organization effort under TC92/SC4 on Fire Safety Engineering.

1. Bukowski, R.W., Tanaka, T., "Toward the Goal of a Performance Fire Code," Fire and Materials, 15, 1991 pp. $175-180$

2. Peacock, R.D., Jones, W.W., Bukowski, R.W., "Verification of a Model of Fire and Smoke Transport," Fire Safet: J., 21, 1993 pp 89-129

3. Peacock, R.D., Reneke, P.A., Forney, C.L., Kostreva, M.M., "Understanding Sensitivity Analysis for Complex Fire Models," To be published

4. R. Friedman, An International Survey of Computer Models for Fire and Smoke, Journal of Fire Protection Engineering 4, 81 - 92 (1992).

5. M. Kokkala, letter of 22 June 1994 to task group members.

6. N. Khoudja, Procedures for Quantitative Sensitivity and Performance Validation Studies of a Deterministic Fire Safety Model, NBS-GCR-88-544, (1988) 


\section{Discussion}

James Quintiere: There's at least one contribution from France. There is a model from EDF that's not in the public domain, and I wondered if they were contributing because we saw that model some time ago and it looked very complete.

Walter Jones: Do you know the name of it?

James Quintiere: Magic.

Walter Jones: The answer is no, it's not on the list. It may might not have met one of the criteria. But if it's not something that people can view and use, it's not something that's going to be accepted. There are in fact two models from France. 


\title{
Computations of Enhanced Soot Production in Flickering Diffusion Flames
}

\author{
Kermit C. Smyth and Christopher R. Shaddix \\ Building and Fire Research Laboratory \\ National Institute of Standards and Technology \\ Gaithersburg, MD
}

and

Carolyn R. Kaplan

Chemistry Division

Naval Research Laboratory

Washington, D.C.

\begin{abstract}
Recent experimental measurements of soot volume fraction in a flickering $\mathrm{CH}_{4} /$ air diffusion flame show that for conditions in which the tip of the flame is clipped, soot production is $\sim 5$ times greater than that measured for a steady flame burning with the same mean fuel flow velocity. Numerical simulations of both steady and time-varying flickering $\mathrm{CH}_{4} /$ air diffusion flames are used to examine the differences in combustion conditions which lead to this observed enhancement in soot production in the flickering flames. These simulations successfully predict that the maximum soot concentraition increases by over four times compared to the steady flame and show that flickering flames exhibit much longer residence times during which the local temperatures and stoichiometries are favorable for soot production.
\end{abstract}

\section{INTRODUCTION}

Time-varying diffusion flames exhibit a much broader range of combustion conditions than those observed in steady flames. Investigation of different combinations of residence time, temperature history, local stoichiometry, and strain and scalar dissipation rates can provide new insights into the chemistry-flowfield interactions relevant to practical turbulent combustion conditions. Recent quantitative experimental measurements of the local soot volume fraction in a flickering $\mathrm{CH}_{4} /$ air flame show that for a forcing condition in which the tip of the flame is clipped, the peak local soot volume fraction is 5-6 times greater than that measured for a steady flame burning with the same mean fuel flow velocity [1]. Direct numerical simulations of steady and flickering $\mathrm{CH}_{4}$ /air diffusion flames have been conducted to (a) quantitatively compare experimental soot volume fraction and temperature results for a steady $\mathrm{CH}_{4} /$ air flame with predictions using the integrated soot model of Syed, Stewart and Moss [2], (b) compare experimental measurements and computations of the soot volume fraction in the flickering flames, and (c) elucidate the changes in residence time, temperature, and mixture fraction which lead to the enhanced soot production observed in the flickering flames. 


\section{NUMERICAL METHOD}

The numerical model solves the two-dimensional, time-dependent, reactive-flow Navier-Stokes equations coupled with sub-models for soot formation and radiation transport. The fluid convection [3] and diffusive transport sub-models are the same as those published previously [4], however, this model uses different chemical reaction and soot formation sub-models. The fuel consumption rate is expressed in terms of Bilger's [5] formulation

$$
\omega_{C B_{4}}=-\rho D(\nabla \xi)^{2}\left(d^{2} Y_{C H_{4}} / d \xi^{2}\right),
$$

where $\rho$ is the fluid density, and $D=1.786 \times 10^{-5} T^{-1.662}[6]$. Mixture fraction is expressed in the form, $\xi=\left(\beta-\beta_{2}\right) /\left(\beta_{1}-\beta_{2}\right)$, where $\beta$ is a weighted summation of atomic fractions [7]. The resulting production and consumption rates of $\mathrm{CO}_{2}, \mathrm{H}_{2} \mathrm{O}$, and $\mathrm{O}_{2}$ are then evaluated from their respective stoichiometric coefficients, while the amount of heat released is based on the amount of $\mathrm{CH}_{4}$ depleted times $\Delta H_{\text {comb }}$.

The soot volume fraction and number density are computed as a function of the local gas properties, based upon the simplified rate expressions of Syed et al. [2] developed from measurements in steady $\mathrm{CH}_{4}$ /air flames:

$$
\begin{aligned}
& \frac{d n_{d}}{d t}-C_{\alpha} N_{o} \rho^{2} T^{1 / 2} \chi_{\text {fuel }} e^{-T_{\alpha} / T}-\frac{C_{\beta}}{N_{o}} T^{1 / 2} n_{d}^{2}-\frac{(36 \pi)^{1 / 3} W_{N S C} n_{d}^{4 / 3}}{\rho_{s 000} f_{v}^{1 / 3}}
\end{aligned}
$$

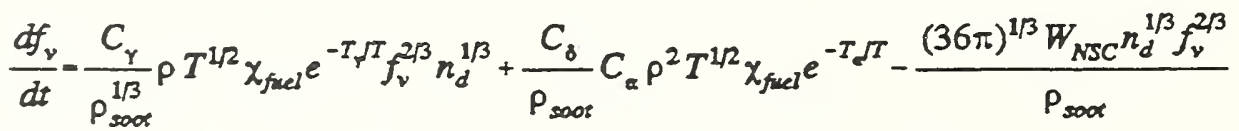

The third term on the right-hand side of each equation represents an oxidation term, based on the Nagle and Strickland-Constable [8] rate of oxidation. Radiation transport is based on an optically-thin assumption, and absorption coefficients are calculated as a function of soot volume fraction, temperature, and mole fractions of $\mathrm{CO}_{2}$ and $\mathrm{H}_{2} \mathrm{O}$.

\section{RESULTS AND DISCUSSION}

\section{Steady Methane/Air Flame}

The experiments and computations are based on an axisymmetric $\mathrm{CH}_{4}$ /air diffusion flame with a fuel tube diameter of $1.11 \mathrm{~cm}$, an air annulus diameter of $10.2 \mathrm{~cm}$, and with $\mathrm{CH}_{4}$ and air velocities of $7.9 \mathrm{~cm} / \mathrm{s}$. Local soot volume fractions have been measured using tomographic reconstruction of extinction data obtained at $632.8 \mathrm{~nm}$ as well as laser-induced incandescence images (LII), with the LII method providing superior results [1]. Temperatures have been obtained from radiation-corrected thermocouple data [9]. Figure 1 shows the experimental configuration for two-dimensional imaging measurements, and Fig. 2 presents images of $\mathrm{OH}$ laser-induced fluorescence (marking the hightemperature reaction zone) as well as scattering from soot particles.

Comparisons between experimental and computed profiles of temperature in the steady flame show good agreement at mid-flame heights, with significant differences occurring at low and high 
axial locations. Soot volume fraction comparisons between the computations and experiments show good agreement for the radial location and shape of the soot profiles, and excellent quantitative agreement for the peak concentration (within 10\%) at heights of 50 to $70 \mathrm{~mm}$.

\section{Flickering Methane/Air Flame}

Experimentally the methane fuel velocity was modulated by applying a variable amplitude sine wave at $10 \mathrm{~Hz}$ to the loudspeaker attached to the fuel plenum (Fig. 1); the mean air and fuel flow velocity were the same as the steady flame conditions $(7.9 \mathrm{~cm} / \mathrm{s})$. Computational simulation of this acoustic forcing is achieved by subjecting the inflow velocity to a $10 \mathrm{~Hz}$ sinusoidal function. Amplitudes corresponding to $25 \%, 50 \%$ and $75 \%$ of the mean flow velocity have been examined, with the $50 \%$ and $75 \%$ conditions giving good qualitative agreement with the experimental flame dynamics. Figure 3 shows computed images of soot volume fraction for the $50 \%$ amplitude case. The shapes of the simulated and experimental flames are similar, and both exhibit tip clipping. The simulations correctly predict increased soot volume fractions in the clipped portions of the flickering flames, $\sim 4$ times greater than that for the steady flame burning with the same mean fuel flow velocity.

Pathline comparisons (Fig. 4) have been made for the soot production rates, temperatures, and local equivalence ratios as a function of residence time in the steady versus flickering flames. Pathlines were obtained by following the history of a fluid parcel which travels through the maximum sooting region in each flickering flame. These results reveal that soot inception and growth occur later in the flickering flames and extend over significantly longer residence times during which favorable temperatures and stoichiometries exist for growth. The pathlines also suggest that soot inception occurs later and at slightly higher temperatures and under leaner conditions in the flickering flames.

\section{ACKNOWLEDGEMENTS}

The computational work was supported by the Office of Naval Research and the Naval Research Laboratory (NRL), and in part by a grant of High Performance Computing (HPC) time from the DoD HPC Shared Resource Center, CEWES Cray-C90. The NIST effort was supported by the Smoke Dynamics Research Group, Fire Science Division.

\section{REFERENCES}

* Present address: Combustion Research Facility, Sandia National Lab., Livermore, CA 94551.

1. Shaddix, C.R., Harrington, J.E., and Smyth, K.C., Combustion and Flame 99, 723 (1994).

2. Syed, K.J., Stewart, C.D., and Moss, J.B., Twenty-Third Symposium (International) on Combustion, p. 1533 (1990).

3. Patnaik, G., Guirguis, R.H., Boris, J.P. and Oran, E.S., Journal of Computational Physics 71, 1 (1987).

4. Kaplan, C.R., Baek, S.W., Oran, E.S. and Ellzey, J.L., Combustion and Flame 96, 1 (1994).

5. Bilger, R.W., Combustion Science and Technology 13, 155 (1976).

6. Bilger, R.W., Combustion and Flame 30, 277 (1977).

7. Bilger, R.W., Twenty-Second Symposium (International) on Combustion, p. 475 (1988).

8. Nagle, J. and Strickland-Constable, R.F., Proceedings of the Fifth Carbon Conference 1, p. 154 (1962).

9. Richardson, T.F. and Santoro, R.J., private communication (1993). 
Figure 1. Experimental set-up for 2-D imaging of axisymmetric diffusion flames which are acoustically excited and phase-locked to the pulsed dye laser system operating at $10 \mathrm{~Hz}$. Images are recorded using an intensified charge-coupled device (ICCD) camera.
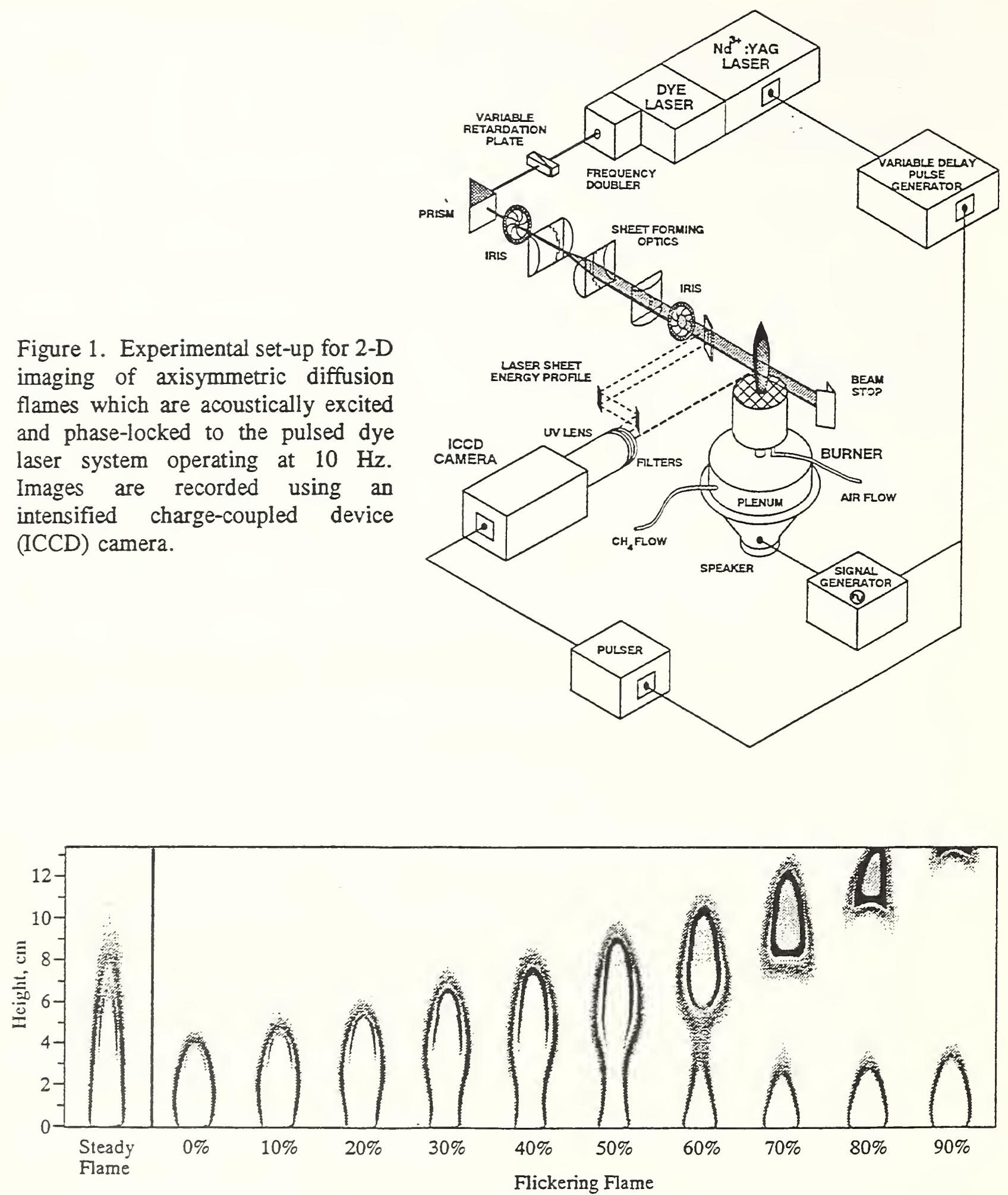

Figure 2. Laser energy-corrected $\mathrm{OH}$ - laser-induced fluorescence and soot scattering images in a steady and time-varying laminar $\mathrm{CH}_{4}$ /air diffusion flame using horizontally polarized light at 283.55 $\mathrm{nm}$. The $\mathrm{OH}$ - fluorescence signals surround the intense scattering from the soot particles and serve as a convenient marker of the high-temperature reaction zone. The visible height of the steady flame is $79 \mathrm{~mm}$ above the fuel tube exit. Ten phase increments separated by $10 \mathrm{~ms}$ are shown with an arbitrary zero phase. 

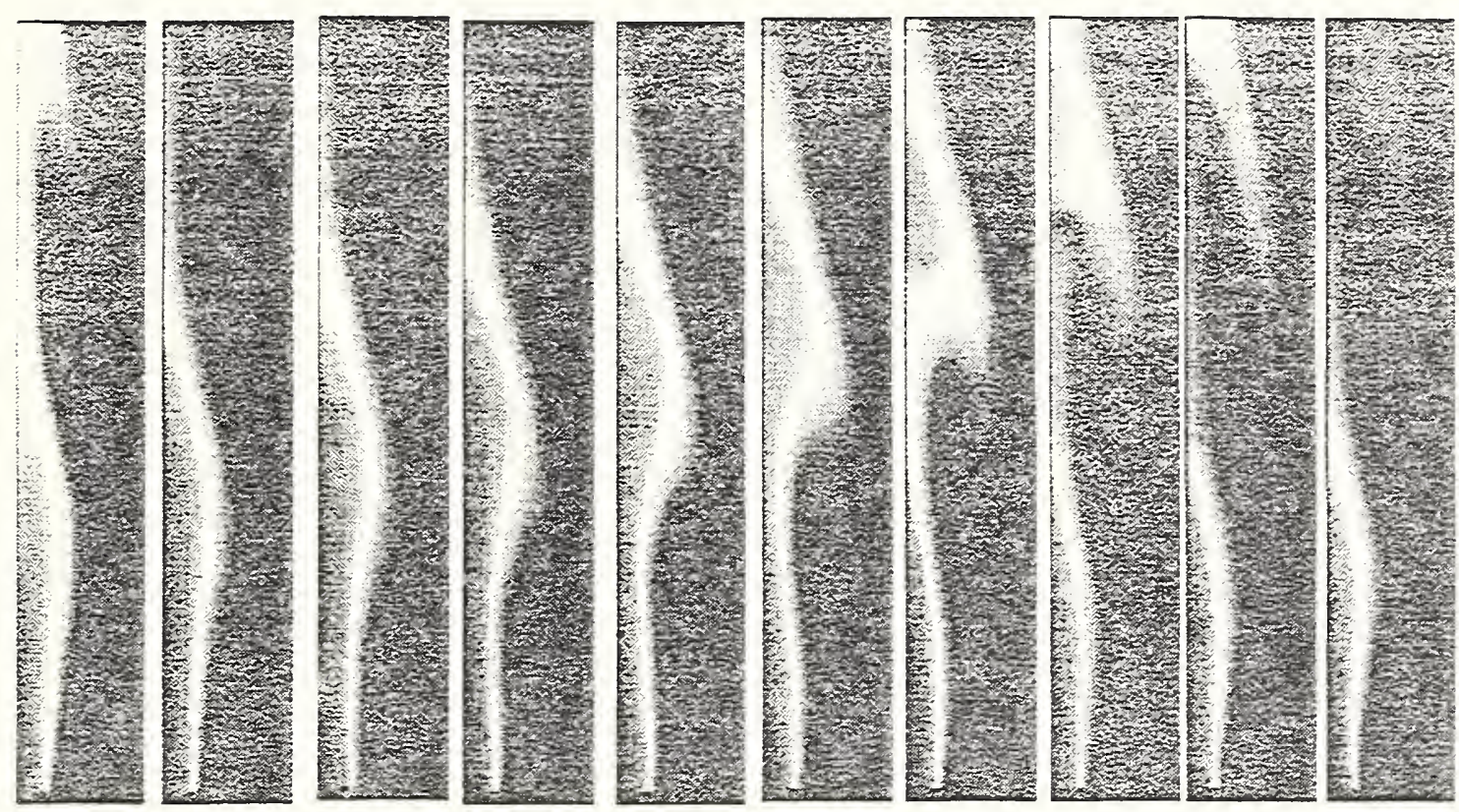

Temperature

$(K)$
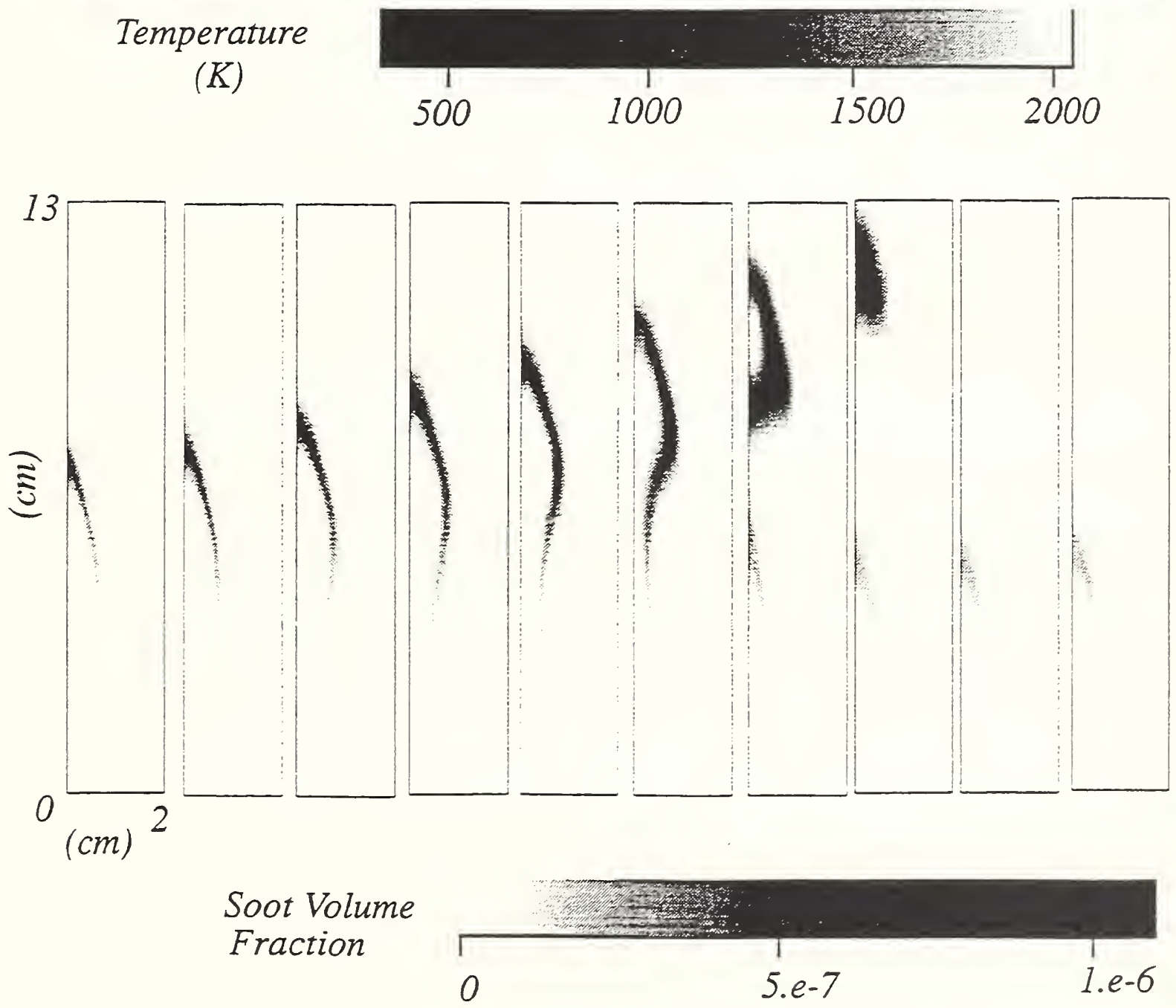

Fig. 3. Temperature and soot volume fraction computations for the flickering $\mathrm{CH}_{4}$ /air diffusion flame ( $50 \%$ amplitude). The time interval between frames is $10 \mathrm{~ms}$. 


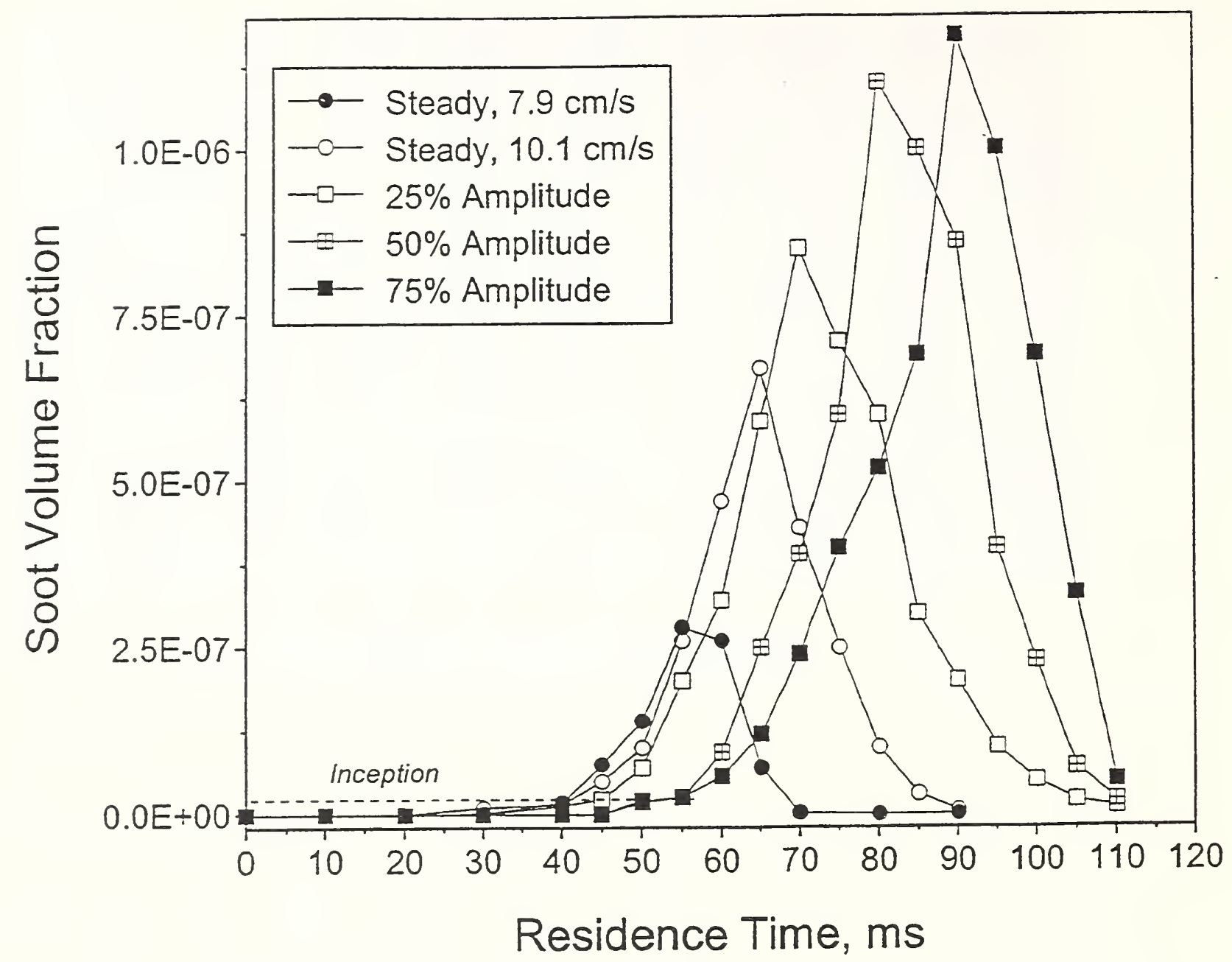

Fig. 4. Pathlines of computed soot volume fraction for two steady flame conditions and three flickering flames where the forcing amplitude of the fuel flow has been varied.

\section{Discussion}

Henri Mitler: I'm a little confused about the oxidation reaction. You said that in the model, $\mathrm{O}_{2}$ is the principle oxidizer. I had the impression that it's the hydroxyl radical that does the damage.

Kermit Smyth: This is a very good point, in fact, I've done work that suggests that $\mathrm{OH}$ is the predominant oxidizer in this simple laminar diffusion flame. What we think is happening is that the Negel Strickland Constable rate overestimates soot oxidation at temperatures above $1800 \mathrm{~K}$, and we have compensating errors here.

Patrick Pagni: I am very excited about your laser measurements. How do you know that the laser does not influence the size of the particles?

Kermit Smyth: In fact, the laser induced incandescence method is very likely to perturb the particle field, so repeat measurements on the same soot particles cannot be carried out with confidence. But if you're not doing that, which is certainly true here, one can make proper calibrations and get very good measurements. 


\title{
Experimental Study of the Exchange Flow through a Horizontal Ceiling Vent in Atrium Fires \\ Criteria of Supply Air and Pressure necessary for Uni-directional Flow
}

\author{
Dr. Tokiyoshi YAMADA \\ Fire Research Institute \\ Fire Defense Agency, Ministry of Home Affairs \\ 14, 1, Nakahara 3-chome, Mitaka, Tokyo, JAPAN
}

\begin{abstract}
This study is directed at understanding the exchange flow through a single top vent in an atrium-type of compartment via fire trials, and obtaining fundamental knowledge of physical mechanism necessary to develop a comprehensive atrium fire model. This study focuses on the supply air and pressure boundary conditions required to prevent flow entering thorough the top vent from outside of the compartment, when the supply air into the compartment is limited. A series of experiments were conducted in a scaled cubical compartment with a natural gas burner as a heat source. Under various heat release rates with different forced air supply, the criteria distinguishing the flow between bidirectional flow and uni-directional out going flow were examined. The result indicates that the criteria can be characterized by dimensionless parameters of the Froude Number and/or the pressure difference, which are functions of the temperature difference between inside and outside of the compartment .

Also, The data obtained in this experiment agrees with the existing equation proposed in the past, however, some discrepancies are found in the pressure difference boundary. The modification and definition of the pressure difference are remained as the future task.
\end{abstract}

\section{INTRODUCTION}

Many buildings with atria spaces have been build for the last decade in Japan as well as in other countries, and adjacent rooms to atrium tends to become more open to the atrium to design spectacular space. From the view point of fire safety, rapid smoke spread through atrium is one of the major concerns in case of fire. Even if there are smoke barriers between surrounding spaces and atrium, smoke layer will be falling down gradually and the whole atrium space will be contaminated with fire smoke at last, unless sufficient ventilation is installed.

Usually, natural ventilation is used to prevent the smoke layer falling as shown in Fig. 1(a). This chimney type smoke ventilation can work effectively, while sufficient top vent and supply air from lower part are given. However when there are not enough supply air, fresh air may thrust the upper hot layer and it make smoke spread rapidly in the lower area as shown in Fig. 1(b). This causes problems for fire fighters' activity as well as occupants' evacuation. Recent buildings with atrium are getting larger and tend to be difficult to get sufficient supply air. For the above reason, criterion of the necessary supply air or pressure difference at the top vent to prevent incoming flow is one of the important information for fire safety design of atrium space.

A series of scaled model experiments have been carried out to study the flow through a horizontal single top vent with a fire inside the atrium. A part of the results has been reported on the exchange flow rate through the top vent under no air supply condition[1]. This study focuses on the horizontal top vent flow behavior with forced air supply vent from the bottom of the model compartment, especially criteria distinguishing the flow between bi-directional and uni-directional flow. A few theoretical 
studies have been reported on this problem. Dr. L. Cooper[2] addressed this phenomena recently and proposed a model of "combined buoyancy- and pressure- driven flow through a horizontal vent". There are small number of experimental data available, and his model was based on the limited number of experiments in the past [3][4]. Some comparisons between experimental data and proposed model were made and examined the correlation between the temperature difference and dimensionless parameters, i.e. the Froude Number and Pressure difference.

\section{DESCRIPTIONS OF THE EXPERIMENTS}

\subsection{Reduced-Scale Experiments}

Reduced scale tests were conducted to study the conditions distinguishing between uni-directional and bi-directional flow through a circular, horizontal, top-centered vent (diameter, $0.15 \mathrm{~m}$ ). A cubic test compartment $(0.8 \mathrm{~m}$ inside) was constructed of $0.05 \mathrm{~m}(0.025 \mathrm{~m}$ at ceiling, $0.0125 \mathrm{~m}$ at ceiling near the vent) thick ceramic fiber board and it was covered by aluminum metal plate to get higher air tightness. This compartment was installed above an air supply chamber as shown in Fig. 2. One $0.15 \mathrm{~m}$ diameter natural gas $\left(11,000 \mathrm{Kcal} / \mathrm{Nm}^{3}\right)$ burner was located flush with the floor and at the center of the compartment. To avoid the direct influence from air supply flow to the fire source, the burner is located on the center of the $0.3 \mathrm{~m}$ square ceramic plate. The gas flow rate was controlled to get heat release rate ranging from 1.1 to $3.5 \mathrm{~kW}$. Under each fixed heat release rate condition, fresh air was supplied at the floor surface. This floor was made of glass fiber covered with meshed metal plate to give an uniform velocity profile ventilation. This forced ventilation rate was set to make unidirectional flow through top vent at first, then reduced step by step to change the flow to the bidirectional flow. The range of ventilation rate was between .300 and $.180 \mathrm{~m}^{3} / \mathrm{min}$. in ambient temperature. The measurements as follows were carried out under quasi-steady state. For careful measurements, it took more than two hours to start measurements after changing the supply air flow rate.

\subsection{Measurements' Installation}

Temperature distribution :

Inside the test compartment were five horizontal thermocouple of trees ( type $\mathrm{K}$ with $0.127 \mathrm{~mm}$ beads) with 15 measuring points at different levels height, and one vertical thermocouple with 17 measuring points to measure gas temperature profile. The data was recorded every 12 seconds during 10 minutes after one monitor temperature installed at the center of the top vent became stable. Also ambient temperature was measured at $5 \mathrm{~cm}$ above the compartment as shown in Fig.2.

Pressure difference between inside and outside of the compartment :

The pressure difference between inside and outside was measured with pressure transducer, which resolution was within $0.01 \mathrm{~Pa}$. The pressure taps made of $1.6 \mathrm{~mm}$ i.o. metal tube were installed at the same level of immediately below the ceiling. The out put of the transducer was recorded every 25 msec. during 2 minutes by 6 times for each test run. The pressure difference reported hereafter is the time averaged data during 12 minutes' data.

Observation of uni-directional/bi-directional flow through top vent :

To distinguish the vent flow between uni-directional and bi-directional flow, temperature fluctuation was measured with very thin thermocouple of $13 \mu \mathrm{m} \phi$ at the ceiling level, $1 \mathrm{~cm}$ apart from the top vent edge. The temperature was measured every $25 \mathrm{msec}$ in the same manner as the pressure difference measurements. And the bi-directional flow mode is decided by the temperature to reach ambient temperature. Also, visualization with smoke of incense at the top vent edge was refereed for final 
decision.

The conditions of each test run and some of the principle data are shown in Table 1, and it is found that the Grashof Number is in the range between $2.1 \times 10^{7}$ and $4.5 \times 10^{7}$ in this experiment.

\section{DISCUSSION}

\subsection{Temperature Profile and Normalized Temperature Difference}

Table 1 shows both conditions of uni- and bi-directional flows under each of the heat release rate condition. Also time and space averaged temperature in side the compartment at level A ( $3 \mathrm{~cm}$ below the ceiling) are listed. The Fig. 3 shows examples of the average temperature profile inside the compartment when the heat release rate is $1.08 \mathrm{~kW}$. Fig. 3(a) is the case of uni-directional flow under relatively high air supply condition. Whereas, (b) is a typical temperature profile under no air supply condition which makes bi-directional flow at the top vent. Fig. 3(b) indicates that the fresh air enters the compartments along the edge of the top vent, and it was observed that fresh air thrust through the hot smoke layer and was well mixed quickly. As the result, temperature profile became relatively flat except fire plume region. The profile became more flat in the case of uni-directional, and the temperature varies a little and relatively stable. For the following discussion, a dimensionless temperature rise is defined by the following equation.

$$
\varepsilon=\left(T_{f}-T_{a m b}\right) /\left(\left(T_{f}+T_{a m b}\right) / 2\right)
$$

where $T$ is the absolute Temperature, the subscript $a m b$ and $f$ refer to ambient temperature and averaged temperature at the level $\mathrm{A}$ in the compartment respectively.

\subsection{Dimensionless Flow Rate and Pressure Difference}

The phenomena of the combined buoyancy- and pressure- driven flow through a horizontal vent was studied by L. Cooper[2]. The following two dimensionless parameters of flow rate (Fr) and pressure difference (II) are adopted as to distinguish the flow between uni- and bi-directional flow. This dimensionless flow rate is known as the Froude Number.

$$
\begin{aligned}
& F r,_{F L O O D}=\left(V_{F L O O D} / A\right) /(2 g D \varepsilon)^{1 / 2} \\
& \Pi_{F L O O D}=\Delta P_{F L O O D} /(4 g \varepsilon \rho D)
\end{aligned}
$$

Where $\mathrm{V}\left[\mathrm{m}^{3} / \mathrm{s}\right]$ is the volumetric flow rate through top vent. This value is estimated by using the supply air flow rate of ambient temperature and the averaged temperature at the level $\mathrm{A}$. A is the area of top vent $\left(0.071\left[\mathrm{~m}^{2}\right]\right), \mathrm{g}$ the gravity acceleration, $D$ the vent diameter $(0.15[\mathrm{~m}])$ and $\rho$ is the average density of the flow $\left[\mathrm{kg} / \mathrm{m}^{3}\right]$. Subscript FLoOD is the boundary between uni- and bi-directional flow mode.

$\Delta \mathrm{P}[\mathrm{Pa}]$ is the pressure difference between bottom and upper side of the horizontal top vent. In this experiment, the pressure difference was measured at the same level height immediately below the ceiling and reference pressure is selected in upper side of the top vent. Then the value is supposed to be positive to make uni-directional flow from bottom to upper side. However the pressure difference was so small that data obtained indicated that statistic pressure inside the compartment was negative against the 
outside at the same level height, even the fresh air was supplied from the bottom of the compartment. This seems to be caused by a buoyant plume above the top vent, which works as if it pumps up the fluids from the compartment. To simplify the mechanism of the flow behavior through the top vent, the flow is assumed to be driven by the pressure difference between bottom and upper sides. Some modification for the pressure difference data obtained is needed by adding the hydrostatic pressure as expressed below.

$$
\Delta P=\Delta P_{E x p_{p}}+\rho_{\text {amb }} g H
$$

Where $H[\mathrm{~m}]$ is vertical distance between bottom and upper reference pressure points. Subscript $\mathrm{Exp}_{\text {is }}$ of the readings in this experiment. $\mathrm{H}$ seems to depend on the depth of the top vent, however the definition of the reference pressure points are ambiguous. To proceed the further discussion, the value $\mathrm{H}$ is set to be 3.5 times of depth of the top vent empirically, which gives similar magnitude pressure difference by using the theoretical correlation proposed in the past[2]

\subsection{Criteria Characterizing Flow Mode}

Fig. 4 shows the correlation between both the Froude Number ( $\mathrm{Fr})$ and the dimensionless pressure difference $(\Pi)$ versus dimensionless temperature difference $(\varepsilon)$. Solid symbols stand for the unidirectional flow and open symbols bi-directional flow. The figure indicates that the criteria distinguishing two flow mode is in the narrow range. The criterion of the Froude number increases gradually with increasing temperature difference. The experimental equation is obtained by using the least square method as follows.

$$
F r{ }_{F L O O D}=0.274 \exp (0.6987 \varepsilon)
$$

The value of the criterion is almost $60 \%$ higher than the equation proposed in the past[2], which is expressed as $0.175 \exp (0.544 \varepsilon)$, however an expression of the correlation is similar. This difference seems to be caused by the definition of the bi-directional flow. In this experiments, when relatively small transient inflow is observed, it is classified as the bi-directional flow. This may gives higher Froude number. Whereas the curve of dimensionless pressure (I) versus $\varepsilon$ is different from the correlation as follows which was proposed in the past [2].

$$
\Pi_{F L O O D}=(1-\varepsilon / 2) F r^{2} F L O O D /\left(4 C^{2} D, F L O O D\right)
$$

where $C_{D_{\text {F Flood }}}$ is the flow coefficients of the top vent and 0.171 is proposed. When this orifice coefficient is constant, the dimensionless pressure increases as increasing the Froude number. However, experimental data obtained indicates that the dimensionless pressure difference decrease as increasing the temperature difference, even if another hydrostatic pressure is assumed. Fig. 5 is the plots between the parameter $(1-\varepsilon / 2) \mathrm{Fr}^{2}$ versus the dimensionless pressure difference( $\left.\Pi\right)$. This figure shows that the boundary is dependent mainly on the (1-E/2) $\mathrm{Fr}^{2}$ parameter and not the pressure difference. Also the flow coefficient seems to vary. For this reason, the Froude Number is more appropriate than the pressure difference as a design criterion to prevent inflow through the horizontal top vent. Further experiments under different vent sizes are expected to verify the criterion correlation expressed by the above dimensionless parameters.

\section{Concluding Remarks}

A series of experiments were conducted to ascertain the criteria correlation which characterizes the uni- and bi-directional flow pattern through top vent under forced ventilation condition. The 
experimental result indicates the followings.

1. The criteria of the dimensionless flow velocity (Fr) can be expressed as an function of the dimensionless temperature $(\varepsilon)$ such as $0.27 \exp (0.70 \varepsilon)$. This is similar to the equation obtained in the previous work. However, the value of this experiment is almost $60 \%$ higher .

2. The behavior of combined buoyancy and pressure driven flow through a horizontal top vent is somewhat complex, especially due to flow coefficient change. Then it is not practical to adopt the pressure difference as a design criterion.

3. The flow through the top vent seems to depend on more macroscopic flow, i.e. plume above the top vent. It causes a negative statistic pressure inside the compartment, even if a certain fresh air is supplied from the bottom. The effect of the plume should be taken into account for examining this physical phenomena in future.

\section{References}

1) T.Yamada, L.Cooper, "Experimental Study on the Exchange Flow Through a Horizontal Ceiling in Atrium Fires ", Proc. of the 3rd International Symposium on Fire Safety Science, 1992

2) L.cooper, "Combined Buoyancy and Pressure Driven Flow through a Horizontal Vent", NISTIR 5384, 1994

3) G.Heskestad, R.Spaulding, "Inflow of Air Required at Wall and Ceiling Apertures to Prevent Escape of Fire Smoke", Proc.of the 3rd International Symposium on Fire Safety Science, pp.919928,Elsevier, 1991

4) M.Epstein, M.Kenton, "Combined Natural Convection and Forced Flow through Small Openings in a Horizontal Partition with Special Reference to Flows in Multi-compartment Enclosure", J.of Heat Transfer, Vol.111, p.980-987,1989 


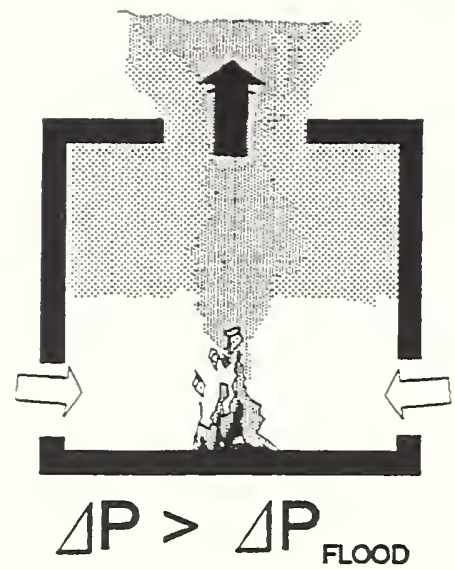

(a) Uni-directional Flow

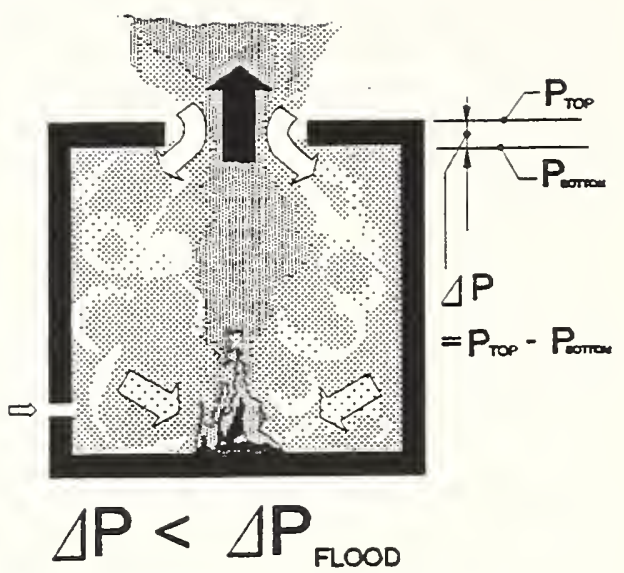

(b) Bi-directional Flow

Figure 1. Schematic flow mode at a horizontal top vent in case of atrium fire (c.f. $\Delta \mathrm{P}_{\text {Flood }}$ is the pressure difference critical boundary between uni-directional (floodind) and bi-directional flow)

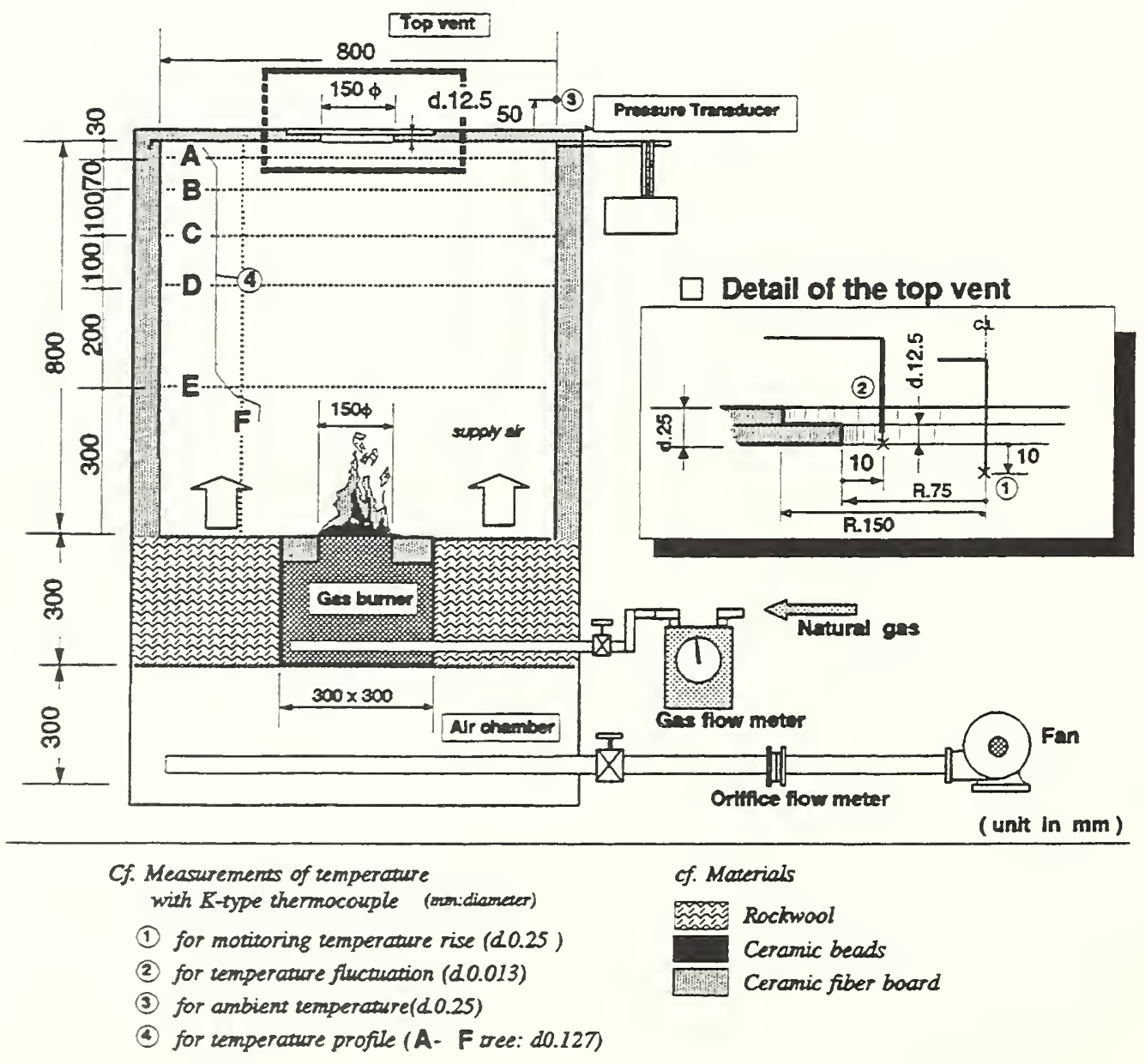

Figure 2. Sketch of experiment 
Table1. Data from the present experiments

\begin{tabular}{|c|c|c|c|c|c|}
\hline \multirow{2}{*}{$\begin{array}{c}\text { Heat Release } \\
\text { Rate }\left(Q_{t}\right) \\
{[K W]}\end{array}$} & \multirow{2}{*}{$\begin{array}{c}\text { Air Supply } \\
\text { Rate }\left(V_{f}\right) \\
{[S L M]}\end{array}$} & Flow Direction & \multicolumn{2}{|c|}{ n Temperature } & \multirow{2}{*}{$\begin{array}{r}\text { Pressure } \\
\text { Difference } \\
{[\mathrm{Pa}]}\end{array}$} \\
\hline & & $\begin{array}{l}\text { Direction } \\
\quad \text { (uni- / bi-) }\end{array}$ & $\begin{array}{r}\text { Ambient } \\
{\left[{ }^{\circ}\right]}\end{array}$ & $\begin{array}{l}\text { Inside } \\
{\left[{ }^{\circ} \mathrm{C}\right]}\end{array}$ & \\
\hline 1.08 & no supply & bi & 21.0 & 172.1 & -0.276 \\
\hline$" \prime$ & 187 & bi & 19.2 & 127.9 & -0.211 \\
\hline$" \prime$ & 240 & bi & 20.0 & 127.9 & -0.165 \\
\hline .... & 251 & uni & 19.6 & 122.5 & -0.145 \\
\hline 1.41 & 252 & $\mathrm{bi}$ & 13.9 & 144.3 & -0.173 \\
\hline .1! & 274 & uni & 11.0 & 140.5 & -0.158 \\
\hline 1.94 & 290 & $\mathrm{bi}$ & 19.8 & 198.2 & -0.183 \\
\hline ." & 314 & uni & 20.9 & 192.8 & -0.148 \\
\hline 2. 42 & 291 & $\mathrm{bi}$ & 22.8 & 225.0 & -0.132 \\
\hline ..! & 310 & uni & 22.0 & 217.9 & -0.153 \\
\hline 3.11 & 307 & $\mathrm{bi}$ & 22.7 & 280.6 & -0.174 \\
\hline ..!. & 320 & uni & 20.8 & 273.8 & -0.130 \\
\hline 3.48 & 287 & $\mathrm{bi}$ & 24.6 & 303.5 & -0.225 \\
\hline$\prime \prime$ & 313 & uni & 24.6 & 292.6 & -0.200 \\
\hline
\end{tabular}

cf. 1) Volumetric flow rate at $15^{\circ} \mathrm{C}$.

2) bi-/uni- directional flow.

3) Time and 15 measuring points averaged data at $3 \mathrm{~cm}$ below the ceiling.

4) Pressure difference between inside and outside at the same level hight of immediately below the ceiling. Negative pressure means that the pressure is negative against the outside.

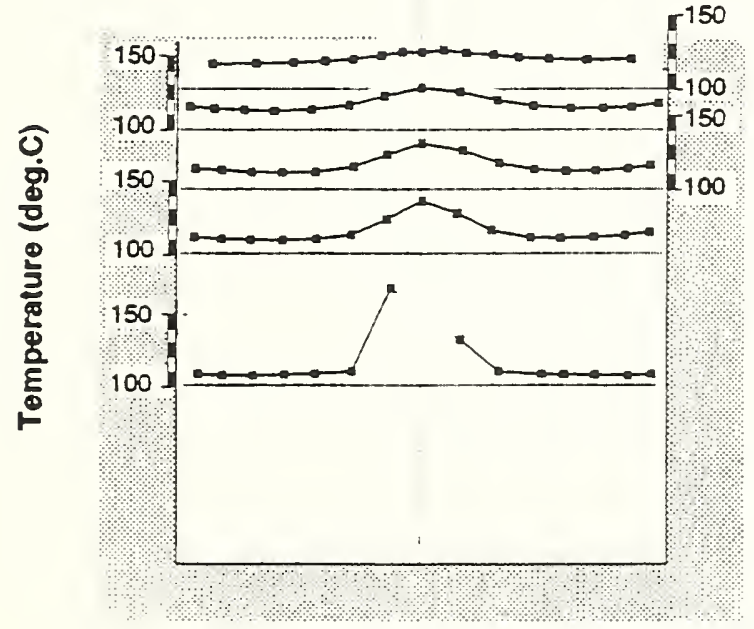

(a) Uni-directional Flow No air supply)

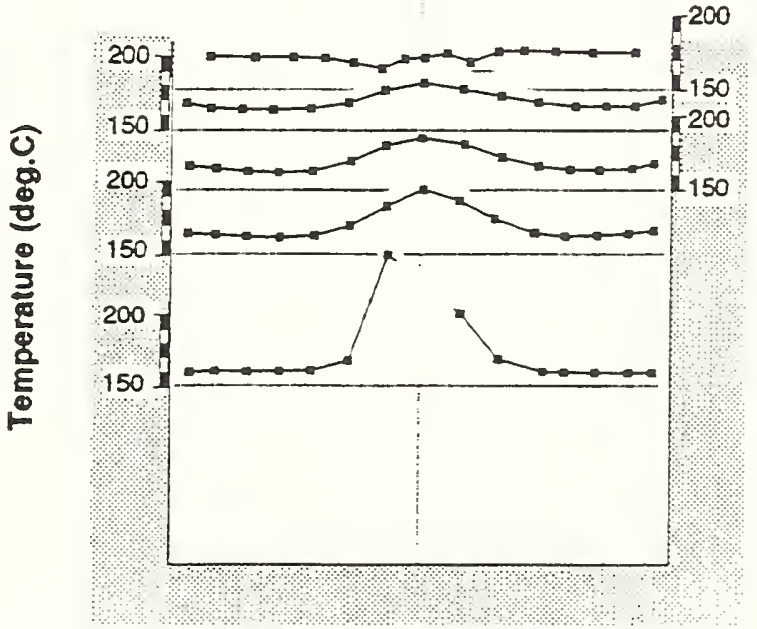

(b) Bi-directional Flow (Supply air $=251 \mathrm{slm}$ )

Figure 3. Example of temperature profile in test compartment :

(Heat release rate : $Q_{r}=1.08 \mathrm{~kW}$ ) 


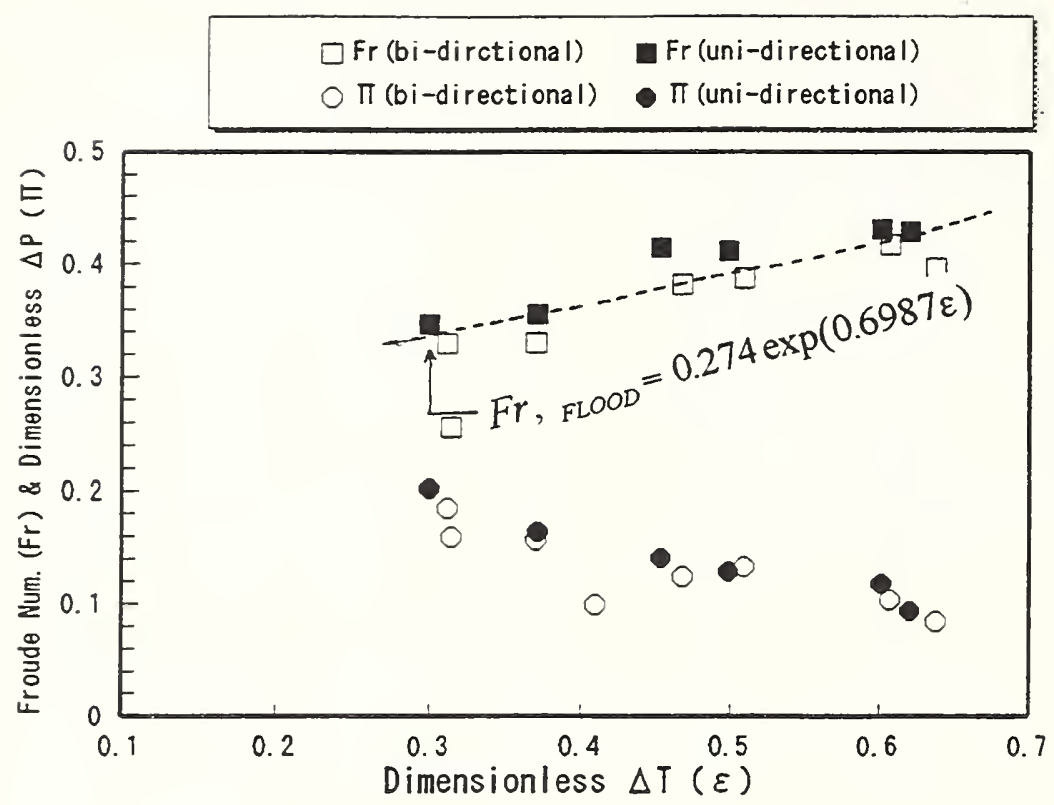

Figure 4. Correlations between dimensionless parameters ( $\mathrm{Fr}, \Pi)$ vs dimensionless temperature differnce $(\varepsilon)$

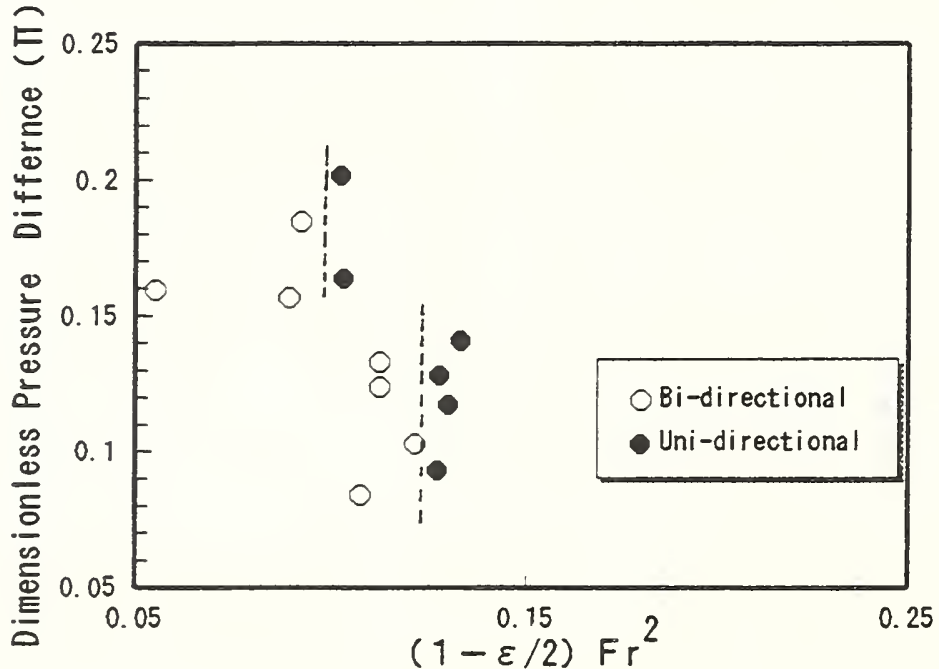

Figure 5. Plots of dimensionless parameter $\left((1-\varepsilon / 2)^{\star} \mathrm{Fr}^{2}\right)$ vs dimensionless pressure difference $(I)$ 


\section{Discussion}

Edward Zukoski: I just did a back-of-the-paper computation of the velocity and the plume inside. A free standing plume would have a velocity of about $1 \mathrm{~m} / \mathrm{s}$ on the center line. Would that cause some problems?

Tokiyoshi Yamada: At this moment, I have no idea. But it may.

Howard Emmons: In my paper, I indicated many points that needed more data. I wish I'd had this about two months ago.

Tokiyoshi Yamada: Actually, this data existed two months ago and this made me aware of how important data exchange is.

Patrick Pagni: Let me close with one simple, practical question. If the air supply is equal to the volume flow out the ceiling vent, is it unidirectional flow?

Tokiyoshi Yamada: Not necessarily. In terms of volume, what goes in is not equal to what comes out because what goes in would be expanded and thus we have to take the expansion into consideration. 


\title{
Modeling on Temperature and Ventilation induced by a Model Fire in a Tall and Narrow Atrium Space
}

by

H. Satoh*, O. Sugawa** and H. Kurioka*

* Kajima Technical Research Institute, Kajima Corp.

2-19-1 Tobitakyu, Chofu-shi, Tokyo 182, Japan

**Center for Science and Technology, Science University of Tokyo, 2641 Yamasaki, Noda, Chiba 278, Japan

\begin{abstract}
Temperature rise and ventilation caused by a fire in a tall and narrow atrium are successfully estimated by an engineering model considering the atrium configuration (floor area, height, opening arrangements), dimensionless heat release rate, and Froude number. These two dimensionless numbers are estimated based on inlet-opening width, heat loss to walls, and inlet-to-outlet opening ratio. Measurements of temperature and upward velocity in a flame, plume and atrium space, and velocity of flow induced at openings were carried out. Experiments were done in a reduced-scale model and three different full scale buildings. Comparison of the estimated values of temperature rise, $\Delta \mathrm{T}$, and ventilation rate using the model proposed with the results of experiments indicated that the model can be used for the estimation of these values as a simple tool. The model helps to evaluate the fire safety design of a tall and narrow atrium.
\end{abstract}

Key Words: Atrium, temperature rise, Froude Number, Dimensionless heat release rate,

Nomenclature

A floor area $\left(\mathrm{m}^{2}\right)$

$\mathrm{A}_{\mathrm{T}} \quad$ total surface area $\left(\mathrm{m}^{2}\right)$

Ad inlet area $\left(\mathrm{m}^{2}\right)$

Ae outlet area $\left(\mathrm{m}^{2}\right)$

C space factor (as defined in this paper) (-)

$C_{D} \quad$ orifice constriction coefficient $(-)$ $(=0.7)$

$\mathrm{Cp} \quad$ specific heat of gas $(\mathrm{kJ} / \mathrm{kg} \cdot \mathrm{K})$

$\mathrm{Dd} \quad$ characteristic door length $\left(=\mathrm{Ad}^{\mathrm{I} / 2}\right)$ (m)

Do characteristic floor length $\left(=A^{1 / 2}\right)$ (m)

$\mathrm{Fr}_{\mathrm{d}} \quad$ Froude number $=\mathbf{F r}_{\mathbf{d}}=\mathbf{V \mathbf { d } ^ { 2 }} / \mathbf{g} \cdot \mathbf{D}_{\mathbf{d}}$

g gravitational acceleration $(9.8$ $\mathrm{m} / \mathrm{s}^{2}$ )

$\mathrm{H}$ height of ceiling above source (m)

he overall heat transfer coefficient $\left(\mathrm{kW} / \mathrm{m}^{2} \cdot \mathrm{K}\right)$
$\mathrm{K}^{2} \quad=(\mathrm{Ta} / \mathrm{Ts})(\mathrm{Ae} / \mathrm{Ad})^{2}(-)$

$\mathrm{Md} \quad$ inlet mass flow rate $(\mathrm{kg} / \mathrm{s})$

$\mathrm{Me}$. outlet mass flow rate $(\mathrm{kg} / \mathrm{s})$

Mair inlet mass flow rate during forced ventilation $(\mathrm{kg} / \mathrm{s})$

Ms outlet mass flow during forced ventilation $(\mathrm{kg} / \mathrm{s})$

$\mathrm{m} \quad=$ DdDo $(-)$

$\mathrm{Q}_{\mathrm{f}} \quad$ heat release rate $(\mathrm{kW})$

Qloss rate of heat loss through walls $(\mathrm{kW})$

$\mathrm{Q}_{\mathrm{fd}}{ }^{*} \quad$ non-dimensional, Froude like door source characterization:

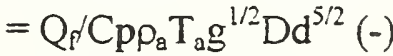

Ta ambient temperature $(\mathrm{K})$

Ts room temperature $(\mathrm{K})$

$\Delta \mathrm{T}$ rise of room temperature above ambient temperature $(\mathrm{K})$

$\mathrm{Vd} \quad$ inletvelocity of air $(\mathrm{m} / \mathrm{sec})$ 
$\begin{array}{llll}\mathrm{Y}^{*} & \text { non-dimensional room heat loss } & \rho_{\mathrm{s}} & \text { room gas density }\left(\mathrm{kg} / \mathrm{m}^{3}\right. \\ \rho_{\mathrm{a}} & \text { ambient air density }\left(\mathrm{kg} / \mathrm{m}^{3}\right) & & \end{array}$

1. Introduction

Buildings having

atrium of various scales, forms and uses increased in Japan. Figure 1 gives the space volume and the aspect ratio, $\mathrm{H}^{2} / \mathrm{A},[1]$ for the eighteen buildings [2]. This figure indicates that the atrium spaces fall roughly into three groups: the shopping-mall type, approximately cubic type, and talland-narrow type. The ASHRAE Design Guide, based on the study of Heskestad [3], gives appropriate guidelines on fire safety for the first two groups. However, no firm guidelines for the tall and narrow atrium building is

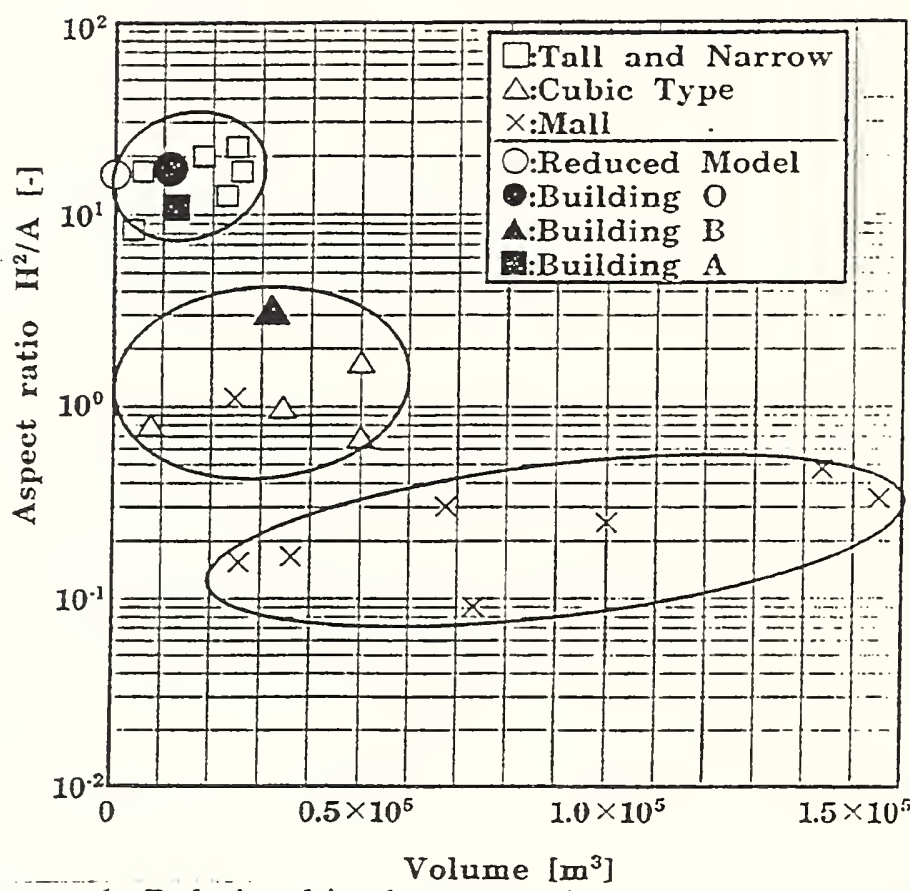

Figure 1 Relationship between the aspect ratio and volume atrium space. Three atrium space used for the experiments and $(\boldsymbol{O}, \mathbf{\Delta}, \mathbf{\square})$ reduced model $(O)$ are illustrated.

given. Several fire tests have been conducted, using reduced model [3 9] and full-scale models $[10,11]$ of compartments. Many researchers [13 15] have proposed models to estimate temperature rise, $\Delta \mathrm{T}$, in a compartment while accounting for heat loss through the surrounding walls covering a wide range of temperature up to $600^{\circ} \mathrm{C}$. However, most cases dealt with a compartment fire and of which have approximately cubic configuration with an opening and which did not deal with an enclosure of a tall and narrow atrium which is the main subject of this study. We pursued to establish a simple method to evaluate temperature rise in a tall and narrow atrium and the inlet-air velocity at openings during the early stage of a fire for natural and/or forced ventilation of the atrium space revealing the relationships among a number of factors; space factor, dimensionless heat release rate, Froude number, and temperature rise in the space. A simplified model which can be used for evaluating the fire prevention measures and smoke management performance of an atrium, given the form and dimensions of the space and a standard fire source, appears the most likely candidate for supplying answers to the questions confronting both designers and regulators.

\section{Simplified Model}

2.1 Basic Expressions

We firstly attempted to define the equations of conservations of mass, energy, and momentum considering the architectural characteristics of an atrium such as floor area, A, height of ceiling, $\mathrm{H}$, and fixed arrangement of openings. 


\subsection{Proposal of simplified model}

\section{1) Formula to predict temperature rise, $\Delta T$}

A formula for predicting temperature rise in a naturally ventilated space can be obtained based on the previous paper. $[10,11]$,

$$
\Delta \mathrm{T} / \mathrm{Ta}=\left(\mathrm{Q}_{\mathrm{fd}}^{*} / \mathrm{Y}^{*}\right) /\left(\mathrm{C}_{\mathrm{D}} \cdot \mathrm{C}^{1 / 2}\right) \text {, }
$$

where the space factor $\mathrm{C}$ is defined as $\mathrm{C}=\left(\mathrm{j} / \mathrm{C}_{\mathrm{D}} \mathrm{m}\right)^{2 / 3}\left(2 \mathrm{~K}^{2} / 1+\mathrm{K}^{2}\right)^{2 / 3}\left(\mathbf{H}^{2} / \mathrm{A}\right)^{2 / 3}$ and this includes $\mathrm{K}^{2}=(\mathrm{Ta} / \mathrm{Ts})(\mathrm{Ae} / \mathrm{Ad})^{2}, \mathbf{Y}^{*}=1+h_{e} \mathbf{A}_{\mathbf{T}} /\left(\mathbf{M}_{\mathrm{d}} \mathrm{C}_{\mathbf{p}}\right)$, and $\left.\mathrm{Q}_{\mathrm{fd}}{ }^{*}=\mathrm{Q}_{\mathrm{f}} / \mathrm{Cp}_{\mathrm{a}} \mathrm{T}_{\mathrm{a}} \mathrm{g}^{1 / 2} \mathrm{Dd}^{5 / 2}\right)$.

A prediction of $\Delta T$ in a mechanically ventilated space can be made directly from $Q_{c}=C_{p} C_{D} A_{d} V_{d} \rho_{a}\left(T_{s}-T_{a}\right)+h_{c} A_{T}\left(T_{s}-T_{a}\right)=\left\{M_{d} C_{p}+h_{e} A_{T}\right\} \Delta T$ if $h_{e}$ is known, but the following formula is more commonly used.

$$
\Delta \mathrm{T} / \mathrm{Ta}=\mathrm{Q}_{\mathrm{fd}}^{*} /\left(\mathbf{Y}^{*} \cdot \mathrm{Fr}_{\mathrm{d}}{ }^{1 / 2}\right) \text { with } \mathrm{Fr}_{\mathrm{d}}=\mathrm{C}\left(\mathrm{Q}_{\mathrm{fd}}^{*} / \mathbf{Y}^{*}\right)^{2 / 3} \text {. }
$$

Formulas for predicting the velocity of air flow at openings and also temperature rise in a naturally ventilated space can be induced from the dimensionless heat release rate as defined with the openings in the space, and the space factor. However, eqs. (1) and (2) contain $\mathrm{Y}^{*}$ so that temperature rise can not be calculated directly using these formulae except when heat loss through the walls is negligible small. These equations must be evaluated from the results of tests. Therefore eq.(2) is transformed into eq.(3) so that $Y^{*}$ may be conveniently obtained from test data. When the value of $\mathrm{Fr}_{\mathrm{d}}$ fluctuates by $20 \%$ to $30 \%$, the value of $\mathbf{Y}^{*}$ fluctuates by the third power. This leads to large error and thus $\mathbf{Y}^{*}$ cannot be used for design purposes.

$$
\mathrm{Y}^{*}=\mathrm{C}^{3 / 2} \mathrm{Q}_{\mathrm{fd}}^{*} / \mathrm{Fr}_{\mathrm{d}}{ }^{3 / 2}
$$

Also, the following formula is obtained by rewriting eq. (2).

$$
Y=Q_{k d}^{*} /\left\{C_{D} C^{12}\left(\Delta T / T_{a}\right)\right\}^{2 / 3}
$$

In eqs.(3) and (4) $\mathrm{Qfd}^{*}$ is a given condition, while $\mathrm{Frd}_{\mathrm{d}}$ and $\Delta \mathrm{T} / \mathrm{Ta}$ are measured values, indicating that $\mathbf{Y}^{*}$ can be predicted in the case of natural ventilation. For the case of forced ventilation, a formula for $\mathbf{Y}^{*}$ is induced from eq.(2).

\section{Verification of the Model}

3.1 Verification by new laboratory tests on scale model

1) Full Scale Atrium

The outline of the full scale models of which size, configuration, and ventilation method are shown in Table 1. Two tests were carried out in tall and narrow atrium of buildings $\mathrm{O}$ and $\mathrm{A}$ under natural and forced convection condition, respectively. The other test was done in cubic-type atrium of building $B$ under natural convection condition. Measurements of temperature and upward velocity were carried out along the center line and also off-center of ascending flow from a model fire source which composed of methanol pools fire. And also upward velocity was measured along the center line in flame zone. Balloon was released near fire source and was traced by video recording system to visualize the convection and the bottom of the hot layer in the atrium space. 
Table 1. Experiment Condition

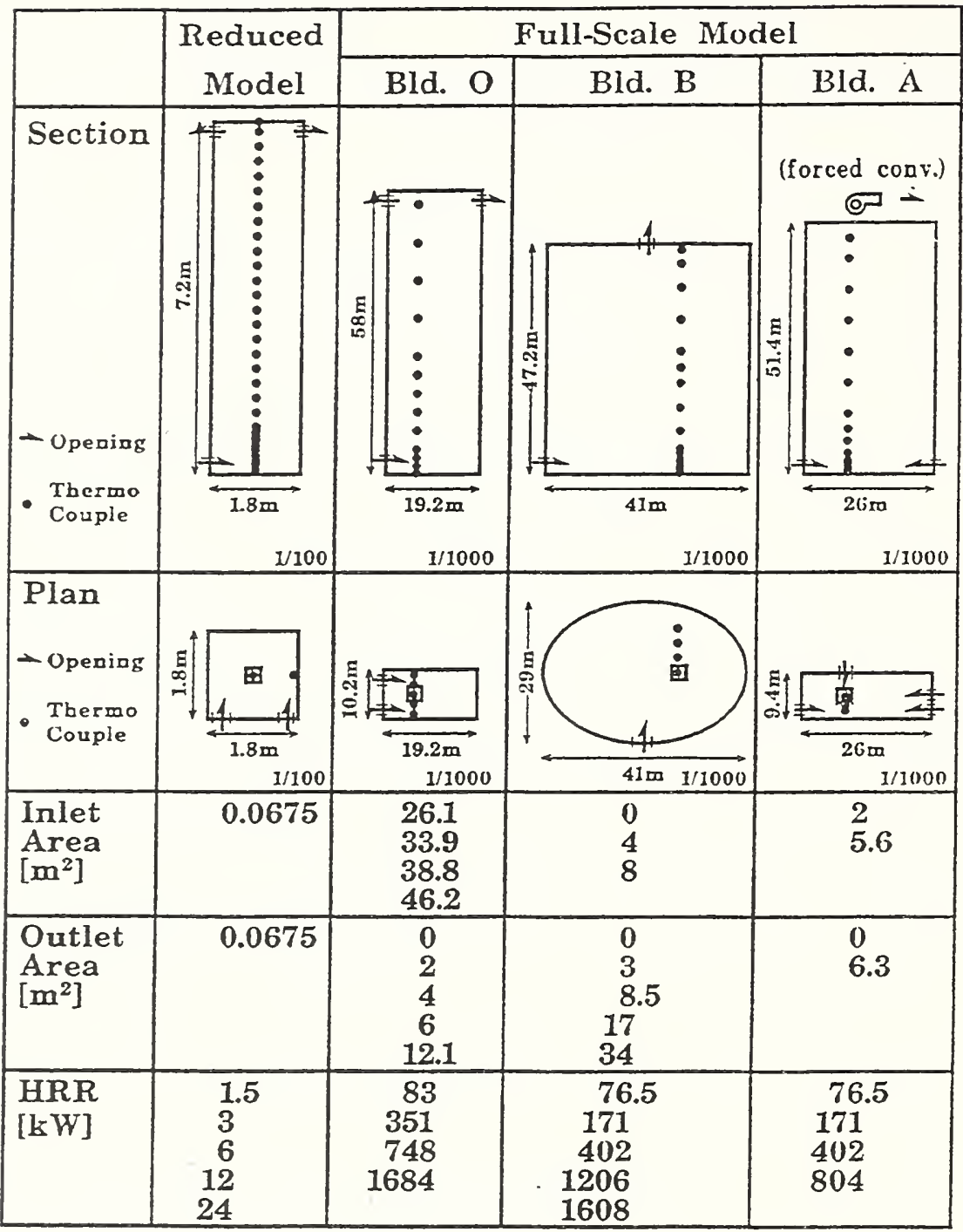

\section{2) Reduced Atrium}

Tests were conducted using the reduced model, $1.8 \mathrm{~m} \times 1.8 \mathrm{~m}$ and $7.2 \mathrm{~m}$ high, of approximately $1 / 10$ scale as reported $[10,11]$. The value for $\boldsymbol{h}_{\mathfrak{c}}$ of the surrounding walls of the model was ca. $0.0594 \mathrm{~kW} / \mathrm{m}^{2} \cdot \mathrm{K}$. The openings for air inlets and outlets were $22.5 \mathrm{~cm}$ high and were fitted with sliding doors made of 6-mm wired glass, allowing width of the openings to be adjusted. The width of these openings was set at a standard $30 \mathrm{~cm}$, making the area of each opening 1/50 the floor area obeying the Japanese Construction Act. The fire source, a propane gas diffusion burner of $20 \mathrm{~cm} \times 20 \mathrm{~cm}$, was set at the center of the floor.

3) Methods of measuring temperature and air flow velocity

Temperatures in the space were measured simultaneously by thermocouples. Velocity of air flow at the air inlets was measured by anemometers. Representative temperature and air flow velocity are also shown in Table 1. 
3.2 Observation and Validity of the Proposal Model

(a) Excess temperature and upward velocity

Figure 2 shows the excess temperatures along the ascending trajectory or along almost the center line of the flow normalized by $Q^{2 / 5}$ which were obtained both in the full and reduced scale tests. Excess temperatures in the full scale and reduced atrium models showed lower than those of open filed, and which may be resulted from the direct striking or active air entrainment into flame zone which was induced through the inlet opening(s). The temperature decreasing modes for vertical direction is similar to those expected from a free flame/plume produced from a diffusion burner.

Figure 3 indicates the representative upward velocity normalized by $\mathrm{Q}^{1 / 5} \quad$ against height corrected by $Q^{2 / 5}$. Upward velocity in flame and intermittent flame zones showed great fluctuations but lower than ones expected for a free plume. In the upper part of the plume, we could get the apparent upward velocity in the main trajectory from the trace of a balloon and which showed

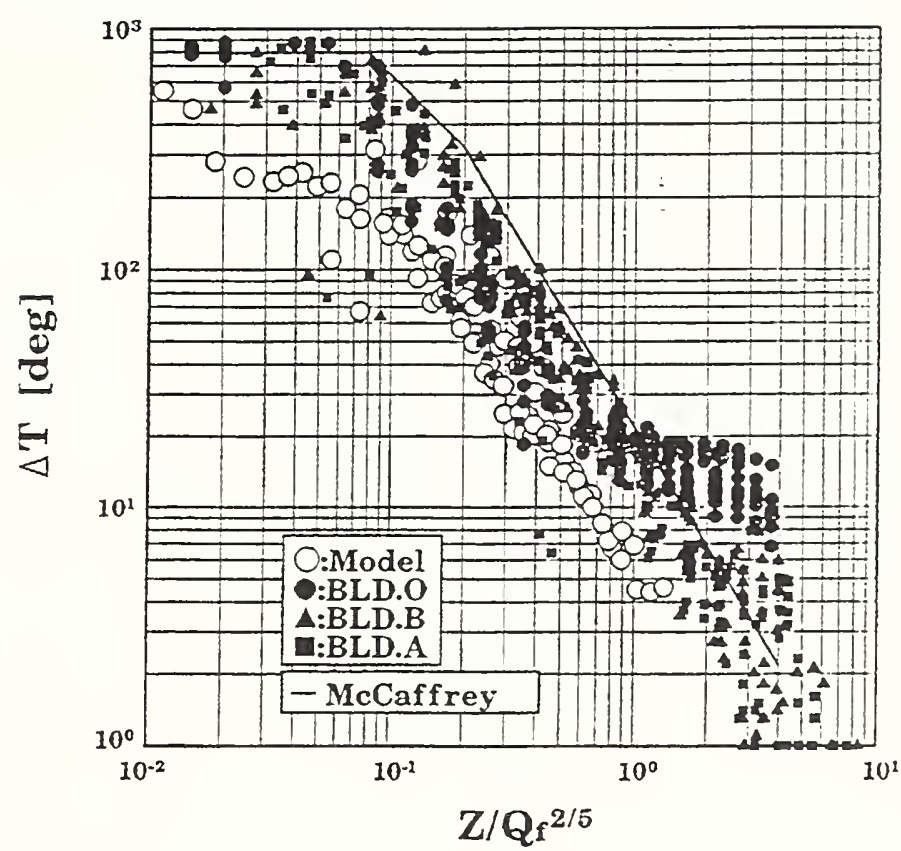

Figure 2 Excess temperature distribution for vertical direction along the ascending trajectory (almost the center line). Verical height is normalized by $\mathrm{Q}^{2 / 5}$.

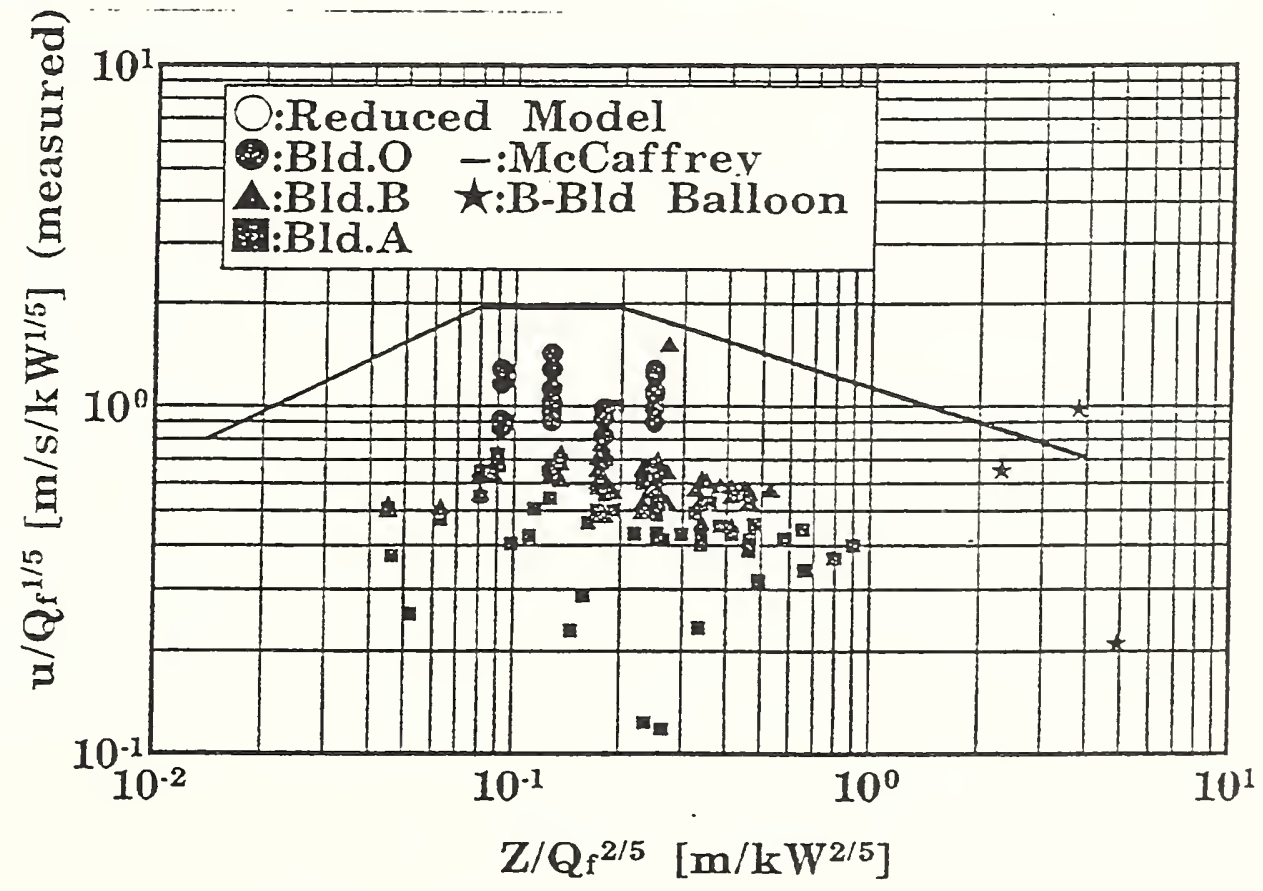

Figure 3 Upward velocity normalized by $Q^{1 / 5}$ are plotted against the verical height is normalized by $Q^{2 / 5}$. 
almost on a line estimated by McCaffrey's paper. [12] It is not so clearly obtained from our data that the decreasing modes of upward velocity for vertical direction shows the same mode as McCaffrey reported [12].

(b) Comparison between Measured and Estimated Values

Figure 4 indicates the relationship between $\mathrm{Fr}_{\mathrm{d}}{ }^{1 / 2}$ determined from the measured air flow velocity and $\mathrm{C}^{1 / 2} \mathrm{Q}_{\mathrm{fd}}{ }^{1 / 3}$ designed from a fire size and space factor of the atrium. Figure 5 illustrates the relationship between measured temperature rise $\Delta \mathrm{T} / \mathrm{Ta}$ and $\mathrm{Q}_{\mathrm{fd}}{ }^{2 / 3} / \mathrm{C}_{\mathrm{D}} \mathrm{C}^{1 / 2}$ under naturally and forced ventilation conditions. The results are arranged in line with the description of the model proposed here. These figures show that all values gained by actual measurement are smaller than theoretical values calculated on the assumption of the adia-

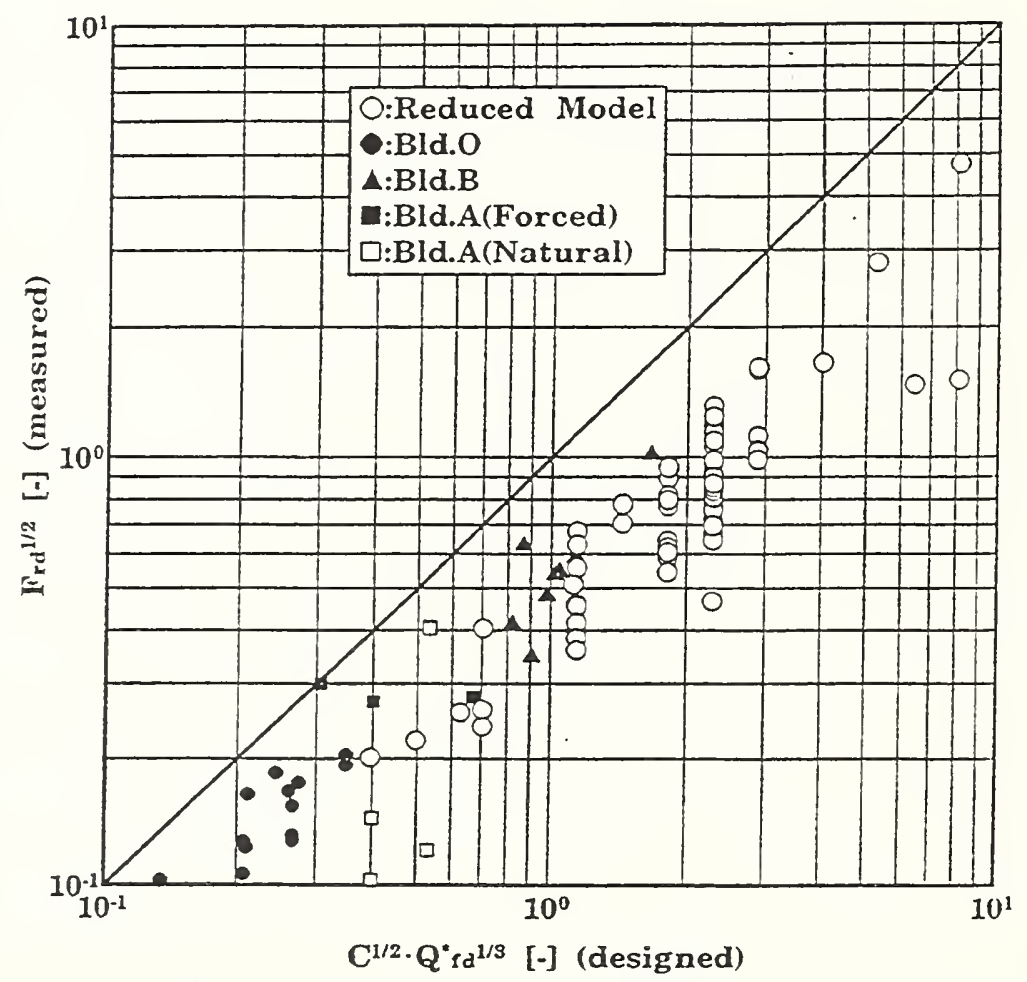

Figure 4 Relationship between Froude number measured as a function of dimensionless heat release rate, Q⿱巳一d ${ }^{*}$, corrected by space factor C. batic condition of $\mathrm{Y}^{*}=1$. The curves of measured values are clustered on the same line and which is parallel to those of theoretical values.

Figure 5 illustrates also the relationship between measured and predicted values of $\Delta \mathrm{T} / \mathrm{Ta}$ conducted in mechanically ventilated full scale atrium space. This figure indicates that, as was found for naturally ventilated space, measured values are consistently smaller than values produced by the model proposed here because of heat loss through the surrounding walls. The results obtained from the tests, shown in Figures 4 and 5 , are smaller than those generated by the model under an assumed condition of thermal insulation that the difference between them cannot be neglected. It is observed, however, that the results of each test reveal regular behavior according to the heat loss characteristic of the atrium used in the test. Therefore it can reasonably be said that when the model is provided with heat loss based on the material used for walls, it can be effectively used to predict with practical accuracy the temperature rise in a space and air flow velocity at inlets, if the space is naturally ventilated. As for application of the model to a mechanically ventilated space, the prediction formula induced by the same theoretical steps as were used to create the prediction formula for a naturally ventilated space. 


\section{Conclusion}

The major points of interest in this study are summarized below.

a) The model presented in this report, which consists of a formula made up of dimensionless heat release rate and the Froude number of the openings, both of which use the openings in the space as their reference point, is able to represent the smoke management performance of an atrium space in the stationary state with heat loss through surrounding walls taken into account, regardless of ventilation system. A distinguishing feature of this allinclusive model is that it evaluates the performance of the space not just with the conventional dimensionless heat release rate or other terms which take the fire source as their reference point, but with terms which take openings in the space as their reference points and, therefore, represent well the characteristics of the space as a whole.

b) This study verified the validity of the model presented here by comparing values produced by the formula to the results of laboratory tests using a tall and narrow full scale and reduced scale atrium. No inconsistencies between the results produced using the formula and knowledge previously reported or newly discovered were revealed.

c) Heat loss through surrounding walls cannot be neglected when predicting temperature rise in an atrium space for purposes of evaluating evacuation safety, which is the object of this study.

\section{References}

1) J. H. Klote, and J. A. Milke; "Design of smoke management systems", ASHRAE, 1992, pp. 107-108.

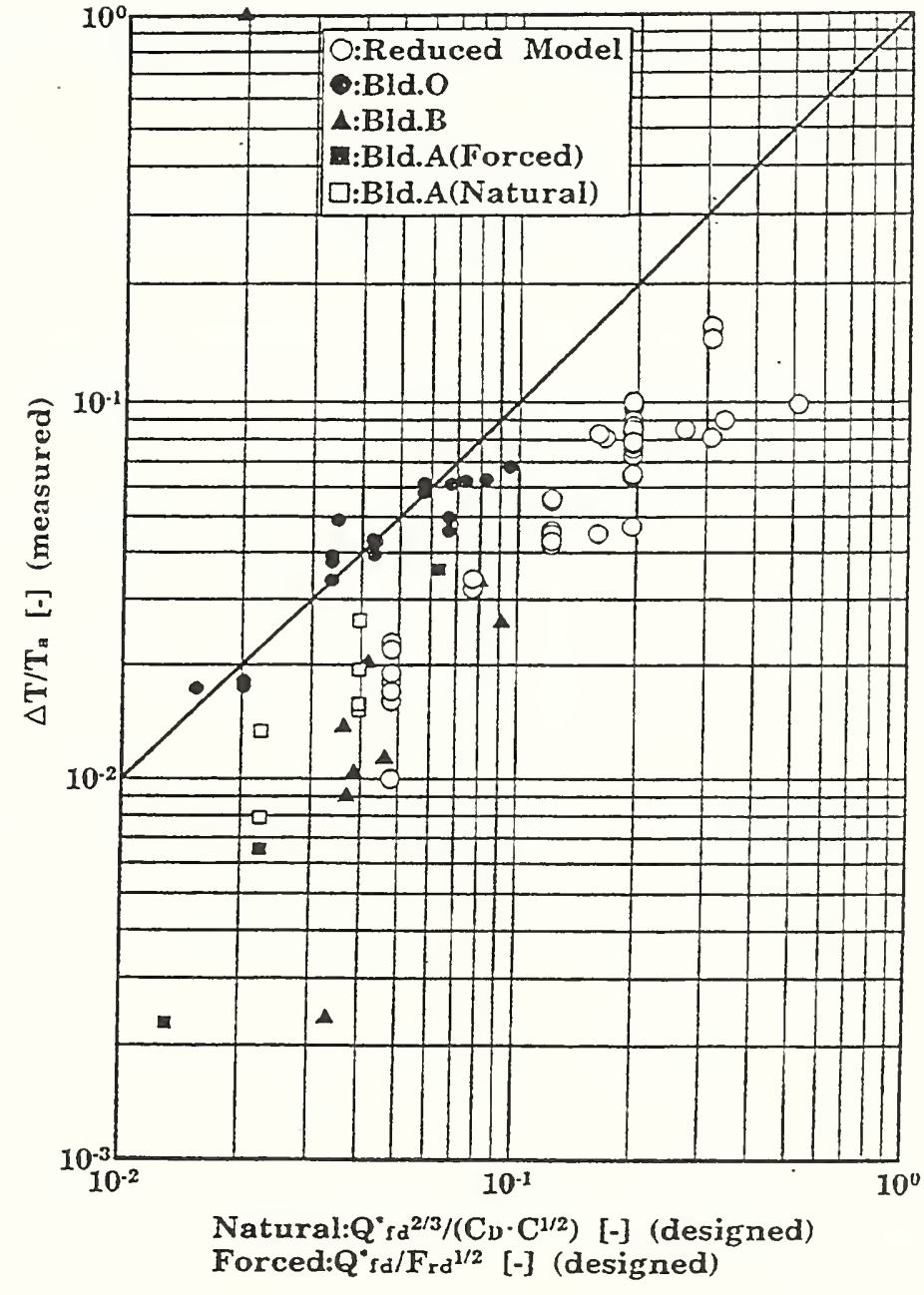

Figure 5 Comparison of measured values of $\Delta \mathrm{T} / \mathrm{Ta}$ and predicted values as $\mathrm{Q}^{*}$ fd corrected with space factor and drag coeeficient at the opening for naturally and forced convection condition which are estimated from the designed fire size and space configuration. 
2) T. Naruse, and S. Sugahara; "Some investigations on fire safety design atriums", Journal of Structural and Construction Engineering (Transactions of AIJ), No. 443, 1993. 1, pp. 147-148.

3) G. Heskestad; see [1].

4) T. Tanaka, M. Yoshida, O. Ishino, K. Kojima, and Y. Kanaya; "Smoke control in atrium space", Japan Association for Fire Science and Engineering Annual Meeting Report, 1991.5, pp. 41-60

5) J. G. Quintiere, W. J. Rinkinen, and W. W. Jones; "The effect of room openings on fire plume entrainment", Combustion and technology, Vol. 26, 19831, pp. 193-201.

6) T. Z. Harmathy; "Ventilation of fully-developed compartment fire", Combustion and flame, 37, 1980, pp. 25-39.

7) K. L. Foote; "Data report of the 1986 LLNL enclosure fire tests", UCID-21236, Lawrence Livermore National Laboratory, 1987

8) K. Kojima, S. Hashimoto, and K. Okajima; "Study on smoke control method (Influence of opening condition for smoke movement)", Japan Association for Fire Science and Engineering Annual Meeting Report, 1993.5, pp. 90-93.

9) E. Yanai, T. Yamada, A. Sekizawa, Y. Watanabe, S. Miyazaki, and N. Saito; "Experimental study on tunnel fire behavior using reduced scale mode", Japan Association for Fire Science and Engineering Annual Meeting Report, 1993. 5, pp. 66-69.

10) H. Satoh, O. Sugawa, and H.Kurioka, "Modeling on Temperature and Ventilation for Fire in a Tall and Narrow Atrium", J. Archit., Plann. Envim. Eng., AIJ, No.463, 1-10, 1994, (in Japanease)

11) H. Satoh, O. Sugawa, and H.Kurioka, "Plume Behavior in a Confined Tall and Narrow Space - as One of Sub-Models of Plume for an Atrium ", Proc. 4th Int. Symp. on Fire Safety Science, pp.551-562, Ottawa, (1994)

12) R. J. McCaffrey, and J. G. Quintiere, and M. F. Harkleroad; "Estimating room temperatures and the likelihood of flashover using fire data correlation's", Fire Technology, $17,2,1981$.

13) K. L. Foote, P. J. Pagni, and N. J. Alvares; "Temperature correlations for forceventilated compartment fires", Fire Safety Science, Proceedings of the First International Symposium, 1985. 11, pp. 139-148.

14) S. Deal, C. Beyler; "Correlation preflashover room fire temperatures", J. of Fire Prot. Engr. 2 (2), 1990, pp. $33-48$.

15) A. Tewarson; "Generation of heat and chemical compounds in fires", The SFPE Handbook of Fire Protection Engineering, SFPE, 1988, 1-179-1-200. 


\section{Discussion}

Edward Zukoski: I have two questions. First, when the fire plume first starts, it won't reach the walls until it goes up a very long way, even with a 10 to 1 diameter to height ratio. Did you take that into account at all?

Osami Sugawa: The ratio is different from a open area plume. It is wider plume in the enclosed space.

Edward Zukoski: When air comes in at the bottom of the atrium, it might introduce some swirl. Did you see any effect of that?

Osami Sugawa: It does not appear as a swirl. 


\author{
Hiroshi Koseki \\ Fire Research Institute \\ 3-14-1 Nakahara Mitaka, Tokyo 181 Japan
}

\begin{abstract}
In order to understand properties of large tank fires and flame structure, external radiation was measured in several size pan fire experiments. In the large fires, radiation was blocked by a huge amount of smoke around the flame, and most of the radiation was emitted from flame base. In order to explain this result other flame properties, smoke emission, fiame temperature, gas velocity and gas concentration in the flame, and the burning rate were also measured. Discussion of the radiation and flame structure is presented base on these data. It is found that most combustion was completed in the low part of flame, and gas flow in the radial direction in the upper part of flame played an important role in emitting smoke.
\end{abstract}

\title{
1. INTRODUCTION
}

In fire fighting and fire safety design of oil tanks, it is very important to understand radiation properties of large tank fires, and it is well known that external radiation is blocked by huge amount of smoke produced around the flame in large tank fires. This is known as the 'smoke blockage effect'. To clarify this phenomenon, external radiation has been measured during FRI large scale pool fire experiments at FRI [for example, 1,2,3,4]. Other flame properties, flame temperature, and burning rate were also measured to understand flame structure by FRI, NIST and others [For example, 9,10]. Currently smoke emission has been measured by Evans, Walton and Mulholland at NIST $[5,6,7,8]$. With these smoke data global study on radiation properties and flame structure of large tank fires have been performed.

\section{EXPERIMENTAL}

A series of experiments were conducted through collaboration between BFRL of NIST, USA and FRI, Japan including a $15 \mathrm{~m}$ square pan fire test using crude oil at the test facilities of the US Coast Guard in the Mobile, A.L. Table 1 is a listing of experiments using crude oil, that is, pan diameter, fuel and test facilities. Most tests were done using the NIST large calorimeter and the FRI large test facility. Figure 1 shows a schematic of the $2.7 \mathrm{~m}$ square pan test, which was done inside the FRI test facilities. For getting local radiant emittance of the crude oil flame, an IR-camera, Nippon Avionics TVS-2000ST was usec. Its specifications are shown in Table 2. The steps in the processing of the IR-camera data reduction are shown in Figure 2. Temperature data obtained with the IR-camera were converted into radiation information by Stefan-Boltzmann's law using a Black-body calibrator. In order to obtain local radiant emittance of flames, slit-attached radiometers were used in the heptane fire test. Using slit-attached radiometers, we can divide the flame into four or five parts vertically at the maximum, the IR-camera gave 10 to 30 slices of the flame easily. Other measurements relating burning rate, gas velocity, temperature, gas concentration were also performed in the tests except for the $15 \mathrm{~m}$ square pan test. Details of measurements are shown previous papers [1,2]. In the $15 \mathrm{~m}$ square pan fire test, the burning rate was measured. In the tests crude oil, popular light crude oil, Arabianlight crude oil, Murban crude oil 
and Louisiana crude oil were used.

\section{RESULTS AND DISCUSSION}

In order to obtain scale dependency on pan diameter, previous data $[1,2,3]$ and FRI large test data [4] were also added into the discussion of the measurements.

\subsection{Total irradiance}

Total irradiance from the whole flame to a target far from flame was measured with wide-angle radiometers and an IR-camera. Figure $3^{i}$ shows difference between irradiances at $L / D=7.1$ measured by both instruments in the $15 \mathrm{~m}$ square pan fire test. Here $\mathrm{L}$ is $a$. distance between flame axis and a target. $D$ is an effective pan diameter, the diameter of circular which the same area as the pool fire as vessel, here $D=17.2 \mathrm{~m}$. In the first few minutes from ignition, both data are in very good agreement, but during the steady state burning the difference was a maximum of about $30 \%$. The difference maybe due to differences in the field of view and the time response of the instruments.

Figure 4 shows that irradiance at $I / D=5$ from various size heptane, kerosene and crude oil fires. Data from Refs. $[1,2,3,4,11]$ are added. Around $D<3 \mathrm{~m}$, average irradiance increased with increasing pan diameter due to emissivity increasing. At $D \fallingdotseq 3 \mathrm{~m}$, emissivity of the flame equals to 1 , and radiant emittance was the maximum, then decreased with pan diameter. This decrease maybe due to so-called smoke blockage effect. Smoke were seen around the flame.

\subsection{Local radiant emittance}

The local radiant emittance of heptane and crude oil fires were measured by wide-angle radiometers and IR-camera. The data are shown in Figure 5 (crude oil) and Figure 6 (heptane). Here the horizontal axis is dimensionless height, $\mathrm{H} / \mathrm{H}$.. Where $\mathrm{H}$ is a height from fuel surface and $\mathrm{H}$ : is the average flame height obtained by IR-camera or video camera.

The $2.7 \mathrm{~m}$ square crude oil pan fire gave the maximum radiant emittance. The peak of irradiance was at $\mathrm{H} / \mathrm{H}_{0}=0.1 \sim 0.2$. Most of the radiation was emitted from flame base. For example about $75 \%$ of total radiation was emitted from the flame base $(0<\mathrm{H} / \mathrm{H},<0.4)$ in the crude oil $15 \mathrm{~m}$ pan fire. The maximum radiant emittance was only $35 \mathrm{~kW} / \mathrm{m}^{2}$ at $\mathrm{H} / \mathrm{H}_{2}=0.08$ and about $120 \mathrm{~kW} / \mathrm{m}^{2}$ at $\mathrm{H} / \mathrm{H}_{\varepsilon}=0.12$ in $2.7 \mathrm{~m}$ square pan fire. A kind of fireball appeared through the black smoke at about $\mathrm{H} / \mathrm{H}_{e}=0.8$, regularly, but it did not make a large contribution for average external radiation.

In the heptane fire, the tendency was similar to crude oil fire. The highest local radiant emittance was $220 \mathrm{~kW} / \mathrm{m}^{2}$ at $\mathrm{H} / \mathrm{H}_{2}=0.3$ in the $2.7 \mathrm{~m}$ square pan fire. The height of peak local radiant emittance was slightly higher than that of crude oil fire, but most radiation was emitted from the continuous flame zone.

3.3 Smoke emission, pulsation of flame height, isotherms, gas and air flow inside the flame, and gas concentration in the flame

In order to understand the above radiation results, other data which were obtained during the experimentals will be discussed.

(1) Smoke emission

It is believed that smoke emission from flame blocked external radiation. Therefore the relationship between smoke emission and radiation is important 
and will be discussed here. As a smoke emission parameter, the dimensionless smoke produce rate, smoke yield (mass smoke production / mass burning rate) as defined by Mulholland et al. [6], was adopted here. Scale dependency of smoke emission has been reported by Mulholland et al. [6,7,8]. External radiation and smoke yield increased with pan diameter until $D=3 \mathrm{~m}$. Over $D>3 \mathrm{~m}$, smoke yield may not increase with increasing pan diameter but irradiance at $L / D=5$ decreased drastically (Figure 3). Therefore smoke yield does not seem to directly to radiation decrease in the large scale fires.

Mulholland et al. [8] also measured smoke particle size and found that the primary particle size changed with pan diameter. Primary particle size is related to the resident time of smoke in the flame. When smoke is emitted at a lower height than the flame tip, the resident time would be shorter and the primary particle size would be smaller, so its distribution would be larger. In a large fire, primary particle size and diameter distribution became larger. The results of average diameter and diameter distribution changed from $1 \mathrm{~m}$ pan fire to $2.7 \mathrm{~m}$ square pan fire. That is, smoke production mode might be change at about $D=2 \mathrm{~m}$. Visual observations are that, most smoke was seen above flame tip in $1 \mathrm{~m}$ pan fire and in the upper part of flame in $2.7 \mathrm{~m}$ square pan fire. Therefore in fires larger than about $2 \mathrm{~m}$ diameter, smoke is emitted from flame tip and upper part of flame is present around most of the flame surface, and blocks radiation emissions.

(2) Pulsation of flame height

Average flame height was obtained using a video camera, $35 \mathrm{~mm}$ camera or IR-camera. The flame height changed regularly. Figure 7 shows the pulsation of flame height obtained with the IR-camera in the heptane $0.60 \mathrm{~m}$ pan fire. Following McCaffrey's definition [9], the flame can be divided into the continuous zone and the intermittent zone. The continuous zone height $\mathrm{H}_{\mathrm{f}} \mathrm{cos}=\mathrm{s}$; is about $65 \%$ of average flame height $\mathrm{H}_{\rho}$. In large fire, $\mathrm{H}_{f}\left(\mathrm{cos}_{0}=\right.$.) is $70 \sim 90 \%$ of $\mathrm{H}_{\mathrm{f}}$, but it is difficult to determine this ratio due to smoke existence around upper part of flame. Calculation by Hesketad's equation gave good agreement with the test result for the intermittent flame height [10] which was $20 \sim 40 \%$ larger than average flame height, H. It is easy to guess from flame pulsation that radiation from continuous flame zone is much larger than intermittent flame zone, which was the result of the measurement of local radiant emittance.

\section{(3) Isotherms}

Figure 8 shows the temperature inside the $6 \mathrm{~m}$ heptane flame, which was obtained using about 60 thermocouples. It is found that there is a high temperature ridge from the pan edge to the flame center which means air flow along this zone strongly. The highest temperature zone existed about the height of $\mathrm{H} / \mathrm{H}_{0}=0.3$ at the fire axis. It is guessed that below this ridge there existed fuel vapor, and above this ridge there existed burned gases. Figure 9 shows the temperature distribution along the flame axis for several size flames. Flame temperature increased with pan diameter. Huge pan fire data implied this results continuation to $50 \mathrm{~m}$ pan fire test, that is, $1380^{\circ} \mathrm{C}$ in $30 \mathrm{~m}$ and $50 \mathrm{~m}$ kerosene fire [11]. Therefore we understand temperature inside the large flame is higher than small flame, but external radiation is smaller due to the smoke around the flame larger than $3 \mathrm{~m}$ in diameter.

(4) Gas flow and air flow

Horizontal gas and air flows were obtained. Air entrainment from the flame base to flame tip was more than five times as much as the calculation from the fuel burning data $[1,2]$.

Figure 10 shows the horizontal flow above the $0.3 \mathrm{~m}$ heptane pan fire. It was measured along the pan edge vertically using bi-directional tube. 
$0<\mathrm{H} / \mathrm{H}_{0}<0.6$ ( $\fallingdotseq$ continuous flame zone), it is mostly steady flow, $0.6<\mathrm{H} / \mathrm{H}_{e}<1.2$ $\doteqdot$ intermittent flame zone) flow from the flame to outside were sometimes observed, and H:>1.2 ( $\fallingdotseq$ plume zone) steady flow were observed again. The horizontal flow observed at the height of $0.6<\mathrm{H}_{2} \mathrm{H}_{2}<1.20$ played an important role of smoke growth and transportation from inside the flame to the around the flame. This flow must be stronger in larger pan fire, and carries smoke to outside of flame.

(5) Gas concentration

Gas concentration along with the flame axis in several size heptane fires were measured. In order to understand combustion in the flame, dimensionless $\mathrm{CO}_{2}, \mathrm{CO}_{2} *$, was defined $[1,2]$. That is, $\mathrm{CO}_{2} *=\mathrm{C}_{\mathrm{co}} /\left(\mathrm{CcO}_{2}+\mathrm{C}_{\mathrm{co}}+\mathrm{C}_{\mathrm{rz}}+\sum \mathrm{C}_{\min }\right)$. When $\mathrm{CO}_{2} *=0.681$ in heptane fire, the combustion must be completed. Figure 11 shows the results using heptane. About $\mathrm{H} / \mathrm{H}_{2}=0.3, \mathrm{CO}_{2} *$ was equal to $0.55 \sim 0.60$, therefore most combustion is finished and most of the heat of combustion was produced below the height of $\mathrm{H} / \mathrm{H}_{2}=0.3$. Therefore the lower part of the continuous flame zone, $\mathrm{H} / \mathrm{H}: \leqq 0.3$, is the most important region for combustion and radiative heat release.

\subsection{Flame structure}

With the above data, it is understood that most air flow penetrate into the flame base and most combustion is finished and most of the heat of combustion is produced below the height of $\mathrm{H}_{2} \mathrm{H}_{2}=0.3$. Then a part of reacted gas is emitted from intermittent flame zone, $\mathrm{H}_{\mathrm{H}}=0.6 \sim 1.2$. This flow may be stronger in larger pan fires, and may be important in the reacted gas emission. Smoke is emitted along with the reacted gas. Therefore 'Smoke blockage' was predominant in $15 \mathrm{~m}$ square pan fire though the smoke yield might not change drastically from $2.7 \mathrm{~m}$ square pan to $15 \mathrm{~m}$ square pan.

\section{CONCLUSIONS}

Total and local radiant emittances of hydrocarbon pool fires were measured. 'Smoke blockage' was explained with data of several size heptane and crude oil fires, up to a $15 \mathrm{~m}$ square pan. In the large pan fires, a huge amount of smoke existed around the flame, and only flame base, $\mathrm{H} / \mathrm{H}_{0}<0.3$, could be seen. This is the reason that most radiation is emitted from flame base of large fires. Data on gas and air flow, isotherms and gas concentration were in good agreement with radiation results. That is, most combustion was completed lower than $\mathrm{H} / \mathrm{H}_{5}=0.3$.

\section{Acknowledgements}

The author wishes to acknowledge the cooperation with Drs. David Evans and Willian D. Walton at NIST in conducting experiments in Japan and in the U.S. The largest test was conducted at the US Coast Guard Test Facility in Mobile, AI, so author also thanks the US Coast Guard for conducting experiment and their help.

\section{References}

1) Yumoto, T, and Koseki, H., FRI Report 59, p.1 (1985) (in Japanese)

2) Koseki, H. and Yumoto, T., Fire Technology 24(1) p.33 (1988)

3) Koseki, H. and Yumoto, T., Fire Safety Science 2 p.231 (1988)

4) FRI Technical Report No.8 (1976) (in Japanese)

5) Evans, D. et al, Proceeding of 15 th AMOP Technical seminar p.593 (1992)

6) Mulholland et al., Fire Safety Science 2 p.347 (1989)

7) Koseki, H. and Mulholland, Fire Technology 27(1) p.54 (1991)

8) Mulholland et al, Proceedings of 13 th UJNR Meeting (March 1996) 
9) McCaffrey, B., NBS-IR 79-1910 (1979)

10) Heskestad, G., Fire Safety Journal 5, p.103 (1983)

11) JASE, Oil Tank Fire Test (1981) (in Japanese)

Table 1 Outline of experimental using crude oil

\begin{tabular}{|l|l|l|}
\hline Pan Diameter & Place & Fuel \\
\cline { 1 - 2 } $0.4 \mathrm{~m}$ & NIST & \multirow{2}{*}{ Arabian } \\
\cline { 1 - 1 } & Nouisiana \\
\cline { 1 - 1 } & FRI & \\
\cline { 1 - 1 } & FRI & \\
\hline $15 \times 15 \mathrm{~m}$ & Mobile & Louisiana \\
\hline
\end{tabular}

Table 2 Speculations of IR-camera

\begin{tabular}{|l|l|}
\hline Type, Manufacture & $\begin{array}{l}\text { TVS 2000ST, Nippon } \\
\text { Avionics Co. Ltd. }\end{array}$ \\
\hline Detector & In-Sb \\
\hline Spectral Range & 3.0 to $5.4 \mu \mathrm{m}$ \\
\hline Focus Range & $254 \mathrm{~mm}$ to $\infty$ \\
\hline Time constant & $11 \mu \mathrm{m}$ \\
\hline Spatial resolution & $2.18 \mathrm{~m} \mathrm{rad}$ \\
\hline
\end{tabular}




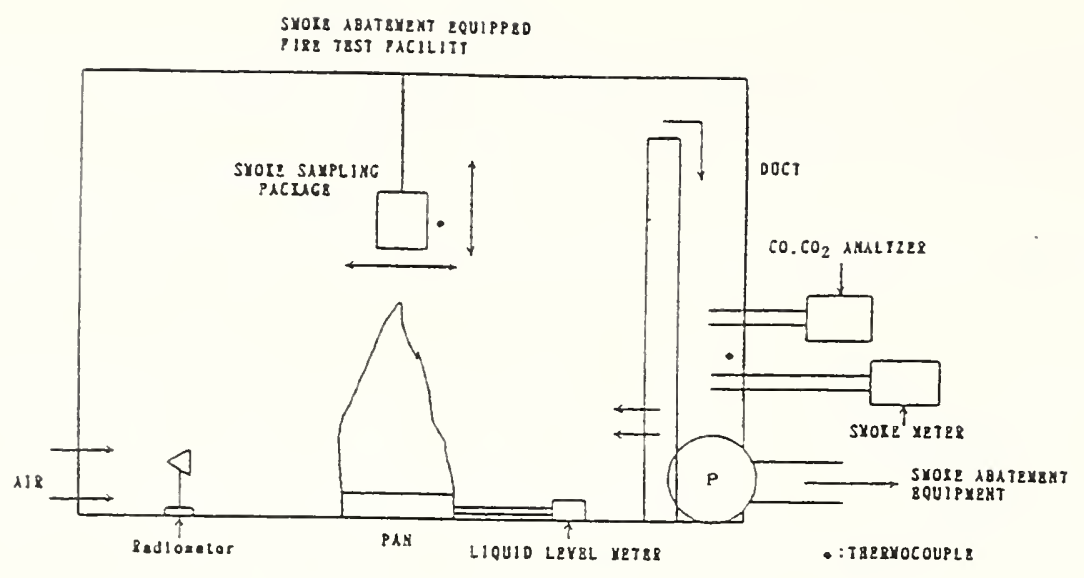

Figure 1 Schematic illustration of the experiment at FRI large test facilities maximum experiment is effective pan diameter $=3 \mathrm{~m}$

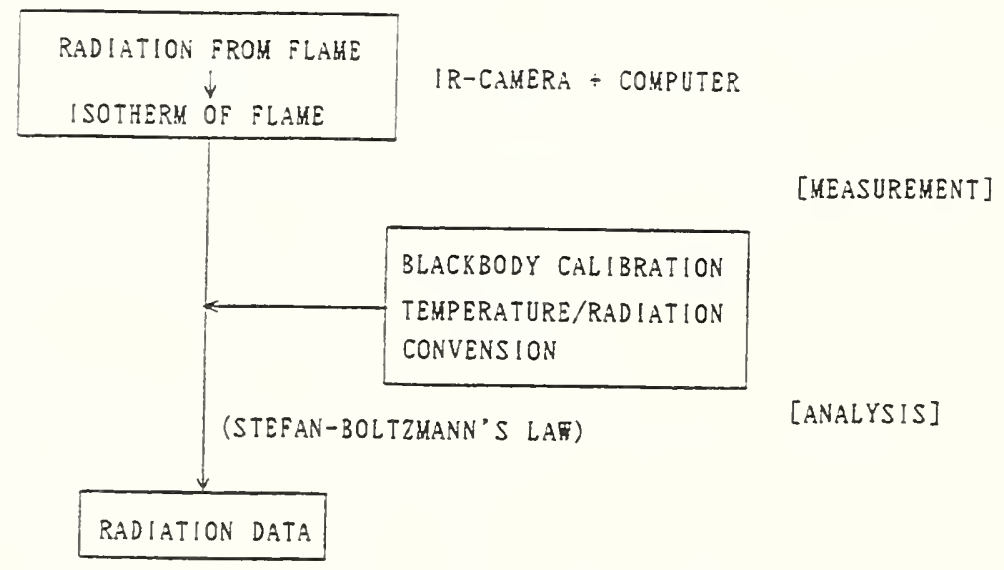

Figure 2 Process of IR-camera data reduction

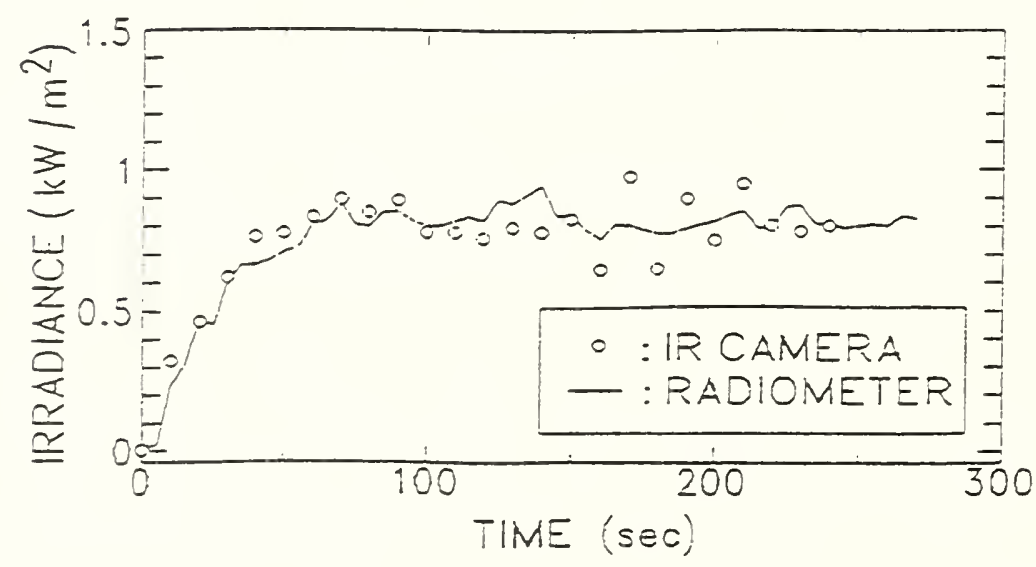

Figure 3 Comparison of time history of irradiance by IR-camera and wide angle radiometer Equipments were set at $L / D=7.1$ 


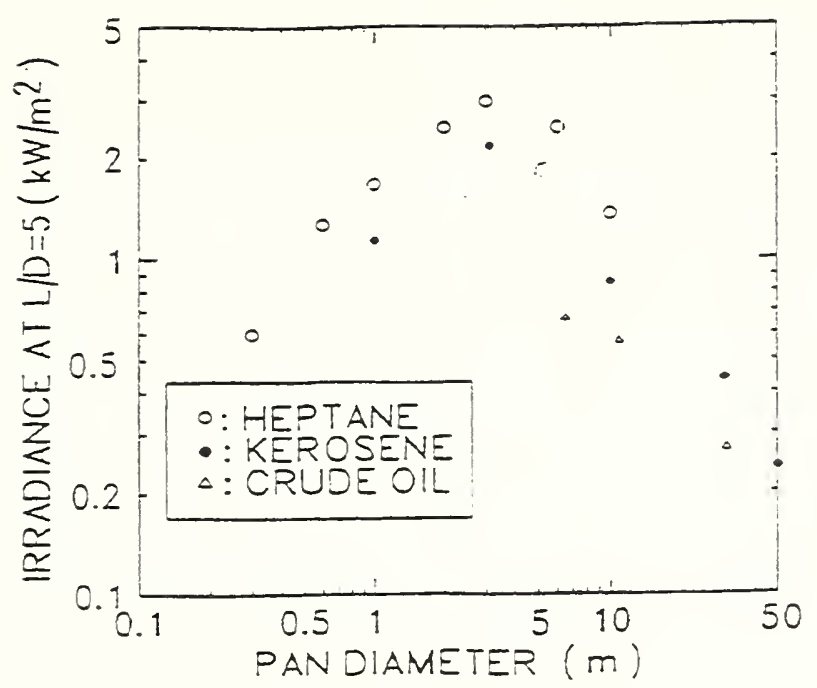

Figure 4 Scale dependency of irradiance at $L / D=5$ for heptane, kerosene and crude oil fires.

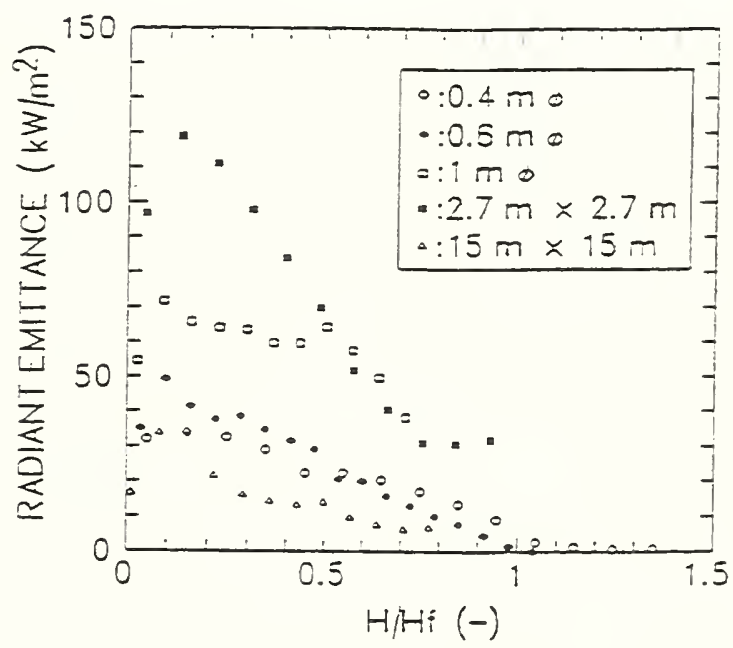

Figure 5 Local radiant emittance of several size crude oil fire

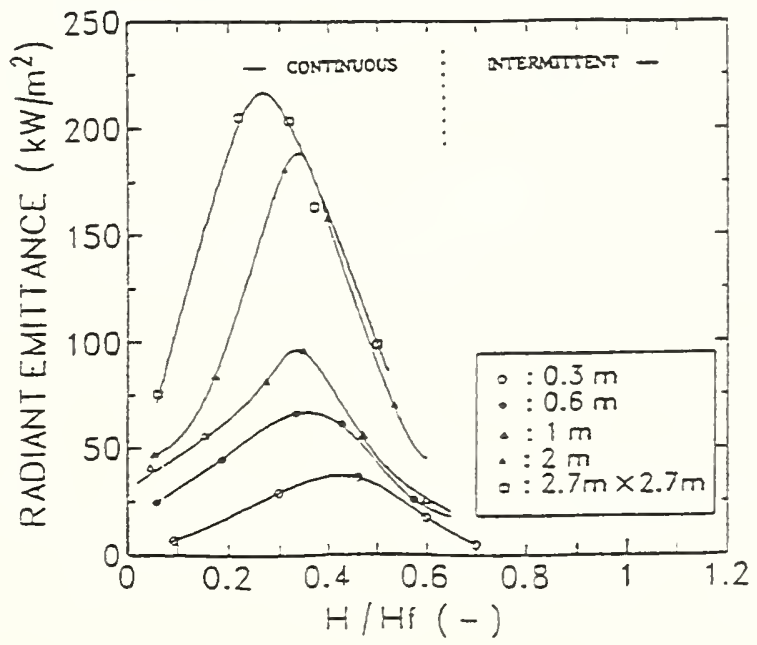

Figure 6 Local radiant emittance of several size heptane fire 


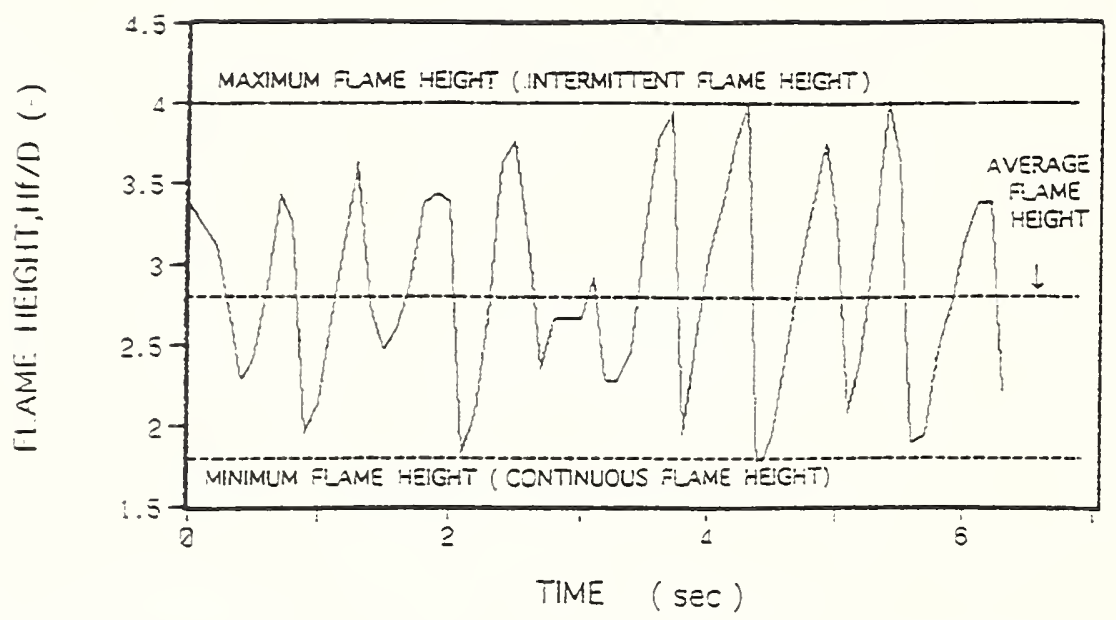

Figure 7 Pulsation of heptane $0.6 \mathrm{~m}$ flame height Flame height was decided using by IR-camera

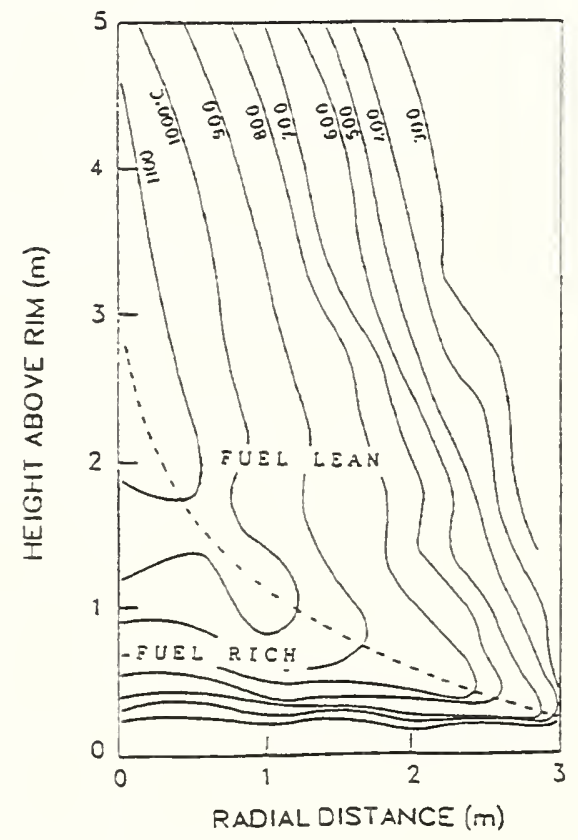

Figure 8 Isotherms of heptane $6 \mathrm{~m}$ flame Temperature was measured by about $60 \mathrm{~K}$-type thermocouples which diameter was $0.32 \mathrm{~mm}$ 


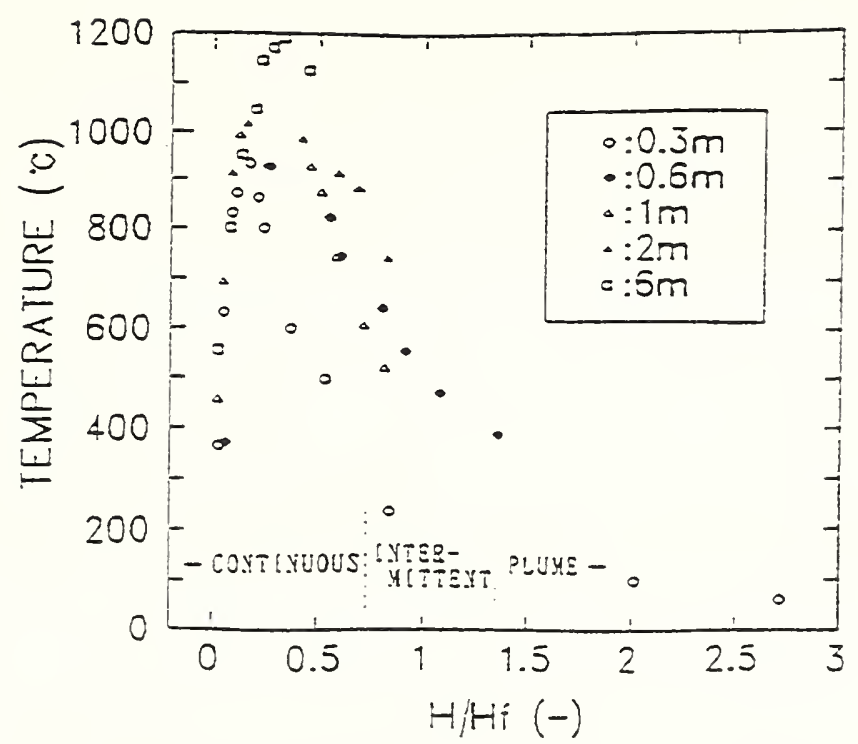

Figure 9 Temperature along several size heptane flame axis

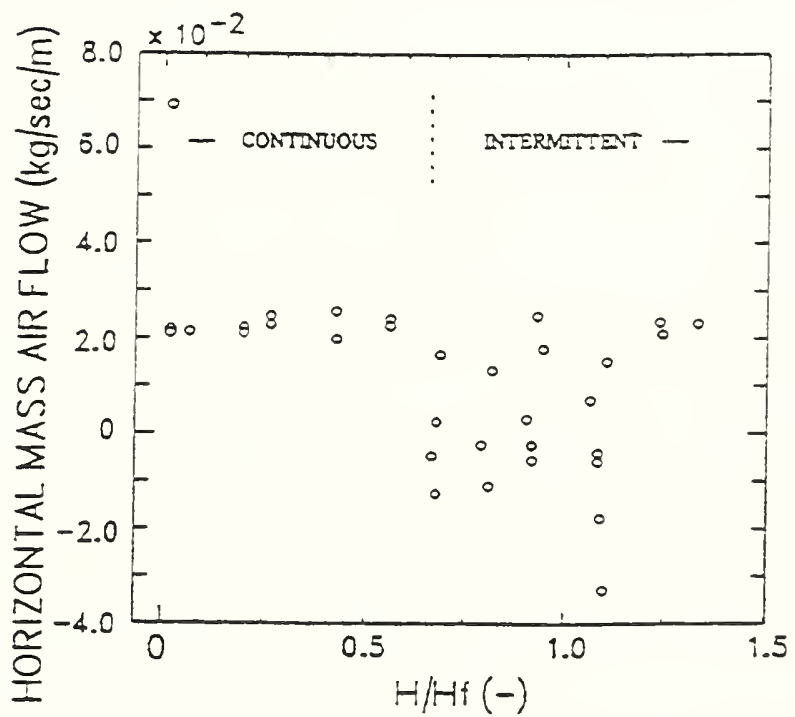

Figure 10 Horizontal mass air and gas flow in heptane $0.3 \mathrm{~m}$ fire measured by bi-directional tube

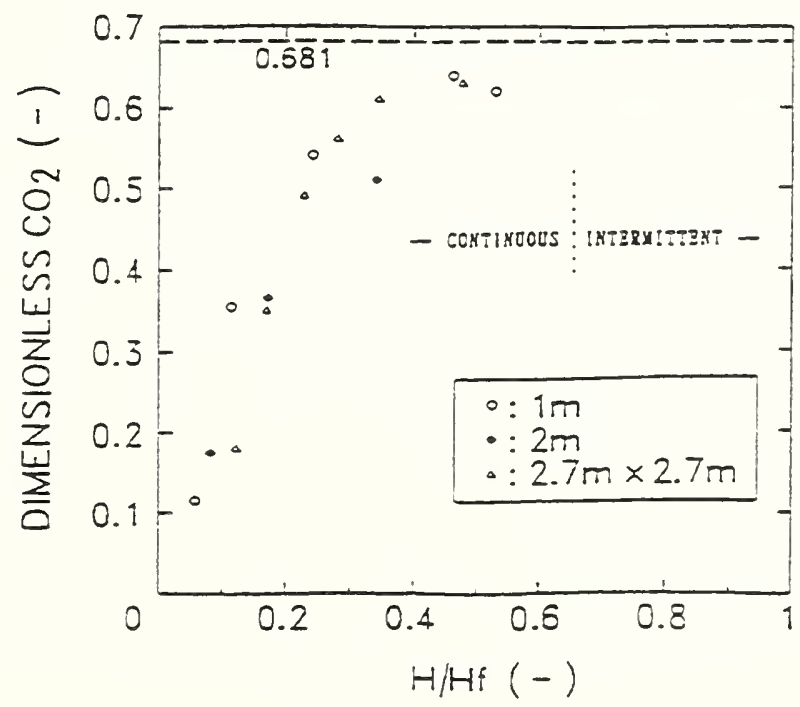

Figure 11 dimensionless $\mathrm{CO} 2 *$ along with flame axis measured with gas chromatograph 


\section{Discussion}

Walter Jones: Would it be possible in this case to extend the work that's been done for smaller scale fires?

Hiroshi Koseki: Actually, the entrainment rate for the vertical direction has already been calculated and presented in another paper.

Patrick Pagni: This was very interesting in that there was an extreme in the radiation with the diameter. Our intuition from small scale is that radiation increases as the scale increases. How did you measure the radiant fraction?

Hiroshi Koseki: In this graph, the flames were sliced into small portions and in each portion, the radiation amount was calculated based upon temperature. 


\title{
CARBON MONOXIDE FORMATION ALGORITHM
}

\author{
William M. Pitts \\ Building and Fire Research Laboratory \\ National Institute of Standards and Technology \\ Gaithersburg, MD 20899
}

\begin{abstract}
This paper discusses an engineering approach for estimating the generation rate of carbon monoxide (CO) within a room containing a fire. The algorithm incorporates four $\mathrm{CO}$ formation mechanisms which have been identified in past investigations.

\section{INTRODUCTION}

At the present time there is no generally accepted model for the formation of carbon monoxide $(\mathrm{CO})$ by enclosure fires. The need for such a model is crucial since roughly two thirds of all deaths resulting from such fires can be attributed to smoke inhalation [1],[2]. $\mathrm{CO}$ is the dominant toxicant in smoke [3]. Here we present an engineering algorithm for predicting $\mathrm{CO}$ formation in enclosure fires. It is intended to be the first step in the development of effective models for this complex process.
\end{abstract}

The experimental basis for the algorithm is a long-term effort in BFRL--the Carbon Monoxide Production and Prediction Priority Project--which has been ongoing for six years. The research plan under which this research has been performed is available [4].

The research has focused on the scenario of an intense fire within a room which is flashed over [5]. It has been shown that this scenario is responsible for the vast majority of fire deaths [3]. The algorithm which is presented is intended for this type of fire. In the following sections current approaches for $\mathrm{CO}$ estimation are discussed (2), experimental findings from a number of studies are summarized (3), the experimental findings are used as the basis of an algorithm for estimating $\mathrm{CO}$ formation within the room of fire origin (4), and a summary is provided (5).

\section{CURRENT APPROACHES FOR CO ESTIMATION}

Three basic approaches have been used in BFRL for modeling the generation of $\mathrm{CO}$ during enclosure fires. The first is that the user simply specifies the grams of $\mathrm{CO}$ generated per gram of fuel consumed. After reviewing a number of full-scale tests, George Mulholland suggested a second approach, which is referred to as the "zeroth-order approximation", which recommends that production rates of $0.002 \mathrm{~g} \mathrm{CO} / \mathrm{g}$ fuel consumed for fully ventilated fires and $0.3 \mathrm{~g} \mathrm{CO} / \mathrm{g}$ fuel consumed for underventilated fires be used [6],[7]. The third approach [3] recommends using values derived from experiments in which flame gases are quenched in a hood located above a fire burning in an open laboratory [8],[9],[10],[11]. These experiments have shown that concentrations of gases in the upper layer are correlated with the global equivalence ratio, $\phi_{\mathrm{g}}$, which is defined as the 
mass ratio of gases in the layer derived from fuel and from air normalized by the mass ratio of fuel and air required for stoichiometric burning. The existence of these correlations has been termed the global equivalence ratio (GER) concept. The correlations are fuel dependent, but have been obtained for a number of different fuel types.

\section{SUMMARY OF EXPERIMENTAL FINDINGS}

Most of the relevant research concerning $\mathrm{CO}$ formation in fires has been summarized in a NIST Monograph [12] and a subsequent review article [13]. Review of the studies which form the basis of the GER concept are included in these documents. It is shown that the GER concept is quite robust and applicable to a wide range of fuels. A slight dependence of the correlations on upper-layer temperature has been identified, but the correlations seem to achieve asymptotic values for temperatures characteristic of upper layers in actual enclosure fires. Pitts has used detailed chemical kinetic modeling to show that a temperature effect should exist, but that once all oxygen is removed from the upper layer by reaction, the gases should be effectively nonreactive until very high temperatures are reached [14],[15].

Gottuk et al. at Virginia Polytechnic Institute and State University have investigated the combustion gases generated in an enclosure fire designed to burn in a configuration similar to the hood experiments [16]. This enclosure had separate vents for air inflow and combustion gas exhaust which ensured that the fire plume in the lower layer had equal access to air from all directions and that the lower-layer air and combustion gases did not directly mix significantly. The results of this study indicated that the GER concept provided good predictions for concentrations of combustion gases, including $\mathrm{CO}$, when the temperature effect was taken into account.

Bryner et al. studied CO formation in a reduced-scale enclosure (RSE, 2/5-scale model of a standard ISO/ASTM room [17],[18]) containing a single doorway [12],[13],[19],[20]. The fire was formed by a single natural gas burner centered in the room. Upper-layer concentrations of $\mathrm{CO}, \mathrm{O}_{2}$, and $\mathrm{CO}_{2}$ as well as vertical temperature profiles were measured for locations in the front and rear of the RSE.

The results for the RSE showed that very low concentrations of $\mathrm{CO}$ were generated when the fire was overventilated, i.e., $\phi_{\mathrm{g}}<1$, but that the concentrations of $\mathrm{CO}$ increased rapidly once $\phi_{\mathrm{g}}$ became greater than one. Concentrations of combustion gases observed in the rear of the enclosure were very similar to those reported by Toner [21] in hood experiments using natural gas as fuel. For large $\phi_{\mathrm{g}}$, the concentrations of $\mathrm{CO}$ were roughly $2 \%$. Unfortunately, concentrations in the front of the layer $(\approx 3 \%)$ were observed to be roughly $50 \%$ higher than predicted by the GER concept. Strong temperature nonuniformities were also observed in the upper layer with higher temperatures measured in the front than the rear.

The degree of nonuniformity observed in the temperature and concentration fields of the upper layer in the RSE for underventilated burning was surprising. Analysis of all of the available data indicated that the observations were the result of air entering and rapidly 
mixing in the front of the upper layer directly from the lower layer instead of being entrained into the fire plume [12],[13],[19],[20]. Such direct entrainment into an upper layer is not believed to occur in the hood experiments or the VPISU enclosure fires. Air entrained directly into a rich upper layer is expected to react with excess fuel to produce $\mathrm{CO}$ in preference to $\mathrm{CO}_{2}$, thus explaining the higher $\mathrm{CO}$ concentrations observed in the front of the upper layer [12]-[15]. The heat release as a result of the additional reaction provides a partial explanation for the higher temperatures observed in the front of the enclosure.

The direct entrainment and mixing of air into a rich, high-temperature upper layer of an enclosure fire is a mechanism for CO formation which is not included in the GER concept. The rich combustion gases created in a hood experiment are only the result of the quenching of reaction when a combusting buoyant plume enters a vitiated upper layer. It is therefore to be expected that when direct entrainment of air into the upper layer occurs, the GER concept will fail to predict upper-layer concentrations.

Much higher concentrations of CO than predicted by the GER concept have been observed in many fires involving wood as fuel. A good example is the study of Levine and Nelson who experimentally reconstructed a fire in Sharon, PA where CO was implicated in several fire deaths [22]. This fire took place in an area with a very heavy fuel loading of wood. During the fire simulation, $\mathrm{CO}$ concentrations as high as $8 \%$ were observed. This value should be contrasted with a value of roughly $3 \%$ which was observed in the hood experiment of Beyler [9] using wood as fuel, as well as the enclosure experiments at VPISU designed to mimic the hood experiments [16]. This is another case where the GER concept fails to predict observed CO concentrations.

Pitts et al. hypothesized that the high concentrations of CO observed in the Sharon fire test resulted from the direct formation of $\mathrm{CO}$ by wood undergoing high temperature pyrolysis in a highly vitiated environment [12],[23]. In order to test this hypothesis, a series of experiments were run in the RSE for which the ceiling and upper walls ( $36 \mathrm{~cm}$ from ceiling) were lined with $6.3 \mathrm{~mm}$ thick plywood. Natural gas fires having various heat-release rates (HRRs) were then burned and concentrations of $\mathrm{CO}$ and other combustion gases were monitored in the upper layer. For these experiments the concentrations of $\mathrm{CO}$ increased rapidly, reaching levels as high as $14 \%$ in the rear of the upper layer and $6 \%$ in the front. These results provide direct evidence that pyrolysis of wood in a high temperature, anaerobic environment does generate significant concentrations of $\mathrm{CO}$.

By using measured HRRs for the fires and an estimate for the heat release per gram of wood pyrolyzed, it was possible to estimate the mass-loss rate of the pyrolyzed wood per unit area of exposed surface. The mass-loss rate increased monotonically with time during the fire and reached a maximum on the order of $10 \mathrm{gs}^{-1} \mathrm{~m}^{-2}$ just before the wood collapsed. Temperature measurements in the enclosure demonstrated that the overall pyrolysis process is endothermic.

The wood-lined-enclosure results demonstrate that a third mechanism for $\mathrm{CO}$ formation in enclosure fires, namely pyrolysis of wood in a high-temperature anaerobic environment, 
exists. The GER concept alone will not be able to make accurate predictions of CO formation when significant anaerobic pyrolysis of wood is occurring.

Much of the discussion thus far has been based on measurements recorded in reduced-scale enclosures. In order to test whether or not these results are valid for real-scale fires, a very recent series of fires has been burned at NIST in a standard size room having dimensions of $2.44 \mathrm{~m} \times 2.44 \mathrm{~m} \times 3.05 \mathrm{~m}$. A natural gas burner was again used as the fuel source. The following discussion is based on preliminary analysis of the results for these burns performed in the late spring of 1994.

The experimental results indicate that the full-scale fires became underventilated for a flow of natural gas corresponding to a HRR of roughly $1.5 \mathrm{MW}$. This value is in good agreement with a prediction based on the corresponding value for the RSE and the expected ventilation based on the known doorway sizes for the two enclosures.

Smaller HRR fires (i.e., 500 and $900 \mathrm{~kW}$ ) should be fully ventilated. Measured upper-layer concentrations are consistent with this expectation. Significant oxygen concentrations were observed, carbon dioxide concentrations were relatively low, and $\mathrm{CO}$ concentrations were very close to zero.

Fires having HRRs corresponding to $\phi_{\mathrm{g}} \approx 1$ (i.e., 1.1 and $1.3 \mathrm{MW}$ ) yielded results which were somewhat different than observed in the RSE. $\mathrm{CO}_{2}$ concentrations in the rear were on the order of $10 \%$ to $12 \%$ while values of $8.0 \%$ to $10.0 \%$ were observed in the front. These values suggest that the gases in the rear of the enclosure have a local equivalence ratio $\left(\phi_{\ell}\right)$ which is close to 1 while the front is somewhat lower. CO concentrations behaved in a manner consistent with this conclusion. In the rear of the enclosure values of $1.5 \%$ $2.5 \%$ were observed, while values in the front were close to zero as expected for fully ventilated burning. As would be expected, oxygen concentrations of $2 \%-3 \%$ were observed in the front of the enclosure while values in the rear were very close to zero. These results demonstrate clearly that values of $\phi_{\ell}$ are significantly lower in the front of the enclosure than in the rear. A similar conclusion was reached for the RSE [12],[13],[19],[20], but the experimental observations were not as well defined as in the full-scale tests.

$\mathrm{CO}$ concentrations observed for fires having HRRs sufficient to ensure underventilated burning with $\phi_{\mathrm{g}}>1.5$ were very different than observed in the RSE. Immediately following the start of the fire, $\mathrm{CO}$ concentrations increased to roughly $3 \%$ in the front and rear of the upper layer. For the remainder of the fire period the $\mathrm{CO}$ concentrations continued to increase, approaching values of $6 \%-7 \%$ for both locations by the end of the burn period. $\mathrm{CO}$ concentrations in the full-scale enclosure reached much higher levels than observed in the RSE, and differences between the front and rear locations were much less obvious than observed for the RSE.

For both enclosures, upper-layer temperatures were observed to increase with time. However, higher upper-layer temperatures were observed in the full-scale tests than in the RSE for comparable $\phi_{\mathrm{g}}$. This can be understood by recognizing that the wall-surface-area to volume ratio of the RSE is greater than for the full-scale enclosure, and a larger fraction 
of the released heat should be lost to the walls for the smaller enclosure. Additionally, the scaling of the doorways for the two enclosures is such that a larger fraction of the energy in the upper layer can be radiated from the smaller enclosure. Both effects should lead to a higher fraction of the energy being trapped in the upper layer of the real-scale enclosure, and hence higher temperatures for scaled HRRs.

Due to the temperature maxima reached and the limits of the chromel/alumel thermocouples used, the maximum temperatures in the upper layer of the full-scale room are uncertain. Limited measurements with platinum/platinum-rhodium thermocouples indicated upper-layer temperatures in the front of the full-scale enclosure for fires with $\phi_{\mathrm{g}}>1$ approached $1400 \mathrm{~K}-1500 \mathrm{~K}$.

The growth of $\mathrm{CO}$ concentrations in the upper layer with time in the real-scale facility seems to be associated with the increasing upper-layer temperatures. The time behaviors are similar. Furthermore, Pitts has shown that mixtures of combustion gases which are kinetically frozen at lower temperatures begin to react and approach thermodynamic equilibrium concentrations at higher temperatures [15]. In this temperature range the formation of $\mathrm{CO}$ is strongly favored, and equilibrium concentrations of $\mathrm{CO}$ approach $16 \%$ for $\phi_{\mathrm{g}}=3$. Therefore, the most plausible explanation for the high CO concentrations observed in these experiments is that the upper-layer temperature has increased to the point where the rich combustion-gas mixture begins to react and approach equilibrium. The ultimate concentrations attained will be a function of residence time and reaction rate.

The approach of a nonequilibrium gas mixture towards thermodynamic equilibrium represents still another mechanism for $\mathrm{CO}$ in enclosure fires and, when it occurs, can lead to much higher concentrations of $\mathrm{CO}$ than predicted by the GER concept.

A single experiment was conducted in which the ceiling and upper walls down to $76 \mathrm{~cm}$ from the ceiling of the full-size enclosure were lined with $12.7 \mathrm{~mm}$ thick plywood. A $2.0 \mathrm{MW}$ natural gas fire was used. Enhanced CO concentrations (8\% in the front of the room and $12 \%$ in the rear) which were very similar to those measured in the RSE were observed. Temperature levels were significantly suppressed from those for underventilated fires in the absence of wood. The formation of CO by anaerobic pyrolysis of wood does not seem to depend on enclosure size.

To summarize, the research thus far has indicated that there are at least four potential mechanisms for $\mathrm{CO}$ formation in enclosure fires. These are:

1) Quenching of a turbulent fire plume upon entering a rich upper layer. This is the mechanism considered by the hood experiments.

2) Mixing of oxygen directly into a rich, high-temperature upper layer.

3) Pyrolysis of wood in high-temperature, vitiated environments. 
4) Approach to full-equilibrium combustion product concentrations in a rich, hightemperature upper layer.

It is important to note that the only mechanism which is captured by the GER concept is the first one. It is particularly disconcerting that each of the other mechanisms results in the formation of additional CO. On this basis, it can be concluded that the GER concept provides an estimate for the least amount of $\mathrm{CO}$ which is likely to be generated by an enclosure fire. More reliable estimates must consider the additional formation mechanisms.

\section{ENGINEERING ALGORITHM FOR PREDICTING CO FORMATION IN ENCLOSURE FIRES}

The findings of the experimental and analytical studies discussed in the last section have been used to construct the engineering algorithm for $\mathrm{CO}$ production rates shown in Figure

1. In order to use this algorithm an engineer will need to be able to make engineering estimates for a number of fire behaviors. Some of the parameters which may need to be specified include:

1) Time behavior of $\phi_{\mathrm{g}}$ for the enclosure as a function of time.

2) The correlations of species yields and/or concentrations for the upper layer as a function of $\phi_{\mathrm{g}}$.

3) Temperature as a function of time for the upper layer.

4) Amount of air directly entering the upper layer as a function of time.

5) The surface area of wood which is located in anaerobic regions of the room.

6) Estimate for the pyrolysis rate of wood as a function of temperature and time when subjected to nonreactive gases or intense flame radiation.

7) Estimate for the mass of $\mathrm{CO}$ generated per mass of wood pyrolyzed under anaerobic conditions.

8) Estimates for equilibrium concentrations of upper-layer gases, conversion rates for nonequilibrium gas mixtures towards equilibrium as a function of temperature, and combustion gas residence times within the enclosure.

\section{Summary}

This report has provided a summary and references for an extensive research program designed to characterize $\mathrm{CO}$ formation mechanisms within enclosure fires. The findings of these investigations have been used to develop an engineering algorithm for the prediction of $\mathrm{CO}$ formation during enclosure fires for use in fire models.

\section{References}

[1] Harland, W. A.; Anderson, R. A. Causes of death in fires. Proceedings, smoke and toxic gases from burning plastics; 1982 Jan. 6-7; London, England; 15/1 to 15/19.

[2] Harwood, B.; Hall, Jr., J. R. What kills in fires: smoke inhalation or burns? Fire J. 83: 29-34; 1989 May/June. 
[3] Babrauskas, V.; Levin, B. C.; Gann, R. G.; Paabo, M.; Harris, Jr., R. H.; Peacock, R. D.; Yusa, S. Toxic potency measurement for fire hazard analysis. National Institute of Standards and Technology Special Publication 827; 1991 December. 107 p.

[4] Pitts, W. M. Long-range plan for a research project on carbon monoxide production and prediction. National Institute of Standards and Technology Internal Report NISTIR 89-4185; 1989 October. 40 p.

[5] Babrauskas, V. Upholstered furniture room fires--measurements, comparison with furniture calorimeter data, and flashover predictions. J. Fire Sciences 2(1): 5-19; 1984 January/February.

[6] Mulholland, G. W. Position paper regarding CO yield. Letter report to Richard G. Gann, Chief, Fire Measurement and Research Division, Center for Fire Research; 1988 June 16.8 p, unpublished.

[7] Mulholland, G. W. Comparison of predicted $\mathrm{CO}$ yield with results from fire reconstruction of Sharon, PA fire. Letter report to William Pitts, Leader, CO Priority Project; 1989 November 1.13 p.

[8] Beyler, C. L. Major species production by diffusion flames in a two-layer compartment fire environment. Fire Safety J. 10(1): 47-56; 1986 January.

[9] Beyler, C. L. Major species production by solid fuels in a two layer compartment fire environment. Grant, C. E.; Pagni, P. J. eds. Fire safety science--proceedings of the first international symposium. New York, NY: Hemisphere; 1991. 431-440.

[10] Zukoski, E. E.; Toner, S. J.; Morehart, J. H.; Kubota, T. Combustion processes in two-layered configurations. Grant, C. E.; Pagni, P. J. eds. Fire safety science-proceedings of the first international symposium. New York, NY: Hemisphere; 1988. 295-304.

[11] Zukoski, E. E.; Morehart, J. H.; Kubota, T.; Toner, S. J. Species production and heat release rates in two-layered natural gas fires. Combust. Flame 83(3 and 4): 325-332; 1991 February.

[12] Pitts, W. M. The global equivalence ratio concept and the prediction of carbon monoxide formation in enclosure fires, National Institute of Standards and Technology Monogr. 179; 1994 May. 156 p.

[13] Pitts, W. M., The global equivalence ratio concept and the formation mechanisms of carbon monoxide in enclosure fires. Prog. Energy Combust. Science 21: 237-197; 1995.

[14] Pitts, W. M. Reactivity of product gases generated in idealized enclosure fire environments. Twenty-fourth symposium (international) on combustion; Pittsburgh, PA: The Combustion Institute; 1992. 1737-1746.

[15] Pitts, W. M. Application of thermodynamic and detailed chemical kinetic modeling to understanding combustion product generation in enclosure fires. Fire Safety J. 23: 271-303; 1994.

[16] Gottuk, D. T.; Roby, R. J.; Peatross, M.; Beyler, C. L. Carbon monoxide production in compartment fires. J. Fire Protection Eng. 4(4): 133-150. 1992 October-December.

[17] Fire tests--full scale room test for surface products. Draft International Standard ISO/DIS 9705; International Organization for Standardization. 1990.

[18] Proposed standard method for room fire test of wall and ceiling material assemblies, Annual Book of ASTM Standards, Part 18, American Society for Testing and Materials; Philadelphia, PA. 1982. 
[19] Bryner, N.; Johnsson, E. L.; Pitts, W. M. Carbon monoxide production in compartment fires--reduced-scale enclosure test facility. National Institute of Standards and Technology Internal Report NISTIR 5568; 1994 December. 202 p.

[20] Bryner, N. P; Johnsson, E. L.; Pitts, W. M. To be submitted for publication.

[21] Toner, S. J. Entrainment, chemistry and structure of fire plumes, Doctor of Philosophy Thesis, California Institute of Technology; 1986. 259 p. Also available as: Toner, S. J.; Zukoski, E. E.; Kubota, T. Entrainment, chemistry and structure of fire plumes. National Bureau of Standards Government Contractor's Report GCR-87528; 1987 April. 222 p.

[22] Levine, R. S.; Nelson, H. E. Full scale simulation of a fatal fire and comparison of results with two multiroom models. National Institute of Standards and Technology Internal Report NISTIR 90-4268; 1990 August. 101 p.

[23] Pitts, W. M.; Bryner, N. P.; Johnsson, E. L. Carbon monoxide formation in fires by high-temperature anaerobic wood pyrolysis. Twenty-fifth symposium (international) on combustion; Pittsburgh, PA: The Combustion Institute; 1994. 1455-1462.

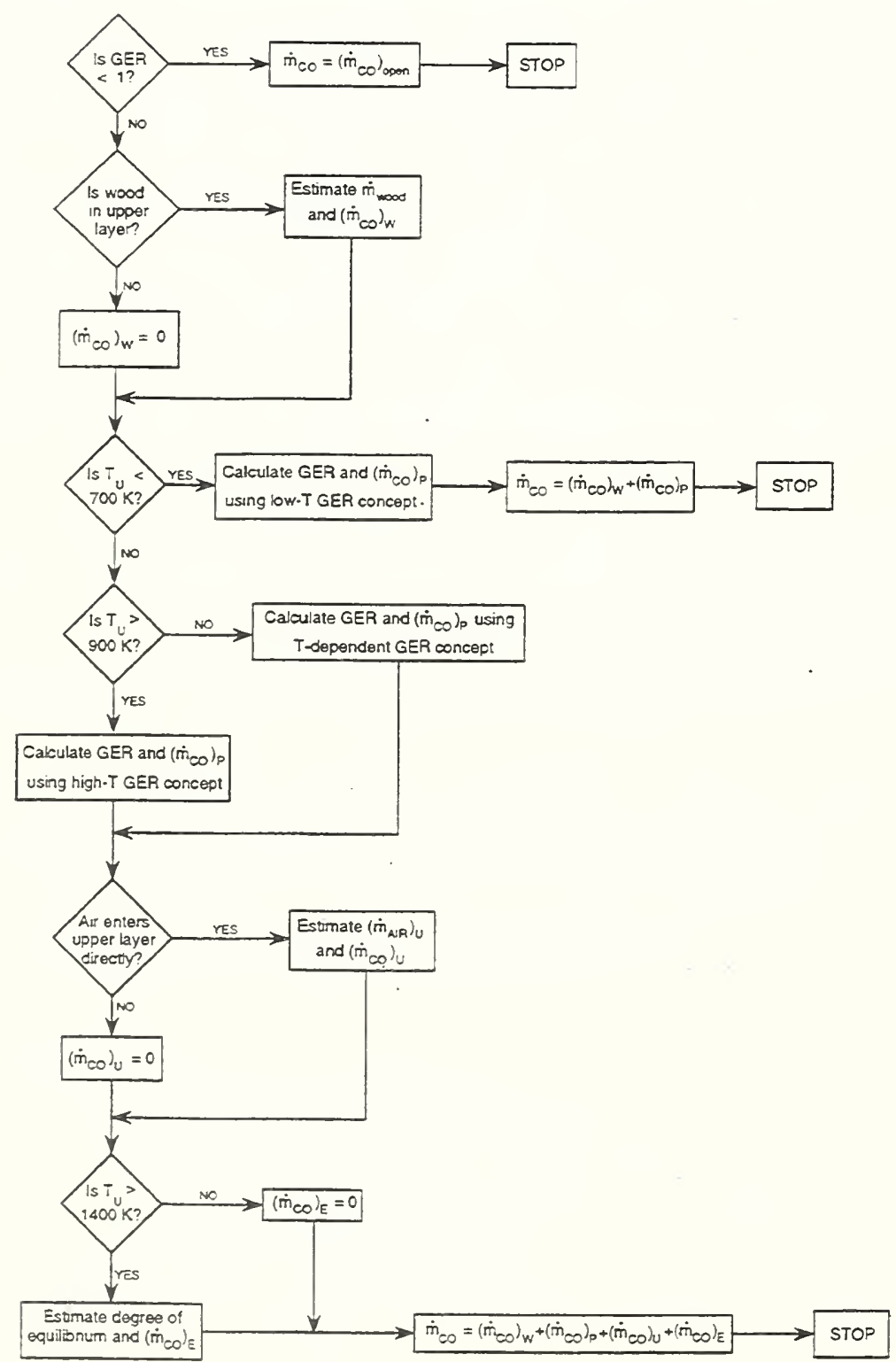

Figure 1. An algorithm for predicting the generation of carbon monoxide during enclosure fires is shown. The symbols are defined as follows: GER is the global equivalence ratio, $T_{u}$ is the upper-layer temperature, $\dot{\mathrm{m}}$ are mass generation or entrainment rates with subscripts representing carbon monoxide (CO) formation, pyrolysis products of wood decomposition (wood), and air mixing into an upper layer of a fire (AIR). Subscripts associated with the $\mathrm{CO}$ mass generation rates refer to $\mathrm{CO}$ formation by fires burning in an open environment (open), by wood pyrolysis in anaerobic environments (W), by quenching of a turbulent fire plume upon entering a rich upper-layer above a fire $(P)$, by mixing air directly into a rich high temperature upper layer of a fire with subsequent reaction to generate $\mathrm{CO}(\mathrm{U})$, and by the upper layer of a fire becoming hot enough for the rich gases to begin to approach thermodynamic equilibrium (E). 


\section{Discussion}

Question: Do you have flame shape data?

William Pitts: We do have data on several things. These include local equivalence ratio measurements, front and rear. We find experimentally that they are lower in the front. That means that more air by whatever path has reached the front as opposed to the rear. The second piece of evidence has to deal with some very simple modeling that we've done of these flow systems. What they indicate is exactly what I described, air going into the upper layer without passing through the flames. They suggested this air ultimately ends up in the front of the enclosure exactly where we see the higher concentrations of $\mathrm{CO}$. These have not been done carefully, but I feel that they're indicative of what's happening. I think the evidence indicates that there is indeed this mechanism for air to indirectly to the upper level.

I did not mention temperature measurements today but we do, indeed, see $200-300 \mathrm{~K}$ higher in the front than in the rear. We attribute it to a combination of additional heat releases associated with the oxygen being entrained directly into the upper layer and to residence time effects. There are recirculation zones in the rear of the enclosure which makes the residence time much longer than for gases passing directly out of the front of the enclosure, so we think heat loss in contributing there as well. 
$6 \underline{0}$ 


\title{
The Fire Hazard Assessment Methodology
}

\author{
Walter W. Jones \\ Fire Modeling and Applications Group \\ Building and Fire Research Laboratory \\ National Institute of Standards and Technology, 20899, USA
}

\section{Introduction}

The United States alone spends about $\$ 850 \mathrm{~B}$ per year on new and renovated construction[1]. About $1 / 5$ of this is to assure safety from unwanted fires. This presents a major opportunity for the introduction of new fire safe products to the building and transportation industries and new products such as advanced detectors and suppression systems and fire fighting equipment for the fire safety industry. These industries need measures of performance for their products and mechanisms to show that these products can be safely and quickly introduced. In order to derive this benefit it is necessary to have tools to evaluate building systems performance which then provide a metric for the effectiveness of design and material use. The Fire Hazard Assessment Methodology provides the first component of a performance evaluation system.

Hazard I is a prototype of a general purpose fire hazard assessment method. The scope of this prototype, its data base and the example cases are focussed on single family residential occupancies. The product helps to make the results of fire research available in a usable way. The hope is that the tedium associated with applying a multiplicity of formulae to solve a problem will be alleviated to some extent.

The methodology consists of a set of procedures combining expert judgment and calculations to estimate the consequences of a specified fire. These procedures involve four steps: 1) defining the context, 2) defining the scenario, 3) calculating the hazard, and 4) evaluating the consequences. Steps 1,2, and 4 are largely judgmental and depend on the expertise of the user. Step 3, which involves use of the extensive HAZARD I software, requires considerable expertise in fire safety practice. The core of HAZARD I is a sequence of procedures implemented in computer software to calculate the development of hazardous conditions over time, calculate the time needed by building occupants to escape under those conditions, and estimate the resulting loss of life based on assumed occupant behavior and tenability criteria. These calculations are performed for specified buildings and fire scenarios of concern.

\section{History}

The first release of the methodology was Hazard I, version 1.0, in the Summer of 1989. Hazard I version 1.1 was released in the spring of 1992 . Versions 1.2 was published in the spring of 1994. Many improvements have been made in the documentation which accompanies the software. These improvements are a result of the experiences fire protection engineers and others have had in using the methodology. 
The centerpiece underlying all of Hazard $I$ is a zone model of fire growth and smoke transport. The Hazard Methodology surrounds this with models of egress and tenability, auxiliary computer codes, databases and tables to enable efficient use of the model. Over the past decade the Building and Fire Research Laboratory (formerly the Center for Fire Research) has developed computer based models as a predictive tool for estimating the environment which results in a building when a fire is present. In the beginning, there were three of these models: FAST, FIRST and ASET. Originally there was supposed to be a benchmark fire code, with all algorithms of fire phenomena available for experimentation. A change in direction was made in 1986 and it was subsequently developed as a prototype of a well structured model. In 1989, another decision was made that development of many computer programs was not the best possible course. The modeling program evolved to two programs from that decision. The one underpinning HAZARD is CFAST. The other is FPETool, which will be discussed later.

CFAST is intended to operate on many platforms, be as error free as is humanly possible, be simple to run for simple problem, yet allow complexity where needed. The code is extremely fast. It is faster than any code of comparable completeness and complexity. It works on laptop personal computers, Unix workstations and supercomputers. It provides for extensive graphics for analysis with pre- and post-processing modules. It is extremely fast on single compartment cases, and with the data editor, there is tremendous flexibility for parameter studies, "what if" testing and so on. It is intended to be a complete, yet very fast, computer code for calculating the effects of fire on the environment of a building. It is particularly well suited for doing parameter studies of changes, both subtle and large, within a single compartment.

\section{Overview of recent changes to the HAZARD software package:}

This section discusses the changes which have occurred in the various modules which constitute the HAZARD package. The discussion is focussed on those who use the components of the package individually, but everything that is stated applies to the combined HAZARD package.

There are a number of additional phenomena which have been added, based on this increased capability to version 2.0. For example, we have implemented a ceiling jet algorithm[2] which takes into account heat loss from a fire placed in an arbitrary position within a compartment. The algorithm describes the theory and implementation of the algorithm which accounts for the off-center placement of the fire and its effect on heat transfer to the room surfaces. This allows us to include the $3 \mathrm{D}$ location of a fire in a room. The natural continuation of this work would be to include smoke and heat detectors in the model so that such studies can be conducted in a systematic manner, both for within compartment detection, as well as remote detection, that is for detector siting in adjacent compartments. A flame spread model now exists in CFAST. At present it is for vertical spread only, but the extension to horizontal (surface and lateral) is being studied. Finally, a general radiation model is now utilized. This is a ten wall model for the four upper wall segments, four lower wall segments, ceiling and floor. Numerically it is simplified to four segments, based on symmetry of the rectangular parallelepiped used in our zone model[3]. It is just slightly slower than the earlier extended ceiling algorithm, but the improvement in accuracy is significant. 
The routines for predicting egress of people and the effect of the fire on human behavior have been combined into a single entity called Survival. The salient difference in Survival is that incapacitation or death will prevent further movement of a person. The original thrust of Exitt and Tenab, which allows one to see relative effects of toxic insults, has been incorporated into Survival.

\section{A View of the Evolution of Fire Modeling:}

As we continue to improve the methodology, there are four avenues to follow: increase the number and improve the capability of the phenomena which are modeled, improve the usability of the package, provide derivative applications, and expand the scope of the use of the methodology.

As the concept of calculating fire safe structures takes hold, the question will arise of how much does some improvement in safety cost, how much will it save. One area we have not discussed explicitly is the valuation of a building or system subject to a fire, and what the worst or most probable fire and concomitant dollar loss would be. Such a capability would be on top of that for estimating the effect of fire.

The concept of general building/people/fire interactions could be included. There are three aspects which we would need to be addressed. The first is the people/building interaction. The second is an integrated model for highrise and residential. The third is an editor for people movement rules. The fire model is sufficiently fast that the run time graphics is almost irrelevant. It should be possible to develop Survival so that the people interact with the fire by having Survival call the CFAST kernel.

The front end graphical user interface (GUI) is a vast improvement over the text based interface currently in use. We intend to extend this to all aspects of modeling, including the use of the FireForm tools as a utility within HAZARD. Our concept of a GUI will be embodied first in FASTLite and the CFAST shell. The CFAST shell embodies a more holistic view of the interaction of a fire with the building environment and FASTLite is the continuation of FPETool in a windowing mode. In some ways this goes beyond our original goal of providing a simple filter to prevent egregious mistakes. However, there are several databases associated with fire modeling: thermophysical properties, fire curves, validation data and so on. It allows us to make these databases much more versatile and accessible without encumbering the user of the system too much. By extending the GUI concept to include the graphics output as well as the people placement and specification of those items which affect the behavior of people it will be possible to provide a uniform basis (look and feel) for all of our modeling effort. This would, in turn, provide a general framework for cooperation in fire research. For example, our post processing tools are being extended by us and others for use with CFD models.

New phenomena: There are many new phenomena which could be incorporated. Those under active consideration include:

Compartment to compartment heat transfer via conduction, Flow within compartments (hybrid), Burning at corners (furniture, adjoining walls), Structural effects (barriers to smoke and fire spread as well as load bearing capability), Improved pyrolysis model (based on more fundamental physical aspects of materials), Construction 
design files (databases used for building and ship design), Improved understanding of species generation such as $\mathrm{CO} / \mathrm{CO}_{2}$ and its source, Two directional heat transfer in walls (non-congruent thermocline), Better detector and other sensor activation, Deposition and agglomeration of smoke and other species, Suppression - include fire size, drop size and distance effects, geometry of the fire, evaporation/cooling, modifications to all modules to utilize FDMS[4],[5] databases, Corrosion - add on for $\mathrm{HCl}$ - important for semiconductor industry and warehouses. Smoke movement in tall shafts, stairways and atria.

Limitations: There are phenomena for which the algorithms are adequate, but could more accurate:

- General - Pyrolysis (and flame spread) models still depend on test methods. No heating/cooling in HVAC ducts, and reverse flow in fans not allowed

- Entrainment - fire plume and doorway jet entrainment are based on the same experimental correlations. The fire plume (for large spaces) and the doorway jet (in general) are often used outside the normal range of validity of these correlations.

- User specification - the level of agreement is critically dependent upon careful choice of the input data for the model. A better understanding of typical fire induced leakage in buildings would facilitate more accurate description of the building environment.

- Statistical treatment of the data - presentation of the differences between model predictions and experimental data are intentionally simple. With a significant base of data to study, appropriate statistical techniques to provide a true measure of the "goodness of fit" should be investigated.

- Experimental measurements - measurement of leakage rates, room pressure, or profiles of gas concentration are atypical in experimental data. These measurements are critical to assessing the accuracy of the underlying physics of the models because the flow characteristics of the model depend critically on the structure being modeled.

An important part of our work is developing into providing various types of databases. This is an important underpinning of the UJNR cooperative program. At present we are redoing the FDMS concept. There are two reasons: 1 ) it is very difficult to add new types of tables. This has resulted in many people abandoning its use; and 2) for the fire modeling work we need a consistent and well defined database structure for data which is used for validation, the various data sets we use within the models, and so on. We are developing the new structure and modules with the caveat in mind that previous work should fit into and be usable.

\section{Extensions to the methodology:}

There are several possible extensions to this work. For real time fire fighting, a portable computer (hand held) would allow one to walk through a building (before or after a fire) and catalog the contents of a building. This could be brought back to the office and used directly as input to the model for geometric specification and data initialization. As the Cellular Digital Packet Data becomes more prevalent, onsite inspections will allow such hand held computers to interact directly with desk bound servers for maintaining databases and ascertaining code compliance. As the model codes become more sophisticated, and their 
complexity increases, researchers, code officials, and everyone else will be aided by on such stratagems. There simply is not enough time to bother with all of the details. This is the arena which should allow us to pursue the goal of a better qualitative understanding of fires, well as doing more of it faster.

All large buildings have annunciator panels for various alarms. Indeed, some fire departments can display floor plans of buildings in the command center at a fire. It is a logical next step to plug these display into the alarm system to see the current status of a building and then make a prediction of the next five minutes.

Another area is that of risk. Risk is the next step up from a hazard calculation, and requires a much more general understanding of the parameters which affect the outcome of a fire and its impact on humans and structures. This application would require an automated application of the model over types of fires, day and night scenarios, position of the fire and so on. The number of such calculations can become enormous. Some means of doing this in finite time will need to be found. Also, in order to provide performance evaluation tools, it is necessary to know how often something does not happen, as well as what to do when a catastrophe occurs. As part of this work, we are developing a mechanism to ascertain the sensitivity of the outcome to the parameters themselves (fine variation) as well as their variation (gross variation). A critical point will be to decide upon a reasonable extent of variation.

As we extend the capability of the zone models, we are encountering the inherent limitations of these types of models. The general concept of a zone or control volume model uses a volume as one of the variables. Inherently there is no spatial information available. The first deviation from this viewpoint was the necessity of including height $v s$. width information in order to calculate flow through a normal vent, such as a door. The second came when flow through a ceiling/floor opening and mechanical ventilation were included. We have extended the concept for the position of the fire. The next step would be to define the spatial component of a compartment so that we could include more sophisticated interactions. This latter is important, for example, in dealing with detection and suppression problems.

The automatic transfer of information from one set of calculations to another is important to avoid unnecessary errors and repetitive data entry. The quest is to provide a tool which will aid rather than hinder. This is not an attempt to make the application of such methods trivial, but rather to provide a mechanism to allow researchers, fire protection engineers, code officials and so on access to the most current understanding of the behavior of fires.

Finally, we have the human factors aspect. How much does fire really cost? Since our knowledge of a situation is not perfect, what range of results might one expect given a most likely scenario. This is the "human factors and cost."

The ability to provide these and other improvements to the hazard assessment technology will depend on the reception and support given to this effort. User feedback is crucial to the process of identifying the most needed. Through this process, research priorities can be established to address the needs of the community in the most efficient 
manner. In addition, we challenge the fire safety community to review and comment on this effort. The gaps in knowledge identified herein can then help guide our work toward resolving these issues. As we continue to plumb the depths of this problem, both the direction and scope of the methodology will be influenced by what users say is needed as well as the results which evolves naturally from the BFRL's research efforts.

\section{New Focus:}

Data and sample runs: We now deliver HAZARD with some sample cases. It would be useful to provide a set of cases from start to finish with a test case, a video of the actual case being burned in a facility such as BFRL Large Scale Fire Test Facility, and the accompanying data.

Survival and the kernel: The concept of general building/people/fire interactions needs to be included. This would allow realistic evacuation scenarios to be studied. There are three aspects which we need to address. The first is the people/building interaction. The second is an integrated model for highrise and residential. The third is an editor for people movement rules. It should be possible to develop Survival so that the people interact with the fire by having Survival call the CFAST kernel.

Parameter variation and estimation of probabilities: One of the most important extensions of HAZARD I would be the concept of automated parameter variation, which include incorporating probability of actual events to ascertain the relative effect of particular scenarios. This capability would increase the usefulness of our models many fold. As part of this work it is necessary to develop a mechanism to ascertain the sensitivity of the outcome to the parameters themselves (fine variation) as well as their variation (gross variation). A critical point will be to decide upon a reasonable extent of variation. For example, if we consider a door that will be open or closed, should we consider it to be absolutely closed, with leakage, a crack $1 / 8,1 / 4,1 / 2$ and fully open, or some other combination?

\section{Conclusions}

The intent is to provide tools which will help improve the understanding of fires. This is an attempt to provide a mechanism to allow researchers, fire protection engineers, and others access to the most current understanding of the behavior of fires. These engineering tools could then be the basis for meeting performance standards.

\section{References}

1. Construction and Building: Federal Research and Development in Support of the US Construction Industry, Subcommittee on Construction and Building, Committee on Civilian Industrial Technology, National Science and Technology Council, 1995.

\section{Cooper, L. Y., Fire-Plume Generated Ceiling Jet Characteristics and Convective} Heat Transfer to Ceiling and Wall Surfaces in a Two-Layer Zone-Type Fire Environment: Uniform Temperature Ceiling and Walls, National Institute of Standards and Technology (USA) Internal Report 4705 (1991). 
3. Forney, G.P., Computing Radiative Heat Transfer Occurring in a Zone Fire Model, National Institute of Standards and Technology (USA) Internal Report 4709 (1992).

4. Portier, R., A Programmer's Reference Guide to FDMS File Formats, National Institute of Standards and Technology Internal Report 5162 (1993).

5. Portier, R., Fire Data Management System, FDMS 2.0, Technical Documentation, National Institute of Standards and Technology Technical Note 1407 (1994).

\section{Discussion}

John Hall: Walter, in last overhead, the third conclusion says better algorithms and improved understanding of the preferred root as opposed to what?

Walter Jones: As opposed to dropping phenomena out and trying to finesse the problem. I think it's more important to understand the problem and solve it that way. To amplify a little bit, certainly the tools that we have are not perfect. We try to do the best we can with the knowledge that we have, but the way to the future, I think, is better understanding as opposed to simplification.

Moni Dey: In terms of your goal for a national fire performance code, is the plan to have such a code endorsed in a guideline like the NFPA code or SFPE?

Walter Jones: Let me divide the world up into three parts: there is the code which is a building code; there are the standards which is what I think you are referring to, which is properly an NFPA function; and there are the tools to evaluate whether a particular building configuration will meet those codes based on those standards. I think we all have to work together to do this. All those aforementioned organizations, code bodies, and NIST all have to work together to do that. 


\title{
A ROOM FIRE MODEL IN VIEW OF PREDICTING FIRE SPREAD BY EXTERNAL FLAMES
}

\author{
Yoshifumi OHMTYA \\ Graduate student, Faculty of Science and Technology, Science University of Tokyo \\ 2641 Yamasaki, Noda-shi, Chiba-ken, 278 Japan \\ Takeyoshi TANAKA \\ Head, Smoke Control Division, Building Research Institute, Ministry of Construction \\ 1 Tatehara, Tsukuba-shi, Ibaraki-ken, 305 Japan \\ Takao WAKAMATSU \\ Professor, Faculty of Science and Technology, Science University of Tokyo \\ 2641 Yamasaki, Noda-shi, Chiba-ken, 278 Japan
}

\section{ABSTRACT}

A model for predicting room fire in fully developed stage is developed for the purpose of estimating the heat release due to the combustion of the excess fuels involved in the external flames ejecting from the windows of fire rooms. In this model, the burning rate is predicted as a function of the predicted heat transfer to the fuels. The model does not need to introduce adjusting factors, such as the "complete combustion rate $(=0.6)$ " by Kawagoe et al., to get reasonably good prediction of room fire temperature. The predictions of the temperatures and the burning rates are compared with the results of preceding experiments to demonstrate fair agreement.

\section{INTRODUCTION}

A significant number of models have been developed to date for the prediction of room fire behavior, for such purposes as assessing the fire impact to structural members or the risk of the fire spread to adjacent spaces. In many of them, however, the burning rate is not predicted but given independently from the heat transfer conditions within the room.

A typical example can be seen in the model by Kawagoe \& Sekine, which is well known and widely used in the fire resistance community in Japan[1]. This model numerically solves the energy conservation equation given as follows:

$$
\mathrm{Q}_{C}=\mathrm{Q}_{W}+\mathrm{Q}_{R}+\mathrm{Q}_{S}
$$

where $Q_{C}, Q_{W}, Q_{R}$ and $Q_{S}$ are the rates of the heat release by the combustion, the heat transfer to the boundary walls, the radiative heat loss from the opening and the heat convected out by the opening flow, respectively. In this model the heat release rate $Q_{C}$ is given by the product of the heat of combustion $\triangle H_{C}$ and the mass burning rate $M$, for the latter of which the famous relationship:

$$
M=0.1 A \sqrt{H}
$$

empirically derived by Kawagoe et al. is invoked. However, the prediction by this method yields unreasonably high temperature, hence so-called "complete combustion rate" with fixed value of 0.6 has been introduced to fill up the gap with the experimentally measured temperatures.

It is considered that this discrepancy is caused by that the model by Kawagoe et al. does not take into account the portion of the fuel volatile that cannot burn within the room due to the shortness of 
available air so is ejected out to burn as external flames. This portion will not contribute to the elevation of the temperature of the room but will affect the temperature of the opening flames and plumes.

Although the distance between the openings on adjacent floors are often controlled by building codes to prevent upstairs fire spread, it is not clear if the provisions is sufficiently rational. The hazard of upstairs fire spread must be a complex function of opening dimension, room size, fire load etc. as well as the distance between the openings. In this study, a room fire model incorporating the prediction of mass burning rate is developed so that the heat release rate due to the combustion of the excess fuels in opening flames can be estimated under different conditions of fire room and fuels.

The intention is to estimate the heat release rate $Q_{e x}$ due to the combustion of excess fuel by

$$
\mathrm{Q}_{\text {ex }}=\Delta H_{\text {fuel }} M-\Delta H_{\text {air }} R_{O F}
$$

using the mass burning rate $M$ predicted by the model.

\section{ROOM FIRE MODEL}

This model is a single room fire model which assumes that the physical properties is uniform within the fire room, like most of the other models for similar purpose. The concept of the model is illustrated in Figure 1.

\subsection{Conservation and State of Room Gases}

Considering the conservation of the mass, the energy and the species for the gases in the fire room, the following equations can be obtained.

(1) Mass Conservation

$$
\frac{d}{d t}(\rho V)=R_{O F}-R_{F O}+M
$$

(2) Energy Conservation

$$
\frac{d}{d t}\left(c_{P} \rho T V\right)=\mathrm{Q}_{C}-\mathrm{Q}_{W}-\mathrm{Q}_{R}-\mathrm{Q}_{\text {fuel }}+C_{P} R_{O F} T_{O}-C_{P} R_{F O} T+C_{P} T_{P} M
$$

Note that the rate of the heat transfer to fuels $Q_{\text {fuel }}$ is included in Eqn.(5) separately from the heat transfer to room boundary walls.

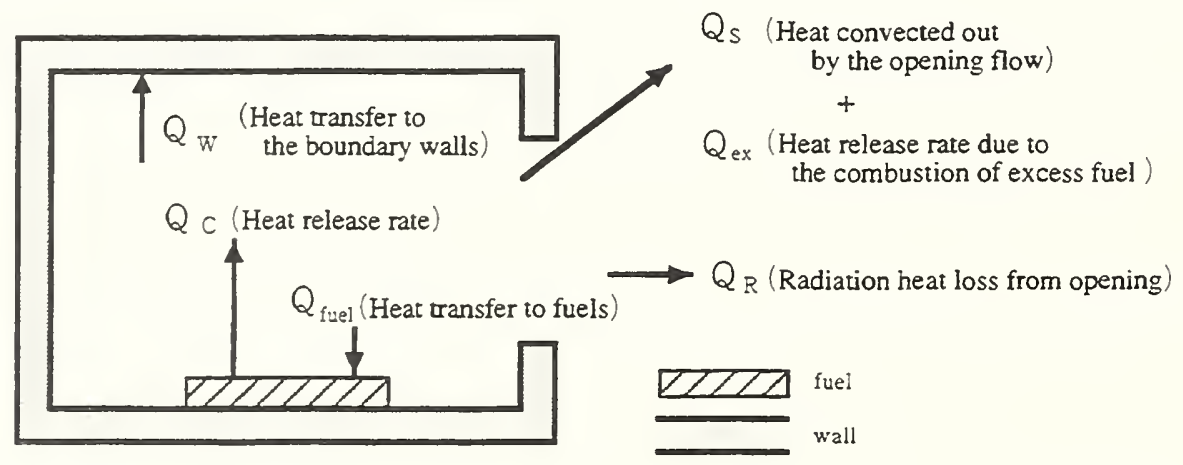

Figure 1. Concept of model 
(3) Species Conservation

$$
\frac{d}{d t}\left(\rho V Y_{l}\right)=R_{O F} Y_{l, O}-R_{F O} Y_{l}+M Y_{l, f u e l}+\Gamma_{l}
$$

\subsection{Heat Transfer Models}

(1) Heat transfer to the boundary walls

The room boundary walls are assumed to be uniform in thermal properties and in temperature, and the rate of heat transfer $Q_{W}$ is calculated using an ordinary equation as:

$$
\frac{\mathrm{Q}_{W}}{A_{W}}=\varepsilon_{W} \varepsilon_{G} \sigma\left(T^{4}-T_{W}^{4}\right)+h\left(T-T_{W}\right)
$$

The surface temperature of the wall $T_{W}$ is calculated by solving the one dimensional thermal conduction equation for the wall.

(2) Heat transfer to fuels

The rate of the heat transfer to the fuels is calculated by the same equation as Eqn.(7-2) except that fuel gasification temperature $T_{P}$ is used instead of wall surface temperature $T_{W}$

$$
\frac{Q_{\text {fuel }}}{A_{\text {fuel }}}=\varepsilon_{\text {fuel }} \varepsilon_{G} \sigma\left(T^{4}-T_{P}^{4}\right)+h\left(T-T_{P}\right)
$$

However, the rate of the heat transfer for wood fuels is assumed to be $1 / 4$ of $Q_{\text {fuel }}$ thus calculated by Eqn. (7-2) based on the results of the preceding experiments[2].

(3) Radiation heat loss from opening

The heat loss due to the radiation through the opening of a room $Q_{R}$ is simply given as:

$$
\mathrm{Q}_{R}=\varepsilon_{G} \sigma\left(T^{4}-T_{0}^{4}\right) A
$$

(4) Convective heat transfer coefficient

No established method to estimate the convective heat transfer coefficient $h$ included in Eqns.(7-1) and (7-2) exists to date, but the following formula is used for its rough estimation[3]

$$
h= \begin{cases}5 \times 10^{-3} & \left(T^{*} \leq 300 \mathrm{~K}\right) \\ \left(0.02 T^{*}-1\right) \times 10^{-3} & \left(300 \mathrm{~K}<T^{*}<800 \mathrm{~K}\right) \\ 15 \times 10^{-3} & \left(800 \mathrm{~K} \leq T^{*}\right)\end{cases}
$$

where $\mathrm{T} *$ is defined as

$$
T^{*}= \begin{cases}\left(T+T_{W}\right) / 2 & \text { (for wall }) \\ \left(T+T_{P}\right) / 2 & (\text { for fuel })\end{cases}
$$




\section{(5) Emissivity of room fire gases}

The fire gases in a real scale room can be regarded as black, but in this model the emissivity of the fire gases is calculated for the convenience of making comparison with the results of the preceding experiments, for which a somewhat small scale room was used.

(a) Emissivity due to smoke particles

The emissivity of the fire gases due to smoke particles in the fire room is calculated using the equation given by Quintiere[4] as follows:

$$
\varepsilon_{G}=1-e^{-k_{G} L_{G}}
$$

where the effective absorption coefficient is given as

$$
k_{G}=1.3(\gamma+1) \frac{M}{M+R_{O F}}
$$

(b) Emissivity due to $\mathrm{CO}_{2}$ and $\mathrm{H}_{2} \mathrm{O}$ gases

The emissivity of the fire gases due to $\mathrm{CO}_{2}$ and $\mathrm{H}_{2} \mathrm{O}$ is calculated simply as the sum of the emissivity of each gas, namely:

$$
\varepsilon_{G}=\varepsilon_{\mathrm{CO}_{2}}+\varepsilon_{\mathrm{H}_{2} \mathrm{O}}
$$

where the emissivities of $\mathrm{CO}_{2}$ and $\mathrm{H}_{2} \mathrm{O}$ are calculated by Schack's equations [5] given as

$$
\varepsilon_{\mathrm{CO}_{2}}=0.7\left(P_{\mathrm{CO}_{2}} \cdot L_{G}\right)^{0.5} /(T / 100)^{0.5} \text { and } \varepsilon_{\mathrm{CO}_{2}}=7.0\left(P_{\mathrm{H}_{2} \mathrm{O}} \cdot L_{G}\right)^{0.8} /(T / 100) \cdots
$$

\subsection{Mass Burning Rate}

In this model, an initial fire source with given mass burning rate is employed to initiate the fire in the room, and the mass burning rate afterwards is calculated as a function of the heat transfer conditions in the room.

(1) Mass burning rate of initial fire source

The mass burning rate of the initial fire source $M_{l}$ is set as:

$$
M_{1}=m_{\text {free }}^{\prime \prime} A_{\text {fuel }}
$$

In the computation, the burning rate is increased linearly from 0 to this value in one minute, since this model focuses on the behavior of vigorous stage of fire so does not concern the behavior in the initial stage.

(2) Mass burning rate in fuel controlled stage

It is assumed that the burning rate in fuel controlled stage $M_{2}$ is affected by the heat from the room gases as well as the heat from the flames formed above the fuels. When the temperature of the room is higher than the gasification temperature of the fuel, is calculated by

$$
M_{2}=m_{\text {free }}^{\prime \prime} A_{\text {fuel }}+Q_{\text {fuel }} / L_{V}
$$

It is doubtful that the heat from the fire room gases can reach the fuel surface penetrating the flames above the fuels. However, it will reasonable to consider that the temperature rise of the room 
reduces the net heat loss of the flames to the surrounding and thus indirectly increases the heat transfer to the fuel.

(3) Mass burning rate in ventilation controlled stage

In ventilation controlled stage, the combustion heavily depends on the air inflow through openings and the flames no longer locate in the vicinity of the fuel but often whirl around in the room. The mass burning rate at such a stage $M_{3}$ is considered to be controlled mainly by the heat transfer from the room fire gases, hence is calculated by

$$
M_{3}=Q_{\text {fuel }} / L_{V}
$$

\section{(4) Mass burning rate in transitional stage}

Figure 2 shows the transition of mass burning rate that may take place within fire room. In Figure 2, transition from $M_{1}$ to $M_{2}$ (1) begins when the room temperature reaches to the fuel gasification temperature, and $M_{2}$ increases as the net incident heat flux to the fuel increase. For ventilation controlled stage it will be reasonable to assume that the mass burning rate is given by $M_{3}$. However, switching from $M_{2}$ to $M_{3}$ at point (2) causes gaps by $m^{\prime \prime}{ }_{\text {free }} A_{\text {fuer }}$ In this model, Eqn.(18) is used to manipulate the mass burning rate in the transitional stage from (2) to (3), namely.

$$
M_{2 \rightarrow 3}=\frac{\Delta H_{\text {air }} R_{O F}}{\Delta H_{\text {fuel }}}
$$

This manipulation may be partly justified. Although the transition from fuel controlled stage to ventilation controlled stage takes place at point in terms of the heat release rate, the transition takes place somewhat more gradually in terms of mass burning rate. Therefore, the stage from (2) to (3) is the stage where the behavior of the mass burning rate is changing gradually from that of the fuel controlled stage to that of the ventilation controlled stage. In any case, this is a relatively short period of time.

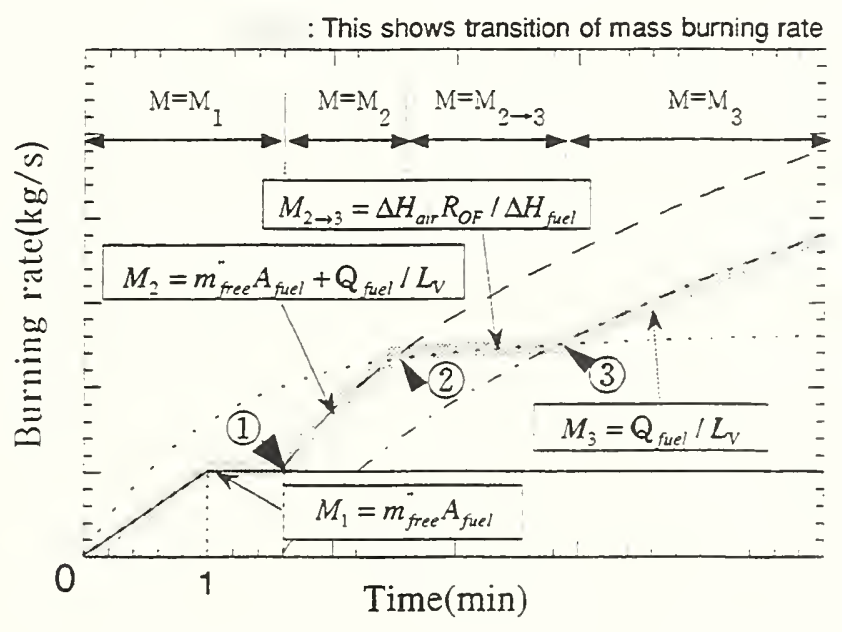

Figure 2. Transition of mass burning rate

\subsection{Heat Release Rate within Fire Room}

Since sufficient air is available to burn all the fuel volatile produced in the fire room in fuel controlled stage, the heat release rate is governed by the mass burning rate. On the other hand, since much more fuel volatile than can be burned by the air supplied into the room by the ventilation is produced in ventilation controlled stage, the heat release rate within the fire room is governed by 
the air supply rate, hence

$$
\mathrm{Q}_{C}= \begin{cases}\Delta H_{\text {fuel }} M & \text { (for fuel control }) \\ \Delta H_{\text {air }} R_{O F} & (\text { for ventilation control })\end{cases}
$$

Note that the heat of combustion per unit mass of air consumed $\Delta H_{\text {air }}$ is $3 \mathrm{MJ} / \mathrm{kg}$ in sufficient accuracy regardless the fuel type.

\subsection{Species Generation Rate}

Since it is known that the heat of combustion per unit mass of air consumed is virtually constant for many fuels encountered in fire, the generation (consumption) rate of oxygen can be expressed as

$$
\Gamma_{O_{2}}=-\frac{Q_{C}}{\Delta H_{O_{2}}}
$$

hence, when the chemical equation of the combustion of the fuel can be written as

$$
\text { fuel }+v_{\mathrm{O}_{2}}^{\prime} \mathrm{O}_{2} \rightarrow v_{\mathrm{CO}_{2}}^{\prime \prime} \mathrm{CO}_{2}+v_{\mathrm{H}_{2} \mathrm{O}}^{\prime \prime} \mathrm{H}_{2} \mathrm{O}
$$

the generation rates of $\mathrm{CO}_{2}$ and $\mathrm{H}_{2} \mathrm{O}$ are given as follows:

$$
\Gamma_{\mathrm{CO}_{2}}=\frac{\mathrm{Q}_{\mathrm{C}}}{\Delta H_{\mathrm{O}_{2}}} \times \frac{44}{32} \times \frac{v_{\mathrm{CO}_{2}}^{\prime \prime}}{v_{\mathrm{O}_{2}}^{\prime}} \text { and } \Gamma_{\mathrm{H}_{2} \mathrm{O}}=\frac{\mathrm{Q}_{\mathrm{C}}}{\Delta H_{\mathrm{O}_{2}}} \times \frac{18}{32} \times \frac{v_{\mathrm{H}_{2} \mathrm{O}}^{\prime \prime}}{\dot{v}_{\mathrm{O}_{2}}}
$$

\section{Zone Equations}

From Eqns.(4) - (6), the ordinary differential equations for the temperature and the species concentration of the fire room, and the algebraic equation for governing the ventilation of the room are obtained as follows:

(1) Room temperature

$$
\frac{d T}{d t}=\frac{T}{C_{P} \rho_{O} T_{O} V}\left[\left(\mathrm{Q}_{C}-\mathrm{Q}_{W}-\mathrm{Q}_{R}-\mathrm{Q}_{\text {fuel }}\right)-C_{P}\left\{R_{O F}\left(T-T_{O}\right)+M\left(T-T_{P}\right)\right\}\right]
$$

(2) Species mass concentration

$$
\frac{d Y_{l}}{d t}=\frac{T}{\rho_{O} T_{O} V}\left\{R_{O F}\left(Y_{l, O}-Y_{l}\right)+M\left(Y_{l, \text { fuel }}-Y_{l}\right)+\Gamma_{l}\right\}
$$

In this model, the concentration of species $I$ in fuel $Y_{l, \text { fuel }}$ is regarded as

$$
Y_{l, f u e l}= \begin{cases}1 & (l=\text { fuel }) \\ 0 & \left(l=\mathrm{O}_{2}, \mathrm{CO}_{2}, \mathrm{H}_{2} \mathrm{O}\right)\end{cases}
$$

(3) Ventilation

$$
\frac{T_{O}}{T} R_{O F}-R_{F O}+\frac{T_{P}}{T} M+\frac{1}{C_{P} T}\left(\mathrm{Q}_{C}-\mathrm{Q}_{W}-\mathrm{Q}_{R}-\mathrm{Q}_{\text {fuel }}\right)=0
$$


Here, Eqns.(23) and (24) are numerically integrated using Runge-Kutta method and Eqn.(26) is solved using Regula-Falsi method.

\section{COMPARISON BETWEEN PREDICTIONS AND EXPERIMENTS}

The predictions by the room fire model are compared with the results of the experiments[6] previously conducted to examine the validity of the model. In the experiments, methanol pools, PMMA panels, and wood panels and cribs with several different surface area were burned in the fire room with the dimensions of $1.1 \times 1.8 \times 1.1(\mathrm{~m})$ under different opening conditions. And the room temperatures, the ceiling and the floor heat fluxes, and the mass burning rates were measured.

\subsection{Conditions for Prediction and Experiments}

The conditions of the calculations, which are essentially the same as the conditions of the preceding experiments, are shown in Table 1. Although the size of the model fire room is fixed for every case, some number of different conditions are tried for the opening. Methanol, PMMA and wood with different surface area are employed as the fuels.

\subsection{Fire Room Temperature}

Figure 3 shows a example of the transient temperatures predicted by the model and measured in the preceding experiments. In this example, the fuel is methanol with three different liquid surface areas and the value of the opening factor is $0.2 \mathrm{~m}^{5 / 2}$. The temperatures in Figure $3(\mathrm{~b})$ are the average of the temperature measured in the experiments by the 10 thermocouples arrayed vertically with the same spacing. It is considered that the fire with fuel A1 is completely fuel controlled, the fire with fuel A4 is completely ventilation controlled and the fire with fuel A2 is around the limit of

Table 1. Conditions of calculations[5][7]

\begin{tabular}{|c|c|c|c|c|c|c|c|c|c|c|}
\hline & Unit & \multicolumn{9}{|c|}{ Values } \\
\hline \multicolumn{11}{|l|}{ (Opening conditions) } \\
\hline $\begin{array}{l}\text { Width } \\
\text { Height }\end{array}$ & $\mathrm{m}$ & \multicolumn{4}{|c|}{$0.4,0.6,0.8$} & \multicolumn{5}{|c|}{$\begin{array}{c}0.2 \sim 0.9 \\
(0.1 \mathrm{~m} \mathrm{spacing}) \\
1 \\
\end{array}$} \\
\hline \multicolumn{11}{|l|}{ (Compartment conditions) } \\
\hline $\begin{array}{l}\text { Width } \\
\text { Depth } \\
\text { Height }\end{array}$ & $\begin{array}{l}\mathrm{m} \\
\mathrm{m} \\
\mathrm{m}\end{array}$ & \multicolumn{9}{|c|}{$\begin{array}{l}1.1 \\
2.8 \\
1.1 \\
\end{array}$} \\
\hline \multicolumn{11}{|l|}{ (Wall conditions) } \\
\hline $\begin{array}{c}\text { Thickness } \\
\text { Thermal conductivity } \\
\text { Thermal capacity } \\
\text { Density }\end{array}$ & $\begin{array}{c}\mathrm{m} \\
\mathrm{kW} / \mathrm{m} / \mathrm{K} \\
\mathrm{kJ} / \mathrm{kg} / \mathrm{K} \\
\mathrm{kg} / \mathrm{m}^{3}\end{array}$ & \multicolumn{9}{|c|}{$\begin{array}{c}0.1 \\
0.0002 \\
1.1 \\
750 \\
\end{array}$} \\
\hline \multicolumn{11}{|l|}{ (Fuel conditions) } \\
\hline & (Type) & $A(1)$ & $A(2)$ & $A(4)$ & $P(1)$ & $P(2)$ & $P(4)$ & $W(1)$ & $W(2)$ & $W(C)$ \\
\hline Fuel & & \multicolumn{3}{|c|}{ Methanol } & \multicolumn{3}{|c|}{ PMMA } & \multicolumn{3}{|c|}{ Wood } \\
\hline Surface area & $\mathrm{m}^{2}$ & 0.2 & 0.41 & 0.81 & 0.13 & 0.22 & 0.42 & 1.33 & 2.41 & 2.62 \\
\hline Weight0 & $\mathrm{kg}$ & & 3.17 & & & 2.4 & & 13 & 13 & 8 \\
\hline Latent heat of gasification & $\mathrm{kJ} / \mathrm{kg}$ & & 1170 & & & 1630 & & & 1700 & \\
\hline Heat of combustion & $\mathrm{kJ} / \mathrm{kg}$ & & 22300 & & & 25200 & & & 18400 & \\
\hline Stoichiometric air/fuel ratio & & & 6.47 & & & 8.28 & & & 5.7 & \\
\hline Gasification temperature & $\mathrm{K}$ & & 338 & & & 543 & & & 623 & \\
\hline Outside temperature & $\mathrm{K}$ & \multicolumn{9}{|c|}{293} \\
\hline
\end{tabular}




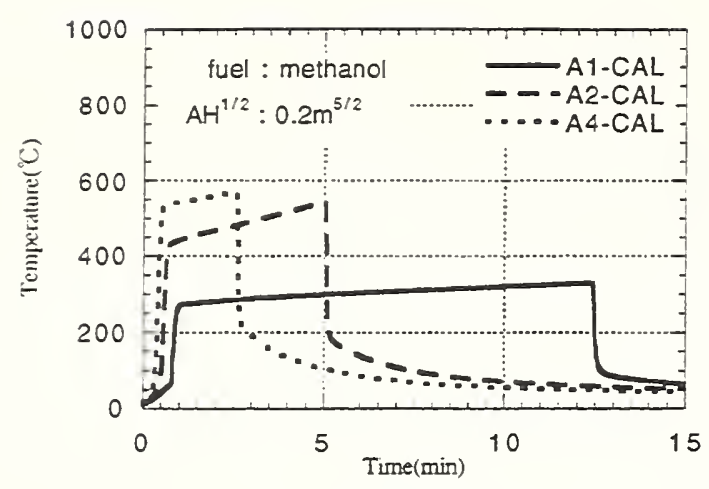

a) Prediction

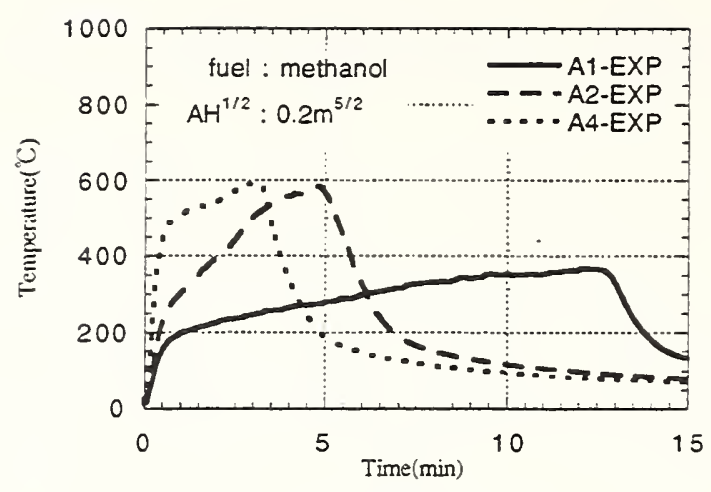

b) Measurement

Figure 3. Temperature

the two regimes. From Figure 3, prediction seem to agree reasonably well with measurement, despite of liquid surface area.

Figure 4 compares the predicted and the measured temperature at vigorous burning stages. The temperatures of the predictions were taken from the values at the last moment of the fire duration, and the value of the experiments are the average during the vigorous burning stage. The open symbols indicate the cases where external flames were not observed in the experiment while the solid symbols indicate the cases where external flames were observed. Although the agreement between the predictions and measurements is only fair for methanol and PMMA, the agreement for wood, which is most common fuel in ordinary fires, seems satisfactory. The predicted temperatures generally tend to be a little higher than the measured temperatures. This may be partly due to the way the comparison is made: the latter is the average while the former is the highest.

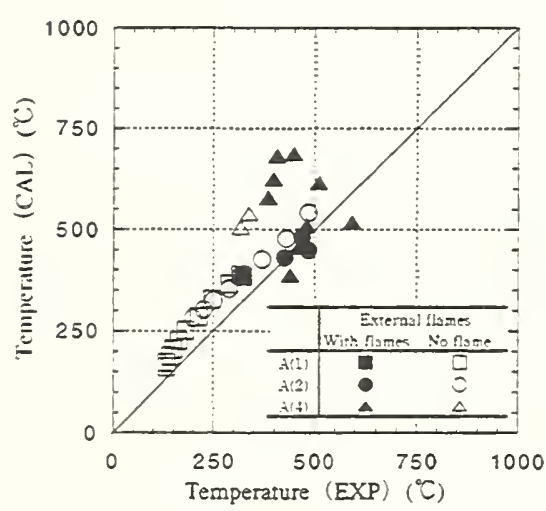

a)Methanol

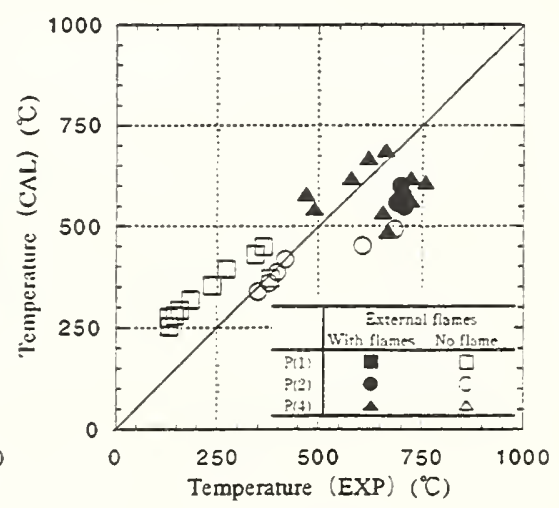

b)PMMA

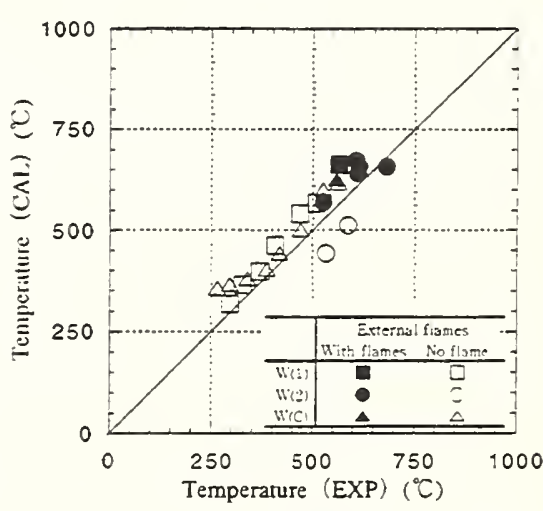

c)Wood

Figure 4. Comparison the predicted and the measured temperature

\subsection{Mass burning rate}

Figure 5 shows a example of the transient mass burning rates predicted by the model and measured in the preceding experiments. The calculation and experimental conditions of the example are the same as in the cases in Figure 3. In Figure 5, in case the liquid surface area is small, prediction seem to agree well with measurement. In case the liquid surface area is large, the prediction tend to be a little higher than the measurement. 


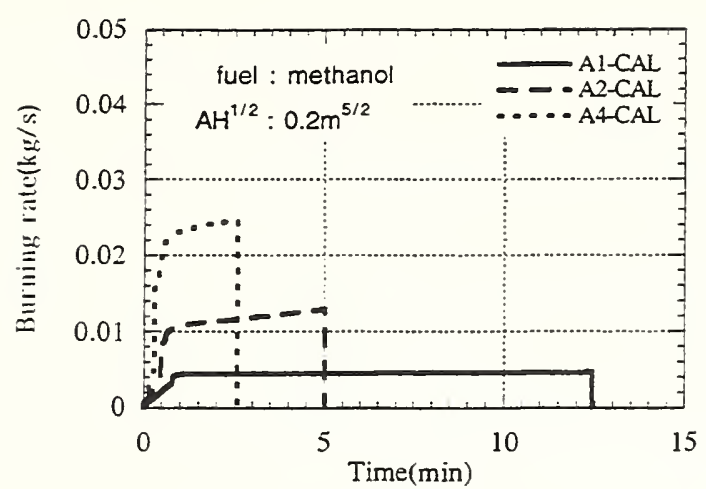

a) Prediction

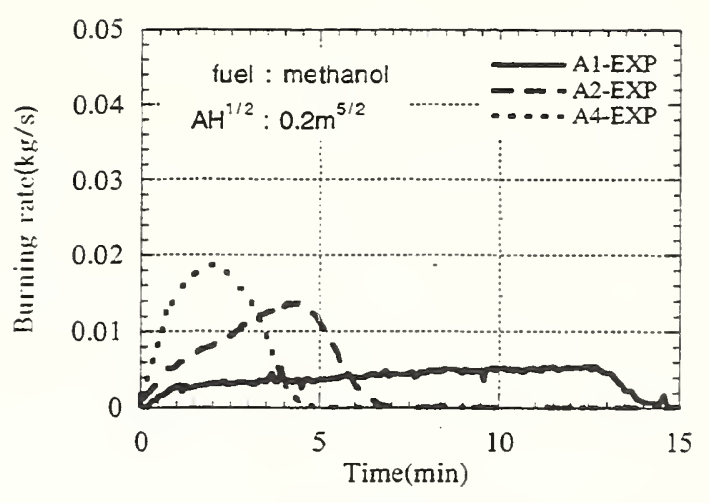

b) Measurement

Figure 5. Mass burning rate

Figures. $6-8$ compares the predicted and the measured burning rates at vigorous burning stages. The burning rates of the predictions were taken from the values at the last moment of the fire duration, and the value of the experiments are the average during the vigorous burning stage. Again, the open symbols indicate the cases with external flames and the solid symbols indicate the cases without external flames. The solid line in each figure stands for Eqn.(2), i.e. the relationship by Kawagoe et al. and the broken line stands for the rate of fuel which can be burned within the fire room by stoichiometric combustion with the air supplied by the ventilation through the opening,

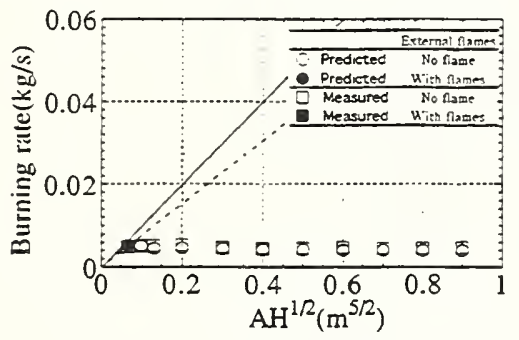

a)A(1)Type

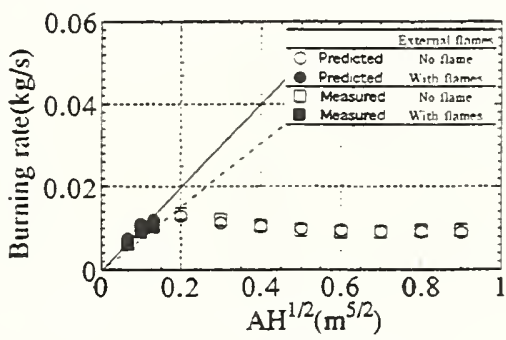

b)A(2)Type

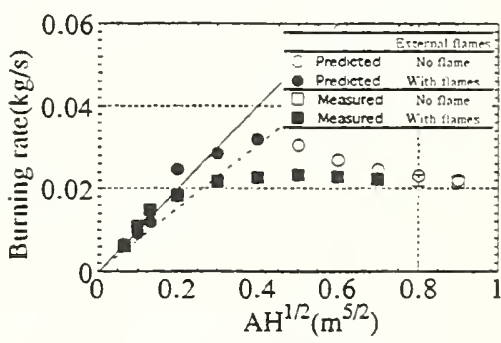

c)A(4)Type

Figure 6. Comparison the predicted and the measured burning rates (Methanol)

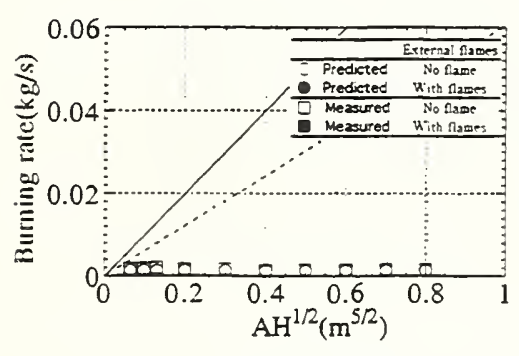

a)P(1)Type

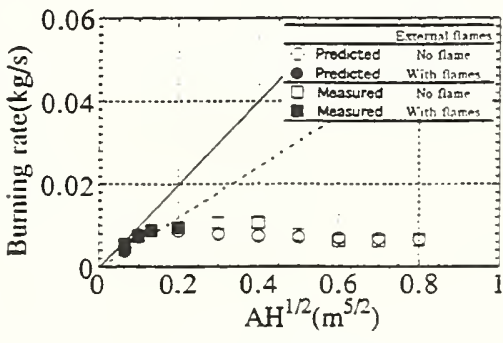

b) $P(2)$ Type

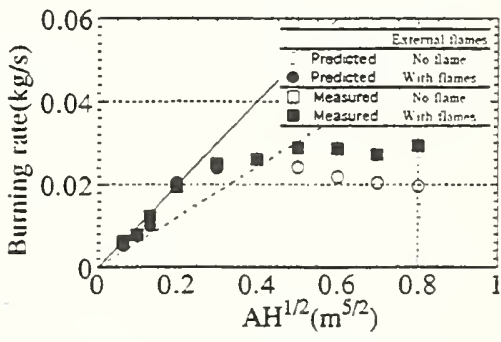

c) P(4)Type

Figure 7. Comparison the predicted and the measured burning rates (PMMA)

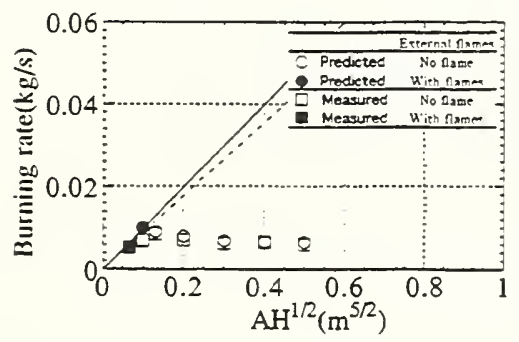

a)W(1)Type

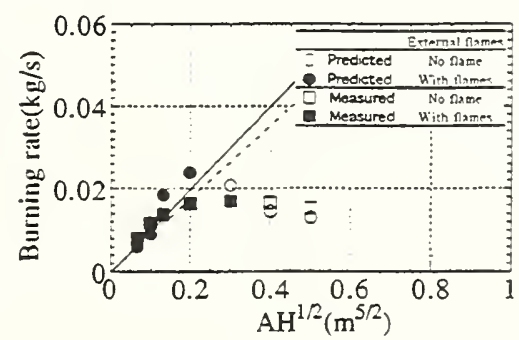

b)W(2)Type

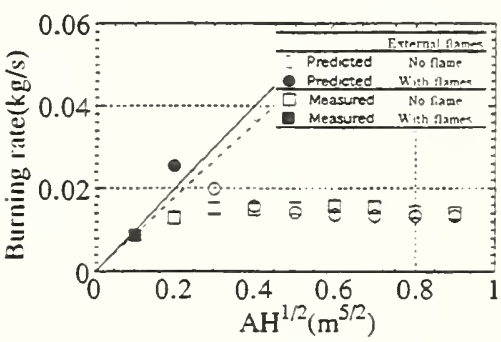

c)W(c)Type

Figure 8. Comparison the predicted and the measured burning rates (Wood) 
i.e:

$$
M_{\text {stoi }}=M_{\text {air }} / \gamma
$$

In this particular case, the stoichiometric burning rate of each fuel $M_{\text {stoi }}$ is given as

$$
M_{\text {stoi }}= \begin{cases}0.077 A \sqrt{H} & \text { (methanol) } \\ 0.061 A \sqrt{H} & (P M M A) \\ 0.089 A \sqrt{H} & (\text { wood })\end{cases}
$$

where the ventilational air supply rate is estimated by

$$
M_{\text {air }}=0.5 \mathrm{~A} \sqrt{\mathrm{H}}
$$

Generally, both of the predicted and measured burning rates tend to increase along the line of Kawagoe et al. regardless the fuel type where the ventilation factor is small and decrease down to the respective value of free burning of each fuel as the ventilation factor becomes large. It seems the maximum burning rates often appear on the broken line, i.e. the stoichiometric burning rate line.

In a couple of the figures, some discrepancies are observed between the predicted and measured burning rates, particularly around the peak burning rates. Partly, this should be attributed to the crudeness of the model, but it should be remained that the measured values are average while the predicted values are the maximum. Considering the complexity of the phenomena involved and the simpleness of the model, the discrepancy to this extent may be acceptable.

\section{CONCLUSION}

In this study, a room fire model incorporating the prediction of the mass burning rate was developed. The room temperature and the mass burning rate predicted by the model were compared with the results of the preceding reduced scale room fire experiment.

The model seem to agree reasonably well with the test results, despite of its simplicity. The relationship on the mass burning rate of ventilation controlled room fires empirically obtained by Kawagoe et al. for wood crib fire was reproduced by the model regardless the fuel type.

\section{REFERENCES}

[1] K.Kawagoe and T.Sekine: Estimation of Fire Temperature Rise Curves in Concrete Buildings (Part1,2), Transactions of the Architectural Institute of Japan, No.85, 86, 1963

[2] Y.Ohmiya, M.Satoh, T.Tanaka and T.Wakamatsu: Incident Heat Flux to Fuel and Burning Rate in Ventilation Controlled fires,J.Struct. Constr. Eng., AIJ, No.472, 169 176, Jun., 1995

[3] T.Tanaka and K.Nakamura: A Model for Predicting Smoke Transport in Building -Based on Two Layers Zone Concept- ,Report of The Building Research Institute, NO123, 1989

[4] J.G.Quintiere and B.J.McCaffrey: The Burning of Wood and Plastic Cribs in an Enclosure Volume 1, Final Report, NBS, November, 1980

[5] JSME Date Book - Heat Transfer- 4th Edition, Japan Society of Mechanical Engineers, 1986

[6] Y.Ohmiya, M.Satoh, T.Tanaka and T.Wakamatsu : Burning Rate of Fuel in Enclosure and Generation Limit of the External Flame,J.Struct. Constr. Eng., AIJ, No.469, 149 158, Mar., 1995

[7] A Handbook of Fires, Japan Association for Fire Science and Engineering , 1984 


\section{NOMENCLATURE}

\begin{tabular}{|c|c|}
\hline A & : Area of opening $\left(\mathrm{m}^{2}\right)$ \\
\hline$A_{\text {fuel }}$ & : Area of fuel $\left(\mathrm{m}^{2}\right)$ \\
\hline$A_{\text {wall }}$ & : Area of wall $\left(\mathrm{m}^{2}\right)$ \\
\hline$c_{p}$ & : Thermal capacity at constant pressure $(\mathrm{kJ} / \mathrm{kg} / \mathrm{K})$ \\
\hline $\mathrm{H}$ & : Height of opening(m) \\
\hline$\Delta \mathrm{H}_{\mathrm{air}}$ & : Heat of combustion per unit mass of air consumed $(\mathrm{kJ} / \mathrm{kg})$ \\
\hline$\Delta \mathrm{H}_{\text {fue! }}$ & : Heat of combustion per unit mass of fuel consumed $(\mathrm{kJ} / \mathrm{kg})$ \\
\hline k & : Effective absorption coefficient $(\mathrm{I} / \mathrm{m})$ \\
\hline L & :Thickness(m) \\
\hline M & : Mass burning rate $(\mathrm{kg} / \mathrm{s})$ \\
\hline$M_{\text {sroi }}$ & : Stoichiometric burning rate $(\mathrm{kg} / \mathrm{s})$ \\
\hline $\mathrm{m}^{\prime \prime} \mathrm{free}$ & : Mass burning rate per unit surface area in the free $(\mathrm{kg} / \mathrm{s})$ \\
\hline $\mathrm{P}$ & : Partial pressure(atm) \\
\hline $\mathrm{Q}_{\mathrm{c}}$ & : Rate of heat release by combustion $(\mathrm{kW})$ \\
\hline$Q_{\text {fuel }}$ & : Rate of the heat transfer to fuel $(\mathrm{kW})$ \\
\hline$Q_{e x}$ & : Heat release rate due to the combustion of excess fuel $(\mathrm{kW})$ \\
\hline$Q_{R}$ & : Radiative heat loss from the opening $(\mathrm{kW})$ \\
\hline$Q_{S}$ & : Heat convected out by the opening flow $(\mathrm{kW})$ \\
\hline$Q_{w}$ & : Heat transfer to the boundary walls $(\mathrm{kW})$ \\
\hline $\mathrm{L}_{\mathrm{v}}$ & : Latent heat of gasification $(\mathrm{kJ} / \mathrm{kg})$ \\
\hline $\mathrm{R}_{\mathrm{OF}}$ & : Rate of air inflow $(\mathrm{kg} / \mathrm{s})$ \\
\hline $\mathrm{R}_{\mathrm{FO}}$ & : Rate of air outflow $(\mathrm{kg} / \mathrm{s})$ \\
\hline $\mathrm{T}$ & $:$ Fire room temperature(K) \\
\hline $\mathrm{T}_{\mathrm{o}}$ & : Outside (ambient) temperature $(\mathrm{K})$ \\
\hline$T_{P}$ & : Fuel gasification temperature $(\mathrm{K})$ \\
\hline $\mathrm{T}_{\mathrm{w}}$ & : Surface temperature of the wall(K) \\
\hline V & : Volume $\left(\mathrm{m}^{3}\right)$ \\
\hline$Y_{1} \cdot 0$ & : Species mass concentration(outside) \\
\hline$Y_{1}$ & : Species mass concentration(inside) \\
\hline h & : Convective heat transfer coefficient $\left(\mathrm{kW} / \mathrm{m}^{2} / \mathrm{K}\right)$ \\
\hline$\gamma$ & : Stoichiometric air/fuel ratio \\
\hline$\Gamma_{1}$ & : Species generation(consumption) rate $(\mathrm{kg} / \mathrm{s}$ ) \\
\hline$\varepsilon_{G}$ & : Emissivity of room fire gases \\
\hline$\varepsilon_{\mathrm{w}}$ & : Emissivity of wall surface \\
\hline$\rho$ & : Density of air $\left(\mathrm{kg} / \mathrm{m}^{3}\right)$ \\
\hline$\sigma$ & : Stefan-Boltzmann constant $\left(=5.67 \times 10^{-11} \mathrm{~kW} / \mathrm{m}^{2} / \mathrm{K}^{4}\right)$ \\
\hline
\end{tabular}




\section{Discussion}

John Rockett: One of the attractive features of the simple models is the possibility that they can be programmed using software such as Mathmatica. One can arrive at solutions with little programming effort. I commend you on your model and I will have a look to see if I can make it run with Mathmatica.

As an old modeler, it would be interesting to compare the results of your model with what you would get with running the Harvard single room fire model. 


\title{
EVALUATION OF COMPLEX FIRE MODELS
}

\author{
Richard D. Peacock, Paul A. Reneke, and Walter W. Jones \\ Building and Fire Research Laboratory, NIST, Gaithersburg, MD 20899, USA
}

\begin{abstract}
Several methods for studying predictive capability and sensitivity have been applied to fire models, but with limited utility. These range from explicit evaluation of the equations used in simple models such as ASET to evaluation of complex models from numerous computer runs of a model along with usually quantitative comparison to laboratory experiments. This paper presents a discussion of the issues involved in conducting an evaluation of a complex room fire model. Examples using currently available room fire models are presented.
\end{abstract}

Analytical models for predicting fire behavior have been evolving since the 1960's. During this time, the completeness of the models has grown. In the beginning, the focus of these efforts was to describe in mathematical language the various phenomena which were observed in fire growth and spread. These separate representations have typically described only parts of a fire. When combined though, they can create a complex computer code capable of giving an estimate of the expected effects of a fire based upon given input parameters. Analytical models have progressed to the point of providing predictions of fire behavior with an accuracy suitable for most engineering applications. Two obvious questions arise concerning the use of these models for engineering calculations:

- How good do the inputs to the model need to be (How do changes in the model inputs affect the model outputs)?

- How good is the output of model (How close are the actual conditions to those predicted by the model)?

To address the former question, this paper presents a summary of the issues involved in conducting a sensitivity analysis of a complex room fire model. Examples using one fire model are provided. For the latter question, some examples are presented illustrating comparisons for both simple correlations and complex fire models. Both of these discussion highlight the strengths and weaknesses of our current level of understanding of evaluation of complex fire models. More complete investigations are available $e^{1,2}$.

\section{SENSITIVITY ANALYSIS}

A sensitivity analysis is a study of how changes in model parameters affect the results generated by the model. Model predictions may be sensitive to uncertainties in input data, to the level of rigor employed in modeling the relevant physics and chemistry, and to the accuracy of numerical treatment. Among the purposes for conducting a sensitivity analysis are to determine:

- the important variables in the models,

- the computationally valid range of values for each input variable, and

- the sensitivity of output variables to variations in input data.

Conducting a sensitivity analysis of a complex fire model is a difficult task. Many models require extensive input data and generate predictions for numerous output variables over a period of simulated time. Several methods of sensitivity analysis have been applied to fire models, but most have had limited utility. These range from explicit evaluation of the equations used in simple models such as $\mathrm{ASET}^{3}$ to pointwise evaluation of complex models from numerous computer runs of the model ${ }^{4}$. The technique chosen for use will be dependent on the objectives of the study, the required results, the resources available and the complexity of the model being analyzed. 
Earlier efforts: Khoudja ${ }^{4}$ has studied the sensitivity of an early version of the FAST model with a fractional factorial design involving two levels of 16 different input parameters. The statistical design, taken from the texts by Box and Hunter ${ }^{5}$, and Daniel ${ }^{6}$ reduced the necessary model runs from more than 65000 to 256 by studying the interactions of input parameters simultaneously. His choice of values for each input parameter represented a range for each parameter. His analysis of the FAST model (a precursor to the CFAST model used for this paper) showed a particular sensitivity to the inclusion of conduction in the calculations and lesser, though consistent sensitivities to the number of compartments included in a simulation and the ambient temperature. Without the inclusion of surface thermophysical properties, this model treats surfaces as adiabatic for conductive heat transfer. Thus, this consistent sensitivity should be expected. Sensitivity to changes in thermal properties of the surfaces were not explored.

For a steady-state model of a liquid pool fire, Ndubizu ${ }^{7}$, et. al. used a Fourier Amplitude Sensitivity Test to study the relative importance of model inputs. With appropriate transformation of input parameters, the model outputs define a periodic function of the transformed inputs. This resulting function is then Fourier analyzed with the Fourier coefficients directly corresponding to the sensitivity of each input parameter.

The ASTM guide for evaluating the predictive capability of fire models ${ }^{8}$ identifies model sensitivity analysis as an important part of model evaluation and identifies two methods which may be applied to perform a sensitivity analysis - a partial differential method and a response surface method. Further details are left to other sources.

Application to a current fire model: Fire growth models are typically based on a system of ordinary differential equations of the form

$$
\frac{d z}{d \tau}=f(z, p, \tau) \quad z(\tau=0)=z_{0}
$$

where $\mathrm{z}\left(\mathrm{z}_{1}, \mathrm{z}_{2}, \ldots, \mathrm{z}_{\mathrm{m}}\right)$ is the solution vector for the system of equations (for example, mass, temperature, or volume) and $\mathrm{p}\left(\mathrm{p}_{1}, \mathrm{p}_{2}, \ldots \mathrm{p}_{\mathrm{n}}\right)$ is a vector of input parameters (for example, room area, room height, heat release rate) and $\tau$ is time ${ }^{9}$. The solutions to these equations are, in general, not known explicitly and must be determined numerically. To study the sensitivity of such a set of equations the partial derivatives of an output $z_{j}$ with respect to an input $p_{i}$ (for $\mathrm{j}=1, \ldots, \mathrm{m}$ and $\mathrm{I}=1, \ldots, \mathrm{n})$ are examined.

Although numerous scenarios could be chosen for study, a single one was used in this paper to illustrate the analysis of a single complex fire model, CFAST ${ }^{18}$. To obtain a complete picture of a model's sensitivity, a number of scenarios representing the entire range of the model would have to be studied. The scenario chosen includes a range of phenomena which can be simulated with this model. The building geometry included four rooms on two floors with horizontal, vertical, and mechanical vents connecting the rooms and venting to the outdoors. The fire source in one of the rooms on the lower floor is a medium growth rate $t^{2}$ fire ${ }^{11}$ chosen to simulate a mattress fire ${ }^{12}$.

Sensitivity to small changes in model inputs: To investigate the sensitivity of the model, a number of simulations were conducted varying the input parameters for CFAST about this base scenario. Both small $( \pm 10 \%)$ and larger (up to an order of magnitude) variations for selected inputs were studied. Varying most of the inputs by small amounts had little effect on the model outputs.

An example, figure (1), shows the results of a $10 \%$ change in room volume (effected by changing the floor area) on several model outputs. The figure shows a somewhat constant relative difference for the changes as a function of time. Ignoring the effects at very early times where upper layer volume and pressure are very nearly zero, the graph shows that temperature and pressure are less sensitive to changes in the volume of the fire room since the $10 \%$ change in room volume-led to smaller relative changes in layer temperature and room pressure for all times. 
Upper layer volume can be considered neutrally sensitive (a 10\% change in room volume led to about a $10 \%$ change in layer volume). Further, this implies that there is negligible effect on layer interface height. This is consistent with both experimental observations in open compartment room fires ${ }^{13}$ and analytical solutions for single compartment steadystate fires ${ }^{14}$. In essence, this implies that reasonable uncertainties in room dimensions would have little effect on the results predicted by the model for this scenario. As suggested by Iman and Helton $^{15}$, an average relative difference could be used to characterize the model sensitivity for comparing individual inputs and outputs.

\section{Sensitivity to larger changes in model} inputs: To investigate the effects of much larger changes in the inputs, a series of simulations where the inputs were varied from 0.1 to 4.0 times the base value was conducted. Simulations changing the heat release rate (HRR) inputs are shown in figure 1. Each set appears as families of curves with similar functional forms. This indicates that multiples of the HRR have a simple monotonic effect on the layer temperatures. Again, it may be possible to describe the sensitivity with a single characteristic number. The choice of heat release is particularly interesting since it appears to be one of the inputs to the model which has a greater effect on the model outputs than other inputs.

Simple "response-surface "correlations: A next step beyond the simple plots presented in figure 1 is a cross-plot of outputs of interest against HRR. The shaded areas on figure 2 shows the locus of individual data points representing more than 100,000 single-time values for layer temperature. For each room, a regression fit to all the data for that room overlays the locus. The temperature curves for both upper and lower layer temperature in all four rooms (figure 2) show a strong functional dependence on HRR. Even for the wide variation in inputs, the HRR provides a simple predictor of the temperature in the rooms. In addition, this relationship allows calculation of the sensitivity of the temperature outputs to the HRR inputs as a simple slope of the resulting correlation between HRR and temperature. Figure 4 shows this sensitivity for the four-room scenario studied. Except for relatively low HRR, the upper layer temperature sensitivity is less than $1 \mathrm{~K} / \mathrm{kW}$ and usually below $0.2 \mathrm{~K} / \mathrm{kW}$. Not surprisingly, the layer that the fire feeds directly is most sensitive to changes. The lower layer in the fire room and all layers in other rooms have sensitivities less than $0.2 \mathrm{~K} / \mathrm{kW}$. This implies, for example, that if the HRR for-a $1 \mathrm{MW}$ fire is known to within $100 \mathrm{~kW}$, the resulting uncertainty in the calculation
Figure 1. An example of time dependent sensitivity of fire model outputs to a $10 \%$ change in room volume for a single room fire scenario
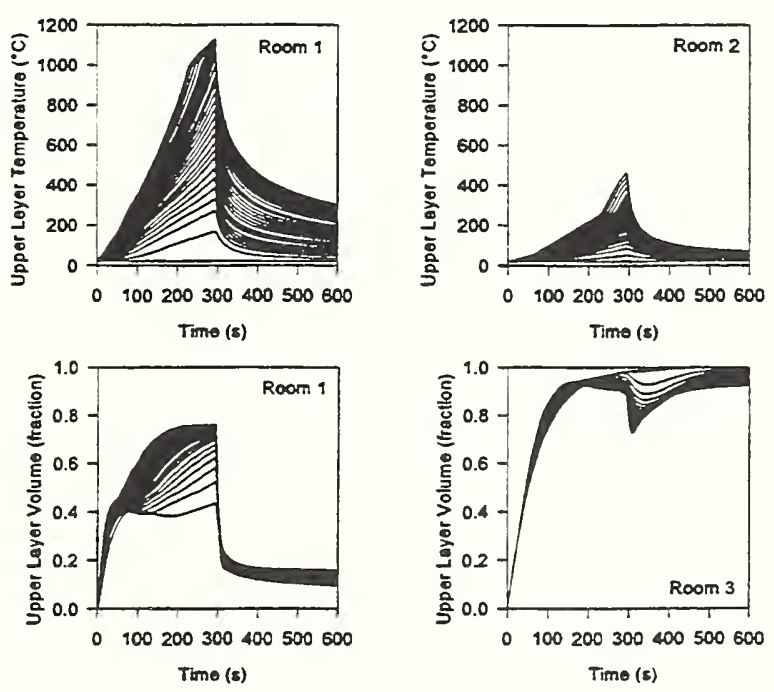

Figure 2. Layer temperatures and volumes in several rooms resulting from variation in heat release rate for a four-room growing fire scenario 
of upper layer temperature in the fire room is about $\pm 30 \mathrm{~K}$.

Evaluating sensitivity by single values:

Many phenomena of interest in fire modeling are transient events that are best represented as time history curves. Examples are HRR, gas temperature, smoke density, and CO concentration. To evaluate the sensitivity of multiple outputs, it would be desirable to have a single number to characterize each output. For the example scenario used in this paper, several choices are available. From figure (1), an average relative difference could be used. Again from figure 4, an average sensitivity calculated from a simpler model (in this case, a simple correlation) could be used. Other possibilities include time to critical events (for example, flashover), average value, or peak value.

Figure 4 presents the effect of both HRR and vent opening (in the fire room) on the upper layer temperature. In this figure, actual model calculations, normalized to the base scenario values are indicated by circles overlaid on a surface grid generated by a spline interpolation between the data points. At high HRR and small vent openings, the fire becomes oxygen limited and the temperature trails off accordingly, but for the most part, the behavior of the model is monotonic in nature. Although more laborious, the approaches used to calculate sensitivities for single variable dependencies illustrated earlier are thus equally applicable to multivariate analyses.

From the surface, it is clear that HRR has more of an effect on the peak temperature than does the vent width. Until the fire becomes oxygen limited, the trends evident in the surface are consistent with expectations temperature goes up with rising HRR and down with rising vent width. The effects are not, of course, linear with either HRR or vent opening. Plume theory and typically used calculations for estimating upper layer temperature in a single room with a fire ${ }^{16,17}$ suggest that the dependence is on the order of $q^{2 / 3}$ for HRR and $A \sqrt{h}$ for the vent opening where $A$ is the area of the vent and $h$ is the height of the vent. Although these correlations are based on a simple analysis of a single room fire, the dependence suggested is similar to that illustrated in figure 4.

\section{EVALUATION OF THE PREDICTIVE CAPABILITY OF FIRE MODELS}

Several researchers have studied the level of agreement between computer fire models and realscale fires ${ }^{2}$. These range from comparisons using simple correlations ${ }^{18}$ to intricate field models ${ }^{19}$. The comparisons made to date are mostly qualitative in nature. The level of agreement between the models and experiment is typically reported as "favorable," "satisfactory," "well predicted," "successful," =or "reasonable." This section provides an overview of some comparisons made as 
part of a program to better understand the evaluation process in concert with research to provide a level of quantification to the comparisons.

Prediction of flashover: A number of simple correlations and the CFAST model were used to simulate a range of geometries and fire conditions to predict the development of the fire up to the point of flashover. The simulation represent a range of compartment sizes from $8 \mathrm{~m}^{3}$ to $1327 \mathrm{~m}^{3}$, with ceiling height varying from $2.4 \mathrm{~m}$ to $12.2 \mathrm{~m}$ and vent openings from $10 \%$ to $100 \%$ of the length of the short wall (plus a "standard" door, $0.76 \mathrm{~m}$ in width). For most of the simulations, the surface lining material was gypsum wallboard, $12.7 \mathrm{~mm}$ in thickness, consistent with the values used in the correlations. A simple constant fire size was varied until the calculated upper layer temperature reached $600{ }^{\circ} \mathrm{C}$ at the end of the simulation. For some simulations, the surface linings ranged from aluminum to

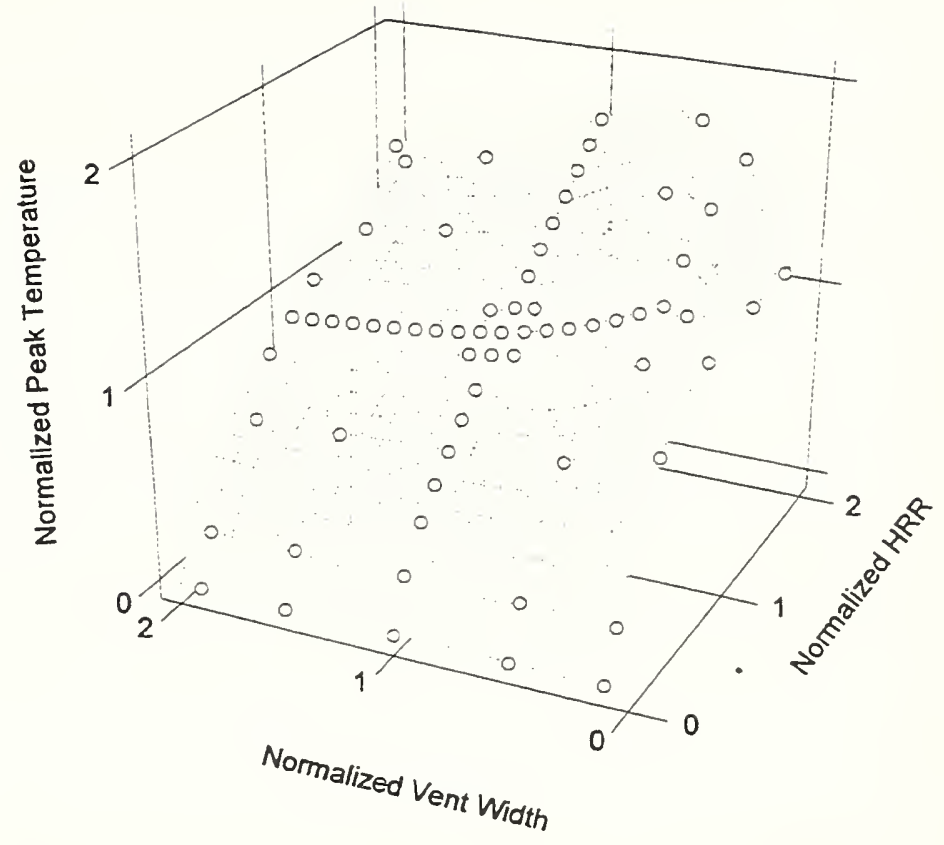
a highly insulating foam and the fire source diverged from the simple steady-state fire to more complex shapes.

The important test of all these prediction methods is in the comparison of the predictions with actual fire observations. Figure 6 presents estimates of the minimum energy required to achieve flashover for a range of room and vent sizes. This figure is an extension of the earlier work of Babrauskas ${ }^{17}$ and includes additional experimental measurements from a variety of sources, most notably the work of Deal and Beyler ${ }^{18}$. In addition, it includes predictions from a current generation zone fire model, CFAST that will be discussed in more detail below.

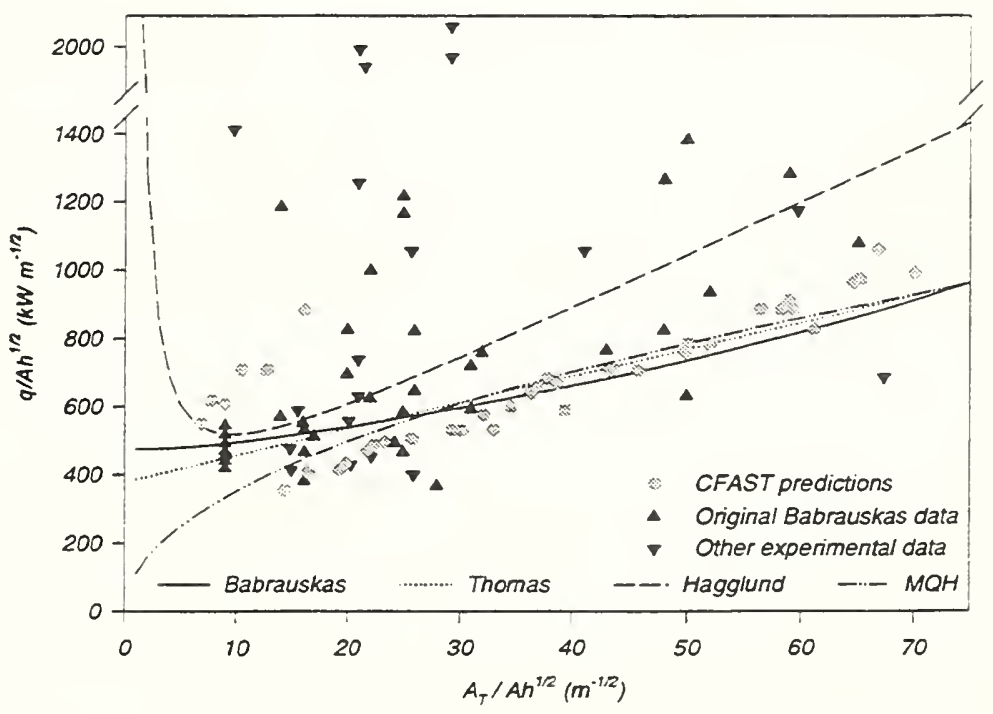

Figure 6. Comparison of correlations, CFAST predictions, and experimental data for the prediction of flashover in a compartment fire.

As with some of the experimental data defining flashover as an upper layer temperature reaching $600^{\circ} \mathrm{C}$, many experimental measures were reported as peak values rather than minimum values necessary to achieve flashover. Thus, ideally all the predictions should provide a lower bound for the experimental data. Indeed, this is consistent with the graph - the vast majority of the experimental observations lie above the correlations and model predictions. For a considerable range in the ratio $A_{T} / A \sqrt{h}$, the correlations of Babrauskas, Thomas, and McCaffrey, Quintiere, 
and Harkelroad provide nearly identical estimates of the minimum energy required to produce flashover. The estimates of Hägglund yields somewhat higher estimates for values of $A_{T} / A \sqrt{h}$ greater than 20.

The results from the CFAST model for this single compartment scenario provide similar results to the experiments and the correlations for most of the range of $A_{T} / A \sqrt{h}$. For small values of $A_{T} / A \sqrt{h}$, the CFAST values rise somewhat above the values from the correlations. These small values of $A_{r} / A \sqrt{h}$ result from either very small compartments (small $A_{T}$ ) or very large openings (large $A \sqrt{h}$ ), both of which stretch the limits of the assumptions inherent in the model. For very small compartments, radiation from the fire to the compartment surfaces becomes more important, enhancing the conductive heat losses through the walls. However, the basic two-zone assumption may break down as the room becomes very small. For very large openings, the calculation of vent flow via an orifice flow coefficient approach is likely inaccurate. Indeed, for such openings, this limitation has been observed experimentally ${ }^{17}$. Still, the estimates are close to the ranges provided by the correlations which also diverge at very small values of $A_{T} / A \sqrt{h}$.

Perhaps most significant in these comparisons is that all the simple correlations provide estimates similar to the CFAST model and all the models are consistent with a wide range of experimental data. For this simple scenario, little is gained with the use of the more complex models. For more complicated scenarios, the comparison may not be as simple.

Other comparisons: Arguably the most frequent question asked about a fire is "How hot did it become?" Temperature in the rooms of a structure is an obvious indicator to answer this question. Peak temperature, time to peak temperature, or time to reach a chosen temperature tenability limit are typical values of interest. Papers by Peacock, Jones, and Bukowski ${ }^{2}$, Beard ${ }^{19}$, Deal and Beyler ${ }^{18}$, and Reneke et.al. ${ }^{20}$ are illustrative.

Figure 7 shows a comparison of measured and predicted upper layer temperature for several tests studied ${ }^{2}$. For the single-room tests, predicted temperatures show obvious similarities to the measured values. Peak values occur at similar times with comparable rise and fall for most comparisons. Peak values are typically higher for upper layer temperature and lower for lower layer temperature and layer interface position. For all the tests, including the singleroom tests, times to peak values and times to $100^{\circ} \mathrm{C}$ predicted by the model average within $25 \mathrm{~s}$ of experimentally measured values.

Systematic deviations exist for the remaining three data sets. Differences between model predictions and experimental measurements change monotonically over time (rising for the three-room test and falling for the fourrooms tests. Modeling of heat conduction
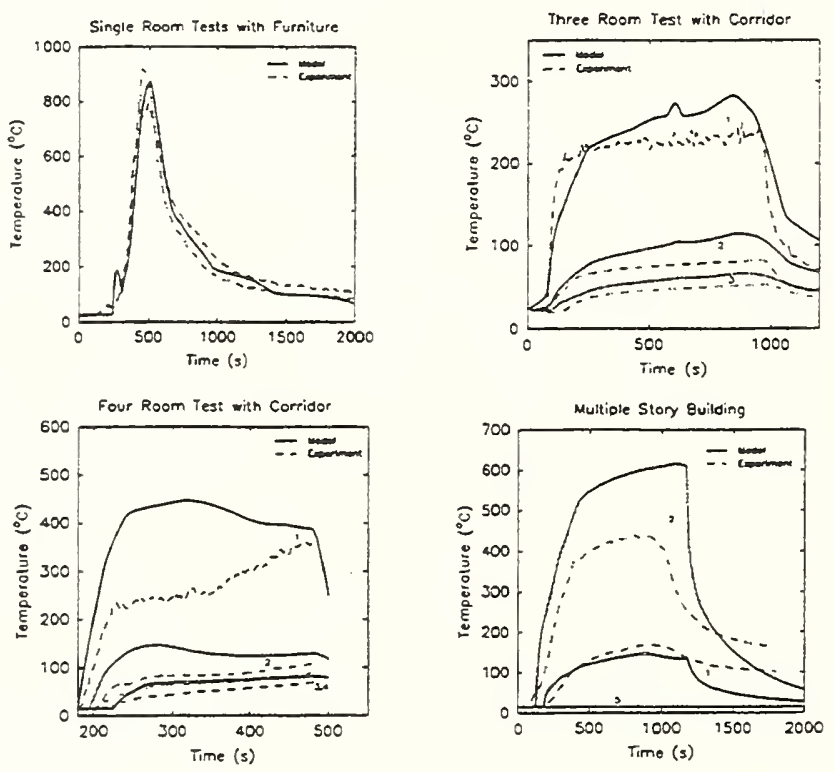

Figure 7. Comparison of measured and predicted upper layer temperatures for several tests. (Numbers indicate comparable rooms in the test structure.)

(losing too much or too little heat to the

surfaces) or lack of modeling of leakage (rooms are presumed perfectly sealed unless vents are included to simulate leakage) may account for the trends.

In general, upper layer temperatures predicted by the model are higher than experimental measurements, with the differences ranging from -46 to $230^{\circ} \mathrm{C}$. Conversely, the lower layer 
temperature is somewhat lower for the model than for the experiments $\left(-60^{\circ} \mathrm{C}\right.$ to $\left.5^{\circ} \mathrm{C}\right)$. Presuming conservation of energy (an underlying assumption in all fire models), these observations are consistent. Limitations inherent in the model also account partially for these trends. In the current version of CFAST, energy exchange in the lower layer is only by mixing or convection from surfaces. Adding radiative exchange to the lower layer would reduce the upper layer temperature and increase the lower layer temperature. Layer interface position is primarily affected by entrainment by the fire or at vents. Plume entrainment in CFAST is based on the work of McCaffrey on circular plumes in relatively small spaces. For large fires in small spaces where the fire impinges on the ceiling (such as the single room tests with wall burning) or very small fires in large spaces (such as atria), these correlations may not be as valid.

Several areas which need additional research are apparent in order to be able to perform broader analyses:

- Presenting the results of a sensitivity analysis - For a complex fire model with $m$ inputs and $n$ outputs, a complete sensitivity analysis will result in a matrix of $m \times n$ time series. It is unlikely that this much information will be of general use. It may be appropriate to develop threshold values for important outputs to alert the model user of particularly sensitive effects for a given test case.

- Calculating sensitivity functions for a complex fire model - In order to apply analytical techniques for sensitivity analysis of a complex fire model, the sensitivity equations need to be included in the equation set solved directly by the model. Even though it is desirable to obtain an overall picture of model performance, the broad range of application of current models demonstrates that whatever range of study is chosen, applications outside this envisioned range will continue to be of interest.

- Statistical treatment of the data - presentation of the differences between model predictions and experimental data to date have been intentionally simple. With a significant base of data to study, appropriate statistical techniques to provide a true measure of the "goodness of fit" should be investigated.

Experimental measurements - Most interest in applying current generation models is in a non-residential setting. Little experimental data is available for these scenarios.

Measurement of leakage rates, room pressure, or profiles of gas concentration are atypical in experimental data. These measurements are critical to assessing the accuracy of the underlying physics of the models or of the models ability to predict toxic gas hazard.

\section{CONCLUSIONS}

This paper has presented a number of alternatives for evaluating both simple and complex room fire models. For the models and test cases examined, the heat release rate is dominant in determining the behavior of the models. Other model inputs, including room volume and vent size have lesser effects on a range of predicted ouputs.

Comparison of model prediction with available experimental measurements show agreement and differences which are understandable given the limitations inherent in the models and experiments. Although a range of applications have been reported in the literature, to use the models with assurance for any purpose, the user must understand the underlying science, assumptions, and limitations inherent in the models and from this decide the applicability for a particular application. Such an understanding allows the trained professional to assure use of the models where applicable does not exceed the capabilities of a particular model. 


\section{REFERENCES}

1. Peacock, R. D., Reneke, P. A., Forney, C. L., and Kostreva, M. M., Sensitivity Analysis for Complex Fire Models, to be published.

2. Peacock, R. D., Jones, W. W., and Bukowski, R. W., Verification of a Model of Fire and Smoke Transport, Fire Safety J. 21, 89-129 (1993).

3. Jarvis, J. P., Kostreva, M. M., Forney, C. L., Tools for Validation and Analysis of Fire Models, Combustion Instiute/Eastern States Section. Chemical and Physical Processes in Combustion. 20th Fall Technical Meeting. Abstracts. November 2-5, 1987, Gaithersburg, MD, 103/1-4 pp. 1987.

4. Khoudja, N., Procedures for Quantitative Sensitivity and Performance Validation of a Deterministic Fire Safety Model. Ph.D. Dissertation, Texas A\&M University, NBS-GCR-88-544, Natl. Inst. Stand. Technol. 1988.

5. Box, G. E. P., Hunter, W. G., and Hunter, J. S., Statistics for Experimenters, An Introduction to Design, Data Analysis and Model Building, John Wiley \& Sons (1978).

6. Daniel, C., Applications of Statistics to Industrial Experimentation, John Wiley \& Sons (1976).

7. Ndubizu, C. C., Ramaker, D. E., Tatem, P. A. and Williams, F. W., The Sensitivity of Various Physical Parameters Upon Fire Model Predictions. Proceedings of Chemical and Physical Processes in Combustion, 1982 Technical Meeting, The Eastern Section of the Combustion Institute, December 14-16, Atlantic City, New Jersey (1982).

8. Standard Guide for Evaluating the Predictive Capability of Fire Models, ASTM E 1355, Annual Book of ASTM Standards, Vol. 04.07, American Society for Testing and Materials, Philadelphia (1990).

9. Forney, G. P., and Moss, W. F., Analyzing and Exploiting Numerical Characteristics of Zone Fire Models. NISTIR 4763, Natl. Inst. Stand Technol. 1992.

10. Peacock, R. D., Forney, G. P., Reneke, P. A., Portier, R. W., and Jones, W. W., CFAST, the Consolidated Model of Fire and Smoke Transport, Natl. Inst. Stand. Technol., Tech. Note 1299, 104 p (1993).

11. NFPA 72, National Fire Alarm Code, National Fire Protection Association, Quincy, Massachusetts (1993).

12. Babrauskas, V. and Krasny J. F., Fire Behavior of Upholstered Furniture, Natl. Bur. Stand. (U. S.), Monograph 173 (1985).

13. Peacock, R. D., Davis, S., and Babrauskas, V., Data for Room Fire Model Comparisons, J. Res. Natl. Inst. Stand. Technol. Vol. 96, 4, 411-462 (1991).

14. Drysdale, D. An Introduction to Fire Dynamics, John Wiley and Sons, pp. 310 (1985).

15. Iman, R. L. and Helton, J. C., An Investigation of Uncertainty and Sensitivity Analysis Techniques for Computer Models, Risk Analysis, Vol. 8, No. 1, 71-90 (1988).

16. McCaffrey, B. J., Quintiere, J. G., and Harkleroad, M. F., Estimating Room Temperatures and the Likelihood of Flashover Using Fire Tests Data Correlations, Fire Technology, Vol. 17, No. 2, 98-119 (1981).

17. Babrauskas, V., Upholstered Furniture Room Fires - Measurements, Comparisons with Fumiture Calorimeter Data, and Flashover Predictions, J. Fire Sci., Vol. 2, 5-19 (1984).

18. Deal, S. and Beyler, C., Correlating Preflashover Room Fire Temperatures, J. of Fire. Prot. Engr.., 2 (2), $33-48$. 1990.

19. Beard, A., "Evaluation of Deterministic Fire Models: Part I - Introduction," Fire Safety J. $19295-3061992$.

20. Reneke, P. A., Peatross, M. J., Jones, W. W., Beyler, C. L., and Richards, B., A Comparison of CFAST

Predicctions to USCG Real-scale Fire Tests, to be published. 


\section{Discussion}

Patrick Pagni: We decided that comparison with data was the major task for the computational models for this meeting. While sensitivity analyses are interesting, I don't think they accomplish that goal. Let me give you a negative comparison since you didn't think there were any out there. We have compared the results of CFAST for the temperature in the upper layer in a long, steady heat release rate experiment and we find consistent overprediction of the upper layer temperature. In the comparisons you showed us today, I think I saw the same trend. I cannot be sure since you didn't dwell on the temperature comparison, but could you tell me if that was the case?

Richard Peacock: Overprediction of temperature by CFAST is fairly classic. We are aware of that and need to look at it. We suspect it's related to two things: one is plume entrainment, which drives practically everything else in the model; and the second is mixing between the layers, because going along with this overprediction of upper layer temperature is an underprediction of lower layer temperature. That's somewhat forced by the mass balance. The dichotomy of overprediction of upper layer temperature and underprediction of lower layer temperature is somewhat forced by mass and energy balance in a compartment. It's something that we are addressing.

Patrick Pagni: I am very happy that you agree with the phenomenon. Let me give you another mechanism. We think there may be something wrong with the heat transfer algorithm at the wall. By running several different programs, we thought we were able to determine that the plume entrainment was correct, but there was something wrong with the wall heat transfer. The paper will appear in the forthcoming issue of the Fire Safety Journal.

Walter Jones: I asked Nick to send the data along when the paper's published. We are certainly interested in finding problems in the model. That's a general request which I will make later today in talking about the database for comparison of models and data. In order to fix problems in models of that sort, one has to have the relevant data sets which show the discrepancies.

John Hall: As a statistician, I tend to think first of all of statistical checks for goodness of fit of mean squared deviation. I'm wondering what you might have done to pursue that concept's applicability here.

Richard Peacock: We have looked at it. The catch is not so much what technique do I use for a specific problem, but rather the fact that I have roughly an infinite amount of data that I'd like to look at and from the offset, don't know what that data looks like. So if we would like to look at an arbitrary problem, before we know what that problem looks like, we'd like to know what appropriate statistical treatment to use. It's sort of a problem of the cart following the horse: I can choose an appropriate technique if I know the problem I'm interested in but not necessarily vice versa. 
$\underline{9} \underline{0}$ 


\section{EXPERIMENTS ON SMOKE BEHAVIOR IN CAVITY SPACES}

Part 3 In case of the cavity space which has an opening at the bottom

\section{Takeyoshi TANAKA}

Building Research Institute, Ministry of Construction

1 Tatehara, Tsukuba-shi, Ibaraki-ken, 305 Japan

Teruhisa FUKUDA and Takao WAKAMATSU

Science University of Tokyo

2641 Yamasaki, Noda-shi, Chiba-ken, 278 Japan

\section{ABSTRACT}

It was demonstrated by the previous experiments using the cavity space which has no bottom opening that the temperature rise of plume at the height of the cavity opening is well scaled by the nondimensional temperature defined as $\Theta \equiv\left(\overline{\Delta T} / T_{\infty}\right) / \dot{Q}^{-2 / 3}$ where $\dot{Q}^{*} \equiv \dot{Q} / \rho_{\infty} C_{p} T_{\infty} \sqrt{g D} D^{2}$, and the temperature is successfully correlated by $\Theta=\alpha(H / D)^{\beta}$, where $\mathrm{b}=-5 / 3,-1$ and $-1 / 3$ for shallow, intermediate and deep cavity, respectively.

In this study, the smoke behavior in cavity spaces is further investigated by the small scale experiments for the cavity space which has an opening at the bottom. It was found that the larger the area of the bottom opening, the more stable the fire plume, but still the temperature is well correlated by the same manner as in the case of cavity with no bottom opening. Also, the effects of bottom opening on the temperature and the pressure difference produced in the cavity are analyzed.

KEYWORDS: smoke behavior, cavity space, fire plume, bottom opening, plume temperature, pressure difference, air inflow rate

\section{INTRODUCTION}

The primary issue of cavity spaces of buildings concerning fire safety is the potential hazard that such spaces may become a dominant passage of smoke spread throughout the buildings. Some means to predict the smoke behavior in cavity spaces will be necessary for rational measures for evacuation safety in the buildings having such cavity like spaces.

In the previous papers, the formulas for predicting the temperature elevation of the plumes as a function of heat release rate of fire and cavity dimensions were obtained for the cases where no opening exist at the bottom of the space. On the other hand, some cavity spaces in buildings have openings near the bottom. In Japan, building officials and fire department suspect that the air supply through such an opening contribute to mitigate the smoke hazard, so tend to insist on the equipment of opening for the cavity space exceeding certain depth. However, virtually no solid data is available for assessing how effective the openings are or how large they should be. 
While all the air entrained to a fire plume is supplied through the opening at the top in case of the cavity having no bottom opening, the air will be supplied both from the top and the bottom in case of the cavity having bottom openings. The portion of the air supplied from the bottom may increase as the area of the bottom opening increases. Consequently, the existence of a bottom opening may significantly affect the smoke behavior in a cavity. In this study, reduced scale experiments are conducted for the cases where an opening exists at the bottom of a cavity, and the effects of the bottom opening size on the plume temperature and the pressure profile in the cavity are investigated.

\section{EXPERIMENTAL SETUP}

The cavity space model used in this experiment is the same as in the preceding experiments[1]: It has the square floor of $0.8 \mathrm{mx} 0.8 \mathrm{~m}$ whose height can be changed from $0 \mathrm{~m}$ to $3 \mathrm{~m}$ using wires and a pulley. The tests are carried out for the depth of the cavity increased by every $0.25 \mathrm{~m}$ from $0.25 \mathrm{~m}$ to $3.0 \mathrm{~m}$.

A $7 \mathrm{~cm}$ diameter diffusion burner is used as the fire source and the heat release rate is set at $0.5,1.0$, $2.0,3.0$ and $4.0 \mathrm{~kW}$ according to the test conditions by adjusting the rate of supply of methane. The fire source in this experiment is set on the center of the floor, as shown in Figure 1.

The bottom opening in each test is arranged on the floor about uniformly around the fire source as shown in Figure 1 to maintain symmetric condition as best as possible and also to avoid the brown down of flames due to the draft induced through the opening. The area of the opening is $0.03,0.067$ or $0.163 \mathrm{~m} 2$ depending on the test conditions.
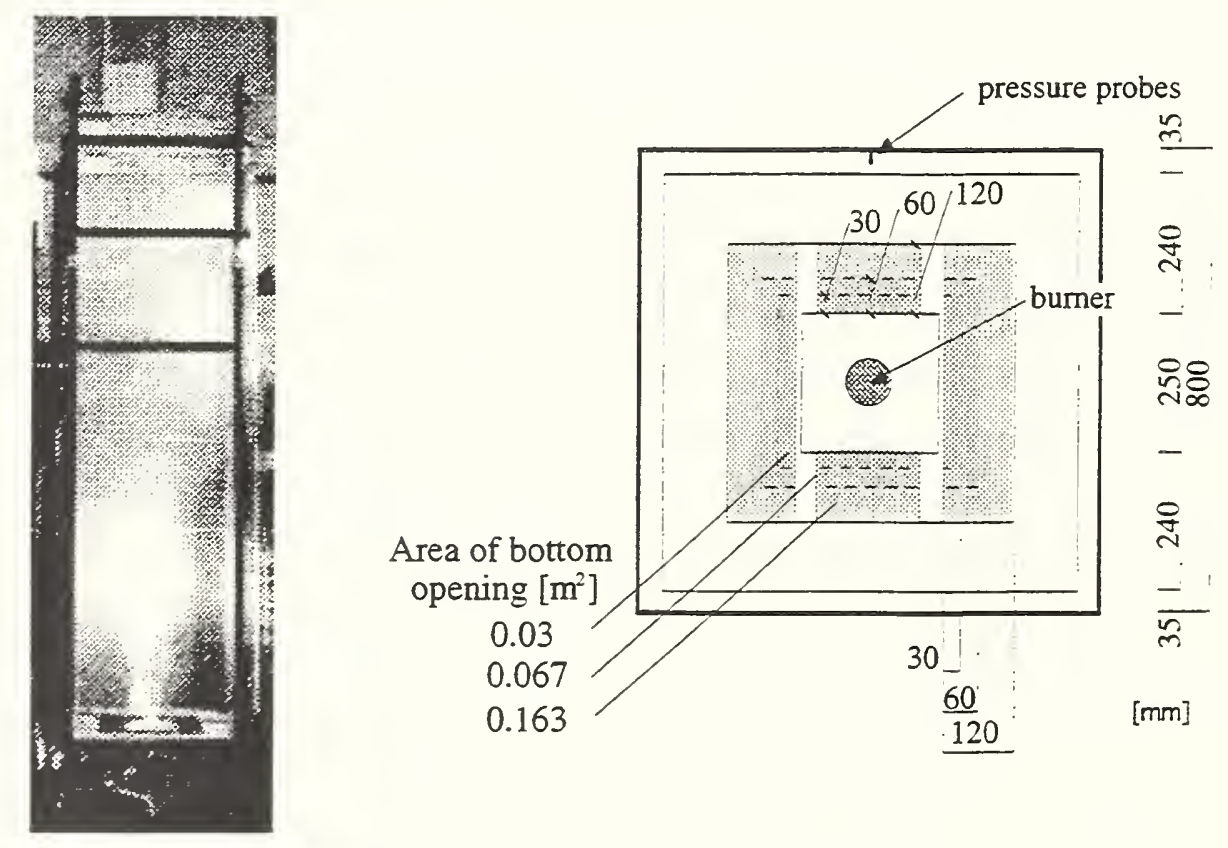

Figure 1 Arrangement of Bottom Opening 
The measurements made are also the same as in Ref.[1]: 85 thermocouples are arrayed about uniformly over the opening at the top of the space to measure the temperatures of the gases flowing out of and the air flowing into the space; Pressure probes arranged at 10 positions on the rear wall for the measurement of the pressure difference between the inside of the cavity and the outside of the cavity space. The data acquisition is started 15 minutes after the ignition of the fire source and in each condition, the data recording is carried out for 5 minutes with 5 second interval.

\section{RESULTS OF THE EXPERIMENTS}

\subsection{Temperature Profile at Top Opening}

Figure 2 compares the typical temperature profiles at the opening at the top of the cavity for different area of the bottom opening. The heat release rate of the source for the examples in Figure 2 is $2.0 \mathrm{~kW}$ and the cavity depth is $0.5 \mathrm{~m}$ or $3.0 \mathrm{~m}$.

As can be seen from the figures for $H=0.5 \mathrm{~m}$, the larger the bottom opening area, the steeper the temperature profile, when cavity depth is small. On the other hand, the larger the bottom opening area, the more dull the temperature profile tend to be, when cavity depth is large.
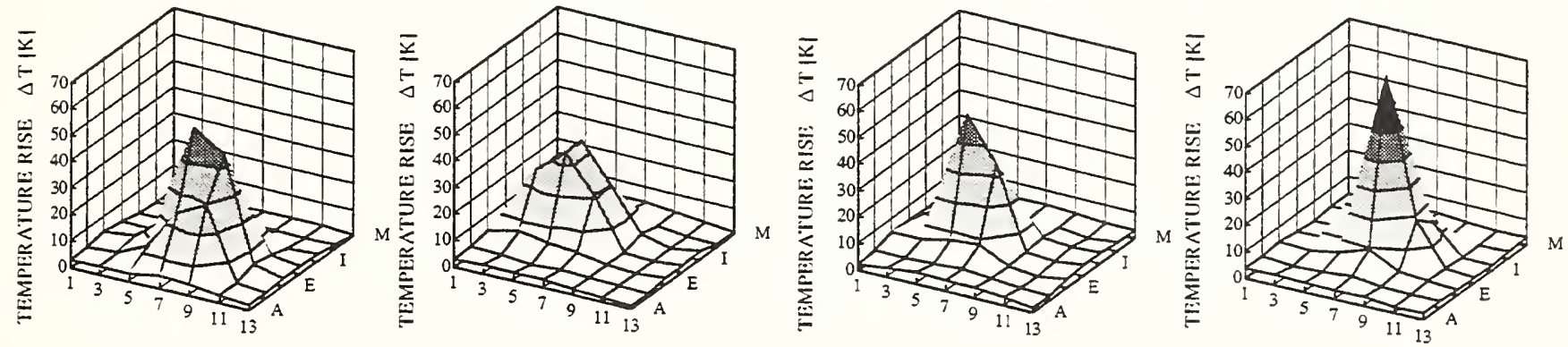

$$
H=0.5 \mathrm{~m} \quad \dot{Q}=2.0 \mathrm{~kW}
$$
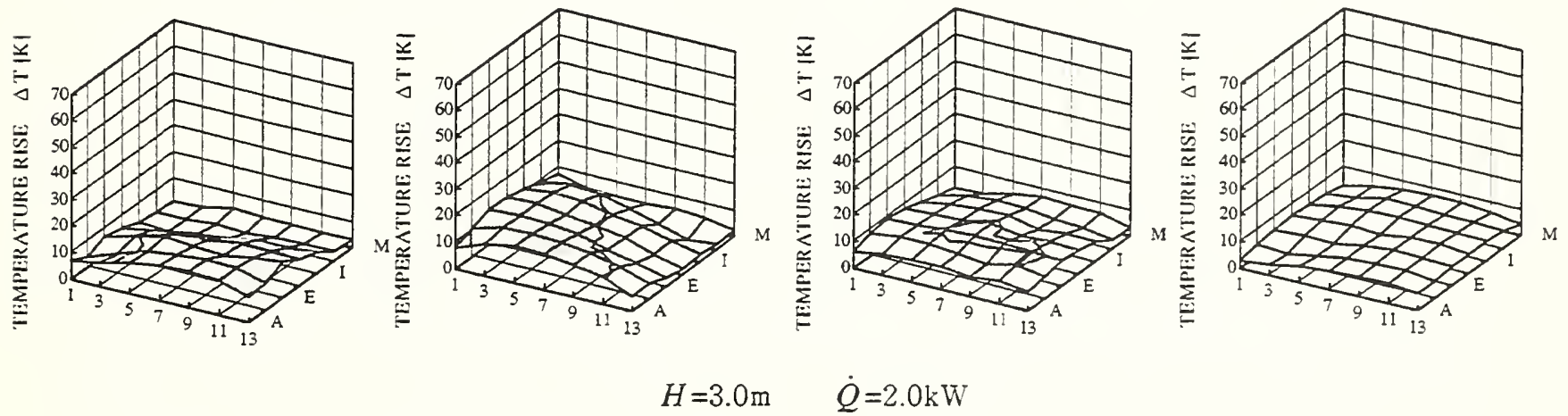

$$
H=3.0 \mathrm{~m} \quad \dot{Q}=2.0 \mathrm{~kW}
$$

$$
\begin{aligned}
& A_{\text {borom }}=0.00\left[\mathrm{~m}^{2}\right] \\
& A_{\text {borom }}=0.03\left[\mathrm{~m}^{2}\right] \\
& A_{\text {bortom }}=0.067\left[\mathrm{~m}^{2}\right] \\
& A_{\text {bonom }}=0.163\left[\mathrm{~m}^{2}\right]
\end{aligned}
$$

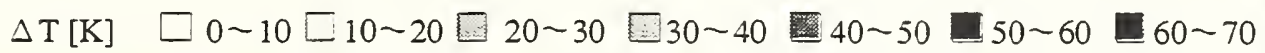




\subsection{Fluctuation of Plume Axis}

Figure 3 shows some examples of the frequency that each of the 85 thermocouples arrayed at the cavity opening recorded the highest temperature in the 60 times measurements, which were made during the 5 minutes of the data acquisition period. The examples are taken from the cases where the heat release rate is $2.0 \mathrm{~kW}$ and the cavity depth is $1.0 \mathrm{~m}$ and $2.0 \mathrm{~m}$.

Here we assume that the plume axis exists around the position of the thermocouples at which the highest temperature is recorded. It can be seen from Figure 3 that the increase of the bottom opening area contributes to he stability of the location of the fire plume axis. This effect is particularly remarkable when the cavity depth is large, but still apparent when the cavity depth is small.
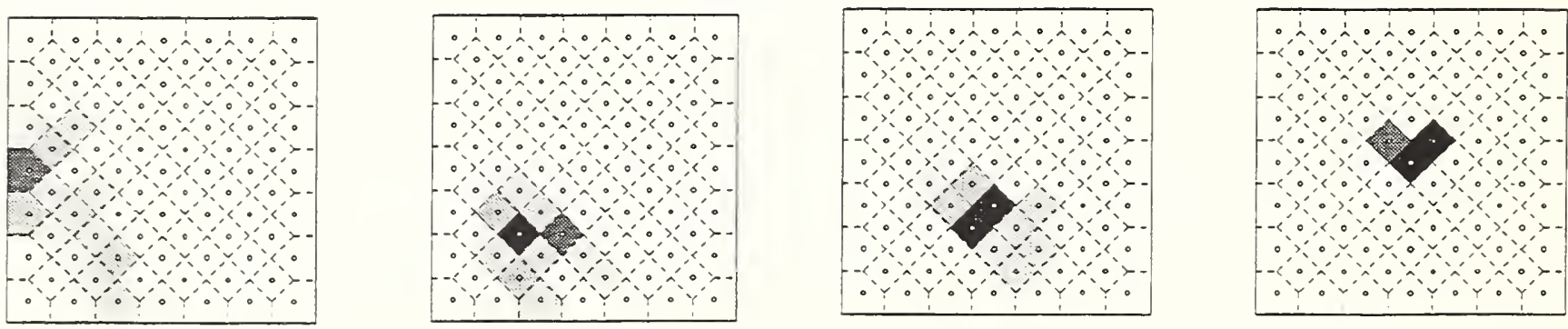

$$
H=1.0 \mathrm{~m}
$$

$\dot{Q}=2.0 \mathrm{~kW}$
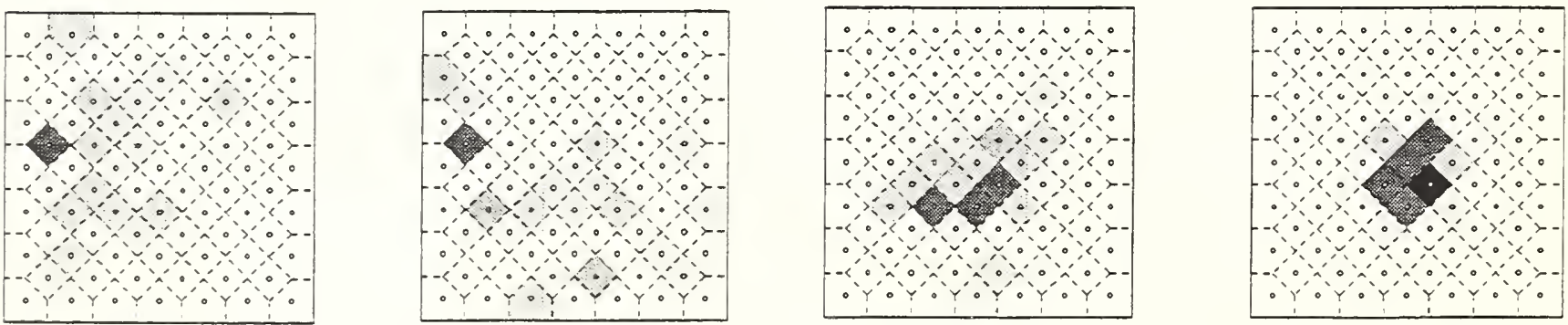

$$
H=2.0 \mathrm{~m} \quad \dot{Q}=2.0 \mathrm{~kW}
$$

$$
A_{\text {bortom }}=0.00\left[\mathrm{~m}^{2}\right] \quad A_{\text {bortom }}=0.03\left[\mathrm{~m}^{2}\right]
$$

\begin{tabular}{|c|c|c|c|}
\hline $0 / 60$ & $2 / 60$ & $6 / 60 \sim 9 / 60$ & $15 / 60 \sim$ \\
\hline $1 / 60$ & $2 / 60 \sim 5 / 60$ & $10 / 60 \sim 14 / 60$ & \\
\hline
\end{tabular}

$$
A_{\text {bonom }}=0.067\left[\mathrm{~m}^{2}\right]
$$$$
A_{\text {borrom }}=0.163\left[\mathrm{~m}^{2}\right]
$$

FIGURE 3 Fluctuation of Plume Axis 


\subsection{Vertical Profile of Pressure Difference}

Figure 4 shows the profile of the pressure difference which develops between the inside and the outside of the $3.0 \mathrm{~m}$ deep cavity space. Each value of the pressure difference is the average of the 60 times of data recordings in each test. The pressure difference of the cavity space relative to the outside space is nearly zero or slightly negative at the height of the cavity opening and increases about proportionally to the distance from the opening, but the increase seems to deter around the middle height of the cavity.

The pressure difference increases with the heat release rate when the area of the bottom opening is the same, and decreases as the bottom opening area increases if the heat release rate is the same.

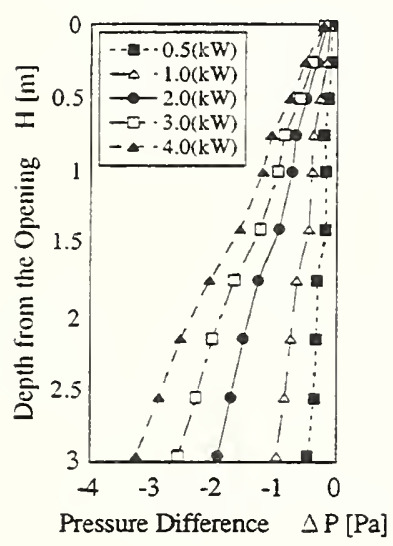

$$
A_{\text {borom }}=0.00\left[\mathrm{~m}^{2}\right]
$$

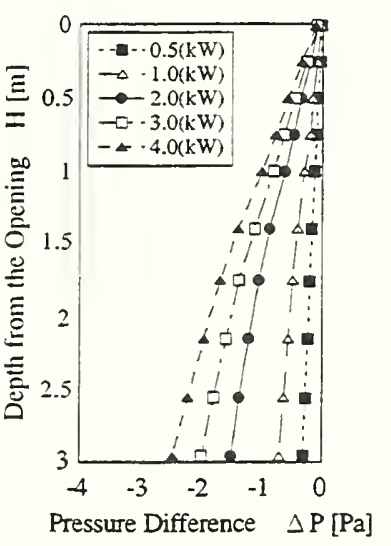

$$
A_{\text {borrom }}=0.03\left[\mathrm{~m}^{2}\right]
$$

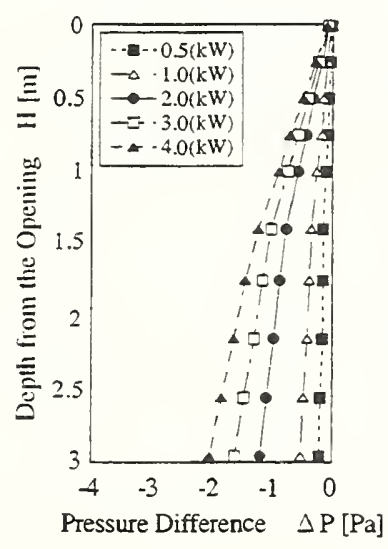

$A_{\text {borom }}=0.067\left[\mathrm{~m}^{2}\right]$

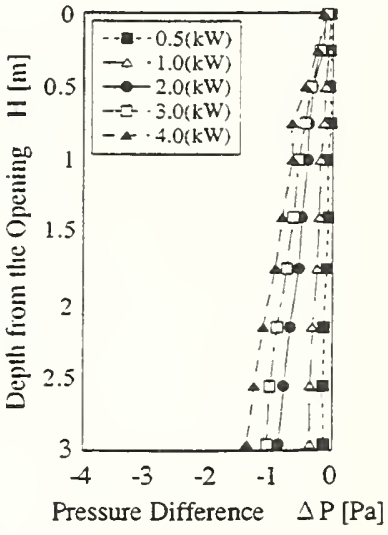

$A_{\text {bonom }}=0.163\left[\mathrm{~m}^{2}\right]$

FIGURE 4 Pressure Difference

\subsection{Plume Area}

The proportion of the plume horizontal area at the height of the opening of the cavity to the floor area of the cavity (plume area ratio) is plotted versus the depth of the cavity in Figure 5. The plume area was assumed as the area in which the thermocouple readings fall in the condition as follows:

$$
\Delta T \geq\left(T_{\max }-T_{\infty}\right) \times k
$$

with $k=0.25$, where $T_{\max }, T_{\infty}$ and $\Delta T$ are the highest temperature reading of the thermocouples, the ambient air temperature and the temperature difference of a thermocouple from the ambient air.

Similarly with the findings previously reported for the cavity space having no bottom opening, the plume area does not depend on the heat release rate and tends to increase with the cavity depth $H$ approximately in proportional to $H^{2}, H^{1}$ and $H^{0}$ when the depth is small, intermediate and large, respectively. The plume area ratio for large cavity depth tends to increase with the increase of the bottom opening area, but the depth at which the transition from intermediate to deep region takes place seem to increase. 

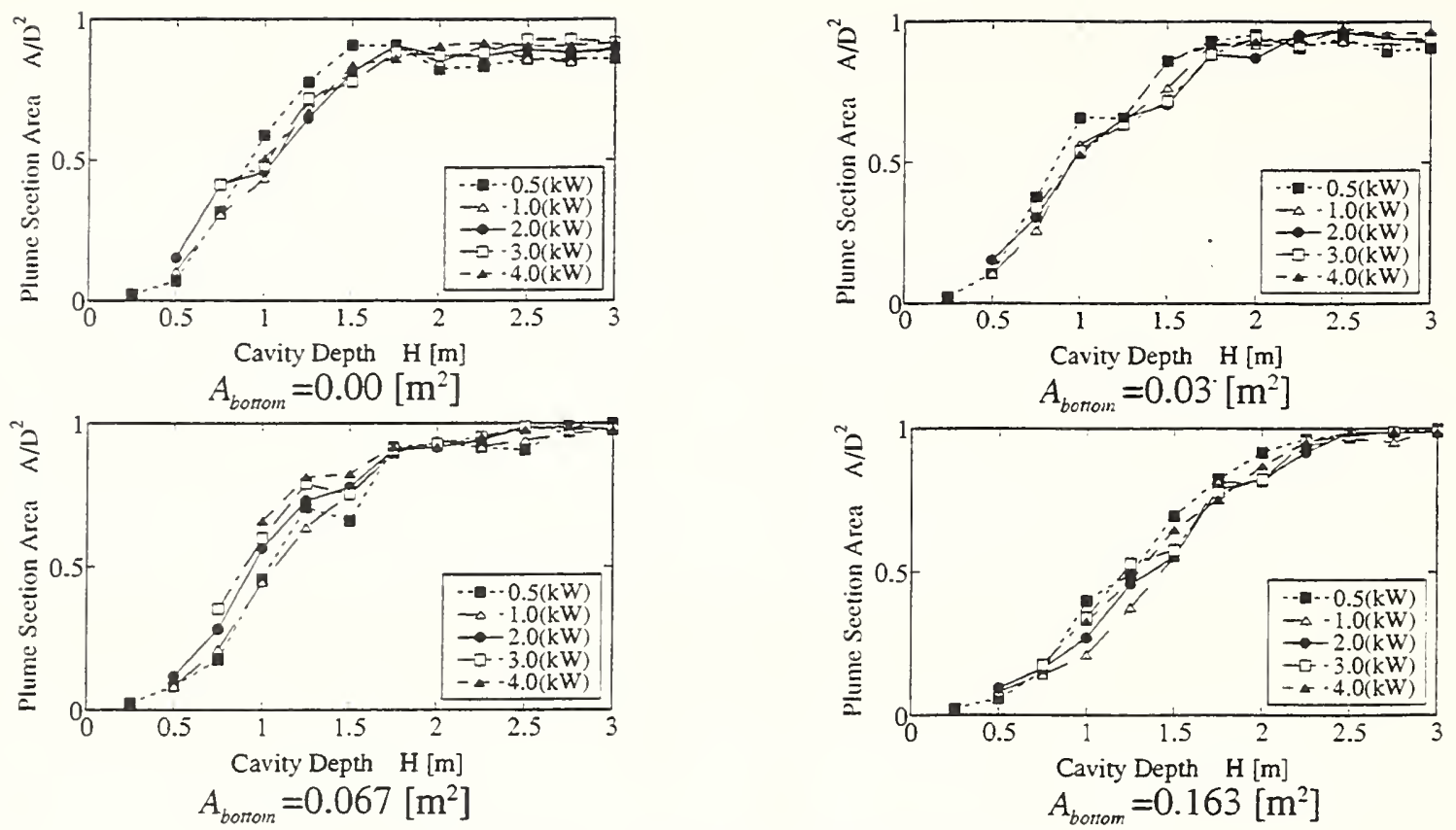

FIGURE 5 Plume Section Area A/D²

\subsection{Average Temperature Rise of the Fire Plume}

The average temperature within the plume area defined by Eqn.(1) with $k=0.25$ is plotted versus the depth of the cavity in Figure 6. Each value of the temperatures in the figure is the average of the plume average temperatures for the 5 minutes of data acquisition period, each of which is obtained at every 5 seconds by averaging the temperatures within the plume area that is defined at each time according to Eqn.(4).

A similar tendency is observed in the temperature rise regardless the difference in bottom opening area as shown in Figure 7, i.e.: the temperatures fall significantly with the cavity depth while the depth is small, but it changes only slightly when the depth is large. The larger the bottom opening area, the lower the temperatures for large depth seem, if the heat release rate is the same.
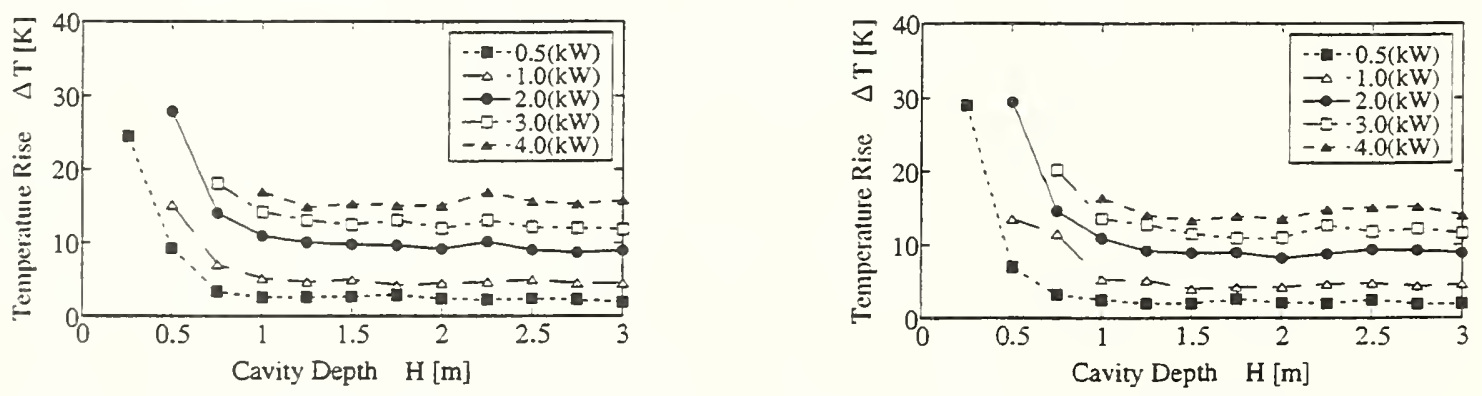

$A_{\text {borom }}=0.00\left[\mathrm{~m}^{2}\right]$

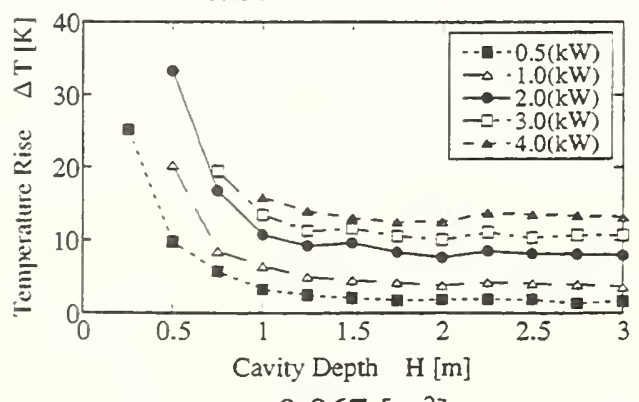

$A_{\text {bonom }}=0.03\left[\mathrm{~m}^{2}\right]$

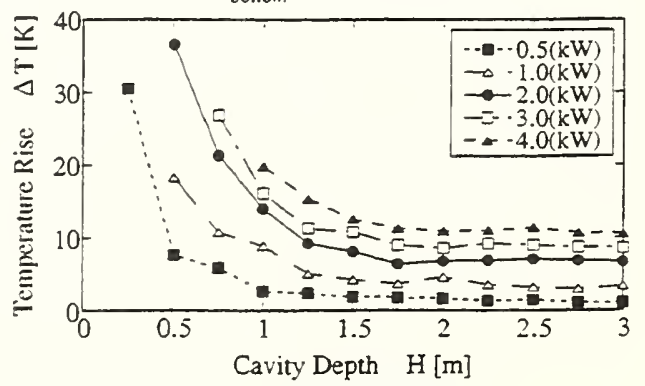

$A_{\text {borom }}=0.067\left[\mathrm{~m}^{2}\right]$ 


\section{EQUATIONS FOR PLUME TEMPERATURE}

In the preceding paper[1],[2], it was theoretically predicted and experimentally verified for the cavity having no bottom opening that the temperature of the fire plume in cavity spaces is well correlated as

$$
\Theta=\alpha(H / D)^{\beta}
$$

where $\beta$ is $-5 / 3,-1$ and $-1 / 3$ for shallow, intermediate and deep cavity, respectively, $\Theta$ is the nondimensional temperature defined as

$$
\Theta \equiv\left(\overline{\Delta T} / T_{\infty}\right) / \dot{Q}^{* 2 / 3}
$$

and $\dot{Q}^{*}$ is the nondimensional heat release rate defined as

$$
\dot{Q}^{*} \equiv \dot{Q} / \rho_{\infty} C_{P} T_{\infty} \sqrt{g D} D^{2}
$$

Since it is considered that essentially the same theoretical consideration holds for the cavities with bottom openings, the equations for correlating the plume temperature are derived having the same relationship as Eqns.(2) -(4) in mind.

\subsection{Equations for Plume Average Temperature}

The plume average temperature here means the average of the temperatures within the area that is defined by Eqn.(1) with $k=0.25$. The temperatures are nondimensionalized in the form of Eqn.(2) and plotted versus $H / D$ as shown in Figure 7. The solid and the broken lines in the figures indicate the regression lines when the theoretical and the experimental values are employed for $\beta$ in Eqn.(2), respectively. The values $\alpha$ and $\beta$ of the theoretical and experimental regression equations are summarized in Table 1 . Note, however, that a cannot be theoretically obtained in either case. The values of $\alpha$ in the column of "theoretical value" are in reality the experimental values that fit well with the test data provided the theoretical $\beta$ is used. Since it does not make meaningful difference in the accuracy whichever of the values may be used for $\beta$, as is recognized from the insignificant difference between the solid and broken lines, the equations adopting the theoretical $\beta$ may be sufficiently adequate for plume average temperature.
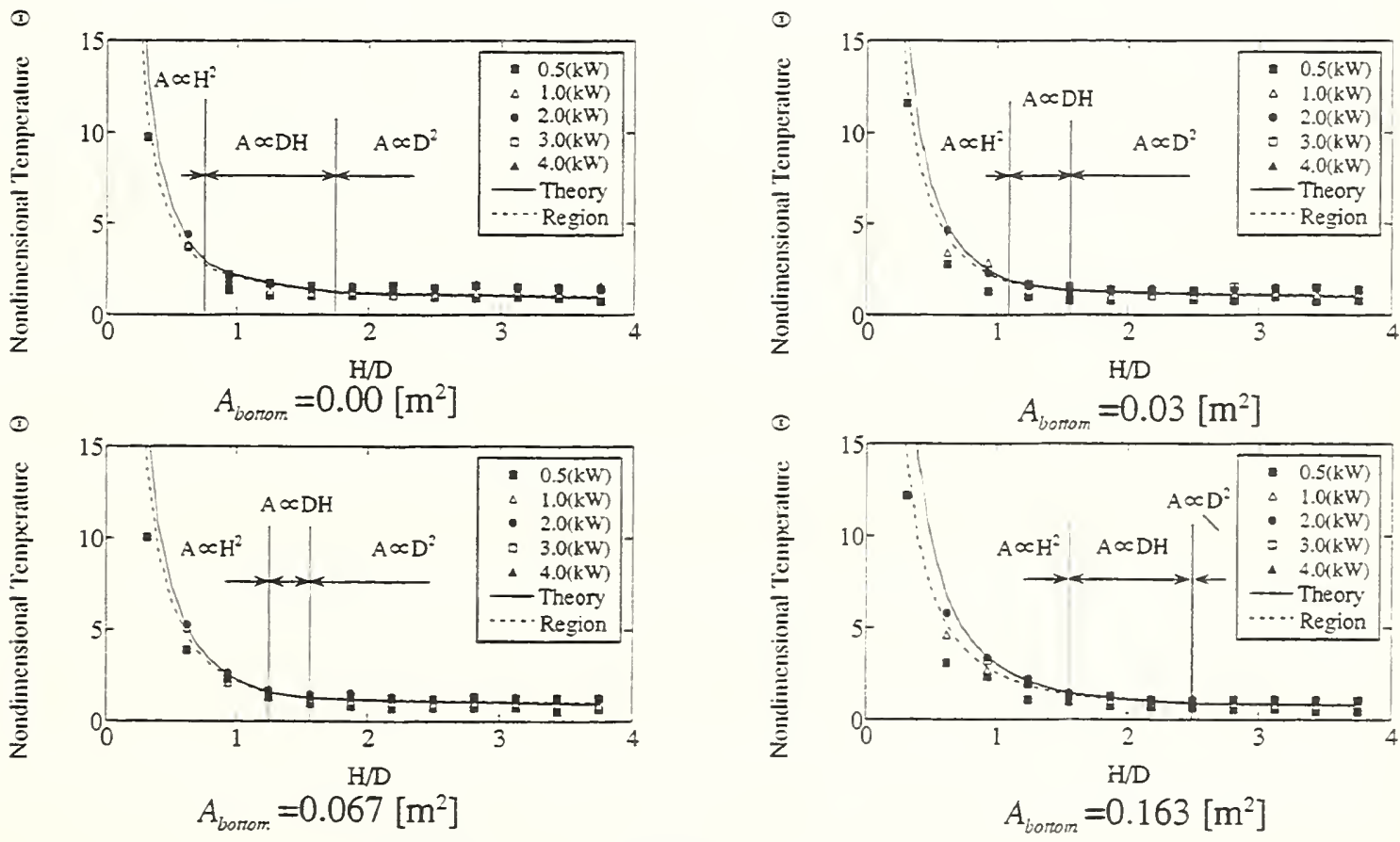

FIGURE 7 Nondimensional Temperature 
Table 1 Comparion with the values $\alpha$ and $\beta$ of the theoretical and experimental regression

\begin{tabular}{|c|c||c|c|c|c|}
\hline $\begin{array}{c}\text { Opening } \\
\text { Area [m }\end{array}$ & \multirow{2}{*}{ Region } & \multicolumn{2}{|c|}{ Experiments } & \multicolumn{2}{c|}{ Theory } \\
\cline { 2 - 6 } & & $\alpha$ & $\beta$ & $a^{*}$ & $\beta$ \\
\hline \multirow{4}{*}{0.00} & $\mathrm{H} / \mathrm{D} \leqq 0.78$ & 1.74 & -1.55 & 1.86 & $-5 / 3$ \\
\cline { 2 - 6 } & $0.78<\mathrm{H} / \mathrm{D} \leqq 1.77$ & 2.09 & -0.90 & 2.19 & -1 \\
\cline { 2 - 6 } & $1.77<\mathrm{H} / \mathrm{D}$ & 1.48 & -0.28 & 1.48 & $-1 / 3$ \\
\hline \multirow{4}{*}{0.03} & $\mathrm{H} / \mathrm{D} \leqq 1.09$ & 2.07 & -1.53 & 2.23 & $-5 / 3$ \\
\cline { 2 - 6 } & $1.09<\mathrm{H} / \mathrm{D} \leqq 1.56$ & 1.93 & -0.81 & 2.09 & -1 \\
\cline { 2 - 6 } & $1.56<\mathrm{H} / \mathrm{D}$ & 1.51 & -0.26 & 1.56 & $-1 / 3$ \\
\hline \multirow{4}{*}{0.0672} & $\mathrm{H} / \mathrm{D} \leqq 1.25$ & 2.23 & -1.56 & 2.33 & $-5 / 3$ \\
\cline { 2 - 6 } & $1.25<\mathrm{H} / \mathrm{D} \leqq 1.56$ & 1.91 & -0.86 & 2.01 & -1 \\
\cline { 2 - 6 } & $1.56<\mathrm{H} / \mathrm{D}$ & 1.45 & -0.26 & 1.49 & $-1 / 3$ \\
\hline \multirow{3}{*}{0.1632} & $\mathrm{H} / \mathrm{D} \leqq 1.56$ & 2.60 & -1.45 & 3.08 & $-5 / 3$ \\
\cline { 2 - 6 } & $1.56<\mathrm{H} / \mathrm{D} \leqq 2.50$ & 2.07 & -0.93 & 2.29 & -1 \\
\cline { 2 - 6 } & $2.50<\mathrm{H} / \mathrm{D}$ & 1.16 & -0.30 & 1.24 & $-1 / 3$ \\
\hline
\end{tabular}

\subsection{Dependence of Plume Temperature for Large Depth on Bottom Opening Area}

It is considered that the smoke hazard in cavity spaces is particularly serious for deep cavity: When $H / D$ is small the contamination by smoke will be confined to a limited part of the space just like by window jets ejecting to outdoor. But when $H / D$ is large the space can be extensively subjected to the influence of smoke.

As can be recognized from the values of coefficient a for large $H / D$ in Table 1 , the temperatures for large depth decrease with the increase of the bottom opening area, so providing bottom opening may help mitigate the smoke hazard. Here we introduce the bottom opening ratio $\gamma$ : the ratio of bottom opening area to horizontal section area of cavity, namely

$$
\gamma \equiv A_{\text {botrom }} / D^{2}
$$

The reason why the temperature for large depth decrease with the increase of the bottom opening area is suspected to be because the rate of the air supply through the opening at the top of the cavity space decreases as the bottom opening area increases, so more fresh air is entrained into the fire plume. Nevertheless, the air supplied from the top of the cavity has a significant effect on diluting the fire plume gases even when $A_{\text {borom }}$ is zero. Since it is considered that both of the air supply from the top and the bottom have significant effect on the temperatures, the values of coefficient $\alpha$ for large $H / D$ are plotted in Figure 8 versus

$$
\frac{A_{\text {borom }}+D^{2}}{D^{2}}=\gamma+1
$$

instead of simply plotting versus $\gamma$. Note that the value of for $\gamma=0$ (no bottom opening) is taken from the previous paper. The experimental correlation between $\alpha$ and $\gamma$ can be established as

$$
\Theta=1.67(\gamma+1)^{-4 / 3}
$$

Note, however, that the discrepancy of the value of for $\gamma=0$ from the regression line was disregarded.

Substituting Eqns.(7) into Eqn.(2) yields the generalized formula for the plume temperature for large $H / D$ as follows:

$$
\Theta=1.67(\gamma+1)^{-4 / 3}(H / D)^{-1 / 3}
$$

The application of Eqn. (8) should be conservatively confined within $0<\gamma<0.25$, since the applicability beyond this region has not been confirmed either experimentally nor theoretically. However, 


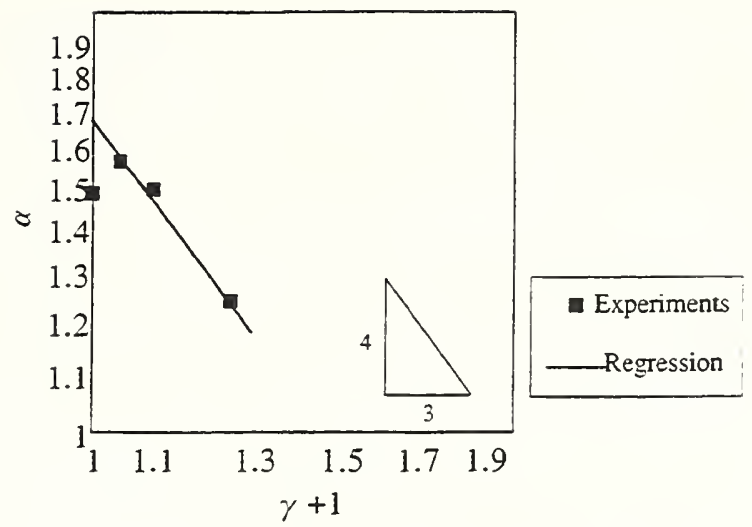

FIGURE 8 Bottom opening ratio and $\alpha$

considering the bottom opening area ratio of the existing cavity spaces, the application of Eqn.(8) will not be so limited.

\section{PRESSURE DIFFERENCE}

\subsection{Nondimensional Pressure Difference}

The pressure difference which develops between the inside and the outside of a cavity space affects the measures for protecting the floor area adjacent to the cavity space from the infiltration of smoke. According to Figure 4, which shows the pressure difference profile for the cavity with $3 \mathrm{~m}$ depth, the pressure difference is nearly zero at the height of the top opening regardless the difference in the heat release rate and the bottom opening area, but differs depending on the conditions.

Although the exact mechanism of the pressure development is not obvious, since the pressure difference increases with the distance from the top, at least down to the middle height, the pressure difference at the bottom $\Delta P_{\text {borom }}$ may be assumed as

$$
\Delta P_{\text {borom }} \propto \Delta \rho g H
$$

Dividing the both side of Eqn.(9) by $\Delta \rho_{\infty} g H$ and noting that for large $H / D$ the relationship as

has been obtained, we have

$$
\frac{\Delta T}{T_{\infty}} \propto \dot{Q}^{\cdot 2 / 3}\left(\frac{H}{D}\right)^{-1 / 3}
$$

$$
\frac{\Delta P_{\text {bonom }}}{\rho_{\infty} g H} \propto \frac{\Delta \rho}{\rho_{\infty}} \approx \frac{\Delta T}{T_{\infty}} \propto \dot{Q}^{\cdot 2 / 3}\left(\frac{H}{D}\right)
$$

However, this presumption may fail because the temperature of the lower part of a cavity space cannot be represented by the plume temperature. Hence, based on the above consideration, we introduce the nondimensional pressure $\pi$ defined as

$$
\pi \equiv\left(\Delta P_{\text {botom }} / \rho_{\infty} g H\right) / \dot{Q}^{* 2 / 3}
$$

and investigate the dependence of $\pi$ on cavity depth.

In Figure 9, the nondimensional pressure $\pi$ for cavity with different depth are plotted versus $H / D$ of cavity. According to Figure 9, the data for 2.0,3.0 and 4.0kW fire are collapse to a single line in each bottom opening size, which implies that the pressure difference is well scaled by $\pi$. Although the data for 0.5 and $2.0 \mathrm{~kW}$ fire are not necessarily in good agreement, this is thought to be because the fire sizes are too small to induce the pressure large enough for the accurate measurement. 

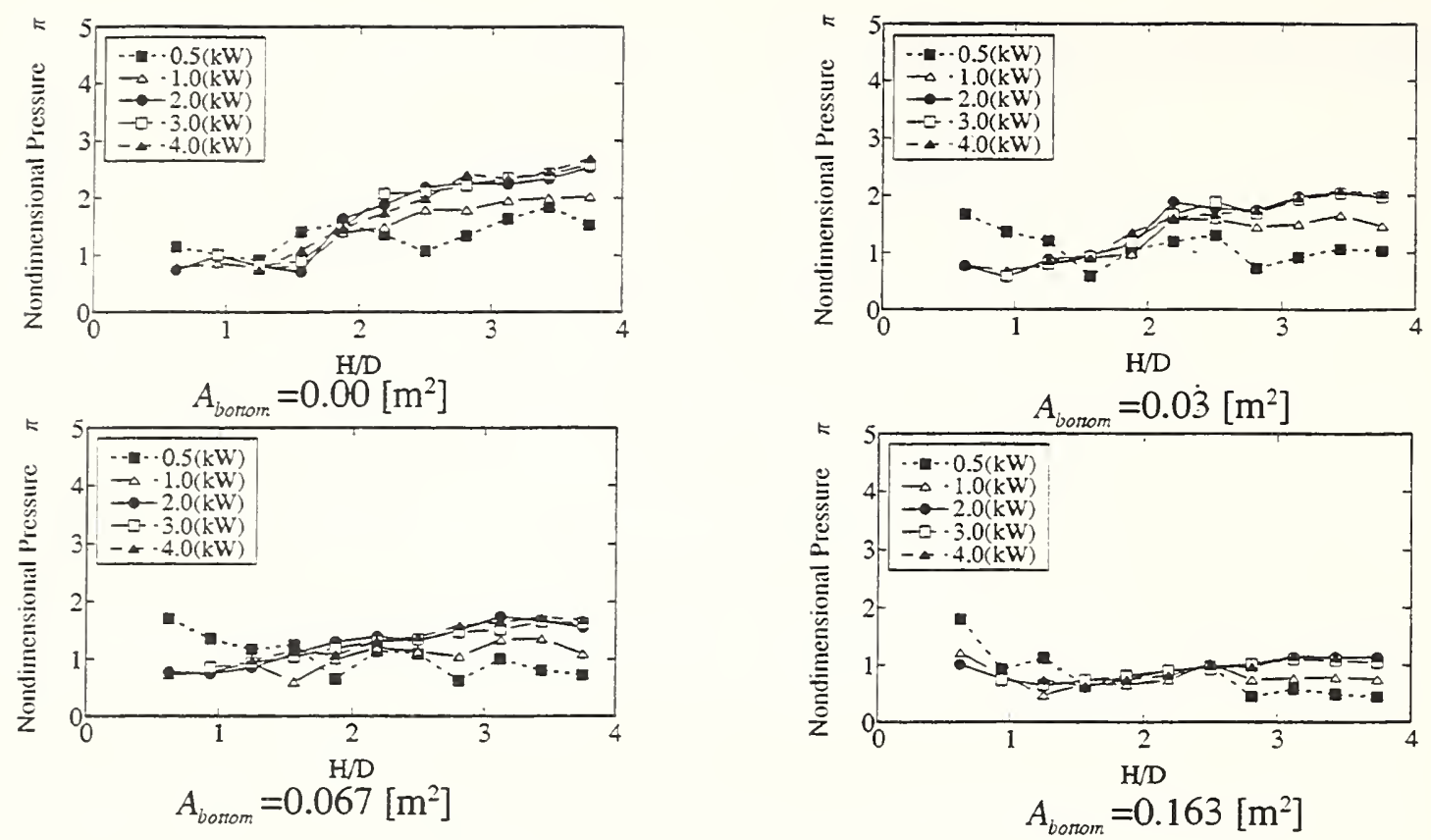

FIGURE 9 Nondimensional Pressure

\subsection{Dependence of Pressure Difference on Bottom Opening Area}

Looking at Figure 9, $\pi$ is about constant where $H / D$ is large and proportional to $H / D$ where $H / D$ is intermediate, while a consistent tendency cannot always be observed where $H / D$ is small. In order to investigating the dependence of the pressure difference on bottom opening area, $\pi$ for cavity space with large $H / D$ and $2.0,3.0$ and $4.0 \mathrm{~kW}$ fire is plotted versus $\gamma+1$ in Figure 10 . The regression line can be expressed as

$$
\pi=2.3(\gamma+1)^{-10 / 3}
$$

Since the boundary of intermediate and large depth seem to be somewhere around $H / D=2.5$, let's regard Eqn.(11) apply for $H / D>2.5$. Then, where cavity depth is intermediate, noting that $\pi$ increases about proportionally to $H / D^{\prime}, \pi$ may be given as

$$
\pi=0.92(\gamma+1)^{-10 / 3}(H / D)
$$

From Figure 11, which compares the experimental data and Eqns.(11) and (12), Eqn.(12) is considered to be applicable roughly for $1.2<H / D<2.5$.

Incidentally, Eqns.(11) and (12) should be considered to be applicable for $0<\gamma<0.25$ with respect to the bottom opening area.

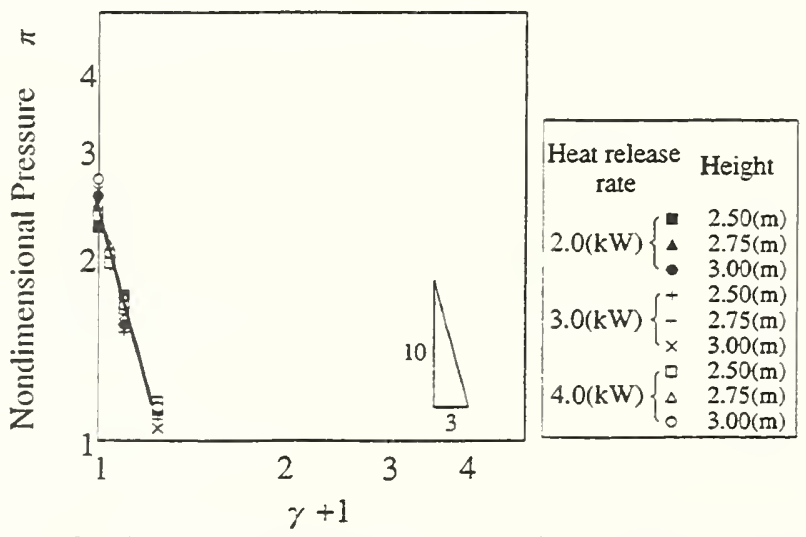

FIGURE 10 Bottom opening ratio and Nondimensional Pressure 


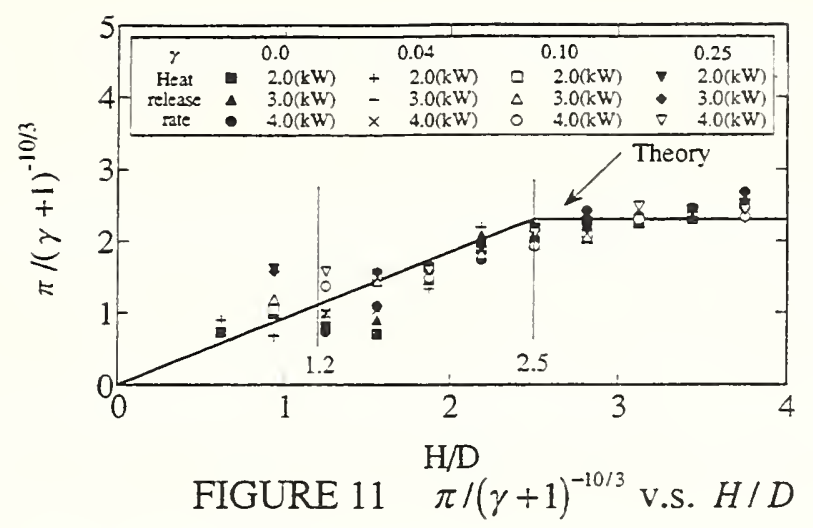

\section{RATE OF AIR INFLOW THROUGH BOTTOM OPENING}

The rate of air inflow through the bottom opening $m_{\text {borom }}$ is given by

$$
m_{\text {borom }}=C_{D} A_{\text {borom }} \sqrt{2 \rho_{\infty} \Delta P_{\text {borom }}}
$$

Substituting Eqn.(10) into Eqn.(13), we have

$$
m_{\text {borom }}=C_{D} \rho_{\infty} A_{\text {bortom }} \sqrt{2 g H \pi} \dot{Q}^{-1 / 3}
$$

Introducing the nondimensional flow rate defined as

$$
\mu \equiv\left(m_{\text {bortom }} / \rho_{\infty} \sqrt{g D^{5}}\right) / Q^{-1 / 3}
$$

and substituting this into Eqn.(14) yields

$$
\mu=C_{D} \sqrt{2}\left(A_{\text {borom }} / D^{2}\right) \pi^{1 / 2}(H / D)^{1 / 2}=C_{D} \sqrt{2} \gamma \pi^{1 / 2}(H / D)^{1 / 2}
$$

Using Eqns.(11) and (12), and letting $C_{D}=0.7$, we have

$$
\mu= \begin{cases}0.95 \frac{\gamma}{(\gamma+1)^{5 / 3}}\left(\frac{H}{D}\right) & (1.2<H / D<2.5) \\ 1.50 \frac{\gamma}{(\gamma+1)^{5 / 3}}\left(\frac{H}{D}\right)^{1 / 2} & (2.5 \leq H / D)\end{cases}
$$

Figure 12 shows the comparison of Eqn.(17) and the nondimensional air inflow rate from the measurement. Notice that the latter is calculated by using the pressure difference measured at the bottom to Eqn.(17), hence the comparison of air inflow rates in Figure 12 is nothing but a different form of the comparison of the pressure difference in Figure 11.

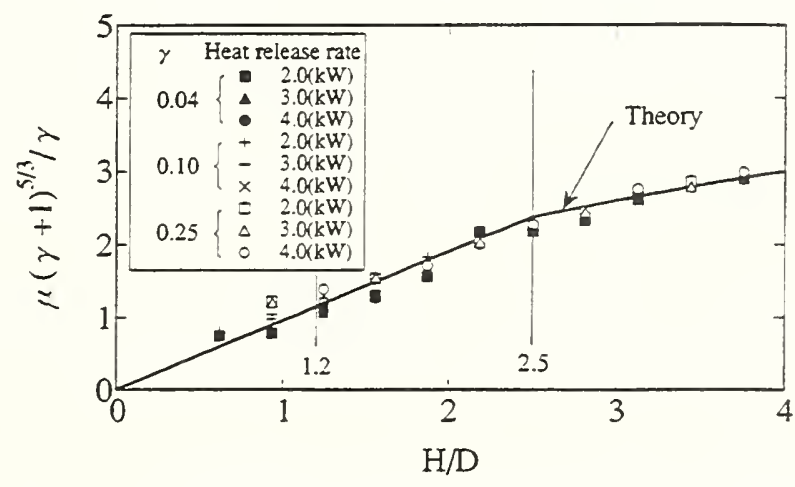

FIGURE $12 \mu(\gamma+1)^{5 / 3} / \gamma$ v.s. $H / D$

\section{CONCLUDING REMARKS}

The experiments were conducted for elucidating the behavior of fire plume in the cavity which has an opening at the bottom. It was found that the larger the bottom opening, the more stable the fire 
plume, but still the temperature is correlated by the same manner as in the case of cavity with no opening.

The effects of the bottom opening area on the plume temperature, the pressure difference induced in the cavity space and the mass inflow rate of air through the bottom opening are investigated and the experimental comelations were obtained.

\section{NOMENCLATURE}

$\begin{array}{ll}A & \text { Horizontal section area of fire plume }\left(\mathrm{m}^{2}\right) \\ A_{\text {borom }} & \text { Area of bottom opening }\left(\mathrm{m}^{2}\right) \\ C_{P} & \text { Specific heat of air }(\mathrm{kJ} / \mathrm{kgK}) \\ C_{D} & \text { Flow Coefficient } \\ D & \text { Length of the side of cavity space }(\mathrm{m}) \\ g & \text { Acceleration due to gravity }\left(\mathrm{m} / \mathrm{s}^{2}\right) \\ H & \text { Depth of cavity }(\mathrm{m}) \\ m_{\text {boron }} & \text { Rate of air inflow through bottom opening }(\mathrm{kg} / \mathrm{s}) \\ \gamma & \text { Bottom opening ratio } \\ \Delta P & \text { Pressure difference }(\mathrm{Pa}) \\ \Delta P_{\text {bonon }} & \text { Pressure difference at the bottom of cavity space }(\mathrm{Pa}) \\ \dot{Q} & \text { Heat release rate of fire source }(\mathrm{kW}) \\ \dot{Q}^{*} & \text { Nondimensional heat release rate } \\ T & \text { Temperature }(\mathrm{K}) \\ T_{\infty} & \text { Ambient temperature }(\mathrm{K}) \\ \Delta T & \text { Temperature difference }(\mathrm{K}) \\ \alpha & \text { Coefficient of Eqn. }(2) \\ \beta & \text { Factor of Eqn.(2) } \\ \rho_{\infty} & \text { Ambient air density } \\ \pi & \text { Nondimensional pressure } \\ \mu & \text { Nondimensional flow rate } \\ \Theta & \text { Nondimensional temperature }\end{array}$

\section{REFERENCES}

[1] Takeyoshi TANAKA and Sunao KUMAI : Experiments on Smoke Behavior in Cavity Spaces,

[2] Takeyoshi TANAKA, Sunao KUMAI, Teruhisa FUKUDA, Akihiko YOSHIZAWA, Osamu ISHINO, and Takao WAKAMATSU: Smoke Behavior in Cavity Spaces, Part 1 In case where the fire sources are located at the center of the cavity floor,

[3] Teruhisa FUKUDA, Akihiko YOSHIZAWA, Sunao KUMAI, Osamu ISHINO, Takeyoshi TANAKA and Takao WAKAMATSU: Smoke Behavior in Cavity Spaces, Part 2 In case where the fire sources are located by a wall or at a corner, 


\section{Discussion}

Edward Zukoski: Is the wind direction important in the original problem, whether the opening to the lower air cavity is upwind or downwind?

Takeyoshi Tanaka: I haven't looked at that problem yet. Whether or not wind is important will be determined by the relationship between the size of heat release and the height of the cavity.

Tokiyoshi Yamada: There is some pressure difference and I think the pressure difference was zero at the top. When at lower part is closed, I believe that vertical flow occurs but could you tell us where the flood occurred.

Takeyoshi Tanaka: I do not know. 
$10 \underline{4}$ 


\title{
A PROTOTYPE FDMS DATABASE FOR MODEL VERIFICATION
}

\author{
Rebecca W. Portier, Richard D. Peacock, and Walter W. Jones \\ Building and Fire Research Laboratory, NIST, Gaithersburg, MD 20899, USA
}

\begin{abstract}
Fire Data Management System, FDMS, is a system designed to store and retrieve fire test results obtained from bench-scale and real-scale tests as well as fire simulation programs. By storing available fire test values in a common format, this data is readily available to computer models, plotting programs, and report generators. An implementation of the FDMS for data ranging from bench-scale test results to real-scale fire tests is discussed.
\end{abstract}

\section{INTRODUCTION}

A unified method of accessing data is desirable for both experimental and modeling efforts in the development of the science of fire. For experimentalists, easy to use data organization and retrieval tools facilitates analysis of the data. For modelers, access to experimental data is important for both model input and evaluation of the predictive capability of the models. FDMS, the Fire Data Management System, provides a system for organizing and presenting fire data obtained from bench-scale and real-scale tests as well as fire simulation programs. Implementation of databases in the FDMS format are intended to be used in individual laboratories to store, edit, and retrieve their own test results, as well as a central repository for contributed data. By storing available fire test values in a common format, this data is readily available to computer models, plotting programs, and report generators.

The goal for FDMS is to provide uniform access to test values generated from a variety of sources within the fire community. An initial version of a database implementing the FDMS was developed under the direction of the Fire Research Station according to the design proposed by Babrauskas, Janssens, Peacock, and Batho ${ }^{1}$. The resulting software, FDMS 1.0, provides for storage and retrieval of Cone Calorimeter and Furniture Calorimeter test data ${ }^{2}$. Further development at the Building and Fire Research Laboratory has extended this database to provide an open file design that will easily accommodate future test apparatus formats.

After completion of the analysis of the initial implementation of FDMS, it was determined that a redesign of the physical file formats was necessary to accommodate additional test methods. Technical documentation providing details for proposed database file formats was generated as the first step in this second stage of development ${ }^{3.4}$. The second stage of development also involved the generation of a centralized database using data contributed from individual FDMS 1.0 databases along with numerous real-scale tests which could not be accommodated by the FDMS 1.0 software with modifications resulting from user feedback on both the design of the database as well as to the completeness of the test data available in the centralized database.. A text-oriented interface, entitled FIREDATA, is being developed to provide quick, simplistic query access to the centralized database over the Internet. Development of a 
stand-alone software program which will allow individual laboratories to store, edit, and retrieve their own test results is in progress. Additionally, a CD-ROM database is being considered as an additional means of distribution for the database.

\section{ACCESSING THE FIREDATA DATABASE}

At present, the centralized database can be accessed only through the Internet. Querying functionality is provided to allow selection of tests in order to generate various export file formats containing the selected data. Generated export files can then be transferred to the user site and accessed by other software applications such as spreadsheets for further evaluation. It is not intended that large-scale transfers of the data take place since the database currently occupies more than 100 megabytes of disk storage.

\section{CONTRIBUTING DATA TO THE FIREDATA DATABASE}

Clearly, the integrity of data in any database is of paramount importance to the utility of the database. In this initial test implementation of FDMS 2.0, we have provided a collection of available fire test data from NIST and elsewhere to provide a basis for evaluating the utility of the database and the effort involved in implementing future, more inclusive databases. In general, we have adapted the ASTM guide for database quality indicators for use in FDMS. Data supplied to NIST for inclusion into the FIREDATA database must include an assessment by the testing organization of each of these quality indicators. The following is considered the minimum values of indicators for data the is acceptable for inclusion into the database:

- $\quad$ Source of data - at least a report must be available describing the test

- $\quad$ Statistical basis of data - replicate tests are desirable but not required for standard bench-scale test methods. Single tests are acceptable for larger than bench-scale tests.

- Material development or production status - any category (from the ASTM guide) is acceptable

- Evaluation status - any category (from the ASTM guide) is acceptable

- $\quad$ Certification status - any category (from the ASTM guide) is acceptable

- Completeness of material(s) information - at least partial information on material. See section on Product Description, below, for details.

- Completeness of test procedure description - any category (from the ASTM guide) is acceptable

\section{INFORMATION IN FDMS FORMAT}

To make data in the FDMS format as complete as possible, this section provides guidelines for the information which can be included for a test to be imported into an FDMS format database. 
Test Description: Each test is identified by the test conducted, persons responsible for the test, the test conditions, product or products tested, and comments or observations about the test. These are each related to fields in physical database files. Applicable documents related to the test method or test documentation can also be identified in the description.

Test Conditions: Test conditions describe setup aspects for each test and thus allow the user to more completely specify the testing conditions. The information which should be included depends upon the specific test method to which the data set applies. For the Cone Calorimeter, for example, the orientation, incident flux, pilot ignition, or mounting frame specification could be included.

Product Description: At least one product must be included. For the initial data included in the Internet version of the FIREDATA database, this information is the least complete and perhaps most important to the future utility of the database. A completely identified product (for FDMS product descriptions) includes: Product name (a text description of the product), Product ID, Manufacturer, Catalog number, Main use of the product (from a controlled choice list within FDMS), Density, Length, Height, Thickness, Mass, Thermal Conductivity, Specific Heat, and Emissivity. For layered products, each individual sub-product is described as above with an additional field to indicate the structure in the final product.

Personnel and Organizations: Description of personnel and organizations related to test data within the database provide contact points for additional information about the test or product details which may have been omitted in the database: Full name, Organization name, Address, and Phone, fax, telex, e-mail.

Organizations are described by: Organization type (from a controlled choice list within FDMS), Organization name, Division, Address, and Phone, fax, telex, e-mail

Fire Test Measurements: Vector and scalar data imported into the Internet version of the FIREDATA database must be specified in SI. The NIST "Guide for the Use of the International System of Units (SI)" provides complete details of SI. Within FDMS, the following base units are used:

$\begin{array}{ll}\text { temperature } & \text { Kelvin } \\ \text { absolute temperature } & \text { Kelvin } \\ \text { pressure } & \text { Pascal } \\ \text { length } & \text { meter } \\ \text { energy } & \text { Joule } \\ \text { energy release rate } & \text { Watt } \\ \text { energy absorbtion rate } & \text { Watt } \\ \text { mass } & \text { kilogram } \\ \text { time } & \text { Second }\end{array}$

\section{CONCLUSIONS}

The availability of a wide range of quality experimental data is important to ongoing efforts in fire model evaluation. The FDMS format provides a consistent system for the exchange of 
such data from both bench-scale and real-scale fire experiments. By establishing a protocol for storing and maintaining fire data and by providing an extensible database, the fire community will be encouraged to contribute results of experiments. This should result in improvement of both modeling and experimental techniques and facilitate further comparison of model predictions and experimental measurements.

\section{REFERENCES}

1. Babrauskas, V., Peacock, R. D., Janssens, M., and Batho, N. E., Standardizing the Exchange of Fire Data - The FDMS, Fire and Materials 15, 85-92 (1991).

2. Software distributed by the Fire Research Station, Borehamwood, England.

3. Portier, R. W.,Fire Data Management System, FDMS 2.0, Technical Documentation, Natl. Inst. Stand. Technol., Technical Note 1407 (1995). Also available through the anonymous ftp site candela.cfr.nist.gov in /pub/reports.

4. Portier, R. W., A Programmer's Reference Guide to FDMS File Formats, Natl. Inst. Stand. Technol., NISTIR 5162 (1993). 


\section{SUPPRESSION}

$10 \underline{9}$ 
110 


\title{
PROGRESS REPORT ON FIRE SUPPRESSION RESEARCH IN THE U.S.
}

\author{
Richard G. Gann \\ Fire Science Division \\ Building and Fire Research Laboratory \\ National Institute of Standards and Technology \\ Gaithersburg, MD 20899
}

\begin{abstract}
Dramatic changes in the field of fire suppression have taken place in the $3 \frac{1}{2}$ years since the 12 th meeting of the UNNR Panel on Fire Research and Safety. Research on and qualification of alternatives to halon 1301 are in process. The use of sprinkler systems in the United States has proliferated.
\end{abstract}

\section{HALON ALTERNATIVES}

F. Sherwood Rowland and Mario Molina received the 1995 Nobel Prize for Chemistry for their findings in 1974 that chlorine atoms, transported into the stratosphere, catalyze the destruction of stratospheric ozone. Subsequent calculations showed that bromine atoms were even more harmful. Halon $1301\left(\mathrm{CF}_{3} \mathrm{Br}\right)$, the suppressant of choice where 'clean' fire suppression is needed, was then identified as a significant contributor to this environmental hazard. Under the amendments to the international Montreal Protocol on Substances that Deplete the Ozone Layer and the U.S. Clean Air Act of 1990, production of new halon 1301 stopped on January 1, 1994, with limited allowances made for developing nations. The supply of this suppressant for nonessential systems became scarce, and many users of this technology began searching for other approaches. Because the military has extensive near-term essential fire protection needs, they have been buying halon 1301 from organizations that have discontinued its use. A market in recycled (purified) 1301 has also emerged.

Research on and testing of alternative suppression technologies has been dominated by the U.S. Department of Defense. Many of the systems being protected by halon 1301 are essential to military readiness; and in 1991, the Department of Defense initiated an urgent program to identify near-term alternatives for weapons systems use, mostly by September, 1996. This research focusses on commercially available or currently emerging chemicals and technologies. Most of the current resources are focussed on testing HFCs (chemicals containing hydrogen, fluorine and carbon) and FCs (chemicals containing only carbon and fluorine) and qualifying them for commercial and military use. For instance, HFC-227ea $\left(\mathrm{C}_{3} \mathrm{HF}_{7}\right)$ is being considered for use in the fixed fire suppression systems aboard ships and HFC-125 $\left(\mathrm{C}_{2} \mathrm{HF}_{5}\right)$ is being considered for use in the engine nacelles of developmental aircraft. The National Fire Protection Association (NFPA) has issued the first standard for systems using alternatives to halon 1301.

A lesser amount of research is aimed at new chemicals and approaches. The former include chemically-generated suppressants and phosphorous nitrides; $\mathrm{CF}_{3} \mathrm{I}$ has potential, but for 
unoccupied spaces only due to its cardiac sensitization. The latter include water mist systems and the use of propellants (such as found in automobile air bags) to generate inert gases rapidly. Papers on all of these conventional and new approaches can be found in the proceedings of the annual International CFC and Halon Alternatives Conference and the Halon Options Technical Working Conference. A recent special American Chemical Society symposium volume contains more detailed papers. A number of papers appear in the proceedings of the International Conference on Fire Research and Engineering. Two extensive NIST Special Publications describe a variety of technological advances in the mechanisms and performance of alternative fire suppressants.

The currently available commercial agents occupy two-to-three times the space and volume of halon 1301; using these, the cost of retrofitting aircraft, ground armored vehicles, etc. is estimated in the many billions of dollars. To ensure the availability of more efficient suppression technology, the Department of Defense is initiating a Next Generation Fire Suppression Technology program. The goal is to develop, by 2005 , environmentally-friendly and user-safe processes, techniques and fluids that meet the operational requirements currently satisfied by halon 1301 systems in ships, aircraft, land combat vehicles, and critical mission support facilities. The program includes research on risk assessment and selection methodology, fire suppression principles, technology testing methodologies, new suppression concepts, emerging technology assessment, and suppression optimization. A published description of the program is due this spring.

\section{SPRINKLERS}

A notable advance during the past 3 years has been the expansion of occupancies requiring sprinklers. The list now includes high rise commercial buildings, hotels and motels, and multifamily residences. In large part, this is due to, first, the adoption by local authorities of such provisions in the NFPA Life Safety Code, and second, the allowed construction trade offs (e.g., reduction in fire ratings of walls). In addition, (U.S.) Public Law 102-522 (1992) required the General Services Administration to install sprinklers in Federally-occupied buildings with over 25 employees and established sprinkler requirements for housing under the jurisdiction of the U.S. Department of Housing and Urban Development.

The use of water sprinklers is a mature technology, and the changes over the past few years have been in system design and code acceptance. For instance, the NFPA 13D standard has been modified with a provision for residences not exceeding $186 \mathrm{~m}^{2}\left(2000 \mathrm{ft}^{2}\right)$ in area. NFPA is also developing the first standard for the installation of water mist systems (NFPA 750). This is expected in May, 1996. 


\section{ACKNOWLEDGMENTS}

Dan Madrzykowski and Richard Bukowski of NIST provided the author with the materials for the section on sprinklers.

\section{REFERENCES}

Bukowski, Richard W., and Budnick, Edward K., Guide for the Implementation of PL 102-522 for Fire Alarm and Automatic Sprinkler Installations, U.S. Department of Housing and Urban Development, 1995.

Gann, Richard G., ed., Fire Suppression System Performance of Alternative Agents in Aircraft Engine and Dry Bay Laboratory Simulations, NIST Special Publication 890 (two volumes), National Institute of Standards and Technology, Gaithersburg, MD, 1995.

Grosshandler, William L., Pitts, William M., and Gann, Richard G., eds., Evaluation of Alternative In-Flight Fire Suppressants for Full-Scale Testing in Simulated Aircraft Engine Nacelles and Dry Bays, NIST Special Publication 861, National Institute of Standards and Technology, Gaithersburg, MD, 1994.

International CFC and Halon Alternatives Conference Proceedings, Alliance for Responsible Atmospheric Policy, Frederick, MD, 1993, 1994, 1995.

International Conference on Fire Research and Engineering Proceedings, Society of Fire Protection Engineers, Boston, MA, 1995.

Halon Options Technical Working Conference Proceedings, New Mexico Engineering Research Institute, Albuquerque, NM, 1993, 1994, 1995.

Miziolek, Andrzej W. And Tsang, Wing, eds., Halon Replacements, Technology and Science, ACS Symposium Series 611, American Chemical Society, Washington, DC, 1995.

NFPA 13D, Sprinkler Systems in One- and Two-Family Dwellings and Manufactured Homes, National Fire Protection Association, Quincy, MA, 1994.

NFPA 2001, Clean Agent Fire Extinguishing Systems, National Fire Protection Association, Quincy, MA, 1994. 
114 


\title{
PROGRESS REPORT ON SUPPRESSION IN JAPAN
}

\author{
Naoshi SAITO \\ Fire Research Institute \\ 14-1, Nakahara 3 chome, Mitaka, Tokyo 181, Japan
}

\begin{abstract}
Since last UJNR in 1992, many problems have been caused in the field of fire suppression in Japan, and there was remarkable progress in the employment of halon replacements.
\end{abstract}

\section{Issues on fire suppression after 1992}

There have been many important incidents on the fire suppression in Japan after 1992. The main problems of them are as follows:

(a) Ban on the halon production for the protection of the stratospheric ozone layer and employment of halon alternatives,

(b) Extinguishing difficulty of large fires caused by the grand Hanshin-A waji earth quake on January 17, 1995,

(c) Accidental leakage and fire of coolant sodium metal in the fast breeder reactor, "Monju," in Tsuruga of Fukui Prefecture on December 7, 1995, and

(d) Accidental discharge of a carbon dioxide fire extinguishing equipment of a parking tower in Tokyo on December 1, 1995. There were two victims in the accidental discharge.

\section{Progress in halon replacements}

Since January 1, 1994, the production of halons is banned to prevent depletion of the stratospheric ozone layer. Potential candidates of halon replacements have been reported, and some of them are already employed in new fire extinguishing equipment as a total flooding system.

The study on development of halon al ternative agents is continued in Japan. In National Industry Research Institute of Nagoya, Fukaya et al. [1] investigates the development of synthesis method and the fire extinguishing efficiency of perfluoroalkylamines as candidates of halon replacement. The perfluoroalkylamines show the efficiency enough for fire suppressants, but any methods for mass production of the chemicals have never succeeded. Recently, they tried to explain the fire suppression mechanism of $\mathrm{CF}_{3}$ group of the perfluorocarbons by using Ab initio molecular orbital calculations [2].

In Sophia University, Takahashi et al. $[3,4]$ studied the inhibition effect of perfluoroalkylamines and polyfluorocarbons using a shock tube, and Sekiuji et al. [5] reported the reaction mechanism of $\mathrm{CF}_{3}$ radical with $\mathrm{O}$ atom and $\mathrm{O}_{2}$ molecule to clear the combustion inhibition mechanism of polyfluorocarbons.

In Fire Research Institute, many investigations have been carried out on the fire suppression efficiency and its evaluation methods for the candidates of halon replacements to employing new fire extinguishing agents used in total flooding system. The cup burner scaleeffect on flame extinguishing concentration was investigated and reported by Saso et al. [6]. Saito et al. [7] studied reproducibility of the flame extinguishing concentrations measured by 
glass cup burner systems under the fixed conditions, and they reported also the influence by operators on measurements of a fire extinguishing concentration in the report. Inoue et al. [8], Sakei et al. [9], and Saito et al. [10] reported the flame extinguishing concentrations of representative "new fire extinguishing agents" for several kinds of fuels, using the FRI cup burner system. The data contain the flame extinguishing concentrations of both HFC agents and inert gas agents.

Extinction phenomena of the cup burner flame are complex, so the burner scale and the experimental conditions affect flame extinguishing concentrations. A counterflow diffusion flame is formed in a stretch flow field and has relatively simple flame structure. Thus, it is expected that the physical conditions at extinction of the flame can be fixed easier than the case of cup bumer flame. Saso et al. [11-13] tried to obtain the flame extinguishing concentrations of inert gases and halon replacements using a counterflow diffusion burner.

Inerting efficiencies of inert gases, halon replacements, and halon 1301 were measured by using a new technique, tubular flame burner method. Liao et al. [14] investigated appropriate measurement conditions of the flammability limits using the tubular flame burner, and reported the flammability limits of twenty-three kinds of fuel-air-additive ternary mixtures [15]. The fuels are methane, ethane, propane, butane, heptane, and ethanol. Nitrogen, carbon dioxide, argon, halon 1301, FC 3-1-10, HFC 227ea, and HFC 23 are used as the additives.

Saito et al. [16] reported that the flammability peak concentrations of halon 1301 and three kinds of halon replacements measured by the tubular flame bumer agree well with the inerting data listed in NFPA 2001. Saito et al. [17] studied also the relation between the suppression efficiency of an inert gas mixture and those of the component of the mixture. They found that the fire suppression efficiency on the mixture is represented by simple relation of harmonic mean weighted by the component mole fractions in both cases of flammability limit and flame extinguishing concentration.

In the relation of halon recycling, Otsu et al. [18] investigated the flame extinguishing efficiency of halon 1301 containing nitrogen, and reported that halon 1301 containing within 7 mole $\%$ of nitrogen does not change the flame extinguishing concentration over $0.1 \%$.

\section{Progress in water system}

There are many activities on the water system, especially water mist technology for the fire extinguishing systems, but a few reports on the system have been appeared in the period.

Tokyo Fire Department [19] carried out extinguishing tests of a high tension transformer station using water fire extinguishing systems as halon alternative, and made a guide line on use of the water mist and sprinkler systems for protection of transformer station.

In Fire Research Institute, Takahashi [20] studied the extinguishing of plastic fires with wet water. He found out that the wet water shows excellent fire extinguishing effect for plastic fires, and use of the wet water can reduce water application rate relative to normal water.

\section{References}

[1] Fukaya, H., Hayashi, E., Hayakawa, Y., Baba, H., Taoda, H., Osaki, T., and Abe, T., Synthesis and Evaluation of Perfluoroalkylamines as Halon Alternatives, J. Environmental Chemistry, 3, pp. 271-277 (1993) (in Japanese)

[2] Fukaya, H., Ono, T., and Abe, T., New Fire Suppression Mechanism of Perfluoroalkylamines, J. Chem. Soc., Chem. Commu., pp. 1207-1208(1995)

[3] Takahashi, K., Sekiuji, Y., Inomata, T., Abe, T., Fukaya, H., Hayashi, E., and Inoue, G., Inhibition of Combustion by Bromine-Free Polyfluorocarbons, Comb. Sci. and Tech., 102, pp. 213-230 (1994)

[4] Takahashi, K., Inomata, T., Fukaya, H., and Abe, T., New Halon Replacements Based on 
Perfluoroalkylamines, ACS Symposium Series 611, pp. 139-150, American Chemical Society (1995)

[5] Sekiuji, Y., Takahashi, K., and Inomata, T., High Temperature Reaction of Trifluoromethl radical with $\mathrm{O}$ atom and $\mathrm{O} 2$ molecule, Proc. 33th Japanese Symposium on Combustion, pp. 581-583, Combustion Society of Japan (1995) (in Japanese)

[6] Saso, Y., Saito, N., and Iwata, Y., Experimental Investigation of the Cup Burner ScaleEffect on Flame-Extinguishing Concentrations, Report of Fire Research Institute of Japan, No. 76, pp. 43-50 (1993) (in Japanese)

[7] Saito, N., Saso, Y., Ogawa, Y., Inoue, Y., Sakei, R., and Otsu, Y., Improvement on Reproducibility of Flame Extinguishing Concentration Measured by Cup Burner Method, Proc. Halon Options Technical Working Conference, pp. 299-309, Albuquerque, NM (May, 1995)

[8] Inoue, Y., Saito, N., Saso, Y., and Ogawa, Y., Evaluation of Fire Suppression Efficiency and Practical Applicability of Halon Replacements, Report of Fire Research Institute of Japan, No. 79, pp. 1-7 (1995) (in Japanese)

[9] Sakei, R., Saito, N., Saso, Y., Ogawa, Y., and Inoue, Y., Flame Extinguishing Concentrations of Halon Replacements for Flammable Liquids, Report of Fire Research Institute of Japan, No. 80, pp. 36-42 (1995) (in Japanese)

[10] Saito, N., Sakei, R., Saso, Y., and Ogawa, Y., Some Problems of Measurement of Flame Extinguishing Concentration for High Flash Point Liquid Fuels, Proc. 33th Japanese Symposium on Combustion, pp. 539-541, Combustion Society of Japan (1995) (in Japanese)

[11] Saso, Y., Saito, N., Liao, C., and Ogawa, Y., Evaluation of Fire Suppression Efficiency of Fire-Extinguishing Agents Using Counterflow Diffusion Flame, Report of Fire Research Institute of Japan, No. 77, pp. 11-19 (1994) (in Japanese)

[12] Saso, Y., Saito, N., Liao, C., Ogawa, Y., and Inoue, Y., Evaluation of Fire Suppression Efficiency of Fire-Extinguishing Agents using Counterflow Diffusion Flame, Report of Fire Research Institute of Japan, No. 78, pp. 42-50 (1994) (in Japanese)

[13] Saso, Y., Saito, N., Liao, C., and Ogawa, Y., Extinction of Counterflow Diffusion Flame With Halon Replacements, Fire Safety J., in press

[14] Liao, C., Saito, N., and Iwata, Y., Determination of Peak Concentration Using a Tubular Flame Burner, Report of Fire Research Institute of Japan, No. 76, pp. 33-41 (1993) (in Japanese)

[15] Liao, C., Saito, N., Saso, Y., and Ogawa, Y., Flammability Limits of Combustible Gases and Vapors Measured bv Tubular Flame Burner, Fire Safety J., in press

[16] Saito, N., Saso, Y., Liao, C., Ogawa, Y., and Inoue, Y., Flammability Peak Concentrations of Halon Replacements and Their Function as Fire Suppressant, ACS Symposium Series 611, pp. 243-257, American Chemical Society (1995)

[17] Saito, N., Ogawa, Y., Saso, Y., Liao, C., and Sakei, R., Flame Extinguishing Concentrations and Peak Concentrations of $\mathrm{N}_{2}, \mathrm{Ar}, \mathrm{CO}_{2}$, and Their Mixtures for Hydrocarbon Fuels, to appear in Fire Safety $J$.

[18] Otsu, Y., Saito, N., Saso, Y., Ogawa, Y., and Sakei, R., Measurement of Flame Extinguishing Concentrations for Recycled Halon 1301, Proc. Annual Meeting of Japan Association for Fire Science and Technology, pp. 56-59 (1995) (in Japanese) 
[19] Tokyo Fire Department, Effectiveness Tests of Water Extinguishing Systems on Transformers, Kasai (Journal of Japan Association for Fire Science and Engineering), 45 (6), pp. 21-25 (1995) (in Japanese)

[20] Takahashi, S., Extinguishment of Plastic Fires with Plain Water and Wet Water, Fire Safety J., 22, pp. 169-179 (1994) 


\author{
Anthony Hamins \\ Building and Fire Research Iaboratory \\ National Institute of Standards and Technology \\ Gaithersburg, MD 20899
}

\title{
ABSTRACT
}

A series of experimental measurements were conducted in a baffle stabilized turbulent jet spray flame and a baffle stabilized pool fire in an effort to provide an improved understanding of the influence of various parameters on the processes controling flame stability. The importance of a number of parameters including the agent injection duration, air velocity, air temperature, and system pressure were tested. A comparison of flame stability in pool fires and spray flames showed that for similar air flows and baffle sizes, baffle stabilized pool fires were more difficult to extinguish than baffle stabilized spray fires. For small air flows, the agent required to extinguish the pool fires was similar to the peak flammability limits related to premixed flames.

\section{Introduction}

Ratification of the Montreal Protocol in 1987, has led to limits in the consumption and production of ozone depleting substances. In the Protocol, two common fire extinguishing agents, Halon $1301\left(\mathrm{CF}_{3} \mathrm{Br}\right)$ and Halon $1211\left(\mathrm{CF}_{2} \mathrm{ClBr}\right)$, were identified along with a number of other halogenated compounds, as detrimental to stratospheric ozone. Halons have been commonly used as firefighting agents since the 1940 s mainly because of their ability to extinguish fires at low concentrations with essentially no residue. As these agents are replaced by possibly less effective alternatives, continued effective fire protection becomes a challenge.

At NIST, a series of studies have been conducted in an effort to identify suitable alternatives agents (Pitts et al., 1990; Grosshandler et al., 1994; Gann, 1995). This multi-year investigation has focussed on a wide range of relevant issues including agent thermodynamic properties, fluid dynamics of agent discharge, stability under storage, metal and elastomer seal compatibility, human exposure and environmental impact, and suppression of fires and quasi-detonations. The NIST effort has been directed mainly towards aircraft fire protection, which has many commonalities with other types of applications in the transportation and communication industries.

A key aspect of fire safety on an aircraft involves protection of the engine nacelle, which encases the jet engine compressor, combustors, and turbine. A nacelle fire is typically a turbulent diffusion flame stabilized behind an obstruction in a moderate speed air flow. The fuel source for a fire in the nacelle can be leaking pipes carrying jet fuel or hydraulic fluid that can feed the fire either as a spray or a pool. Extinguishment occurs when a critical amount of agent is transported to the combustion zone. In the NIST flame suppression measurements, the 
effectiveness of candidate replacement agents were tested in a number of combustion configurations including a cup burner, an opposed flow diffusion flame, and two baffle stabilized flame configurations, a spray flame and a pool fire (Grosshandler et al., 1994; Gann, 1995). In addition to agent ranking studies, measurements were conducted in the spray burner to test the impact of operating parameters on agent requirements. These measurements underscored the importance of agent entrainment into the recirculation/combustion zone. The remainder of this paper is a description of some of the suppression measurements conducted on obstacle stabilized flames.

\section{Experimental Method and Apparatus}

Figure 1 shows a cross-sectional view of the spray burner which has been described previously (Gann, 1995). The apparatus incorporated an air delivery system, a fuel delivery system, an agent injection system, and a combustion zone. Air co-flowed around a fuel tube within a $7.3 \mathrm{~cm}$ stainless steel tube. The fuel was injected along the centerline through a pressure-jet nozzle that formed a $45^{\circ}$ solid-cone spray. The flame was stabilized by a steel disk ( $3.5 \mathrm{~cm}$ diameter) attached to the body of the nozzle. An attachment to the burner facilitated tests on the influence of super-ambient pressures on flame stability.

The mass of agent delivered to the air stream was determined by measuring the initial temperature and the transient pressure in the vessel and using the Redlich-Kwong equation of state. The vessel pressure data were collected at a rate of $1000 \mathrm{~Hz}$. Uniform dispersion of agent across the air stream was verified by hot film probe measurements. The amount

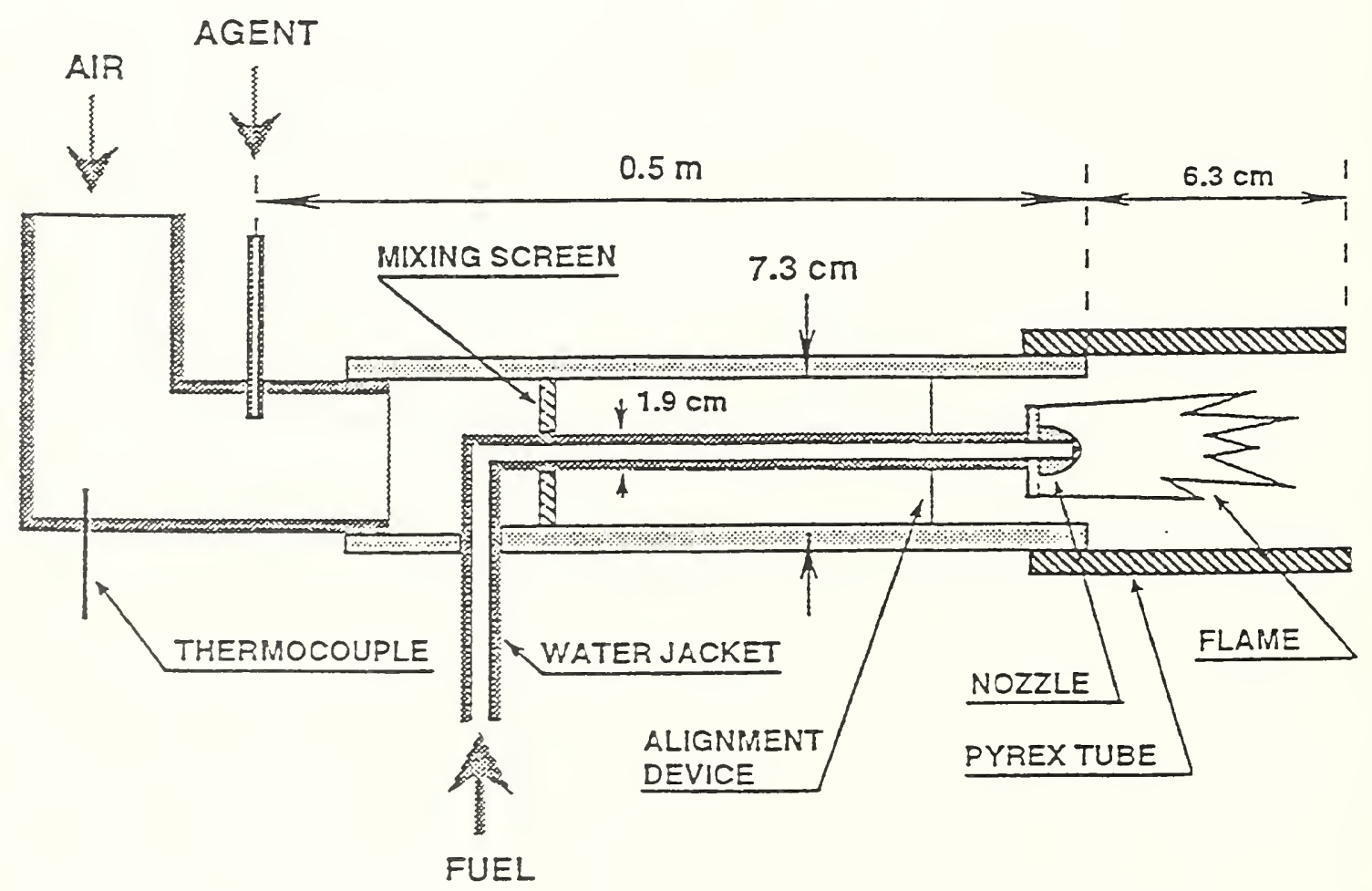

Figure 1. Schematic diagram of the baffle stabilized spray burner used for suppression testing. 
of injected agent was controlled by varying the initial vessel pressure, the time the solenoid valve was open and the valve opening diameter. The agent injection system under idealized conditions was designed to deliver a square-wave pulse of agent to the burner for a controlled amount of time.

The independent parameters which were controlled in the spray burner facility were the air flow, the agent delivery interval or injection duration, the air temperature, the system pressure, the fuel flow, and the agent temperature. The primary dependent experimental parameters were the agent mass, and the rate and duration of agent injection required for suppression. Extinction measurements were performed with $\mathrm{CF}_{3} I, \mathrm{C}_{2} \mathrm{HF}_{5}$ ( $\mathrm{HFC}-125$ ), and $\mathrm{C}_{3} \mathrm{HF}_{7}(\mathrm{HFC}-227$ ), which were selected as candidate halon replacements for engine nacelle applications due to a number of positive attributes (Grosshandler et al., 1994). Measurements were also performed using $\mathrm{CF}_{3} \mathrm{Br}$ (halon 1301) to establish a performance reference.

\section{Experimental Results}

3.1 Effect of Agent Injection Interval. Figure 2 shows the critical mass fraction (B) of $\mathrm{CF}_{3} \mathrm{Br}$ and the three alternative agents at extinction as a function of agent delivery interval for a constant air velocity equal to $7.5 \mathrm{~m} / \mathrm{s}$. For conditions below the data points in Fig. 2, the flames were not extinguished, whereas for conditions above the data points, the flames were extinguished. As the delivery interval increased, the critical $B$ decreased, and approached an asymptote for long delivery intervals. $\mathrm{CF}_{3} \mathrm{Br}$ required the least mass fraction to extinguish the flames, followed by $C_{3} I$, and the other two agents, $\mathrm{C}_{2} \mathrm{HF}_{5}$ and $\mathrm{C}_{3} \mathrm{HF}_{7}$. The shape of the curves for all of the agents in Fig. 2 were nearly identical, but displaced along the $y$-axis.

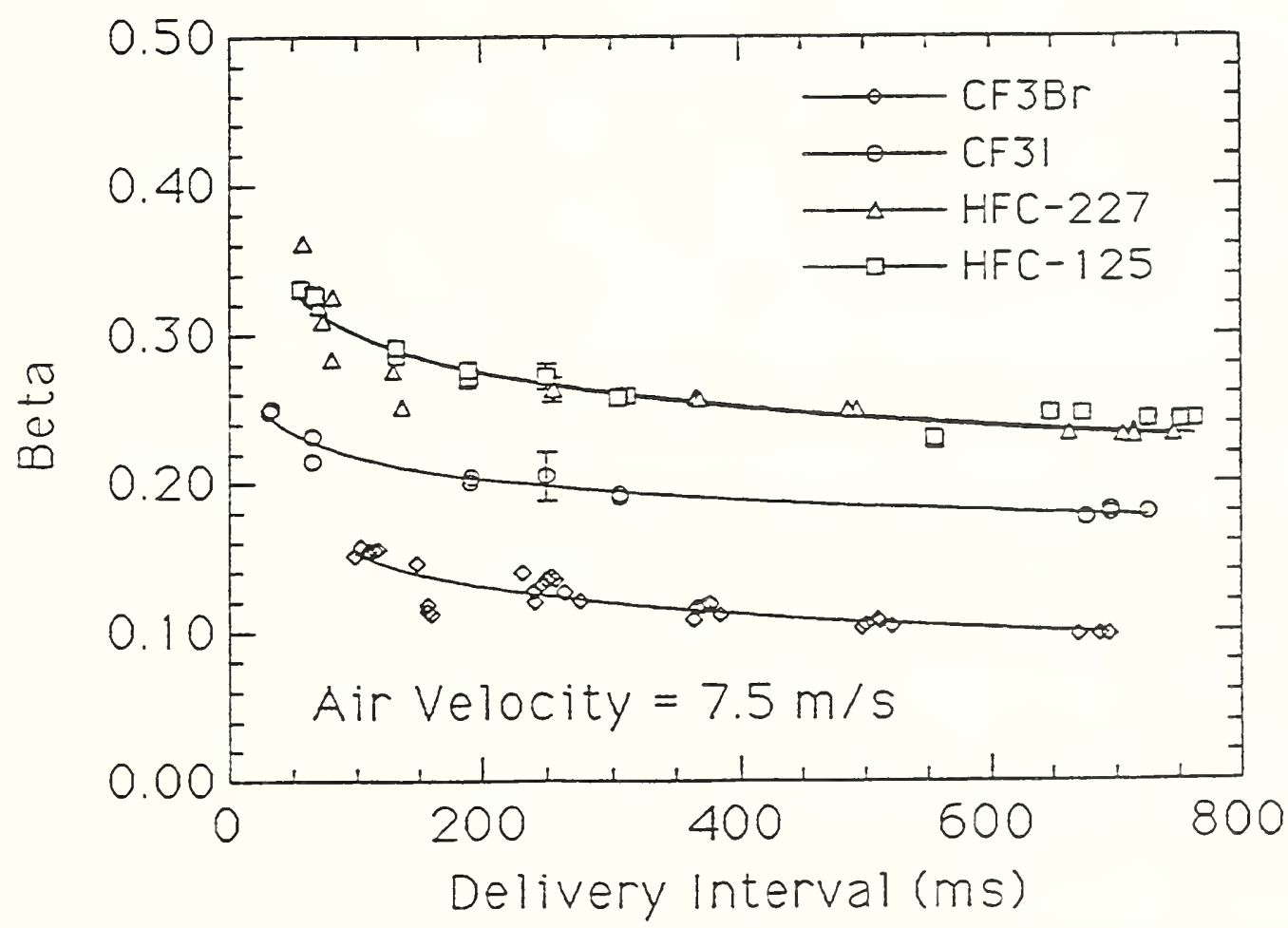

Figure 2. The critical agent mass fraction at extinction as a function of agent delivery interval. 
These data can be explained in terms of a phenomenological model first developed by Longwell et al. (1953) to explain blowoff of premixed flames by treating the recirculation zone as a well-stirred reactor. The characteristic mixing time of reactants to entrain from the free stream into the recirculation zone is a key parameter in the model. Here, the model is extended to treat agent entrainment into the recirculation zone and subsequent flame extinction. The assumptions used to develop the model are as follows. The flame is stabilized in the recirculation zone behind the obstacle. To extinguish the flame, the agent (volume based) concentration (X) must obtain a critical value. The recirculation zone is homogeneous and mixing of the agent in the zone is instantaneous. Spray characteristics are considered unimportant. As agent entrains into the recirculation zone, the concentration there is given by:

$$
X=X_{f}\left[1-e^{(-\Delta t / \tau)}\right]
$$

where $x_{f}$ is the free stream agent mole concentration, $\Delta t$ is the agent injection duration, and $\tau$ is the characteristic mixing time for entrainment into the recirculation zone. For very long injection times $(\Delta t>>\tau)$, the concentration in the recirculation zone will approach the free stream agent concentration, $x_{f}$. Experiments reported by Bovina (1958) confirm the form of Eq. 1 . The well stirred reactor model requires that for flame extinction, the agent concentration in the recirculation zone must obtain the same critical value, regardless of agent injection duration. Thus, the model suggests that the critical agent concentration in the free stream required to achieve extinction, $x_{c}(\Delta t)$, for a finite injection interval ( $\left.\Delta t\right)$ is related to the critical agent concentration in the free stream, $x_{\infty}(\Delta t>>\tau)$, for long injection intervals $(\Delta t>>\tau)$ and an exponential term associated with the extent of mixing:

$$
X_{c}(\Delta t)=\frac{X_{\infty}(\Delta t>\tau)}{1-e^{(-\Delta t / \tau)}}
$$

For long injection durations, the denominator in Eq. 2 takes a value of $\approx 1.0$ and $x_{c}$ is equal to $x_{\infty}$. For short injection intervals, very high agent concentrations are required to obtain extinction.

In addition, $x_{c}$ is constrained such that $x_{c} \leq 1$. This implies that there exists a critical injection duration $\left(\Delta t_{c}\right)$ such that when $x_{c}=1, \Delta t_{c}$ is given by:

$$
\Delta t_{c}=-\tau \ln \left(1-X_{\infty}\right)
$$

For $\tau$ equal to $100 \mathrm{~ms}$, representative of conditions in the spray burner for an air velocity of $3 \mathrm{~m} / \mathrm{s}$, and $x_{\infty}$ equal to $0.1, E q \cdot 3$ yields a value of $\Delta t_{c} \approx 11 \mathrm{~ms}$. Unfortunately, the minimum solenoid opening time was much larger than this value, so the veracity of $\mathrm{Eq.} 3$ was not tested.

Bovina (1958) found that $\tau$ in $E q .2$ is related to the baffle diameter (d) and the upstrgam velocity (V): 


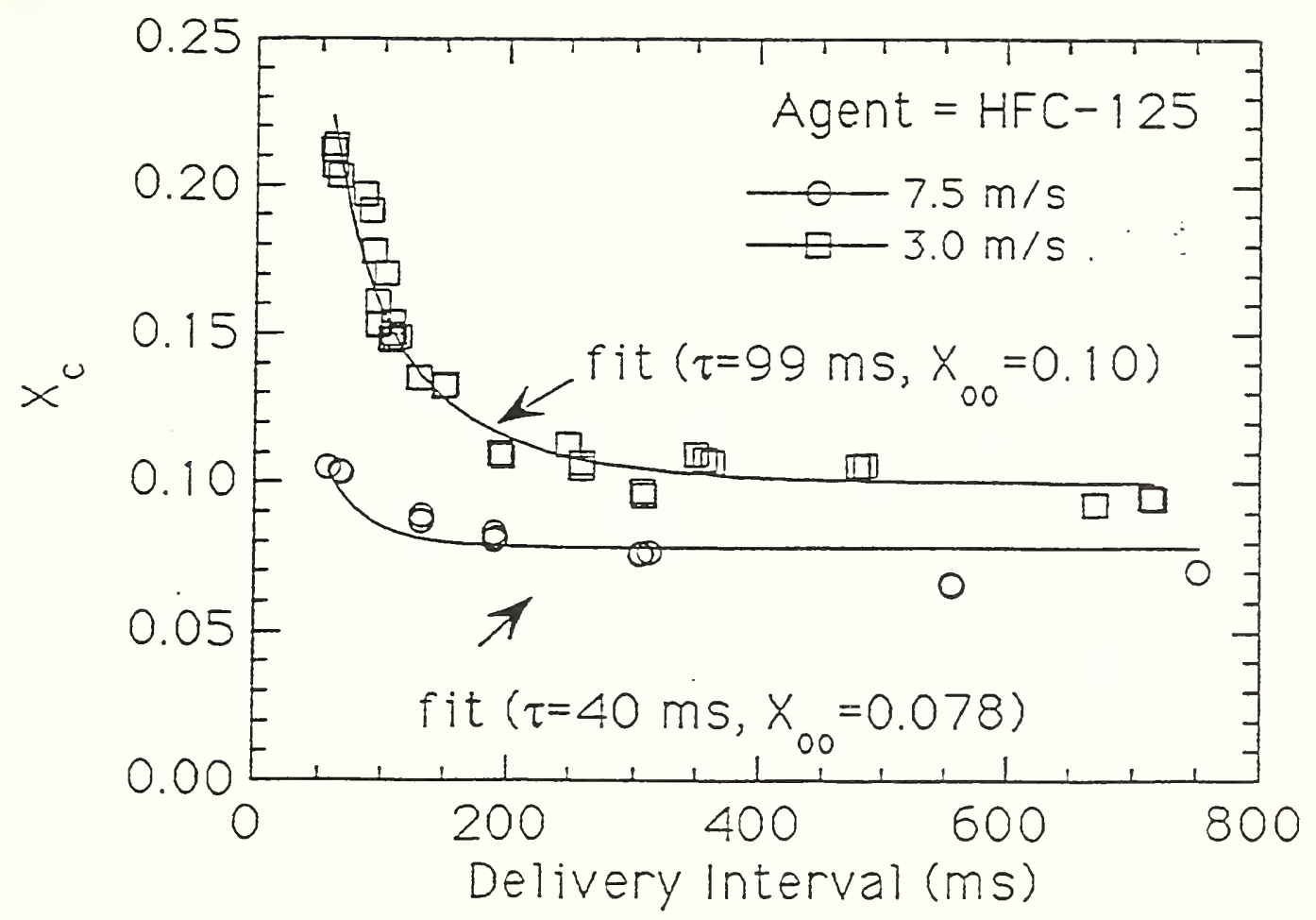

Figure 3. The critical mole fraction of HFC-125 at extinction for air velocities of 3.0 and $7.5 \mathrm{~m} / \mathrm{s}$.

Winterfeld (1965) verified Eq. 4 for both combusting and noncombusting conditions for Reynolds numbers extending from $\approx 1.5 \cdot 10^{4}$ to $2.2 \cdot 10^{5}$. Winterfeld $(1965)$ also found that the time constant was a function of the blockage ratio and the geometry of the flame holder.

A two parameter fit to the extinction data shown in Fig. 2 (after conversion to mole from mass fraction) allows determination of the parameters $X_{\infty}$ and $\tau$ in Eq. 2. The critical mole fraction of HFC-125 at extinction for air velocities of 3.0 and $7.5 \mathrm{~m} / \mathrm{s}$ is shown in Fig. 3 using a portion of the data presented in Fig. 2. Interpreting the curves in terms of Eq. 2 shows that a best two parameter fit for the $V=3.0 \mathrm{~m} / \mathrm{s}$ data yields $\tau=99 \mathrm{~ms}$ and $X_{\infty}=0.10$. Because Eq. 4 suggests that $\tau \propto(1 / V)$, the $7.5 \mathrm{~m} / \mathrm{s}$ data should be well represented by $\tau=40 \mathrm{~ms}(=99 \mathrm{~ms} / 2.5)$. A plot using $40 \mathrm{~ms}$ for $\tau$ leads to a reasonable fit of the $\mathrm{V}=7.5 \mathrm{~m} / \mathrm{s}$ data shown in Fig. 5. The fit yields a value of 0.078 for $\mathrm{x}_{\infty}$. The value of $x_{\infty}$ itself is a strong function of the air velocity and is thus a function of $(1 / \tau)$ as described below.

3.2 Effect of Air velocity. Figure 4 shows the critical mass fraction ( $(B)$ of $\mathrm{CF}_{3} \mathrm{Br}$ and the three alternative agents at extinction as a function of air velocity for a constant injection duration equal to $700 \mathrm{~ms}$. This value of the injection duration was selected because $X_{c} \approx X_{\infty} \cdot C_{3} B r$ required the smallest mass fraction to extinguish the flames, followed by $\mathrm{CF}_{3} I$, and the other two agents, HFC-125 and HFC-227, which were measured to have nearly identical effectiveness. As the air velocity increased from $3 \mathrm{~m} / \mathrm{s}, B$ decreased. At high air velocities, the flames were less stable and easier to extinguish, i.e. less 


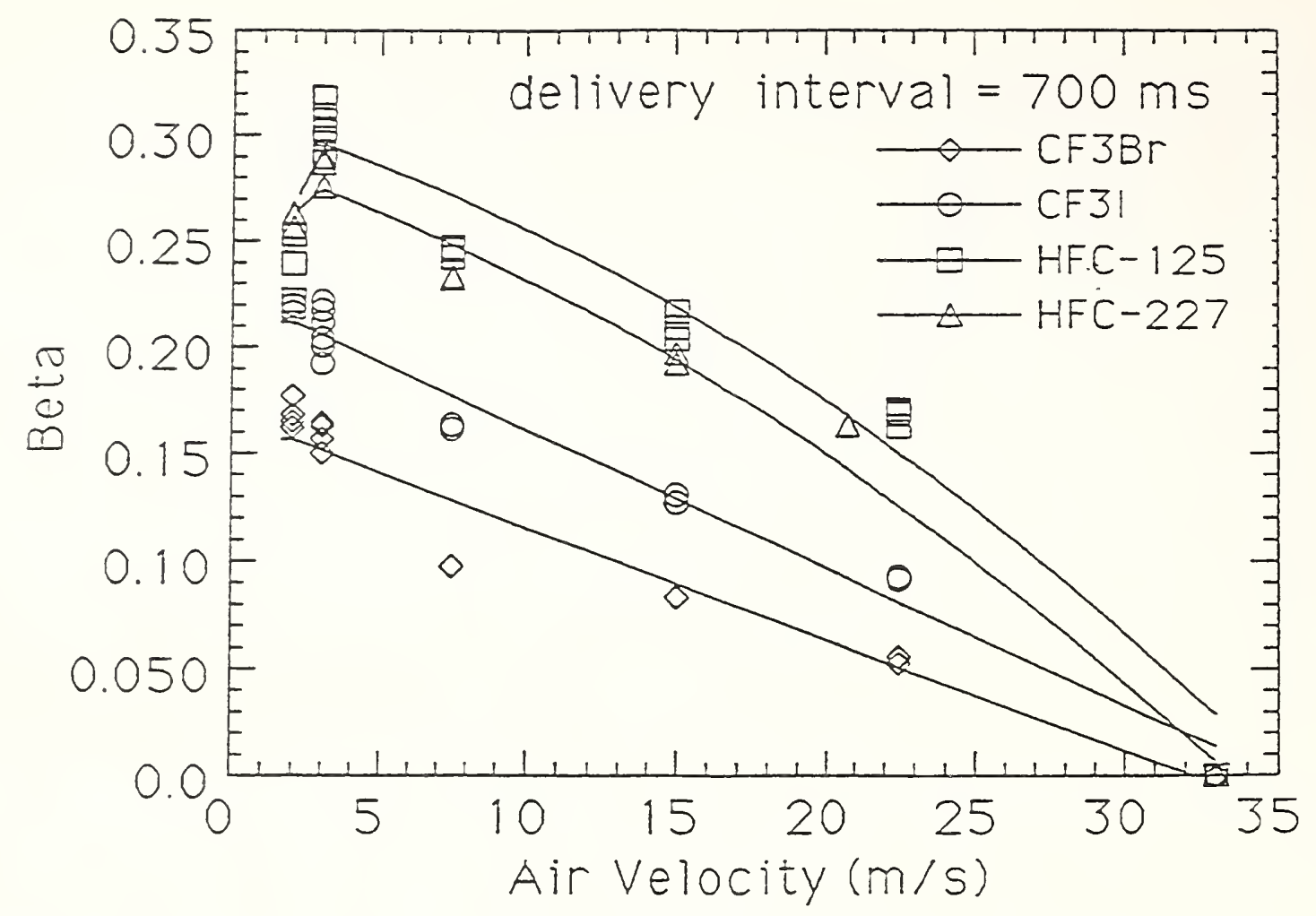

Figure 4. The critical agent mass fraction at extinction as a function of air velocity.

agent was required to extinguish them. At $V=33 \mathrm{~m} / \mathrm{s}$, air with no agent addition caused flame extinction. For very low air velocities ( $2 \mathrm{~m} / \mathrm{s}$ ), $B$ decreased or remained nearly the same as the results for $V=3 \mathrm{~m} / \mathrm{s}$. For all agents, the values of $B$ for the low air velocity spray flame results are very similar to agent extinction concentrations measured in cup burner flames and in opposed flow diffusion flames (OFDF) at low (25 $\mathrm{s}^{-1}$ ) strain rates (Hamins et al., 1994). Table 1 documents the correspondence between the flame extinction measurements in the three burners. All tests were conducted with JP-8 fuel. Table 1 shows that a correspondence also exists between the critical agent mass fractions for moderate $\left(80 \mathrm{~s}^{-1}\right)$ strain rates in the OFDF burner (Hamins et al., 1994) and moderate air velocities $(15 \mathrm{~m} / \mathrm{s}$ ) in the spray burner. The same correspondence holds for high $(22.5 \mathrm{~m} / \mathrm{s})$ air velocities in the spray burner and high $\left(175 \mathrm{~s}^{-1}\right)$ strain rates in the OFDF burner. The practical implication of the results shown in Table 1 is that it is not necessary to rank the suppression effectiveness of agents in every possible configuration, a single test apparatus is sufficient. The correspondence between extinction concentrations in the spray burner, cup burner, and OFDF implies that a relationship exists in terms of the critical Damköhler number criterion for flame extinction.

3.3 Effect of Fuel Flow, Pressure, and Air Temperature. Other experiments showed that more agent mass was required to extinguish flames when the air was heated. This trend was anticipated, since heating the air adds enthalpy to a flame, and a flame with a higher enthalpy is expected to be more stable. 
However, increasing the air temperature altered the agent ranking. For temperatures below $150{ }^{\circ} \mathrm{C}, \mathrm{CF}_{3} I$ was the most effective agent. For temperatures above $150^{\circ} \mathrm{C}$, the three agents, $\mathrm{CF}_{3} \mathrm{I}$, $\mathrm{HFC}-125$ and $\mathrm{HFC}-227$ were approximately equally effective.

Experiments using a butterfly valve placed on the downstream end of the burner showed that the system pressure did not impact the agent concentration required to obtain extinction over the pressure range tested (101-135 kPa). Other suppression measurements showed that the fuel flow had little effect on the agent concentration required to achieve flame extinction.

of the three candidate replacement agents evaluated in the turbulent spray burner, $\mathrm{CF}_{3} I$ was consistently the most effective compound. $\mathrm{CF}_{3} I$ required the least amount of gaseous agent to extinguish the flames on both a mass and volume basis. The other two alternative agents tested, HFC-125 and HFC-227, were measured to have nearly identical suppression effectiveness, and were significantly less efficient than $\mathrm{CF}_{3} I$ in extinguishing the flames. On a mass basis, none of the agents performed as well as halon 1301 .

Table 1 Agent mass fraction at extinction with JP-8 fuel

\begin{tabular}{|c|c|c|c|c|c|c|c|}
\hline \multirow{2}{*}{ Agent } & \multirow{2}{*}{$\begin{array}{l}\text { Cup } \\
\text { Burner }\end{array}$} & \multicolumn{3}{|c|}{$\begin{array}{l}\text { Air Velocity (m/s) } \\
\text { in Spray Burner }\end{array}$} & \multicolumn{3}{|c|}{$\begin{array}{c}\text { Strain Rate }\left(\mathrm{s}^{-1}\right) \text { in } \\
\text { OFDF burner }\end{array}$} \\
\hline & & 3.0 & 15 & 22 & 25 & 80 & 175 \\
\hline $\mathrm{CF}_{3} \mathrm{Br}$ & 0.8 .1 .4 & 0.6 .6 & 0.085 & 0.05 & 0.618 & 0.080 & 0.050 \\
\hline $\mathrm{CF}_{3} \mathrm{I}$ & 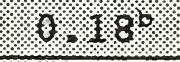 & 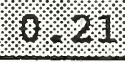 & 0.13 & 0.09 & a & a & $a$ \\
\hline $\mathrm{HFC}-125$ & 0.28. & (1. 30 & 0.21 & 0.17 & 0.28 & 0.22 & 0.16 \\
\hline $\mathrm{HFC}-227$ & (3.2. 2 & 10.28 & 0.20 & 0.15 & 0.26 & 0.20 & 0.14 \\
\hline
\end{tabular}

a Not measured

b Measured with heptane as fuel. The agent concentration required to extinguish heptane and JP-8 cup burner flames are within 48 (Grosshandler et al., 1994).

3.4 Suppression of Baffle stabilized Pool Fires. Measurements on the suppression of baffle stabilized pool fires were conducted at walter Kidde Aerospace under NIST direction. A detailed description of the experimental apparatus can be found in Gann (1995). The test results showed that the mixing time $(\tau)$ was relatively large in baffle stabilized pool fires as compared to the baffle stabilized spray fires. The characteristic mixing times from the data fits were 0.5 for HFC-125 (with the air velocity approximately equal to $3 \mathrm{~m} / \mathrm{s}$ ) and $0.7 \mathrm{~s}$ for HFC-227 (with the air velocity approximately equal to $1.5 \mathrm{~m} / \mathrm{s}$ ). The minimum critical agent concentration required to achieve flame extinction was significantly larger than the concentration required to suppress cup burner flames under similar conditions, consistent with the results of Hirst et al. 
(1976) and Dyer et al. (1977). The minimum critical agent concentrations approximately corresponded to the amount of agent required to suppress hydrocarbon flames at their peak flammability limits.

\section{Conclusions}

A comparison of flame stability in pool fires and spray flames showed that for similar air flows and baffle sizes, baffle stabilized pool fires were more difficult to extinguish than the baffle stabilized spray fires. Larger agent concentrations and longer characteristic agent mixing times were required to achieve suppression in the pool fires due to the structure of the recirculation zone.

Two dimensional isothermal fluid flow calculations showed that the characteristic time for an agent to entrain into a recirculation zone behind a bluff body depends on the location of the baffle in relation to the wall. The characteristic entrainment time is significantly larger for a baffle abutting a wall as compared to a baffle in the center of the flow field. The experimental findings were therefore consistent with the flow calculations.

\section{Acknowledgements}

This study was funded by the U.S. Army, Navy, Air Force and FAA. The author is grateful to $T$. Cleary and $R$. McGrattan for performing the pool fire experiments and flow field calculations, respectively, and C. Presser and I. Melton for assisting with the spray burner suppression measurements.

\section{References}

Bovina, T.A., Seventh sym. (Int.) on Combustion, The Combustion Institute, p. 692, (1958).

126, (1977).

Dyer, J.H., Marjoram, M.J.\& Simmons, R.F., Fire Tech., 13,

Gann, R.G., ed., "Fire Suppression System Performance of Alternate Agents in Aircraft Engine and Dry Bay Laboratory Simulations," NIST Special Publication SP 890 (1995).

Grosshandler, W., Gann, R.G., and Pitts, W.M., eds., "Evaluation of Alternative In-Flight Fire Suppressants for FullScale Testing in Simulated Aircraft Engine Nacelles and Dry Bays," NIST Special Publication Number SP 861, (1994).

Hamins, A., Trees, D., Seshadri, K., Chelliah, H.K., Combust. Flame, 99, 221-230 (1994).

Hirst, R., Farenden, P.J., and Simmons, R.F., Fire Tech., $12,266,(1976)$.

Longwell, J.P., Frost, E.E., and Weiss, M.A., Ind. Eng. Chem., 45, 1629, (1953).

Pitts, W.M., Nyden, M.R., Gann, R.G., Mallard, W.G., Tsang, W., eds., "Construction of an Exploratory List of Chemicals to Initiate the Search for Halon Alternatives," NIST Technical Note 1279, (1990).

Winterfeld, G., Tenth Sym. (Int.) on Combustion, The Combustion Institute, p. 1265, (1965). 


\section{Discussion}

Naoshi Saito: In your paper, you said in order to compare the individual suppressant performance, you can just use one formula. I wonder if this is true. It reminds us of the time when we determined the size of the cup burner, but depending on the fuel, we could not get consistent results.

Anthony Hamins: What we showed is the most dangerous situation. In order to extinguish such a configuration, one must consider final events. 


\title{
Evaluation of Fire Suppression Efficiency of Halon Replacements in Japan
}

\author{
Naoshi SAITO \\ Fire Research Institute \\ 14-1, Nakahara 3 chome, Mitaka, Tokyo 181, Japan
}

\begin{abstract}
"Committee on Evaluation of Fire Suppression Efficiency for Halon Replacements" in Japan discussed methods of evaluation on the fire suppression efficiency to prepare the basis of design concentration. Use of FRI cup burner was recommended to evaluate the efficiency of fire extinguishing agents in Japan by the committee.
\end{abstract}

\section{Introduction}

Halon phase-out program in Japan is composed with following three main policies [1];

(a) Ban on the new installation of halon fire extinguishing system,

(b) Promotion of halon recycling for existing equipment, and

(c) Employment of halon replacements.

When the candidates of halon replacement are employed as new fire extinguishing agents for total flooding system, limits of agent concentration on toxicity and fire extinguishing efficiency must be set up for realizing safe fire extinguishing. In 1993, Fire Research Institute organized "Committee on Evaluation of Toxicity for Halon Replacements." The chair person of the committee was Professor Uehara who works for Yokohama National University. The committee investigated evaluating procedures of toxicity to nominate the candidates of halon replacement and to determine the maximum design concentration of each candidate for the total flooding system $[2,3]$.

In 1994, "Committee on Evaluation of Fire Suppression Efficiency for Halon Replacements" was established by Fire Research Institute, and studied the evaluation method of the suppression efficiency for determining the minimum of design concentrations [4]. The chair person of the committee was Professor Hirano of Tokyo University. Each committee proposed the technical guideline on evaluating procedure of toxicity or fire suppression efficiency of halon replacements in March 28, 1995, respectively. Fire Defense Agency announced the guideline on the use of halon replacements on May 10, 1995, referring to the proposals of the above two committees [5].

The paper is a report on the evaluation method of fire suppression efficiency of halon replacements proposed by Committee on Evaluation of Fire Suppression Efficiency for Halon Replacements.

\section{Evaluation method of fire suppression efficiency}

The objective of evaluation of fire suppression efficiency was restricted to determine design concentration for total flooding system in the committee, because the halon replacements for the total flooding system were required most urgently and seriously in Japan in those days. In the committee, the evaluation methods were discussed from the view point of combustion science. At first, the literature on the flame extinguishing was searched for extract appropriate 
evaluation methods. Then, fire extinguishing concentrations were measured by using cup burner [6] and counterflow burner [7], and peak concentrations of flammability were obtained by tubular flame burner [8]. The committee on halon phase-out program of Japan, organized by Fire Defense Agency, carried out fire extinguishing tests using large scale room fire model, independently. The objective was to obtain the information on the design concentration of potential candidates for real size compartment fires [9].

In the facilities of telecommunication, computer rooms, museums, etc., fires with diffusion flame occur more frequently than explosion accidents. Therefore, the Committee chose to evaluate the fire extinguishing efficiency by testing the extinction behavior of diffusion flame. The cup burner apparatus is only a test method standardized internationally to estimate the fire extinguishing efficiency of carbon dioxide [10]. Any other test methods have never existed internationally for evaluating the efficiency of other fire suppressants. However, the cup bumer test has been widely employed as one of the most typical laboratory scale tests. Flame extinguishing concentration measured by the cup burner is used frequently as a basis for determination of a design concentration of total flooding fire extinguishing system. Thus, the cup burner method is considered one of the potential candidates of evaluation method on fire suppression efficiency of agents.

\section{Flame extinguishing concentrations of cup burner}

It is known that the flame extinguishing concentration is affected by the size of cup burner [11]. To investigate whether the flame extinguishing concentration is reliable as a basis of design concentration or not, the reproducibility of the concentration was tested by measuring the flame extinguishing concentrations of halon 1301 for heptane flame using three FRI glass cup burners. The cup burner apparatus shown in Fig. 1, that has a $30 \mathrm{~mm}$ outer diameter cup in a chimney with an $85 \mathrm{~mm}$ inner diameter. The experiments were conducted under $25^{\circ} \mathrm{C}$ and the atmospheric pressure, and air flow rate was adjusted at 40 liters per minute in all the tests. The flame is allowed to burn for 8 minutes before agent is added to the air stream.

The results are shown in Table 1. There is good agreement among the flame extinguishing concentrations obtained by the three apparatuses even under the small different conditions of burner setting draft chamber, cup shape, and test period. The fact means that the cup burner gives the same flame extinguishing concentration, if the measurement is carried out using same size apparatus under fixed air flow conditions [12].

The flame extinguishing concentration stayed constant, when the measurement was performed by different groups of operator as seen in Table 2. Moreover, there is good correlation between the flame extinguishing concentrations measured by the glass cup burner and the metal cup burner like Fig. 2. Therefore, use of FRI cup burners was recommended for evaluating the efficiency of fire extinguishing agents in Japan.

The flame extinguishing concentrations of new agents for various fuels have been measured and reported in the literature $[6,13,14]$. The up-to-date data are shown in Table 3.

\section{Requirement of safety factor and extinguishing test of large scale model fire}

The flame extinguishing concentration measured by cup burner is affected by not only bumer size [11], but also flow velocity of air-suppressant mixture in a chimney [15]. So, physical meaning of the concentration is ambiguous, and the conditions at fire extinction are not specified in the scenario of real fire growth. This means that the flame extinguishing concentration must be considered as the minimum concentration required for extinguishing the fire. Therefore, a safety factor is needed when a design concentration for total flooding fire extinguishing system is determined by the flame extinguishing concentration.

The design concentration for total flooding system is determined by the cup bumer flame extinguishing concentration multiplied by the safety factor. However, the design concentration is not always enough to extinguish any fires. Therefore, the fire extinguishing test using large scale model fire must be required to prove the fire extinction by the design concentration. 


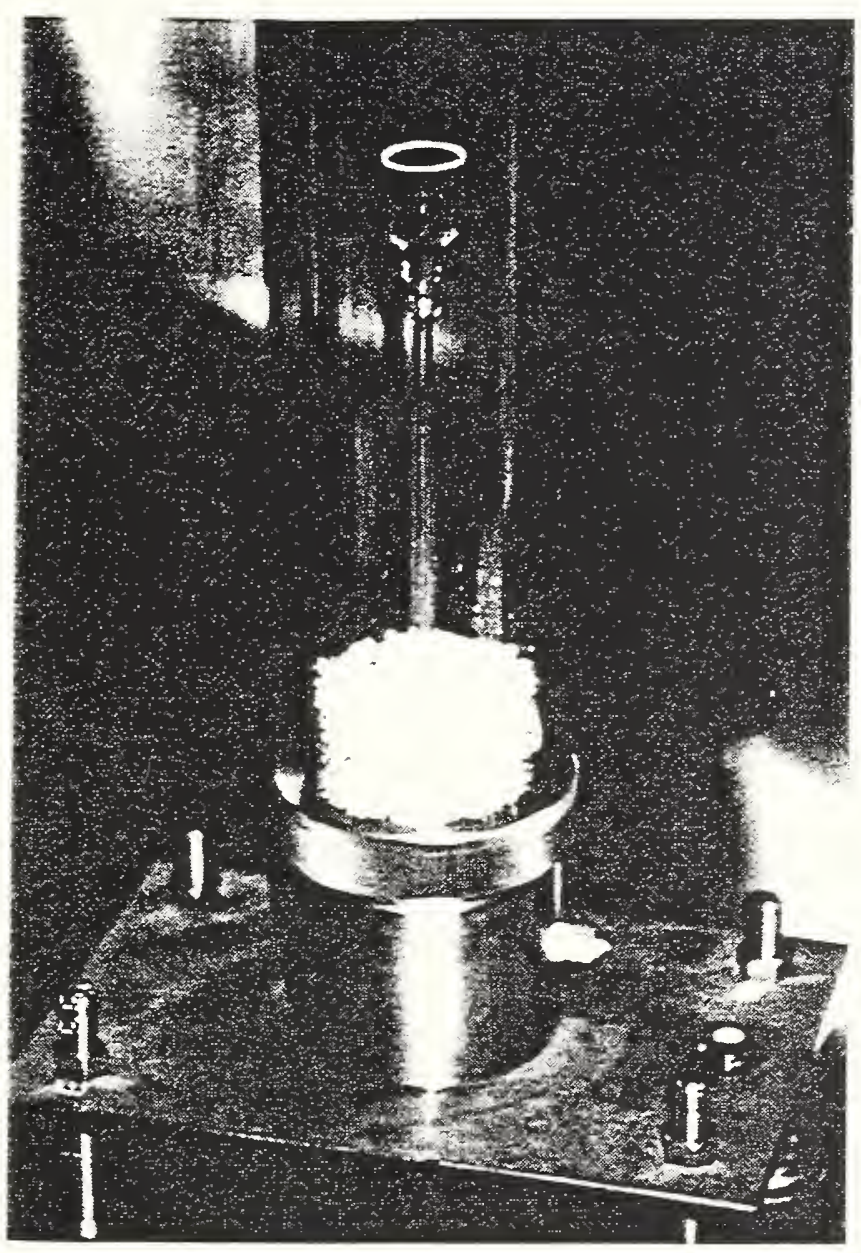

Fig. 1 FRI glass cup burner

Table 1 Extinguishing concentrations of halon 1301 for heptane flame measured by FRI glass cup burners

\begin{tabular}{|c|c|c|c|c|c|c|c|c|}
\hline Test period & \multicolumn{5}{|c|}{ 5 min } & \multicolumn{3}{c|}{ 2.5 min } \\
\hline Burner & FRI-0 & \multicolumn{2}{|c|}{ FRI-1 } & \multicolumn{2}{c|}{ FRI-2 } & FRI-0 & FRI-1 & FRI-2 \\
\hline Cup No. & $\mathbf{1}$ & $\mathbf{1}$ & $\mathbf{2}$ & $\mathbf{1}$ & $\mathbf{2}$ & $\mathbf{1}$ & $\mathbf{2}$ & $\mathbf{2}$ \\
\hline Observed (\%) & & & & & & & & \\
$\mathbf{1}$ & 3.37 & 3.48 & 3.18 & 3.37 & 3.37 & 3.22 & 3.22 & 3.41 \\
$\mathbf{2}$ & 3.41 & 3.52 & 3.33 & 3.41 & 3.33 & 3.22 & 3.41 & 3.22 \\
$\mathbf{3}$ & 3.29 & 3.44 & 3.44 & 3.41 & 3.29 & 3.14 & 3.37 & 3.33 \\
$\mathbf{4}$ & 3.33 & 3.56 & 3.41 & 3.37 & 3.25 & 3.14 & 3.48 & 3.29 \\
$\mathbf{5}$ & 3.33 & 3.56 & 3.44 & 3.44 & 3.41 & & 3.29 & 3.37 \\
\hline Average (\%) & $\mathbf{3 . 3 5}$ & $\mathbf{3 . 5 1}$ & $\mathbf{3 . 3 6}$ & $\mathbf{3 . 4 0}$ & $\mathbf{3 . 3 3}$ & $\mathbf{3 . 1 8}$ & $\mathbf{3 . 3 5}$ & $\mathbf{3 . 3 2}$ \\
\hline Std. div. & $\mathbf{0 . 0 4 6}$ & $\mathbf{0 . 0 5 2}$ & $\mathbf{0 . 1 1 0}$ & $\mathbf{0 . 0 3 0}$ & $\mathbf{0 . 0 6 3}$ & $\mathbf{0 . 0 4 6}$ & $\mathbf{0 . 1 0 2}$ & $\mathbf{0 . 0 7 3}$ \\
\hline
\end{tabular}


Table 2 Flame extinguishing concentrations of halon 1301 for heptane flame measured by dufferent operator groups

\begin{tabular}{|c|ccccc|c|c|}
\hline Groups & \multicolumn{5}{|c|}{ Observed data (\%) } & Average & Std. div. \\
\hline A & 3.42 & 3.46 & 3.42 & 3.35 & 3.39 & $\mathbf{3 . 4 1}$ & $\mathbf{0 . 0 4 1}$ \\
B & 3.36 & 3.40 & 3.36 & 3.51 & 3.29 & $\mathbf{3 . 3 8}$ & $\mathbf{0 . 0 8 1}$ \\
C & 3.44 & 3.40 & 3.30 & 3.37 & 3.44 & $\mathbf{3 . 3 9}$ & $\mathbf{0 . 0 5 9}$ \\
D & 3.44 & 3.30 & 3.37 & 3.33 & 3.37 & $\mathbf{3 . 3 6}$ & $\mathbf{0 . 0 5 3}$ \\
E & 3.33 & 3.30 & 3.40 & 3.40 & 3.44 & $\mathbf{3 . 3 7}$ & $\mathbf{0 . 0 5 7}$ \\
F & 3.37 & 3.44 & 3.56 & 3.37 & 3.44 & $\mathbf{3 . 4 4}$ & $\mathbf{0 . 0 7 8}$ \\
G & 3.42 & 3.46 & 3.42 & 3.35 & 3.39 & $\mathbf{3 . 4 1}$ & $\mathbf{0 . 0 4 1}$ \\
\hline
\end{tabular}

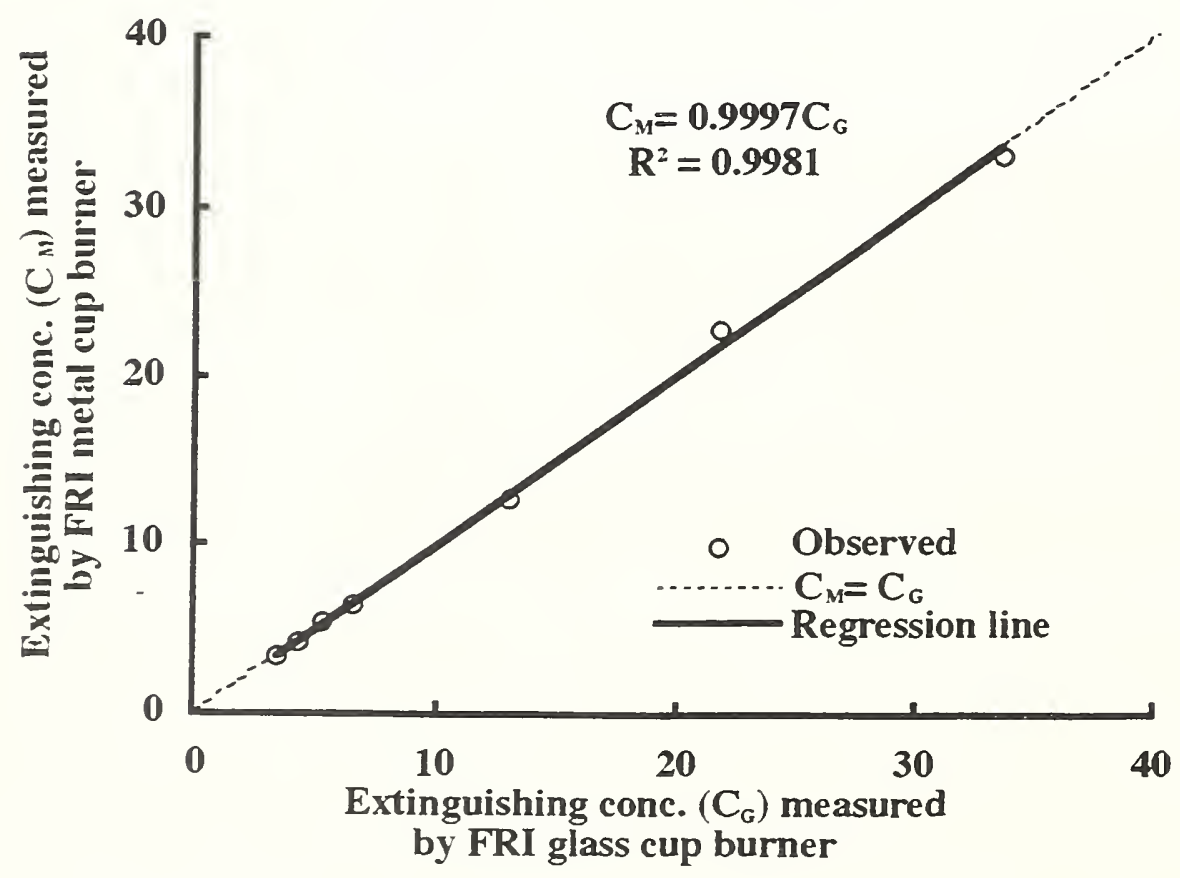

Fig. 2 Comparison of flame extinguishing concentrations measured by glass cup burner and metal cup burner 
Table 3 Flame extinguishing concentrations measured by FRI glass cup burner

\begin{tabular}{|c|cccccccccc|}
\hline \multirow{2}{*}{ Agent } & \multicolumn{10}{|c|}{ Fuels } \\
\cline { 2 - 9 } & heptane & octane & decane & undecane & dodecane & methanol & ethanol & benzene & toluene \\
\hline Halon 1301 & 3.4 & 3.4 & 3.9 & 3.8 & 3.7 & 7.8 & 4.3 & 2.4 & 2.3 \\
FC-3-1-10 & 5.3 & & & & & 8.0 & 6.9 & 3.4 & 3.6 \\
HFC-227ea & 6.6 & & & & & 9.4 & 8.2 & .4 .8 & 4.6 \\
$\mathrm{HFC-23}$ & 12.9 & & & & & 19.0 & 16.0 & 10.6 & 9.7 \\
$\mathrm{~N}_{2}$ & 33.6 & 33.8 & 33.9 & 33.3 & 33.5 & 43.5 & 36.8 & 30.9 & 25.7 \\
$\mathrm{Ar}$ & 43.3 & & & & & & & & \\
$\mathrm{CO}_{2}$ & 22.0 & & 23.4 & 23.5 & 22.8 & 29.3 & 24.3 & 20.2 & 16.9 \\
$\mathrm{IG541}$ & 35.6 & & & & & & & & \\
$\mathrm{IG505}$ & 25.9 & & & & & & & & \\
\hline
\end{tabular}

\section{Consistency of flame extinguishing concentrations measured by FRI cup burner}

The flame extinguishing concentration measured under the fixed conditions showed good reproducibility enough to use a basis for determining a design concentration. Since the design concentration has to be changed by characteristic of combustibles, it requires consistency of the flame extinguishing concentrations measured by the cup burner.

Let's assume that inert gases act only as heat sink individually in flame, and that the flame extinction occurs when the flame temperature decreases and reaches at a certain threshold value. Then, the following expression is derived for the flame extinguishing concentration $C_{M}$ of any mixture $\mathrm{M}$ of inert gases by simple thermodynamic consideration [16].

$$
1 / C_{M}=\sum_{j=1}^{n} X_{j} / C_{j}
$$

In Eq. (1), $n$ is number of components in the inert gas mixture. $X_{j}$ and $C_{j}$ denote mole fraction and flame extinguishing concentration of $j$ th component, respectively. The equation is similar to the famous formula for calculating the flammability limits of any mixture of combustible gases [17].

Since the flame extinguishing concentrations of nitrogen, argon, carbon dioxide, and inert gas mixtures, IG541 and IG505, for heptane are shown in Table 3, we can confirm the verity of the Eq. (1). The calculated flame extinguishing concentrations of IG541 and IG505 mixtures are compared with the observed values in Table 4. Here, the calculation was carried out using the flame extinguishing concentrations of nitrogen, argon, and carbon dioxide in Table 3, and the composition of the mixtures listed in Table 4 . The estimated values agree well with the observed value within $3 \%$ in relative difference to mean value. The fact suggests that the relation of Eq. (1) is correct for the flame extinguishing concentration of any inert gases, and at the same time, there is no contradiction in the flame extinguishing data measured with FRI cup burner.

To confirm the assumptions for Eq. (1), adiabatic flame temperature was calculated for the heptane diffusion flame diluted with nitrogen, argon, carbon dioxide, IG541, or IG505 just under the flame extinction condition. The calculated temperature and mole number of main combustion products generated per unit mole of fuel are listed in Table 5, that shows also the flame extinguishing concentrations measured by FRI cup burner. All the calculated adiabatic flame temperatures agree well, and the mole numbers of each main product are also nearly equal 
to each other, except the case of CO production. In the case where carbon dioxide or IG505 is added, $\mathrm{CO}$ production becomes twice. However, the $\mathrm{CO}$ concentration in burned gas is low enough, so it can change only $0.4 \%$ or less of the total heat capacity of combustion products. The results mean that the assumptions in Eq. (1) are valid for the flame extinction of the FRI cup burner by inert gases. At the same time, this is a proof of the consistency on the flame extinguishing concentrations reported in Table 3.

Table 4 Coparison of calculated flame extinguishing concentrations (\%) of IG541 and IG505 mixtures for heptane flame with obserbed values

\begin{tabular}{|c|c|c|c|c|c|}
\hline \multirow{2}{*}{ Mixture } & \multicolumn{2}{|c|}{ Flame ext. conc. (\%) } & \multicolumn{3}{|c|}{ Compositions of mixure (\%) } \\
\hline & Calc. & Obs. & $\mathrm{N}_{2}$ & $\mathrm{Ar}$ & $\mathrm{CO}_{2}$ \\
\hline IG541 & 35.6 & 35.6 & 51.5 & 41.6 & 6.9 \\
\hline IG505 & 26.6 & 25.9 & 50.0 & 0.0 & 50.0 \\
\hline
\end{tabular}

Table 5 Production of stable species (moles per unit mole fuel) and temperature of heptane diffusion flame at extinction by inert gas mixtures

\begin{tabular}{|c|ccccc|}
\hline \multirow{2}{*}{ Products } & \multicolumn{5}{|c|}{ Inert Gases } \\
\cline { 2 - 6 } & $\mathrm{N}_{2}$ & $\mathrm{Ar}$ & $\mathrm{CO}_{2}$ & IG541 & IG505 \\
\hline $\mathrm{H}_{2} \mathrm{O}$ & 7.97 & 7.96 & 7.97 & 7.97 & 7.97 \\
$\mathrm{H}_{2}$ & 0.02 & 0.03 & 0.02 & 0.02 & 0.02 \\
$\mathrm{CO}_{2}$ & 6.92 & 6.91 & 6.80 & 6.89 & 6.82 \\
$\mathrm{CO}$ & 0.08 & 0.09 & 0.20 & 0.11 & 0.18 \\
\hline Flame Temp.(K) & 1820 & 1835 & 1849 & 1836 & 1858 \\
\hline Ext. Conc. (\%) & 33.6 & 43.3 & 22.0 & 35.6 & 25.9 \\
\hline
\end{tabular}

\section{Conclusion}

In Japan, the FRI cup burner is used to evaluate the fire suppression efficiency of halon replacements. The cup burner is a potential apparatus for evaluation of fire suppression efficiency. It gives the same flame extinguishing concentration, if the measurement is carried out using same size apparatus under fixed air flow conditions.

It was suggested by thermodynamic consideration that flame extinguishing concentration of any mixture of inert gases is represented as a simple relation of mole fraction and flame extinguishing concentration of components in the inert gas mixture. The flame extinguishing concentrations of the inert gas mixtures for heptane flame were observed and compared with the estimated values. There was good agreement between both the values, and the flame extinguishing concentrations measured by the cup burner are consistent. At the same time, this is a proof of the consistency on the flame extinguishing concentrations in the paper. 


\section{Reference}

[1] Prevention Section, Fire Defense Agency, 1990 Annual Report of Committee on Halon Phase-Out (March, 1991) (in Japanese)

[2] Fire Research Institute, 1993 Annual Report of Committee for Evaluation on Toxicity of Halon Replacements (March, 1994) (in Japanese)

[3] Fire Research Institute, Final Report of Committee for Evaluation on Toxicitv of Halon Replacements (Sept., 1995) (in Japanese)

[4] Fire Research Institute, Final Report of Committee for Evaluation on Fire Suppression Efficiency of Halon Replacements (Dec., 1995) (in Japanese)

[5] Suzuki, K., Treatment Procedure on Fire Extinguishing Gas svstems as Halon Replacement, Kasai (Journal of Japan Association for Fire Science and Engineering), 45 (6), 1-6 (1995) (in Japanese)

[6] Inoue, Y., Saito, N., Saso, Y., and Ogawa, Y., Evaluation of Fire Suppression Efficiency and Practical Applicability of Halon Replacements, Report of Fire Research Institute of Japan, No. 79, pp. 1-7 (1995) (in Japanese)

[7] Saso, Y., Saito, N., Liao, C., Ogawa, Y., and Inoue, Y., Evaluation of Fire Suppression Efficiency of Fire-Extinguishing Agents using Counterflow Diffusion Flame, Report of Fire Research Institute of Japan, No. 78, pp. 42-50 (1994) (in Japanese)

[8] Saito, N., Saso, Y., Liao, C., Ogawa, Y., and Inoue, Y., Flammability Peak Concentrations of Halon Replacements and Their Function as Fire Suppressant, ACS Symposium Series 611, pp. 243-257, American Chemical Society (1995)

[9] Prevention Section, Fire Defense Agency, 1993 Annual Report of Committee on Halon Phase-Out (March, 1994) (in Japanese)

[10] ISO 6183, Fire protection eauipment - Carbon dioxide extinguishing xyxtems for use on premises - Desin and installation, (1991)

[11] Saso, Y., Saito, N., and Iwata, Y., Scale Effect of Cup Burner on Flame-Extinguishing Concentrations, Fire Technology, 29, pp. 22-33 (1993)

[12] Saito, N., Saso, Y., Ogawa, Y., Inoue, Y., Sakei, R., and Otsu, Y., Improvement on Reproducibility of Flame Extinguishing Concentration Measured bv Cup Burner Method, Proc. Halon Options Technical Working Conference, pp. 299-309, Albuquerque, NM (May, 1995)

[13] Sakei, R., Saito, N., Saso, Y., Ogawa, Y., and Inoue, Y., Flame Extinguishing Concentrations of Halon Replacements for Flammable Liquids, Report of Fire Research Institute of Japan, No. 80, pp. 36-42 (1995) (in Japanese)

[14] Saito, N., Sakei, R., Saso, Y., and Ogawa, Y., Some Problems on Measurement of Flame Extinguishing Concentration for High Flash Point Liquid Fuels, Proc. 33th Japanese Symposium on Combustion, pp. 539-541, Combustion Society of Japan (1995) (in Japanese)

[15] Bajpai, S. N., An Investigation of the Extinction of Diffusion Flames bv Halons, J. Fire \& Flammability, 5, pp. 225-267(1974) 
[16] Saito, N., Ogawa, Y., Saso, Y., Liao, C., and Sakei, R., Flame Extinguishing Concentrations and Peak Concentrations of N2. Ar, CO2, and Their Mixtures for Hydrocarbon Fuels, Submitted to Fire Safety J.

[17] Coward, H. F. and Jones, G. W., Limits of Flammability of Gases and Vapors, pp. 6, Bureau of Mines Bull. 503 (1952)

\section{Discussion}

William Grosshandler: Could you explain what the standard procedure is in Japan once you have the efficiency. How does one actually go about the design?

Naoshi Saito: In Japan they use the cup burner concentration for the design concentration. Then we multiply an appropriate safety ratio by that flame extinguishing concentration. How to set this safety ratio is left totally to the discretion of the designer of the apparatus. However, if you just stop there, there is always some fear that it might not be able to extinguish the fire so after designing it, you have to do a large scale room experiment.

Richard Gann: Several years ago, we experimented with replacing the heptane by a stick of PMMA to make the procedure simpler. Have you thought of something like that?

Naoshi Saito: Our purpose is to extinguish all the flammable things in the room or in the space. In our opinion, PMMA cannot represent everything which is flammable in the room or space. 


\section{Status Report on Water Mist Fire Suppression Systems - 1996.}

J. R. Mawhinney, P. Eng.

Senior Engineer

Hughes Associates, Inc.

Baltimore, MD
J. K. Richardson, P. Eng.

Head, National Fire Laboratory

National Research Council

Ottawa, Canada

\section{Introduction}

This status report will review a selection of recent developments in water mist fire suppression systems technology. Research and development work with water mist as a fire extinguishing medium began soon after the phase-out of Halon began with the signing of the Montreal Protocol in 1987. Still, in March 1996, the pace of development continues to be rapid and advances are occurring on several fronts. These include: full-scale testing to evaluate water mist on different fire scenarios; the development of test protocols to validate the performance of water mist; laboratory studies to advance the understanding of fire suppression using water mist; and developments in establishing design and installation standards and obtaining approvals listing of hardware associated with mist systems.

In collaboration with J.K. Richardson at the National Research Council in Canada (NRCC), I am coauthoring a summary review of current water mist research worldwide. A survey of water mist research activities was distributed by the NRCC in July 1995 to a broad range of agencies and individuals involved in water mist research. Table 1 is an abridged list of subjects identified in the survey, which shows the directions of interest to researchers, approval authorities, and manufacturers as of the end of 1995.

There are many interesting subjects worthy of detailed discussion in Table 1. For this "status report" I have selected four issues from the Table for comment. They are: the International Maritime Organization (IMO) test protocols, the Factory Mutual approvals for Gas Turbine Enclosures; the use of mist in Heritage buildings, and developments in applying mist to telecommunications facilities. The activity regarding the IMO test protocols is stimulating much international exchange, product development and technology transfer. A great many organizations in Europe and North America are involved in discussing the tests, conducting them or reacting to the results by modifying products. The approval by Factory Mutual of a pre-engineered water mist system for gas turbine enclosures represents a second significant step toward establishing water mist systems in main-stream fire safety engineering. These test protocols are viewed in the context of the recently completed National Fire Protection Association Standard, NFPA 750, Standard on Water Mist Fire Protection Systems [1]. NFPA 750 defers to full scale testing, such as the IMO and the FM protocols, as the only way to validate the performance of a water mist fire suppression system.

The desire to use water mist to protect property that is sensitive to water damage sustains strong interest in using mist in Heritage buildings, and on electronic equipment. The status of work in these two areas is also discussed in the following text. 
Table 1. Partial list of development activities pertaining to water mist fire suppression systems, as of August 1995.

\begin{tabular}{|c|c|}
\hline Subject & Comments \\
\hline $\begin{array}{l}\text { Characterizing droplet sizes and velocities: } \\
\text { - to advance understanding of extinguishment } \\
\text { - to evaluate nozzles for listing purposes } \\
\text { - to assist numerical modelling of sprays }\end{array}$ & $\begin{array}{l}\text { Phased Doppler Anemometers. Listing agencies need to } \\
\text { characterize sprays for listing purposes; CFD modelling } \\
\text { groups need initial drop size and velocity distributions. }\end{array}$ \\
\hline $\begin{array}{l}\text { Spray momentum } \\
\text { Spray dynamics } \\
\text { Additives }\end{array}$ & $\begin{array}{l}\text { A few agencies systematically studying the ability of spray to } \\
\text { penetrate openings, and the effects of striking hot surfaces. } \\
\text { Role of momentum in overcoming plume energy; spray } \\
\text { dynamics revealed with CFD. }\end{array}$ \\
\hline $\begin{array}{l}\text { Water mist on Class A combustibles } \\
\text { IMO cabin/public space test protocols } \\
\text { Libraries, archives, mobile shelving } \\
\text { Heritage Buildings, art work } \\
\text { High-rack warehouses, } 9 \mathrm{~m}\end{array}$ & $\begin{array}{l}\text { Equivalent to sprinkler system performance. } \\
\text { IMO Light and Ordinary hazard tests; smoldering fires; } \\
\text { shielded fires. Full-scale testing in progress. } \\
\text { Rack Height, } 9 \mathrm{~m} \text {; corrugated cartons, polypropylene boxes, } \\
\text { wood pallets. }\end{array}$ \\
\hline $\begin{array}{l}\text { Water mist on Class B hydrocarbon fires } \\
\text { IMO test protocols - full-scale tests } \\
\text { Marine machinery compartments } \\
\text { Gas turbine enclosures }\end{array}$ & $\begin{array}{l}\text { Machinery spaces; pool fires, spray fires, diesel fuel, } \\
\text { lubrication oils; high pressure and low pressure sprays. }\end{array}$ \\
\hline $\begin{array}{l}\text { Standards development } \\
\text { NFPA } 750 \\
\text { IMO tests } \\
\text { ULI - component testing }\end{array}$ & Much international technology exchange occurring. \\
\hline Spray generation methods: nozzle innovations & Manufacturers developing variety of nozzles. \\
\hline Computer Modelling: CFD & $\begin{array}{l}\text { NRCC - TASCflow CFD; FRS - JASMINE ; validated } \\
\text { against sprinklers; testing with water mist planned. }\end{array}$ \\
\hline $\begin{array}{l}\text { Watermist on electronics, telecommunication } \\
\text { equipment }\end{array}$ & $\begin{array}{l}\text { Several research groups working. Need to study non-thermal } \\
\text { damage. }\end{array}$ \\
\hline Spray generation methods: nozzle innovations & Manufacturers developing variety of nozzles. \\
\hline Improved detection/activation, system controls & Zoning requirements to improve efficiency \\
\hline Use of Additives: & $\begin{array}{l}\text { Laboratory scale experiments } \\
\text { Studying the interaction of water/additive droplets on hot } \\
\text { surfaces }\end{array}$ \\
\hline Combined water/inert gas sprays & $\begin{array}{l}\text { Combining mist with nitrogen discharge. } \\
\text { Mixing } \mathrm{CO}_{2} \text { and } \mathrm{H}_{2} \mathrm{O} \text { at discharge - unique cooling/inerting }\end{array}$ \\
\hline Water mist systems for Aircraft & $\begin{array}{l}\text { Aircraft passenger compartments - not applied } \\
\text { Aircraft: Cargo Bay systems - recent experiments } \\
\text { Aircraft: Jet engine nacelles - high challenge }\end{array}$ \\
\hline Manual fire suppression using water mist & $\begin{array}{l}\text { Using zone modeling; studying the efficiency of commercial } \\
\text { fire hose nozzles of different quality sprays; }\end{array}$ \\
\hline Health effects of inhaling water mist & $\begin{array}{l}\text { Health panel formed to review issues; concluded fresh and } \\
\text { sea water mist not a hazard to humans. Concerns remain } \\
\text { about the effects of additives such as surfactants, biocides, } \\
\text { suppression enhancing salts. }\end{array}$ \\
\hline
\end{tabular}




\section{IMO Test Protocols.}

The International Maritime Organization (IMO) is close to finalizing a test protocol to validate the use of water mist as equivalent to sprinkler systems on ships [2] [3] [4]. Test protocols have been developed for machinery spaces, where Class B liquid fuels are involved, and cabins, corridors and public spaces on ships, where Class A combustibles are involved. In the past 8 years, most of the NATO Navies (Norway, Sweden, Denmark, Germany, the Netherlands, United Kingdom, Canada, the US) have conducted full-scale machinery room tests using water mist. These were the tests that established the basic expectations for water mist. Attention has now focused on the details of a set of tests that will confirm performance of water mist in Class I machinery rooms up to $500 \mathrm{~m}^{3}$ volume; and in larger Class II and III engine rooms up to $3000 \mathrm{~m}^{3}$ and larger. Activity in support of the IMO test protocols for both cellulosic fuels (Class A) and liquid fuels (Class B) scenarios is occurring in Finland, at VTT; at the Swedish Testing laboratories (SP) in Borås, Sweden; at the Norwegian fire testing laboratories (SINTEF) in Trondheim; in separate projects funded by the US Department of Defense and the US Coast Guard on board two fire test ships located in Mobile, Alabama; and at Factory Mutual's test facility in West Gloucester, Rhode Island. Virtually all manufacturers of nozzles and equipment for water mist systems have contributed products for testing under these protocols.

It is interesting to note that the starting point for the IMO machinery space test protocols is defined by the engineering constraints on ships, rather than the optimum performance conditions for water mist. That is to say, it is assumed that total flooding of the compartment by water mist is the only practical design approach, because subdivision into smaller "zones" is too complex to be carried out reliably on merchant ships. Further, the IMO protocol requires water mist nozzles to be mounted at ceiling level only, not at "strategic" positions in the compartment which might improve performance. The reason for such a restriction is because it is considered impractical to situate nozzles ideally for all possible fire origins in an engine compartment. Again, it is assumed that under typical maritime conditions, there will be a ventilation opening into the compartment, therefore the water mist system cannot rely on "enclosure effects" of reduced oxygen concentration to achieve suppression. And, the performance objective for the mist system is set at nothing less than full extinguishment, given that, for a merchant ship with limited manual fire fighting capacity, "control" for a limited time may only delay the catastrophe. Although this "practical" approach is understandable, it poses difficulties for water mist, particularly in very large machinery compartments where the conditions needed for water mist to extinguish fires are seldom met.

Testing to the IMO protocol has been performed by the US Navy, US Coast Guard, and Factory Mutual, in North America, and at SP (Sweden), SINTEF (Norway), and VTT (Finland) in Europe. The tests confirm that water mist can be effective against diesel fuel spray and spill fires in marine machinery spaces up to $500 \mathrm{~m}^{3}$, and possibly up to $1000 \mathrm{~m}^{3}$. Various agencies are expressing doubt about the ability to meet the IMO test performance objectives in spaces larger than $1000 \mathrm{~m}^{3}$. The expectations of a low water demand system ("low" as opposed to sprinkler water demand, for example) are not met, because of the inefficiency of applying water in a deluge system mode in a large compartment. The economics of meeting the water storage, pumping energy, and piping requirements for deluge-type water mist systems on merchant ships has not yet been established.

Based on the results of full-scale testing the US Navy has concluded that water mist performance, supplemented by bilge foam system, provides adequate protection. The US Navy estimates that the cost of a water mist system for a large Naval machinery room $\left(>3000 \mathrm{~m}^{3}\right)$ will be competitive with the other Halon replacement alternatives [5]. The US Army is reported to have plans to install water mist systems in machinery compartments $\left(<500 \mathrm{~m}^{3}\right)$ on their inland waterways ships. 
The IMO test protocols extend also to Class $\mathrm{A}$ fire hazards involving cabins, crews quarters and public spaces on passenger ships [3,4]. Water mist systems are tested to show equivalence with a sprinkler system designed to the SOLAS regulations. Testing agencies have been involved in designing the fuel packages and fire scenarios, and testing different mist generating equipment against fires in Class A combustibles. The ability to achieve fire suppression with significantly less water than a standard marine sprinkler system has been demonstrated. Testing is now progressing to many other scenarios involving Class A combustibles, including ordinary hazard fuel loadings in public spaces on ships, and libraries, museums and large wooden structures on land.

\section{Turbine Enclosures.}

The turbine enclosure is a subset of the marine machinery space. The first water mist systems to receive insurance industry "approval" in North America have been for the turbine enclosure scenario. Full-scale testing for this application began with SINTEF in Norway in the late 1980's [6]. Recently, Factory Mutual Research Corporation completed the approval process for a water mist system for turbine enclosures and (similar machinery spaces) up to $80 \mathrm{~m}^{3}$ in volume. Testing is currently underway to evaluate similar systems for compartments up to $260 \mathrm{~m}^{3}$. Testing of turbine enclosure scenarios has also been carried out by John Dyer and Associates for British Gas, in the United Kingdom [7]. Several installations of water mist systems based on the British Gas design are presently in progress.

The following aspects of the turbine enclosure systems are noteworthy.

1. Unlike the IMO constraints on the machinery spaces systems, the turbine enclosure system is not restricted to nozzles at the ceiling in a ventilated space. Nozzles can be placed strategically at several levels to address shielded, hidden spray fires. The room is assumed to have the same limited ventilation as was expected for the performance of Halon 1301.

2. The potential to damage turbine blades by overly-rapid cooling introduces a unique performance criterion to these tests. FM uses the results of cooling tests on a hot steel plate, combined with a computer model, to predict the rate of cooling for a given spray application rate. The results of the plate cooling are compared with the output of a computer simulation of the surface heat transfer. If the cooling rate of the surface does not exceed $25^{\circ} \mathrm{C}$ in 22 seconds, the thermal distortion of the turbine blades is expected to be within acceptable limits. One system design achieves this by using sequential short bursts of mist. Using the same criterion, a British group doing turbine enclosure tests for British Gas achieved the cooling-rate objective without cycling the spray [7]. Differences in turbine design, such as blade clearances, may account for the different outcomes.

3. Testing encompassed total system performance, including the detection and activation equipment; the water mist system component reliability; the effects of the mist on the protected object; and the degree of control or extinguishment provided. This is the level of comprehensive testing contemplated by NFPA 750 [1], which, as noted earlier, defers to full scale testing to validate a design.

4. The first system given approval is the Securiplex twin-fluid system, for volumes up to $80 \mathrm{~m}^{3}$. Now that a test protocol exists, mist system manufacturers can submit their equipment for testing. At least two other manufacturers are seeking to pass the $80 \mathrm{~m}^{3}$ tests, and all candidates are extending application to $260 \mathrm{~m}^{3}$ spaces. The importance of the emergence of a first North American approval listing for a water mist fire suppression system cannot be underestimated. This comprehensive testing approach, involving evaluations of system performance with respect to fire suppression, 
detection and actuation, cooling impact, and system reliability, defines the model for how water mist system designs will be validated in the future.

\section{Heritage Buildings}

Full-scale fire tests have been conducted in Norway and in the US to assess the ability of water mist to extinguish fires but limit water damage to valuable cultural property. The expectation is that if fire control or extinguishment can be achieved using very small total volumes of water, non-thermal (water) damage to the protected property will be minimized. This is appealing to managers of libraries, archives, museums, art galleries, and heritage buildings. In the US, at least one manufacturer is working on tests to demonstrate the ability of water mist to extinguish library fires in fixed shelf systems [8]. Testing is in progress for the fixed library shelving scenario; and plans are being made to do preliminary testing in 1996 on mobile shelving units.

In Norway, full-scale tests were conducted through SINTEF, to demonstrate the potential for water mist to protect small 13th century wood stave churches in remote areas [9]. In addition to being constructed of wood, located in areas of limited water supply and a cold climate, they contain irreplaceable icons painted with water soluble paint. Preliminary tests indicate that flashover can be prevented in the church, using water mist, without irreparable harm to the icons. The Norwegian fire engineering firm, IGP/AS in Trondheim, is planning further tests for 1996 for this application.

\section{Telecommunications and Electronic Equipment}

There continues to be strong interest in adapting water mist for application in telecommunications and electronic equipment facilities. These facilities were exclusively protected by Halons until recently. Although the incentive to find an affordable alternative to Halon sustains interest in the area, there is a strong prejudice against the use of water in electronics or electrical equipment which dampens enthusiasm. The tests to date suggest that water mist can be used on a wide range of electronic switchgear equipment, without incurring unacceptable levels of water damage. Because of the reluctance to apply water to the equipment, the approach to design of a water mist system in an electronic room is the opposite of a total-flooding approach. A study conducted at NRCC in Canada [10] emphasizes the advantages of subdividing a water mist system into zones, and of providing a detection system that supports the zoning of the water mist system. Then, water is only applied in the actual fire location, not on all equipment in the room. The following issues are noteworthy.

1. The fire hazards in telecommunications facilities include electronic cabinets, underfloor cable plenums, overhead cable trays, and general work areas involving Class A combustibles. Fires originating in electronic equipment cabinets are rare, and fire growth is slow when it does occur. Fire in underfloor cable plenums and cable trays is more likely, as with a short circuit followed by ignition from the uninterruptible power supply. Fires in ordinary combustibles (desks, chair, waste paper) can occur. Each of these fire scenarios requires a different approach to suppression with water mist.

2. Several manufacturers of fire suppression equipment and users of telecommunications equipment have conducted demonstration tests in the UK, Europe, and the US, with nozzles placed inside different types of electronic cabinets, or in the aisles between open-door cabinets. The tests show that water mist is capable of extinguishing fires in some circumstances. Although trials continue, progress is slow because of a limited commitment to the concept by owners/users of telecom and 
electronic equipment. There is no consensus that the passive fire control measures built-into cabinets are not enough to exclude the need for in-cabinet active suppression systems.

3. There are very few studies that properly quantify the nature of "water damage" to electronics. Without reputable evaluations of the non-thermal damage to electronics, it is not possible to refute arguments that the damage would be "unacceptable". The few studies that do exist [11] [12], suggest that different types of equipment have different levels of sensitivity to damage, and that in many cases, damage due to water is negligible compared to the damage done by smoke from an unsuppressed fire. The Norwegian firm, IGP/AS is proposing to study the potential for water damage to electronics in 1996. There is a need for more work in this area.

4. Laboratory testing of water mist for telecommunications equipment was conducted in New Zealand, at University of Christchurch, which was funded by NZ Telecom. More recently, testing was done in the US at NIST and FM; in Canada at NRCC; in Norway at SINTEF; in Sweden at SP; and in Finland at VTT. The New Zealand study [11] confirmed that water mist is suitable for use on main distribution frames, and that the use of distilled or de-mineralized water reduced water damage to energized printed circuit boards (PCBs) to negligible levels. A study conducted at NIST [13], in which the ability of water mist to extinguish fires in an array of PCBs was studied, demonstrated that the ability of spray to penetrate between the parallel PCBs is limited, so the range of effectiveness is limited.

5. Recently completed studies at NRCC in Canada observed the same phenomena, in which the arrays of parallel circuit boards limited the ability of spray to penetrate to the seat of a fire [10] [14]. The experimental work at NRCC identified strategies to overcome the challenge imposed by the rigid geometry of printed circuit boards, and described the spray characteristics suitable for in-cabinet fire suppression [11].

6. The NRCC study points out the need for advances in fire detection and signal processing in order to effectively zone a system. The ability to subdivide a system into zones, and then to activate the correct zone for a given fire, becomes increasingly challenging as compartment complexity increases. Further work is required to assemble multi-sensor detectors with an "intelligent" signal processing center that can reliably identify the fire location within a compartment.

7. Safety concerns about using water mist on high voltage electrical equipment are frequently raised. Studies conducted by SINTEF in Norway [15] indicate that conductivity through mist is very low. However, particularly on ships, the accumulation of water on conductive floors in rooms containing an uninterruptible power supply remains a concern. A systematic study of when it is acceptable to use water mist around high voltage equipment is needed.

\section{Conclusion}

As indicated in the introduction, there are many interesting subjects worthy of discussion in Table 1. The four issues discussed in this presentation were selected because they represent significant milestones in the development of water mist fire protection systems technology. The activity regarding the IMO test protocols has stimulated much international exchange, product development and technology transfer. Providing that these international efforts continue, more applications will be validated over the next few years. Considerably more research effort will be required before the application of water mist to a new fire scenario becomes a routine design problem. 


\section{REFERENCES}

1. NFPA 750 - "Standard on Water Mist Fire Protection Systems", First Edition, National Fire Protection Association, Quincy, MA, 1996.

2. International Maritime Organization, (IMO). "Draft Test Method for Equivalent Sprinkler System for Class A Machinery Compartments (up to $500 \mathrm{~m}^{3}$ ) Volume," I.M.O. Fire Test Procedures Correspondence Group submittal for the I.M.O. Fire Protection Sub-committee, July, 1995.

3. International Maritime Organization, (IMO). "Water-Based Fire Extinguishing Systems in Accommodation and Public Space Areas on Board Ships", I.M.O. Fire Test Procedures Correspondence Group submittal for the I.M.O. Fire Protection Sub-committee, June 1994.

4. International Maritime Organization, (IMO). "Draft Test Protocol for Equivalent Sprinkler System for Cabin and Corridor Fires," July, 1995.

5. Personal correspondence, R. Darwin, NAVSEA, Crystal City, VA, July, 1995.

6. Berner, F.; Vemmestad, J., "Development of Fine Water Spray Technology for Application on BP Norwegian Continental Shelf Offshore Installations ULA and GYDA", in Proceedings:

7. Personal correspondence, J. H. Dyer, J.H. Dyer \& Associates, North Lincolnshire, England, January, 1996.

8. Milke, J., "University of Maryland Tests of a Water Mist Fire Suppression System for Library of Congress", in Proceedings of SFPE Symposium, Orlando, FL, September, 1995.

9. Meland, Øystein; Jensen, Geir; Sjur Helseth; "Water Mist to Protect Wooden Historic Structures", in Second International Symposium on Fire Protection of Ancient Monuments, Cracow, Poland, October 17-21, 1994.

10. Mawhinney, J.R. and Taber, B., "Summary Report for the Combined Intelligent Fire Detection and Water Mist Fire Suppression System Project," Client Report A4078.1, National Research Council Canada for National Defense Canada, Ottawa, Canada, January 1996.

11. Botting, R.; Beaumont, P. "Research Project Concerning Fire Protection of BPO Type Main Distribution Frames in Telephone Exchanges," Telecom New Zealand, Limited, August, 1993. (Advanced draft copy).

12. Morgan, R., "The effects of water borne electrolytic corrosion on live electronic equipment, Part II", Research Report, School of Engineering, University of Canterbury, Christchurch, New Zealand, September 1, 1994.

13. Grosshandler, W., Lowe, D., Notarianni, K., Rinkinen, W., "Protection of Data Processing Equipment with Fine Water Sprays", NISTIR 5514, National Institute of Standards and Technology, Gaithersburg, MD. 1994.

14. Mawhinney, J.R.; Taber, B.; Su, J.Z. "The Fire Extinguishing Capability of Mists Generated by Flashing of Super-Heated Water," in Proceedings: American Institute of Chemical Engineers, 1995 Summer National Meeting, Boston, MA., July 30 to August 2, 1995.

15. Verlo, $T$. "The Use of Water as an Extinguishant in Live Electrical Installations", a study conducted for the Norwegian Electric Power Research Institute, by SINTEF, Trondheim, Norway, October 1991. 


\section{Discussion}

Masahiro Morita: I have one question with regard to the water mist and whether the drop should be small or large. When you apply water to methanol, the mechanism of water would be quite different then the conventional sprinkler system. In Japan, we have also studied the difference when you apply water mist in a small room or large room, and each time the mechanism is different. Do you have any study on that?

Jack Mawhinney: Yes. I would refer first to the paper that I prepared with my colleagues at NCL looking at the extinguishing mechanisms from water mist and identifying enclosure effects where you get the benefit of reduced oxygen and the benefit of the recirculation of oxygenated gases at higher evaporation rates. Yes, it was very true that water mechanisms differ depending on the fire scenario and depending on the volume size of the compartment, and depending on the nature of the fuel. 


\title{
Experimental Study on Fire Hazard of Residential Fires Before and After Sprinkler Activation
}

\author{
Ai SEKIZAWA, Akio TAKEMOTO, Daisuke KOZEKI, Eiji YANAI, and Keiko SUZUKI
}

Fire Research Institute

Fire Defense Agency

3-14-1, Nakahara, Mitaka, Tokyo 181

\section{ABSTRACT}

Fire experiments were conducted in a real scale room which is assumed to be a residential living room $(3.8 \mathrm{~m} \times 3.6 \mathrm{~m} \times 2.4 \mathrm{~m})$ under the various opening conditions and locations of wood crib fire source. Concentrations of oxygen and carbon monoxide, smoke density, and temperature were measured: Response time of fire detection by several detection methods such as rate of rise heat detectors, fixed temperature type heat detectors, photoelectric type smoke detectors, residential smoke detectors, and the Intelligent Fire Detection System and activation of residential sprinklers were also examined. From the results of the experiments, the following were obtained.

(1) Maximum mass loss of wooden crib by combustion until activation of sprinklers was less than $1 \mathrm{~kg}$. Therefore, the fires burnt under the fuel control condition. In this condition, it was confirmed that the residential sprinkler systems can control the wooden crib combustion.

(2) In some experiments, the smoke density and the $\mathrm{CO}$ gas concentration increased after activation of sprinklers because of stirring air by water discharge.

(3) In most of the experiments, it was found that the response time of all the fire detection methods used in the experiments are less than the response time of sprinklers.

\section{INTRODUCTION}

Every year, many lives of disaster vulnerable people like elderly, infants, and physically handicapped are lost in residential fires. For example, it is reported in the "White Book on Fire Service in Japan, 1993"1) that 95\% of fire deaths excluding incendiary suicides came from residential fires and $54 \%$ of that were elderly, infants, and ill or physically handicapped persons. Besides this fact, Japan is facing the problem of rapidly aging of a society that is expected to continue to a stage where one fourth of total population are 65 or older around in 2020. Therefore, It is earnestly desired to develop measures for reducing fires and fire deaths in homes.

From this viewpoint, the Fire Defence Agency issued a "Technical guideline on residential sprinkler system and residential detectors"2), as of March 25, 1991. Reflecting this trend of the administration, fire protection equipment makers started the development of a quick response sprinkler head. Also, the Japan Fire Equipment Inspection Institute started experimental research ${ }^{3)}$ on test fires for establishment of approval procedures on residential sprinklers.

As the first step to study the feasibility of residential sprinklers to save persons in a room of fire origin, we carried out fire experiments using wood crib fire source in a real scale test room. We measured and examined the temperature near the ceiling, the vertical temperature distribution, smoke density, and the gas concentration as environmental conditions inside a fire room. Also, to consider conditions for a fire detection system to have 
capability of both fire alarm and sprinkler activator, response time was measured as for heat detectors, smoke detectors, and the multiple sensors for the fire detection system applying Fuzzy Theory 4) 5) ( hereafter we call "Intelligent Fire Detection System" ) developed by the Fire Research Institute.

\section{OUTLINE OF THE EXPERIMENTS}

\subsection{Fire Test Room}

The area of a fire test room is $13.7 \mathrm{~m}^{2}(3.8 \mathrm{~m} \mathrm{x} 3.6 \mathrm{~m})$, and the room height is $2.4 \mathrm{~m}$. The walls and the ceilings is covered with rock wool cement board and the floor is covered with anti-water plywood. There is a slide door in the east side of a fire test room which is used to adjust the opening ratio by changing the width of a door. Next to a fire test room, there is an observation room where measurement apparatuses are located. The outline of the experiment room is shown in Fig. 1.

\subsection{Fire Source and Ignition}

The fire source used is wood crib whose configuration and ignition method are specified in the "Technical guideline on residential sprinkler system and residential detectors". The wood crib is assembled with 6 layers of 58 pieces of cedar crib, $0.03 \mathrm{~m} \times 0.035 \mathrm{~m} \times 0.90 \mathrm{~m}$ for each piece, the total weight of that is about $20 \mathrm{~kg}$. The wood crib was ignited by combustion of $50 \mathrm{ml} \mathrm{n}$-heptane in a heat source pan on the floor beneath the wood crib's center.

\subsection{Measurement of Temperature}

The temperature was measured with $1 \mathrm{~mm} \phi \mathrm{K}$ - type thermocouples. Thermocouples are installed near the ceiling surface for looking into horizontal distribution and also installed in the positions as the center of the fire room (A point), south of A point by $0.9 \mathrm{~m}$ (B point), and near the south window (D point) for looking into vertical distribution. The location of thermocouples are shown in Fig. 2 (a).

\subsection{Measurement of Smoke Density and Gas Concentration}

Smoke density was measured with smoke density meters of $1 \mathrm{~m}$ light pass length. In order to see horizontal distribution of smoke density near the ceiling, six smoke density meters were placed. And, seven smoke density meters were placed along the south window in order to see the vertical distribution of smoke density.

The gases concentration was measured at the center of the fire room, $0.05 \mathrm{~m}$ below the ceiling level. $\mathrm{O}_{2}$ concentration was measured using $\mathrm{O}_{2}$ gas analyzer with the range of $16 \%$ to $21 \%$ and $\mathrm{CO}$ gas concentration was measured using the $\mathrm{CO}$ gas analyzer with the range of $0 \%$ to $2 \%$. The gas sampling position is shown in Fig. 2 (a) and positions of smoke density meters are shown in Fig. 2 (b).

\subsection{Installation Position of Detection Sensors}

As fire detecting sensors, a thermocouple, an analog type photo-electric smoke detector, a CO sensor are installed in a fire room in order to measure temperature, smoke density, and $\mathrm{CO}$ gas concentration. Also, six residential smoke detectors are installed to measure the response time in several position. The locations of the sensors, the residential smoke detectors, wood crib fire source, and sprinklers are shown in Fig. 2 (b).

\subsection{Sprinkler Installation and Water Discharge Condition}

Sprinkler heads with the nominal release temperature of $72^{\circ} \mathrm{C}$ were installed at the center of a fire room (A point), and at the two positions ( $F$ point and $G$ point ), $0.8 \mathrm{~m}$ away 
south and north respectively from A point, as shown in Fig. 2 (a). The conditions of water discharge of sprinkler heads were as follows.

(1) In case that only the sprinkler at A point is available.

Water discharge flow : $28 \mathrm{l} / \mathrm{min}$. Water pressure : $1.08 \sim 1.17 \mathrm{kgf} / \mathrm{cm}^{2}$.

(2) In case that the sprinklers at $F$ point and $G$ point are available, but the sprinkler at $A$ point is not available.

Water discharge flow : $57 \mathrm{l} / \mathrm{min}$. Water pressure : $1.19 \sim 1.24 \mathrm{kgf} / \mathrm{cm}^{2}$.

The water discharge flow in the case when only one of $F$ and $G$ sprinkler activated was 34 $36 \mathrm{l} / \mathrm{min}$.

\subsection{The Conditions of Experiments}

The experiment conditions of the 15 fire tests are shown in Table 1 . The experiment No. 1 to No. 6 in Table 1 are the cases that the fire source was placed in the center of a fire room with variety of opening ratio. The experiment No. 7 to No. 12 are the cases that the opening ratio was fixed (all closed) with variety of location of the fire source. The experiments No. 13 to No. 15 were done with no sprinklers.

\section{RESULTS AND DISCUSSIONS}

\subsection{The Temperature Profile and The Fire Behavior}

(1) The weight loss of the wood crib fire source

The weight change of the fire source for the experiments with no sprinklers, No. 13 to No. 15 are shown in Fig.3. The weight was normalized with the wood crib's initial weight. In these three cases, the weight loss curves trace nearly the same tendency. The weight loss rate became constant around 5 minutes after the ignition. From this constant weight loss rate and the heat of wood combustion, $18,900 \mathrm{~kJ} / \mathrm{kg}$, the heat release rate was estimated approximately $170 \mathrm{~kW}$.

At some point on the decreasing curve, the weight increases suddenly. This was caused by the water drop on the crib for suppression to prevent a fire from growing to be incontrollable state. Therefore, this sudden jump of weight should be ignored.

The data of weight loss, heat release rate, and the total heat release amount at sprinkler activation for the experiments, No. 1 to No. 12, with sprinklers are listed in Table 2. From this table, in all of these experiments, sprinklers responded in the early stage of the combustion of wood crib. This weight loss was at most $1 \mathrm{~kg}$. For example, when the fire source was placed in the center of the fire room, sprinklers activated at the weight loss of $240 \mathrm{~g}$ to $280 \mathrm{~g}$ regardless of the open ratio.

(2) The temperature near the ceiling level above the fire source

The temperature-time curve near the ceiling level for the cases that the fire source is at the center of the room (Exp. No. 1 to Exp. No. 6) traces three stages as a preheating stage of the wood crib by the buming of $n$-heptane where the temperature flattens after slight increase, then a drastic temperature rising stage after the ignition of wooden crib, and a cooling stage where the temperature drops due to the sprinkler's water discharge. As all of the cases followed the same pattern, the reproducibility of the crib fire is considered to be obtained, although temperature-time profiles shown in Fig. 4 were the data of different opening ratio.

It is seen that there is a difference in the initial temperature rising point, ranging from 10 seconds to about 1 minute among the curves. As the preheating time of wood crib differs by the wooden crib's moisture, the room's temperature, and humidity, so the preheating time must be disregarded when the comparison of fire behavior among experiments is done. 
(3) Vertical temperature distribution

The vertical distribution of temperature before sprinkler activation at the A, B, and D points for the Exp. No. 8, where the fire source was set at the north west corner and the opening was closed, is shown in Fig. 5. Between $0.4 \mathrm{~m}$ and $2 \mathrm{~m}$ below the ceiling level, the temperature decreases linearly as a measuring point gets closer to the floor, and the vertical distribution of temperature is nearly the same for the points $A, B$, and $D$. This indicates that the smoke layer in the room was stratified until the sprinkler activated when the opening was all closed.

The temperature near the ceiling decreased as a measuring point gets further from the fire source. This feature of the temperature near the ceiling was commonly seen in all of the experiments. Therefore, when setting a sprinkler and/or residential smoke detectors on the wall, fires could be easily detected if set at $0.2 \mathrm{~m}$ to $0.4 \mathrm{~m}$ below the ceiling level.

\subsection{Smoke Density and Gas Concentration}

The change in smoke density and gas concentration as environmental conditions of a fire room before and after the sprinkler activation is discussed here.

(1) The change in smoke density

The Fig. 6 and Fig. 7 shows the change in smoke density at points Gs, As, Bs, and Ds at the ceiling level and also vertical points at Ds (excluding Ds2) for the experiment No. 8. This experiment was done in a condition that was most unfavorable for the activation of sprinklers, because the fire source and the sprinkler location was far apart of all of the experiments in Table1.

From Fig. 6, before the activation of the sprinklers, the change of smoke density was similar among the data for the points on the ceiling except for Ds1. Four and a half minutes after the ignition, the smoke density reached $60 \% / \mathrm{m}$ to $70 \% / \mathrm{m}$ and then flattened. On the other hand, after the activation of sprinklers, the density at all of the points became uniform with time lapse due to the stirring effect of the air by water discharge. And the smoke density rose up to nearly $100 \% / \mathrm{m}$ about two minutes after the sprinkler activation.

From Fig. 7 for measuring points on the Ds vertical line, before the activation of sprinklers, the smoke density rose with lapse of time in the order of higher position of points since the smoke layer lowered at the same period. Although the smoke density, except for the point Ds7, was in the stable state after the increasing state before the sprinkler activation, the smoke density at Ds7 was still in the increasing state. Thus, the smoke boundary layer at this point can be estimated to be in between Ds6 and Ds7. This result corresponds with the results from Fig. 8 which is mentioned in the next paragraph. After the sprinkler activation, the smoke density at all of the points, regardless of the height, became uniform as time elapsed due to the stirring effect of the room's air by discharged water, and then it became almost $100 \% / \mathrm{m}$ two minutes after the sprinkler activation.

\section{(2) The change of the height of the smoke boundary layer}

The Fig. 8 shows the height changes of the smoke boundary layer by time for the three cases (Exp. No. 7, 9, and 12) where the sprinkler head was only one in the center of a fire room and the fire source was not in the center of the room and one case (Exp. No. 14) without sprinklers. For the Exp. No. 14 without sprinklers, which was the case when the fire growth was quite rapid, 4 minutes after the ignition the smoke layer boundary lowered to $0.4 \mathrm{~m}$ above the floor level, and reached the floor level after 7 minutes. For the three cases with sprinklers, whose data are presented up to the sprinkler activation, the smoke layer boundary linearly lowered and reached around $0.6 \mathrm{~m}$ above the floor level at the sprinkler activation time. 
(3) The change of the gas concentration $\left(\mathrm{O}_{2}, \mathrm{CO}\right)$

For the cases shown in Table 1 , as the fire source was placed in the center of the room, the response of sprinklers were quick and the fire was extinguished in the fairly early stage, so the $\mathrm{O}_{2}$ concentration was $19 \%$ to $20 \%$ and $\mathrm{CO}$ concentration was $200 \mathrm{ppm}$ before the sprinkler activation and was $500 \mathrm{ppm}$ after sprinkler activation which was not a so large value.

Therefore, we introduce the data of the Exp. No. 8 which was done in a condition that was most unfavorable for the activation of sprinklers in Fig. 9, because the fire source and the sprinkler head were the most apart among all of the experiments in Table 1.

In this experiment, the sprinkler was activated 7 minutes after the ignition, and the water discharge up to 13 minutes after the ignition controlled he fire. However, when the slide door at the east side of the room was opened 15 minutes after the ignition, the fire started to grow again. This indicates that the imperfect combustion state was thought to continue even after the sprinkler activation. CO gas concentration rose drastically 7 minutes after the ignition, the time that the sprinkler was activated, and it became 2000ppm just before the stop of water discharge by a sprinkler (13 minutes after ignition), and reached 3500ppm at the end of the experiment ( 15 minutes after the ignition).

These values of $\mathrm{CO}$ gas concentration were close to the level as the lethal concentration for 30 minutes exposure, 4000ppm. Although this result is for the case most unfavorable among the all experiments in Table 1, we should note that in some unfavorable cases for the activation of sprinklers, persons in a room of fire origin could be involved in a critical condition for survival. Also, the high smoke density after sprinklers activation should be remarked, when we consider the environment condition of a fire room along with the unification of smoke and gas by the stirring effect of the air by water discharge.

\subsection{Detection Method and Detection Time}

Here, we will discuss the fire detection system that has the capability of the fire alarm and the sprinkler activator. We examined the response time of the heat detector, the smoke detector, residential smoke detectors, and the Intelligent Fire Detection System applying the Fuzzy Theory by the Fire Research Institute ${ }^{13) 14)}$ based on the output signals of multiple sensors such as heat sensors, smoke detectors, and the CO gas sensors.

(1) Each detection methods

We simulated fire detection methods of the rate of rise heat detector, the fixed temperature detector, the photo-electric smoke detector, and the Intelligent Fire Detection System applying the Fuzzy Theory.

The detecting time for the rate of rise heat detector, the fixed temperature detector, and the photo-electric smoke detector was calculated using the alarm conditions shown in Table 3. The response sensitivity for the detectors were set at the same level as those set in general buildings. The response time to identify a fire by the Intelligent Fire Detection System applying the Fuzzy Theory was estimated by using the defuzzification rules for judging a fire/non-fire in Table 4 after first calculating each certainty factor based on the four judging rules as follows;

*Flaming fire judging rule

*Smoldering fire judging rule

* Steam judging rule

* Cigarette judging rule

The calculation of the certainty factor was done by combining the fuzzy membership functions ${ }^{4 / 5}$. 
(2) The detection time by detection method

The detection time for the various detection methods and the activation time of sprinklers are shown in Table 5. Also, the output signal of the sensors and the detecting point of the various detecting methods for the Exp. No. 12 are shown in Fig. 10. From Table 5 , the approximate trend can be seen as the following.

The rate of rise heat detector was likely to respond earlier than the photo-electric smoke detector and the fixed temperature detector. The reason for this is thought to be that the fire source used in the experiments was flaming fire of wood crib's combustion and so the volume of smoke generated was fairly small. In comparison with sprinklers activation, the rate of rise heat detector responded quicker than sprinklers, but the photo-electric smoke detector responded later than sprinklers in some cases (for example, Exp. No. 5). The fixed temperature detector some times did not respond, but this was because the activation of sprinklers lowered the room temperature.

The Intelligent Fire Detection System judged adequately all of the cases as a flaming fire. The detection time was not as quick as that of the rate of rise heat detector, but was quicker than activation of sprinklers. The detection time of the residential smoke detectors were not consistent among J0 to J5.

\section{CONCLUSION}

Fire experiments were conducted in a real scale room using a wooden crib fire source, and measured smoke density, the gas concentration, and the temperature before and after activation of sprinklers to look into environmental conditions for survivors in a room of fire origin. Also, fire detection time for the various fire detection methods and the response time of residential sprinklers were measured and compared. The result were as follows.

1) The maximum mass loss of wooden crib by combustion until the activation of sprinklers were less than $1 \mathrm{~kg}$. The air inside a fire room was enough for the combustion of this size of level wood crib fire, so then did not become ventilation control fire.

2) In some cases, the smoke density and $C O$ gas concentration increased after sprinkler activation because the air inside the room was stirred by the sprinkler's water discharge.

3) It was found that the rate of rise heat detector method, the fixed temperature detector method, the photo-electric smoke detector method, and the Intelligent Fire Detection System responded quicker than the activation of the sprinklers.

4) In all of the experiments, the control of a fire by sprinklers were confirmed.

\section{REFERENCES}

1) Fire Defense Agency : White Book on Fire Service in Japan for 1993.

2) Fire Defense Agency : "Technical guideline on residential sprinkler system and residential detectors", 1991.3.

3) The Japan Fire Equipment Inspection Institute : "Experimental study on residential sprinkler system, 1992.

4) Satoh, K: "Intelligent Fire Detection System Based on Fuzzy Theory - Introduction of Fuzzy Expert System and CO gas Sensor - ", proceedings of '94 Asian Fire Science Seminar, Bali Indonesia, Dec. 1994.

5) Kozeki, D, et al. : "Study of Evacuation Assisting System incorporated into Intelligent Fire Detection System", proceedings of AUBE'95, Duisburg Germany, Apr. 1995. 


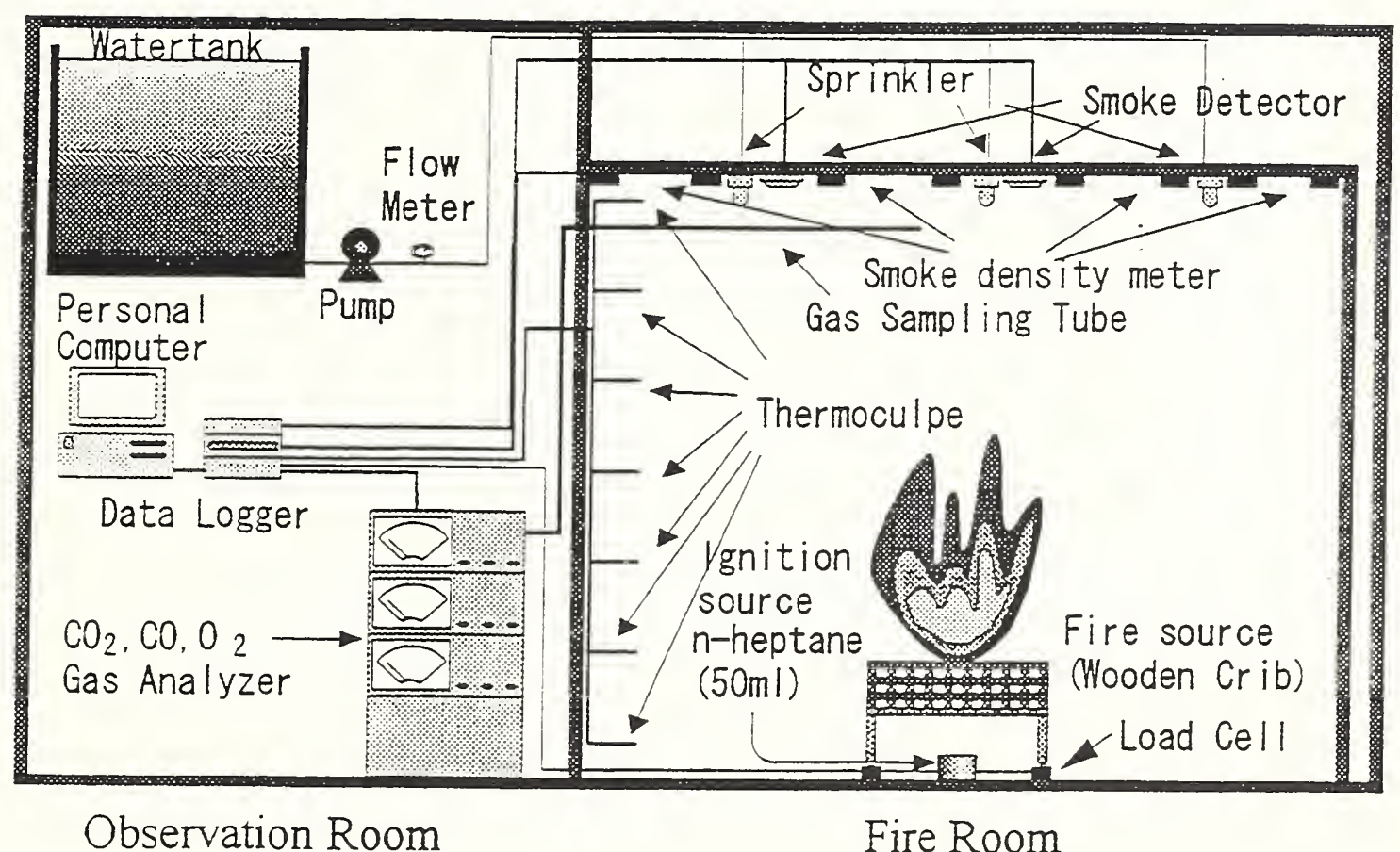

Fig.1 Schematic diagram of fire test room.

Table 1 Experimental conditions.

\begin{tabular}{|c|c|c|c|c|}
\hline $\begin{array}{l}\text { Exp. } \\
\text { No. }\end{array}$ & $\begin{array}{l}\text { Locations of } \\
\text { spriniler } \\
\text { heads }\end{array}$ & $\begin{array}{l}\text { Location of } \\
\text { fire source }\end{array}$ & $\begin{array}{l}\text { Ventilation } \\
\text { conditions }\end{array}$ & $\begin{array}{l}\text { Mooden crib s } \\
\text { moisture } \\
\text { cortent (y) }\end{array}$ \\
\hline$T$ & $\bar{A}$ & A & $1 / 2$ opened & 17.8 \\
\hline 2 & $F, G$ & $\bar{A}$ & $1 / 2$ opened & 16.4 \\
\hline 3 & $F, G$ & A & $1 / 4$ opened & 17.3 \\
\hline 4 & $F, G$ & A & $1 / 8$ opened & 14.2 \\
\hline 5 & $F, G$ & A & $1 / 16$ opened & 12.6 \\
\hline$\hat{6}$ & $F, G$ & $\bar{A}$ & Closed & 13.0 \\
\hline 7 & $\bar{A}$ & J & Closed & 13.4 \\
\hline 8 & $F$ & $J$ & Closed & 12.5 \\
\hline 9 & A & $\mathrm{K}$ & Closed & 10.1 \\
\hline 10 & $\bar{F}, \mathrm{G}$ & $\bar{K}$ & Closed & 12.5 \\
\hline 71 & $F$ & i! & Closed & 10.8 \\
\hline 72 & $\bar{A}$ & $T$ & Closed & 13.1 \\
\hline 13 & No Sprinklers & J & Closed & 14.3 \\
\hline 14 & No Sorinklers & $J$ & Closed & 13.3 \\
\hline 75 & Wo Sorinklers & $J$ & Closed & 13.4 \\
\hline
\end{tabular}

Wooden crib's moisture percentage $(\%)$ represents the average of four measurements.
Table 2 Weight loss, Heat release rate and total heat release amount at sprinkler response.

\begin{tabular}{|c|c|c|c|}
\hline $\begin{array}{c}\text { Exp. } \\
\text { No. }\end{array}$ & $\begin{array}{c}\text { Height } \\
\text { loss } \\
(\mathrm{g})\end{array}$ & $\begin{array}{c}\text { Heat release rate } \\
\text { at sprink ler } \\
\text { response (kW) }\end{array}$ & $\begin{array}{c}\text { Total heat release } \\
\text { amount at sprinkler } \\
\text { response(kJ) }\end{array}$ \\
\hline 1 & 0.7 & 33 & 2.800 \\
\hline 2 & 1.4 & 57 & 5.900 \\
\hline 3 & 1.2 & 47 & 5.200 \\
\hline 4 & 1.4 & 57 & 6.000 \\
\hline 5 & 1.2 & 57 & 5.400 \\
\hline 6 & 1.2 & 57 & 5.100 \\
\hline 7 & 2.8 & 90 & 11.000 \\
\hline 8 & 4.3 & 100 & 18.000 \\
\hline 9 & 2.8 & 85 & 12.000 \\
\hline 10 & 3.3 & 99 & 14.000 \\
\hline 11 & 4.4 & 130 & 18.000 \\
\hline 12 & 2.6 & 90 & 11.000 \\
\hline
\end{tabular}

Height Loss $(\%)$ represents the percentage of the weight lost from the initial weight. 


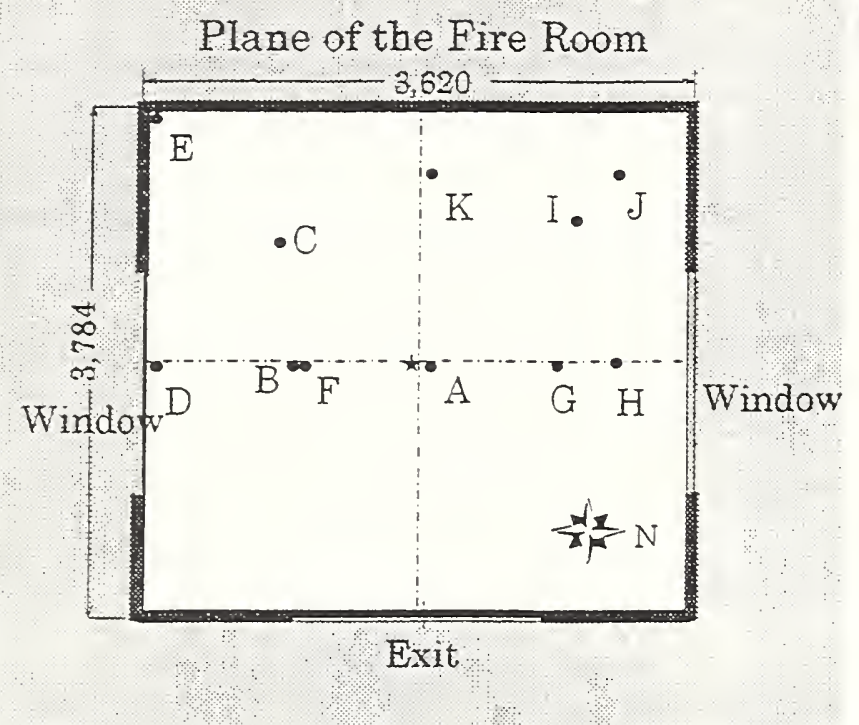

Tertical measuring points of
temperature and gas conc.

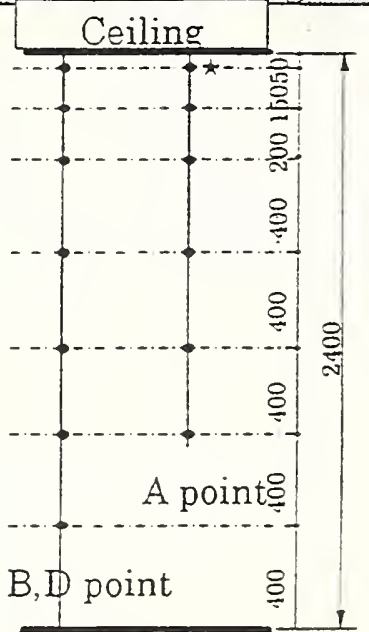

\section{floor}

* : Setup location of gas sampling

- : Setup locations of the thermo couples

(a) Setup locations of thermocouples and gas sampling
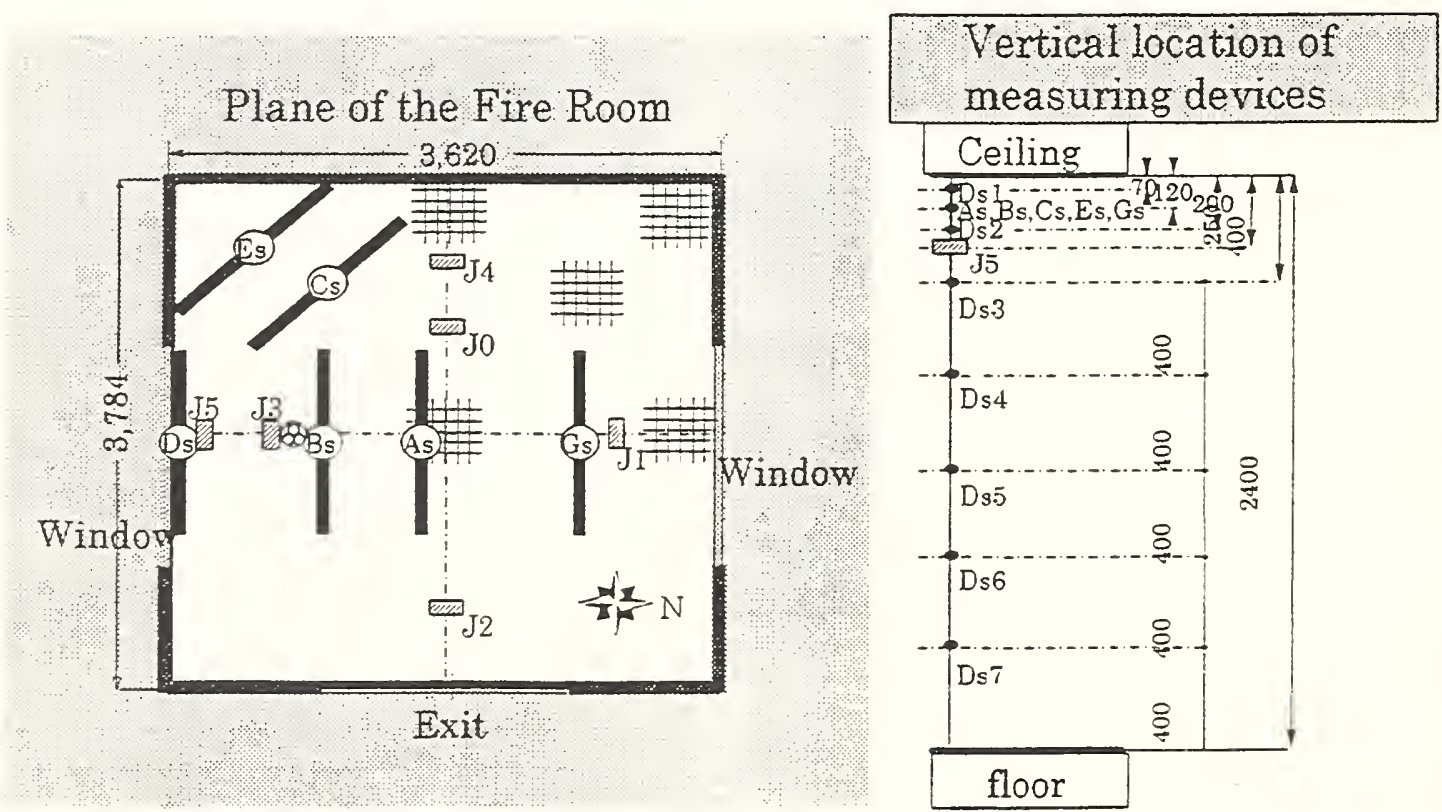

- Smoke density meter

(8) : Analog type smoke detector

Thermo couple

$\mathrm{CO}$ gas sensor

: Fire source

Photoelectric smoke detection type Stand alone home fire alarm

(b) Setup locations of Smoke density meter, Analog smoke detector and Fire alarm

Fig.2 Set up locations of thermocuples, gas sampling, smoke density meters, analog smoke detector and fire alarms. 


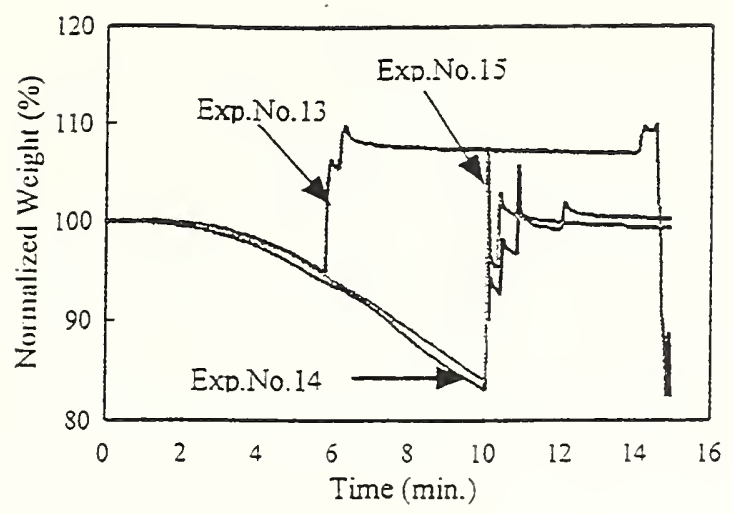

Fig.3 Change of Nomalized weight expressed by percentages as the initial weight is to be $100 \%$

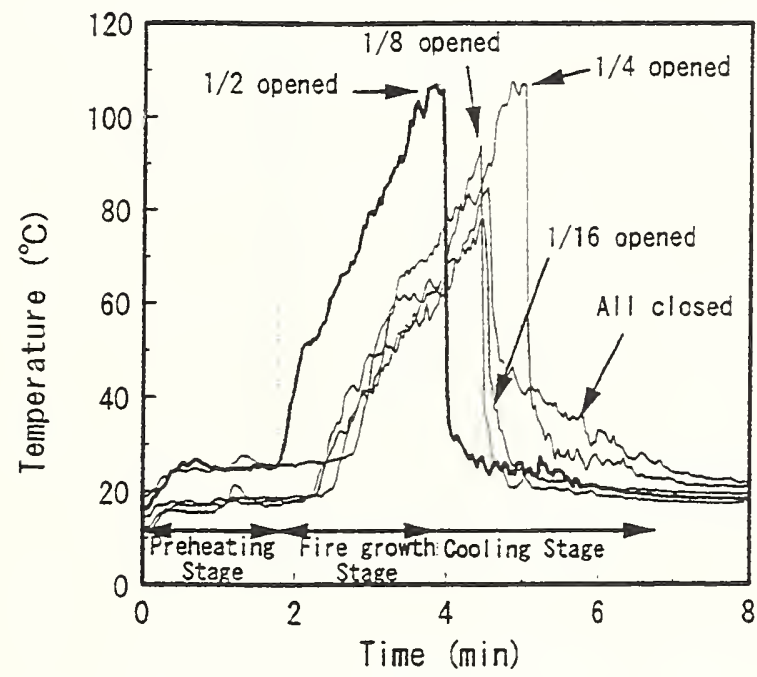

Fig.4 Relation between temperature near the ceiling level above the fire source and elapsed time.

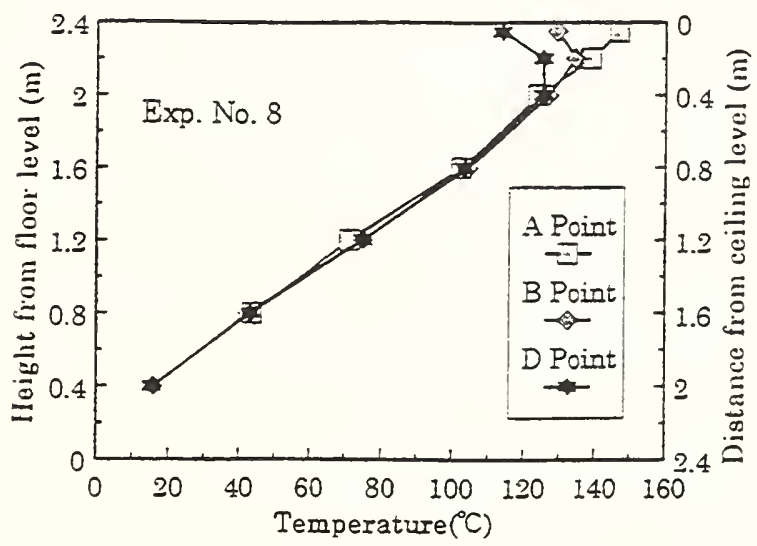

Fig.5 Vertical distribution of temperature in the fire test room for the experiment No8. 


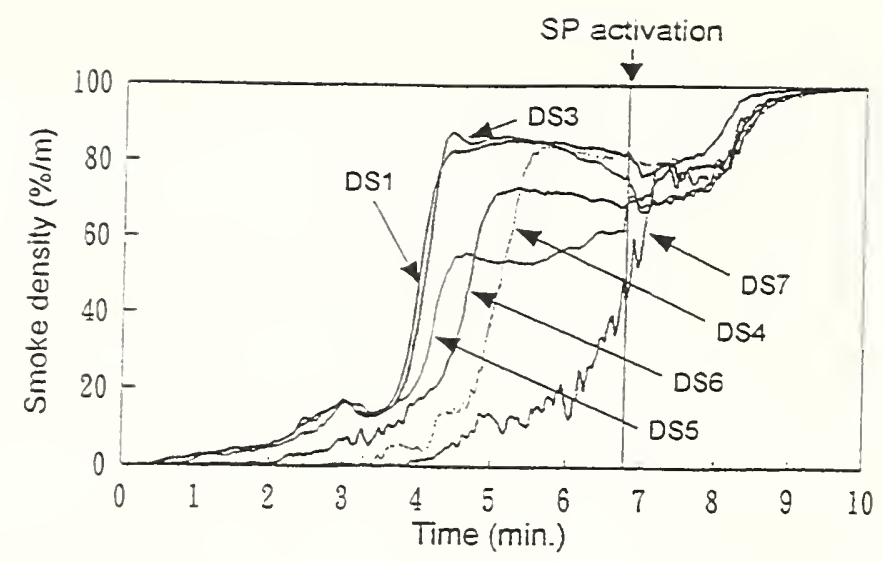

Fig.6 Smoke density at different points on the ceiling in lapse of time for the experiment No8.

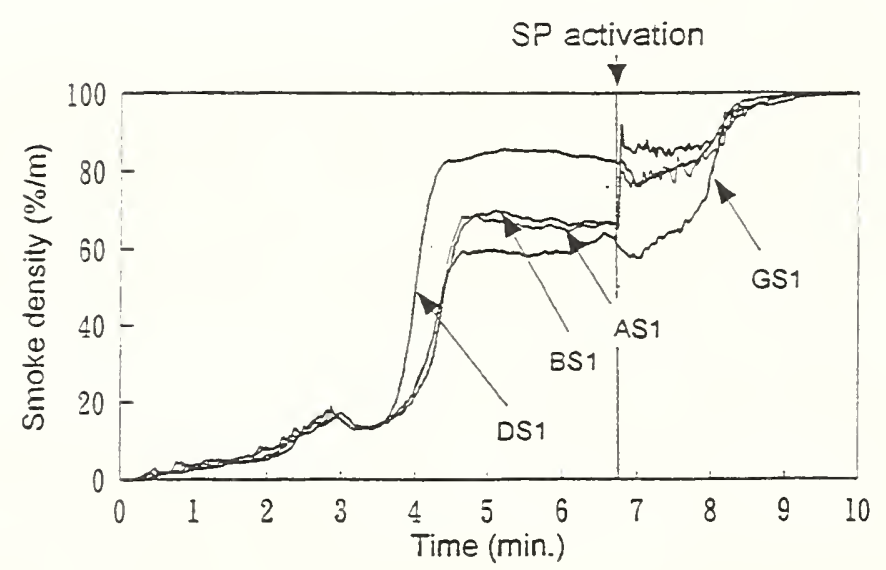

Fig.7 Smoke density at different points on the wall in lapse of time for the experiment No8.

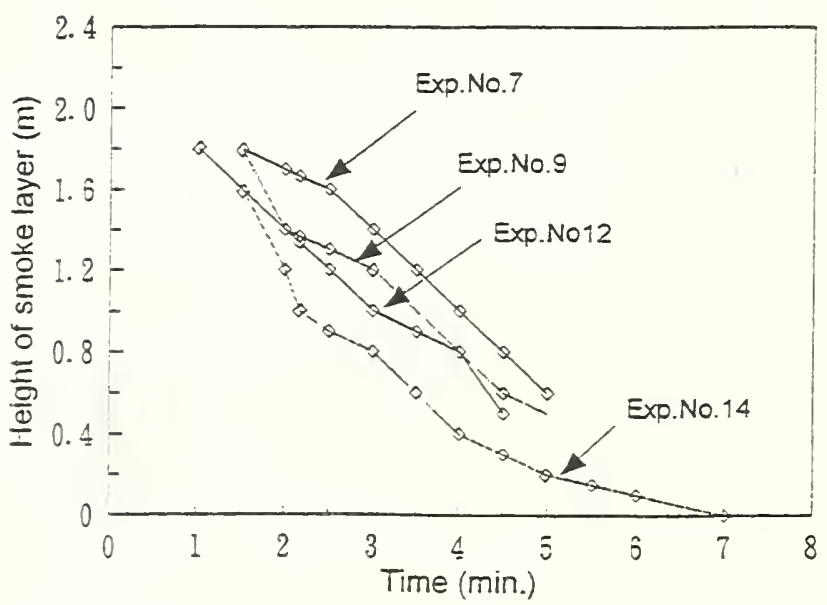

Fig.8 Height of smoke layer from the floor in lapse of time. 


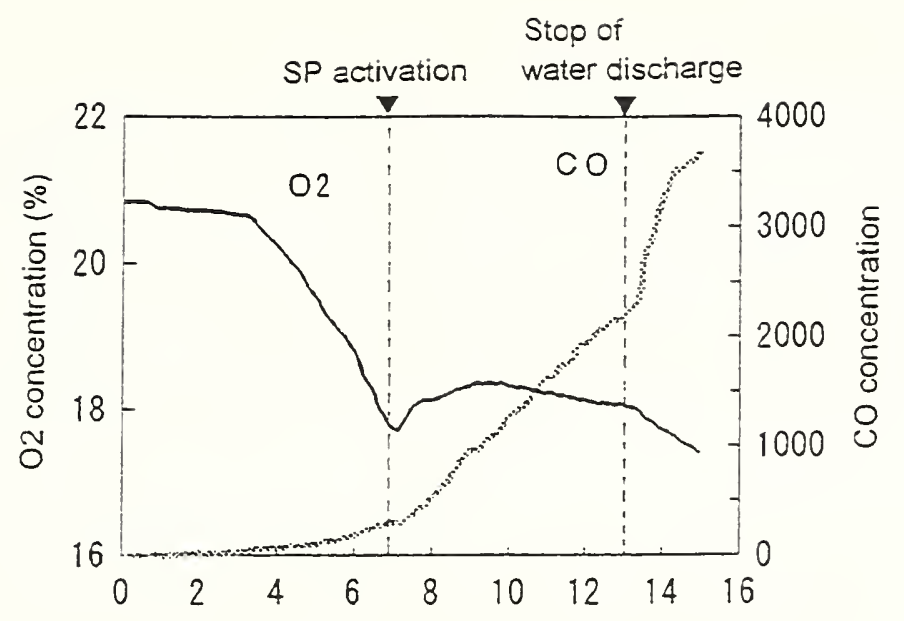

Fig.9 $\mathrm{O}_{2}$ and $\mathrm{CO}$ concentrations in lapse of time for the experiment No8.

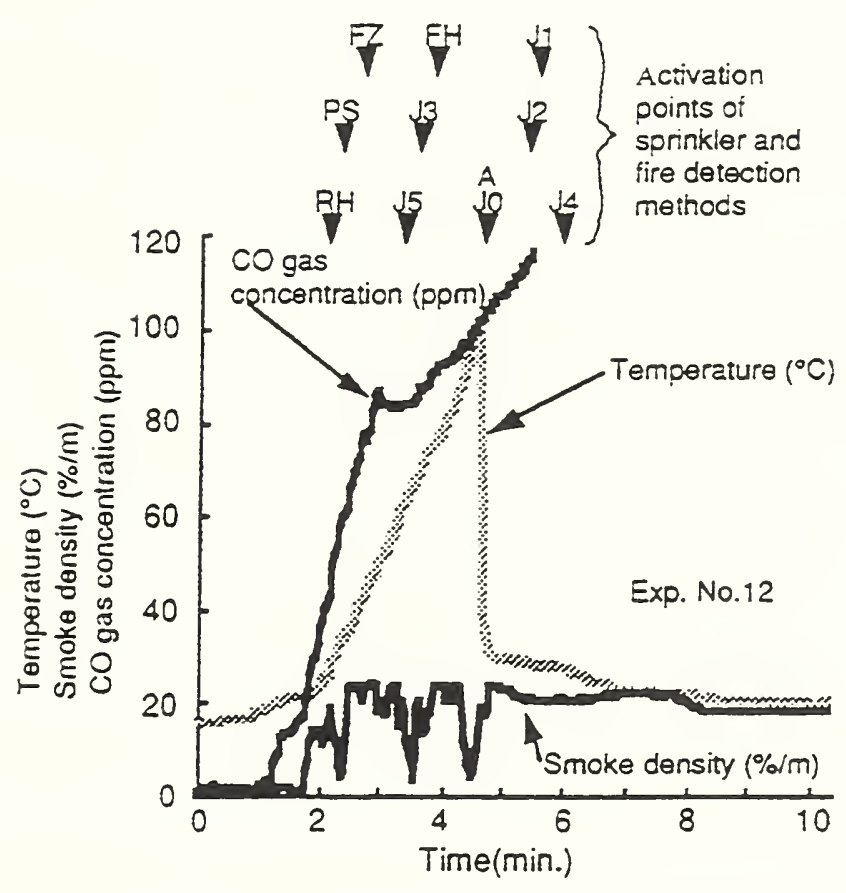

Fig.10 An example of sensor signal outputs and activation points of sprinkler and fire detection methods.

$A$ : residential sprinkler ' $A$ ' activation $R H$ : rate-of-rise heat detection

FH : fixed-temperature type heat detection PS : photoelectric rype smoke detection $F Z$ : multi sensor with fuzzy reasoning J0-J5 : residential smoke detectors 
Table 3 Alarm condition of heat detection and smoke detection methods.

\begin{tabular}{|l|c|}
\hline $\begin{array}{c}\text { Fire detection } \\
\text { method }\end{array}$ & Alarm condition \\
\hline $\begin{array}{l}\text { Rate-of-rise heat } \\
\text { detection }\end{array}$ & $15^{\circ} \mathrm{C} /$ min. \\
\hline $\begin{array}{l}\text { Fixed-temperature } \\
\text { type heat detection }\end{array}$ & $\begin{array}{c}70^{\circ} \mathrm{C} \\
\text { response }=\text { special class }\end{array}$ \\
\hline $\begin{array}{l}\text { Photoelectric type } \\
\text { smoke detection }\end{array}$ & duration $=10 \mathrm{~m}$ sec. \\
\hline
\end{tabular}

Table 4 Defuzzified rules for judging a fire/non-fire.

\begin{tabular}{|c|c|}
\hline Case 1 & $\begin{array}{c}\text { Value of first rank = over } 90 \% \\
\text { and } \\
\text { value of second rank = under } 60 \% \\
\text { a nd } \\
\text { duration }=4 \text { sec. }\end{array}$ \\
\hline Case 2 & $\begin{array}{c}\text { Value of first rank = over } 80 \% \\
\text { a nd } \\
\text { value of second rank = under } 50 \% \\
\text { a nd } \\
\text { duration }=8 \text { sec. }\end{array}$ \\
\hline Case 3 & $\begin{array}{c}\text { value of first rank = over } 70 \% \\
\text { a nd } \\
\text { value of second rank = under } 40 \% \\
\text { a nd } \\
\text { duration }=12 \text { sec. }\end{array}$ \\
\hline
\end{tabular}

Table 5 Time lapse of sprinkler activation and fire detection.

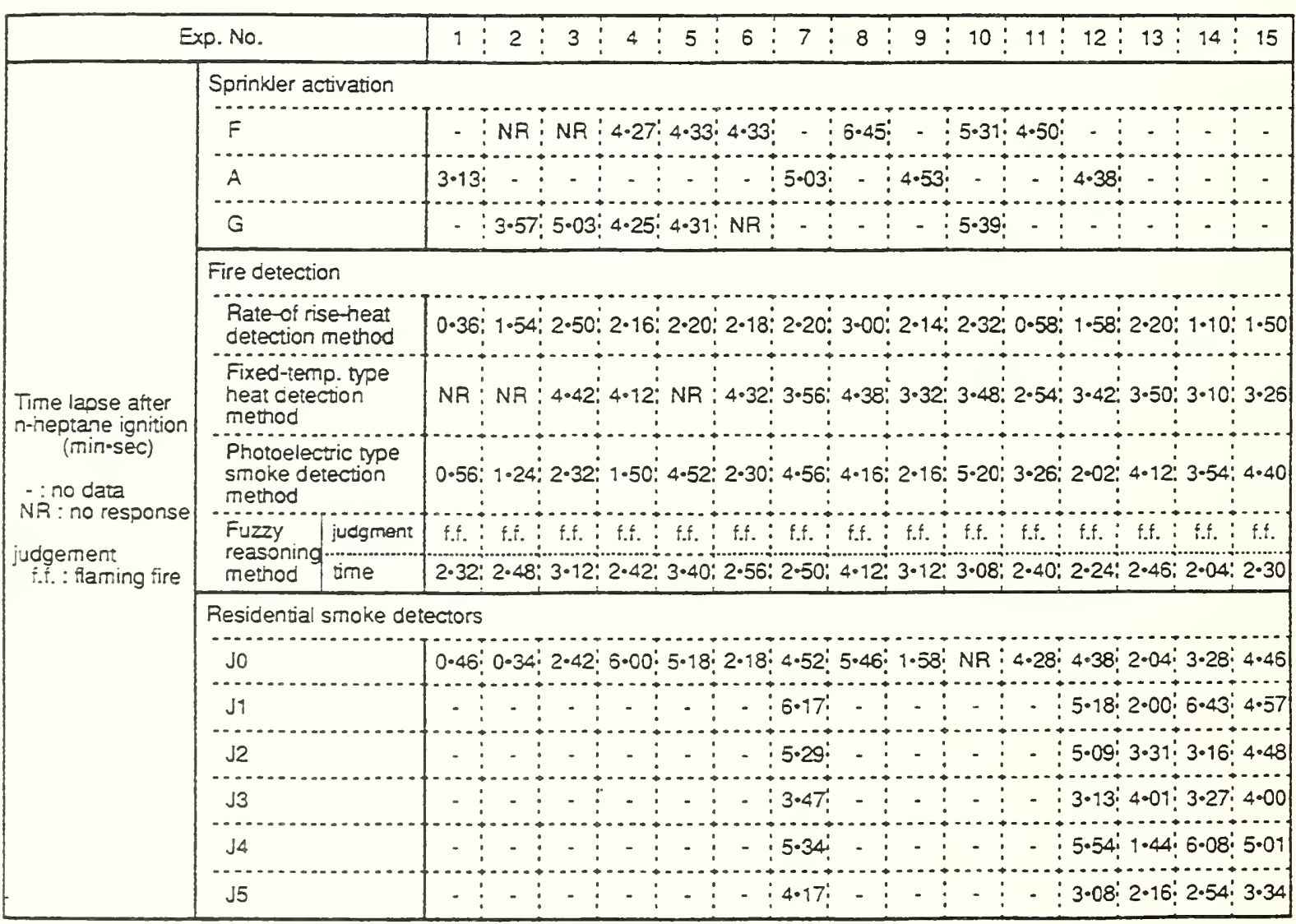




\section{Discussion}

Yuji Hasemi: You measured the gas concentration near the ceiling level but I think it might have been better if you would have measured at a lower level. When you focus on the life safety of the people inside the room, then probably you should have measured the height of where people stand up or people sit down.

Ai Sekizawa: Your are right. After this paper was prepared, we started vertical direction measurement tests. As Dr. Hasemi pointed out, we started to measure the gas concentration at the level people are sleeping and also the level of people standing up. 
$1 \underline{5} 8$ 
MATERIALS AND TESTING

$1 \underline{5} \underline{9}$ 
$1 \underline{6} 0$ 


\section{PROGRESS REPORT ON MATERIALS AND TEST METHODS}

\section{Yuji Hasemi}

Building Research Institute

Tatehara 1, Tsukuba-City

Ibaraki, 305, Japan

This report tries to review briefly Japanese progress in the materials and test methods since the last UJNR meeting. Material is perhaps one of the subfield of fire safety science in which application of engineering concept to fire safety design, regulation and assessment is still very young, while fire safety engineering has already been brought into practice in structural fire safety and smoke control. In the last three years since the previous UNNR meeting, there have been continuous research efforts on materials and testing methods in fire chemistry, building fire safety, and material industry as there had been before. However, perhaps start of the Ministry of Construction's R \& D Project(MOC So-pro) on the development of assessment methods of fire safety performance in 1993 is becoming a locomotive driving research and development on materials and testing methods especially in building area.

\section{Ignition/Ignitability}

Condition for the ignition due to arc welding particles was studied experimentally using high speed video camera(Kinoshita et al, 1995). Arc welding apparatus was operated and arc welding particles were sampled on a gauze sheet soaked with liquid fuel covering a tray located $0.5 \mathrm{~m} \sim$ $2.0 \mathrm{~m}$ away from the arc welding unit. Gauze sheet was used to prevent decay of the arc welding particles before sampling. Since large numbers of particles flew on the gauze sheet, the particle which actually ignited the liquid fuel was identified by the high-speed video camera and was sampled. Dimension and weight of each particle identified as the igniter was analyzed, and the equivalent diameter and weight of the smallest particle thus identified at each distance from the welding unit was defined as the critical diameter and the critical weight for ignition respectively. The critical diameter and the critical weight for ignition thus defined were found to be increasing functions of distance. Equivalent density for this critical condition seemed to be independent of distance.

\section{Noncombustibility}

The current Japanese regulation on building fire safety specifies noncombustible materials according to the results of the noncombustibility test(MOC Notification No.1828) and the surface test(MOC Notification No.1231). The Notification No.1828 noncombustibility test was developed according to a draft British Standard which later became ISO 1182, and its apparatus is essentially very close to the ISO 1182 noncombustibility test apparatus. The both test methods adopt temperature rise as one of the indicators for fire safety assessment, which is however defined in different way between the two tests. Comparison of the Notification No.1828 noncombustibility test and the ISO 1182 noncombustibility test on 14 identical materials was carried out to explore replacement of the Notification No.1828 noncombustibility test in the Japanese building regulation(He et al 1995). According to this comparison, temperature rise value as index for the assessment was always lower at the ISO 1182 than at the Notification No.1828 noncombustibility test. However, except for highly noncombustible materials such as metal, tested materials were graded in very similar order by the two test methods. It seems to suggest high interchangeability between the Notification No.1828 noncombustibility test and the ISO 1182 . Benefits of the ISO 1182 noncombustibility test relative to the No1828 noncombustibility test include resolution of 
apparent heat generation typically for metal due to low density, and its drawback includes relatively long time for manipulation.

Relevance of the results of the Notification No.1828 noncombustibility test with heat release has been studied on asbestos-substitute materials(He et al 1994). Some clear correspondence has been confirmed between the index temperature(maximum temperature in the furnace) and the maximum heat release rate measured with the oxygen consumption method.

\section{Heat Release Rate}

Heat release rate is considered one of the key parameters controlling flame spread in fire. Many fire models for flame spread and room fires use heat release data from the ISO 5660 Cone Calorimeter. However, since the flame heat transfer is a size-dependent process, there has been question if such bench-scale test as the Cone Calorimeter can reproduce heat release in full scale fire. A comparative study between the Cone Calorimeter and an intermediate scale vertical electrical radiant panel has been made to clarify scale effects on the heat release rate(Hasemi et al 1995). According to this result, discrepancy between heat release rate by the Cone Calorimeter and by the intermediate panel becomes significant as the heat flux level to the specimen is reduced.

Another analysis using the Cone Calorimeter with the level of heat flux and the preheating condition as the experimental parameters demonstrated considerable dependence of heat release rate on the preheating condition which was represented by the surface temperature of the specimen just before the ignition(Kikuchi et al 1994).

\section{Gas Toxicity}

Development and popularization of halogen-free fire retardant has been an important subject in fire chemistry during the last decade. However, it is widely recognized that performance of fire retardant often is not consistent with other safety aspects such as corrosivity and toxicity. Naba et al studied generation characteristics of phosphine and other toxic products from plastics containing red phosphorus as fire retardant using a quartz-tube furnace anticipating possible phosphine generation in fire through reaction between red phosphorus and water vapor. They used EVA, Nylon, PVC and LDPE with and without red phosphorus, and evaluated contribution of toxic gas component by the ratio of $\mathrm{C}_{\mathrm{X}} / \mathrm{LC}_{50-\mathrm{x}} /\left(\Sigma \mathrm{C}_{\mathrm{i}} / \mathrm{LC}_{50-\mathrm{i}}\right)$ with literature values of $\mathrm{LC}_{50}$. According to their analysis, contribution of phosphine was rather minor, generally not larger than $6 \%$, for each plastic they used. Generation of HCN and CO was weaker for FR Nylon with red phosphorus than without red phosphorus whereas FR EVA generated more CO than EVA.

\section{Pyrolysis}

Pyrolysis of Polyimido, one of metal substitute polymers, at high temperatures anticipated in fire and influence of oxygen concentration on the pyrolysis was studied with TG-DTA/MS (Asai et al, 1995). $100 \mu \mathrm{m}$ thick Kapton sheets were used as the specimens. At 0\% oxygen atmosphere, pyrolysis started at around $530 \mathrm{C}$, and the maximum weight loss rate appeared at around $580 \mathrm{C}$. Approximately $60 \%$ of the initial weight remained as black residue. In atmospheres with oxygen, although there is a peak weight loss rate at around 580C, there was always a secondary peak of pyrolysis at between $610 \mathrm{C}$ and $800 \mathrm{C}$. Estimated activation energy was far higher at the first pyrolysis step than at the second step, and the activation energy at the first step was found to decrease with increasing the oxygen concentration. 


\section{So-pro Activities on Materials and Testing}

So-pro subprogram on materials is trying to coordinate Japanese fire tests on materials with international standards and make framework of material fire safety assessment consistent with fire safety engineering concept. The So-pro subprogram on materials consists of two working groups, one supported by the material industry and the other supported by construction industry. The first working group started in 1993 deals with testing methods and invites experts mainly from fire testing laboratories, whereas the second working group started in 1995 invites experts from construction companies and deals mainly with application of test data to fire safety design and fire safety evaluation of post-construction conditions of building products. Figure 1 is a framework for the relation between fire safety design and material tests used as the guide for the current So-pro material subprogram, which includes various activities such as

(1)technical survey, development and practice with international standard fire tests

(2) development of tests on reaction-to-fire performances not yet standardized by ISO

(3) quantification of design parameters for the influence of construction, configuration and other postfabricated conditions of building products on fire growth

(4) validation of fire growth models

(5) comparison of test results between Japanese current fire tests and international standard tests

(6) development of matrix method for the classification and assessment of lining materials based on the fire safety engineering concept

The activity (1) includes interlaboratory trials on ISO reaction-to-fire tests which are now carried out as an international cooperation as a CIB W14 subprogram. Results of the previous interlaboratory-trials carried out within the So-pro framework has been published(Marchal et al 1995). A series of fire tests from bench-scale to full-scale including research oriented ones on over 15 materials are run for the purpose of (2),(3),(4), and (5). It is planned that existing fire growth models be validated against the tests. The matrix method for the assessment of material fire safety is planned to be developed by simulating fire growth and evacuation in fire for different conditions of buidings such as use and size. Some of the recent works introduced in respective reaction-to-fire performance have been carried out within the So-pro framework.

\section{REFERENCES}

Hasemi,Y., and Hayashi,Y. Ed.: Fire Safety Design of Buildings and Fire Safety Engineering, Proceedings of the Mini-Symposium, BRI Proceedings No.3, 1995.

Ministry of Construction: MOC Fire Tests So-pro, Development of Assessment Methods of Fire Safety Performance(brochure), 1994.

He,F., Sugahara,S., and Naruse,T.: Fire performance of non-asbestos ceramic composite materials, Proceedings of the 1994 Annual Meeting, Japan Association of Fire Safety Science, p.4- 5, 1994*.

Naba,H., Morikawa,T., and Kobayashi,T.: Phosphine and other toxic gases from plastics containing red phosphorus as fire retardant during combustion, ibid, p.66-69*.

Kikuchi,R., Yasui,N., Takashima,S., Hasemi,Y., and Yoshida,M.: Upward flame spread along a wooden wall - Part II, ibid, p.178- 181*.

Kinoshita,K.,Watanabe,N., and Hagimoto,Y.: Discriminating method of arc welding particles igniting combustibles and ignition properties of the particles, Proceedings of the 1995 Annual 
Meeting, Japan Association of Fire Safety Science, p.232- 235, 1995*.

Asai,T., Maekawa,M., Nohmi,T., Tezuka,Y., Yajima,H., and Ishii,T.: Characteristics of thermal degradation of polyimido in different oxygen concentration, ibid, p.252- 253*.

Hasemi,Y., Yoshida,M., Goto,T., Kikuchi,R., Hosomi,M., and Yamamoto,E.: On the predictability of turbulent upward flame spread based on material fire tests, ibid, p.286- 289*.

He, F., Goto,T., and Yoshida,M.: A study on non-combustibility tests, Proceedings of the 1995 Annual Meeting, Architectural Institute of Japan, Fire Safety Division, p.39- 40, 1995*.

Marchal,A., Yoshida,M., and Hasemi,Y.: Technical Report on Interlaboratory-Trials on Reactionto-Fire Tests, Building Research Institute, September, 1995.

* The references thus marked are written only in the Japanese language. 
Figure 1

\section{So-pro Proposed Firesafety Assessment Framework}

\section{on Lining Materials}

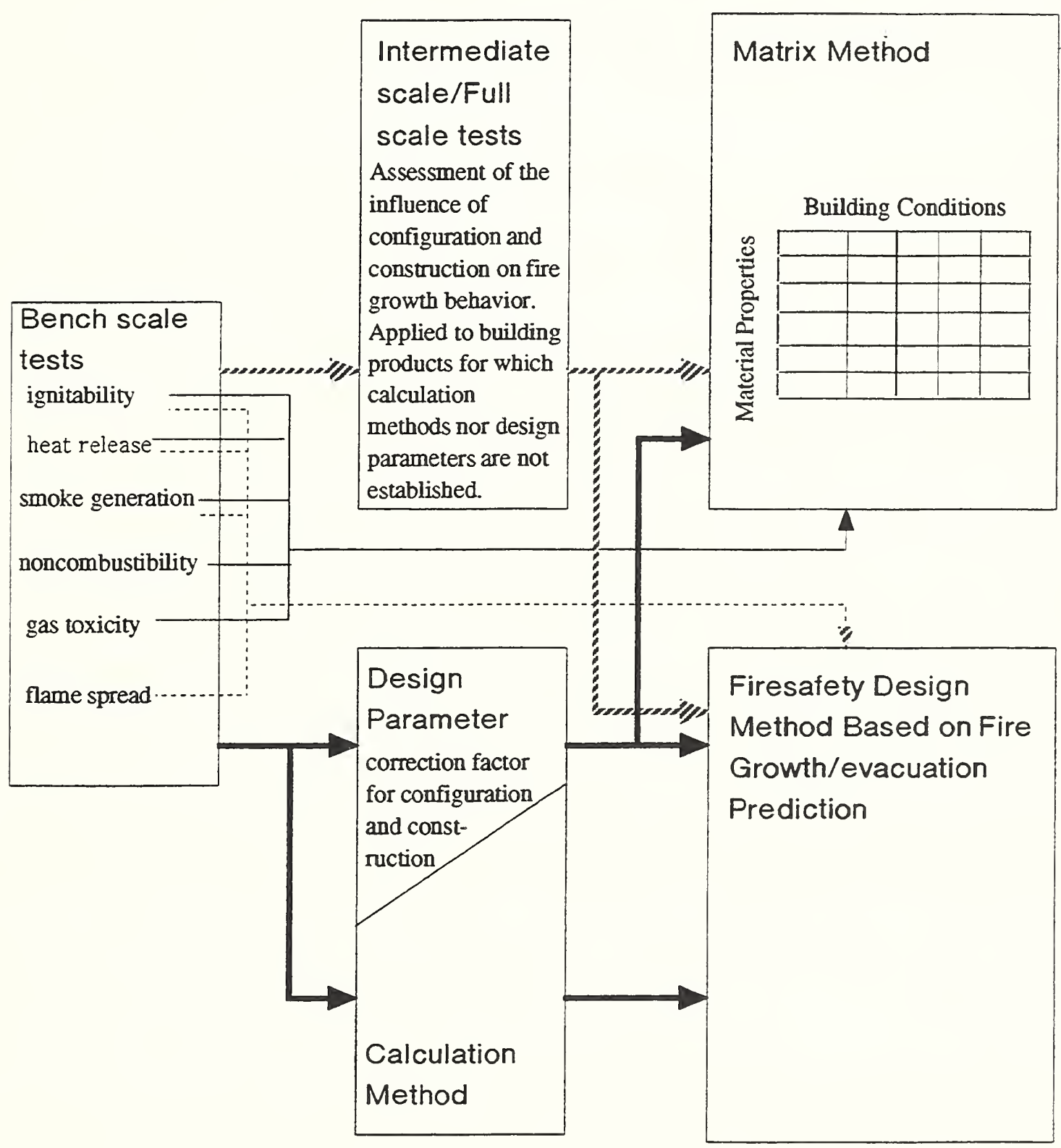

The following two approaches of fire safety desing are considered.

(1)Application of prediction method

(2) Matrix method

The first approach will perhaps be applied only to special design or big construction projects, and most of practical design will be done according to the second approach. Both design methods should basically use data from same tests. Screening tests such as the noncombustibility and gas toxiciy tests are to be used essentially for the second approach. The first approach may use additional tests necessary for FSE calculation. Assessment should primarily be based on benchscale tests ( $\rightarrow$ in the diagram). Intermediate and full scale tests will be applied only to building products for which bench-scale tests cannot lead to rational assessment of fire safety. 
$16 \underline{6}$ 
Progress Report on U.S. Research on Test Methods and Materials

\author{
Marcelo M. Hirschler \\ GBH International
}

and

Takashi Kashiwagi

Building and Fire Research Laboratory

National Institute of Standards and Technology

\title{
1. TEST METHODS
}

In the United States, the most important fire standards writing organization is the American Society for Testing and Materials (ASTM). Fire standards are also, however, developed by the National Fire Protection Association (NFPA), and Underwriters Laboratories (UL), but they are often similar to the ones issued by ASTM.

ASTM has issued over 100 standards associated with various aspects of fire, including guides, terminologies and specifications as well as test methods. Within ASTM, committee E5 is specifically dedicated to writing generic fire standards. However, many other committees, which have different primary responsibilities, also write fire standards which are specific to the materials, products or occupancies of their concern. For example, committees D7 (on Wood), D9 (on Electrical Insulation Materials), D11 (on Rubber), D13 (on Textiles) and D 20 (on Plastics) all write fire test methods for their materials, as do committees F15 (Consumer Products) and F23 (Protective Clothing). Committees interested in Aircraft (F7), Ships (F25) or Correctional Facilities (F33), also write test methods that deal with fire issues. The process by which ASTM develops consensus test methods is public, and every concern expressed must be addressed, through a relatively strenuous procedure.

At NFPA, all fire tests are developed by the Fire Test committee, the members of which are appointed, and the results of whose deliberations are published. Comments can then be made by the public and the final recommendations are voted on in public meetings by the entire membership. Such standards generally tend to contain pass-fail criteria, while those of ASTM tend to be generic. UL standards are not generated by public consensus. UL is a private organization which canvasses industrial opinions, and makes its own decisions.

All of these organizations create various types of fire standards, mainly guidance documents and test methods addressing most of the major fire properties. Only newly adopted test methods during the last several years are described here.

\section{SMOKE OBSCURATION}

ASTM E662, often known as the NBS smoke density chamber, is extensively referred to in specifications and requirements, and quoted in published data. However, this test method has several limitations such as the use of white light (uncertainty over integrating over a broad emission spectrum), vertical sample mounting configuration (dripping problem for certain materials), change 
in oxygen concentration in the test chamber during an experiment, deposition of soot and condensable species on walls and optical components, and others. A modification of this test method has been drafted in ASTM (draft ASTM E5.21.3) and has also been standardized internationally (ISO 5659, Part 2), which differs from the original in that the heat source is a conical radiant heater, similar (but not identical) to the cone heater in ASTM E1354 (Cone Calorimeter Test), piloted ignition is achieved by means of a spark igniter, the specimen is oriented horizontally, and there is an optional capability for a load cell, which measures sample mass loss continuously during a test. The incident heat flux can be set at any value, but values of 25 and $50 \mathrm{~kW} / \mathrm{m}^{2}$ are required in the standard. The repeatability and reproducibility of this test method are better than those of ASTM E662, and it also solves some of the limitations of that procedure.

\section{HEAT RELEASE RATE}

\section{Bench-scale:}

ASTM E1474 (or NFPA 264A) is an application of the Cone Calorimeter (ASTM E1354) specifically for use with upholstered furniture or mattress composite specimens. It determines the same flammability characteristics as ASTM E1354, but specifies a particular incident heat flux of $35 \mathrm{~kW} / \mathrm{m}^{2}$, and a specific specimen preparation and mounting procedure [1]. In fact, the standard allows two specimen preparation procedures, with one of them suggested for screening purposes only. The method deemed acceptable for final testing was developed as a result of work funded by the European Commission, and conducted by a testing conglomerate under the designation "Combustion Behavior of Upholstered Furniture" [2]. ASTM F1550 is based on ASTM E1474, for direct applicability to correction and detention facilities. It addresses the testing of upholstered furniture or mattress composite specimens, but in a vandalized fashion, by slashing through the fabric and any potential interliner present. The objective of this test method is to prevent the use, in correction and detention facilities, of paddings posing excessively high fuel loads. Such paddings may be simply protected by a pierceable barrier, so that they do not ignite easily under normal circumstances, but cause a severe fire hazard when they do eventually burn. Other applications of the Cone Calorimeter also exist: ASTM E1740 was approved in 1995, for use with wall covering composites (including the backing material) and a new standard is being drafted for use with electrical wires and cables and their coating materials.

Real-scale:

ASTM E1537 (or NFPA 266), ASTM E1590 (or NFPA 267) and a draft test for stacking chairs represent a new generation of fire tests: a real-scale item (either an upholstered furniture piece, a mattress, or a stack of chairs) is placed on a load cell in a furniture calorimeter, or in a room, and ignited by a gas burner that is on for a fixed period of time. The release rates of heat, smoke, and combustion products are determined by measurements in the exhaust duct. It has been shown that, for peak heat release rates of less than $600 \mathrm{~kW}$, heat release is not affected by the re-radiation from the walls, so that testing in a small or in an open furniture calorimeter should give similar results [3]. Therefore, ASTM E1537 involves upholstered furniture, which can be tested under the hood in a furniture calorimeter or inside a small room, either of dimensions 2.4 by $3.7 \mathrm{~m}$ or 3.0 by $3.7 \mathrm{~m}$, with a $2.4 \mathrm{~m}$ height and a standard door opening. The ignition burner is square shaped, and the flame is tumed on for $80 \mathrm{~s}$, at a propane flow rate of $13 \mathrm{l} / \mathrm{min}$. The application for this test method is contract occupancies, of higher than average risk, particularly in the absence of sprinklers. The test is based on the concepts put forward in California Technical Bulletin TB 133 and contains a set of pass/fail 
criteria, based on heat release: $80 \mathrm{~kW}$ peak rate of heat release and $25 \mathrm{MJ}$ total heat released in the first $10 \mathrm{~min}$. of test. NFPA 266 is equivalent to ASTME1537, except that testing must be conducted in a furniture calorimeter. This test method has been adopted for regulation in some states, and has been incorporated into the generic sections of the National Life Safety Code, NFPA 101, as well as into the specific sections related to detention and correction occupancies. The requirements set are a maximum rate of heat release of $250 \mathrm{~kW}$ and total heat release of no more than $40 \mathrm{MJ}$ in the first 5 min of test. Similarly, ASTME1590 has also been adopted by various states and by NFPA 101 .

Room-corner tests: The heat release rate of wall lining materials is determined in full scale, by oxygen consumption calorimetry, by lining walls (or walls and ceiling) of a small room (2.4 by 3.7 $\mathrm{m}$ by $2.4 \mathrm{~m}$ high) with the products under consideration. In ASTM E-5.13.2 Draft, wall lining materials lining three walls are tested (all the walls except the one containing the door opening); it utilizes a gas burner, placed flush against walls in the corner at a height of $305 \mathrm{~mm}$ from the floor. The burner is set at an incident power of $40 \mathrm{~kW}$ for 5 minutes, followed by a setting of $160 \mathrm{~kW}$ for a further $10 \mathrm{~min}$. The measurements, all made in the exhaust duct, include heat and smoke release, but the principal decision to be made is whether the wall lining is able to prevent the flames from reaching the outer extremities of the test specimen and the room from reaching flashover.

Large Scale Tests for Cables: ASTM has issued two vertical cable tray tests for cables to be used in industrial facilities. They are ASTM D5424 and ASTM D5537 and they measure smoke release (D5424) and heat release (D5537) together with flame spread and mass loss on $2.4 \mathrm{~m}$ lengths of cables.

\section{Smoke Toxicity}

Both ASTM and NFPA have been working for many years in developing a standard test for toxic potency of smoke. ASTM has recently issued ASTM E1678, a test method for determining toxic potency. The apparatus consists of a $200 \mathrm{~L}$ plastic exposure chamber communicating, through a connecting chimney, with a combustion chamber. Sample combustion results from radiant exposure to a flux of $50 \mathrm{~kW} / \mathrm{m}^{2}$, generated by a set of quartz lamps, for $15 \mathrm{~min}$. Concentrations of the major gaseous toxicants are monitored over a $30 \mathrm{~min}$ period, with concentration-time products for each being determined from integration of the areas under the respective concentration-time plots. They are then used to determine the preliminary analytical toxic potency $\left(\mathrm{LC}_{50}\right)$ by using $\mathrm{N}$-gas model equations, where toxic potency is calculated as an additive function of the toxic potencies of the individual gases. Unfortunately, the standard only addresses pre-flashover fire situations, and toxic potency values for many products are separated only by less than a factor of three. Work is in progress to develop an alternative standard, or changes to E1678, to address toxic potency measurements for post-flashover scenarios, with the same test apparatus (by requiring correction for the carbon monoxide concentration inevitably present in post-flashover scenarios). NFPA 269 includes both the test method and the corrections needed for post-flashover scenarios.

\section{Smoke Corrosivity}

ASTM has issued the cone corrosimeter (ASTM D5485). In it the cone calorimeter is used to burn small samples of materials or products, and an aliquot of the resulting smoke is passed through an exposure chamber where copper circuit boards are exposed for 1 hour. The targets are then removed from the exposure chamber and kept for 24 hours post-exposure, at room temperature and $75 \%$ 
relative humidity. Another test method is in the process of being developed, using the same test apparatus as the smoke toxicity standard E1678, and the same corrosion targets and post-exposure protocol as ASTM D5485.

\section{Fire Safe Materials}

A new major research thrust has been put forward for the development of advanced fire safe materials applicable to a commercial aircraft interior by the Technical Center of the Federal Aviation Agency, FAA. The National Research Council has recently published a report describing recommendations on this matter [4]. The research activity at this center on this topic will be presented by Dr. Lyon. Many universities are participating in this thrust and their projects are briefly described below.

- Case Western Reserve University: Synthesis and characterization of polybenzoxazines - a new thermally-stable, high char yield polymer. The polybenzoxazines are superior in thermal stability and comparable in price and ease of processing to phenolic resins without the drawbacks of free formaldehyde or gaseous product generation during curing.

- Cornell University: Thermal stability enhancement by molecular-level reinforcement and its relationship to flammability will be studied for engineering polymer nanocomposites.

- Richard Stockton State College of New Jersey: Synthesis of new cyanate ester monomers leading to addition-cured triazine resins for interior panels and adhesives with high thermal stability, low heat of combustion, and low combustion efficiency will be conducted.

- Rutgers University: Mechanical properties and fracture behavior of carbon-fiber reinforced polysialate primary and secondary aircraft composites will be studied at ambient and elevated temperatures and related to constituent material interactions and process variables.

- Pennsylvania State University: Linear polychlorophosphazenes using an economical synthetic route will be synthesized and evaluated for miscibility and fire resistance in blends and composites.

- University of Massachusetts: Molecular design of fire safe polymers and composites for various interior applications will be conducted using computational and synthetic chemistries. Mechanistic approaches to improve fire resistance include thermodynamic phase changes and chemical reactions during fire exposure which consume heat, generate flame suppressants, or increase char formation.

- University of Michigan: A family of inorganic-organic polymer will be synthesized for various aircraft applications requiring thermally stable resins, low viscosity liquid crystalline materials, high silicon-content elastomers, and low density thermal insulation.

- University of Akron: Molecular dynamic simulations and experimental kinetic studies of polymer pyrolysis in the bulk, at surfaces, and with coupled diffusion of small molecules (degradation products, flame retardants, oxygen) will help researchers to design materials which will provide a totally fire resistant aircraft cabin. 
- University of South Carolina: A new class of fire safe polymeric materials containing no halogens or heteroatoms will be synthesized; it is based on soluble and processable oligomeric and polymeric alkyne-functionalized polyphenylenes and fullerenes.

- Virginia Polytechnic Institute and State University: Synthesis, characterization, and fire evaluation of phosphine oxide copolymers of polyetheretherketone, polyetherketone, polypheneylene sulfide, and polyimide.

At NIST, new non-halogenated flame retardant approaches are being explored mainly for large volume commodity polymers such as polyethylene, polypropylene, Nylon, polystyrene, and others. One approach is to enhance char formation by an appropriate combination of additives to these polymers. Reduction in heat release rate by forming char has been demonstrated by the use of potassium carbonate and silica gel combination in these polymers [5]. Another approach using silicon compounds is also being explored. The molecular dynamic model to calculate thermal degradation behavior of polymers has been further extended by combining its code with the DISCOVER code of Biosym/Molecular Simulations. This extension allows it to apply to many different polymers[6]. A new model is being developed to describe physical and chemical processes yielding an intumescent char. The model is numerically solved for the time-dependent behavior of growth and transport of numerous bubbles in a polymer slab and subsequent swelling of the polymer sample. With the addition of carbonization chemistry and its physical effect, the model can be used to explore how to design intumescent materials at the optimum condition to reduce gasification rate/heat release rate most effectively [7].

\section{References}

1. Babrauskas, V. and Wetterlund, I., "Fire Testing of Furniture in the Cone Calorimeter. The CBUF Test Protocol", Swedish National Testing and Research Institute", SP Report 1994:32, Boras, Sweden, 1994.

2. Sundstrom, B. (Ed.), "CBUF: Fire Safety of Upholstered Furniture - the Final Report on the CBUF Research Program", European Commission Measurements and Testing Report, EUR 16477 EN, Interscience Communications, London, UK, 1995.

3. Parker, W.J., Tu, K.-M., Nurbakhsh, S., and Damant, G.H., "Furniture Flammability: an Investigation of the California Technical Bulletin 133 test. Part III: Full Scale Chair Burns", NISTIR 90-4375, NIST, Gaithersburg, MD, 1990.

4. "Fire- and Smoke-Resistant Interior Materials for Commercial Transport Aircraft" Publication NMAB-477-1, National Academy Press, Washington, D.C. 1995.

5. Gilman, J.W., Kashiwagi, T., and Lomakin, S.M. "Environmentally Safe Fire Retardants for Polymeric Materials, 1: Silica Gel-Potassium Carbonate Additive" to be presented at 41st International SAMPE Symposium and Exhibition, March 24-28, 1996, Anaheim CA. 
6. Nyden, M., Coley, T.R., and Mumby, S. "Applications of Molecular Dynamics to the Study of Thermal Degradation in Aromatic Polymers; 1 Polystyrene" to be presented at SPE ANTEC'96, May 5-9, 1996, Indianapolis, $\mathbb{N}$.

7. Butler, K.M., Baum, H.R. and Kashiwagi, T. "Three-Dimensional Modeling of Intumescent Materials" to be presented at SPE ANTEC'96, May 5-9, 1996, Indianapolis, $\mathbb{N}$. 
Ministry of Construction, Building Research Institute.

Report on

\section{Asia-Oceania \\ I.S.O. 5660 Cone Calorimeter Inter-laboratory trials}

Prepared by

A. Marchal, M. Yoshida, Y. Hasemi

September 1995 


\section{Coordinators' Preface}

Heat release rate is becoming considered as the most important property for the fire hazard assessment of materials. The oxygen consumption principle has led to the development of heat release measurement for the different scales and configurations under simulated fire environments during the 1980's. Especially the Cone Calorimeter, currently under the process of international standardization as ISO 5660, is becoming a bench-scale laboratory standard for reaction to fire assessments.

However, the Cone Calorimeter is a sophisticated apparatus in comparison with conventional fire test apparatuses. Heat release rate of a material under specific irradiance is obtained only after the combination of very different measurements, namely gas concentrations, gas flow rate and heat flux. The oxygen consumption principle needs fairly complicated instrumentation and calculation, many of the procedure and other technical components of which are not yet standardized in practical way. Calibration of the heating conditions with heat flux gages needs careful treatment and maintenance of the instrumentation, for which, nevertheless, most of fire laboratories are not yet able to check the results against real values of irradiance.

In view of the importance of the Cone Calorimeter as a basic tool for fire hazard assessment and as a candidate for the future key regulatory reaction-to-fire test, several interlaboratory trials have been conducted in the late 1980's. Most of the participants of the Cone Calorimeter round robin were from Western Europe and North America since there were very few laboratories using the Cone Calorimeter equipments in Asia-Oceania until around the beginning of 1990's. However, many of the Cone Calorimeter users in this district suffer from technical difficulties of the equipments and from difficulties in establishing confidence in their own data.

In 1993, the Ministry of Construction of the Japanese Government started a five-year research \& development programme for the possible renovation of the fire test methods. As part of this programme, interlaboratory trials with ISO reaction-to-fire tests were initiated first with ISO 5657 Ignitability test in 1993. The interlaboratory trial was extended to include the ISO 5660 Cone Calorimeter in 1994. Considering the growing interest in international fire research coordination in the Asia-Oceania, the invitation to the Cone Calorimeter round robin was circulated to all fire laboratories using Cone Calorimeter available in time for the round robin in this district. Also fire laboratories outside the district who were interested in the participation were similarly invited. Ten fire laboratories including two from outside Asia-Oceania finally decided to participate in the round robin. The participation from outside Asia-Oceania is appreciated not only because their own experiences have led to the improvement of the quality of the interlaboratory trials but also because their participation could be a germ of a wider international framework for cooperation of fire laboratories which is believed to be a key for a world-wide coordination of the fire-growth testing in the future. 
Relation and connection in the participating laboratories in this round robin, or even participating countries, are believed to be far weaker than those in the previous Cone Calorimeter round robins which were run within some more formal framework as ASTM or ISO. This fact has resulted in some difficulties in keeping consistent testing conditions in the participants, and this difficulty could be reflected in the result of the round robin. However, this difficulty is considered rather an essential characteristic in Asia-Oceania, and is a potential problem to be overcome when a wider inter-district round robin is intended in the future. The coordinators wish that the experience through this project become a basis for further cooperation of the participating laboratories, and also wisdom and ideas for a wider interlaboratory cooperations be derived from this experience. A new Cone Calorimeter round robin with wider scope is already planned on the basis of the experience of this round robin as a CIB W14 (Fire) subprogramme.

\section{Masashi Yoshida, General Coordinator Senior Research Member, Code \& Evaluation Research Center, Building Research Institute.}

Yuji Hasemi, International Coordinator Head, Fire Safety Division, Building Research Institute.

\section{Acknowledgment}

The coordinators would like to acknowledge technical support of Dr. M.L. Janssens, American Forest and Paper Association, and Dr. V. Babrauskas, Fire Science and Technology Inc., throughout the round robin projects and the efforts of Dr. A. Marchal, STA fellow at Building Research Institute, who made the major contribution to the analysis and is the substantial author of this report. The coordinators thank all participants and the committee members of this project. The international Coordinator especially thanks Prof. W.K. Chow, the Hong Kong Polytechnic University, for the arrangement of the international participants meeting in Hong Kong in March i995. For the majority of the round robin participants, it was the first opportunity to meet together. 


\section{Table of Contents}

\section{Preface}

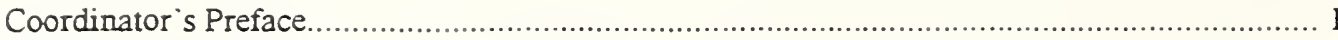

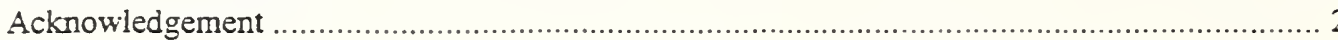

\section{Introduction}

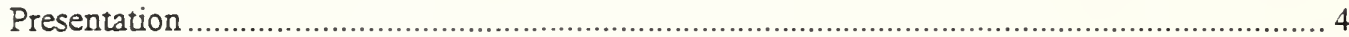

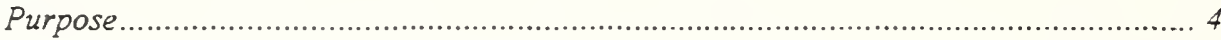

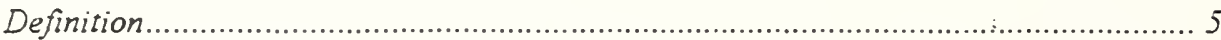

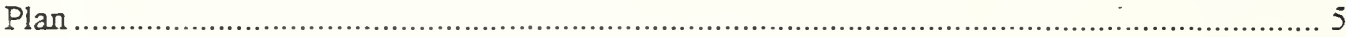

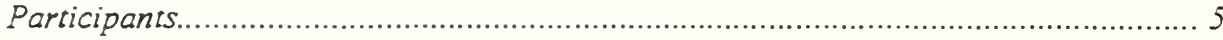

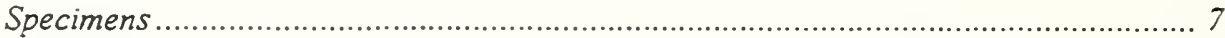

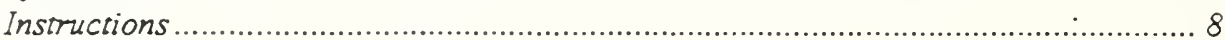

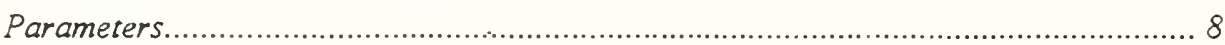

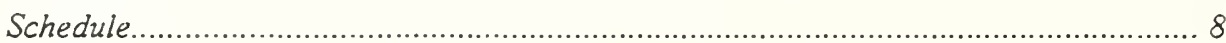

Analysis of the data

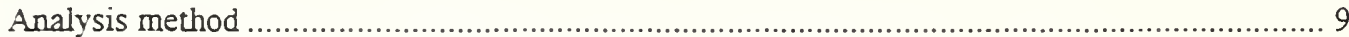

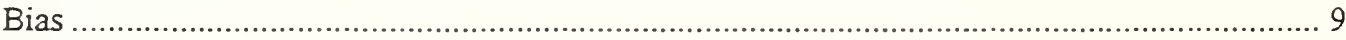

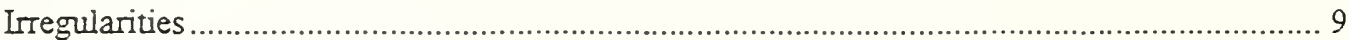

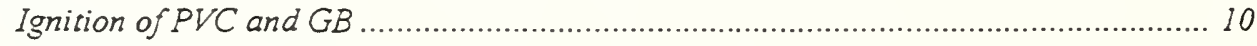

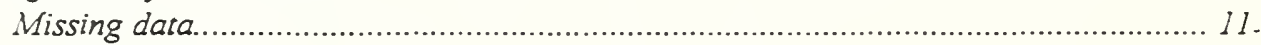

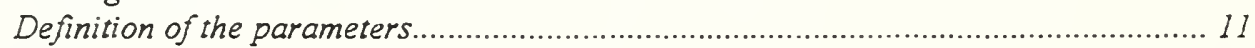

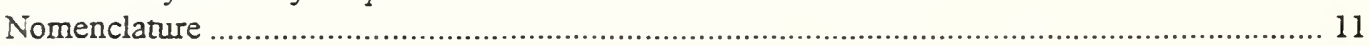

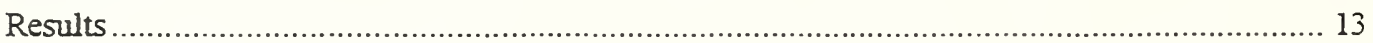

\section{Comments}

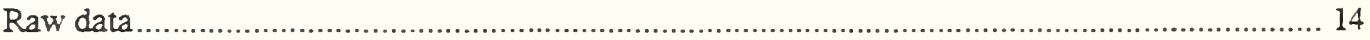

Mass loss...................................................................................................... 14

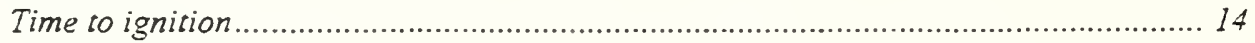

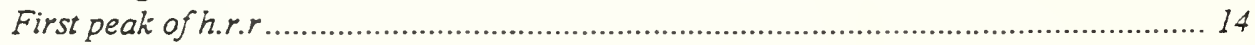

Time to first peak of h.r.r..................................................................................... 15

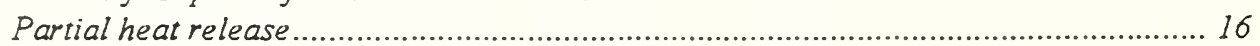

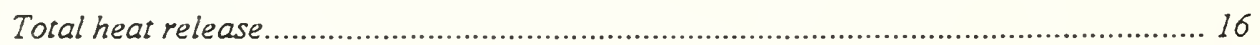

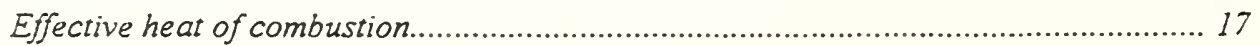

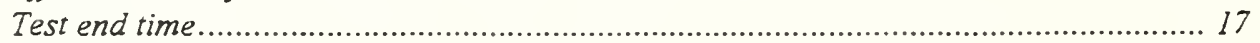

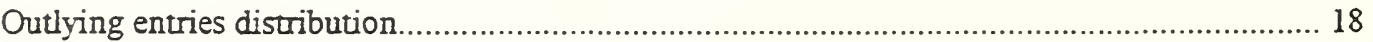

Distribution according to the type of data ................................................................ 18

Distribution according to the different level \& heat fluxes............................................ 20

Distribution according repeatability and reproducibility........................................ 22

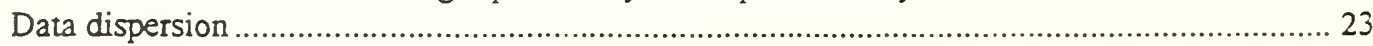

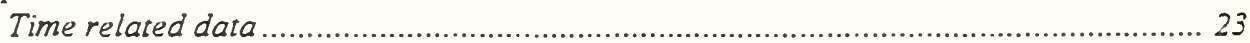

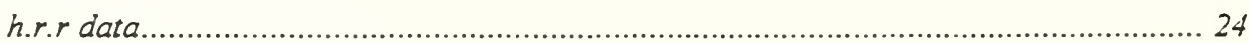

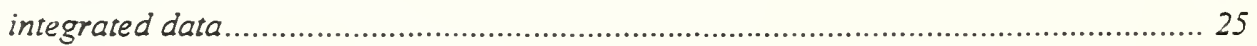

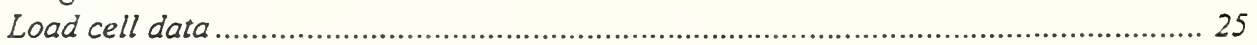

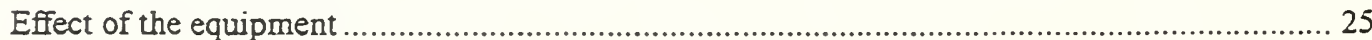

Comparison

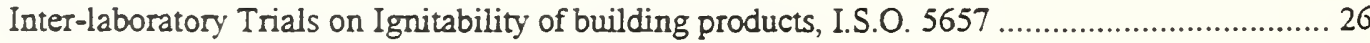

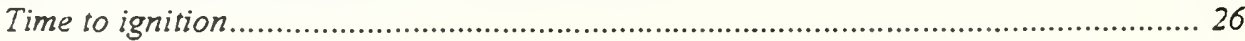

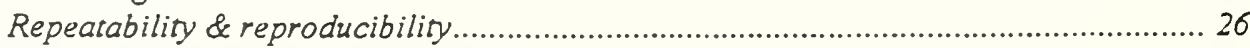

Inter-laboratory Trials on Cone Calorimeter from A.S.T.M. \& I.S.O........................................... 27

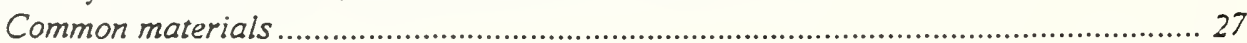

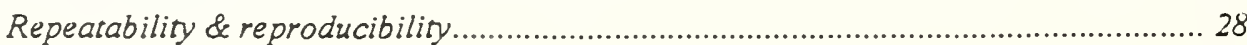

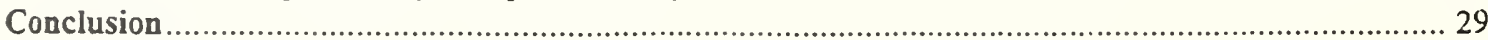

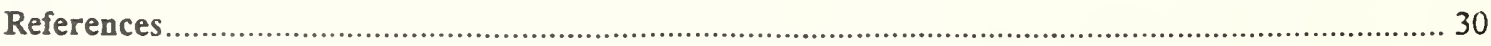

Annex : Tables III to XII......................................................... 31 


\section{Introduction}

\section{Presentation}

\section{Purpose}

As part of a large inter-laboratory calibration program initiated by the Ministry of Construction of the Japanese Government, the I.S.O. 5660 Cone Calorimeter has been submitted to an international round robin, including mainly Asian countries. If different cooperation programs were ever launched, through EU and USA, to bring a harmonization of fire tests, Asian laboratories still stayed out from such calibration platform. This round robin, conducted under the auspices of the Building Research Institute of Japan, aims to improve the measurements' quality of the participating laboratories and develop the reliability and accuracy of fire tests data.

The present document reports a statistical analysis of the first I.S.O. 5660 Cone Calorimeter inter-laboratory trials in Asia-Oceania, carried out in 1994-1995. The main objectives which have been sought were : participants.

- To check the consistency of the Cone Calorimeter's outputs between the

- To point out the major deviations and troubleshootings which could appear through the data processing, try to identify and to link them to their potential origins.

- To assess statistically suitable statements of the repeatability and the reproducibility achievable by the method.

- To enable recommendations to the participating laboratories in order to improve their operating experience in conducting Cone Calorimeter tests and to design some quality control procedures.

During the execution and the analysis of this round robin, but unluckly after its initiation, data and related reports about previous similar inter-laboratory trials on Cone Calorimeter (by A.S.T.M.) and on other fire tests (I.S.O. 5657) became available to the coordinators. In addition to the exposed initial objectives, those documents allow us to test another important item, the coherence and consistency between Cone Calorimeter round robins results or more specifically between some fire parameters. 


\section{Definition}

The definition of the principles used in this report can be found in Section 3.1 of I.S.O 5725 titled "Precision of test method". The most important ones are :

- Coefficient of variation: The standard deviation (of a particular statistic), divided by the mean value (of the same statistic).

- Repeatability conditions : Conditions where mutually independent test results are obtained with the same method on identical test material in the same laboratory by the same operator using the same equipment within short intervals of time.

- Repeatability value ( $r$ ): The value below which the absolute difference between two single test results obtained under repeatability conditions may be expected to lie with a probability of $95 \%$.

- Reproducibility conditions: Conditions where test results are obtained with the same method on identical test material in different laboratories with different operators using different equipment.

- Reproducibility value $(R)$ : The value below which the absolute difference between two single test results obtained under reproducibility conditions may be expected to lie with a probability of $95 \%$.

\section{Plan}

\section{Participants}

Invitation for participation was circulated by August, 1994 to all fire laboratories in Asia-Oceania using the Cone Calorimeter equipment available in time for the round robin. Fire laboratories outside Asia-Oceania interested in this project were also invited to the round robin. As the result, the 10 fire laboratories listed below decided to participate in this round robin.

- Architecture and Building Research Institute

13th Floor No. 333 Tun-Hua S. Rd Sec. 2,

Taipei

Taiwan.

Mr. Sam Chou, Dr. Alec M.Y. Lei

- Building Research Institute

Tatehara 1, Tsukuba-City,

Ibaraki-Pref., 305

Japan.

Mr. M. Yoshida, Dr. Y. Hasemi 
- Forestry and Forest Products Research Institute Matsunosato, Kukizaki-Town, Inashiki-Gun, Ibaraki-Pref.,305 Japan.

Mr. T. Harada

- Hokkaido Forest Products Research Institute 1-10 Nishikagura, Asahikawa-City, Hokkaido, 071-01 Japan.

Mr. S. Kikuchi

- Hong Kong Polytechnic University

Department of Building Services Engineering, Hung Hom, Kowloon

Hong Kong. Prof. Chow Wan Ki

- Institute of Building Fire Research China Academy of Building Research, 9, Xiao-Huang-Zhuang, An-Wai, P.O. Box 752, 100013 Beijing P.R. China.

Mr. Li Yin-Qing, Mr. Chen Jinghui, Mr. Ji Guangqi

- L.S.F. Laboratorio di studi e ricerche sul fuoco

GRANDATE (CO),

Via Vetreria, 1

Italy.

Mr. S. Messa, Mr. P. Acciarri

- Research Institute of Marine Engineering I-5-12 Fujimi-Cho, Higashimurayama-City, Tokyo, 189 Japan.

Mr. K. Yoshida, Mr. S. Nagasawa

- State Forest Products Research Institute

Lamacska cesta 1, 83330 Bratislava Slovakia.

Dr. O. Grexa

- Tianjin Fire Research Institute

P.O. Box 27, 92 Jin Zi Road, Tianjin 300381

P.R. China.

Ms. Du Lanping 
Summary and analysis of the data have been prepared using the data from 8 laboratories out of the 10 participants which became available to the secretariat by the end of May, 1995. In this report, participants are only identified as alphabetical characters as Laboratory A, B, and so on. The identifications do not reflect the order of the names in the list above.

Planning and coordination of the round robin program were conducted by the executive committee consisting of M. Yoshida*, Y. Hasemi*, T. Harada, S. Kikuchi, K. Yoshida, Alec M.Y. Lei, I. Nakaya, Y. Tanaike (Japan Testing Center for Construction Materials) and S. Tasaka (General Building Research Corporation of Japan). Messrs Tanaike and Tasaka are not Cone Calorimeter users, but were invited to the project as experienced fire test experts. Dr. A Marchal (STA fellow at Building Research Institute) joined the project in 1995.

* Respectively General \& International Coordinator, Building Research Institute.

\section{Specimen}

The following materials were selected as the specimens. All specimens of each material were arranged by Building Research Institute using the products from a same lot directly shipped by factories. $100 \mathrm{~mm} \times 100 \mathrm{~mm}$ specimens were sent to the participants. Thickness depends on the material.

- PMMA sheet (referred to as PMMA) transparent, $10 \mathrm{~mm}$ thick, density $1180 \mathrm{~kg} / \mathrm{m}^{3}$.

Product from Mitsubishi-Kasei, Ltd. Japan, identified as "Aclylite".

- Medium Density Fiber board (referred to as MDF) untreated (natural wood color), $12 \mathrm{~mm}$ thick, density $640 \mathrm{~kg} / \mathrm{m}^{3}$.

Produced from tropical wood through arrangement of the Forestry and Forest Product Research Institute, Japan.

- polyVinyl chloride Coated Steel Plate (referred to as VCSP)

black, $0.6 \mathrm{~mm}$ thick, average density $6870 \mathrm{~kg} / \mathrm{m}^{3}$.

Product from Toyo Kohan Ltd., Japan.

- Gypsum Board (referred to as GB) covered by paper sheets on both sides, $9.5 \mathrm{~mm}$ thick, average density including the paper sheets $650 \mathrm{~kg} / \mathrm{m}^{3}$, paper $230 \sim 240 \mathrm{~kg} / \mathrm{m}^{2}$. Product from Yoshino Gypsum Ltd., Japan, identified as "Tigerboard". Graded as "quasi-noncombustible material" in the Japanese Building Standard Law.

- PolyVinyl Chloride sheet (referred to as PVC) white, $2 \mathrm{~mm}$ thick, average density $1420 \mathrm{~kg} / \mathrm{m}^{3}$.

Product from Takiron, Ltd., Japan, identified as "Takiron Plate". 


\section{Instructions}

Two different irradiance levels $\left(30 \mathrm{~kW} / \mathrm{m}^{2}\right.$ and $\left.50 \mathrm{~kW} / \mathrm{m}^{2}\right)$ were chosen. Only horizontal orientation has been selected. At least three replicate tests were to be conducted for each heat flux level for each material in randomized order for different materials. A two seconds interval was suggested for data sampling. Medium density fiber board specimens were to be conditioned before test. Calibration of heat release rate was to be made for two levels of heat release using methanol (100cc and $200 \mathrm{cc}$ ) without radiation. Calibration of heat flux gage was to be done against either virgin heat flux gage or appropriate calibration furnace*.

* All Japanese participants were able to compare their own heat flux gage with a reference heat flux gage. It was regretful that this circulation was impossible for other participants because of the relatively tight schedule and potential importcustom problems.

\section{Reported parameters}

The following data were to be reported on each test.

- Initial mass and total mass loss during each test.

- Time to ignition.

- Heat release rate as a function of time.

- First peak of heat release rate and time to first peak.

- Average heat release rate at 180 s.

- Total heat release at 180 s, 300 s and over the burning period.

- Average effective heat of combustion over the burning period.

- Burning period.

\section{Schedule}

September 1994

December 1994

January 1995

March 1995

May 1995
Shipping of specimens and instructions

First deadline for data submission (Four laboratories delivered data) Committee meeting, preliminary analysis of the data, delivery of summarized data to participants

Participants meeting

Final deadline for data submission 


\section{Analysis of the data}

\section{Analysis method}

The I.S.O. 5725 "Precision of test methods - Determination of repeatability and reproducibility for a standard test method by inter-laboratory tests" has been adopted to calculate and analyze the data. The report to A.S.T.M. on Cone Calorimeter inter-laboratory trials has been used as a guideline as well. All the definitions and calculations used in this report can be found in those two documents. This method has been chosen here for two main reasons. First of all, the I.S.O 5725 provides a very important guidance about the data analysis procedures and especially recommendations on how to deal with the outlying observations (this point will be discussed later).

The second goal which has been aimed by choosing this standard concerns the comparison ability between the different round robins which have already been published on Cone Calorimeter purpose. The A.S.T.M. and the I.S.O. inter-laboratory trials have actually been proceeded by the same data analysis procedure and so, even if the chosen parameters as well as the products are not exactly the same, overall results can be compared.

\section{Bias}

The general average of a material's property, called "level of the test property", is not necessarily equal to the "true" value. The difference between these two data, when it exists, is called the "bias of the test method". In the case of Cone Calorimeter, bias seems difficult to report insofar that an independent true assessment of a variable's value remains hardly reachable. However, all data associated with heat release measurement (first peak of heat release rate, average heat release rate, partial heat evolved, total heat evolved, and so on) are reduced using the $\Delta h d r_{0}$ constant which is given with $=5 \%$ of uncertainty. Thus, a bias of around $\pm 5 \%$ can be expected for these heat release related variables.

\section{Irregularities}

As prescribed in the I.S.O. 5725, gross errors have to be removed from the data analyses. Even if, as they have to be purged, they will not enter in consideration in the further data treatment, the presence of such important deviation must be staved off by an accurate operating protocol and a careful attention must be paid on the obtained observations. We could propose, as it has been written in the 'Memorandum for US Laboratories participating in the Cone Calorimeter round-robin testing', dated from April 28, 1988 that if any of the mean heat release values for the first $60,180,300$ seconds after ignition, among the three replicates of one material at one set of conditions, differs by more than $10 \%$ from the average for the three replicates, an additional series of three tests has to be executed. The data that does not conform to the $10 \%$ variability should, of course, be identified and returned to the round-robin's organization. 
This measure must decrease the numbers of gross errors detected and, on the same way, improve the reliability of the data together with the repeatability values. During the present round robin, this instruction may not have been applied.

In spite of the one-test-gross-errors, three general kinds of 'curiosities' have been noticed and are reported below.

\section{Ignition of $P V C$ and $G B$}

\begin{tabular}{|c|c|c|c|c|c|c|c|c|c|}
\hline \multirow{2}{*}{ Mat. } & Irr. & \multicolumn{10}{|c|}{ Laboratory reference } \\
\cline { 2 - 10 } & $\mathrm{kW} / \mathrm{m}^{2}$ & $\mathrm{~B}$ & $\mathrm{D}$ & $\mathrm{E}$ & $\mathrm{F}$ & $\mathrm{G}$ & $\mathrm{H}$ & $\mathrm{I}$ & $\mathrm{J}$ \\
\hline $\mathrm{GB}$ & 30 & $\mathrm{NI}$ & - & - & $\mathrm{NI}$ & - & - & $\mathrm{NI}$ & $\mathrm{NI}$ \\
\hline $\mathrm{PVC}$ & 30 & $\mathrm{NI}$ & $\mathrm{NI}$ & - & $\mathrm{NI}$ & - & - & $\mathrm{NI}$ & $\mathrm{NI}$ \\
& 50 & - & $\mathrm{NI}$ & - & - & - & - & $\mathrm{NI}$ & $\mathrm{NI}$ \\
\hline
\end{tabular}

Table I. PVC \& GB's ignition occurrence $N I$ : No Ignition).

The results of the ignition 'success' are quite confusing to analyze since the ratio of 'noignition laboratories' on 'ignition-laboratories' could not lead to discard one or the another laboratory. The explanation of such discordant data should be inquired. The interpretation of the definitions of 'flash ignition' and 'sustained surface ignition' could be a key in the comprehension of this phenomenon.

Moreover one of the main factor influencing ignition time, and therefore, flame outbreak, that is to say just 'ignition', is by far the irradiance level. Some miscalibrations of the heat flux gage leading to an overestimate of the irradiance and consequently to an operating weaker radiant exposure, could thus be suspected. That potential overestimate may explain the scattering of ignition results, especially in the case of the $30 \mathrm{~kW} / \mathrm{m}^{2}$ runs.

In order to realize the importance of this ignition occurrence variability, it should be noticed that in the scope of the Asian inter-laboratory trials on I.S.O. 5657 'Ignitability test' conducted on exactly the same products, PVC under an exposure of $30 \mathrm{~kW} / \mathrm{m}^{2}$ has always been ignited and, thus, must obviously always be able to be ignited at higher heat fluxes $\left(50 \mathrm{~kW} / \mathrm{m}^{2}\right.$ for instance in our case).-

Concerning GB, just one among the six laboratories taking part in the I.S.O. 5657 'Ignitability test' round robin did not state any 'ignition time', defined as 'sustained surface ignition', for the gypsum board sample at $30 \mathrm{~kW} / \mathrm{m}^{2}$. However, its values of 'flash ignition' for that specimen agreed perfectly with the ignition time reported by the five other laboratories. This means that, even this 'outlying' laboratory succeeds somehow in igniting the GB sample under a $30 \mathrm{~kW} / \mathrm{m}^{2}$ irradiance, and makes a drastic contrast with the present results.

The heating process being quite analogous in both standards, the high percentage of noignition obtained here could appear as a major irregularity, and consequently must be carefully watched over. In the following study, the lack of data due to no-ignition runs will just be considered as missing data, without any statistical processes.

Another irregularity, or inconsistency rather, linked to the ignition occurrence variability lies in the fact that some laboratories reported different parameters (such as first peak of heat release rate or total heat evolved) even if they did not state any ignition time for the same run. If these data are consistent with the other laboratories results, that is to say in reproducibility condition, they will be processed as regular data, assuming that the lack of ignition time data was just due to a visual difficulty of the operator to assess the proper instant of flame appearance. If not, they will be discarded. However, as the use of such kind of observations could lend itself to suspicion, it will always be mentioned in the following analyses. 


\section{Missing data}

Despite the ignition/no-ignition problem, just two laboratories computed the all ten parameters requested by the round robin organization as it is indicated in Table II. The others just skip over some variables, even if these data could be reachable (no physical or technical troubles with samples were notified). As there are no visible reasons which could explain this lack of data, the 'empty' parameters will be just regarded as missing data.

\begin{tabular}{|c|c|c|c|c|c|c|c|c|}
\hline & \multicolumn{8}{|c|}{ Laboratory reference } \\
\hline & $\mathrm{B}$ & $\mathrm{D}$ & $E$ & $\mathrm{~F}$ & $G$ & $\mathrm{H}$ & $\mathrm{I}$ & $\mathrm{J}$ \\
\hline $\mathrm{N}^{\circ}$ of unstudied para. & 3 & 0 & 0 & 3 & 2 & 3 & 7 & 6 \\
\hline
\end{tabular}

Table II. Number of variables which have never been studied (Total requested: 10).

Nevertheless, this default of answers is unfortunate for several reasons, especially for the accuracy of the analysis and the relevancy of the conclusions. The comparison, for instance, between the performances of laboratory $I$ or $J$ and laboratory $D$ or $E$ becomes actually very critical, even in relative figures, insofar that the statistical analysis will not recover by far the same amount of entries.

\section{Definition of the parameters}

In the case of MDF's runs (at both levels of irradiance), laboratory H showed some clearly outlying values of first heat release rate peak and especially of time to first heat release rate peak (between 9 and 15 times larger than the average time value of the other laboratories). Looking more closely to their heat release curves, a misunderstanding of the parameter's definition has been pointed out. The given results were not the coordinates from the 'first' heat release rate peak, but the one from the 'maximum' heat release rate peak. Such errors did not occur for the other materials tested because first peaks were also maximum peaks. So, even if the parameter's definition was misunderstood, the results were however correct.

Considering that there was no reasons to keep these erroneous data, since they did not correspond to the requested parameters, they have been removed and replaced by the values we could have read from the heat release rate profiles. The graphical readings were, of course, not as accurate as data acquisition unit could be and it is the reason why these data must be marked and used carefully.

If other parameters definitions misunderstanding could be suspected (concerning mainly times and calculations), this is the only one which have been proved and corrected.

\section{Nomenclature}

According to the I.S.O. 5725 data analysis method and taking into account the previous remarks, the values obtained are analyzed in Tables III to XII (at the end of the present report), corresponding to the 10 requested parameters on the 5 materials tested at two levels of irradiance. The nomenclature used is :

- Mass loss: Remaining mass of the sample at the end of the test expressed in percentage of the initial mass (\%).

- $T_{\text {ig }}$ : Time to ignition (sustained flaming), expressed in seconds (s). 
- f.h.r.r peak: First heat release rate peak (first extreme value of heat release rate), expressed in kilowatt per square meter $\left(\mathrm{kW} / \mathrm{m}^{2}\right)$.

- $T_{f}$ : Time to first heat release rate peak (time of occurrence of the first extreme value of heat release rate), expressed in seconds (s).

- $\mathrm{PHE}_{180}$ : Total heat evolved during the first 180 seconds after ignition (integration of the heat release rate profile from time to ignition to 180 seconds), expressed in megajoule per square meter $\left(\mathrm{MJ} / \mathrm{m}^{2}\right)$.

- $A H R_{300}$ : Average heat release rate computed over the period starting at time to ignition and ending at 300 seconds, $\left(\mathrm{kW} / \mathrm{m}^{2}\right)$.

- $P H E_{300}$ : : Total heat evolved during the first 300 seconds after ignition (cf. $P H E_{180}$ ).

- THE: Total heat evolved during the entire test period, $\left(\mathrm{MJ} / \mathrm{m}^{2}\right)$.

- e.h.c. : Effective heat of combustion (ratio of heat release rate on corresponding mass loss rate), expressed in megajoule per kilogram (MJ/kg).

- Tend: $\quad$ Test ending time, the end of the test being defined as when the mass loss rate drops below $2.5 \mathrm{~g} / \mathrm{s}$ and the average mass loss rate over the next minute does not exceed $2.5 \mathrm{~g} / \mathrm{s}$.

- No. of Labs: Number of laboratories from whom data analyses have been completed, that is to say, from whom data was available and after removing the statistical outliers (This last point differs from the definition used in A.S.T.M. inter-laboratory trials in which this number includes the statistical outliers).

- $\mathrm{m}, \mathrm{r}, \mathrm{R}$ : The mean (or 'level of the test property'), repeatability (inside one : laboratory) and reproducibility (between laboratories) respectively, and as defined before.

- $(a 3 ; b 3)$ : the coefficients of a regression line to fit a possible functional relationship between the repeatability $r$ and the mean $m(r=a 3 \div b 3 * m)$. From $a$ statistical viewpoint, the fitting of a straight line is complicated by the fact that both $\mathrm{r}$ and $\mathrm{m}$ are estimates and thus subject to error. The I.S.O. 5725 recommends an iterative procedure to calculate the coefficients of such an equation. $(a 3 ; b 3)$ are the results of the three steps' calculation (Most of time, a two step's calculation could be considered as the final result).

- $(\mathrm{A} 3 ; \mathrm{B} 3)$ : the coefficients of a regression line to fit a possible functional relationship between the reproducibility $R$ and the mean $m(R=A 3+B 3 * m)$. These are the corresponding coefficients to $(a 3 ; b 3)$ for the reproducibility.

- Ave. r, R: the overall averages of repeatability and reproducibility values for the same parameter. In term of fitting with the $r$ (or $R$ ) versus $m$ curves, this represents horizontal lines and gives then an overall repeatability and reproducibility values of the studied parameter. These values have to be used when the model for error is considered as independent of the mean. 
- V.C.: the overall average by laboratory of the variation coefficient defined previously as the ratio of the standard deviation on the mean value of the same statistic

\section{Results}

After purging the gathered data from obvious irregularities (or inconsistencies) inside their three replicates runs, relative means and their standard deviations, Cochran's one-sided outlier's test was used to determine outlying laboratories in repeatability at each level. Dixon's test was used, at each level as well, for the reproducibility. For both tests, I. S.O. 5725 describes two types of outlying observations, based on their probabilities of occurring $\mathrm{P}$ :

- $\mathrm{P}>5 \%$ (i.e. Cochran's or Dixon's test statistic is less than its $5 \%$ critical value) the item tested is accepted as correct.

- $5>\mathrm{P}>1 \%$ (i.e. the test statistic lies between its $5 \%$ and $1 \%$ critical values) the item tested is called Straggler and have to be marked. Stragglers could be retained as correct.

- $1 \%>\mathrm{P} \quad$ (i.e., Cochran's or Dixon's test statistic is greater than its $5 \%$ critical value) the item tested is called Outlier and has to be marked. Outliers should be discarded.

Both types of outlying data are notified for each level. Stragglers have been retained as correct, while outliers have been removed from further calculations. Once an outlying entry has been identified, tests have been reconducted on the remaining data in order to check the presence of 'hidden' outlying observations.

Calculations of the mean, repeatability and reproducibility have been then computed for all levels and on each parameter, except when the number of retained set of data (initial data minus irregularities and outliers for one particular level of one variable) was less than 3 . In such a case, it was considered that too few data were available to produce a relevant statistical analysis, and results have been replace by a horizontal stroke in the tables.

Once the mean, repeatability and reproducibility values have been assessed with regard to the previous remarks, a functional relationship between them (i.e. $r=f(m)$ and $R=f(m)$ ) has been sought. The fitting equations have been proceeded by a 3 iterative steps' method, as prescribed in the I.S.O. 5725, part 15.6 and have been plotted for each studied parameters. In addition to the coefficients of these regression lines, the averages of repeatability and reproducibility values over the whole set of materials for each variable have been indicated in order to choose, according the plot of $I$ (and $R$ ) against $m$, the best satisfactory final estimation of repeatability and reproducibility, if ever.

Tables of overall coefficient of variation (V.C.) has been added for each parameter. Main notes have been made on each page, but some comments could be added. 


\section{Comments}

\section{Raw data}

\section{Mass loss (Table III)}

The shape of the diagram $r, R=f(\mathrm{~m})$ leads us to prescribe a constant average value for the repeatability and the reproducibility instead of choosing the regression lines. The negative second coefficients of the latest (b3 and B3) confirm that choice. It is actually very seldom to get such negative slopes, the repeatability and reproducibility increasing, logically and most of time, with the mean. A constant value of 0.03 for the repeatability and 0.11 for the reproducibility could therefore be proposed.

Remembering the typical figures of the load cell accuracy (around $\pm 1 \%$ ), the obtained results are very good. However, the weight range of the tested products (starting from about $30 \mathrm{~g}$ for the PVC samples to nearly $120 \mathrm{~g}$ for the PMMA samples) might be too narrow to interpolate safely these precision figures to heavier (thicker) samples.

\section{Time to ignition (Table $\mathrm{IV}$ )}

A graphical good agreement is observed between the regression line and the calculated repeatabilities and reproducibilities. However, the very high value of the reproducibility ( $151 \%$ of the mean) of PVC under a $50 \mathrm{~kW} / \mathrm{m}^{2}$ irradiance has to be noticed (point far from the $\mathrm{R} 3$ line). During the I.S.O. 5657 round robin an analogous behavior of this same product has already been pointed out and was suspected to be due to the numerous transient flaming that PVC's ignition can produce.

As it has been done in these Ignitability test inter-laboratory trials, a best-estimate relationship between $\mathrm{R}$ and $\mathrm{m}$ may be obtained by removing that point. A more complete comparison between the two standards will be report in next chapter.

\section{First peak of h.r.r (Table V)}

As first remark, if the figures of repeatability could be considered as correct (around 10\% of the mean) for most of materials, the reproducibility values stay very high. And this is of first importance knowing that heat release rate is the major data given by the Cone Calorimeter and somehow one of the most useful data to handle with fire behavior of materials (entry data for several fire propagation and flame spread models). It is thus hardly prejudicial for models harmonization and corroboration to obtained so large difference in the h.r.r assessment between laboratories. 
Such a difference between repeatability and reproducibility may be reduced by improving the accuracy of the calibration factor $(C)$. In fact, this factor, calculated by a methane calibration, intervene in h.r.r calculations as a constant multiplicative factor inside a laboratory and, thus, has quite no influence on repeatability conditions but could be an important item on reproducibility conditions (between laboratories). A harmonization of the calibration factor calculation process (methane specification, heat flux gage, ...) should be a clue to decrease the reproducibility values.

An other way to improve the consistency of h.r.r results between laboratories could be the harmonization of the drying agent located on the oxygen gas sampling line. Previous studies have shown that according to the nature and the quality of that chemical filter, some variations could occur in the oxygen concentration evaluation and thus in the h.r.r data.

These remarks are valid for all materials except PVC for which both repeatability and reproducibility are almost intolerable (around $40 \%$ of the mean for the repeatability and $260 \%$ in reproducibility!). Together with the previous statistical analysis of ignition time results from that specimen, we can already conclude that a Cone Calorimeter agreement on PVC inside and between this round robin participating laboratories is a failure, as, from many point of view, ignition time and rate of heat release are the main parameters which are looking for during a Cone Calorimeter experiment.

The statistical results of first peak of heat release rate of GB could be compared with the PVC's ones. That point could lead us to say that materials with a single peak shape of h.r.r profile give some difficulties to be correctly tested. But in the case of VCSP which presents a single narrow peak of h.r.r as well, the results, although high, are much smaller, in term of repeatability and reproducibility, than the two previous materials. We should thus emphasize on the ignition's occurrence variability ( $c f$. Irregularities) that several laboratories encountered to ignite GB and PVC. This phenomenon could explain a further instability in the heat release profiles and consequently in the first peak value.

Even if its $\mathrm{r}$ and $\mathrm{R}$ values are, in average, the smallest of the whole set of materials, not so low reproducibility figures can surprisingly be observed for PMMA at both levels of irradiance. 'Surprisingly' because PMMA, and that is due to its degradation mode, is often said to be one of the best calibration material. However, these results are, by far, much more better than the analogous figures obtained during the A.S.T.M. round robin ( $R$ around $58 \%$ of the mean for the American inter-laboratory trials versus $28 \%$ for the present one).

\section{Time to first peak of h.r.r (Table VI)}

For that parameter, roughly the same remark than the previous one concerning the h.r.r peak parameter of PVC and GB, should be brought, i.e large reproducibility values certainly due to the ignition troubles. However, the repeatability range of that time variable lies in correct borders (around $11 \%$ of the mean in average), even better than the repeatability range of the other important timing data, the ignition time (around 15\% of the mean in average). The comparison between these two times is quite interesting insofar that if we can consider that, at the first glance, ignition time is mainly governed by the external heat flux, the time to first peak of h.r.r also included an evaluation of oxygen analyzer response latency.

As for the calibration factor (C), the analyzer response delay time could be considered as a specification of each Cone Calorimeter device and so should not have any tremendous effect on the repeatability conditions. This fact could explain the good repeatability results we obtained, but we can wonder why they are even better, in average, than the ignition time repeatability values. This difference might prove that it is more delicate to judge the exact moment of the outbreak of the flame than to draw a vertical line on the heat release rate curve. We should thus emphasize on 
the main and sensible importance of an accurate definition of "Ignition Time". A second hypothesis could be that this time to first heat release peak might be more 'stable', more featuring, less subject to fluctuations than the previous one. However, the repeatability improvement between the two parameters is definitively not huge enough to secure such an assumption.

If the delay time must not have any influence on repeatability conditions, figures show, as we can expect, that this is not the case for reproducibility conditions. We can notice that, in the case of PMMA, where the first peak of heat release occurs at a late stage of the manipulation (mean time $>300 \mathrm{~s}$ ), the $\mathrm{R}$ coefficients are the best of the all studied materials, even better than the analogous figures concerning ignition time (in percentage of the mean), but it is the only case. On the opposite, for early stage peak occurrence, that is to say for all the other samples, results are worse. The PMMA's heat release curve does not present a real triangle peak shape as the other one (at the beginning of the manipulation at least) and one could wonder if the characteristic physical or chemical phenomena conducting to that particular and smooth shape, phenomena which must be featuring from PMMA degradation process, could explain such good results. But, as a matter of fact, this idea has to be given up insofar that, as we previously mentioned, the repeatability values of any of the samples are very good and thus do not allow any assumption on the 'stability' of one or the other material degradation processes.

In other words, and as it is logical to think, the oxygen analyzer delay time decreases heavily the between-laboratories' agreement for 'rapid' peak, i.e. during the initial stage of the run, but its effect fades for 'late' peak, just as if it was diluted by the time of occurrence. This observation could lead us to conclude that, as for the calibration factor and in the same time (during the same calibration), a mutual, and especially accurate, process of oxygen analyzer response latency assessment should be brought in order to decrease the reproducibility values.

\section{Partial and average heat release (Table VII to IX)}

For that series of parameters, attention must be paid on the fact that four laboratories, maximum, took part in the study. Thus, results could not have the same "weight" for drawing conclusions. Nevertheless, for the three studied variables, the distributions of repeatability and reproducibility values in respect to the mean seem to abide by the regression lines. Moreover the slope coefficients of their respective equations lie in the same range of order (around 0.05 in repeatability and 0.25 in reproducibility).

The concentration of outlying data for the two partial cumulative parameters (total h.r.r until 180 seconds \& total h.r.r until 300 seconds) from D laboratory is noticeable. In addition, this same laboratory has been identified three times as straggler for the average heat release parameter. Except $E$ which have been identified straggler just one time, $D$ is the only laboratory for these three parameters in which outlying data have been found (and 9 times!). That observation could point out laboratory $\mathrm{D}$ as a total 'outlying laboratory' as described in Par. 11.2.3.d of the I.S.O. 5725, and thus lead us to discard its whole data. Laboratory D has, nevertheless, been kept in this study.

\section{Total heat release (Table X)}

Laboratory $D$ has still been identified twice as outlier. Together with the previous remark, it can be guessed that $\mathrm{D}$ has undoubtedly some troubles with its integrated data. Being already identified twice outlier on 'time to first peak of h.r.r' and twice as well on 'h.r.r peak', these bad performances seem, at least, to make sense. In the case of laboratory $\mathrm{H}$, three times outlier on that 
parameter but never on parameters which could be related to total heat release, the results are quite puzzling. For both laboratories, the explanation of such discordant data should be inquired.

If the 'calculated' points do not seem to follow with a good agreement the proposed regression lines, this is certainly due to the VCSP and GB specimens. Both of these materials do not include a large proportion of 'combustible' elements as it is proved by their mass loss (just around $5 \%$ for the first one and $15 \%$ for the 'quasi-noncombustible' second one). Their resulting total heat releases are thus very small and involve the curve to pass by the origin.

It is allowed to think that a so low total heat release may give rise to some irrelevancies. GB and VCSP show actually some very large values of repeatability and reproducibility in term of percentage in respect to the mean. Moreover, as we mentioned previously in the case of GB and PVC, the ignition troubles can still be suspected to affect that parameter. Removing these points, (GB and VCSP) the slope coefficients of the fitting equation become very close to the A.S.T.M. results $(\mathrm{b} 3=0.06$ and $\mathrm{B} 3=0.08$ for the present study without $\mathrm{GB}$ and VCSP, versus 0.056 and 0.075 for the A.S.T.M. round robin).

Gathering all data about cumulative heat release, that is to say total heat release until 180 seconds, 300 seconds and over the total buming period, an overall graphic can be drawn in order to see how repeatability and reproducibility are evolving. If the regression coefficients of that line lie in the same range of order than the split data, no featuring evolution has been found.

\section{Effective heat of combustion (Table XI)}

Just three laboratories computed that value. If the VCSP's run is excepted, the values of repeatability and reproducibility seem to be constant around 1.6 and 2 respectively (disregarding the VCSP value), results in good agreement with the I.S.O. inter-laboratory trials in which constant bounds are obtained as well. The 'out of average' statistics of VCSP might be explained by a perturbation due to the steel plate (incombustible and which could act as a heat reflector) included in the samples.

The effective heat of combustion must not vary, theoretically, according to the external heat flux if degradation modes are constant (same energies of activation). For each of studied materials we can presume, according to the literature, that, at $30 \mathrm{~kW} / \mathrm{m}^{2}$ and $50 \mathrm{~kW} / \mathrm{m}^{2}$ levels of irradiance, their respective degradation processes have to be identical. Looking at the means, we can notice that this law is perfectly respected and this is a good indication of the reliability of the computed results, in repeatability conditions at least.

\section{Test end time (Table XII)}

If the repeatability value of that last parameter could be considered as acceptable in the majority of the case, the reproducibility values overtake quite always the mean. It seems that there is no reachable reproducibility value at all for that parameter. The regression lines attempts fail (with some negative coefficients) and an average value of repeatability or reproducibility is, according to the plot, impossible to accept.

Moreover, the results from Laboratories $D$ and $E$ seem definitively suspicious insofar that for all the runs of one same level, a constant value have been found out. Remembering how looks like a Cone Calorimeter run, this behavior is very improbable. Some confusions in the meaning of the ending test time could be suspected, and an accurate definition of the burning period (when the mass loss rate drop below $2.5 \mathrm{~g} / \mathrm{s}$ and the average mass loss rate over the next minute does not exceed $2.5 \mathrm{~g} / \mathrm{s}$ ) should be used and precisely respected. 


\section{Outlying entries distribution}

The distribution of the outlying entries, in others words 'where' are the outliers and stragglers, has been studied in order to focus on the difficulties the participating laboratories encountered. This should bring some keys to understand the major causes of troubles and point out the improvements that should be done.

\section{Distribution according to the type of data}

In table XIII (next page), the distribution of the outliers and stragglers are given according to the type of data. The set of the ten requested parameters of the present round robin could be divided into three main groups : the 'simple', corresponding to a single apparatus, the 'cumulative', or integrated data, and the 'combined', or calculated data. Each main group could then be subdivided according to the dimension of each parameter (time, mass, heat, ....).

The row and the column called ' $N^{\circ}$ of part' indicate the number of manipulations which have been made (row : by laboratory, column : by parameter). The dashes denote that the parameter has not been studied at all by the laboratory. As the number of manipulations constantly varies, the outlying observations 'concentration', or 'ratio', have to be expressed in respect to the total amount of runs (two last rows and columns titled 'Tot rel.'). No distinction has been brought between repeatability outlying entries and reproducibility outlying entries. This point will be discussed later.

The last two rows could be, somehow, considered as a "reliability" ranking of the laboratories. $E, F$ and $G$ seem to give the best result, while laboratory $D$ (with $13.6 \%$ of outliers) stays far away from the average percent of the seven other laboratories.

We also could notice that laboratory $\mathrm{H}$ would considerably increase its performances by solving its integration troubles ( $3 / 4$ of its outlying entries). Unluckily, no data from its partial cumulative heat release were available. It is thus difficult to assess the cause of such outlying data. These unstudied parameters could have guide us to check if the only integration calculation process could be involved in the underestimates of total heat release, or if some other reasons have to be sought (burning time period definition, h.r.r base line, ...).

B laboratory seems to have difficulties with the time parameters and especially with ignition time. As its heat related variables remain correct, we can assume that it may not just be due to a cone irradiance misadjustment. However, no outlying values in a parameter assessment do not imply a perfect evaluation of that parameter.

The major part of outlying values of laboratory $E$ concern time variables as well. However half of laboratory E's outliers come from 'Ending time', parameter which seems, according to the previous chapter, highly difficult to control. Moreover, there are some reasons to think that Laboratory $E$ may have use an 'unusual' definition of that parameter insofar that for the three replicates of each run, exactly the same value to within a second has been found. These outliers must easily be eliminated using the proper definition of the burning period.

So few parameters have been computed by I and J laboratories that it is quite impossible to analyze further their results, even in percent. Nevertheless their number of outlying values is not negligible and some additional data should be provided to assume their possible causes of occurrence. 


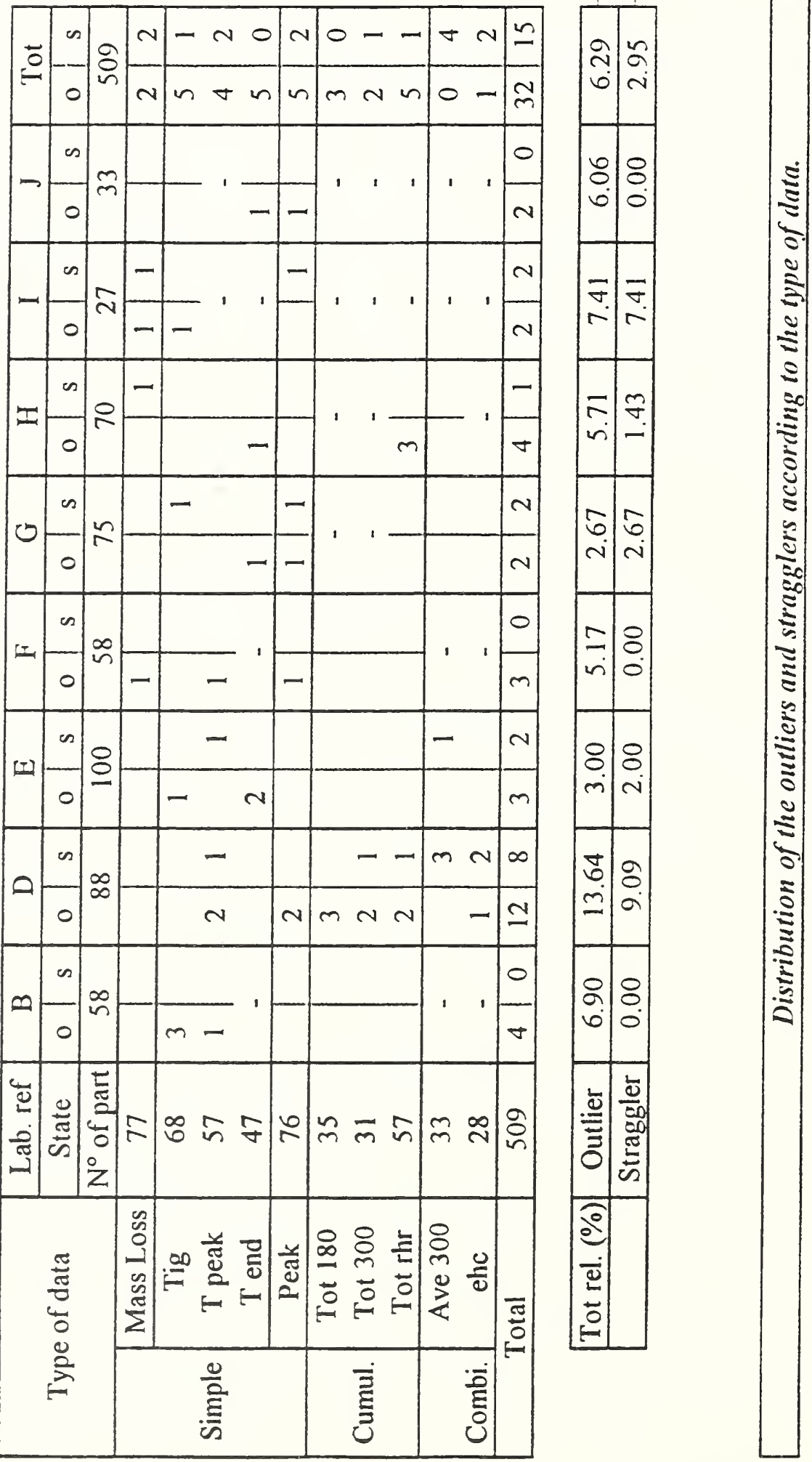


Concerning the two last columns, that is to say the relevancy of each parameter, as it has been guessed, the ending test time shows the worst results. Surprisingly, best percentages are found for mass loss and, to a lower degree, e.h.c. (linked to mass loss) although the regular accuracy of the load cell is around $=1 \%$.

Two of the most important parameters of the Cone Calorimeter, ignition time and especially peak of heat release, lie around $7 \%$ of outlying runs. This figure seems too high, even if the major influence of the numerous outliers from $D$ on h.r.r peak and those from $B$ on ignition time can be felt. However, no outlying variable (a parameter which would implicate a large number of outliers) has be found.

\section{Distribution according to the different levels.}

In table XIV, we tried to check the distribution of outliers and stragglers according to the levels of irradiance. We could suspect that for low heat flux, as ignition may be more delicate, the number of outlying data would increase.

\begin{tabular}{|c|c|c|c|c|c|c|c|c|c|c|c|c|c|c|c|c|c|c|c|c|c|}
\hline \multirow{2}{*}{\multicolumn{2}{|c|}{$\frac{\text { Lab. ref }}{\text { State }}$}} & \multicolumn{2}{|c|}{$B$} & \multicolumn{2}{|c|}{$D$} & \multicolumn{2}{|c|}{$E$} & \multicolumn{2}{|c|}{$\mathrm{F}$} & \multicolumn{2}{|c|}{$\bar{G}$} & \multicolumn{2}{|c|}{$\mathrm{H}$} & \multicolumn{2}{|c|}{$I$} & \multicolumn{2}{|c|}{$\mathrm{J}$} & \multicolumn{2}{|c|}{ Tot } & \multirow{2}{*}{\multicolumn{2}{|c|}{ Tot. rel. }} \\
\hline & & 0 & s & 0 & s & 0 & $\mathrm{~s}$ & 0 & $\mathrm{~s}$ & 0 & $\mathrm{~s}$ & 0 & $\mathrm{~s}$ & 0 & $\mathrm{~s}$ & 0 & $\mathrm{~s}$ & 0 & $\mathrm{~s}$ & & \\
\hline Flux & $N^{\circ}$ par & \multicolumn{2}{|c|}{58} & \multicolumn{2}{|c|}{88} & \multicolumn{2}{|c|}{100} & \multicolumn{2}{|c|}{58} & \multicolumn{2}{|c|}{75} & \multicolumn{2}{|c|}{70} & \multicolumn{2}{|c|}{27} & \multicolumn{2}{|c|}{33} & \multicolumn{2}{|c|}{509} & Out. & Strag. \\
\hline 30 & 242 & & & 5 & 4 & 1 & 1 & 2 & & 1 & 1 & 4 & & 1 & 1 & & & 14 & 7 & 5.79 & 2.89 \\
\hline 50 & 267 & 4 & & 7 & 4 & 2 & 1 & 1 & & 1 & 1 & & 1 & 1 & 1 & 2 & & 18 & 8 & 6.74 & 3.00 \\
\hline Tot & 509 & 4 & 0 & 12 & 8 & 3 & 2 & 3 & 0 & 2 & 2 & 4 & 1 & 2 & 2 & 2 & 0 & 32 & 15 & 6.29 & 2.95 \\
\hline \multicolumn{2}{|c|}{ Outlier } & \multicolumn{2}{|c|}{6.90} & \multicolumn{2}{|c|}{13.64} & \multicolumn{2}{|c|}{3.00} & \multicolumn{2}{|c|}{5.17} & \multicolumn{2}{|c|}{2.67} & \multicolumn{2}{|c|}{5.71} & \multicolumn{2}{|c|}{7.41} & \multicolumn{2}{|c|}{6.06} & \multicolumn{2}{|c|}{6.29} & & \\
\hline \multicolumn{2}{|c|}{ Straggler } & \multicolumn{2}{|c|}{0.00} & \multicolumn{2}{|c|}{9.09} & \multicolumn{2}{|c|}{2.00} & 0.0 & & 2. & & 1.4 & & 7. & & 0.0 & & 2.5 & & & \\
\hline
\end{tabular}

Table XIV. Distribution of outlying values according to the external heat flux irradiance.

It seems to be the case for Laboratory $\mathrm{H}$ ( 4 outliers), but we already know that $3 / 4$ of its outlying data derived from the total heat release parameter and so are unlikely to be due to any ignition troubles. Moreover, the table XIV's two last columns shows an opposite overall behavior. Overall behavior which might lead us to conclude, on the contrary of our first idea, that the higher heat flux, the larger number of outliers.

This assumption could make sense insofar that higher the irradiance, quicker the manipulation, so smaller the time variables are (which represent $1 / 3$ of the studied parameters) and consequently more difficult are their assessments. Laboratory B could be a good example of this presumed phenomenon with all its outliers occurring during the $50 \mathrm{~kW} / \mathrm{m}^{2}$ runs and, as we previously noticed, all for time variables.

However the results expressed in percent just differ from one point and, thus, a statistical distribution between the two levels can be guessed. According to these figures, it appears that external irradiance must not have any effect on the outlying values concentration.

In Table XV (next page), the above table XIV has been detailed by including the different type of specimen. The distribution of the outliers and stragglers is so given according to the products and the irradiance they were submitted to (in other words, the different levels). The same nomenclature with the previous tables has been used. The two last rows are, of course and obviously, exactly identical in the three tables. 
Table XV.

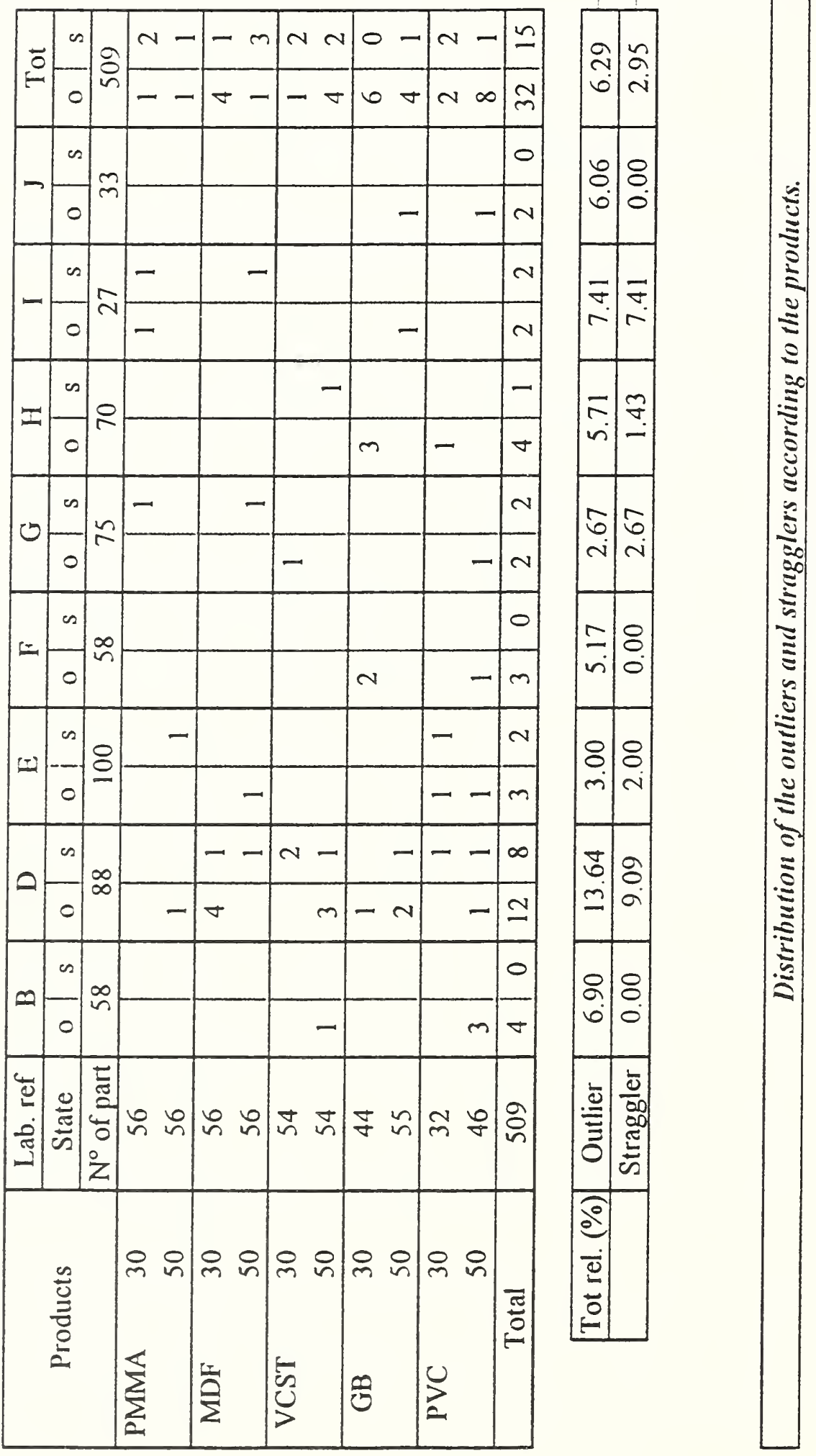


Some precision can be added, now, to the previous comment about H's way to improve its results. They must actually check not only their integration process, but also their gypsum board samples (with still $3 / 4$ of its outliers). This concentration of outlying values could lead us to think that, as all the samples were given to be issued from the same batches, the conditioning operating system of GB samples in laboratory $H$ has to be verified. Previous studies have shown that Cone Calorimeter's answers of materials as GB and MDF could be very sensitive to moisture contents and thus to prior test conditioning.

On the same point of view, laboratory B concentrates its outliers on PVC samples. And that makes sense with the previous remarks concerning its difficulties to assess properly the time variables. We already mentioned that PVC is inclined to give some transient flaming which could perturb the timing evaluation, and especially ignition time.

From the laboratory D columns of results, we can notice that there is no particular product on which outlying observations are focused, meaning that the cause of the D's outliers generation is unlikely to be linked to one or another product. A general inquiry must so be brought more on the experimental device and its whole operating conditions than on any particular result.

Despite what has been said previously about reproducibility values of some PMMA's parameters, PMCMA shows in this table the best overall results and that could somehow justify its designation as 'calibration material'.

On the other hand, GB and, especially PVC take a large part to increase the outlying concentration of these inter-laboratory trials. We have already touched on the reasons for which most of the participants had difficulties to test them. All together, PVC and GB represent about $2 / 3$ of the total amount of outliers found out during this round robin.

At last, on tables XIII and XV, A.S.T.M. Cone Calorimeter inter-laboratory trials overall results about outlying entries concentration have been indicated to allow a comparison. Without analyzing too far the figures (the A.S.T.M. round robin using different parameters and different products), two major points can be seen. The outliers concentration of the present round robin is much more higher than the A.S.T.M. one. But, on the other hand, stragglers percentages are in the opposite balance.

This comparison tends to prove that the results of the present study are much more scattered around the mean values, while A.S.T.M. observations stay centralized around them, as two concentric circles. It is authorized to think that a kind of 'auto-control' as described in the chapter 'Irregularities' of the present report and which have been applied during the A.S.T.M. round robin would painlessly reduce the number of outliers and thus reduce the diameter of the 'scattering circle' to more acceptable one.

However, if we consider laboratory $D$ as an outlying laboratory (causing $2 / 3$ of the amount of outlying values) and, so, if we discard all its data, this percentage jumps from 6.29 to 3.93. Keeping laboratory $\mathrm{D}$, but removing all the PVC runs, the outliers concentration falls to 4.32. In both cases, it is obviously still more than three times the $1.26 \%$ of A.S.T.M., but these calculations show that a lot of improvements could be easily done.

\section{Distribution according repeatability and reproducibility}

No distinction has been brought between repeatability and reproducibility outlying observations since now. This categorization may not be of first general importance insofar that outlying entries have anyhow to be removed from further analyses. On the other hand, it could be useful to examine, according to laboratories, if improvements efforts have to be done just inside 
the laboratory or if the variation of apparatus, operating experiences and so on, weaken the consistency of the results.

\begin{tabular}{|c|c|c|c|c|c|c|c|c|c|c|c|}
\hline \multicolumn{2}{|c|}{ Lab. ref. } & B & $\mathrm{D}$ & $E$ & $F$ & G & $\mathrm{H}$ & I & J & Tot. & partial total \\
\hline \multirow[t]{2}{*}{ Reprod. } & outlier & 1 & 0 & 0 & 1 & 1 & 1 & 0 & 0 & 4 & $12.50 \%$ of outliers \\
\hline & straggler & 0 & 0 & 0 & 0 & 1 & 1 & 1 & 0 & 3 & $20.00 \%$ of stragglers \\
\hline \multirow[t]{2}{*}{ repeat. } & outlier & 3 & 12 & 3 & 2 & 1 & 3 & 2 & 2 & 28 & $87.50 \%$ of outliers \\
\hline & straggler & 0 & 8 & 2 & 0 & 1 & 0 & 1 & 0 & 12 & $80.00 \%$ of stragglers \\
\hline \multicolumn{2}{|c|}{ Total Reprod. } & 1 & 0 & 0 & 1 & 2 & 2 & 1 & 0 & 7 & \\
\hline \multicolumn{2}{|c|}{ Total repeat. } & 3 & 20 & 5 & 2 & 2 & 3 & 3 & 2 & 40 & \\
\hline
\end{tabular}

As shown in the above Table XVI, the great majority of the outlying observation are due to a lack of repeatability, meaning agreement inside one laboratory (repeatability condition). The case of Laboratory D is exemplary, all its 20 outlying data holding in repeatability and confirm our previous recommendation to check their Cone Calorimeter apparatus and their operating conditions.

On that point, we are on total agreement with the American round robin during which most of the outlying data were due to repeatability condition as well. So, all the classical advice to improve repeatability of any tests can here be made (cf. Irregularities). But, we have to be aware that improvements of repeatability will not necessarily decrease the reproducibility figures.

\section{Data dispersion}

The four graphs of Figure I are the representations of the ratio between the laboratory average value and the overall mean of several types of parameters regrouped by dimension (for instance, the Time data dispersion graph gathers all the timing data : ignition time, time to first peak of h.r.r, ending test time). The aim of such diagram is to point out the general tendency of any laboratories to under- or over-estimate data. Outlying values have been removed from the data used here.

\section{Time related data}

This graph has been drawn in order to determine and assess the presence of 'rapid' or 'slow' laboratories. The standard deviation observed is mainly due to the ending test time, data included in the calculation except for B, F and I laboratories which did not provide with that parameter. Moreover, the laboratory I representative point must not be directly compared to the others insofar that its average point and standard deviation line represent just the ignition time variable, the only timing parameter he provided.

According to the diagram, Laboratory $F$ seems to be a 'slow' laboratory (standard deviation line does not cross the $100 \%$ line). On the opposite, even if its standard deviation interval crosses the average line, Laboratory $G$ seems to be a quite 'rapid' laboratory. The operator's ability to detect instantaneously the moment of ignition may not be involved in such 'rapid/slow' categorization, but, as we previously mentioned, it is likely to be just a question of cone irradiance adjustment. Without paying attention to outlying values, a more accurate calibration of the heat flux could be necessary in order, for $F$ and $G$ laboratories, to reduce their 
deviation tendency. As we previously mentioned, moisture contents and thus samples conditioning may affect ignition time assessment as well, especially in the case of MDF and GB specimens. It is why, along with the heat flux calibration, conditioning system has to be checked as another possible cause of discrepancy.

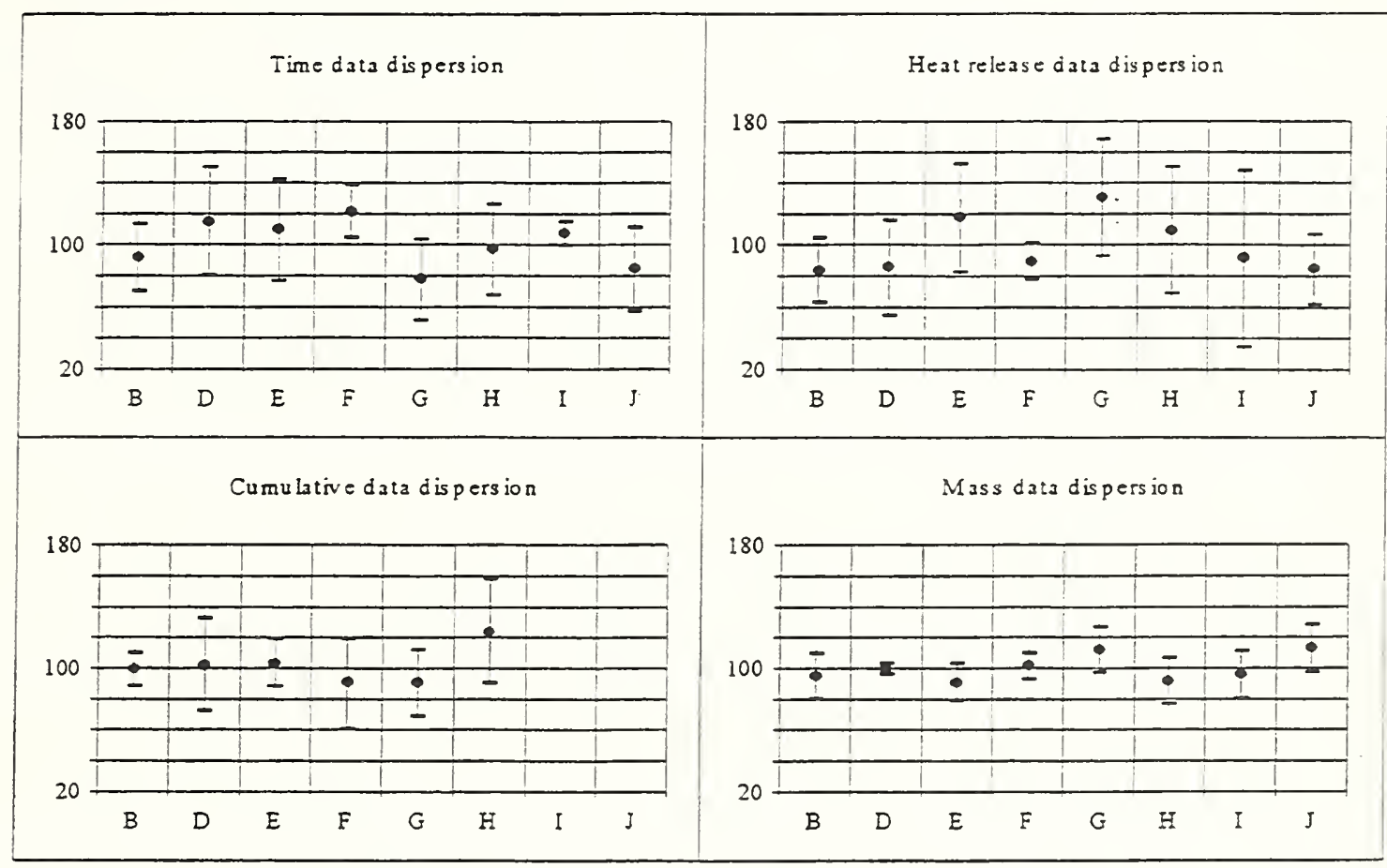

Figure I. Overall deviation to the general mean.

\section{h.r.r data}

All the standard deviation intervals are crossing the $100 \%$ line, but, however, G laboratory seems to overvalue heat release in average and $F$ underevaluate it. If we compare these two last results with the two previous one concerning the time variables, we notice that they are going in opposite sense (underevaluation of time/overevaluation of h.r.r and vice-versa). Laboratories $F$ and $G$ are not the only laboratories in such configuration. Five out of the eight participating laboratories find their results in a 'symmetrical' location about the $100 \%$ line.

That remark could actually confirm our hypothesis about the cone heater calibration effect on the time variable and, in addition on heat release. In the range of studied irradiance levels, if the heat flux is set higher than prescribed, it will obviously decrease the ignition time (1/Tig ${ }^{1 / 2}$ prop. to extemal heat flux) and increase the h.r.r peak value (materials answer to the thermal stress). And an overevaluation of the heater calibration will increase ignition time (and definitively other related time variables) and decrease the first maximum h.r.r values. That 'symmetrical' behavior thus makes sense and points out the major role of the thermal calibration for the consistency of Cone Calorimeter data. Some almost analogous reasoning could be drawn for conditioning system variation, insofar that moisture contents' vaporization could be considered to act as a "heat remover", but this effect could occur only for few of the chosen specimens.

In the case of $B$ and $E$ laboratories, for which the respective representative plots of time data and heat release data are on the same side of the $100 \%$ line, additional hypotheses have to be sought. Nevertheless, even if outlying data were not taken into account in plotting the figure I, we have to remember that these two laboratories encounter the main part of their difficulties in assessing the time variables ( $c f$. Table XIII).

Another important remark which has to be done on that diagram is the huge interval line of I laboratory which indicates a wide sharing out of its h.r.r results. It has to be explained, at least, and consequently to be reduced. A so large standard deviation interval is hardly acceptable, h.r.r being one of the most important data being searched by using Cone Calorimeter. 


\section{integrated data}

This graph gathers all data concerning total heat release (total heat release up to $180 \mathrm{~s}, 300 \mathrm{~s}$ and total heat release). Except for $\mathrm{H}$ laboratory, for which remarks about its integrated data have already been made, the concentration of the results around the $100 \%$ line is better than in the two previous diagrams. It can be guessed that the fact to integrate data fades somehow their disparity and balances their average values. No clear relation between the cumulative data dispersion graph and the time and heat release data dispersion graphs have been found.

\section{Load cell data}

The diagram just confirms our previous conclusions about the load cell data which appears as one of the most reproducible and repeatable variable among the set of parameters which have been requested.

\section{Effect of the equipment}

The laboratories participating in this round robin have used different styles of Cone Calorimeters. Some characteristics of the apparatuses which have been used are sum up in table XVII.

\begin{tabular}{|c|c|c|c|c|c|c|c|c|c|}
\hline \multicolumn{2}{|c|}{ Laboratory ref. } & $\mathrm{B}$ & $D$ & $E$ & $E$ & $G$ & $\mathrm{H}$ & $I$ & $\mathrm{~J}$ \\
\hline \multirow{2}{*}{$\begin{array}{c}\text { Cone } \\
\text { environment }\end{array}$} & wall & 1 wall & 1 wall & \multirow{2}{*}{$\begin{array}{l}4 \text { open } \\
\text { sides }\end{array}$} & 1 wall & & 1 wall & 1 wall & 1 wall \\
\hline & glasses & 3 & 3 & & 3 & & 3 & $3 s$ & free \\
\hline \multirow{3}{*}{$\mathrm{O}_{2}$ Analyzer } & range & $0-25$ & $0-21$ & $0-21$ & $0-25$ & & $0-25$ & $0-25$ & \\
\hline & delay time & 35 & 30 & 15 & 54 & & 40 & 18 & \\
\hline & Initial & & & & * & & * & $* *$ & \\
\hline Heat gage & & A & $\mathrm{A}$ & A & A & & $\mathrm{A}$ & B & \\
\hline Remarks & & & & & $\#$ & & $\#$ & $\#$ & \\
\hline & \multicolumn{9}{|c|}{$\begin{array}{l}\text { *: Average for } 60 \text { seconds before the start of measurement. } \\
* * \text { : Oxygen concentration assumed as } 20.95 \% \text {. } \\
\# \text { : Own software. } \\
\# \text { : Software computed by the manufacturer, equations unkr }\end{array}$} \\
\hline
\end{tabular}

Table XVII. Some characteristics of the laboratories' Cone Calorimeter.

Despite the different attempts made to link any of these characteristics to the outlying concentration or the deviation tendency of one or the other laboratories, no clear relations have been found out. However, we should notice that this absence of simple relation does not imply that there are no possible links between apparatuses' specifications and inaccuracy or potential systematic deviation as, as a matter of fact, the number of tests points we computed was quite restricted. The only thing which could be noticed, but just as a remark, is that F laboratory which has got the larger oxygen analyzer delay time ( 54 seconds) has also the bigger time data deviation (around 120\% of the overall general mean). Nevertheless this fact does not represent any law neither tendency. 


\section{Comparison}

\section{Inter-laboratory Trials on I.S.O. 5657 Ignitability test.}

A large number of inter-laboratory trials have been conducted on various fire tests Unluckily, for most of them, data will be difficult to compare since different analysis methods have been employed. However, the I.S.O. Ignitability of building products, I.S.O. 5657, is an example that is directly comparable. Moreover, this test is especially interesting to compare since it uses a conical heater somewhat similar to the one used on Cone Calorimeter, and the I.S.O. 5657 round robin has been proceeded by the same I.S.O. 5725 data analysis method.

\section{Time to ignition}

Since that test is only a test for ignitability, only one variable is examined, the ignition time. As the same materials were used in both round robins, a five points comparison can be made. Unluckily, among the two levels of irradiance which were used, just one was common.

\begin{tabular}{|c|c|c|c|c|c|c|c|c|c|c|}
\cline { 2 - 10 } \multicolumn{1}{c|}{} & \multicolumn{2}{c|}{ PMMA } & \multicolumn{2}{c|}{ MDF } & \multicolumn{2}{c|}{ VCSP } & \multicolumn{2}{c|}{ GB } & \multicolumn{2}{c|}{ PVC } \\
\cline { 2 - 10 } \multicolumn{1}{c|}{} & Igni. & Cone & Igni. & Cone & Igni. & Cone & Igni. & Cone & Igni. & Cone \\
\hline N $^{\circ}$ labs & 5 & 7 & 5 & 8 & 7 & 8 & 6 & 4 & 7 & 2 \\
\hline m & 78.50 & 72.87 & 85.69 & 82.18 & 29.67 & 23.9 & 127.95 & 100.7 & 138.354 & - \\
\hline I & 7.18 & 10.7 & 10.34 & 8.95 & 6.19 & 4.94 & 15.71 & 13.81 & 50.6483 & - \\
\hline R & 19.12 & 32.41 & 18.20 & 42.14 & 8.07 & 7.51 & 33.16 & 36.84 & 74.7523 & - \\
\hline
\end{tabular}

Table XVIII. Mean and related data of Ignition time in both I.S.O. 5657 and I.S.O. 5660.

The above table XVIII compares the ignition time obtained during the I.S.O. 5657 round robin of 1994-95 and the present Cone Calorimeter inter-laboratory trials, under $30 \mathrm{~kW} / \mathrm{m}^{2}$. For the three first products (PMMA, MDF, VCSP) a very good correlation is shown in terms of mean and repeatability. We can notice that average ignition times obtained from Cone Calorimeter seem always be a little bit lower than the one obtained by the ignitability test.

Concerning gypsum board, even if the reproducibility intervals overlap one to another, the agreement between the mean value stays poor. This could be explained by the difficulties that $4 / 8$ laboratories encounter to ignite GB during this Cone Calorimeter round robin. The same remark has to be done in the case of PVC. The dashes do not mean no results, but just not enough results to process a statistical analysis.

We could propose, in order to reduce the uncertainty and transient flaming that some materials could give during Cone Calorimeter experiments, to let the spark ignition device during 10 seconds after the first flame outbreak in order to ensure ignition.

\section{Repeatability \& reproducibility}

As the results of the I.S.O. 5657 trials were analyzed according to the same model for error as used to analyze the Cone Calorimeter data, the same kind of relation between $r$ (or $R$ ) and $m$ have been established in both round robins (figure $I$ ), and thus can be compared.

It can be seen that if the repeatability curves are quite close, the reproducibility for Ignitability test is substantially better (smaller) than for the Cone Calorimeter. 


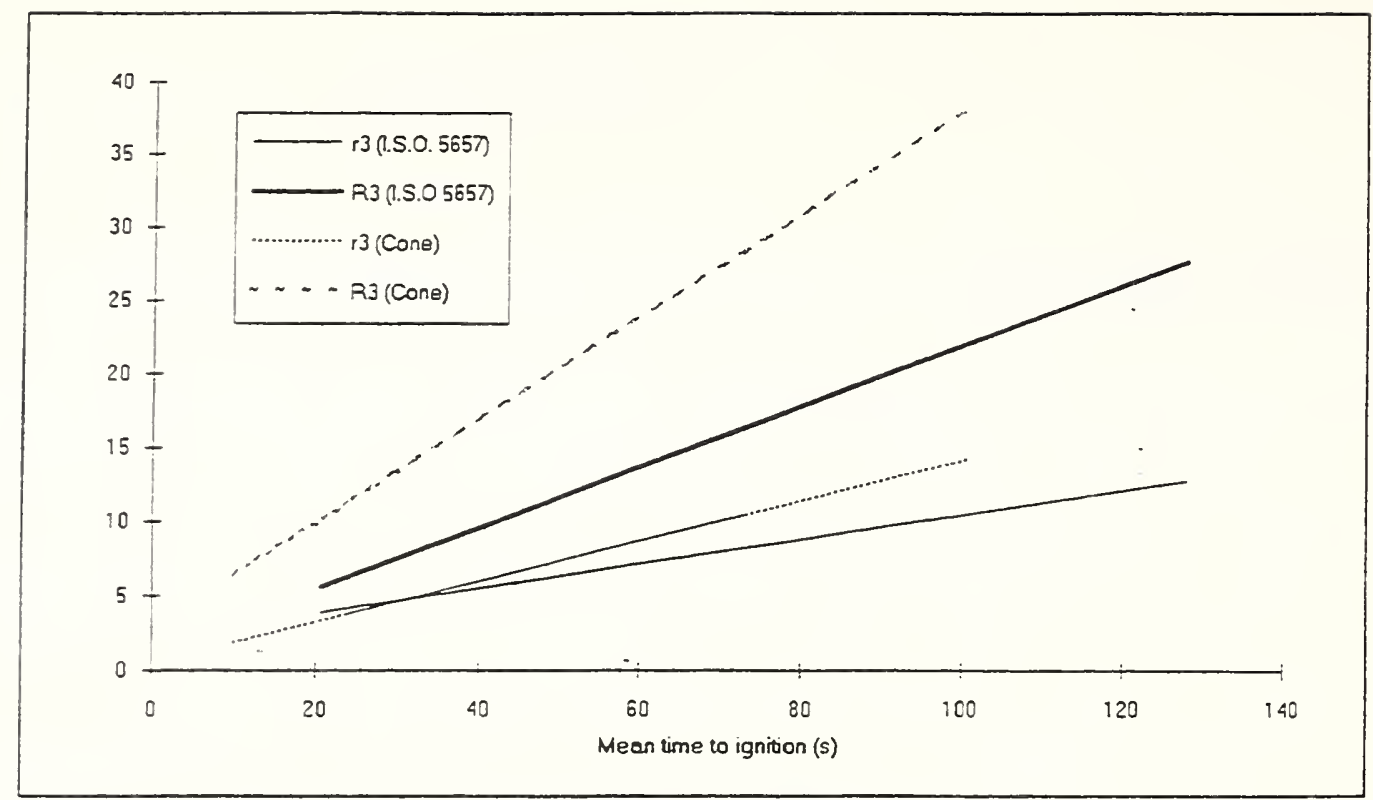

Figure II. Cone Calorimeter \& Ignitability test results.

\section{Inter-laboratory Trials on Cone Calorimeter from A.S.T.M. \& I.S.O.}

Along with 'other' fire tests, it seems logical to compare our results with some very similar inter-laboratory trials which have been published. As mentioned previously, two Cone Calorimeter round robins have already been conducted, both under the auspices of I.S.O, one directly by I.S.O. and the second one by A.S.T.M.. Since the protocol being tested was quite the same, the data should be comparable.

\section{Common materials}

The Table XIX provides a comparison of some parameters of particle board and PMMA in the A.S.T.M. and present round robins. Unluckily, MDF is the only material tested in both inter-laboratory trials with some similar characteristics (material, thickness and density). In the case of PMMA, the respective samples did not have the same thickness neither the same color, nevertheless some parameters which are given to be unaffected by thickness could be compared.

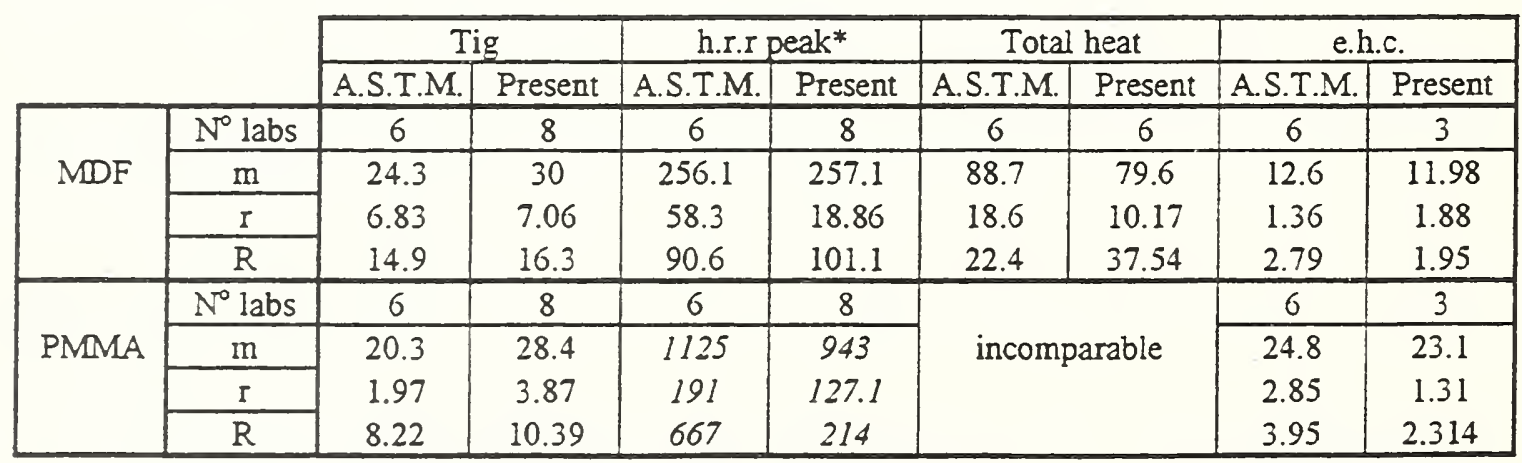

Table XIX. Comparison of some variables in A.S.T.M. and the present round robin.

The chosen comparative parameters are the only common in both round robins. The star on the head of the h.r.r peak column means that this variable is actually not defined on the same way in both trials. A.S.T.M. prescribed to report the maximum h.r.r peak and the present study 
asked for the first h.r.r peak. But, as a matter of fact, for these two materials, the shape of the heat release curves makes the definition similar and thus comparable.

Even if the particle boards do not have the same origin neither the same batch, a very good agreement is observed for each common parameter which has been studied. The agreement does not only concern the mean values, but also repeatability and reproducibility.

For PMMA, the comparison is more delicate as samples did not have the same size, which, for instance, forbids the comparison of total heat release, and may be prejudicial for the h.r.r peak variable. However, ignition time and effective heat of combustion should not be affected. If e.h.c. parameters are in good agreement, the reproducibility interval of ignition time overlaps at the last end. A better correlation could have been expected even if the difference of color may disturb the comparison.

\section{Repeatability \& reproducibility}

A direct comparison with the ISO round robin on materials runs, as previously made with the A.S.T.M. inter-laboratory trials, was impossible since the only available data were just given on repeatability and reproducibility fitting equations terms.

As for all the round robins which have been taken into reference in this report, the two antecedent Cone Calorimeter trials were analyzed according to the same I.S.O. 5725 model for error, and thus can be compared.

\begin{tabular}{|c|c|c|c|c|c|c|c|c|c|}
\hline & \multicolumn{3}{|c|}{ Tig } & \multicolumn{3}{|c|}{ Total heat } & \multicolumn{3}{|c|}{ e.h.c. } \\
\hline & A.S.T.M. & I.S.O. & Present & A.S.T.M. & I.S.O. & Present & A.S.T.M. & I.S.O. & Present \\
\hline 23 & 4.5 & 2.6 & 0.53 & 11 & 3.9 & 0.7 & 1.02 & $2 *$ & $1.6^{*}$ \\
\hline b3 & 0.158 & 0.102 & 0.14 & 0.056 & 0.07 & 0.07 & 0.064 & & \\
\hline $\mathrm{A3}$ & 7.0 & 7.4 & 2.88 & 16.3 & 7.4 & 3.5 & 2.06 & $3.4^{*}$ & $2.0^{*}$ \\
\hline $\mathrm{B} 3$ & 0.247 & 0.196 & 0.35 & 0.075 & 0.092 & 0.20 & 0.07 & & \\
\hline
\end{tabular}

The table above allows a comparison between the different coefficients of the $r$ (or $R$ ) and $\mathrm{m}$ regressions in the 3 round robins. The h.r.r peak coefficients are not compared here since the definition may not be always comparable (according to the product and their heat release curves).

If the values of the intercept ( 23 or $A 3$ ) do not match very well, most of time, the slope (b3 or B3) lies in the same range of order, though always slightly higher in the present trials. The slope coefficients of the total heat release parameter (in italic) computed during this round robin could appear as an exception to the above remark, but as we mentioned in the 'comments' chapter, GB and VCSP are suspected to distort these figures ( $c f$. Comments. Total heat release rate) and removing these data, the slope coefficients of the fitting equation become very close to the A.S.T.M. and I.S.O. results ( $\mathrm{b} 3=0.06$ and $\mathrm{B} 3=0.08$ ), and thus confirm our assumptions about the potential irrelevancies generate by products which produce very low total heat release.

The stars denote the fact that for the e.h.c criterion, the best estimates fitting equations which have been chosen during I.S.O. trials as well as during the present one, are a horizontal line (constant values of repeatability and reproducibility), independent of the mean. We gave here as figures for the present round robin, the values of repeatability and reproducibility computed without the VCSP specimen ( $c f$. Comments. Effective Heat of Combustion). By mean of that calculation, results of the present trials are altogether comparable, and even better than the I.S.O.'s one. 


\section{Conclusions}

Among the purposes of these first Asia-Oceania I.S.O. 5660 Cone Calorimeter interlaboratory trials data analyses, one of the major intention was to underline the delicate points and potential pitfalls of the test procedure that participating laboratories met or could meet. The data processing was instructive from several points of view and enables us to provide some recommendations in order to improve accuracy, reliability and consistency of Cone Calorimeter test operating protocol and consequently of computed data. Without entering any audit of particular laboratory case, three main general conclusions can be derived from this report.

First of all, the relative concentration of outliers needs definitely to be reduced. Even when laboratories which could be suspected to take a large part in that value are removed, the concentration of outlying entries stays very high, especially in repeatability conditions. A general attention must be paid on that problem. A kind of 'auto-control', or more precisely the establishment of a criterion according to which additional testing is required should be brought.

We can propose, as a simple auto-regulation, that if any of the mean heat release values for the first $60,180,300$ seconds after ignition, among the three replicates of one material at one set of conditions, differs by more than $10 \%$ from the average for the three replicates, an additional series of three tests has to be executed. That procedure must remove rapidly and easily, most of the numerous outliers we found out.

Secondly, it is guessed that some misreading or misunderstanding of parameters' definition could be the cause of defective results. Main troubles were found for time parameters, and mostly for ignition time and test ending time. The definition of 'ignition time' is from great importance since most of the calculations, inside Cone Calorimeter applications and outside, take it in reference. As it is now common to say, the term of 'ignition' should be understood, for cone purpose, as 'sustained flaming'. Concerning test end time, the definition related to the mass loss rate as given in the chapter 'Nomenclature' must be the more appropriate to ensure data consistency.

And thirdly, the tremendous impact of a right and accurate calibration of the irradiance level has been pointed out throughout the report, especially in reproducibility conditions and for data deviation tendency. All the governing factors leading to calibrate or to set the external heat flux, from the temperature controller to the heat flux gage, should be carefully checked and harmonized. Moreover, as it has been prescribed during the ASTM round robin, the need for a reference heat flux meter, distinct from the one used for daily calibrations seems obvious.

It is thought that a specific attention must be paid on the calibration factor assessment (C) and on oxygen analyzer response time, by means of methane purity specification, flow meters accuracy, drying agent harmonization, and so on.

Finally, the aim to reach, now, is to encourage the laboratories to increase their general reliability and to improve their methodology in order to prepare a second round robin. As we previously mentioned, it can be guessed that with few efforts great improvement can be expected. 


\section{References}

- Precision of test methods - Determination of repeatability and reproducibility for a standard test method by inter-laboratory test, I.S.O. 5725. International Organization for Standardization, Geneva, 1986.

- A.S.T.M. Task Group E5.21, T.G. 60, "Report to A.S.T.M. on Cone Calorimeter Interlaboratory Trials.", January, 1990.

- Fire tests - Reaction to Fire - Ignitability of building products, International Standard I.S.O. 5657, International Organization for Standardization, Geneva, 1986.

- Proposed Test Method for Heat and Visible Smoke Release Rates for Materials and Products using an Oxygen Consumption Calorimeter, E-5 Proposal P 190, 1986 Annual Book of A.S.T.M. Standards, Vol. 04.07, pp. 1221-1237, 1986.

- Babrauskas V., Grayson S.J., "Heat Release in Fires", Elsevier Science Publishers Ltd, Barking, England, 1992.

- Asia-Oceania I.S.O. 5657 - Ignitability of building products Test - Inter-laboratory Trials Report to Building Research Institute, Building Research Institute, Tsukuba, Japan, 1995.

- Fire tests - Reaction to Fire - Rate of Heat Release from building products, Draft International Standard I.S.O. 5660, International Organization for Standardization, Geneva 1991. 


\section{Annex}

\section{Statistical Analysis Results}

Tables III to XII

Repeatability \& Reproducibility Raw Results. 


\section{Mass loss (Mass initial-Mass final)}

\begin{tabular}{|c|c|c|c|c|c|c|c|c|c|c|}
\hline Products & \multicolumn{2}{|c|}{ PMMA } & \multicolumn{2}{c|}{ MDF } & \multicolumn{2}{c|}{ VCST } & \multicolumn{2}{c|}{ GB } & \multicolumn{3}{c|}{ PVC } \\
\hline Flux $\left(\mathrm{kW} / \mathrm{m}^{2}\right)$ & 30 & 50 & 30 & 50 & 30 & 50 & 30 & 50 & 30 & 50 \\
\hline Number of labs. & 0 & 0 & 8 & 8 & 8 & 8 & 8 & 7 & 6 & 6 \\
Notes. & $\mathrm{a}$ & $\mathrm{a}$ & - & $\mathrm{b}$ & - & $\mathrm{c}$ & $-*$ & $\mathrm{~d}$ & $\mathrm{e}^{*}$ & $\mathrm{f}^{*}$ \\
\hline mean (m) & - & - & 0.205 & 0.174 & 0.953 & 0.949 & 0.840 & 0.837 & 0.233 & 0.106 \\
repeatability (r) & - & - & 0.038 & 0.046 & 0.008 & 0.020 & 0.027 & 0.008 & 0.066 & 0.038 \\
Reproducibility (R) & - & - & 0.116 & 0.134 & 0.028 & 0.069 & 0.125 & 0.106 & 0.156 & 0.175 \\
\hline
\end{tabular}

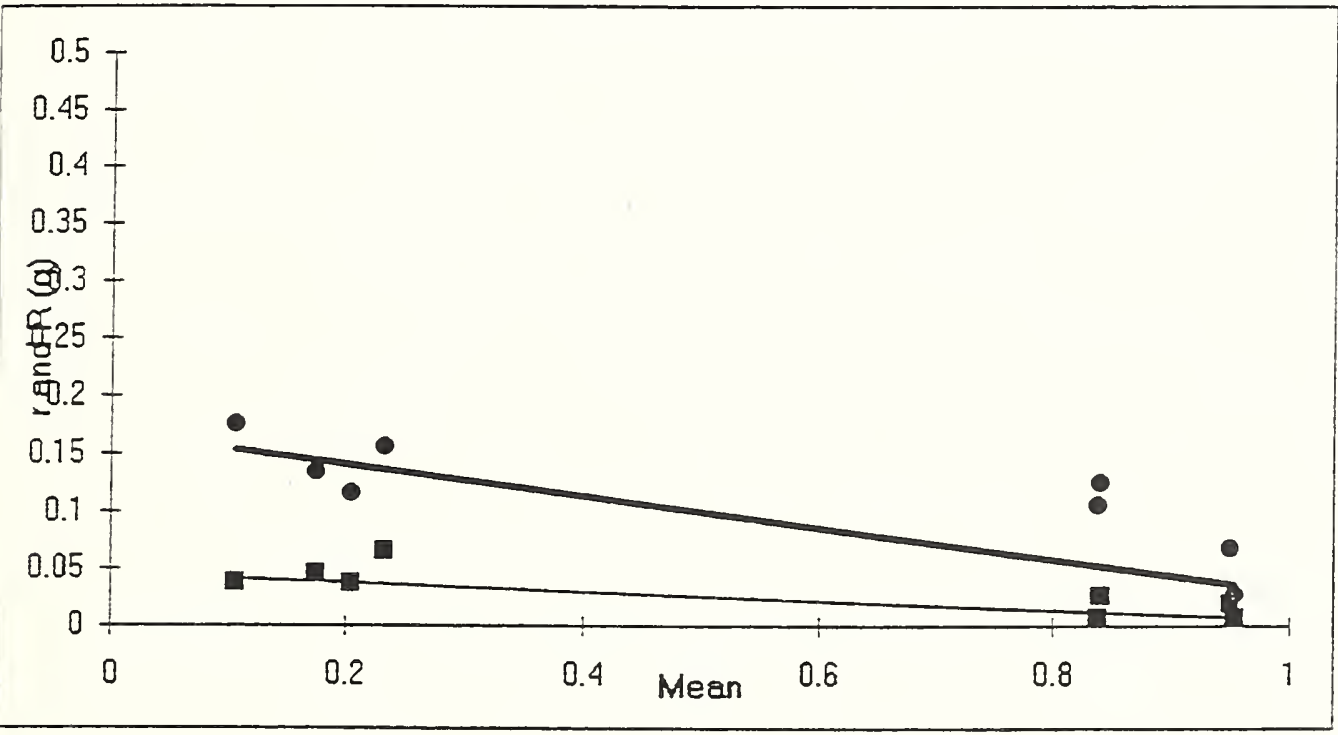

\begin{tabular}{|lr|}
\hline Fitt \\
\hline Ave. & $\mathbf{r}=0.03$ \\
$\mathbf{R}$ & $=0.11$ \\
\hline Repeat. & $\mathrm{a} 3=0.05$ \\
& $\mathrm{~b} 3=-0.04$ \\
Reprod. & $\mathrm{A} 3=0.17$ \\
& $\mathrm{~B} 3=-0.14$ \\
\hline
\end{tabular}

\begin{tabular}{|c|cccccccc|}
\hline Lab. & B & D & E & F & G & H & I & J \\
\hline V.C. & 1.4391 & 0.5656 & 1.0294 & 1.4692 & 1.2832 & 1.4456 & 3.59 & 2.60 \\
\hline
\end{tabular}

Notes.

* These figures must be handled with precaution insofar that for 4 of the 9 laboratories no ignition was reported, but a mass loss was recorded.

a : PMMA being totally burned out, there is no mass left at the end of the manipulation.

Results are so uninterpretable and were not taken into account.

b : Laboratory I was identified as straggler (repeatability)

c : Laboratory $\mathrm{H}$ was identified as straggler (reproducibility)

d : Laboratory I was removed as outlier (repeatability)

e : $\quad$ Missing data from Laboratories D \& F (No ignition)

f : $\quad$ Missing data from laboratory D (1 ignition/ 3 trials)

and Laboratory $\mathrm{F}$ was removed as outlier (repeatability) 


\section{Time to Ignition (s)}

\begin{tabular}{|c|c|c|c|c|c|c|c|c|c|c|}
\hline Products & \multicolumn{2}{|c|}{ PMMA } & \multicolumn{2}{c|}{ MDF } & \multicolumn{2}{c|}{ VCST } & \multicolumn{2}{c|}{ GB } & \multicolumn{2}{c|}{ PVC } \\
\hline Flux $\left(\mathrm{kW} / \mathrm{m}^{2}\right)$ & 30 & 50 & 30 & 50 & 30 & 50 & 30 & 50 & 30 & 50 \\
\hline Number of labs. & 7 & 8 & 8 & 8 & 8 & 7 & 4 & .8 & 2 & 5 \\
Notes. & $\mathrm{a}$ & - & - & $\mathrm{b}$ & - & $\mathrm{c}$ & $-*$ & - & $\mathrm{d}^{* *}$ & $\mathrm{e}^{* * *}$ \\
\hline mean (m) & 70.752 & 28.367 & 82.183 & 29.996 & 23.904 & 9.7833 & 100.74 & 32.496 & - & 25.906 \\
repeatability (r) & 6.2918 & 3.875 & 8.9518 & 7.0565 & 4.9361 & 1.5976 & 13.815 & 6.8678 & - & 4.8077 \\
Reproducibility (R) & 28.911 & 10.392 & 42.138 & 16.302 & 7.5079 & 11.883 & 36.841 & 18.686 & - & 39.36 \\
\hline
\end{tabular}

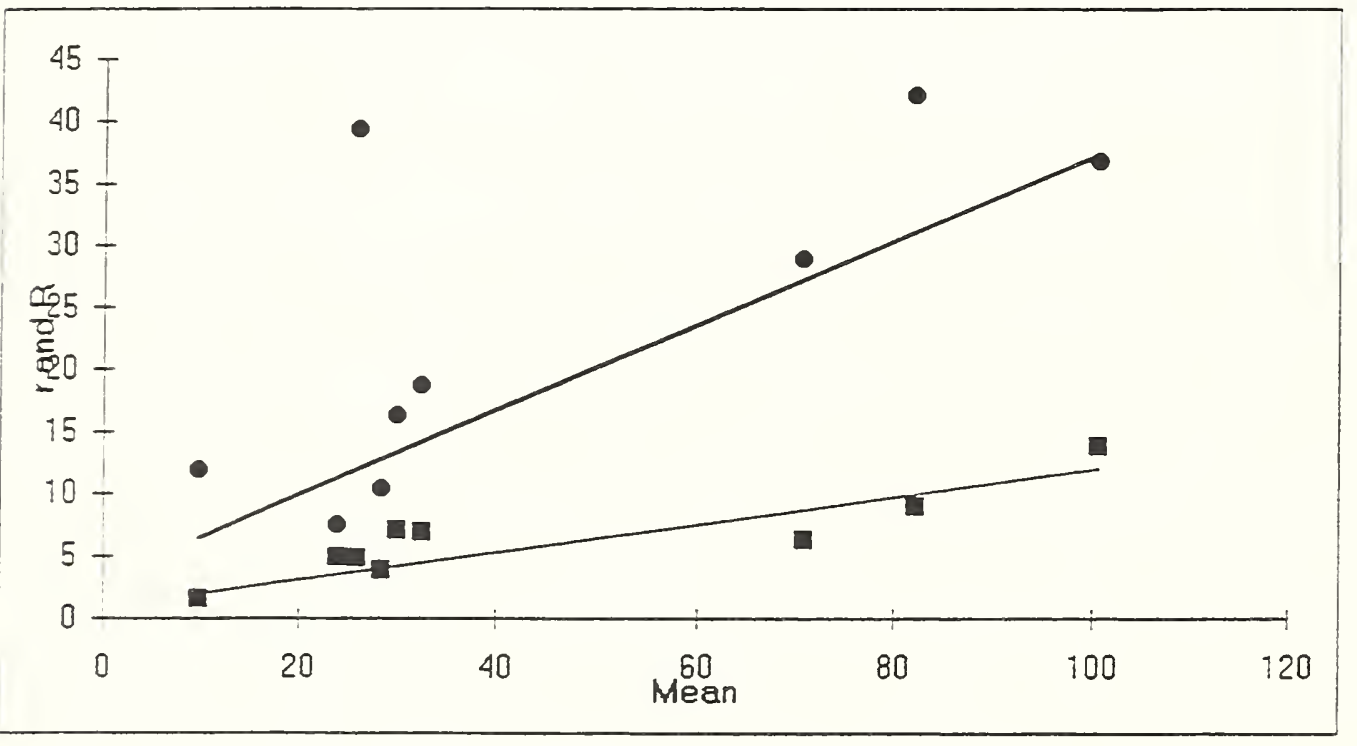

Fitt

\begin{tabular}{|ll|}
\hline Ave. & $\mathrm{r}=6.47$ \\
& $\mathrm{R}=23.56$ \\
\hline Repeat. & $\mathrm{a} 3=0.88$ \\
& $\mathrm{~b} 3=0.11$ \\
Reprod. & $\mathrm{A3}=3.11$ \\
& $\mathrm{~B} 3=0.34$ \\
\hline
\end{tabular}

\begin{tabular}{|r|}
$r$ \\
$R$ \\
$r 3=a 3+b 3 . m$ \\
$R 3=A 3+B 3 . m$ \\
\hline
\end{tabular}

\begin{tabular}{|c|cccccccc|}
\hline Lab. & B & D & E & F & G & H & I & J \\
\hline V.C. & 5.2384 & 5.5399 & 4.5182 & 3.8366 & 4.0303 & 3.9253 & 7.2604 & 4.8478 \\
\hline
\end{tabular}

Notes.

\footnotetext{
* : Missing data from laboratory B, F, I, J (No ignition)

** : Missing data from laboratory B,D, F, I, J (No ignition) : No data enough, results disgarded.

*** : Missing data from laboratory I, J (No ignition)

a : laboratory I was removed as outlier (repeatability)

b : laboratory $G$ was identified as straggler (reproducibility)

$c$ : laboratory B was removed as outlier (repeatability)

$d$ : laboratory $E$ was removed as outlier (repeatability)

e : laboratory B was removed as outlier (repeatability \& reproducibility)
} 


\section{First peak of R.H.R. $\left(\mathrm{kW} / \mathrm{m}^{2}\right)$}

\begin{tabular}{|c|c|c|c|c|c|c|c|c|c|c|}
\hline Products & \multicolumn{2}{|c|}{ PMMA } & \multicolumn{2}{c|}{ MDF } & \multicolumn{2}{c|}{ VCST } & \multicolumn{3}{c|}{ GB } & \multicolumn{2}{c|}{ PVC } \\
\hline Flux (kW/m $\left.{ }^{2}\right)$ & 30 & 50 & 30 & 50 & 30 & 50 & 30 & 50 & 30 & 50 \\
\hline Number of labs. & 8 & 8 & 8 & 8 & 8 & 8 & 5 & 7 & 5 & 6 \\
Notes. & $\mathrm{a}$ & $\mathrm{b}$ & $\mathrm{c}$ & $\mathrm{c}$ & - & - & $\mathrm{d}$ & $\mathrm{e}^{*}$ & $-*$ & $\mathrm{f}$ \\
\hline mean (m) & 685.37 & 942.7 & 199.26 & 257.14 & 139.3 & 153.95 & 53.193 & 90.208 & 76.68 & 54.658 \\
repeatability (r) & 46.485 & 127.08 & 19.37 & 18.856 & 28.06 & 17.458 & 6.6041 & 6.8108 & 44.054 & 17.511 \\
Reproducibility (R) & 224.44 & 214.21 & 74.475 & 101.1 & 97.344 & 135.84 & 128.93 & 1.20 .32 & 206.06 & 137.34 \\
\hline
\end{tabular}

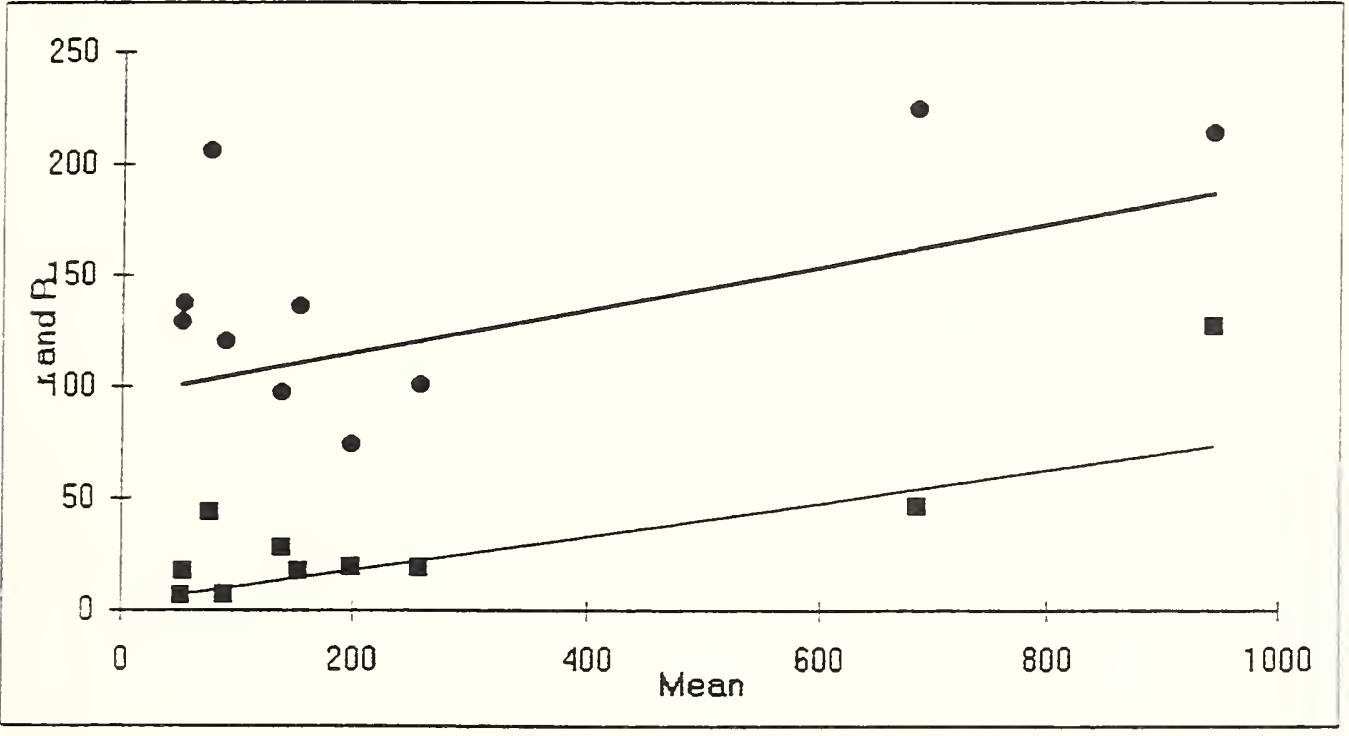

\begin{tabular}{|c|c|c|}
\hline \multicolumn{3}{|c|}{ Fitt } \\
\hline \multirow[t]{2}{*}{ Average } & & 33.2284 \\
\hline & $R=$ & 144.006 \\
\hline \multirow[t]{2}{*}{ Repeat. } & $a 3=$ & 3.07179 \\
\hline & $b 3=$ & 0.07452 \\
\hline \multirow[t]{2}{*}{ Reprod. } & $\mathrm{A} 3=$ & 95.492 \\
\hline & $\mathrm{B} 3=$ & 0.09696 \\
\hline
\end{tabular}

$r$
$R$
$-r 3=a 3+b 3 . m$
$-R 3=A 3+B 3 . m$

\begin{tabular}{|c|cccccccc|}
\hline Lab. & B & D & E & F & G & H & I & J \\
\hline V.C. & 2.6416 & 3.7546 & 5.043 & 3.8814 & 3.475 & 3.6821 & 4.6239 & 1.7617 \\
\hline
\end{tabular}

Notes.

* : Missing data due to non-ignition

a : laboratory $G$ was identified as straggier (repeatability)

b : laboratory I was identified as straggler (repeatability)

$c$ : With corrected data from Laboratory $\mathrm{H}$ (first peak/maximun peak)

d : laboratory $D$ was removed as outlier (repeatability)

laboratory $F$ was removed as outlier (repeatability), data without ignition.

e : laboratory $D$ was removed as outlier (repeatability)

f : laboratory $G$ was removed as outlier (reproducibility), 4 times the averall average! laboratory J was removed as outhier (repeatability), data without ignition 


\section{Time to first peak of R.H.R. (s)}

\begin{tabular}{|c|c|c|c|c|c|c|c|c|c|c|}
\hline Products & \multicolumn{2}{|c|}{ PMMA } & \multicolumn{2}{c|}{ MDF } & \multicolumn{2}{c|}{ VCST } & \multicolumn{3}{c|}{ GB } & \multicolumn{3}{c|}{ PVC } \\
\hline Flux $\left(\mathrm{kW} / \mathrm{m}^{2}\right)$ & 30 & 50 & 30 & 50 & 30 & 50 & 30 & 50 & 30 & 50 \\
\hline Number of labs. & 6 & 5 & 5 & 6 & 6 & 6 & 4 & 6 & 4 & 5 \\
Notes. & $-*$ & $\mathrm{a}^{*}$ & $\mathrm{~b}^{* 1}$ & $-*$ & $-*$ & $-*$ & $\mathrm{c}^{*}$ & $-*$ & $\mathrm{~d}^{*}$ & $\mathrm{e}^{*}$ \\
\hline mean (m) & 510.94 & 383.8 & 69.778 & 47.111 & 27.222 & 19.667 & 92 & 37.389 & 243.1 & 55.059 \\
repeatability (r) & 47.935 & 18.432 & 5.465 & 5.6358 & 4.7024 & 3.6425 & 11.582 & 3.8569 & 46.065 & 6.9459 \\
Reproducibility (R) & 185.14 & 68.349 & 114.73 & 33.206 & 16.176 & 15.386 & 150.64 & 40.76 & 100.1 & 118.41 \\
\hline
\end{tabular}

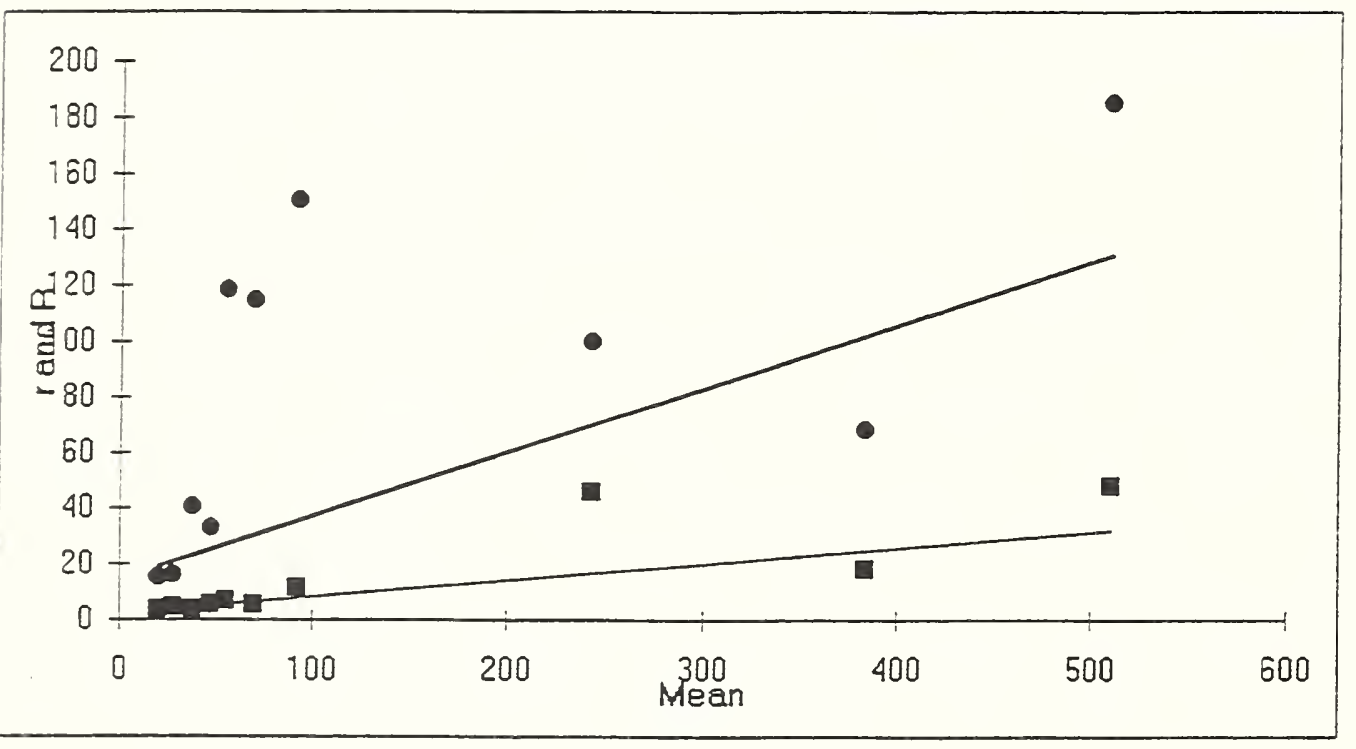

Fitt

\begin{tabular}{|ll|}
\hline Average & $\mathrm{r}=15.4263$ \\
& $\mathrm{R}=84.2908$ \\
\hline Repeat. & $\mathrm{a} 3=2.50116$ \\
& $\mathrm{~b} 3=0.05848$ \\
Reprod. & $\mathrm{A} 3=14.5898$ \\
& $\mathrm{~B} 3=0.22764$ \\
\hline
\end{tabular}

\begin{tabular}{|c|}
$\mathrm{I}$ \\
$\mathrm{R}$ \\
$\mathrm{r} 3=\mathrm{a} 3+\mathrm{b} 3 . \mathrm{m}$ \\
$\mathrm{R} 3=\mathrm{A} 3+\mathrm{B} 3 . \mathrm{m}$ \\
\hline
\end{tabular}

\begin{tabular}{|c|cccccccc|}
\hline Lab. & B & D & E & F & G & H & I & J \\
\hline V.C. & 4.5989 & 5.7729 & 1.4341 & 2.8257 & 1.0866 & 3.7761 & - & - \\
\hline
\end{tabular}

Notes.

* : Missing data from I, J laboratories, no obvious reasons

' : with corrected data from laboratory H (first peak/maximun peak)

a : Laboratory $\mathrm{D}$ was removed as outlier (repeatability)

b : Laboratory $\mathrm{D}$ was removed as outlier (repeatability)

c : Laboratory $F$ was removed as outlier (reproducibility)

d : Laboratories D \& E was identified as straggler (repeatability)

e : Laboratory B was removed as outlier (repeatability) 


\section{Total Heat Release from Tig to $180 \mathrm{~s}\left(\mathrm{MJ} / \mathrm{m}^{2}\right)$}

\begin{tabular}{|c|c|c|c|c|c|c|c|c|c|c|}
\hline Products & \multicolumn{2}{|c|}{ PMMA } & \multicolumn{2}{c|}{ MDF } & \multicolumn{2}{c|}{ VCST } & \multicolumn{3}{c|}{ GB } & \multicolumn{2}{c|}{ PVC } \\
\hline Flux $\left(\mathrm{kW} / \mathrm{m}^{2}\right)$ & 30 & 50 & 30 & 50 & 30 & 50 & 30 & 50 & 30 & 50 \\
\hline Number of labs. & 4 & 4 & 3 & 4 & 4 & 3 & 3 & 3 & 1 & 3 \\
Notes. & $-*$ & $-*$ & $\mathrm{a}^{*}$ & $-*$ & $-*$ & $\mathrm{~b}^{*}$ & $\mathrm{c}^{*}$ & $\mathrm{~d}^{*}$ & $\mathrm{e}^{*}$ & $\mathrm{f}^{*}$ \\
\hline mean (m) & 58.025 & 85.558 & 21.978 & 26.383 & 2.2167 & 2.025 & 1.7 & 2.0917 & - & 10.125 \\
repeatability (r) & 4.2327 & 7.8884 & 1.0896 & 1.6291 & 1.0337 & 0.1771 & 0.9063 & 0.2892 & - & 4.6452 \\
Reproducibility (R) & 18.465 & 26.135 & 6.7917 & 5.5954 & 2.2259 & 3.7901 & 2.4797 & 3.9396 & - & 4.6575 \\
\hline
\end{tabular}

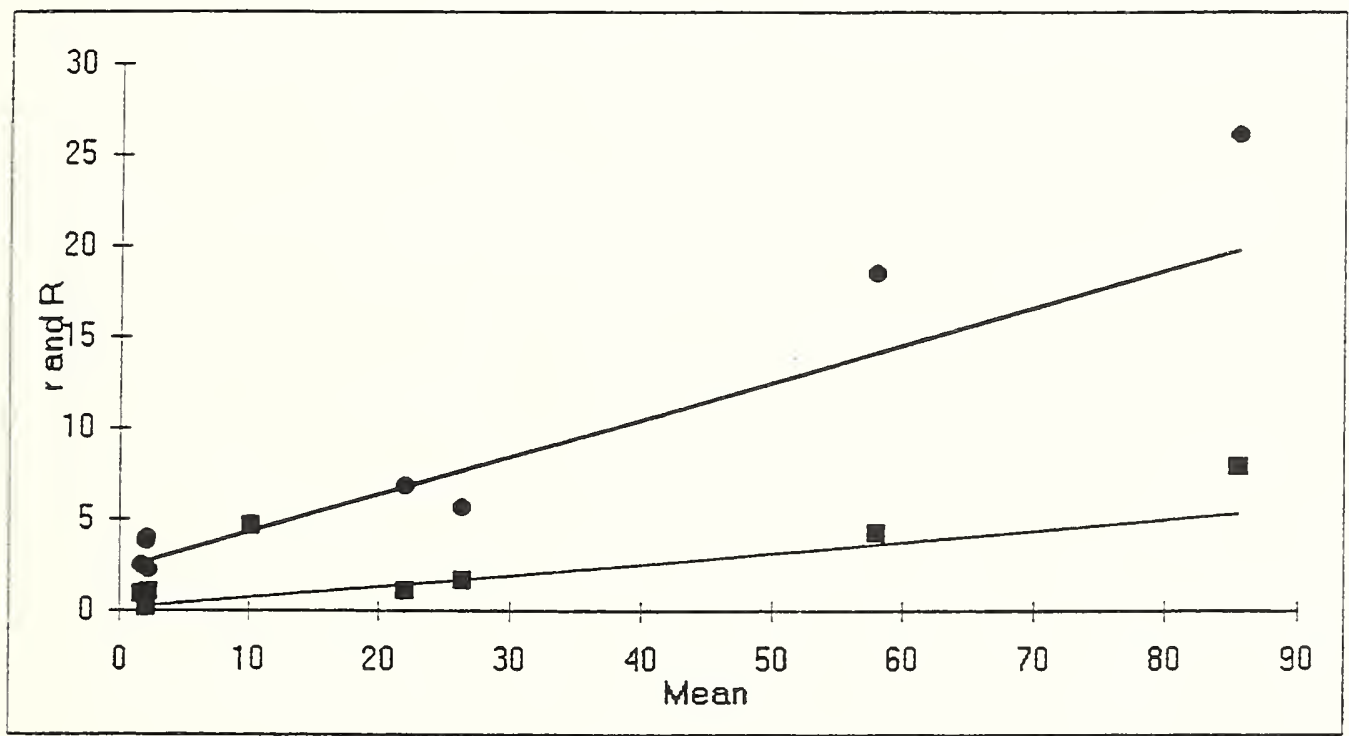

\begin{tabular}{|lrl|}
\hline Fitt \\
\hline Average & $\mathrm{r}=2.43235$ \\
$\mathrm{R}=$ & 8.2311 \\
\hline Repeat. & $\mathrm{a} 3=0.11818$ \\
& $\mathrm{~b} 3=0.06043$ \\
Reprod. & $\mathrm{A} 3=2.28033$ \\
& $\mathrm{~B} 3=0.2042$ \\
\hline
\end{tabular}

$\mathrm{T}$
$R$
$\mathrm{r} 3=\mathrm{a} 3+\mathrm{b} 3 . \mathrm{m}$
$\mathrm{R} 3=\mathrm{A} 3+\mathrm{B} 3 . \mathrm{m}$

\begin{tabular}{|c|cccccccc|}
\hline Lab. & B & D & E & F & G & H & I & J \\
\hline V.C. & 3.9811 & 2.7588 & 4.656 & 1.4213 & - & - & - & - \\
\hline
\end{tabular}

Notes.

* : Missing data from G, H, I, J laboratories, no obvious reasons.

a : Laboratory $\mathrm{D}$ was removed as outlier (repeatability)

b : Laboratory D was removed as outlier (repeatability)

$c$ : Missing data from Laboratory B, no ignition.

d : Laboratory $D$ was removed as outlier (repeatability)

e : Missing data from Laboratories B, C, F, no ignition : data discarded.

f : Missing data from $D$ laboratory 


\section{Average Heat release from Tig to $300 \mathrm{~s}\left(\mathrm{~kW} / \mathrm{m}^{2}\right)$}

\begin{tabular}{|c|c|c|c|c|c|c|c|c|c|c|}
\hline Products & \multicolumn{2}{|c|}{ PMMA } & \multicolumn{2}{c|}{ MDF } & \multicolumn{2}{c|}{ VCST } & \multicolumn{3}{c|}{ GB } & \multicolumn{2}{c|}{ PVC } \\
\hline Flux (kW/m²) & 30 & 50 & 30 & 50 & 30 & 50 & 30 & 50 & 30 & 50 \\
\hline Number of labs. & 4 & 4 & 4 & 4 & 3 & 3 & 3 & 4 & 2 & 2 \\
Notes. & $-*$ & $\mathrm{a}^{*}$ & $\mathrm{~b}^{*}$ & $-*$ & $-*$ & $\mathrm{c}^{*}$ & $-*$ & $-*$ & $\mathrm{~d}^{*}$ & $\mathrm{e}^{*}$ \\
\hline mean (m) & 390.38 & 590.53 & 101.12 & 125.84 & 8.95 & 10.444 & 7.1889 & 9.15 & - & - \\
repeatability (r) & 18.158 & 51.039 & 10.333 & 10.536 & 6.8896 & 5.4712 & 3.4532 & 4.52 & - & - \\
Reproducibility (R) & 138.14 & 221.49 & 24.545 & 31.451 & 12.104 & 6.8765 & 3.5281 & 3.9435 & - & - \\
\hline
\end{tabular}

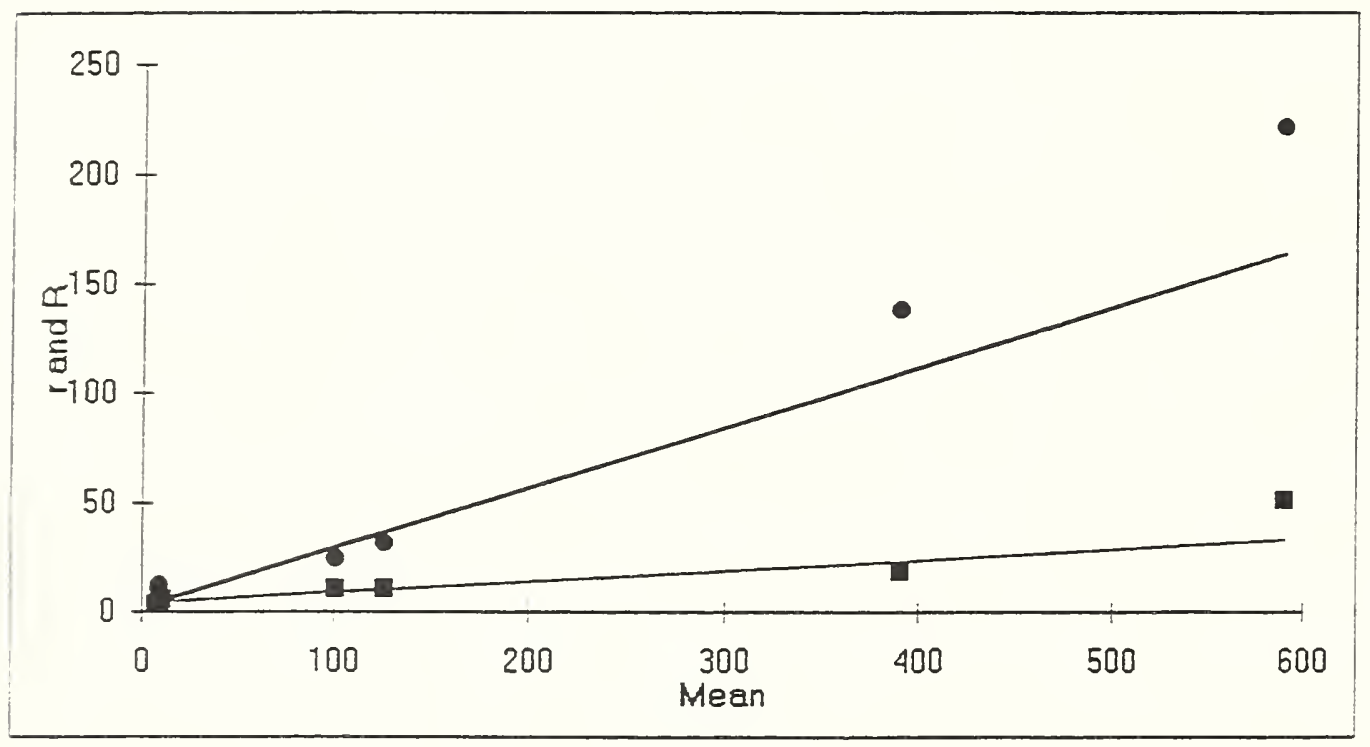

\begin{tabular}{|lrl|}
\hline \multicolumn{1}{l}{ Fitt } \\
\hline Average & $\mathrm{r}=13.7999$ \\
& $\mathrm{R}=55.2589$ \\
\hline Repeat. & $\mathrm{a} 3=4.10373$ \\
& $\mathrm{~b} 3=0.04865$ \\
Reprod. & $\mathrm{A} 3=2.07228$ \\
& $\mathrm{~B} 3=0.27371$ \\
\hline
\end{tabular}

\begin{tabular}{|c|}
$r$ \\
$R$ \\
$r 3=a 3+b 3 . m$ \\
$R 3=A 3+B 3 . m$
\end{tabular}

\begin{tabular}{|c|cccccccc|}
\hline Lab. & B & D & E & F & G & H & I & J \\
\hline V.C. & - & 3.4548 & 4.7063 & - & 1.9628 & 1.582 & - & - \\
\hline
\end{tabular}

Notes.

* : Missing data from B, F, I, J laboratories, no obvious reasons

a : Laboratoy $E$ was identified as straggler (repeatability)

b : Laboratoy D was identified as straggler (repeatability)

c : Laboratoy D was identified as straggler (repeatability)

d : Missing data from D, G laboratories, not enough data to analyse : level discarded.

e : Laboratoy $D$ was identified as straggler (repeatability)

Missing data from D, G laboratories, not enough data to analyse: level discarded. 
Table $X X$

\section{Total Heat Release from Tig to $300 \mathrm{~s}\left(\mathrm{MJ} / \mathrm{m}^{2}\right)$}

\begin{tabular}{|c|c|c|c|c|c|c|c|c|c|c|}
\hline Products & \multicolumn{2}{|c|}{ PMMA } & \multicolumn{2}{c|}{ MDF } & \multicolumn{2}{c|}{ VCST } & \multicolumn{3}{c|}{ GB. } & \multicolumn{2}{c|}{ PVC } \\
\hline Flux $\left(\mathrm{kW} / \mathrm{m}^{2}\right)$ & 30 & 50 & 30 & 50 & 30 & 50 & 30 & 50 & 30 & 50 \\
\hline Number of labs. & 4 & 4 & 3 & 4 & 3 & 2 & 2 & 2 & 1 & 3 \\
Notes. & $-*$ & $-*$ & $\mathrm{a}^{*}$ & $-*$ & $\mathrm{~b}^{*}$ & $\mathrm{c}^{*}$ & $\mathrm{~d}^{*}$ & $\mathrm{e}^{*}$ & $\mathrm{f}^{*}$ & $\mathrm{~g}^{*}$ \\
\hline mean (m) & 113.24 & 169.43 & 29.878 & 36.633 & 2.8111 & - & - & - & - & 12.367 \\
repeatability (r) & 6.3384 & 15.868 & 1.0441 & 2.467 & 1.9206 & - & - & - & - & 4.832 \\
Reproducibility (R) & 28 & 53.213 & 10.424 & 8.007 & 2.8365 & - & - & - & - & 15.469 \\
\hline
\end{tabular}

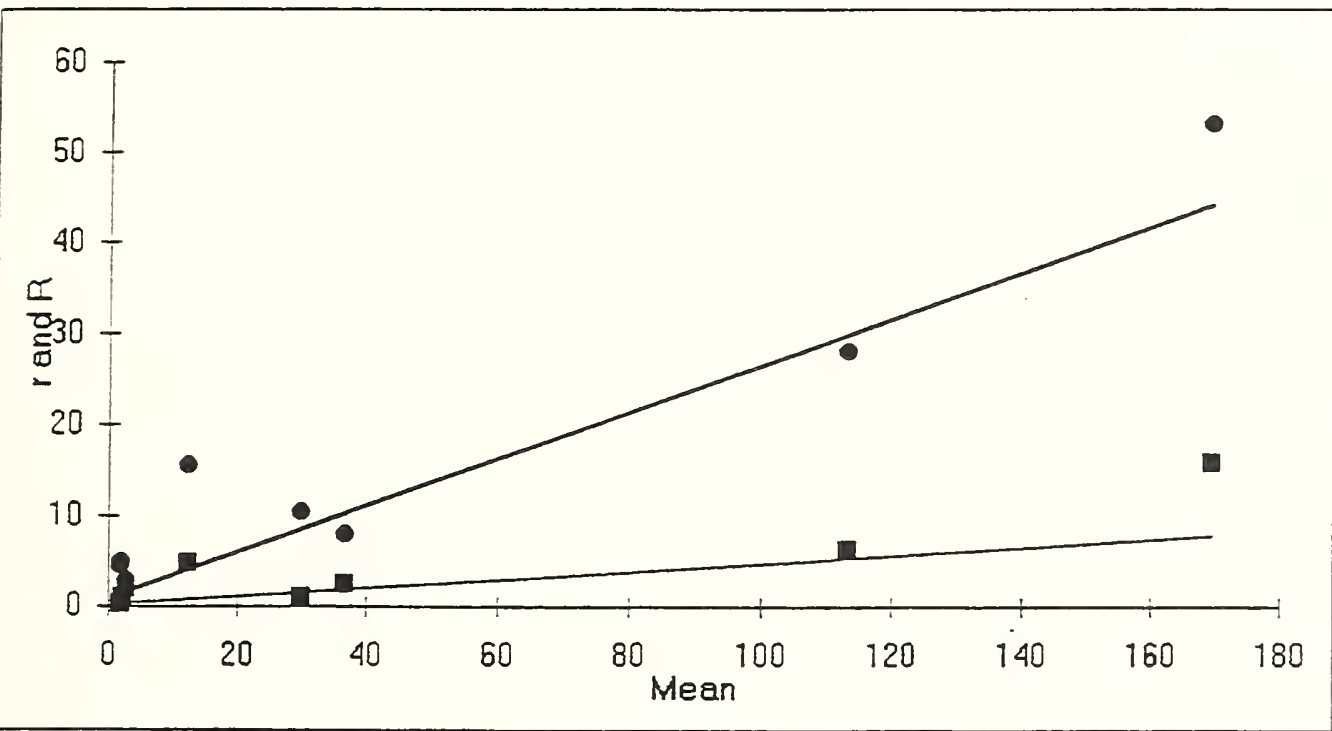

\begin{tabular}{|lrl}
\multicolumn{2}{l}{ Fitt } \\
\hline Average & $\quad \mathrm{I}=5.41161$ \\
$\mathrm{R}=19.6582$ \\
Repeat. & $\mathrm{a} 3=0.23584$ \\
& $\mathrm{~b} 3=0.04511$ \\
Reprod. & $\mathrm{A} 3=0.92367$ \\
& $\mathrm{~B} 3=0.25532$
\end{tabular}

\begin{tabular}{c}
$\quad r$ \\
$R$ \\
$-r 3=a 3+b 3 . m$ \\
\hline$R 3=A 3+B 3 . m$
\end{tabular}

\begin{tabular}{|c|cccccccc|}
\hline Lab. & B & D & E & F & G & H & I & J \\
\hline V.C. & 3.2263 & 2.697 & 4.7079 & 1.0713 & - & - & - & - \\
\hline
\end{tabular}

Notes.

* : Missing data from $G, H, I, J$ laboratories, no obvious reasons

a : Laboratory $D$ was removed as outlier (repeatability)

$\mathrm{b}$ : Missing data from $F$ laboratory

c : Missing data from F laboratory \&

Laboratory D was removed as outlier (repeatability) : no enough data : leveldiscarded.

d : Missing data from F, B laboratories

e : Laboratory $D$ was identified as straggler (repeatability)

$f$ : No data except from Laboratory $E$ : level discarded.

$\mathrm{g}$ : Missing data from $\mathrm{D}$ laboratory 
Table X

\section{Total Heat release $\left(\mathrm{kW} / \mathrm{m}^{2}\right)$}

\begin{tabular}{|c|c|c|c|c|c|c|c|c|c|c|}
\hline Products & \multicolumn{2}{|c|}{ PMMA } & \multicolumn{2}{c|}{ MDF } & \multicolumn{2}{c|}{ VCST } & \multicolumn{3}{c|}{ GB } & \multicolumn{2}{c|}{ PVC } \\
\hline Flux $\left(\mathrm{kW} / \mathrm{m}^{2}\right)$ & 30 & 50 & 30 & 50 & 30 & 50 & 30 & 50 & 30 & 50 \\
\hline Number of labs. & 6 & 6 & 6 & 6 & 6 & 5 & 4 & .6 & 3 & 5 \\
Notes. & $-*$ & $-*$ & $-*$ & $-*$ & $\mathrm{a}^{*}$ & $\mathrm{~b}^{*}$ & $\mathrm{c}^{*}$ & $-*$ & $\mathrm{~d}^{*}$ & $\mathrm{e}^{*}$ \\
\hline mean (m) & 278.28 & 274.94 & 74.756 & 79.606 & 2.8 & 2.6944 & 1.92 & 4.1667 & 7.1231 & 16.1 \\
repeatability (r) & 18.753 & 13.64 & 8.2093 & 10.169 & 1.8421 & 0.5533 & 1.1297 & 2.9428 & 1.7816 & 2.9271 \\
Reproducibility (R) & 50.461 & 46.096 & 32.418 & 37.539 & 3.1828 & 5.0675 & 3.1653 & 5.82 & 23.728 & 31.647 \\
\hline
\end{tabular}

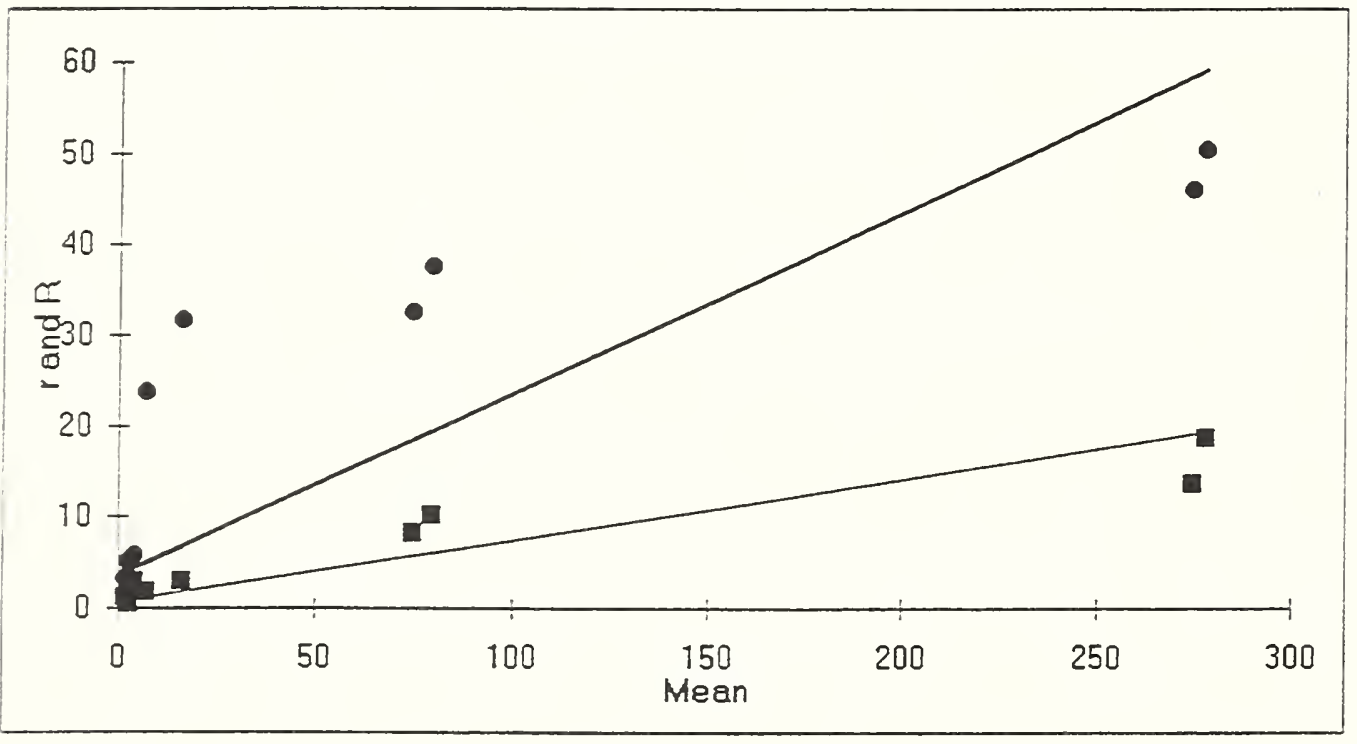

Fitt

\begin{tabular}{|ll|}
\hline Average & $\mathrm{I}=6.19468$ \\
& $\mathrm{R}=23.9125$ \\
\hline Repeat. & $\mathrm{a} 3=0.70908$ \\
& $\mathrm{~b} 3=0.06703$ \\
Reprod. & $\mathrm{A} 3=3.50246$ \\
& $\mathrm{~B} 3=0.20013$ \\
\hline
\end{tabular}

\begin{tabular}{c}
$r$ \\
$R$ \\
$-r 3=a 3+b 3 \cdot m$ \\
\hline$R 3=A 3+B 3 \cdot m$
\end{tabular}

\begin{tabular}{|c|cccccccc|}
\hline Lab. & B & D & E & F & G & H & I & J \\
\hline V.C. & 1.9129 & 3.8855 & 2.1664 & 1.2135 & 4.2559 & 1.9402 & - & - \\
\hline
\end{tabular}

Notes.

\footnotetext{
* : Missing data from I, J laboratories, no obvious reasons.

a : Laboratory D was identified as straggler (repeatability)

b : Laboratory D was removed as outlier (repeatability)

c : Laboratory $\mathrm{H}$ was removed as outlier (repeatability \& reproducibility)

d : Laboratory $\mathrm{H}$ was removed as outlier (repeatability)

e : Laboratory D was removed as outlier (repeatability)
} 


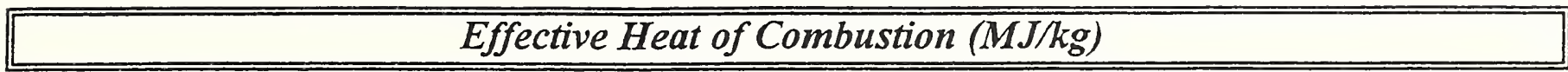

\begin{tabular}{|c|c|c|c|c|c|c|c|c|c|c|}
\hline Products & \multicolumn{2}{|c|}{ PMMA } & \multicolumn{2}{c|}{ MOF } & \multicolumn{2}{c|}{ VCST } & \multicolumn{3}{c|}{ GB } & \multicolumn{3}{c|}{ PVC } \\
\hline Flux $\left(\mathrm{kW} / \mathrm{m}^{2}\right)$ & 30 & 50 & 30 & 50 & 30 & 50 & 30 & 50 & 30 & 50 \\
\hline Number of labs. & 3 & 3 & 2 & 3 & 3 & 3 & 3 & .3 & 2 & 2 \\
Notes. & $-*$ & $-*$ & $\mathrm{a}^{*}$ & $\mathrm{~b}^{*}$ & $\mathrm{c}^{*}$ & $-*$ & $-*$ & $-*$ & $\mathrm{~d}^{*}$ & $\mathrm{~d}^{*}$ \\
\hline mean (m) & 23.233 & 23.089 & - & 11.978 & 14.033 & 13.722 & 2.8556 & 2.7444 & - & - \\
repeatability (r) & 1.3442 & 1.3048 & - & 1.8793 & 10.53 & 5.905 & 1.3879 & 1.9818 & - & - \\
Reproducibility (R) & 2.098 & 2.3139 & - & 1.948 & 12.403 & 8.4614 & 1.5611 & 1.6244 & - & - \\
\hline
\end{tabular}

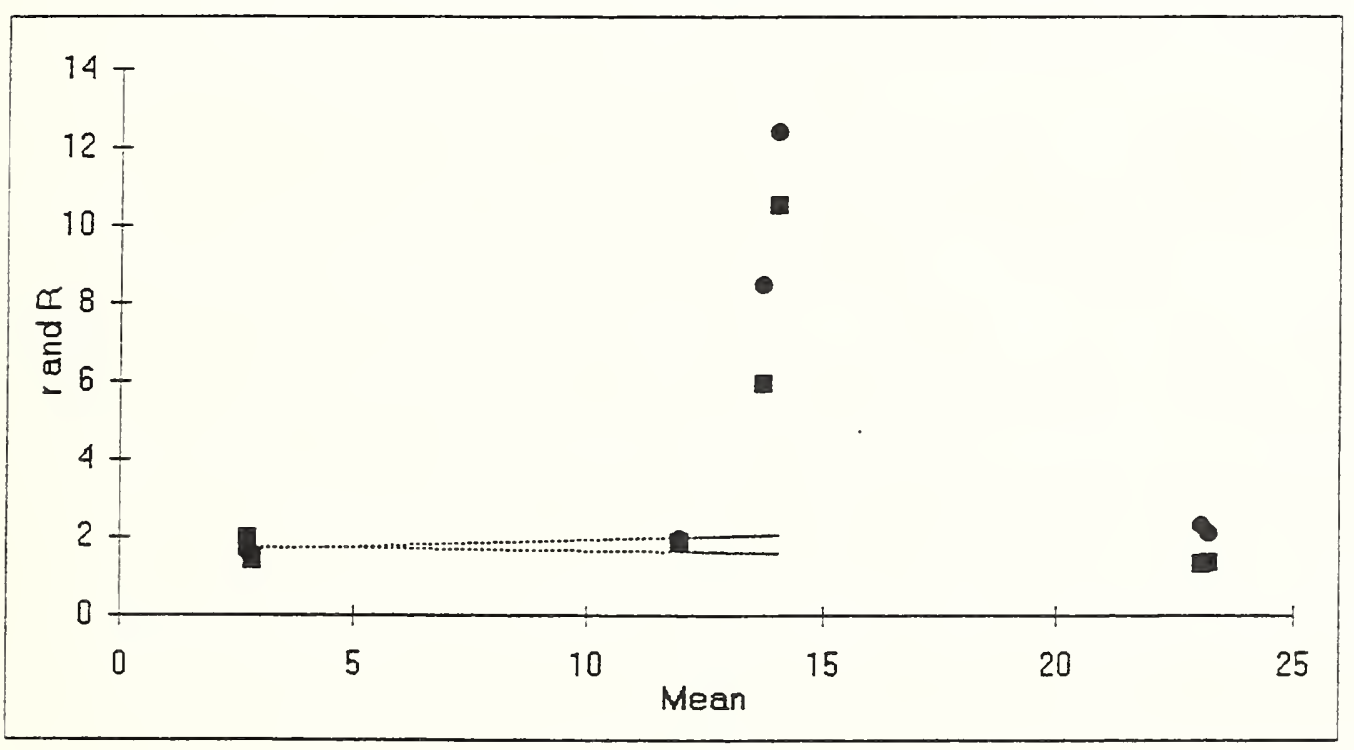

\begin{tabular}{|c|c|c|}
\hline \multicolumn{3}{|c|}{ Fitt } \\
\hline \multirow[t]{2}{*}{ Average } & $r=$ & 3.47609 \\
\hline & $\mathbf{R}=$ & 4.34429 \\
\hline \multirow[t]{2}{*}{ Repeat. } & $a 3=$ & 1.79994 \\
\hline & $\mathrm{b} 3=$ & -0.01512 \\
\hline \multirow[t]{2}{*}{ Reprod. } & $\mathrm{A} 3=$ & 1.57582 \\
\hline & $B 3=$ & 0.035 \\
\hline
\end{tabular}

\begin{tabular}{|c|cccccccc|}
\hline Lab. & B & D & E & F & G & H & I & J \\
\hline V.C. & - & 14.116 & 4.863 & - & 4.2788 & - & - & - \\
\hline
\end{tabular}

Notes.

* : Missing data from B, F, H, I J laboratories, no obvious reasons.

a : Laboratory $D$ was removed as outlier (repeatability), no data enoughto analyze : level discarded

b : Laboratory D was identified as straggler (repeatability)

c : Laboratory D was identified as straggler (repeatability)

d : Missing data from $D$ laboratory, no data enough to analyze: level discarded

Rq. The maximun number of laboratories taking part in that analysis is only three.

Results must so be handle with precaution. 


\section{Test End Time (s)}

\begin{tabular}{|c|c|c|c|c|c|c|c|c|c|c|}
\hline Products & \multicolumn{2}{|c|}{ PMMA } & \multicolumn{2}{c|}{ MDF } & \multicolumn{2}{c|}{ VCST } & \multicolumn{2}{c|}{ GB } & \multicolumn{2}{c|}{ PVC } \\
\hline Flux $\left(\mathrm{kW} / \mathrm{m}^{2}\right)$ & 30 & 50 & 30 & 50 & 30 & 50 & 30 & 50 & 30 & 50 \\
\hline Number of labs. & 5 & 5 & 5 & 4 & 4 & 5 & 3 & .4 & 4 & 3 \\
Notes. & $-*$ & $-*$ & $-*$ & $\mathrm{a}^{*}$ & $\mathrm{~b}^{*}$ & $-*$ & $\mathrm{c}^{*}$ & $\mathrm{~d}^{*}$ & $-^{*}$ & $\mathrm{e}^{*}$ \\
\hline mean (m) & 664.73 & 484.8 & 795.8 & 510.07 & 143.67 & 82.8 & 177.33 & 194.67 & 403.42 & 212.42 \\
repeatability (r) & 37.661 & 38.626 & 47.302 & 23.033 & 1.3199 & 4.4673 & 4.3976 & 5.4422 & 310 & 13.793 \\
Reproducibility (R) & 269.15 & 186.29 & 591.23 & 907.24 & 482.71 & 225.35 & 417.05 & 365.33 & 872.29 & 595.4 \\
\hline
\end{tabular}

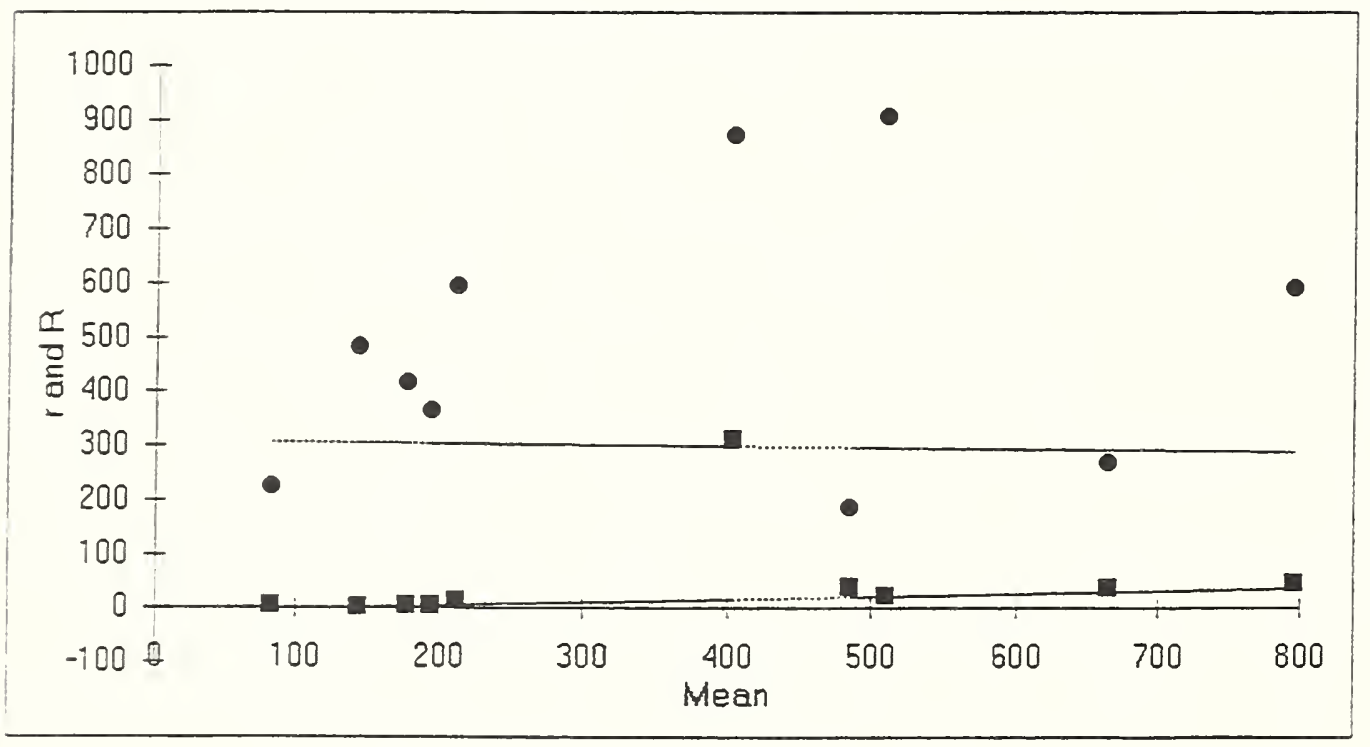

Fitt

\begin{tabular}{|lr|}
\hline Average & $\mathrm{r}=48.6037$ \\
& $\mathrm{R}=491.204$ \\
\hline Repeat. & $\mathrm{a} 3=-5.59274$ \\
& $\mathrm{~b} 3=0.05308$ \\
Reprod. & $\mathrm{A} 3=309.011$ \\
& $\mathrm{~B} 3=-0.02705$ \\
\hline
\end{tabular}

\begin{tabular}{|l}
$I$ \\
$R$ \\
$r 3=a 3+b 3 . m$ \\
$-R 3=A 3 \div B 3 . m$
\end{tabular}

\begin{tabular}{|c|cccccccc|}
\hline Lab. & B & D & E & F & G & H & I & J \\
\hline V.C. & - & 2.9149 & 5.5953 & - & 3.0286 & 2.4034 & - & 2.8483 \\
\hline
\end{tabular}

Notes.

* : Missing data fromB, F, I, no obvious reasons.

- a : Laboratory $E$ was removed as outlier (repeatability)

b : Laboratory $G$ was removed as outlier (repeatability)

c : Laboratory $\mathrm{H}$ was removed as outlier (repeatability)

d : Laboratory J was removed as outlier (repeatability)

e : Laboratory E was removed as outlier (repeatability)

Rq. Results seem to be unstable, no fitt possible.

Puzzling results obtained by $D \& E$ laboratories (no dispersion of the results). 


\title{
FLAMMABILITY OF UPHOLSTERED FURNITURE
}

\author{
T. J. Ohlemiller \\ National Institute of Standards and Technology \\ Building and Fire Research Laboratory
}

\begin{abstract}
Upholstered furniture flammability is both an ignitability and a heat release rate issue. Limited work on ignitability has been done recently. The major focus in three recent studies is the intensity of burning which follows exposure to a source strong enough to assure ignition. No adequate model of the post-ignition fire growth process exists. Examination of the attempts to correlate Cone Calorimeter data with full-scale heat release rate shows mixed results. Reasonably good correlations have been obtained but it is not clear that they have broad applicability and thus clear usefulness in designing furniture with known fire performance.
\end{abstract}

\section{The Nature of the Problem}

A substantial fraction of U. S. fire fatalities continues to be attributable to situations in which upholstered furniture is the first item ignited [1]. Significant reductions have occurred in this furniture-related death toll over the past twenty years, evidently as a consequence both of the widespread use of smoke detectors and of a voluntary industry standard on resistance to cigarette ignition. Cigarettes and small flames continue to constitute the bulk of the ignition sources, however, with a smattering of other sources such as electrical shorts or misused heating appliances. Once ignited to flaming by any of these means, the typical upholstered item, with its large volume of polyurethane foam, is capable of a rapidly developing fire that can exceed a megawatt in size.

\section{General Approaches to the Problem.}

Two broad approaches to this persistent flammability problem are possible: decrease the likelihood of ignition or decrease the impact of ignition. The ignition event itself, if it is a consequence of the most common scenario of a dropped cigarette, can be prevented by changes in the upholstery (mainly the fabric) or by changes in the cigarette. The former approach is at the heart of the voluntary program by the Upholstered Furniture Action Council. While this program has been in effect for two decades, a quantitative and objective measure of its effectiveness has been lacking for the last ten years. The Consumer Products Safety Commission is currently engaged in sampling and testing representative upholstered chairs from the residential furniture market. A recent program at NIST showed the feasibility of altering cigarettes to reduce their furniture ignition propensity and an appropriate test method for this purpose was developed $[2,3]$. At present there are no regulatory requirements of this nature on cigarettes.

The Consumer Products Safety Commission is also now addressing the question of the ignition of upholstery by small, match-size flames. Their statistical studies show that dropped smoker's matches and children playing with matches are substantial parts of the problem. Thus they are studying the ignitability of upholstered items in three locations: the seat/back crevice (a worst 
case location for a dropped smoker's match), the bottom of the stiffened fabric skirt found around the base of many furniture designs and the dust-cover fabric that typically covers the bottom surface of a chair or sofa. The last two locations are thought to be typical areas of interest for children playing with matches.

To decrease the impact of ignition of upholstered furniture, either by a cigarette or a flaming source, one needs to sharply limit the rate of heat release from the subsequent fire. Typically this implies a need to either modify (flame retard) the polyurethane foam or protect it from direct flame impingement by the use of a barrier layer. These are relatively costly solutions, not known to work with all possible fabrics. The enormous diversity of fabrics, due to varied fiber blends and weave designs, greatly complicates the search for assured solutions. This has led to recent studies, summarized below, which seek to understand and quantify the relation between upholstery composition and its rate of heat release behavior.

\section{3) The Rate of Heat Release from Upholstered Furniture.}

Furniture manufacturers need quantitative information on the relation between the properties of their materials and the fire performance of the chairs they manufacture from them. Room fire modelers need quantitative models of the development of fires involving the room contents and the interaction with the room, particularly for upholstered furniture. Both needs should be satisfied, if possible, by inputs derived from inexpensive tests on small samples of the materials of interest, thus precluding the need to burn a sample of each new chair design. This has led to several studies that examined the relation between behavior exhibited by furniture composites in the Cone Calorimeter and that shown by full-scale mock-ups or chairs.

Most of the recent interest in this area has been driven by regulatory issues. In the US, several states have adopted a stringent requirement on furniture to be placed in public occupancies (hotels, hospitals, etc.). In this test, known as California Technical Bulletin 133, an item of upholstered furniture (or a closely modeled mock-up of it) is subjected to an $18 \mathrm{~kW}$ gas burner playing flames on its seat area for 80 seconds. The resulting fire must not exceed $80 \mathrm{~kW}$ (including the igniter). NIST as well as a textile industry group have recently completed studies of materials effects in this test [4]. In Europe, the EC recently sponsored a large furniture flammability study, known as the CBUF study, aimed at harmonizing regulations across member countries [5]. Various chairs and sofas were subjected to the same gas burner design, but it was run at $30 \mathrm{~kW}$ for 120 seconds. In all of the above studies, the upholstery material combinations were also tested in the Cone Calorimeter, typically at $35 \mathrm{~kW} / \mathrm{m}^{2}$.

Ideally, one would be able to plug the Cone Calorimeter measurements into a well-developed predictive model of fire growth and calculate the heat release rate as a function of time for the full-scale furniture item. Unfortunately, the fire behavior of upholstered furniture items can be among the most non-ideal imaginable due to the strong role played by material movement in response to heat. Thermoplastic materials flow under the influence of gravity, moving fuel and heat to new, ill-defined locations. No model is able to handle these kinds of complications at present. A significant conclusion from the CBUF study was that, of the several dozen chair and material combinations studied, only a small proportion, perhaps one third or less, behaved in a manner consistent with a "simple" physical model in which material movements could be ignored 
prior to the peak in heat release rate. Thus it appears that the majority of real upholstered furniture will not be describable by a model that ignores the complexities of thermoplastic behavior. This has led to other, much more simplified approaches to relating Cone and full-scale behavior.

In the recent NIST study, the focus was on fabric and barrier effects in mock-ups tested according to the CB-133 protocol. A barrier is a nominally incombustible fiber layer placed between the fabric and polyurethane foam to minimize the participation of the latter in the burning process. The fire behavior during the gas burner exposure and afterward was sufficiently different as to suggest the controlling mechanisms are not the same for the two situations; the former involves mainly the fabric and the latter mainly the foam. It is worth noting that if the barrier layer were fully successful, there would be no significant heat release rate after the burner exposure but this was frequently not the case. Thus the behaviors during and after the burner exposure were assessed separately.

The fire growth problem during the gas burner exposure is dominated by lateral and upward flame spread processes on the fabric. The fundamentals of these processes are essentially similar to those in a room corner test, if thermoplastic material effects are minimal. Thus a dimensional analysis analogous to that performed by Thomas, et al. $[6,7]$ was made for this problem. The result, for a fixed chair geometry and igniter, is as follows:

$$
\frac{Q(t)}{Q_{\max }}=\left(\frac{(k p C)^{1 / 2} \Delta T}{\sqrt{t} Q_{\max }}\right)^{\alpha}\left(\frac{t_{B}}{t}\right)^{\beta}\left(\frac{q_{i g n}}{Q_{\max }}\right)^{\gamma}\left(\frac{\Phi}{Q_{\max }^{2} I_{B}}\right)^{\delta} \ldots \ldots
$$

Here $Q(t)$ is the rate of heat release behavior of interest, i. e., the growth toward a peak, $Q_{\text {max }}$ is the height of the first heat release peak seen in the Cone Calorimeter at a flux of $35 \mathrm{~kW} / \mathrm{m}^{2}$ for a mock-up of the chair materials (fabric, barrier, foam), $(\mathrm{k} \rho \mathrm{C})$ is the effective thermal inertia of the mock-up during ignition, $\Delta \mathrm{T}$ is the temperature increase of the mock-up surface at ignition, $t_{B}$ is the duration of the first heat release peak in the Cone test, $q_{i g n}$ is the heat flux from the spreading flames to the unignited chair surfaces during fire growth, $\Phi$ is the lateral flame spread parameter normally measured in a LIFT apparatus (ASTM E-1321), $l_{B}$ is a reference length, taken here as the width of the gas burner. The exponents on these dimensionless groups, $\alpha, \beta$, etc., are determined, in principle, by matching this expression to experimental data.

It is highly desirable to simplify this, if possible, by using surrogate parameters that may be more readily available. A surrogate is a parameter that is in large measure proportional to the original. A surrogate for the numerator in the first group is the ignition delay time in the Cone test. A partial surrogate for $\mathrm{q}_{\mathrm{ign}}$ is the heat of combustion which influences flame temperature. We also confine our interest to the value of the full-scale peak in heat release rate. Then the above becomes a dimensional proportionality: 


$$
Q_{\text {paak }} \sim t_{i g n}^{a} t_{B}^{b} Q_{\max }^{c} \Phi^{d} H_{c o m b}^{o} \ldots \ldots \ldots
$$

All of these parameters are available from the Cone Calorimeter except for $\Phi$, the LIFT heat flux parameter. Since this parameter value was not available for the materials in this study, the fitting process between the full scale results and Equation (2) omitted this; if reasonable fits are still found without it, this implies that the role of this parameter is minor.

Since simplicity is clearly desirable and not all of the above parameters will necessarily have a substantial role to play in correlating full-scale data, one starts with a limited subset. The nonlinear, least squares curve-fitting routine in the SigmaPlot* software package was used to evaluate the exponents and the proportionality constant in the above that makes it into a predictive equation. One seeks to minimize the norm which is the square root of the sum of the squared residuals (difference between a predicted and an actual point). This is a purely mathematical process. The exponent values which are generated tend to be closely coupled to each other and sensitive to noise in the data. Thus no physical significance should be assigned to the particular values which emerge.

When this approach is applied to the full data set obtained in the recent NIST study [4], the result is mixed; see Figure 1. All but two of the twenty-eight data points fall fairly close to the $45^{\circ}$ line which indicates equality between predicted and measured full-scale heat release rate peak (during burner exposure). However, there is no evident reason to dismiss the two outlying points and they are non-conservative (the actual heat release peak is greater than that predicted).

If the correlation is limited to charring fabrics, clustering along the $45^{\circ}$ line is much tighter suggesting that omission of ill-behaved thermoplastic fabrics could be beneficial to this form of correlation [4]. However, the number of charring fabrics in this data set was quite limited (seven points).

The recent textile industry study included twenty-two charring fabrics. Figure 2 shows the result of the best fit obtained with this data set; note that an additional surrogate parameter (from the Cone Calorimeter) is included here, the test average smoke extinction area. The estimated $95 \%$ (i.e., $\pm 2 \sigma$ ) confidence limits are included on a typical data point on this graph to make it clear that there is significant inherent noise in both the correlation function and the measured full-scale peak heat release rate. Thus, one should not expect the points to fall much closer to the $45^{\circ}$ line than they do in Fig. 2. This would thus seem to be a very good correlation. However, there is a worrisome element. The smoke extinction area was included in the correlation as a surrogate for the flame flux (instead of heat of combustion which gives a somewhat lesser degree of clustering on the $45^{\circ}$ line) with the thought that soot concentration may be a controlling factor for a predominantly radiative heat flux. The exponent for this parameter is negative. Thus the implied role for soot is one of radiation attenuation, not enhancement. It is not possible to discern if this is a real effect or a peculiarity of the limited data available.

\footnotetext{
* Certain commercial products are mentioned for clarity and completeness; this does not imply any endorsement by NIST nor does it imply that they are the best for this purpose.
} 
Before looking at the correlation efforts made in the European Community furniture flammability study, it is of interest to look briefly at the heat release peak that often occurs after the gas burner exposure. When a barrier is present, as it was in the NIST and textile industry studies, this peak occurs after a lull of a few minutes or more. The NIST study found this peak to be a result of the ultimate failure of the barrier to protect the foam [4]. Failure occurred when a fire became established on the base of one or more cushions and transferred enough heat through the intact barrier to melt the foam out through the base of the cushion, thus assuring a continuing flow of fuel to the fire. No attempt was made to correlate the strength of the resulting heat release peak because the controlling parameters cannot yet be quantified. Some suggestions as to how to prevent such fires were made [4].

The furniture used in the EC CBUF program [5] was largely residential in character and thus most of it did not include a barrier between the fabric and the foam. The study involved open market and specially-constructed furniture, not mock-ups, as in the two studies above. In the absence of a barrier, furniture tends to give a single heat release peak occurring at times intermediate between the two peaks seen in furniture with a barrier. This single peak is typically much higher, often exceeding one megawatt.

Two types of effort were made in the EC study to relate Cone Calorimeter data to the observed full-scale fire data [5]. The first was a largely heuristic statistical correlation study. Two parameters were defined as follows:

$$
\left.x_{1}=\left(m_{\text {soft }}\right)^{1.25} \text { (Style Factor }\right)\left(Q_{\max }+\overline{Q_{300}}\right)^{0.7}\left(15+t_{i g n}\right)^{-0.7} \ldots \ldots
$$

and

$$
x_{2}=880+500\left(m_{\text {soft }}\right)^{0.7}(\text { Style Factor })\left(H_{\text {comb }} / q_{\text {tot }}\right)^{1.4} \ldots
$$

Here $m_{\text {soft }}$ is the readily flammable mass of the furniture item (essentially the fabric and foam mass); "Style Factor" is an empirical coefficient accounting for such things as the differences in mode of burning between a chair and a sofa made of the same materials; $\mathrm{Q}_{\max }$ is the peak heat release rate in the Cone, at $35 \mathrm{~kW} / \mathrm{m}^{2}$ (as in Eqn. 2 above); $t_{\text {ign }}$ is the ignition delay time in the Cone at this same flux; $\mathrm{H}_{\text {comb }}$ is the test average effective heat of combustion in the Cone and $\mathrm{q}_{\text {tot }}$ is the total heat release from the sample in the Cone at $35 \mathrm{~kW} / \mathrm{m}^{2}$. Clearly, there are some parameters in common between the dimensional analysis approach used above and this heuristic approach. The predicted full-scale peak heat release rate from an item of furniture is a somewhat complex, conditional function of these two parameters [5].

The comparison of predicted and actual data from the CBUF study is shown in Fig.3. Note that the range goes from zero to 2 megawatts. The prediction is good to about $\pm 200 \mathrm{~kW}$ for most test items in this series; it is conservative when outside this range. Such a correlation clearly is useful as a general guideline but it poses something of a problem for manufacturers trying to be assured of staying below some regulatory limit such as $400 \mathrm{~kW}$. To be sure of success, they 
must actually design the furniture so that this correlation predicts its behavior to be $200 \mathrm{~kW}$ less than the limit. This uncertainty could force the manufacturer to use more expensive materials (e.g., a barrier layer) when it is not really necessary.

Given that the CBUF correlation is based largely on furniture that did not include barriers, it is of interest to see if it predicts the behavior seen in the NIST study where barriers were used. Fig. 4 shows such a comparison where here, as in the CBUF study, the absolute peak is used for a given test, regardless of whether it occurred during or after the burner exposure. All points shown satisfy the CBUF criterion for "propagating fires" [5] though the open circles had very weak spread after the gas burner exposure. The comparison is marginal, at best, with the average real behavior being a significantly less intense fire than predicted.

This last result, coupled with the discussion above of worrisome aspects of the dimensional analysis approach, raises the question of whether these correlations are truly valid beyond the data set for which they were derived. This is an unresolved issue.

Finally, it is of interest to briefly describe a second approach to heat release prediction that arose from the CBUF study. The heat release rate from any spreading fire can be calculated, in principle, from the following integral.

$$
q_{F S}(t)=\int_{0}^{t}\left(d A_{B} / d t^{\prime}\right) Q\left(t-t^{\prime}\right) \dot{m}\left(t-t^{\prime}\right) d t^{\prime} \ldots \ldots \ldots \ldots(5)
$$

Here the first term under the integral is the rate of growth of fire area. The product of the next two terms is the local heat release rate per unit area. The integral thus sums the heat release contributions from successively ignited areas up to the time of interest. To correctly use this expression, one needs to know the history of fire growth and the feedback flux from point-topoint in order to calculate the local heat release rate. In other words, one really needs a complete model of the fire growth process, then one can apply Eqn. (5) as a definition of the total heat release rate that the model predicts. In the absence of the necessary model, a more empirical approach was employed in the CBUF study. The heat release history from the Cone for a flux of $35 \mathrm{~kW} / \mathrm{m}^{2}$ was inserted into the right hand side of Eqn. (5) and the measured full-scale history was inserted on the left (for a series of times). The effective fire area as a function of time was thus inferred for a series of chairs of differing materials and constructions by a de-convolution process. For a given chair geometry, an average normalized effective area versus time function was deduced by averaging over results for several materials of construction. There are $100 \%$ deviations from the inferred average at some points in time. In spite of this and the fact that most of the real physical complications of the burning process are buried in the effective area function, the results work moderately well when used in a predictive mode. One plugs the area function back into Eqn. (5) along with the Cone data (at $35 \mathrm{~kW} / \mathrm{m}^{2}$ ) for a new combination of interest to predict the entire heat release history for a chair made from these materials. The predictions shown in Ref. 5 range from excellent agreement with actual measurements to mediocre agreement. Unfortunately, it is not entirely clear what causes this variability in the results though the behavior of the floor fire appears to be an element of it. It is worth noting that 
this approach is a hybrid of bench-scale and full-scale based prediction, requiring up to ten fullscale tests to characterize the area function for a given furniture style.

\section{Conclusions}

There have been significant advances in the quantification of the flammability of furniture, particularly its rate of heat release behavior. However, the ultimate goal of predicting full-scale heat release rate based on bench-scale measurements remains elusive. Modeling of fire growth from first principals appears impractical for the majority of furniture until the role of thermoplastic fabric and foam behavior is better quantified. The use of correlations to relate Cone Calorimeter and full scale data is moderately successful inasmuch as one can deduce such correlations by various approaches from a given data set. However, there have not been sufficient tests of these correlations against new data from other laboratories. There are hints that the correlations may not be very broadly useful. It seems probable that this approach has to be more narrowly limited in its scope. Thus, a correlation for charring fabrics over barriers in one limited set of chair geometries may work across laboratories but removal of the barrier would point to the need for a new empirical correlation for charring fabrics in this geometry. Excessive fragmentation of correlations for various situations could become unwieldy, of course, particularly if construction dimensions and details cannot be unified in some manner. Further study of the available data, especially that from the large CBUF program, is needed to clarify this situation.

\section{References}

1) Miller, A., "The U. S. Home Product Report, 1988-1992 Forms and Types of Materials First Ignited in Fires," National Fire Protection Association, Quincy, Mass., Feb., 1995

2) Gann, R., Harris, R., Krasny, J., Levine, R., Mitler, H. and Ohlemiller, T., "The Effect of Cigarette Characteristics on the Ignition of Soft Furnishings", National Bureau of Standards Technical Note 1241, January, 1988

3) Ohlemiller, T., Villa, K., Braun, E., Eberhardt, K, Harris, R., Lawson, J. and Gann, R., "Test Methods for Quantifying the Propensity of Cigarettes to Ignite Soft Furnishings", National Institute of Standards and Technology Special Publication 851, August, 1993

4) Ohlemiller, T. and Shields, J., "Behavior of Mock-Ups in the California Technical Bulletin 133 Test Protocol: Fabric and Barrier Effects," National Institute of Standards and Technology NISTIR 5653, May, 1995

5) Sundstrom, B. (ed.), "Fire Safety of Upholstered Furniture - The Full Report of the European Commission Research Programme CBUF," SP, Sweden, June, 1995

6) Thomas, P., "Dimensionless Quantities in Fire Growth: The Weighting of Heat Release Rate," Lund University, Sweden, Dec., 1990

7) Kokkala, M., Thomas, P. and Karlsson, B., "Rate of Heat Release and Ignitability Indices for Surface Linings," Fire and Materials, 17, Sept, 1993, p. 209 

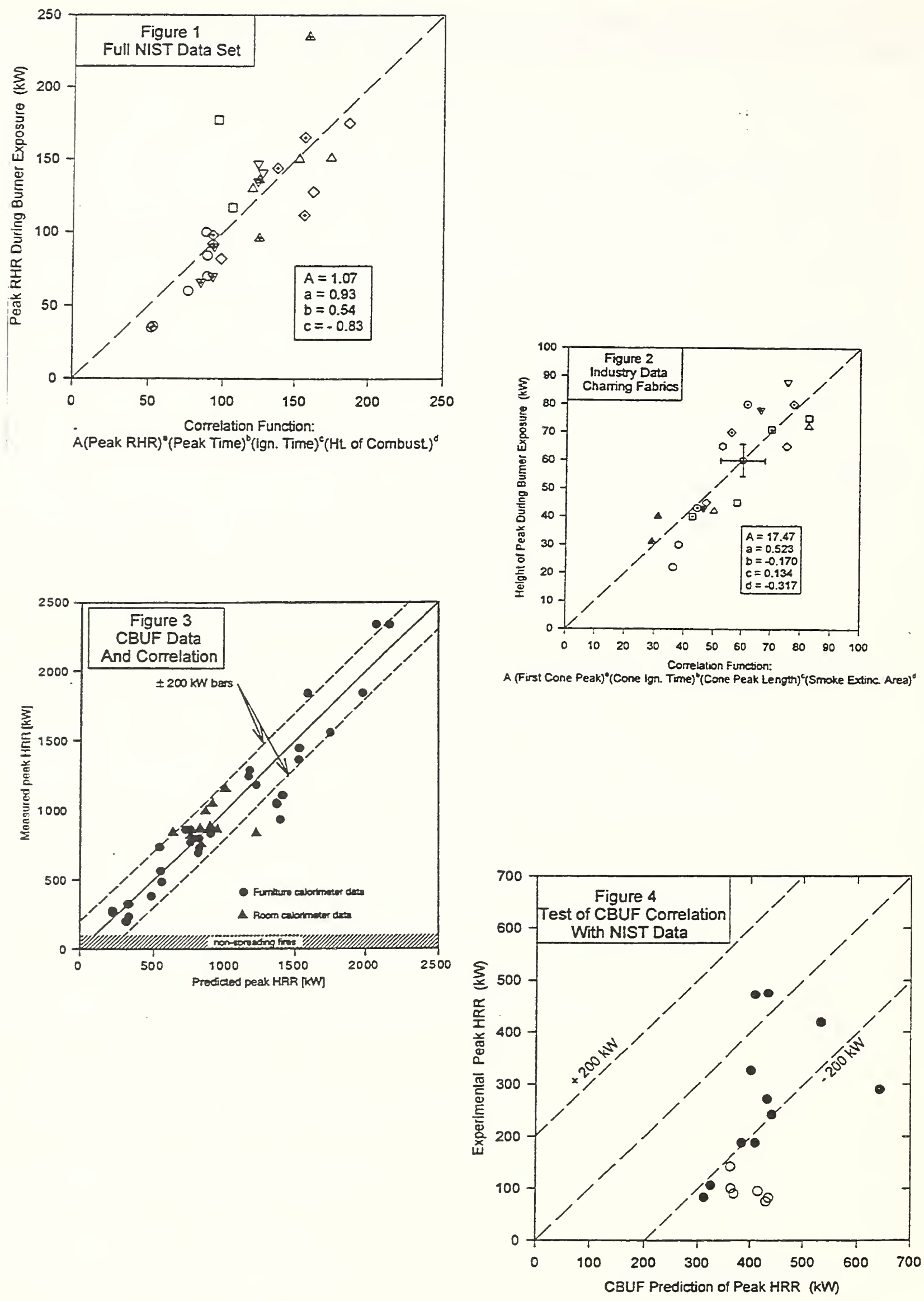


\section{Discussion}

Yuji Hasemi: When we decided to move our institute from the city, there was an abundance of chairs left over, and we used those. If you just ignite a chair itself, it does not burn too much. However, if you add some heat to the chairs before ignition, then they burn very vigorously. Of course how to evaluate the flammability of chairs depends on the fire area. I think there are two different situations. One is ignition of the chairs so that the fire actually starts with the chair; another scenario is when a chair gets exposed to flames from other furniture in other parts of the room. I think they burn quite differently. It's quite natural to think about the first scenario where the chair itself ignites, but not consider the second situation.

Thomas Ohlemiller: First of all, there have a couple of studies done here with chairs igniting themselves, looking at the extent to which the heat release behavior depends on how the chair was ignited. They both showed that the heat release was essentially independent of how they were ignited, but the time at which the peak occurred shifted very much. So I would expect the chair behavior in the 133 test probably would be valid for reaction to fire types of concern.

Eiji Yanai: In Japan there are some chairs which have a fire treatment on them, and if there is no radiation, then they will not burn.

Thomas Ohlemiller: That is very dependent on how intense a fire there is. The chairs that I have been discussing do not need radiation to burn. 
2 24 


\title{
HEAT RELEASE RATES MEASURED BY THE CONE CALORIMETER AND INTERMEDIATE SCALE ELECTRICAL RADIANT PANELS
}

\author{
Yuji Hasemi and Masashi Yoshida \\ Building Research Institute
}

Ryoichi Kikuchi, Emi Yamamoto, and Ryosuke Takaike

Science University of Tokyo, School of Science and Technology

\section{INTRODUCTION}

Heat release rate is widely considered one of the most dominant material properties on the growth of fire. Its measurement in simulated fire environment was made possible by the introduction of oxygen consumption method, and the Cone Calorimeter is becoming the most standard tool to evaluate heat release rate of building materials. There are already numbers of mathematical fire growth models using Cone Calorimeter data as input. However, there is considerable experimental evidence that heat flux to burning surface is dependent on the size of the flame, and since heat flux to a burning surface is the driving force of heat release in fire, some size effect is anticipated on the heat release characteristics of materials. In order to investigate into the possible size effect in heat release rate, comparative study has been made using the Cone Calorimeter and an intermediate scale vertical electrical radiant panel.

\section{EXPERIMENTAL DESCRIPTION}

Efforts have been made to reproduce identical experimental conditions other than the size in the two series of experiments. Particle board and Medium Density Fiberboard(MDF) has been used as specimens; specimens of each material were prepared from a same lot and were delivered directly from manufacturers. Surface and backsurface temperature of specimen and heat flux to the surface were monitored with type- $\mathrm{K}$ thermocouples and Schmidt-Boelter heat flux gages respectively at both series of the test. Heat flux levels from the radiation sources were between 5 and $25 \mathrm{~kW} / \mathrm{m}^{2}$. From previous vertical flame spread tests at BRI, any significant upward flame spread had not been observed on this material for external heat flux weaker than $5 \mathrm{~kW} / \mathrm{m}^{2}$, whereas for external heat flux higher than $15 \mathrm{~kW} / \mathrm{m}^{2}$ upward flame spread was too fast for visual observation. If flame spread is so fast, it is believed that surface ignition dominates the life safety and there is not any strong need to predict flame spread at least from the firesafety point of view. The level of external heat flux for the present tests was chosen to reproduce fire exposure at which surface flame spread is considered the most relevant to life safety. Tests at heat flux levels not higher than $20 \mathrm{~kW} / \mathrm{m}^{2}$ were carried out in FY 1994, and those at $25 \mathrm{~kW} / \mathrm{m}^{2}$ were run in FY 1995. Partly because of the limitation in the power capacity of the intermediate scale electrical radiant panels, uniform heat flux distribution was not achieved on the specimen at heat flux level higher than $25 \mathrm{~kW} / \mathrm{m}^{2}$ with the intermediate scale tests. At several tests, heating of the specimen preceded the ignition as previous flame spread tests by the authors suggest importance of the initial surface temperature or preheat condition on the time history of local heat release rate. Identical heat flux gage was used for the Cone Calorimeter and for the intermediate scale tests. The heat flux gage was calibrated against a black body cavity at every interval of the tests. Temperature of the electrical heater was kept constant at both of the Cone Calorimeter tests and the intermediate scale tests.

\section{Cone Calorimeter Tests}

Specimens were placed in the vertical orientation in order to make it possible to measure surface 
heat flux to the specimen although the draft international standard(ISO5660) specifies only the horizontal orientation. Since the selected range of heat flux was too weak for the spark igniter, ignition was made using solid alcohol arranged on the specimen holder at the level of the lower edge of the specimen. Figure 1 shows arrangement of the specimen. The ignition source made an uniform flame layer covering the whole surface of the specimen. At some of the tests, an invertor was used to achieve stable weak heat flux level.

\section{Intermediate-scale Heat Release Measurements}

Two $0.5 \mathrm{~m}$ wide $1.9 \mathrm{~m}$ tall electrical radiant panels as shown in Figure 2 were placed beneath a smoke collection hood originally built for the ISO 9705 Room Corner Test. A $0.53 \mathrm{~m} \times 0.57 \mathrm{~m}$ specimen was mounted vertically on the BRI's wall flame heat transfer apparatus, and was placed in front of the radiant panels. Level of external heat flux was controlled by changing the distance of the specimen from the radiant panels. Heat flux was monitored at the center of the specimen. The specimen was ignited with a methane porous line burner at the lower edge level of the specimen which makes a flame layer covering the specimen surface. Blank tests both on the Cone Calorimeter and the intermediate-scale radiant panels using fiber cement board have confirmed that heat flux to the specimen due to the igniter in the two series of the tests is very close.

\section{TEST RESULTS AND DISCUSSION}

Figure 3 and 4 compare time histories of heat release rate for different external heat flux levels obtained from intermediate scale radiant panels and the Cone Calorimeter. Figure 3 represents low level of external heat flux and Figure 4 does higher heat flux level. Heat release rate per unit area measured with the Cone Calorimeter is always lower than that with the intermediate scale radiant panels. Flaming combustion was not sustained enough long in some of the Cone Calorimeter tests, especially at low heat flux levels, although combustion seemed to be stabler in the intermediate scale tests. With approximation of the time history of heat release rate after ignition on a charring material by $q_{0} \cdot \exp (-\lambda \mathrm{t})$, the peak heat release rate, $\mathrm{q}_{0}$, is smaller and the decay coefficient, $\lambda$, is larger for the Cone Calorimeter than for the intermediate scale apparatus as seen in Figures 5 and 6. It means that use of heat release data from the Cone Calorimeter may always lead to safer side prediction than that from the intermediate scale apparatus. The heat release data were applied to the Baroudi-Kokkala diagram for upward flame spread(Figure 7). Experimental xpoff/xpo values for the identical heat flux conditions were (1) $\mathrm{xpoff} / \mathrm{xpo}=14$, (2) $\mathrm{xpoff} / \mathrm{xpo}_{\mathrm{po}}=18$, (3) $\mathrm{xpoff} / \mathrm{xpo}_{\mathrm{po}}>40$, (4) $\mathrm{xpoff} / \mathrm{xpo}_{\mathrm{p}}>26$, (5) $\mathrm{xpoff} / \mathrm{xpo}>40$, and (6) $\mathrm{xpoff} / \mathrm{xpo}>40$. Results of the flame spread tests were always better explained by the heat release data from the intermediate scale tests than by the Cone Calorimeter data.

Surface and backsurface temperatures and surface heat flux measured at the same test with Figure 3 are summarized in Figures 8 and 9. There is not notable difference in temperature nor in surface heat flux until around the peak of heat release between the Cone Calorimeter and the intermediate scale test. However, decay of surface heat flux starts earlier at the Cone Calorimeter than at the intermediate scale test, and this earlier decay of heating seems to be a main cause for the unstable burning behavior observed in the Cone Calorimeter tests.

During the FY1994 tests with relatively low heat flux levels, this considerably lower heat release rate from smaller testing apparatus was attributed to the possible lower combustion efficiency at smaller tests as the total CO generation per unit specimen surface area was always noticeably higher at the Cone Calorimeter measurement than at the intermediate scale test. If it is the reason, increase of external heat flux should result in closer heat release rate output between the Cone Calorimeter and the intermediate scale test apparatus. The comparison for higher heat flux was done partly to verify this assumption. As shown in Figure 6, the peak Cone heat release rate to the 
peak intermediate scale heat release rate increases with the level of external heat flux, and at the heat flux level, $25 \mathrm{~kW} / \mathrm{m}^{2}$, difference in measured heat release rate became less than $20 \%$. This result at least suggests increase of the extemal heat flux level can compensate the size effect in heat release.

\section{CONCLUSIONS}

From the comparison of the heat release data obtained with the Cone Calorimeter(vertical orientation) and those with the intermediate scale electrical radiant panels, the following conclusions could be drawn.

(1) The Cone Calorimeter(vertical orientation) gives generally lower heat release rate than the intermediate scale radiant panels.

(2) Results of large scale upward flame spread tests can be better explained by the intermediate scale tests than by the Cone Calorimeter(vertical orientation).

(3) Difference in heat release rate is reduced as the external heat flux level is increased.

The Cone Calorimeter tests were conducted only in the vertical orientation in order to measure surface heat flux to the specimen. Therefore the above conclusions should not be applied directly to the ISO 5660 which specifies only the horizontal sample orientation. It is perhaps worth comparing the present data with those in the horizontal orientation at identical heat flux levels.

\section{REFERENCES}

1. Hasemi,Y., Yoshida,M., Goto,T., Kikuchi,R., Hosomi,M., and Yamamoto,E.: On the predictability of turbulent upward flame spread based on material fire tests, Proceedings of the 1995 Annual Meeting of the Japan Association of Fire Science and Engineering, p.286- 289, 1995(in Japanese).

2. Hasemi,Y., Yoshida,M., Yasui,N., and Parker,W.J.: Upward Flame Spread Along a Vertical Solid for Transient Local Heat Release Rate, Proceedings of the Fourth International Symposium on Fire Safety Science, Ottawa, 1994. 


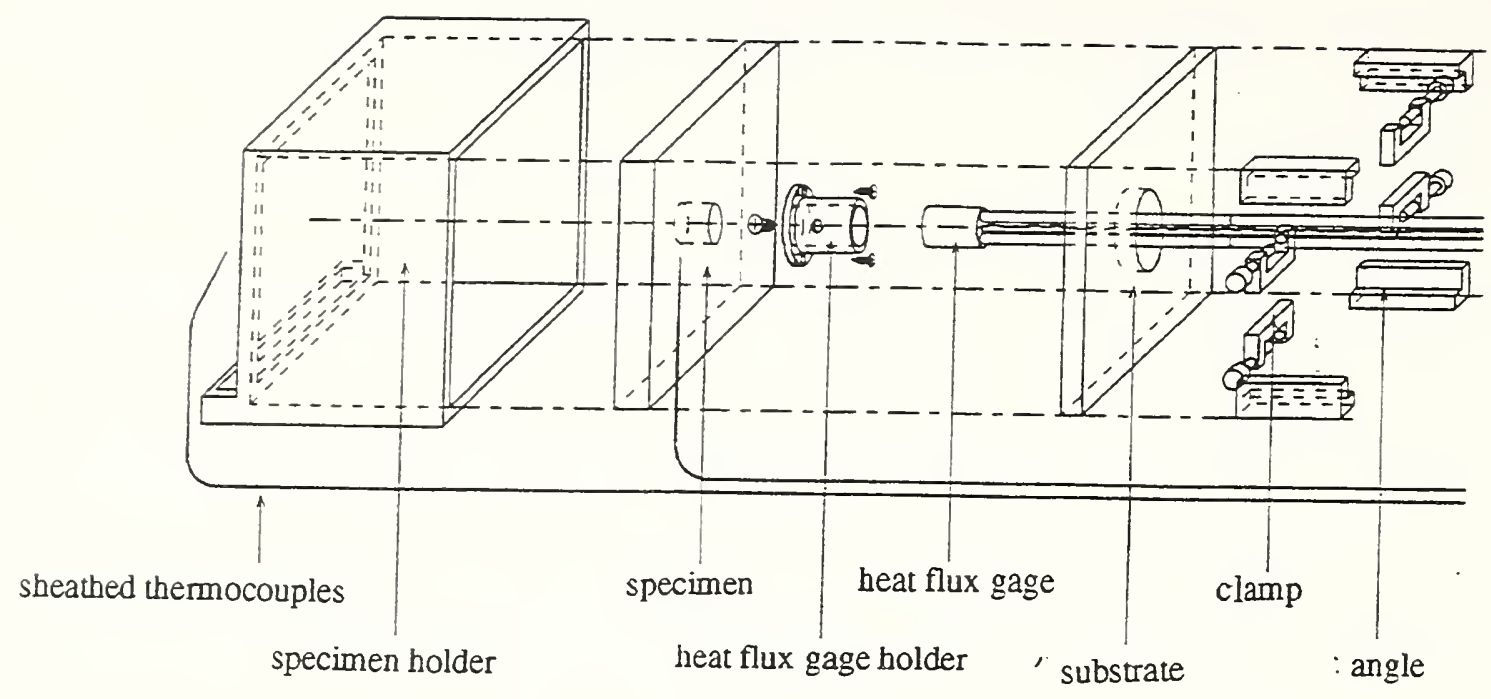

Figure 1 Arrangement of specimen and heat flux gage, Cone Calorimeter vertical orientation
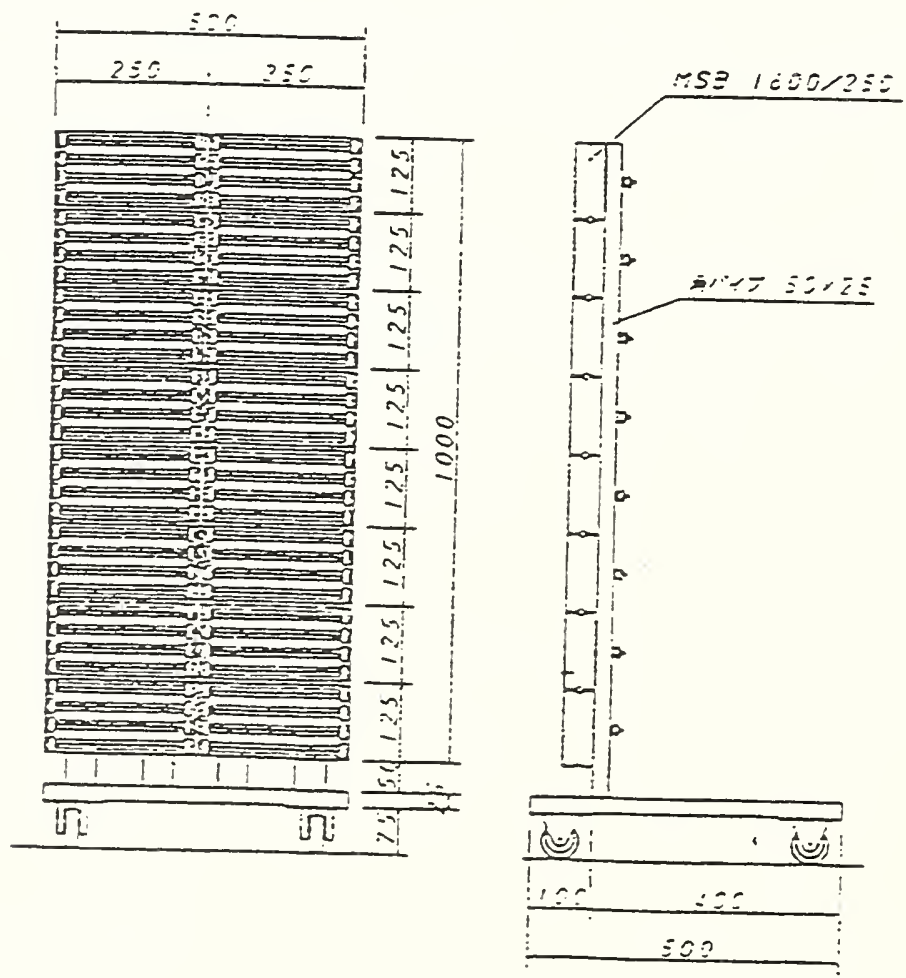

Figure 2 Intermediate scale electrical radiant panel 
Figure 3(a) Heat release rate

Intermediate scale electrical panels
Particle board, $q e=5 \mathrm{~kW} / \mathrm{m}^{2}$ Tini $=130 \mathrm{C}$
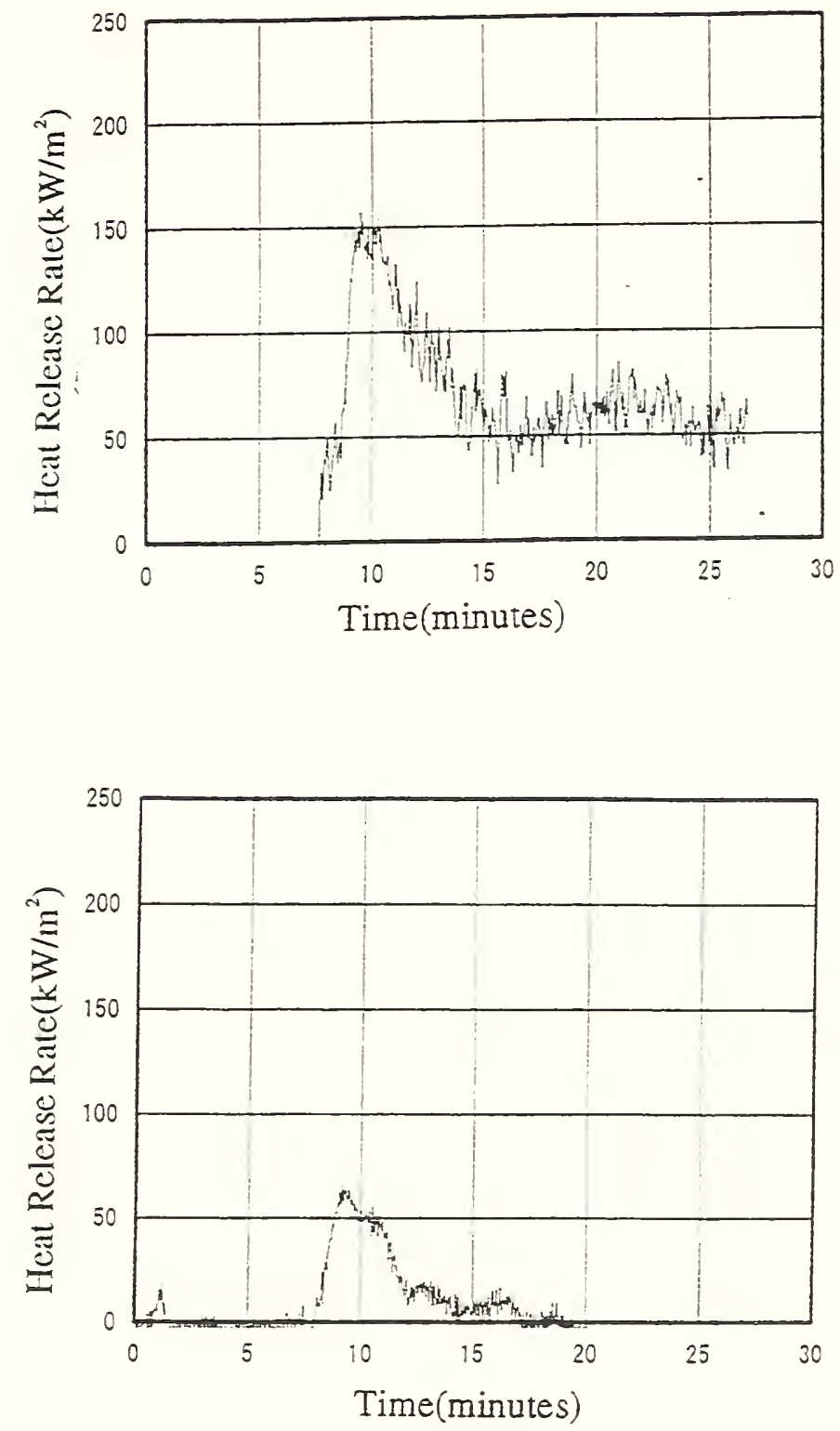

Figure 3(b) Heat release rate Cone Calorimeter(vertical)

Particle board, qe $=5 \mathrm{~kW} / \mathrm{m}^{2}$ Tini $=130 \mathrm{C}$

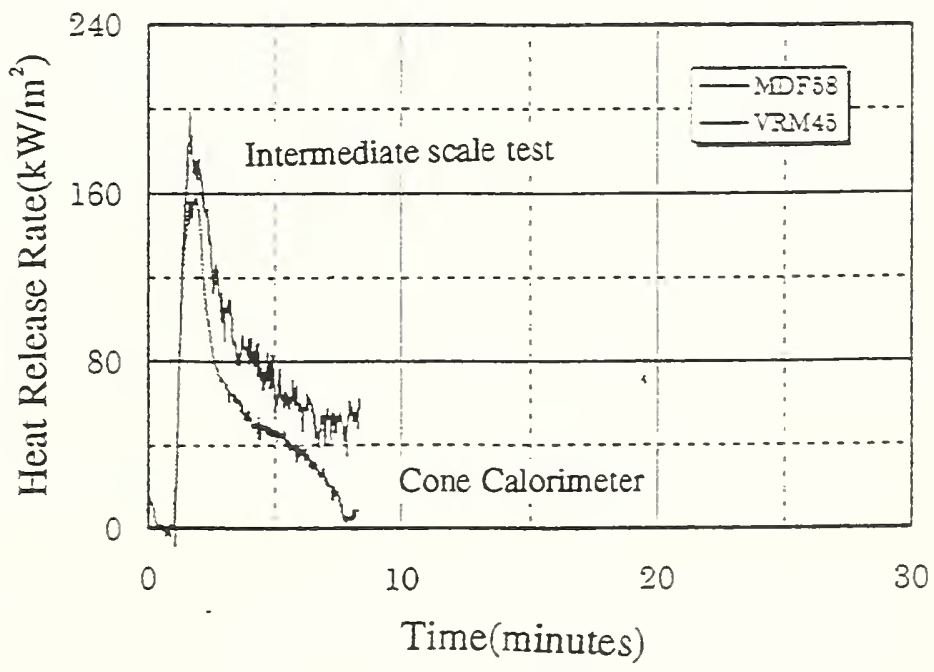

Figure 4 Heat release rate Intermediate scale electrical panels \& Cone Calorimeter

Time(minutes) 


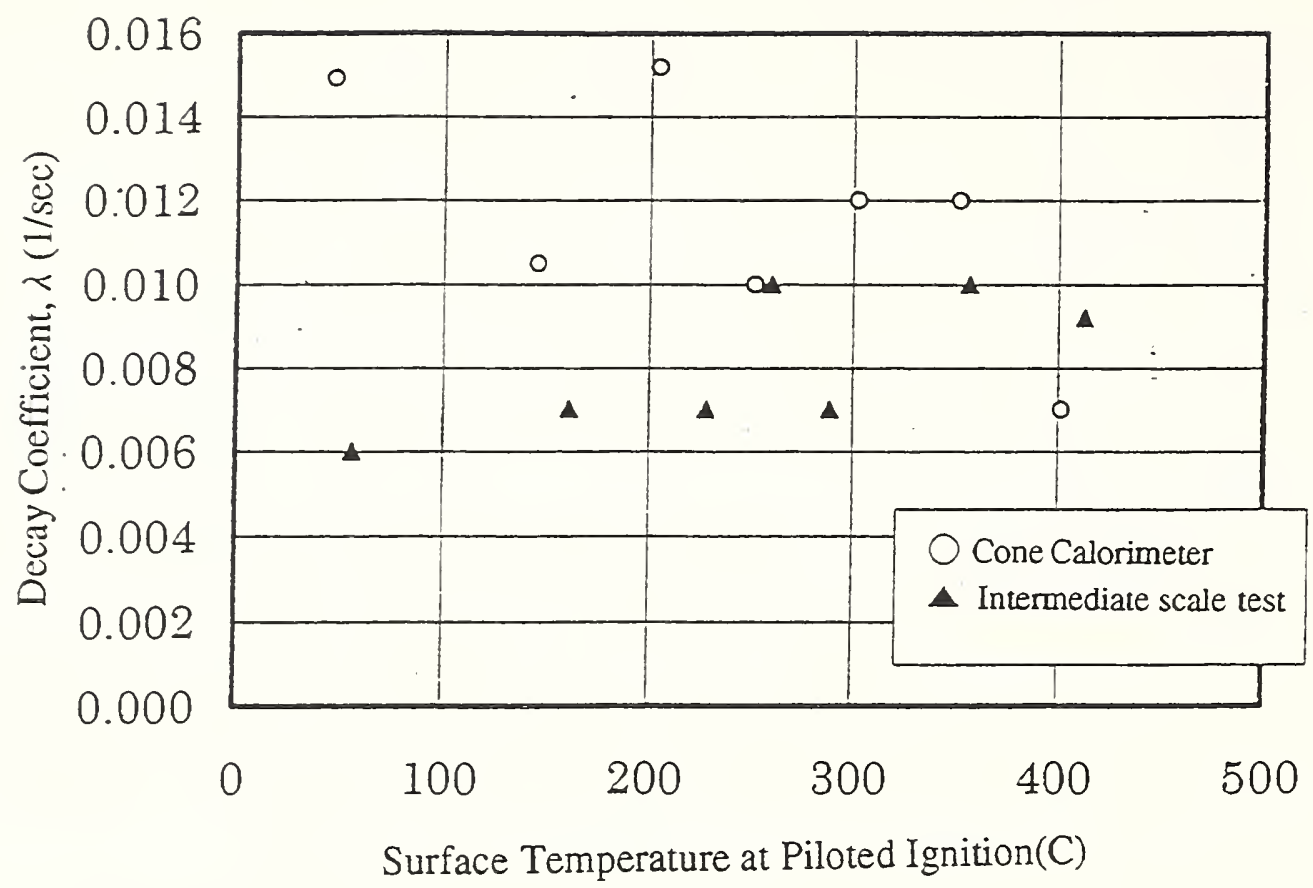

Figure 5 Heat release rate decay coefficient vs. Surface temperature just before piloted ignition

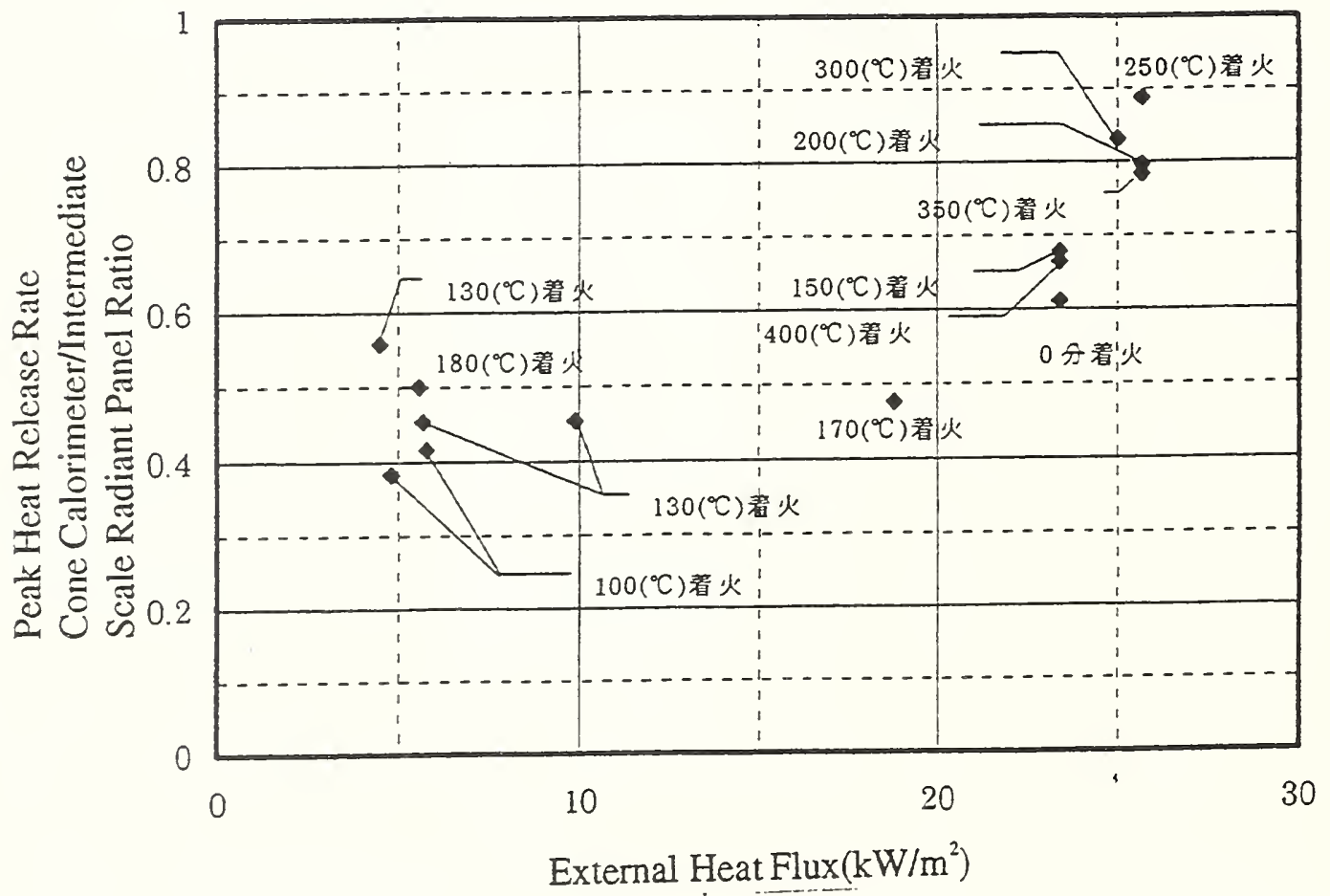

Figure 6 go from Cone Calorimeter to qo from intermediate scale test ratio 


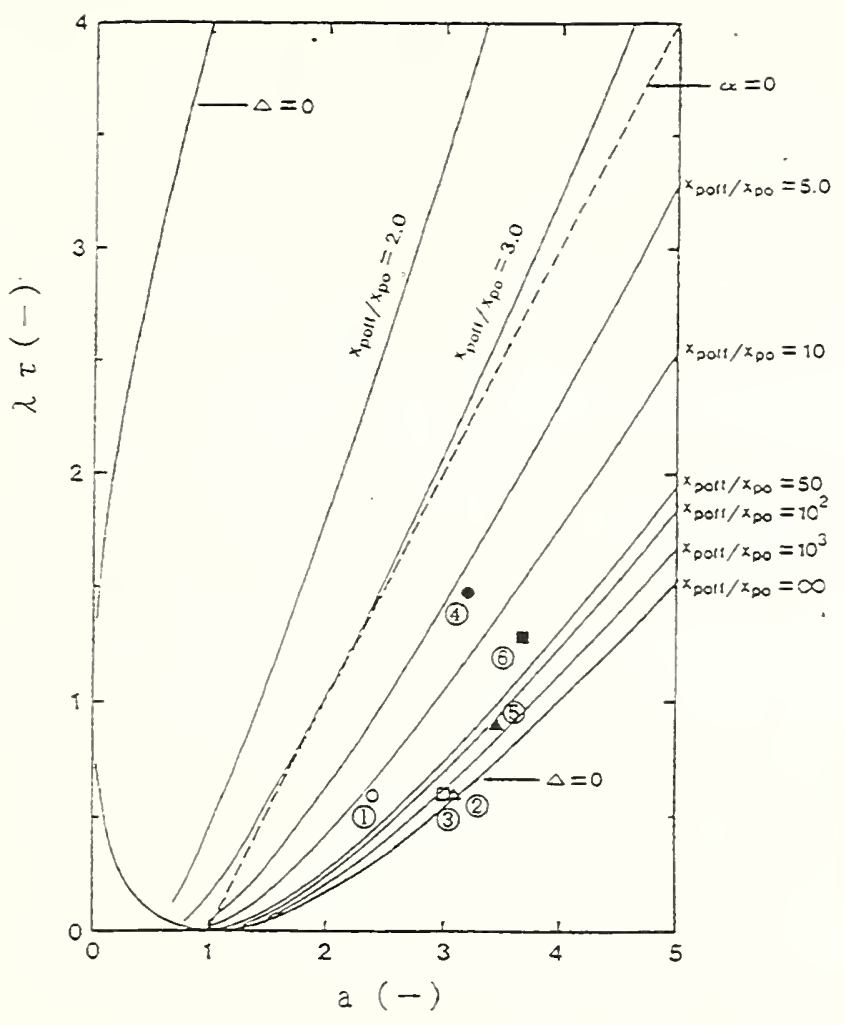

(1) particleboard, $q \mathrm{e}=6 \mathrm{~kW} / \mathrm{m}^{2}$, Tini $=100 \mathrm{C}$

(2) $\triangle$ particleboard, $q e=6 \mathrm{~kW} / \mathrm{m}^{2}$, Tini $=130 \mathrm{C}$

(3) $\square$ particleboard, qe $=6 \mathrm{~kW} / \mathrm{m}^{2}$, thermal equilibrium

(4) $\mathrm{MDF}, \mathrm{qe}=6 \mathrm{~kW} / \mathrm{m}^{2}, \mathrm{Tini}=100 \mathrm{C}$

(5) $M D F, q e=6 \mathrm{~kW} / \mathrm{m}^{2}$, Tini $=130 \mathrm{C}$

(6) $\mathrm{MDF}, \mathrm{qe}=6 \mathrm{~kW} / \mathrm{m}^{2}$, thermal equilibrium

Figure 7(a) Application of heat release data to the Baroudi-Kokkala diagram, Intermediate scale test

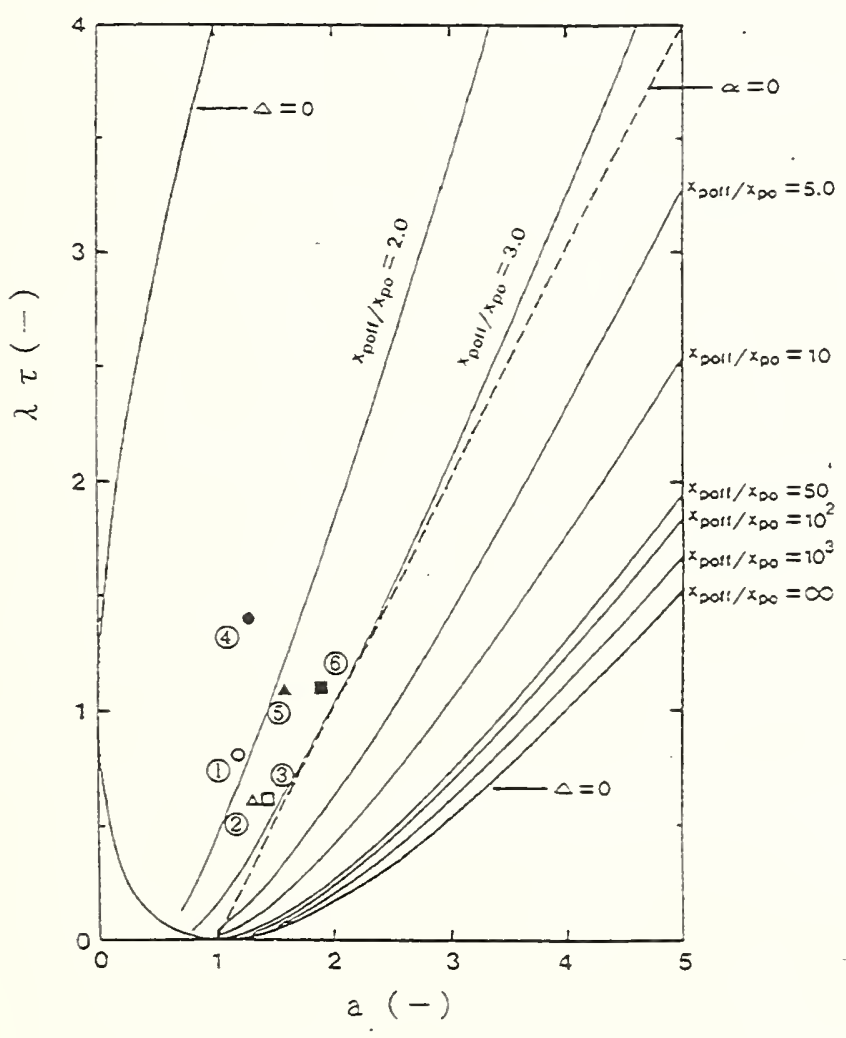

(1) particleboard, $q \mathrm{e}=6 \mathrm{~kW} / \mathrm{m}^{2}$, Tini $=100 \mathrm{C}$

(2) $\triangle$ particleboard, $q \mathrm{e}=6 \mathrm{~kW} / \mathrm{m}^{2}, \operatorname{Tini}=130 \mathrm{C}$

(3) $\square$ particleboard, $q e=6 \mathrm{~kW} / \mathrm{m}^{2}$, thermal equilibrium

(4) $\mathrm{MDF}, \mathrm{qe}=6 \mathrm{~kW} / \mathrm{m}^{2}$, Tini $=100 \mathrm{C}$

(5) $\mathrm{MDF}, q \mathrm{~g}=6 \mathrm{~kW} / \mathrm{m}^{2}$, Tini $=130 \mathrm{C}$

(6) $\mathrm{MDF}, \mathrm{qe}=6 \mathrm{~kW} / \mathrm{m}^{2}$, thermal equilibrium

Figure 7(b) Application of heat release data to the Baroudi-Kokkala diagram, Cone Calorimeter 


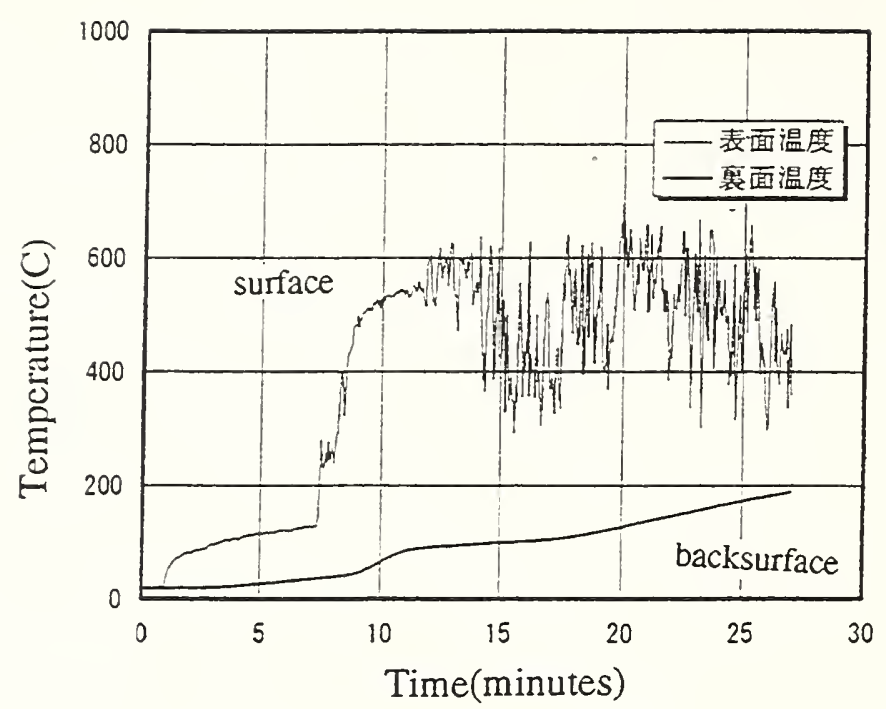

Figure 8(a) Surface and backsurface temperatures, Intermediate scale test (thin curve: surface, thick curve: backsurface)

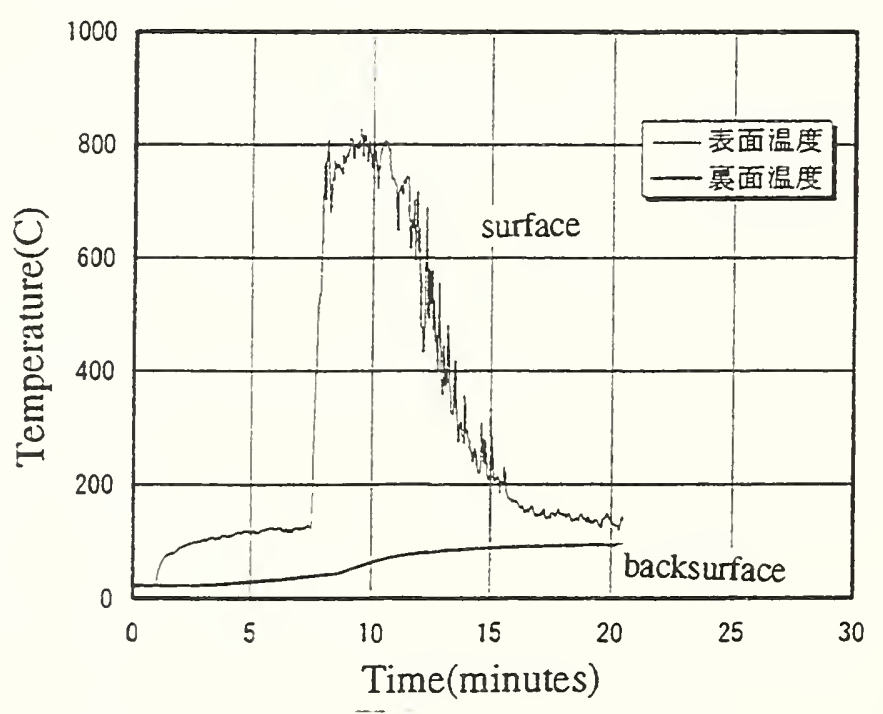

Figure 8(b) Surface and backsurface temperatures, Cone Calorimeter (thin curve: surface, thick curve: backsurface) 


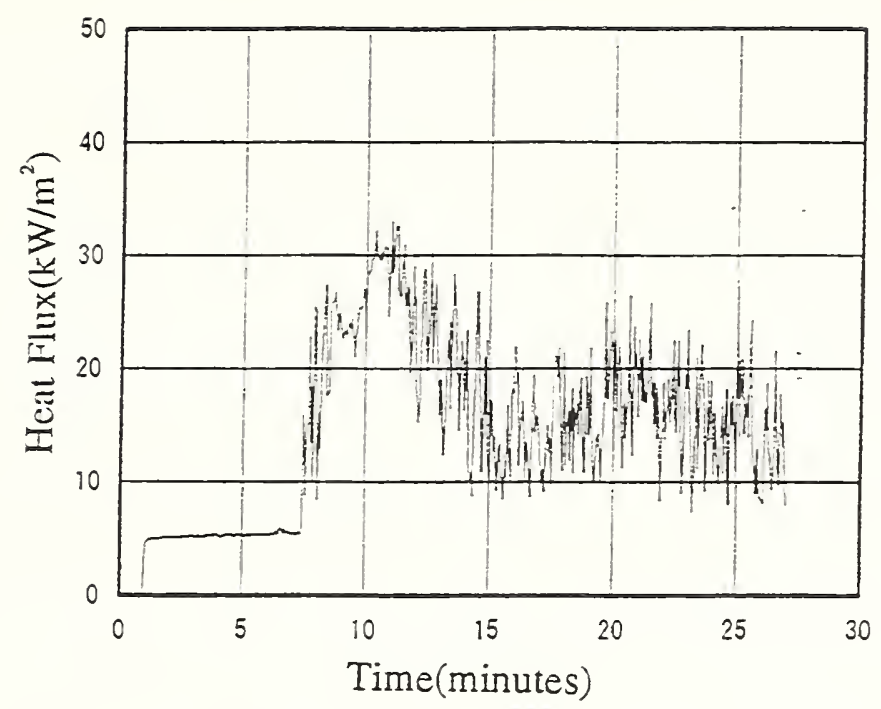

Figure 9(a) Surface heat flux, Intermediate scale test

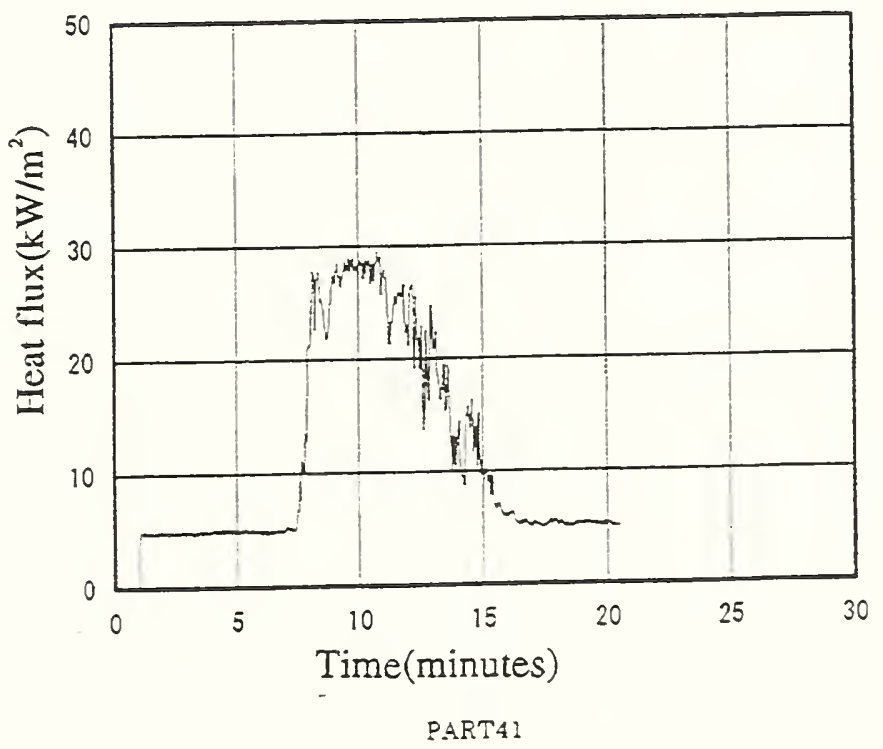

Figure 9(b) Surface heat flux, Cone Calorimeter 
Intermediate scale tests
MDF Tini $=100 \mathrm{C}$
OMDF Tini $=130 \mathrm{C}$
$\triangle M D F$ Tini $=200 \mathrm{C}$
Cone calorimeter
$\square \mathrm{MDF}$ Tini $=100 \mathrm{C}$
OMDF Tini $=130 \mathrm{C}$
$\triangle \mathrm{MDF}$ Tini $=160-180 \mathrm{C}$
$\checkmark \mathrm{MDF}$ Tini $=200 \mathrm{C}$

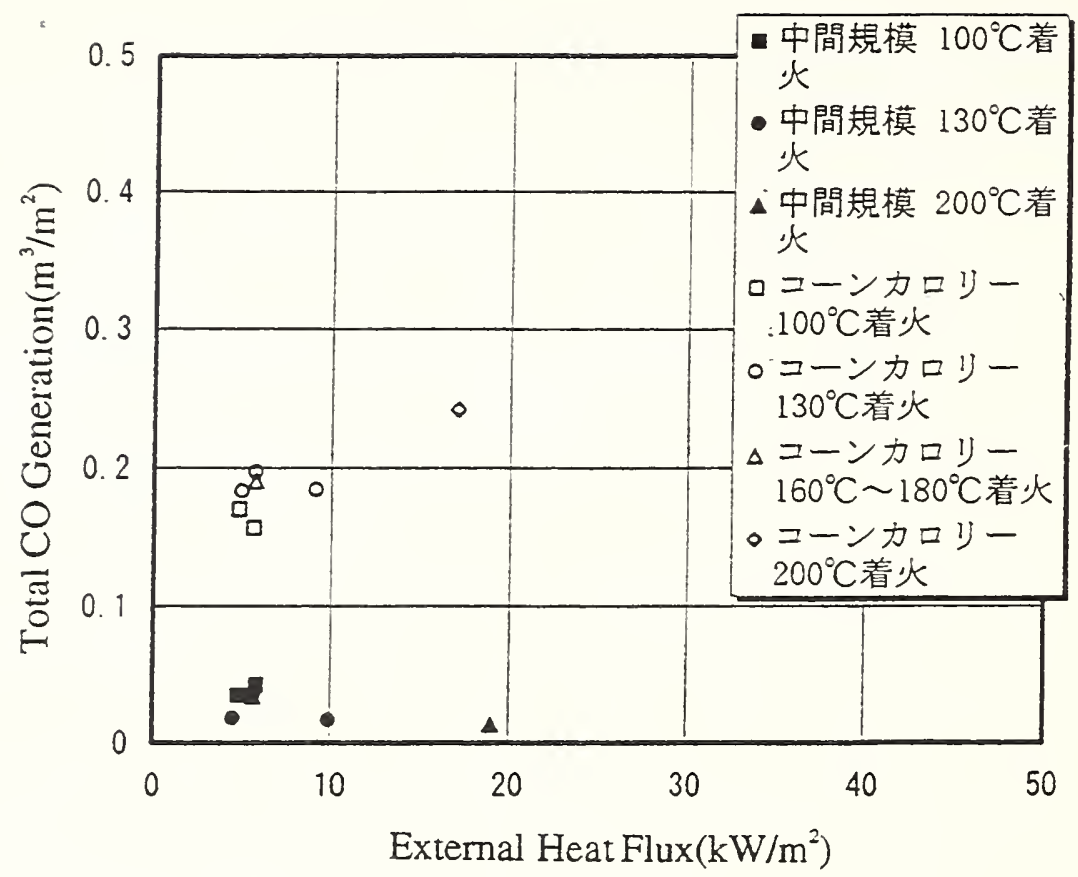

Figure 10 Comparison of $\mathrm{CO}$ generation between the Cone Calorimeter and the intermediate scale test 


\title{
A Flammability test for Granular Synthetic Resins using a Modified Oxygen Index Method
}

Eiji YANAI and Yusaku IWATA

Fire research Institute of Japan

3-14-1, Nakahara, Mitaka, Tokyo 181, Japan

\begin{abstract}
A new flammability test has been developed for granular synthetic resins, based on a modified Japanese Industrial Standard (JS) oxygen index test. In addition, an oxygen index has been determined for several different materials using the newly proposed method. The Appropriateness of the new test method is examined by comparison to oxygen indexes as measured by the conventional test.
\end{abstract}

\section{Introduction}

Materials for which fire spreads rapidly, or which are difficult to extinguish, are classified in Japan as appointed flammable materials. Some synthetic resins fall into this category. In many cases these synthetic resins are stored and handled in the form of beads, granules, pastes, etc.

The oxygen index method 1), a prescribed Japanese Industrial Standard, is usually used as the flammability test method for synthetic resins. However, this test has been devised for sample in the form of sticks or textiles. It is therefore difficult to assess the flammability of synthetic resins in granular form using this standard oxygen index method. In addition, there are problems when applying the test to materials whose chemical composition is changed on heating, like thermosetting resins, or for materials which melt by slight heating, such as reactivity resins.

Tewarson et al. 2,3) studied the flammability of polymeric materials using apparatus equipped with an external radiant heat panel, similar to that found in oxygen index testing equipment. This equipment has some advantages, because it can be used for granular polymer and measure the flammability of polymeric materials exposed to fire. However, the equipment is not in wide use, being confined to research studies.

In contrast, experimental studies of oxygen indexes has been regularly performed using existing oxygen index flammability test equipment 4,5). However, the oxygen index has been determined for film, textile and stick forms, and as far as the authors are aware, the oxygen index has not been measured for samples in granular form.

The prime purpose of this research has been to improve the oxygen index method when used to evaluate the flammability of granular synthetic resins. Moreover, a range of synthetic resin samples in stick or membranes forms were 
fabricated and the new test method applied to allow comparison to oxygen index values as measured by the conventional oxygen index test.

\section{Experiment}

\section{I Test equipment}

The equipment (ON-1 type) which was used to measure oxygen index is equivalent to the equipment stipulated in JS K 7201. Reproducibility of results was confirmed by testing the material PMMA (Polymethylmethacrylate), which has a definite oxygen index value.

\subsection{Sample holder}

A sample holder made of stainless steel was fabricated to support granular polymer samples within the burning tube of the oxygen index equipment, shown in figure 1. This supporting implement was used instead of the existing sample holder for stick and textile, while also allowing granular sample to be tested.

\subsection{Sample cap}

A cap of quartz was made to contain the granular samples and the influence on oxygen index versus the size of the cap was examined. Caps diameter were 10,15,20 and 25 $\mathrm{mm}$ respectively, thickness is $1 \mathrm{~mm}$, height is $5 \mathrm{~mm}$.

\subsection{Ceramic paper}

Ceramic paper of thickness $1 \mathrm{~mm}$ was placed on sample support between the sample support and the sample cap. The heat loss from the sample was thereby reduced as much as

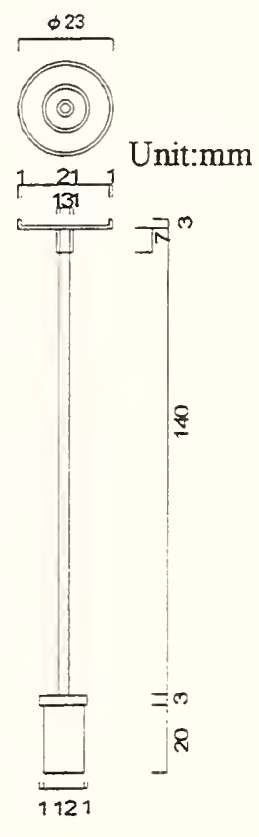
possible. Ceramic paper used had the following properties; Fig.1 Sample holder $0.15 \mathrm{~g} / \mathrm{cm}^{3}$ for density, $1260{ }^{\circ} \mathrm{C}$ for the highest used temperature, and $0.06 \mathrm{kcal} / \mathrm{mh}^{\circ} \mathrm{C}\left(400^{\circ} \mathrm{C}\right), 0.08 \mathrm{kcal} / \mathrm{mh}^{\circ} \mathrm{C}\left(600^{\circ} \mathrm{C}\right), 0.10 \mathrm{kcal} / \mathrm{mh}^{\circ} \mathrm{C}$ $\left(800^{\circ} \mathrm{C}\right)$ and $0.13 \mathrm{kcal} / \mathrm{mh}^{\circ} \mathrm{C}\left(1000^{\circ} \mathrm{C}\right)$ respectively for heat conductivity.

\subsection{Ignition source}

Urban gas was used as an ignition source for the sample and the flame length was adjusted to be $20 \sim 30 \mathrm{~mm}$. The flame contact time was varied for each sample, and the flame was allowed to contact the sample until it was ignited. To ensure reproducibility of this flame contact condition, the distance between the burner front and the edge of the sample cap was essentially fixed.

\subsection{Samples}

27 different of samples were used; liquid paraffin, aromatic hydrocarbon resin, polyethylene (4 types of granule and 1 type of film), polypropylene ( 2 types of granule), polystyrene ( 3 types of granule), polyvinyl chloride (3 types of granule, 1 type of powder, 3 types of stick), polycarbonate, polyamide, 
polybutylene terephthalate, polymethyl methacrylate, ABS resin, polyvinylidene fluoride and fluorocarbon resin ( 2 types). All the samples, except liquid paraffin and polyvinyl chloride (powder), are raw materials which have actually appeared in the market. In addition, 20 pieces of sample grain were picked out randomly and the maximum diameter was measured using a micrometer. Grain diameters shorn in table 1 are the average of 20 values.

Sample no. 1, liquid paraffin, was used to examine the necessary size of the cap for testing thermoplastic materials.

\subsection{Test procedure}

(1) Method for supporting the samples

Each sample was packed level to the top surface of the sample cap and placed on the sample holder. Ceramic paper was laid between the sample holder and sample cap, to minimize heat loss. By using this method, the support of the samples could be united without having to consider the thermal properties of the samples. An outline of the sample support method is shown in figure 2.

(2) Experimental method and measurement of the oxygen index

1) Sample is put into a quartz sample cap filled level to the top surface of the cap, and the sample weight is measured.

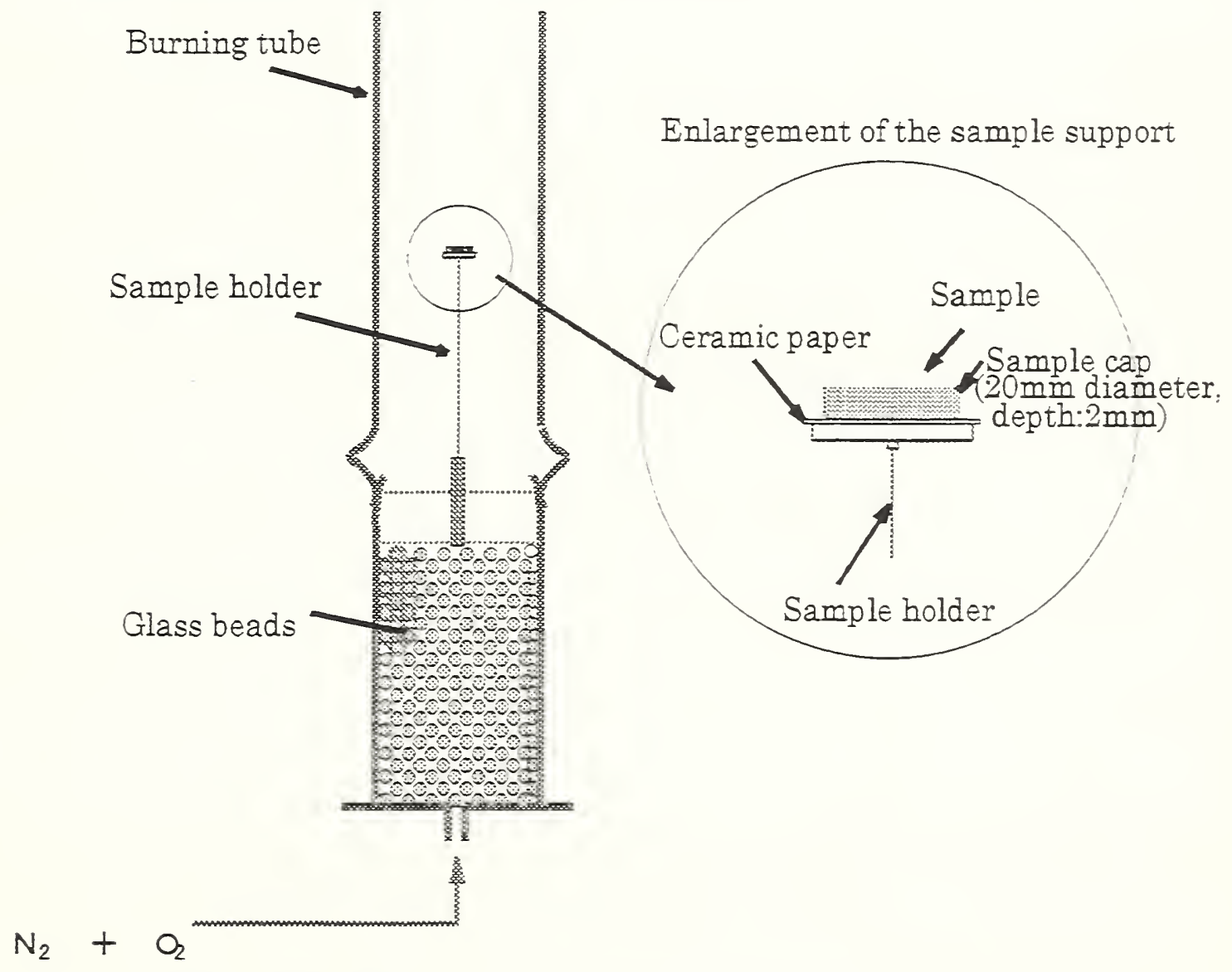

Fig.2 Method for supporting granular samples 
2) A circular ceramic paper, $25 \mathrm{~mm}$ in diameter, was placed on the sample holder, and the cap and sample weighed in 1), placed at its center.

3) The sample is located in the oxygen index apparatus and the oxygen atmosphere in the burning tube is adjusted for the test.

4) The sample is ignited by a burner.

5) A residual weight after burning is measured, and the burn-up fraction is calculated using the following expression.

$$
\text { Burn -up fraction }=\frac{\text { sample weight }- \text { residual weight }}{\text { sample weight }} \times 100
$$

6) Change the oxygen concentration in intervals of about $1 \%$ between the start point when burn-up fraction is increasing, and the end point when it attains roughly a fixed value: The operational procedure from 1) to 5) is repeated.

7) A maximum burn-up fraction is determined and the conversion burn-up fraction is calculated from the following expression.

$$
\text { Conversion burn - up fraction }=\frac{\text { burn }- \text { up fraction }}{\text { maximum burn - up fraction }} \times 100
$$

8) The conversion burn-up fraction is plotted against oxygen concentration and an S-type curve fitted to the data. The conversion burn-up fraction at $50 \%$ is taken as the oxygen index of the sample.

In addition, a stick and film samples were tested following the method prescribed in JS K 7201 .

Furthermore, burning behavior was recorded on video and photographs were taken during the tests.

The method to determine the oxygen index is shown graphically in figure 3 .

\section{Results}

It can be expected that the relation between oxygen concentration and burn-up fraction will be an S-type curve. This is based on the assumption that the curve is a logistic curve.

The Results for oxygen index as measured by the new method are shown in table 1. Oxygen index values determined by fitting an S-type curve to the relation between oxygen concentration and conversion burn-up fraction are compared to oxygen index values from the literature.

The following text explains how cap size was determined by using liquid paraffin, discuses the burning behavior of each sample, and compares oxygen indexes measured by the new method proposed here to indexes measured by the conventional technique.

\subsection{Liquid paraffin}

Using liquid paraffin as sample No. 1, the influence on oxygen index of the size of the quartz cap was investigated. The results are shown in figure 4 . The oxygen index was also determined by the conventional method. 
Table 1 Oxygen index for granular synthetic resins

\begin{tabular}{|c|c|c|c|c|c|c|}
\hline $\begin{array}{c}\text { Sample } \\
\text { No. }\end{array}$ & Sample name & Configuration & $\begin{array}{c}\text { Diameter } \\
(\mathrm{mm})\end{array}$ & $\begin{array}{c}\text { Oxygen index } \\
\text { (1) }\end{array}$ & $\begin{array}{c}\text { Oxygen index } \\
(2)\end{array}$ & Reference $^{7.8)}$ \\
\hline 1 & Liquid paraffin & Liquid & - & 16.7 & 16.7 & - \\
\hline 2 & Aromatic hydrocarbon resin & Granular & 5.5 & 19.8 & 19.8 & - \\
\hline 3 & Polyethylene A & Granular & 3.6 & 20.2 & 20.3 & \multirow{5}{*}{$18.8 \sim 19.3$} \\
\hline 4 & Polyethylene A & Film & - & 21.3 & - & \\
\hline 5 & Polyethylene B & Granular & 3.6 & 18.6 & 18.2 & \\
\hline 6 & Polyethylene C & Granular & 3.8 & 19.4 & 19.3 & \\
\hline 7 & Polyethylene D & Granular & 4.0 & 18.9 & 18.8 & \\
\hline 8 & Polypropylene A & Granular & 4.0 & 18.3 & 18.2 & \multirow[t]{2}{*}{19.0} \\
\hline 9 & Polypropylene B & Granular & 3.9 & 19.2 & 19.1 & \\
\hline 10 & Polystyrene A & Granular & 3.3 & 20.8 & 20.7 & \multirow{3}{*}{18.1} \\
\hline 11 & Polystyrene B & Granular & 2.9 & 23.4 & 23.4 & \\
\hline 12 & Polystyrene $\mathrm{C}$ & Granular & 2.9 & 21.6 & 21.7 & \\
\hline 13 & Polymethyl methacrylate & Granular & 3.2 & 18.6 & 18.3 & $18.6 \sim 18.8$ \\
\hline 14 & Polyamide & Granular & 2.5 & 24.3 & 24.3 & 24 \\
\hline 15 & Polybutylene terephthalate & Granular & 3.4 & 24.5 & 24.3 & - \\
\hline 16 & ABS resin & Granular & 2.7 & 19.6 & 19.4 & $19 \sim 20$ \\
\hline 17 & Polycarbonate & Granular & 3.0 & 31.2 & 31.8 & $26 \sim 28$ \\
\hline 18 & Polyvinyl chloride $\mathrm{A}$ & Granular & 3.3 & 39.5 & 39.4 & \multirow{7}{*}{$45 \sim 49$} \\
\hline 19 & Polyvinyl chloride A & Stick & - & 33.0 & - & \\
\hline 20 & Polyvinyl chloride B & Granular & 2.9 & 39.2 & 39.1 & \\
\hline 21 & Polyvinyl chloride $B$ & Stick & - & 32.2 & - & \\
\hline 22 & Polyvinyl chloride C & Granular & 3.2 & 38.5 & 39.7 & \\
\hline 23 & Polyvinyl chloride C & Stick & - & 34.0 & - & \\
\hline 24 & Polyvinyl chloride D & Powder & - & 40.8 & 43.7 & \\
\hline 25 & Polyvinylidene fluoride & Granular & 3.5 & 58.0 & 56.1 & - \\
\hline 26 & Flluorocarbon resin A & Granular & 3.6 & $>90$ & $>90$ & \multirow[t]{2}{*}{95.0} \\
\hline 27 & Flluorocarbon resin B & Granular & 3.2 & $>90$ & $>90$ & \\
\hline
\end{tabular}

Oxygen Index (1): Values determined by fitting an S-type curve to the relation between oxygen concentration and conversion burn-up fraction as a rough estimate.

Oxygen Index(2): Values determined by fitting a logistic curve to this relation using a least squares method.

Each point in figure 4 is the average value of the oxygen index measured 3 separate times. This figure indicates that when the diameter of the quartz cap is small, the heat loss is large and oxygen index increases. When the cap size is $20 \mathrm{~mm}$, the oxygen index is approximately constant. It was therefore concluded that it is suitable to use a quartz cap of $20 \mathrm{~mm}$ diameter.

The influence of depth of the cap was not checked. For deeper caps, the sample quantity increases, and the flame-contact time is longer.

Based on the above, the oxygen index of the remaining samples was measured using a quartz cap of $20 \mathrm{~mm}$ diameter, depth $2 \mathrm{~mm}$.

\subsection{Aromatic hydrocarbon resin}



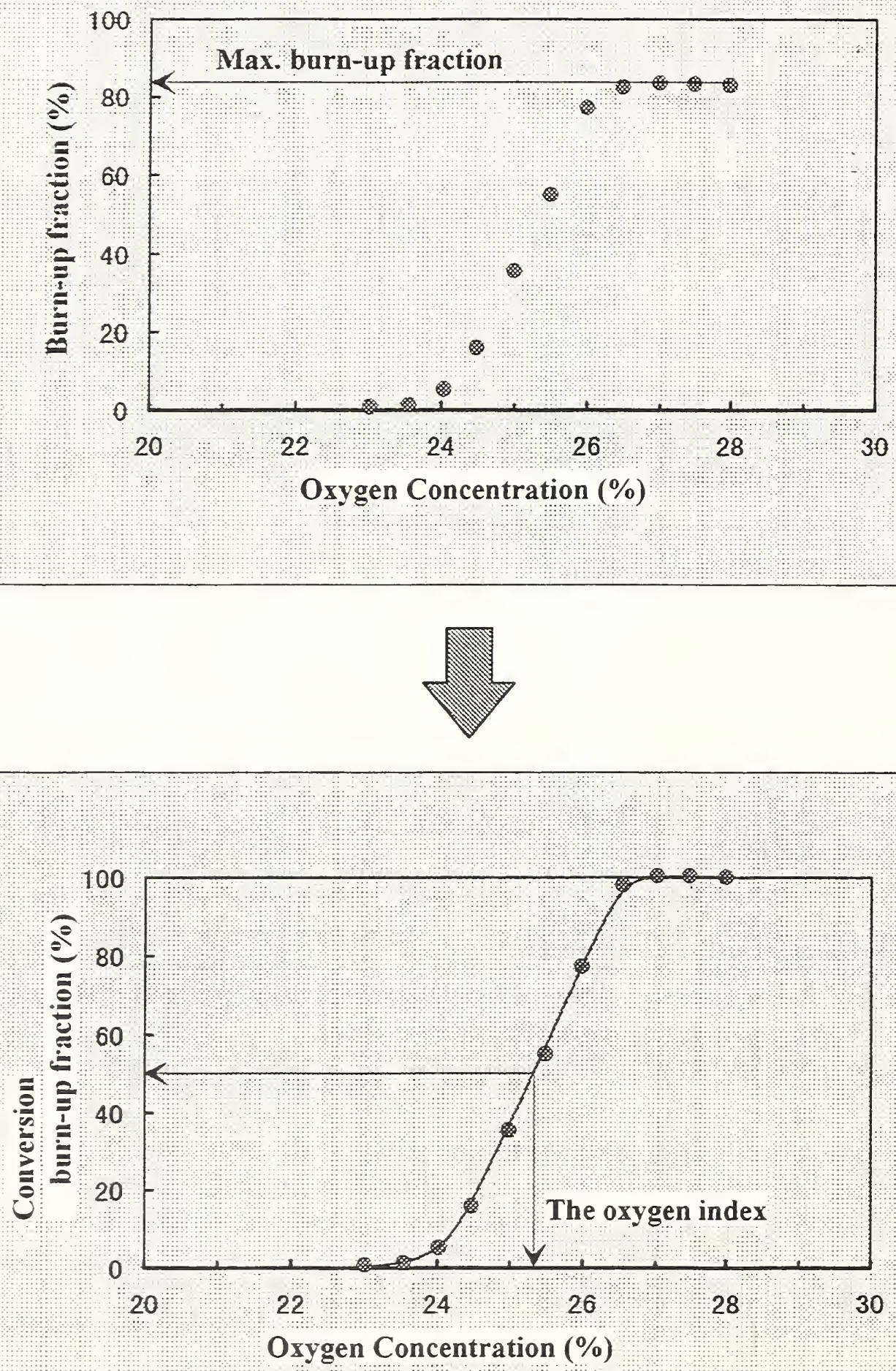

Fig 3 Determination of the oxygen index 


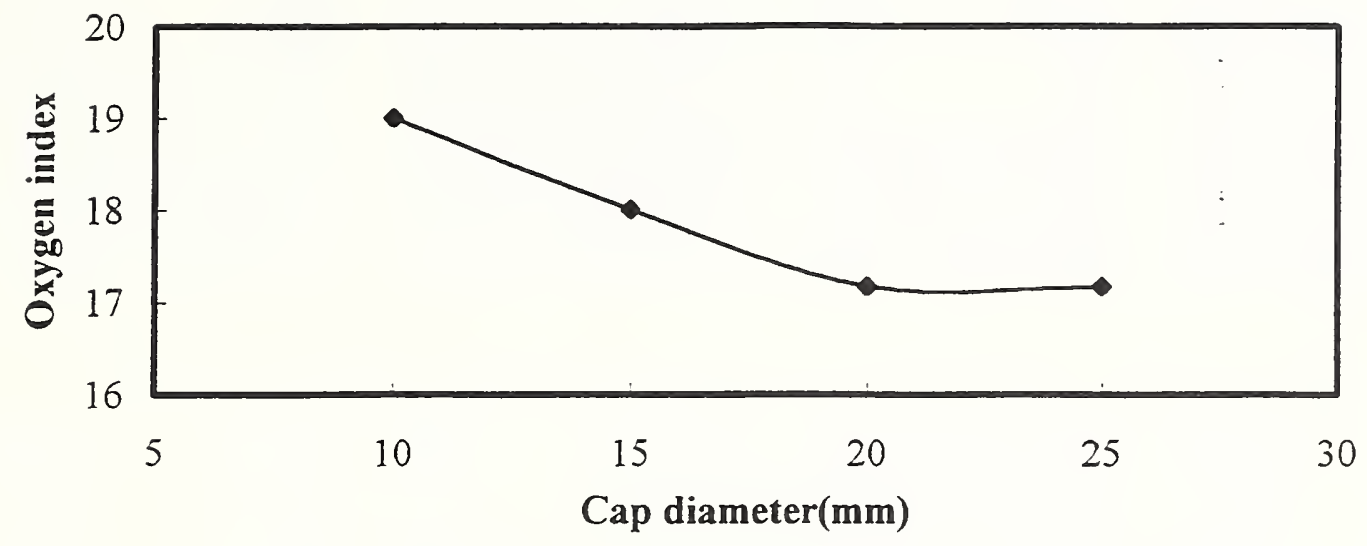

Fig.4 The effect of cap diameter on the oxygen index

Polymerizing resins of materials such as indene, vinyltoluene and the present sample are used as the raw materials such as paints, rubber combination agents, printing ink, an adhesives. The melting point of this resin is low, and it changed readily into the liquid state when a pilot flame was placed in contact with the sample.

After melting entirely, on contact with the pilot flame, it ignited. In some cases the sample took abut 5 minutes to form a stable flame on contact with the pilot flame. The flame had a yellow luminosity and generated soot from its tip. Burning behavior near the oxygen index is shown in figure 5 (b). The oxygen index value was 19.8 .

\subsection{Polyethylene}

5 types of polyethylene No. 3 7 were prepared as samples. No.4 is the only film-shaped sample, the others were all granular samples. No.5 is a high-density polyethylene from a different manufacture to that of No.3 and No.4. No.6 is a low-density polyethlene. No.7 is different again from the above-mentioned polyethylenes.

Granular samples melted on contact with the pilot flame and changed into a liquid state. The time from flame contact to the formation of a stable flame, that is, the flame-contact time, was the same as for the aromatic hydrocarbon resin above. The flame had little yellow luminosity, but was pale and nearly transparent. Burning behavior near the oxygen index for sample No. 3 is shown in figure 5 (c).

Oxygen indexes of samples No.3 $\sim 7$ were found to be $20.2,21.3,18.6$, 19.4 , and 18.9 , respectively. When the oxygen index of No. 3 was compared with that of No.4, the value of No.4 (film-shaped sample) was about 1 point higher than that of No.3. This may well be due to its different form. The oxygen index of No.3 was slightly higher than the literature value of polyethlene; $18.8 \sim 19.3{ }^{7)}$. The others were the same as the literature values. 


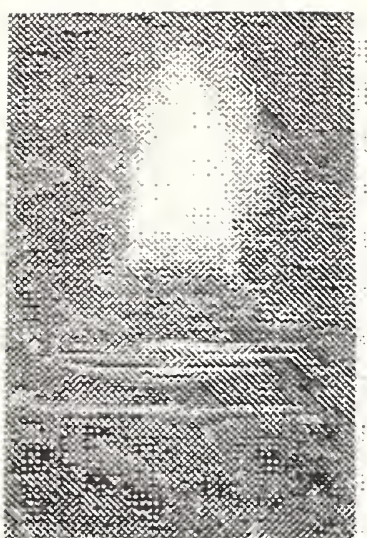

(a)

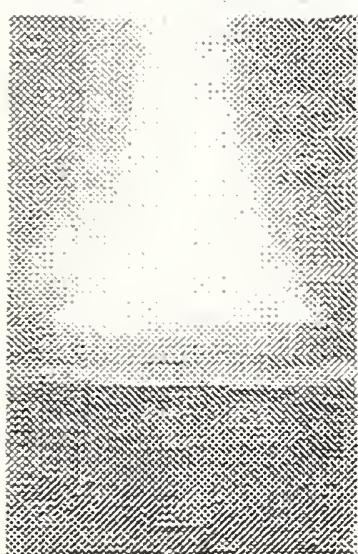

(e)

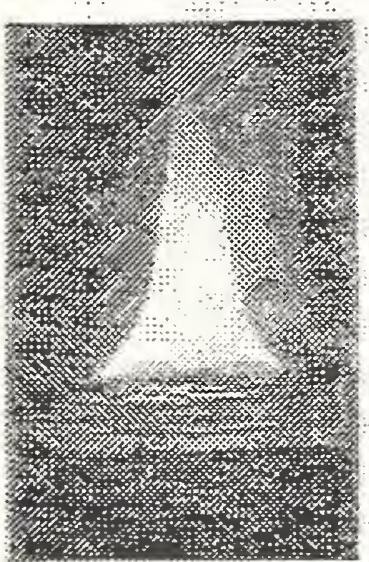

(i)

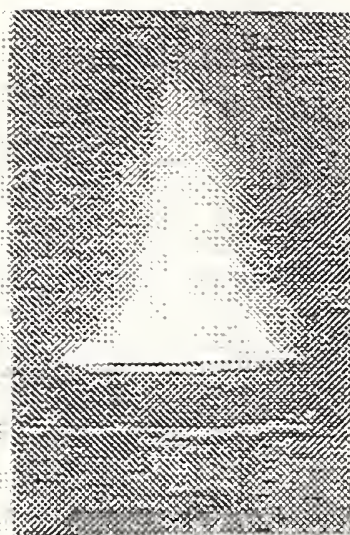

(b)

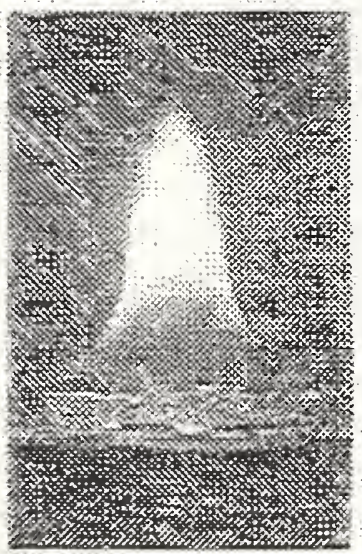

(f)

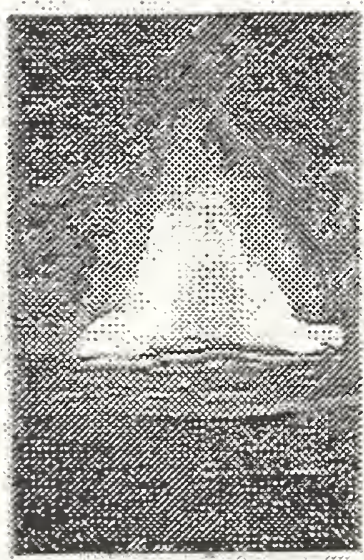

(j)

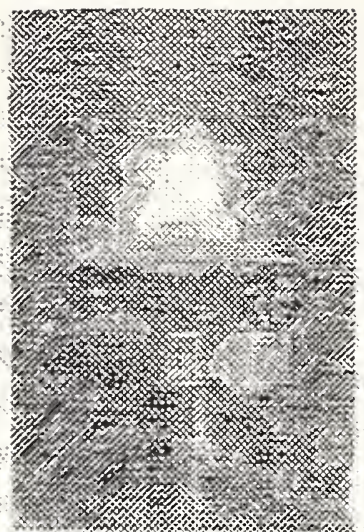

(c)

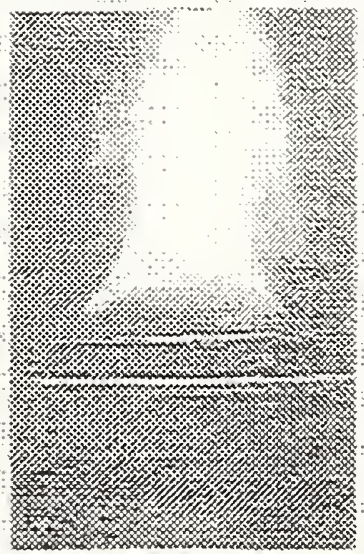

(g)

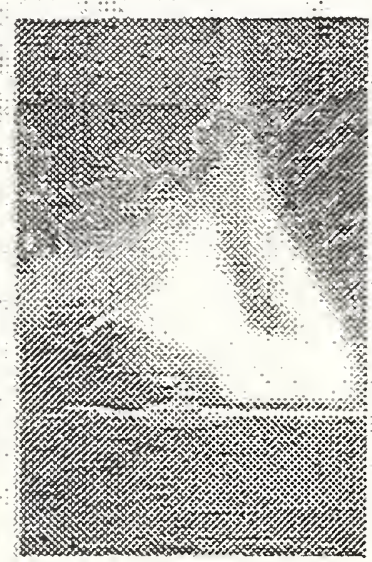

(k)

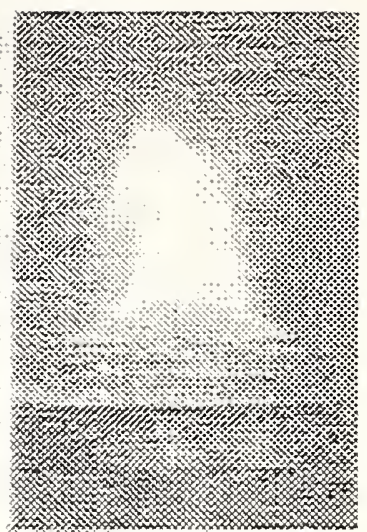

(d)

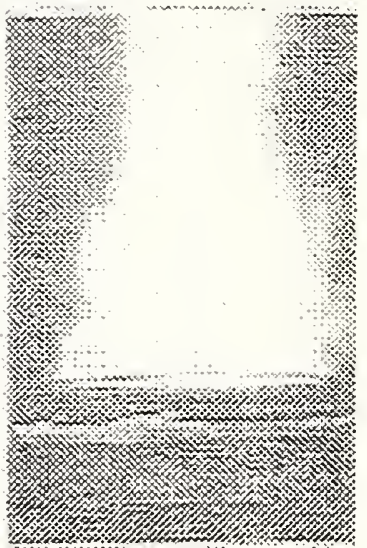

(h)

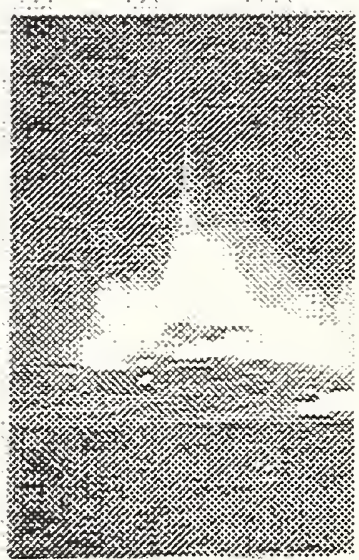

(1)

Figure 5 Burning behavior near the oxygen index for granular synthetic resins.

(a) Liquid paraffin (b) Aromatic hydrocarbon resin (c) Polyethylene

(d) Polypropylene (e) Polystyrene (f) Polymethyl methacrylate

(g) Polyamide (h) Polybutylene terephthalate (i) ABS resin

(j) Polycarbonate (k) Polyvinyl chloride (1) Polyvinylidene fluoride 


\subsection{Polypropylene}

2 types of Polypropylene, No.8 and No.9, were prepared as samples. These samples melted on contact with the pilot flame and changed into a liquid state in the same way as the polyethylene. The flame-contact time and the burning behavior were also similar to polyethylene. Burning behavior near the oxygen index for sample No. 8 is shown in figure 5 (d).

The oxygen indexes of samples No. 8 and No. 9 were 18.3, and 19.2, respectively. The oxygen index values were the same as the literature values.

\subsection{Polystyrene}

3 types of Polystyrene, including a styrene monomer about 400ppm $650 \mathrm{ppm}$ as an impurity, were prepared as samples. The ignition behavior of polystyrene differed from that of the polyethylene. It ignited easily, before melting on contact with the pilot flame. The flame had a yellow luminosity and produced soot in large quantities. Burning behavior near the oxygen index for sample No.10 is shown in figure $5(e)$.

Oxygen indexes of samples No. 10, No.11 and No.12 were 20.8, 23.4, and 21.6 , respectively. There are about $3 \sim 5$ points higher than the literature values.

\subsection{Polymethyl methacrylate}

1 type of sample No. 13 was prepared. Ignition behavior was similar to that of polystyrene. That is, before it had entirely melted on contacting with the pilot flame, it readily ignited. Ignition time was within 3 minutes. Burning behavior near the oxygen index for sample No. 13 is shown in figure 5 ( $f$ ).

The oxygen index of sample No.13 was measured as 18.6. It was almost the same as the literature value, $18.6 \sim 18.8$ 8).

\subsection{Polyamide}

1 type of sample No.14 was prepared. Ignition behavior was like that of polyethylene. After it had entirely melted on contact with the pilot flame, it ignited. The flame had a yellow luminosity and produced soot. Burning behavior near the oxygen index for sample No.14 is shown in figure $5(\mathrm{~g})$.

The oxygen index of sample No.14 was measured as 24.3. It was almost the same as the literature value, $24.0^{8}$.

\subsection{Polybutylene terephthalate}

1 type of sample No.15 was prepared. Ignition behavior was like that of polyethylene. After it had entirely melted on contact with the pilot flame, it ignited. The flame had a yellow luminosity and produced soot. Burning behavior near the oxygen index for sample No. 15 is shown in figure 5 (h).

The oxygen index of sample No.15 was measured as 24.5.

\subsection{ABS resin}

1 type of sample No.16 was prepared. Ignition behavior was similar to that of polystyrene. Before it had melted perfectly on contact with the pilot flame, it readily ignited. The flame had a yellow luminosity and produced soot in large 
quantities. Burning behavior near the oxygen index for sample No.16 is shown in figure 5 (i).

The oxygen index of No.16 was measured as 19.6. It was almost the same as the literature value, $19 \sim 20{ }^{8)}$.

\subsection{Polycarbonate}

1 type of sample, No.17 was prepared. It ignited easily on contact with the pilot flame. After the sample had burned, a carbonized residue was generated on the surface of the sample. Burning behavior near the oxygen index for sample No. 17 is shown in figure $5(\mathrm{j})$.

The oxygen index of No.17 was measured as 31.2. It was about $3 \sim 5$ points higher than the literature value, $26 \sim 28{ }^{7}$.

\subsection{Polyvinyl chloride}

6 types of sample No. 18 23 were prepared. No.18, No.20, and No.22 are granular samples. No. 19, No.21 and No.23 are stick-shaped samples. Moreover, No. 18 and No.19, No. 20 and No.21, and No. 22 and No.23 are the same materials, respectively.

They ignited easily on contact with the pilot flame. The flame emitted a white flash and generated a carbonized residue. Burning behavior near the oxygen index for sample No.18 is shown in figure $5(\mathrm{k})$.

The oxygen indexes of samples No.18, No.19, No.20, No.21, No.22 and No.23 were measured as $39.5,33.0,39.2,32.2,38.5,34.0$, respectively. When the oxygen index of granular samples were compared with the values of stick-shaped sample for the same material, it was found to be about $4.5 \sim 7$ points higher. This is mainly caused by the different forms. After the sample had burnt, a carbonized residue was generated within the cup. Further burning was prevented by the carbonized residue. When tested in a stick-shaped form, carbonized residue is generated, but, this does not prevent the sample from burning further. For samples generating a carbonized residue, as measured using the proposed oxygen index method, the oxygen index of granular sample can be expected to be higher than the values of stick-shaped samples. This is a characteristic of granular carbonized synthetic resins and is considered to be an essential flammability property of these resins.

\subsection{Polyvinylidene fluoride}

1 type of sample No. 25 was prepared. It ignited easily, in the same way as polyvinyl chloride on contact with the pilot flame. The flame had a yellow luminosity and generated a carbonized residue on the sample surface. Burning behavior near the oxygen index for sample No.25 is shown in figure 5 (1).

The oxygen index of No.25 was measured as 58.0.

\subsection{Flluorocarbon resin}

2 types of sample No. 26 and No.27 were prepared. Though they were tested in the largest oxygen concentration of the equipment, $90 \%$, they merely evaporated and did not ignite. Measurement of an oxygen index was therefore not 
possible for these samples. The oxygen index of fluorocarbon resin was 95.0 from the literature ${ }^{\text {) }}$.

\section{Discussion}

\subsection{Fitting the S-type curve}

This relation is concerned with the oxygen index (1) as determined by fitting an S-type curve to the relation between oxygen concentration and conversion burn-up fraction, as a rough estimate, and oxygen index (2) determined by fitting a logistic curve to these relations using a least squares method. The logistic curve is given by the following equation.

$$
Y=\frac{100}{1+\exp [-b(X-a)]}
$$

Where $\mathrm{a}$ and $\mathrm{b}$ are calculated by the least squares method, $\mathrm{Y}$ is the conversion burn-up fraction and $\mathrm{X}$ is oxygen concentration.

Oxygen index (2) was compared with oxygen index (1). The relation between these oxygen index values is shown in figure 6 . The dotted straight line in the figure shows the relation between oxygen index (1) and oxygen index (2) to be 1:1. These data almost lies on this straight line. Therefore, it is concerned that the method for determining oxygen index by fitting an S-type curve as a rough estimate, is sufficient for practical purpose. Naturally there will be a difference between operators of the test, so in order to reduce scatter we must take care of the following.

1) When conversion burn-up fraction changes rapidly, the oxygen concentration must be altered slowly.

2) Samples with large scatter in oxygen index must be tested $3 \sim 5$ times with identical oxygen concentrations.

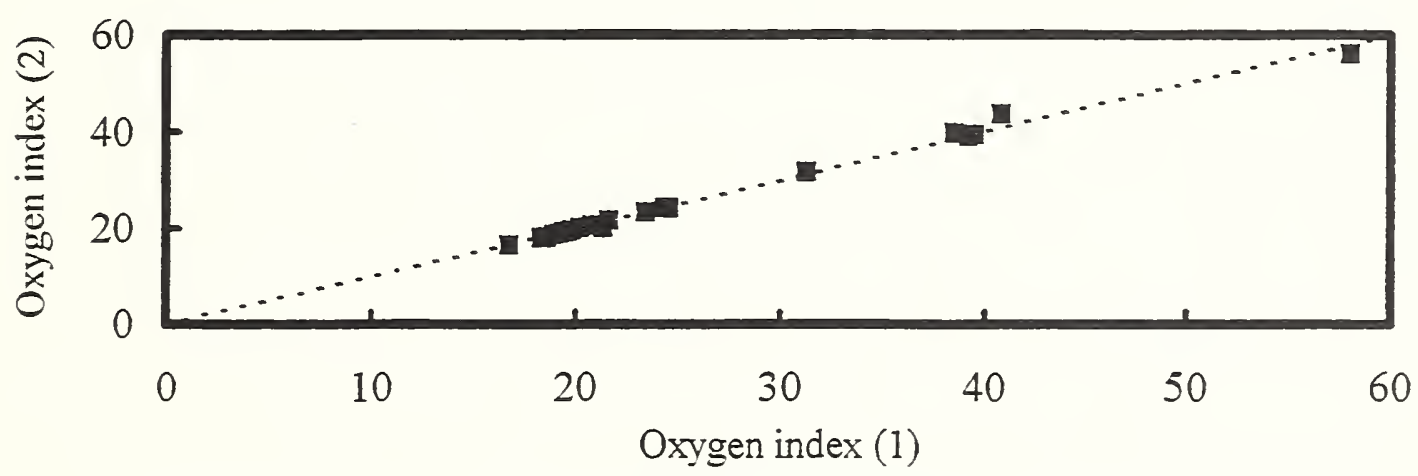

Fig. 6 Relation between oxygen index determined by fitting an S-type curve, as a rough estimate, and fitting a logistic curve using the least squares method.

\subsection{Classification by heat property of the resin}

The samples mentioned in this paper can be classified into three groups by their heat properties on contact with the pilot flame. Aromatic hydrocarbon resin, polyethylene, polypropylene, polyamide and polybutylene terephthalate fall into 
the first group. These begin to ignite after entirely melting on contact with the pilot flame. Their oxygen index values were nearly the same as the literature values. This is because their burning behaviors is similar to that of stick samples tested using the conventional oxygen index method.

Polymethyl methacrylate, polystyrene and ABS resin fall into the second group. These materials are readily ignited before entirely melting. Their oxygen index values were almost the same as the literature values or slightly higher. It appears that they burn without losing granular form.

Polyvinyl chloride, polycarbonate and polyvinylidene fluoride fall into the third group. These materials are ignited also readily on contact with the pilot flame and generate a the carbonized residue on the sample surface. Their oxygen index values were considerably higher than the literature values. This is caused by the covering of the sample surface by the carbonized residue, considered to reflect the flammability property of these granular carbonized synthetic resins.

\section{Conclusion}

The conventional oxygen index method was modified for testing granular samples. 27 types of granular resin were tested using this modified test method. The result are as follows:

1) Oxygen index values of melting granular samples, such as polyethylene, are almost the same as those of their stick sample.

2) Oxygen index values of the easily ignitable and melting granular samples, such as polymethyl methacrylate, are almost the same as, or slightly higher, than those of their stick sample.

3) Oxygen index values of carbonizing granular samples, such as polyvinyl chloride, are considerably higher than those of their stick sample.

\section{Reference}

1) JS K 7201, Testing Method for Flammability of Polymeric Materials Using the Oxygen Index Method, (1976) (in Japanese)

2) A.Tewarson and R.F.Pion, Flammability of Plastics - 1. Burning Intensity, COMBUSTION AND FLAME, 26, p.85(1976)

3) A.Tewarson, Heat Release Rate in Fires, FIRE AND MATERIALS, Vol.4, No.4, p.185(1980)

4) J.L.Isaacs, The Oxygen Index Flammability Test, J. FIRE \& FLAMIMABILITY, Vol.1, p.36, (1976)

5) L.Cegielka MA, The Burning Behaviour of Textiles and its Assessment by Oxygen-index Methods, Textile Volume 18 Number 1/2/3, The Textile Institute, (1989)

6) The Catalogue KAOWOOL ACE-PAPER, Isoraito Ltd. (in Japanese)

7) Flammability Handbook for Plastics: CARLOS J. Hilado, TECHNOMIC, p.40(1969)

8) Plastic Data Handbook edited by K. Itou, Kougyoutyousakai,p.111(1980) (in Japanese) 


\section{Discussion}

James Quintiere: Could you comment on the character of the flame, particularly on the Cone flame. Was it turbulence or laminar?

Eiji Yanai: Laminar.

Richard Lyon: Do I understand that you're determining not the oxygen concentration when the flame goes out, but a 50\% weight loss concentration. What is the basis for that assumption?

Eiji Yanai: The definition of oxygen index is the boundary between whether it burns or it does not. If you look at a granular substance, which is not an ordinary sample for determining the oxygen index, we thought it was appropriate to get a $50 \%$ loss of weight.

Richard Lyon: So do you notice that the sample stops burning when it reaches a $50 \%$ weight loss peak? Did you notice that it didn't bum at that point and that was the reason for picking that particular criteria?

Eiji Yanai: Are you asking whether we took into consideration that when we ignited, it was burned already?

Richard Lyon: No. Was the point at which the sample would no longer sustain burning, was that equal to the time point at which the weight loss was $50 \%$ ? Did the flame go out when the weight loss was $50 \%$ ?

Eiji Yanai: The flame doesn't go away. It's not necessary that the flame goes out after $50 \%$. It's a different oxygen index. 
$24 \underline{8}$ 


\title{
ADVANCED FIRE SAFE MATERIALS FOR AIRCRAFT INTERIORS
}

\author{
Richard E. Lyon \\ Fire Research Section, AAR-423 \\ Federal Aviation Administration Technical Center \\ Atlantic City International Airport, N.J. 08405 USA
}

\begin{abstract}
Phenomenological equations for solid material burning in combination with pyrolysis kinetics and uncoupled transient heat conduction provide relationships between material properties and the fire response of polymeric solids. The predicted scaling of ignition temperature, time to ignition, heat of gasification, mass loss rate, and heat release rate with thermodynamic, kinetic, and transport properties is in reasonable agreement with available data for polymeric solids using this simple approach.
\end{abstract}

\section{INTRODUCTION}

Heat release rate is considered to be the single most important fire parameter in determining the fire hazard of a material. Unfortunately, no analytic results for heat release rate in terms of chemical or physical properties of materials are available to guide chemists in their syntheses of fire resistant polymers. Current material fire models are computational loops in computerbased fire codes. Material parameters required by these computational fire models include at a minimum the chemical heat of complete combustion of the material, a combined heat transport parameter (thermal inertia), and a "decomposition temperature" at which the material becomes a gaseous fuel. In a few sophisticated fire codes two additional pyrolysis kinetic parameters may be required to complete the material model. These material fire models have no physical basis outside of the computational regime and are of little value to material scientists seeking a qualitative understanding of how thermodynamic, kinetic, mechanical, and transport properties of polymeric materials effect their fire response. In order to provide some physical insight into the phenomenology of materials combustion, simple scaling relationships between tailorable material properties and the fire response of polymeric solids are developed and verified in the following sections. It is hoped that these analytic results will help guide the development of totally fire resistant materials for next generation aircraft interiors.

\section{RATE-DEPENDENT DECOMPOSITION TEMPERATURE OF POLYMERS}

The peak mass loss rate (decomposition) temperature for a thermally decomposing polymer is obtained from the appropriate kinetic expression. For the first-order pyrolysis of a polymer with volatile mass, $\mathrm{m}$, and non-volatile (char) mass, $\mathrm{m}_{\mathrm{c}}$,

$$
-\frac{\mathrm{dm}}{\mathrm{dt}}=\mathrm{k}\left(\mathrm{m}-\mathrm{m}_{\mathrm{c}}\right)
$$

Assuming an Arrhenius rate constant,

$$
\mathrm{k}=\mathrm{A} \exp \left(-\mathrm{E}_{\mathrm{a}} / \mathrm{RT}\right)
$$


and a constant heating rate, $\mathrm{dT} / \mathrm{dt}=\dot{\mathrm{T}}$, the peak pyrolysis temperature is obtained by setting the time derivative of the mass loss rate in Equation 1 (second time derivative of mass) equal to zero at $\mathrm{T}=\mathrm{T}_{\mathrm{p}}{ }^{\max }$, i.e.,

$$
-\ddot{\mathrm{m}}=\mathrm{k}_{\mathrm{p}} \dot{\mathrm{m}}+\left(\mathrm{m}-\mathrm{m}_{\mathrm{c}}\right) \dot{\mathrm{k}}_{\mathrm{p}}=0 @ \mathrm{~T}=\mathrm{T}_{\mathrm{p}}^{\max } .
$$

The resulting non-dimensionalized equation in terms of $T_{P} \max$ is [1]

$$
\ln \left[\frac{E_{a}}{\mathrm{RT}_{\mathrm{p}}^{\max }}\right]^{2}+\frac{\mathrm{E}_{\mathrm{a}}}{\mathrm{RT}_{\mathrm{p}}^{\max }}+\ln \left[\frac{\mathrm{R} \dot{\mathrm{T}}}{\mathrm{AE}_{\mathrm{a}}}\right]=0
$$

Solution of Equation 3 for the dimensionless root, $\left[E_{a} / R T_{p} \max \right]$ for a particular heating rate, $\dot{T}$, gives the temperature at maximum mass loss rate with the use of the Arrhenius parameters, $\mathrm{A}$, $E_{a}$. A plot of calculated $T_{p} \max$ vs. $T$ for polymethylmethacrylate (PMMA, $A=10^{12} / s, E_{a}=160$ $\mathrm{kJ} / \mathrm{mol}$ ) is shown in Figure 1.

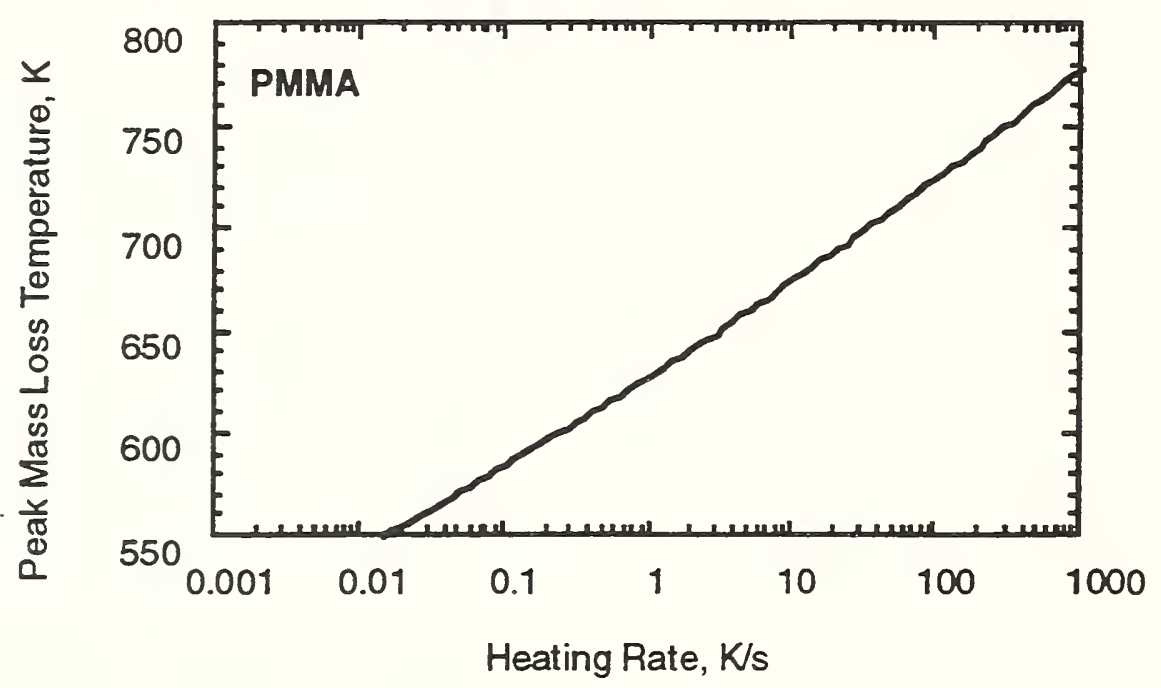

Figure 1. Temperature at peak mass loss rate versus linear beating rate for PMMA according to first-order kinetics.

\section{IGNITABILITY}

The thermal theory of ignition assumes that ignition occurs when the surface temperature of the material reaches its ignition temperature, i.e., $t=t_{i g n}$, at,$T_{s}=T_{i g n}$., at which time and temperature sufficient fuel generation takes place to sustain flaming combustion. Consequently, this ignition temperature is on the order of a thermal decomposition temperature for the material. In the previous section it was shown that the peak decomposition temperature of a polymer has a strong dependence on heating rate in the region 1-100 K/s typically encountered in fire testing.

The surface temperature history for a semi-finite slab with a constant net heat flux to the surface, $\dot{Q}_{n e t}$, is

$$
\mathrm{T}_{\mathrm{s}}(\mathrm{t})=\mathrm{T}_{\mathrm{o}}+2 \mathrm{Q}_{\mathrm{net}}\left[\frac{\mathrm{t}}{\pi \kappa p \mathrm{C}_{\mathrm{p}}}\right]^{1 / 2}
$$


with $T_{s}$ the surface temperature at time $t$, and $T_{o}$ the initial (ambient) temperature of the material. Rearranging Equation 4 with the assumption that the surface temperature at ignition is the (rate dependent) peak pyrolysis temperature, $T_{s}=T_{i g n}=T_{p}{ }^{\max }$, gives for the time to ignition

$$
t_{\text {ign }}=\pi \kappa \rho C_{p}\left[\frac{T_{p}^{\max }-T_{0}}{2 \dot{Q}_{\text {net }}}\right]^{2}
$$

Equation 5 calculates the time to ignition of a thermally thick polymer specimen using the rate (heat flux) dependent peak pyrolysis temperature for a particular $\dot{Q}_{n e t}$. The heating rate at the surface of a thermally thick polymer specimen during radiant heating is not constant, but may be approximated by a single time-average rate of temperature rise, $\langle\mathrm{dT} / \mathrm{dt}\rangle$. The semi-infinite slab result valid up to time, $\tau=\rho C_{p} b^{2} / 4 K$, for a net heat flux, $\dot{Q}_{\text {net }}$ on the face of a slab of thickness, $b$, density, $\rho$, thermal conductivity, $\kappa$, and heat capacity, $C_{p}$, gives for the timeaveraged heating rate [1]

$$
\dot{\mathrm{T}}=\left\langle\frac{\mathrm{dT}}{\mathrm{dt}}\right\rangle=\frac{1}{\tau} \int_{0}^{\tau} \frac{\dot{\mathrm{Q}}_{\text {net }}}{\sqrt{\pi \mathrm{k} \rho \mathrm{C}_{\mathrm{p}}}} \frac{\mathrm{dt}}{\sqrt{\mathrm{t}}}=\frac{4}{\sqrt{\pi}} \frac{\dot{\mathrm{Q}}_{\mathrm{net}}}{\rho b \mathrm{C}_{\mathrm{p}}}
$$

The results of Equations 6 and 3 for poly(methylmethacrylate) (PMMA) exposed to net heat flux levels from 5 to $75 \mathrm{~kW} / \mathrm{m}^{2}$ were substituted into Equation 5 and the results plotted in Figure 2. It is seen that a rate-dependent decomposition temperature correlates the measured time-to-ignition for PMMA over a range of incident heat flux levels [2] using $A=1012 / \mathrm{s}, \mathrm{E}_{\mathrm{a}}=$ $160 \mathrm{~kJ} / \mathrm{mol}$ [3] for the Arrhenius parameters, and $\kappa \rho C_{p}=6 \times 10^{5} \mathrm{~W}^{2}-s / \mathrm{m}^{4}-\mathrm{K}^{2}$ [4] for the thermal capacitance. At external heat fluxes below about $10 \mathrm{~kW} / \mathrm{m}^{2}$ the semi-infinite slab conduction solution (Equation 4) over predicts the surface temperature (under predicts time-totemperature) so that a finite-slab calculation which takes into account heat losses from the rear surface at the longer heating times must be used to obtain an accurate surface temperature history. The finite slab calculation (not shown) indicates that below about $8 \mathrm{~kW} / \mathrm{m}^{2}$ incident heat flux the surface temperature of PMMA never reaches the peak decomposition temperature, in general agreement with a measured critical heat flux of about $10 \mathrm{~kW} / \mathrm{m}^{2}[5]$.

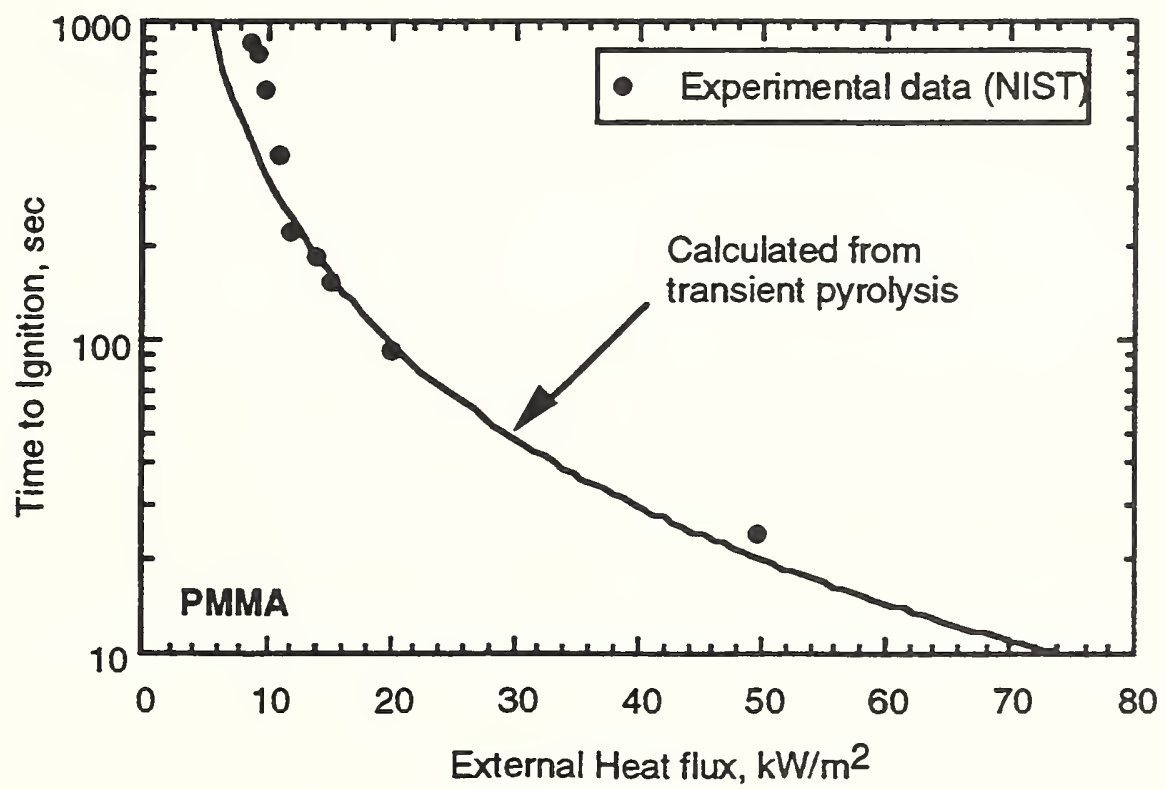

Figure 2. Calculated and measured time-to-ignition for PMMA assuming $T_{i g n}=T_{p}$ max 
Figure 2 demonstrates that the time to ignition of a material (PMMA) can depend strongly on the net heat flux to the surface and that this effect is reasonably well predicted by transient conduction with a heating rate effect on the peak pyrolysis temperature.

In the previous treatment it was assumed that, $T_{p} \max =T_{s}$, for flaming combustion By the same reasoning if $T_{p} \max >T_{s}$, i.e., the pyrolysis temperature of the material is above the equilibrium surface temperature for flaming combustion at a particular incident heat flux, then the fuel volatilization rate may be insufficient to support burning of the sample. Equation 7 gives the equilibrium surface temperature of a specimen in terms of $\dot{Q}_{\text {net }}$, the sample emissivity, $\varepsilon$, and the surface convective heat transfer coefficient, $\bar{h}[5]$

$$
\dot{Q}_{\text {net }}=\varepsilon \sigma\left(T_{\infty}^{4}-T_{0}^{4}\right)+\bar{h}\left(T_{\infty}-T_{0}\right)
$$

For a horizontal specimen with, $\varepsilon=0.8$, and surface convective heat transfer coefficient, $\bar{h}=24$ $\mathrm{W} / \mathrm{m}^{2}-\mathrm{K}$ it is found that, $T_{\infty} \approx 675 \mathrm{C}$ at a net surface heat flux, $\dot{Q}_{\text {net }}=50 \mathrm{~kW} / \mathrm{m}^{2}$. Consequently, materials tested in a cone calorimeter with a peak pyrolysis temperature greater than about $675 \mathrm{C}$ would not be expected to burn at a net surface heat flux of $50 \mathrm{~kW} / \mathrm{m}^{2}$. Figure 3 shows the measured rate of heat release at an external heat flux of $50 \mathrm{~kW} / \mathrm{m}^{2}$ for a variety of polymeric materials for which reasonable values of both the 180 s average heat release rate and the decomposition temperature are available. It is seen that as the pyrolysis temperature of the material approaches the equilibrium surface temperature for a particular surface heat flux the heat release rate approaches zero.

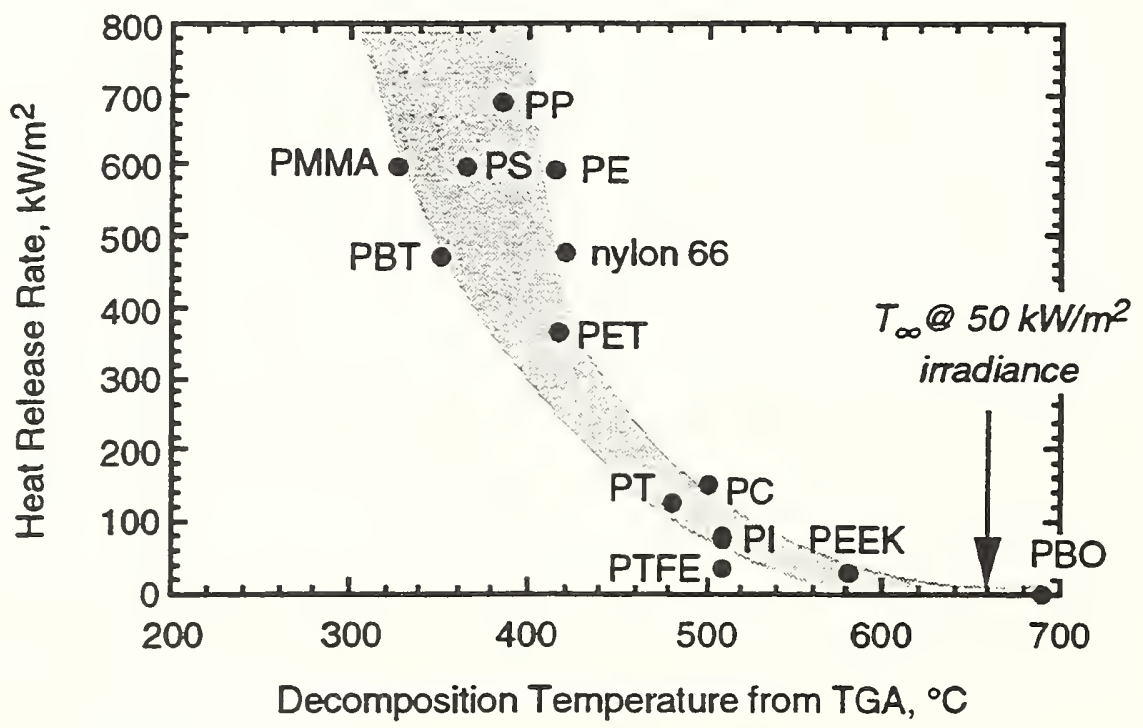

Figure 3. Average heat release rate at $50 \mathrm{~kW} / \mathrm{m}^{2}$ irradiance versus temperature at peak mass loss rate for a variety of polymers.

\section{MASS LOSS RATE}

To provide insight into the physical phenomenon governing heat release and smoke generation rates of materials in fires an analytic equation was developed for the onedimensional, steady-state mass loss rate of a burning polymer. The approach is to propagate the isothermal layer through the material via uncoupled thermal diffusion and calculate the mass loss rate at each time and temperature from isothermal pyrolysis kinetics. The dynamic mass loss problem is formulated as a steady-state process characterized by a constant surface (pyrolysis zone) temperature, $\mathrm{T}_{\mathrm{S}}=\mathrm{T}_{\mathrm{P}} \max$. The simplifying assumption of a constant surface 
temperature during steady-state mass loss (burning) implies dynamic equilibrium at the gassolid interface and eliminates the need to determine the complete energy and species balance to solve the geometric problem.

From Equation 1 with initial conditions; $\mathrm{m}=\mathrm{m}_{\mathrm{o}} @ \mathrm{t}=0$, the isothermal mass loss history for a polymer of initial mass, $m_{0}$, is

$$
\frac{\mathrm{m}}{\mathrm{m}_{\mathrm{o}}}=\mathrm{Y}_{\mathrm{c}}+\left(1-\mathrm{Y}_{\mathrm{c}}\right) \mathrm{e}^{-\mathrm{kt}}
$$

with, $Y_{c}=m_{c} / m_{0}$, the char yield of the polymer. From Equation 8 the fractional mass loss rate is

$$
-\frac{\mathrm{dm}(\mathrm{t})}{\mathrm{dt}}=k \mathrm{~m}_{\mathrm{o}}\left(1-\mathrm{Y}_{\mathrm{c}}\right) \mathrm{e}^{-\mathrm{kt}}
$$

Since the surface area (S) and volume (V) of a planar specimen are related to thickness, $\delta$, as, $\mathrm{S}=\mathrm{V} / \delta$, the areal mass loss rate from Equation 9 is

$$
\dot{\mathrm{m}}_{\mathrm{A}}=-\frac{1}{\mathrm{~S}} \frac{\mathrm{dm}(\mathrm{t})}{\mathrm{dt}}=\mathrm{k} \delta \frac{\mathrm{m}_{\mathrm{o}}}{\mathrm{V}}\left(1-\mathrm{Y}_{\mathrm{c}}\right) \mathrm{e}^{-\mathrm{kt}}=\mathrm{k} \delta \rho\left(1-\mathrm{Y}_{\mathrm{c}}\right) \mathrm{e}^{-\mathrm{kt}}
$$

where $m_{0} / V=\rho$ is the initial material density. We assume that pyrolysis is confined to a quasiisothermal layer or pyrolysis zone of depth, $\delta$, whose average temperature is within $\pm 5 \%$ of the surface temperature and calculate how the mass loss rate within this layer changes with time. For a semi-infinite slab with a constant flaming surface temperature, $T_{s}$, the depth at which the temperature drop through the layer is less than $10 \%$ of the total temperature drop through the material is

$$
\delta \cong \frac{\sqrt{\alpha t}}{5}
$$

where $\alpha=\kappa / \rho C_{p}$ is the thermal diffusivity of the material, assumed to be independent of temperature and conversion. Substituting for the quasi-isothermal layer depth, $\delta$, in Equation 10

$$
\dot{\mathrm{m}}_{\mathrm{A}}=\frac{\rho \sqrt{\alpha}}{5}\left(1-\mathrm{Y}_{\mathrm{c}}\right)\left[\mathrm{kt}^{1 / 2} \mathrm{e}^{-\mathrm{kt}}\right]
$$

The mass loss rate history in the isothermal layer (Equation 11) is plotted in Figure 4 for a polymer of low thermal stability (PMMA) and a high temperature polymer, poly(benzobisoxazole)(PBO), using tabulated values for Arrhenius and physical parameters and approximate surface temperature at ignition (assumed equal to the peak pyrolysis temperature) at $\dot{Q}_{\text {net }}=50 \mathrm{~kW} / \mathrm{m}^{2}$.

PMMA; $\quad A=10^{12}, E_{a}=160 \mathrm{~kJ} / \mathrm{mol}, T=T_{s}=400 \mathrm{C}, \alpha=1.3 \times 10^{-7} \mathrm{~m}^{2} / \mathrm{s}, \rho=1000 \mathrm{~kg} / \mathrm{m}^{3}, Y_{c}=0$ PBO; $A=10^{14}, E_{a}=290 \mathrm{~kJ} / \mathrm{mol}, T=T_{s}=625 \mathrm{C}, \alpha=1.3 \times 10^{-7} \mathrm{~m}^{2} / \mathrm{s}, \rho=1200 \mathrm{~kg} / \mathrm{m}^{3}, Y_{\mathrm{c}}=0.7$

The maximum in Figure 4 corresponds to a balance between the rate of increase of isothermal mass by thermal diffusion and the rate of mass loss due to isothermal pyrolysis. This is the steady-state condition. Setting the derivative of Equation 11 equal to zero and solving

$$
\frac{\partial \dot{\mathrm{m}}_{\mathrm{A}}}{\partial \mathrm{t}}=\mathrm{kp} \sqrt{\alpha}\left(1-\mathrm{Y}_{\mathrm{c}}\right) \mathrm{e}^{-\mathrm{kt}}\left\{\frac{1}{2 \mathrm{t}^{1 / 2}}-\mathrm{kt}^{1 / 2}\right\}=0
$$


gives the characteristic time, $t=1 /(2 \mathrm{k})$, which when substituted back into Equation 11 yields the steady-state mass loss rate for a burning material with a surface temperature, $T_{s}=T_{P}{ }^{\max }$,

$$
\dot{\mathrm{m}}_{\mathrm{A}}=\rho\left(1-\mathrm{Y}_{\mathrm{c}}\right) \sqrt{\frac{\alpha \mathrm{A}}{50 \mathrm{e}}} \exp \left(-\frac{E_{\mathrm{a}}}{2 \mathrm{RT}_{\mathrm{s}}}\right) \text {. }
$$

Equation 13 is plotted in Figure 5 for PMMA using the above property set with $Y_{c}=0$.

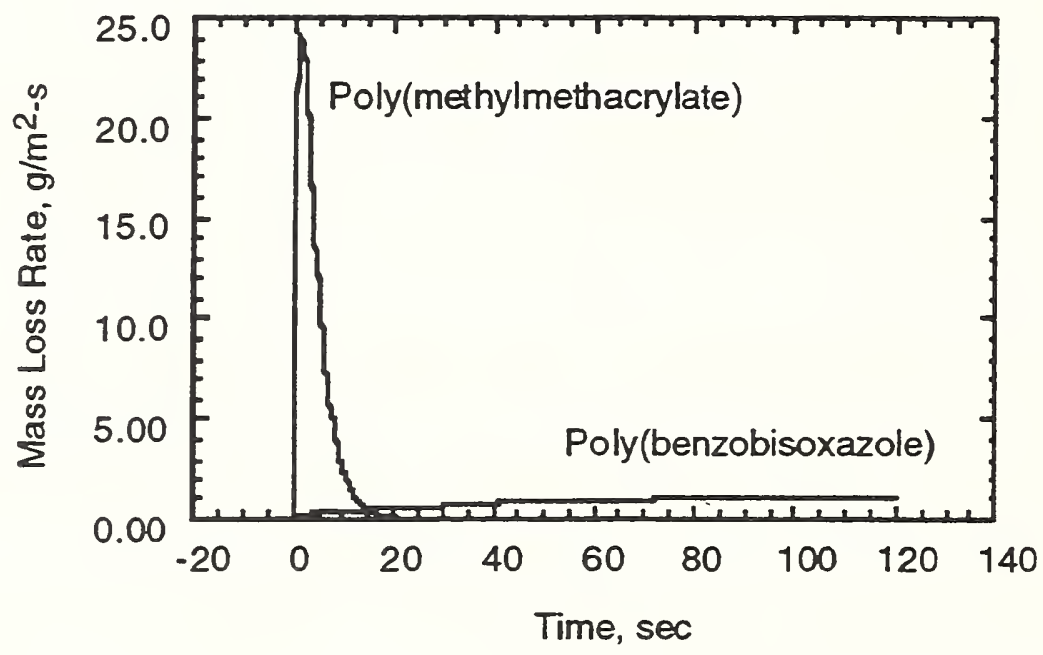

Figure 4. Mass loss rate versus time for isothermal layer in transient pyrolysis model

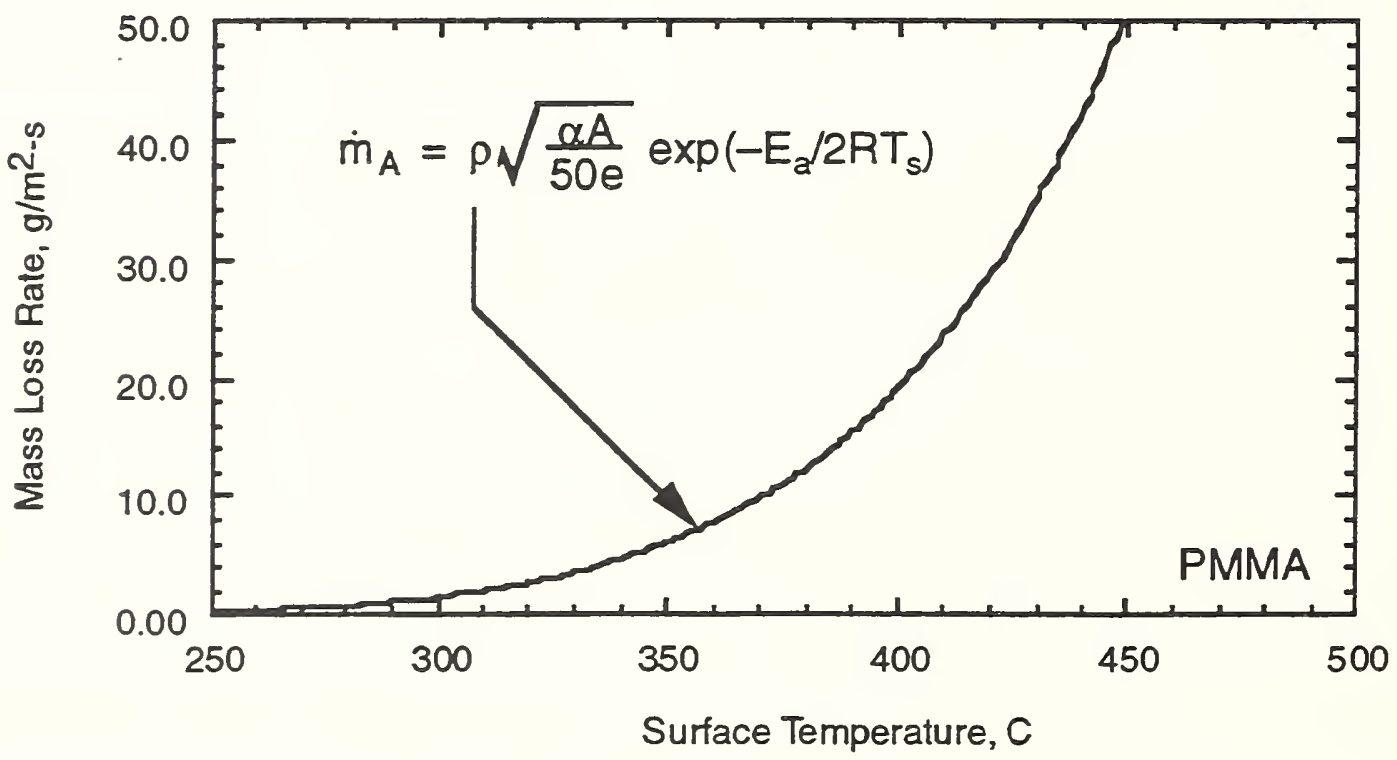

Figure 5. Mass loss rate versus surface temperature for PMMA according to Equation 13.

Since the surface recession velocity, $v$, is related to the areal mass loss rate as $\dot{\mathrm{m}}_{\mathrm{A}}=\rho \mathrm{v}$, the timeindependent recession velocity is 


$$
v=\frac{\dot{\mathrm{m}}_{\mathrm{A}}}{\rho}=\left(1-Y_{\partial}\right) \sqrt{\frac{\alpha \mathrm{A}}{50 \mathrm{e}}} \exp \left(-\frac{\mathrm{E}_{\mathrm{a}}}{2 \mathrm{RT}}\right)
$$

which has the same form as the usual result for the flame front propagation speed, $S_{u}$, of a premixed laminar flame [6], i.e.

$$
S_{\mathrm{u}} \propto \sqrt{\alpha} \exp \left(-\frac{E_{\mathrm{a}}}{2 \mathrm{RT}}\right)
$$

For PMMA the above property set substituted into Equation 14 for a flaming surface temperature, $T_{s}=400^{\circ} \mathrm{C}$, gives a surface recession velocity, $v=20 \mu \mathrm{m} / \mathrm{s}$, from which

$$
\dot{\mathrm{m}}_{\mathrm{A}}=\rho \mathrm{v}=\left(1000 \mathrm{~kg} / \mathrm{m}^{3}\right)(1000 \mathrm{~g} / \mathrm{kg})\left(20 \times 10^{-6} \mathrm{~m} / \mathrm{s}\right) \approx 20 \mathrm{~g} / \mathrm{m}^{2}-\mathrm{s}
$$

which compares favorably with experimental measurements of $\dot{\mathrm{m}}_{\mathrm{A}}=23-28 \mathrm{~g} / \mathrm{m}^{2}$-s for PMMA during flaming combustion at $50 \mathrm{~kW} / \mathrm{m}^{2}$ irradiance where measured surface temperatures are in the $400^{\circ} \mathrm{C}$ range [2].

\section{HEAT OF GASIFICATION}

The Arrhenius relation (Equation 2) was originally derived with the assumption that the reactants are at equilibrium with a high energy state and could proceed to products with no further energy requirements. The assumption of equilibrium between the reactants and activated complex allows us to attach a thermodynamic significance to the constants in the Arrhenius equation. From the definition of the free energy at equilibrium,

$$
\ln \mathrm{K}=\ln \frac{\mathrm{k}_{\mathrm{p}}}{\mathrm{k}_{-\mathrm{p}}}=-\frac{\Delta \mathrm{G}_{\mathrm{a}}}{\mathrm{RT}}=-\left[\frac{\Delta \mathrm{H}_{\mathrm{a}}}{\mathrm{RT}}-\frac{\Delta \mathrm{S}_{\mathrm{a}}}{\mathrm{R}}\right]
$$

where $\mathrm{k}_{\mathrm{p}}, \mathrm{k}_{-\mathrm{p}}$, are the forward (pyrolysis) and backward (recombination) rates of the reaction and, $\Delta \mathrm{G}_{\mathrm{a}}, \Delta \mathrm{H}_{\mathrm{a}}$, and, $\Delta \mathrm{S}_{\mathrm{a}}$, are the molar free energy, enthalpy, and entropy of activation, respectively. It follows that

$$
k_{p}=A e^{-\left(E_{d} / R T\right)}=\left[k_{-p} e^{\Delta S_{z} / R}\right] e^{-\left(\Delta H_{a} / R T\right)}
$$

where the pre-exponential factor now has the identity, $A=\left[\mathrm{K}_{p} \mathrm{e}^{\Delta \mathrm{S}_{\mathrm{a}} / \mathrm{R}}\right]$, and, $\mathrm{E}_{\mathrm{a}}=\Delta \mathrm{H}_{\mathrm{a}}$, is the molar enthalpy of pyrolysis (depolymerization). Kishore and Pai Verneker have shown that the enthalpy of pyrolysis, which they assumed to be equal but opposite in sign to the heat of polymerization, is equal to the activation energy of pyrolysis for a variety of polymers [7].

The phenomenological equation relating areal mass loss rate to net heat flux is [8],

$$
\dot{\mathrm{m}}_{\mathrm{A}}=\frac{\dot{\mathrm{Q}}_{\text {net }}}{\mathrm{L}_{\mathrm{g}}}=\frac{\dot{\mathrm{Q}}_{\text {ext }}}{\mathrm{L}_{\mathrm{g}}}+\frac{\dot{\mathrm{Q}}_{\text {flame }}-\dot{\mathrm{Q}}_{\text {loss }}}{\dot{\mathrm{L}}_{\mathrm{g}}}
$$

where $\mathrm{L}_{\mathrm{g}}(\mathrm{kJ} / \mathrm{g})$ is a quantity called the heat of gasification which relates the net heat flux to the surface to the observed mass loss rate. In Equation $18, \dot{Q}_{\text {net }}$ is seen to be the difference between the incident heat from an external heater, $Q_{\text {ext }}$, or surface flame, $\dot{Q}_{\text {flame }}$, and the heat lost through reradiation to the surroundings, $\mathrm{Q}_{\text {loss. }}$ In practice $L_{\mathrm{g}}$ is determined experimentally as the slope of a plot of peak mass loss rate versus external radiant heat flux, $\dot{Q}_{\text {ext }}$ 
Since it is found that the molar heat of depolymerization (gasification) is numerically equal to the molar activation energy for pyrolysis, $\mathrm{E}_{\mathrm{a}}$, the heat of gasification per unit mass, $\mathrm{L}_{\mathrm{g}}$, is related to the molar activation energy for pyrolysis through the molecular weight of the decomposition products, $\mathrm{M}_{\mathrm{g}}$,

$$
\mathrm{L}_{\mathrm{g}}=\frac{\Delta \mathrm{H}_{\mathrm{a}}}{\mathrm{M}_{\mathrm{g}}}=\frac{\mathrm{E}_{\mathrm{a}}}{\mathrm{M}_{\mathrm{g}}}
$$

Polymers which pyrolyze to monomer (depolymerize) at near-quantitative yield such as polymethylmethacrylate, polyoxymethylene, and polystyrene, should have $\mathrm{M}_{\mathrm{g}}$ equal to the monomer molecular weight, $M_{o}$, i.e., $M_{g} / M_{o} \approx 1$. Polymers such as polyethylene and polypropylene which decompose by random scission to multi-monomer fragments would have $\mathrm{M}_{\mathrm{g}} / \mathrm{M}_{\mathrm{o}}>1$. In contrast, polymers with high molecular weight repeat units $\left(\mathrm{M}_{\mathrm{o}} \geq 200 \mathrm{~g} / \mathrm{mol}\right)$ such as nylon, cellulose, polycarbonate, or with good leaving groups (e.g., polyvinylchloride) are known to yield primarily low molecular weight species (water, carbon dioxide, alkanes, $\mathrm{HCl}$ ) on pyrolysis and should have, $\mathrm{M}_{\mathrm{g}} / \mathrm{M}_{0}<1$.

The Table shows the ratio $M_{g} / M_{0}$ predicted from the relation

$$
\frac{E_{a} / M_{o}}{L_{g}}=\frac{M_{g}}{M_{o}}
$$

for a variety of common polymers for which $L_{g}$ has been measured [8].

Heat of Gasification [8], Thermal Activation Energy [3,9], and Calculated Molecular Weight of Decomposition

\begin{tabular}{|c|c|c|c|c|c|}
\hline POLYMER & $\begin{array}{l}\mathrm{M}_{\mathrm{o}} \\
(\mathrm{g} / \mathrm{mol})\end{array}$ & $\begin{array}{l}\mathrm{L}_{\mathrm{g}} \\
(\mathrm{kJ} / \mathrm{g})\end{array}$ & $\begin{array}{l}\mathrm{E}_{\mathrm{a}} \\
(\mathrm{kJ} / \mathrm{mol})\end{array}$ & $\mathrm{M}_{\mathrm{g}} / \mathrm{M}_{\mathrm{o}}$ & $\begin{array}{l}\text { PYROLYSIS } \\
\text { PRODUCTS }\end{array}$ \\
\hline polypropylene & 42 & 2.5 & 243 & 2.3 & \multirow{2}{*}{$\begin{array}{l}\mathrm{C}_{2}-\mathrm{C}_{90} \text { saturated and } \\
\text { unsaturated } \\
\text { hydrocarbons }\end{array}$} \\
\hline polyethylene & 28 & 2.4 & 264 & 3.9 & \\
\hline & & & & & \\
\hline polystyrene & 104 & 2.2 & 230 & 1.0 & 40-60\% monomer \\
\hline polymethylmethacrylate & 100 & 1.6 & 160 & 1.0 & $100 \%$ monomer \\
\hline polyoxymethylene & 30 & 2.7 & 84 & 1.0 & $100 \%$ monomer \\
\hline nylon 6,6 & 226 & 26 & 160 & 03 & $\mathrm{H}_{3} \mathrm{O} \quad \mathrm{CO}_{2} \mathrm{C}_{5} \mathrm{HC}^{\prime} \mathrm{s}$ \\
\hline cellulose & 162 & 3.2 & 200 & 0.4 & $\mathrm{H}_{2} \mathrm{O}, \mathrm{CO}_{2}, \mathrm{CO}$ \\
\hline polyvinylchloride & 62 & 2.5 & 110 & 0.7 & $\mathrm{HCl}$, benzene, toluene \\
\hline
\end{tabular}
Products for Common Polymers with Known Pyrolysis Modes [9].

The qualitative agreement between the observed modes of pyrolysis (random scission, depolymerization, solid-phase oxidation/fragmentation) and the calculated fragment molecular weight using Equation 20 is strong support for the identity, $\mathrm{L}_{\mathrm{g}}=\mathrm{E}_{\mathrm{a}} / \mathrm{M}_{\mathrm{g}}$. Consequently, $\mathrm{L}_{\mathrm{g}}$ determined experimentally from a plot of peak mass loss rate versus external heat flux has the significance of a thermodynamic property. From Equations 18 and 19 the effective mass loss rate in terms of the net heat flux to the surface, is

$$
\dot{\mathrm{m}}_{\mathrm{A}}=\frac{\dot{\mathrm{Q}}_{\mathrm{net}}}{\mathrm{L}_{\mathrm{g}}}=\frac{\mathrm{M}_{\mathrm{g}}}{\mathrm{E}_{\mathrm{a}}} \dot{\mathrm{Q}}_{\mathrm{net}}
$$




\section{HEAT RELEASE RATE}

The phenomenological equation relating mass loss rate to heat release rate is,

$$
\dot{\mathrm{Q}}_{\mathrm{c}}\left(\mathrm{kW} / \mathrm{m}^{2}\right)=\chi \Delta \mathrm{H}_{\mathrm{c}} \dot{\mathrm{m}}_{\mathrm{A}}
$$

where $\chi, \Delta \mathrm{H}_{\mathrm{c}}$, and $\dot{\mathrm{m}}_{\mathrm{A}}$, are the combustion efficiency, chemical heat of complete combustion, and the mass loss rate per unit area, respectively. Substituting Equation 13 for the areal mass loss rate of a char forming polymer into Equation 22 gives the heat release rate of a burning polymer in terms of its flaming surface temperature as

$$
\dot{\mathrm{Q}}_{\mathrm{c}}=\Delta \mathrm{H}_{\mathrm{c}} \chi \dot{\mathrm{m}}_{\mathrm{A}}=\chi\left(1-\mathrm{Y}_{\mathrm{c}}\right) \Delta \mathrm{H}_{\mathrm{q}}\left[\frac{\mathrm{K \rho}}{\mathrm{C}_{\mathrm{p}}} \frac{\mathrm{A}}{50 \mathrm{e}}\right]^{1 / 2} \exp \left(-\frac{\mathrm{E}_{\mathrm{a}}}{2 \mathrm{RT}_{\mathrm{s}}}\right)
$$

Figure 6 shows the excellent correlation $\left(\mathrm{r}^{2}=0.94\right)$ between steady-state heat release rate calculated from Equation 23 and measured values for a wide variety of polymers for which heat release rate data $(10-13)$ and char yields $(3,9,13)$ were available as well as reliable kinetic parameters $(3,9,10)$ for the calculation of the flaming surface temperature, $T_{s} \approx T_{p}$ max at an external heat flux of $50 \mathrm{~kW} / \mathrm{m}^{2}$.

In terms of the net heat flux to the surface, the heat release rate from Equations 21 and 22 is

$$
\dot{\mathrm{Q}}_{\mathrm{c}}=\frac{\chi \Delta \mathrm{H}_{\mathrm{c}} \mathrm{M}_{\mathrm{g}}}{\mathrm{E}_{\mathrm{a}}} \mathrm{Q}_{\mathrm{net}}
$$

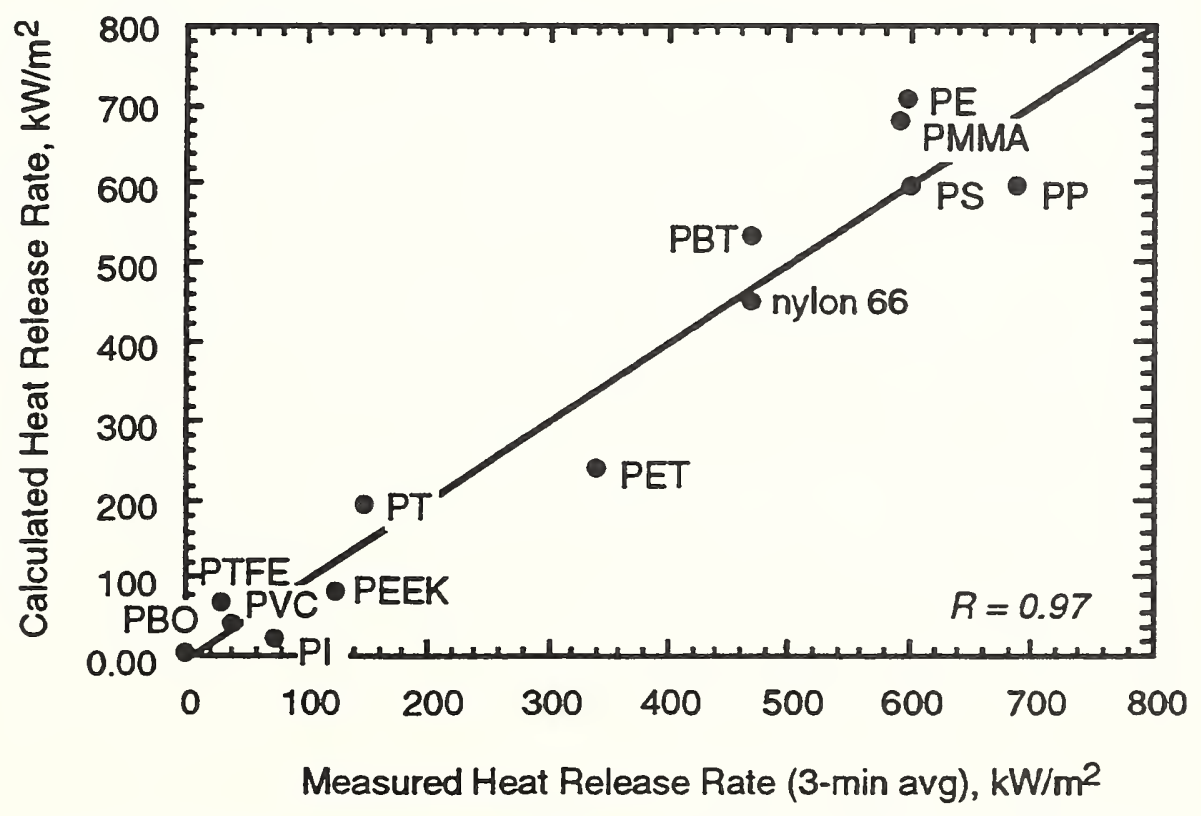

Figure 6. Calculated versus measured heat release rate for a variety of polymers 


\section{SUMMARY}

The objective of this work was to find simple relationships between the fire response of polymeric materials and their thermodynamic, kinetic, and transport properties. Polymer thermal stability was assumed to be completely described by first-order Arrhenius pyrolysis kinetics modified to account for char yield. Analytic expressions for the time-to-ignition, mass loss rate, heat of gasification, and heat release rate were developed from phenomenological equations, transient heat conduction analyses, and the Arrhenius parameters and thermal properties of the material. Qualitative agreement between predicted and experimental fire response data for a wide range of polymeric materials is obtained by this simple approach.

\section{REFERENCES}

1. R.E. Lyon, "Fire-Safe Aircraft Cabin Materials," in Fire and Polymers, ACS Symposium Series Number 599, G.L. Nelson, ed., American Chemical Society, Washington, D.C., p. 618,1995

2. Kashiwagi , T.; Omori, A., Twenty-Second Symposium (International) on Combustion, The Combustion Institute: Pittsburg, PA, pp. 1329-13382, 1988

3. Polymer Handbook, Brandrup, J.; Immergut, E.H. , Eds.; 2nd edition; Wiley-Interscience: NY, 1975

4. Tewarson, A., "Flammability Parameters of Materials: Ingition, Combustion, and Flame Spread," Proceedings of the International Conference for the Promotion of Advanced Fire Resistant Aircraft Materials, Atlantic City, New Jersey, Feb. 9-11, pp. 263, 1993

5. Quintiere, J.G.; Harkelroad, M.T.; "New Concepts for Measuring Flame Spread Properties,"in Fire Safety:Science and Engineering, Harmathy, T.Z, ed; ASTM Special Technical Publication 882: Phila., PA, pp. 239-267, 1985

6. T.Y. Toong, Combustion Dynamics: The Dynamics of Chemically Reacting Fluids, McGraw Hill, New York, 1983

7. Kishore, K., and Pai Verneker, V.R., "Correlation Between Heats of Depolymerization and Activation Energies in the Degradation of Polymers," J.Polym.Sci.Polym.Lett. Ed., 14, 761 (1976)

8. Tewarson, A., "Generation of Heat and Chemical Compounds in Fires," SFPE Handbook of Fire Protection Engineering: P.J. DiNenno, ed.; National Fire Protection Association Press: Quincy, MA, Section 1, Chapter 13, pp. 1-179,1988

9. D.W. Van krevelen, Properties of Polymers, Elsevier Scientific, NY, 1976

10. Kim, P.K., Pierini, P., and Wessling, R., "Thermal and Flammability Properties of Poly(p-phenylene-benzobisoxazole)," Proceedings of the Thirty-Ninth Sagamore Army Materials Research Conference, Plymouth, MA, 14-17 September, 1992, pp. 327-335

11. Hirschler, M.M., "Heat Release From Plastic Materials," in Heat Release in Fires, V. Babrauskas and S. Grayson, Eds., Elsevier Applied Science, New York, Chapter 12(a)., pp. 375-422, 1992

12. Scudamore, M.J., Briggs, P.J. and Prager, F.H., "Cone Calorimetry-A Review of Tests Carried out on Plastics for the Association of Plastic Manufacturers in Europe," Fire and Materials, 15, 65-84 (1991)

13. Grand, A.F., SBIR Phase I Progress Report, 1995, Contract No. DTRS-57-94-C-00172 


\section{Discussion}

Henri Mitler: Do I understand you to say the main difference in your formulation is the assumption of the existence of this charred mode?

Richard Lyon: There are a couple of subtle things, and I'm not sure that any one thing is the most important. What I have done here is assume parallel reactions. I allowed for gas and char formation. Equally important is the assumption of a transition state. That goes back to the physical chemistry theory of reactions. This is a transition state in it's degradation to gas and char.

Masahiro Morita: What kind of atmosphere did you use?

Richard Lyon: It was a small $5 \mathrm{mg}$ sample, no oxygen. 
2 $\underline{6} \underline{0}$ 
FIRE RETARDANT ADDITIVES FOR POLYMERIC MATERIALS - I. CHAR FORMATION from SILICA GEL - POTASSIUM CARBONATE

\author{
Jeffrey W. Gilman,* Steven J. Ritchie, Takashi Kashiwagi \\ Materials Fire Research Group, Building and Fire Research Laboratory, \\ National Institute of Standards and Technology, Gaithersburg, Maryland 20899 \\ and \\ Sergey M. Lomakin \\ Institute of Biochemical Physics, \\ Russian Academy of Sciences, Moscow, Russia
}

\begin{abstract}
Silica gel combined with potassium carbonate is an effective fire retardant for a wide variety of common polymers (at only $10 \mathrm{wt}$. \% total additive) such as polypropylene, nylon, polymethylmethacrylate, poly (vinyl alcohol), cellulose, and to a lesser extent polystyrene and styrene-acrylonitrile. The peak heat release rate is reduced by up to $68 \%$ without significantly increasing the smoke or carbon monoxide levels during the combustion.
\end{abstract}

\title{
INTRODUCTION
}

An alternative to the use of halogenated fire retardants, which control flammability by changing the chemistry in the flame ${ }^{1}$ is to control polymer flammability by manipulating the condensed phase chemistry. Additives that increase the amount of charcoal-like residue or carbonaceous char that forms during polymer combustion are very effective fire retardants ${ }^{2}$. However, very little is understood about the detailed structure of char or how it forms. Our research efforts focus on reducing polymer flammability by promoting char formation through manipulation of the condensed phase decomposition chemistry. Char formation reduces the amount of small volatile polymer pyrolysis fragments, or fuel, available for burning in the gas phase; this, in turn reduces the amount of heat released and feedback to the polymer surface. The char also insulates the underlying virgin polymer, due to the char's low thermal conductivity. It also traps decomposition products and reradiates energy away from the polymer. The physical structure of the char is important in this role. Foamy char structure appears to be more fire resistant than brittle, thin char. This char enhancing approach is most successful when the polymer chars rapidly and early in the burning process 3 . To be useful the charring process must be designed so that it occur between the processing temperature and the polymer decomposition temperature. Our approach to char promotion is to investigate additives which enhance charring and to gain a fundamental understanding of the additive's mechanism of char formation with the goal of optimizing the additive's performance. Recent studies of the flammability of polymers containing silicon based materials have shown these materials to be promising fire retardants, either as additives, in blends with organic polymers or in copolymers 4,5 . This paper reports on the effect that silica gel and potassium carbonate additives have on polymer flammability.

\section{EXPERIMENTAL}

Polymers and additives6: Silica gel (Fisher Scientific Co., 28-200 mesh), potassium carbonate, $\mathrm{K}_{2} \mathrm{CO}_{3}$ (Mallinckrodt, granular) polypropylene, PP (Scientific Polymer Products, Inc., $\mathrm{Mw}=$ 240,000 g/mole), polystyrene, PS (Scientific Polymer Products, Inc., Mw 45,000 g/mole), styrene-acrylonitrile, SAN (GE Polymers), polymethymethacrylate, PMMA (Du Pont, Elvacite), poly(vinyl alcohol), PVA (Scientific Polymer Products, Inc., $\mathrm{Mn}=86,000 \mathrm{~g} / \mathrm{mole}, \mathrm{Mw}=$ 178,000 g/mole, 99.7\% hydrolysed via NaOHaq method), nylon 6,6 (Rhone Poulence) and alpha cellulose (Sigma Chemical Co., fiber, 99.5\%) were all used as received.

Cone Calorimeter: Evaluations of polymer and polymer/additive flammability were done using the Cone Calorimeter ${ }^{7}$. Rate of heat release and heat of combustion data are good to within $10 \%$. The 
carbon monoxide and soot yield data are good to within $10 \%$. The tests were done at an incident heat flux of $35 \mathrm{~kW} / \mathrm{m}^{2}$ using the cone heater. The additives were mixed with the polymers by grinding the powders together in a mortar and pestle. Cone samples were prepared by compression molding the powdered samples ( $40 \mathrm{~g}-55 \mathrm{~g}$ ) into $75 \mathrm{~mm} \times 7-8 \mathrm{~mm}$ disks using a Carver press with a heated mold ( $22 \mathrm{MPa}$ (10 tons) held for $3-5$ minutes at $150^{\circ} \mathrm{C}$ or at $\mathrm{Tg}$ ).

Gasification apparatus: Figure 5 is a schematic diagram of a radiative gasification apparatus. This facility is physically similar to the Cone calorimeter (identical samples, cone heater system, etc.) except that the tests are carried out in a controlled, oxidized-free (nitrogen) atmosphere. This apparatus allows study of the condensed-phase processes decoupled from the influence of gas phase combustion and heat feedback. This is achieved using fire-like incident heat fluxes of 20 to $70 \mathrm{~kW} / \mathrm{m}^{2}$ from the cone heater. In a typical experiment thermocouples are imbedded into the exposed and backside sample surfaces to monitor the temperatures at which the pyrolysis and decomposition processes are occurring. A load cell gives mass loss rate data which can be compared to that from the Cone calorimeter experiments.

Nuclear Magnetic Resonance (NMR): Solid State ${ }^{13}$ C NMR characterization utilized techniques of cross polarization $8(\mathrm{CP})$ and magic angle spinning (MAS) $(25 \mathrm{MHz}, 4 \mathrm{kHz}$ MAS, $1 \mathrm{~ms}$ CP time, 3 sec rep. time). In the interrupted decoupling experiments the decoupler was turned off for $40 \mathrm{~ms}$ prior to acquisition with decoupling 9,10.

Oxygen Index, OI: Oxygen index tests were carried out on $50 \mathrm{~mm}$ long by $3 \mathrm{~mm}$ diameter rods. The polymers and additives were mixed in powder form in a blender and were extruded from a three stage lab scale $(100 \mathrm{~g})$ extruder.

\section{RESULTS AND DISCUSSION}

\section{Thermoplastic Polymers}

\section{CONE CALORIMETER}

\section{Polypropylene}

Polypropylene, like PE and polystyrene, PS, when ignited burns rapidly, completely and leaves little or no char 11 . Reducing the flammability of inherently non-charring polymers through char enhancement presents a particular challenge. In the process of systematically evaluating additives expected to enhance char formation, we found that silica gel when combined with potassium carbonate not only increased char yields but also reduced the flammability of a variety of aliphatic polymers. Figure 1 shows heat release rate data for $\mathrm{PP}$ and a $\mathrm{PP} /$ silica gel/ $\mathrm{K}_{2} \mathrm{CO}_{3}(90 / 6 / 4)$ mixture, from the Cone Calorimeter. The peak heat release rate has been shown to be the most important parameter for predicting fire hazard. The presence of the silica gel/ $\mathrm{K}_{2} \mathrm{CO}_{3}$ additives reduces the peak heat release rate by $58 \%$ and reduces the total heat released by $28 \%$. The rate of heat release curve shows the double maxima characteristic of a material that forms a char layer during combustion 12. The presence of the silica gel/ $\mathrm{K}_{2} \mathrm{CO}_{3}$ additives in $\mathrm{PP}$ produces a residue yield of $19 \%$. The residue is at least $47 \%$ carbonaceous material if we assume that all the additives survived the combustion in the Cone calorimeter and are contained in the residue. The yield of carbonaceous char therefore is $\sim 10 \%$ (see Table 1). All char yields in Table 1 and in the following discussion are corrected for the presence of additive in this manner and are therefore carbonaceous char yields. It appears that the char that forms during the combustion reduces the rate at which fuel is released into the gas phase, this reduction in mass loss rate reduces the rate of heat release.

Unlike halogenated additives silica gel $/ \mathrm{K}_{2} \mathrm{CO}_{3}$ does not significantly effect the specific heat of combustion when added to PP, as seen in figure 2. Furthermore, the $\mathrm{CO}$ yield and soot (specific extinction area) are not significantly effected by silica gel $/ \mathrm{K}_{2} \mathrm{CO}_{3}$ additives. This indicates that these additives most likely act primarily in the condensed phase not in the gas phase. 
The $\mathrm{CO}$ yield results are shown in figure 3 , and indicate an advantage these additives may have relative to halogenated and phosphorous based additives, which commonly tend to increase $\mathrm{CO}$ yield and soot and are typically used in higher concentrations to obtain similar results. The Cone Calorimeter results for PP are summarized in Table 1 along with the data for several other polymers we examined.

\section{Polystyrene}

The effect that silica gel/ $\mathrm{K}_{2} \mathrm{CO}_{3}$ has on the flammability of PS is shown in Table 1 . The results are similar, but reduced in magnitude, to those for PP. Use of silica gel $/ \mathrm{K}_{2} \mathrm{CO}_{3}$ additives cause the otherwise non-char-forming PS to produce a char yield of $6 \%$ (16\% residue yield). The additives reduce the rate of heat release by $31 \%$, reduce the total heat release by $11 \%$, and similar to PP, had little effect on the $\mathrm{CO}$ yield and smoke.

\section{Polymethylmethacrylate}

The Cone calorimeter results for PMMA are also shown in Table 1. PMMA is an inherently nonchar-forming thermoplastic polymer; however, using less than half the usual amount of additives, the reduction in flammability was comparable to PP and PS. Even at this low level (3\% silica gel, $1 \% \mathrm{~K}_{2} \mathrm{CO}_{3}$ ) the additives generated a $15 \%$ carbonaceous char $(24 \%$ residue yield). The rate of heat release was reduced by $42 \%$, the total heat release was reduced by $28 \%$, and like PP and PS there was no effect on smoke. The CO yield, however, was somewhat higher in the presence of the additives. It is reasonable to expect that if the additives were used at the loadings used for PP and PS then the reduction in PMMA's flammability would meet or exceed that of PP.

The efficacy of the additives in each of these inherently non-char-forming thermoplastic polymers (PP, PS, and PMMA) depends on the polymer ( $\mathrm{PP} \approx \mathrm{PMMA}>\mathrm{PS}$ ). It is not clear what polymer property ( $\mathrm{Mw}$, functionality, decomposition mode, etc.) is responsible for the different behavior. This is also true for the inherently char-forming polymers we examined (PVA $>$ cellulose $>$ SAN). These results are discussed below.

\section{Char Forming Polymers \\ Poly(vinyl alcohol)}

PVA is one of the few linear non-halogenated aliphatic polymers that has a significant char yield (3-5\%) when burned. The flammability properties of PVA, the structure of the char and the processes by which it forms, have been studied previously 13. The Cone calorimeter results for PVA are shown in Table 1 . Silica gel/ $\mathrm{K}_{2} \mathrm{CO}_{3}$ has a stronger effect on the flammability of PVA than on any other polymer studied. The additives generated a $43 \%$ carbonaceous char ( $49 \%$ residue yield). The peak heat release rate was reduced by $68 \%$ and the total heat release was reduced by 54\%. Furthermore, in contrast to the results for PP, PS, and PMMA: the heat of combustion was reduced by $27 \%$; the $\mathrm{CO}$ yield was unchanged and the smoke was reduced by $66 \%$ !

\section{Cellulose}

Cellulose like PVA gives a measurable char yield when combusted (3-4\%) and in view of the promising results seen for PVA, and since cellulose is a commercially important polymer, it's flammability properties were examined in the presence of silica gel/ $\mathrm{K}_{2} \mathrm{CO}_{3}$ additive. The results are shown in table 1. Cellulose, in the presence of the additives, like PVA showed a significant increase in the amount of carbonaceous char, 32\% (39\% residue yield). The peak heat release rate was reduced by $52 \%$, and the total heat release was reduced by $66 \%$. Again, in contrast to the results for PP, PS, and PMMA: the heat of combustion was reduced by $53 \%$. The CO yield was increased by $-50 \%$, primarily from incomplete oxidation at the end of the combustion (ca. Fig. 3); and the smoke was decreased by $26 \%$.

The results for PVA and cellulose indicate a combination of condensed phase mechanisms of 
action. In addition to the enhanced charring effect and the resulting reduction in mass loss rate, the additives may also be an increasing the rate of $\mathrm{H}_{2} \mathrm{O}$ elimination from cellulose and PVA. This dilution of the combustable gases may be responsible for the reduction in the specific heat of combustion.

\section{Styrene acrylonitrile}

The Cone calorimeter results for styrene acrylonitrile, SAN, are also shown in Table 1 . The effect of the additives on the flammability of SAN was only moderate, much the same as PS. This was somewhat surprising since SAN is an inherently char-forming polymer. For the SAN studied the char yield was $\sim 2 \%$ for the combustion of the pure polymer and only $\sim 3 \%$ in the presence of the additives. As seen here and in other cases controlling the flammability of styrene and styrene copolymers is difficult.

\section{OXYGEN INDEX}

We also measured the effect of the additives on the oxygen index of PMMA, PS and nylon 6,6. The results are shown in Table 1 . The trend in oxygen index response for these polymers is similar to the trend in the peak and average heat release rate data from the Cone calorimeter. Costa and Camino report similar results in their comparison between Cone calorimeter and traditional tests (oxygen index, glow wire test, etc.) for polypropylene with additives or fillers ${ }^{14}$.

\section{RADIA TIVE GASIFICATION}

Figure 6 shows the mass and mass loss rate data for an experiment aimed at determining the influence of the additives on the decomposition processes. The slope of the mass loss curve for PMMA w/ silica gel and $\mathrm{K}_{2} \mathrm{CO}_{3}$ (95:4:1) first begins to differ from that for pure PMMA at $~ 80$ sec. A video of the pyrolysis experiment reveals that the PMMA begins to char at this time. The thermocouple embedded in the top surface of the sample shows that the temperature is $360^{\circ} \mathrm{C}-440$ ${ }^{\circ} \mathrm{C}$ at this time. This data shows that the silica gel $/ \mathrm{K}_{2} \mathrm{CO}_{3}$ additives affects charring of the PMMA early in the decomposition process and at temperatures as low as $360^{\circ} \mathrm{C}$. This is similar to the results of Benbow where fumed silica reduced the smoke by forming a sintered silica surface layer at temperatures as low as $447^{\circ} \mathrm{C} 15$.

\section{CPIMAS ${ }^{13} \mathrm{C}$ NMR CHARACTERIZATION}

The chars of PVA with silica gel / $\mathrm{K}_{2} \mathrm{CO}_{3}$ (90:6:4) and PVA with silica gel only (90:10) isolated following the combustion in the Cone calorimeter, were analyzed using several solid state $13 \mathrm{C} \mathrm{NMR}$ techniques. The spectra are shown in Figures 7 and 8 for the PVA with silica gel only (90:10) char and for the PVA with silica gel / $\mathrm{K}_{2} \mathrm{CO}_{3}$ (90:6:4) char, respectively. The normal CP/MAS $13 \mathrm{C}$ NMR, shown in the middle of Figure 7, contains a broad resonance in the aromatic-olefinic region from 110 ppm to $150 \mathrm{ppm}$ and two weaker broad signals in the aliphatic region, one centered at $20 \mathrm{ppm}$ and the other at $35 \mathrm{ppm}$. This spectrum shows that the ratio of aromatic-olefinic $\left(\mathrm{sp}^{2}\right)$ carbon to aliphatic $\left(\mathrm{sp}^{3}\right)$ carbon is $\sim 3: 1$. However, due to the uncertainty associated with quantitative measurements of the intensities of $13 \mathrm{C}$ signals in CP/MAS experiments of hydrogen depleted carbonaceous materials and of materials which may contain significant concentrations of paramagnetic centers (e.g., unpaired electrons, ie., free radicals) this spectrum may only be representative of a fraction of the carbons present in the char 16,17. Carbons which are less than $0.5 \mathrm{~nm}$ from protons make the largest contribution to this signal. Within this limitation, the fraction of the cross-polarized signal arising from protonated versus non-protonated carbons can be analyzed quantitatively. An interrupted decoupling (ID)-CP/MAS spectrum of this char, shown in the bottom spectrum in Figure 7, reveals only the non-protonated carbons which have cross-polarized. Comparison of the ID-CP/MAS spectrum (bottom) to the normal CP/MAS spectrum (middle) reveals that the downfield shoulder in the CP/MAS spectrum, centered at $135 \mathrm{ppm}$, is due to non-protonated aromatic-olefinic carbons. The result of subtracting the appropriate intensity of the ID-CP/MAS spectrum from the CP/MAS spectrum, so that the downfield shoulder is removed, is shown in the top of Figure 7 . This difference spectrum (top) reveals that the narrower upfield portion of the aromatic-olefinic resonance, at 110 ppm -135 ppm, is due to protonated carbons. The ratio of non-protonated to protonated aromaticolefinic carbons in the cross-polarized signal of this char sample is approximately 1 to 1 . Comparison 
of the set of NMR data above with that for the char resulting from the pyrolysis of pure PVA reveals that the non-protonated to protonated aromatic-olefinic carbon ratio is $1: 1$ in both cases and that the chars appear to have almost identical structure ${ }^{18}$. However, the presence of silica gel in PVA reduces the peak heat release rate from $609 \mathrm{~kW} / \mathrm{m}^{2}$, for pure PVA, to $250 \mathrm{~kW} / \mathrm{m}^{2}$. The presence of silica gel also increases the char yield from $5 \%$ to $27 \%$. It appears that the silica gel does not change the type of char formed, since the chars have similar structure, but it does change the rate that it forms since the char yield is higher and the flammability is lower.

A similar series of spectra of the char from PVA with silica gel and $\mathrm{K}_{2} \mathrm{CO}_{3}$ (90:6:4) is shown in Figure 8. The normal CP/MAS spectrum (middle) contains the sharp carbonate resonance at $162 \mathrm{ppm}$ and a broad resonance in the aromatic-olefinic region from $115 \mathrm{ppm}$ to $150 \mathrm{ppm}$. In contrast to the spectra for PVA only or for PVA with silica gel (10\%) there is little evidence of any aliphatic $\left(\mathrm{sp}^{3}\right)$ carbon. Comparison of the ID-CP/MAS spectrum (bottom) to the normal CP/MAS spectrum (middle) reveals, as was observed for pure PVA and for PVA with silica gel, that the downfield shoulder in the CP/MAS spectrum, centered at $135 \mathrm{ppm}$, is due to non-protonated aromatic-olefinic carbons. The result of subtracting the appropriate intensity of the ID-CP/MAS spectrum from the CP/MAS spectrum, so that the downfield shoulder is removed, is shown in the top of Figure 8 . The difference spectrum (top) reveals that the upfield portion of the aromatic-olefinic resonance, at $115 \mathrm{ppm}-135$ $\mathrm{ppm}$, is due to protonated carbons. The ratio of non-protonated to protonated aromatic-olefinic carbon in the spectrum of this char sample is approximately 1.5 to 1 , ie., this char contains a greater fraction of non-protonated aromatic-olefinic carbons than the char formed in the absence of $\mathrm{K}_{2} \mathrm{CO}_{3}$. To the extent that the cross-polarized signals in these materials reflect the sample-wide chemistries, this data indicates that the presence of the $\mathrm{K}_{2} \mathrm{CO}_{3}$ has increased the extent of carbon-carbon bond formation and therefore of crosslinking in the char. This may be the reason for the even lower flammability (peak heat release rate: $609 \mathrm{~kW} / \mathrm{m}^{2}$ for pure PVA; $250 \mathrm{~kW} / \mathrm{m}^{2}$ for PVA with silica gel; and 194 $\mathrm{kW} / \mathrm{m}^{2}$ for PVA with silica gel and $\mathrm{K}_{2} \mathrm{CO}_{3}$ ) and for the higher char yield in the presence of $\mathrm{K}_{2} \mathrm{CO}_{3}$ (char yield: 5\% for pure PVA; 27\% for PVA with silica gel; and 43\% for PVA with silica gel and $\mathrm{K}_{2} \mathrm{CO}_{3}$ ).

\section{MECHANISM}

A discussion of the original approach envisioned for this additive system may shed some light on how these additives reduce polymer flammability. The original intention in using silica gel with $\mathrm{K}_{2} \mathrm{CO}_{3}$ was to devise a method of in situ formation of silicon based fire retardants, during the combustion. The reaction of silica gel and organic alcohols in the presence of metal hydroxides has been shown to give multicoordinate organosiliconate compounds 19 . Instead of synthesizing these materials and then combining them with various polymers to evaluate their effect on polymer flammability properties, we envisioned the reaction occurring in the condensed phase of the pyrolyzing polymer beneath the burning surface, by combining a polyhydroxylic polymer, e.g. PVA or cellulose, with silica gel and $\mathrm{K}_{2} \mathrm{CO}_{3}$ (a weaker base but a more palatable additive than a metal hydroxide). If the indicated reaction occurred between the polymer and the additives it should crosslink the polymer, as shown in figure 4, and might assist in forming a silicon-oxy-carbide, SiOC, type protective char during combustion. It is not as likely, however, that this is how the additives affect flammability reduction in the nonhydroxylic polymers such as PP, PS, SAN, and PMMA.

An alternative mechanism of action for these additives is through the formation of a potassium silicate glass during the combustion. In earlier work on fire retardants, silicates were claimed to be quite effective 20 . The pertinent phase diagrams do not show potassium silicate formation until $725^{\circ} \mathrm{C}$. However, if sodium salts are present this temperature drops to $400^{\circ} \mathrm{C}-500^{\circ} \mathrm{C} 21$. Other work on inorganic glass forming fire retardants examined an analogous borate/carbonate system; $\mathrm{B}_{2} \mathrm{O}_{3} / \mathrm{MCO}_{3}$. These formulations were found to form an inorganic glassy foam as a surface barrier which insulated and slowed the escape of volatile decomposition gasses. Unfortunately relatively high loadings of these additives, $40 \mathrm{phr}$ - $100 \mathrm{phr}$ (parts per hundered parts of resin), were required to realize 
significant improvement in fire retardant performance and the loss of mechanical properties precluded application of this system 22 .

\section{CONCLUSIONS}

The results presented here demonstrate that the flammability of a wide variety of polymers is dramatically reduced in the presence of relatively small concentrations of silica gel and $\mathrm{K}_{2} \mathrm{CO}_{3}$. We see that these additives appear to act in the condensed phase but we have only just begun to gain insight into how these additives change the polymer pyrolysis. Future efforts to understand this system will include evaluation of the effect of other types of basic materials and of particle size and internal pore size of the silica gel. We are also characterizing the residues formed from the combustion of the polymers discussed above using solid state $1 \mathrm{H}, 29 \mathrm{Si}$, single pulse (more quantitative) ${ }^{13 \mathrm{C}}$ NMR, and other techniques. This information should help us understand how this additive system increases the char yield and reduces the flammability of such a wide variety of polymers.

\section{ACKNOWLEDGMENTS}

The authors would like to thank Mr. Jack Lee for Cone Calorimeter data, Dr. David L. VanderHart for use of the NMR facilities, and Mr. Roy McLane for construction of the radiative gasification facility. The authors are also grateful for partial support of this work by the Federal Aviation Administration under Interagency Agreement DTFA003-92-Z-0018, and for the discussions with the FAA technical monitor Dr. Richard E. Lyon.

\section{REFERENCES}

1. Cullis, C.F., Hirschler, M.M., The Combustion of Organic Polymers, p. 229, Clarendon Press, Oxford, (1981)

2. Weil, E.D., Hansen, R.H. and Patel, N., in; Fire and Polymer, ACS Symposium Series 425, ed.by Nelson, G. L. p. 97, American Chemical Society, Washington, D. C. (1990).

3. Kashiwagi, T. , Proceedings of the Twenty -Fifth Symposium (International) on Combustion/The Combustion Institute, p. 1423, Irvine (1994).

4. Kashiwagi, T., Fire Calorimetry, Proceedings of a special symposium on Fire Calorimetry, Report no. DOT/FAA/CT-95/46, (1995) 48.

5. Buch, R., R., Fire Calorimetry, Proceedings of a special symposium on Fire Calorimetry, Report no. DOT/FAA/CT-95/46, (1995) 154, and references cited therein.

6. Certain commercial equipment, instruments, materials, services or companies are identified in this paper in order to specify adequately the experimental procedure. This in no way implies endorsement or recommendation by NIST.

7. Babrauskas, V., Peacock, R. D., Fire Safety Journal, 18 (1992) 255.

8. Schaefer, J. Stejskal, E. O. and Buchdahl, R. Macromolecules, 8, 291-296 (1975).

9. O'Donnell, D. J. in NMR and Macromolecules, Sequence, Dynamics and Domain Structure; Randall, J. C. Jr., Ed. ACS Symposium Series 247; American Chemical Society, Washington, D. C. 1984; pp 21-41.

10. Opella, J., Frey, M. H., J. Am. Chem. Soc., 101, 5854 (1979).

11. Cullis, C.F., Hirschler, M.M., in The Combustion of Organic Polymers, p. 217, Clarendon Press, Oxford, (1981).

12. Marchal, A., et al., Polymer Degradation and Stability, 44 (1994) 263.

13. Gilman, J. W., VanderHart, D. L. and Kashiwagi, T. in Fire and Polymer, ACS Symposium Series 599, ed. Nelson, by G. L. p. 161, American Chemical Society, Washington, D. C. (1995).

14. Costa, L., Camino, G., Bertelli, G., and Borsini, G. Fire and Materials 19, 133 (1995).

15. Benbow, A. W. And Chalabi, R. in Proceedings of Interflam '79, Guilford, March, Heyden, London (1979) 87.

16. Zang, M., Maciel, G. E.,Fuel, 69, 557 (1990). 
17. Single pulse (SP) MAS ${ }^{13}$ C NMR experiments which avoid the problems inherent in CP/MAS experiments were attempted; however, suitable signal-to-noise levels were not obtained in either the normal $\left(90^{\circ}\right) \mathrm{SP} / \mathrm{MAS}$ or in the interrupted decoupling (ID) SP/MAS experiments. This may be due to very long ${ }^{13} \mathrm{C}_{1}$ relaxation times relative to the pulse repetition delay we were able to employ.

18. Gilman, J. W., VanderHart, D. L. and Kashiwagi, T. in Fire and Polymer, ACS Symposium Series 599, ed. Nelson, by G. L. p. 168-171, American Chemical Society, Washington, D. C. (1995).

19. Laine, R., M., Nature, 353, (1991) 642.

20. Compton, G., 1988 British patent application; 2,203,157.

21. Cook, L. P., McMurdie, H. F., Phase Diagrams for Ceramists, American Ceramics Society, Inc. Westerville, Ohio, figures 381 and 2020 (1964).

22. Myers, R. E., Licursi, E., Journal of Fire Science, 3, (1985) 415.

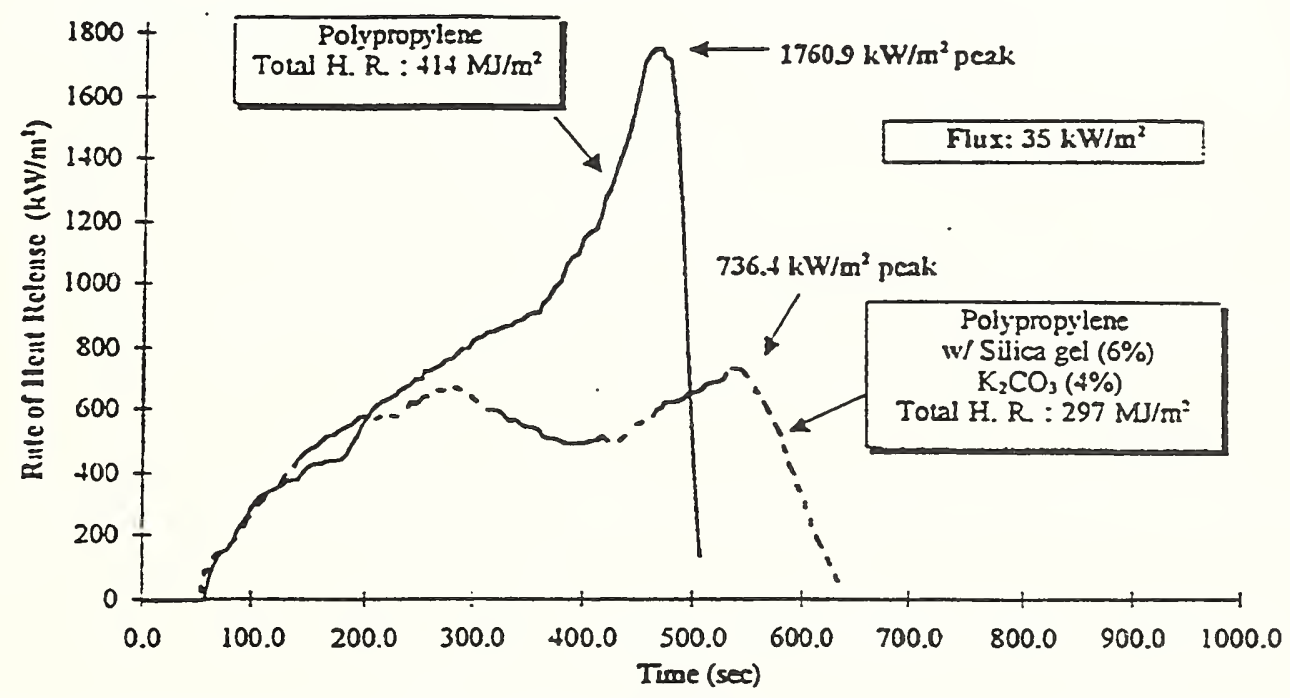

Fig 1. Rate of Heat Release (RHR) cure for PP $\left(35 \mathrm{~kW} / \mathrm{m}^{2}\right)\left(75 \mathrm{~mm} \times 7 \mathrm{~mm}\right.$ disk) and PP/silia gel $/ \mathrm{K}_{2} \mathrm{CO}_{3}$ (90/6/4 ratio, $75 \mathrm{~mm} \times 10 \mathrm{~mm}$ disk) with a $58 \%$ reduction in peak $R H R$ and a $28 \%$ reduction in total $Y . R$ in the presence of the additives.

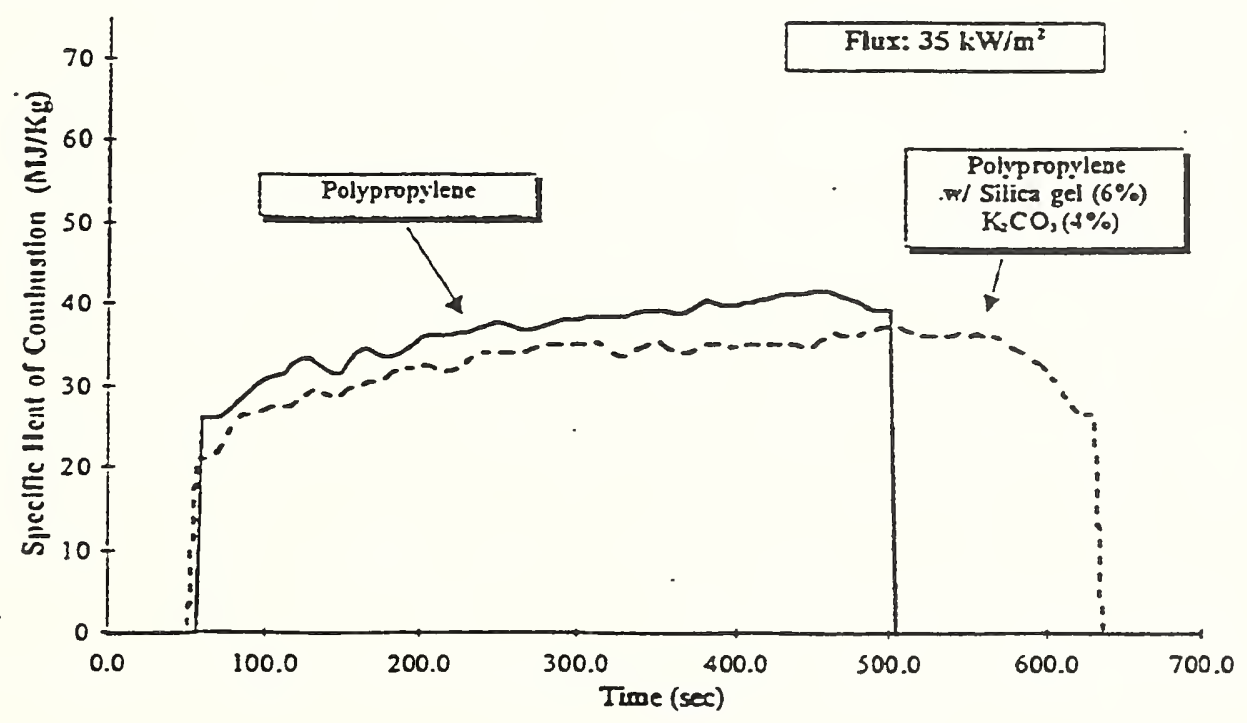

Fig 2 Specific heat of comburion for $P P\left(a t 35 \mathrm{~kW} / \mathrm{m}^{2}\right)$ and $P P / s i l i a$ gel $/ K_{2} \mathrm{CO}_{3}(90 / 6 / 4$ ratio) shouing only a slighi reduction in the presence of the additive. This indicates silica gel $/ K_{2} C O_{3}$ is acting in the condensed phase not is the gas phase as haiogenaled fire reardaris do. 


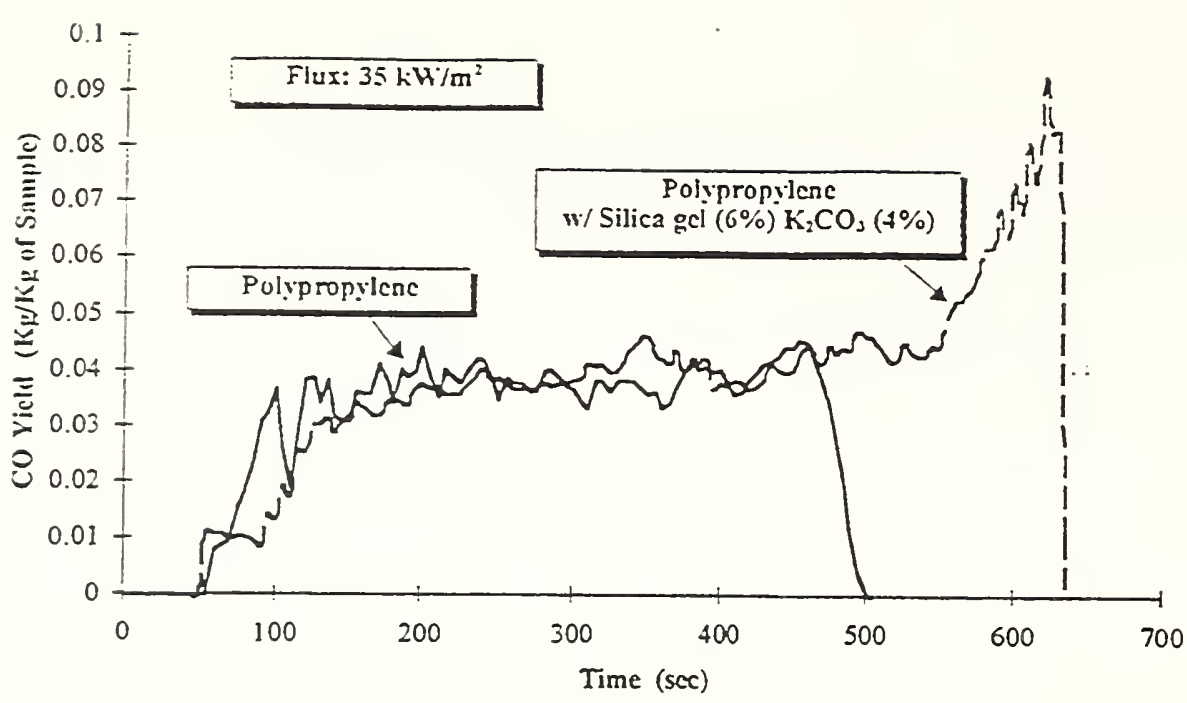

Fig 3. The carbon monoxide yiclds for PP (at $\left.35 \mathrm{~kW} / \mathrm{m}^{2}\right)$ and $\mathrm{PP} / \mathrm{silica} \mathrm{gcl} / \mathrm{K}_{2} \mathrm{CO}_{3}(90 / 6 / 4$ ratio) are ver. similar except at the end of the combustion uhcre very small mass losses may be arificially raising the CO len'el, or since the sample surface is not completely covered by the flame the hot char residue may be gencrating $\mathrm{CO}$ through incomplcte oxidation

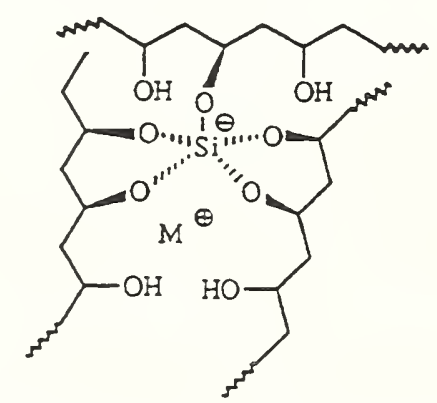

Fig. 4. Pentacoordinate organosilicate crosslinked PVA.

Table 1. Cone Calorimetry Data

\begin{tabular}{|c|c|c|c|c|c|c|c|c|}
\hline $\begin{array}{l}\text { Sample disk } \\
75 \mathrm{~mm} \times 8 \mathrm{~mm}\end{array}$ & $\begin{array}{l}\text { Chas } \\
\text { Yisid } \\
(\%)\end{array}$ & $\begin{array}{l}\text { LOI } \\
\text { (\%) }\end{array}$ & $\begin{array}{c}\text { Pcal } \\
\text { RHR (1) } \\
\left(\mathrm{kW} / \mathrm{m}^{2}\right)\end{array}$ & $\begin{array}{c}\text { Mœan } \\
R H R \\
\left(\mathrm{~kW} / \mathrm{m}^{2}\right)\end{array}$ & $\begin{array}{c}\text { Man } \\
\text { Hcat of } \\
\text { Combustion } \\
(\mathrm{MJ} / \mathrm{kg})\end{array}$ & $\begin{array}{l}\text { Total Hcat } \\
\text { Released } \\
\left(\mathrm{MJ} / \mathrm{m}^{2}\right)\end{array}$ & $\begin{array}{c}\text { Mcan } \\
\text { Ext. Arca } \\
\left(\mathrm{m}^{2} / \mathrm{kg}\right)\end{array}$ & $\begin{array}{c}\text { Mean } \\
\text { CO yield } \\
(\mathrm{kg} / \mathrm{kg})\end{array}$ \\
\hline$P P$ & 0 & - & 1,761 & 803 & 37.9 & 357 & 689 & 0.04 \\
\hline $\begin{array}{c}\text { PP म/ } 6 \% \text { SG \& } \\
4 \% \text { PC }\end{array}$ & 10 & - & $736(58 \%)$ & 512 & 33.1 & 297 & 710 & 0.04 \\
\hline$\overline{P S}$ & 0 & 18 & 1,737 & 1.010 & 27.6 & 277 & 1,422 & 0.07 \\
\hline $\begin{array}{c}\text { PS } 4 / 6 \% \text { SG \& } \\
4 \% \mathrm{PC}\end{array}$ & 6 & 24 & $\begin{array}{l}1,190 \\
(31 \%)\end{array}$ & 725 & 24.7 & 246 & 1.503 & 0.07 \\
\hline PMMA & 0 & 18 & 722 & 569 & 23.1 & 219 & 210 & 0.01 \\
\hline $\begin{array}{c}\text { PMDA } 4 / 3 \% \text { SG } \\
\& 1 \% \mathrm{PC}\end{array}$ & 15 & 25 & $120(42 \%)$ & 246 & 20.9 & 231 & 199 & 0.05 \\
\hline PVA & 4 & - & 609 & 381 & 17.0 & 221 & 594 & 0.03 \\
\hline $\begin{array}{c}\text { PVA \&/ } 6 \% \text { SG \& } \\
4 \% \mathrm{C}\end{array}$ & 43 & - & $194(68 \%)$ & 114 & 12.4 & 101 & 201 & 0.03 \\
\hline Celluiose & 4 & - & 310 & 161 & 11.3 & 101 & 27 & 0.02 \\
\hline \begin{tabular}{|c|} 
Celluiose w/ \\
$6 \%$ SC \& 4\% \\
\end{tabular} & 32 & - & $149(52 \%)$ & 71 & 5.3 & 34 & 20 & 0.04 \\
\hline$\overline{\mathrm{SAN}}$ & 2 & - & 1.499 & 837 & $=5.2$ & 197 & 1.331 & 0.07 \\
\hline $\begin{array}{c}\text { SAN } 4 / 6 \% \text { \%G \& } \\
4 \% \mathrm{PC}\end{array}$ & 3 & - & $\begin{array}{l}1.127 \\
(25 \%)\end{array}$ & 772 & 23.0 & 169 & 1.301 & 0.06 \\
\hline jylon 6.6 & 1 & 30 & 1.131 & 640 & 23.2 & 108 & 234 & 0.02 \\
\hline $\begin{array}{c}\text { Nylon } 6.6 \text { u. } \\
3 \% \text { SG \& } 2 \% \text { PC }\end{array}$ & 5 & 33 & $526(53 \%)$ & 390 & 22.0 & 105 & $\begin{array}{r}171 \\
-\end{array}$ & 0.02 \\
\hline $\begin{array}{c}\text { Nylon } 6.6 \mathrm{w} / \\
6 \% \text { SG } 8.4 \% \mathrm{PC}\end{array}$ & $\overline{6}$ & 30 & $546(52 \%)$ & 370 & 23.5 & 102 & 185 & 0.02 \\
\hline
\end{tabular}




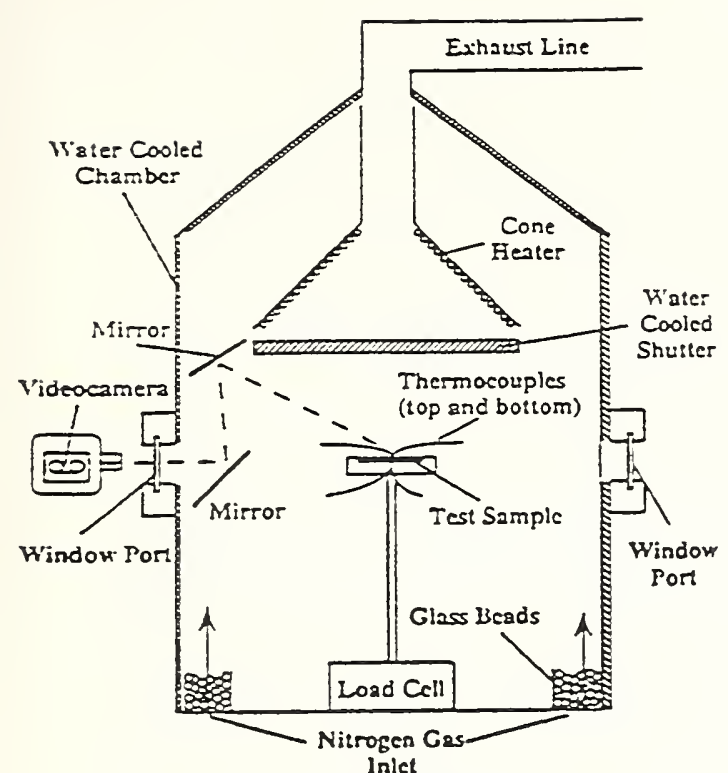

Fig. 5. Gasification apparatus.

Allows study of condensed phase processes in the absence of combustion.

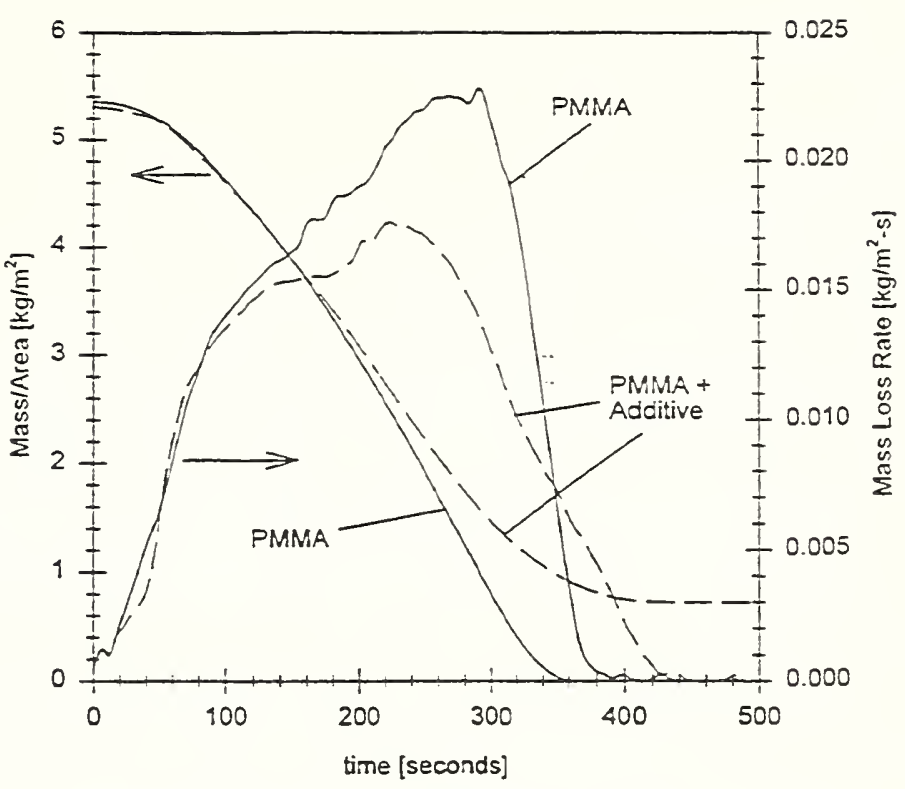

Fig. 6. Gasification experiments for PMMA and PMMA with silica gel $/ \mathrm{K}_{2} \mathrm{CO}_{3}$ (95:4:1)

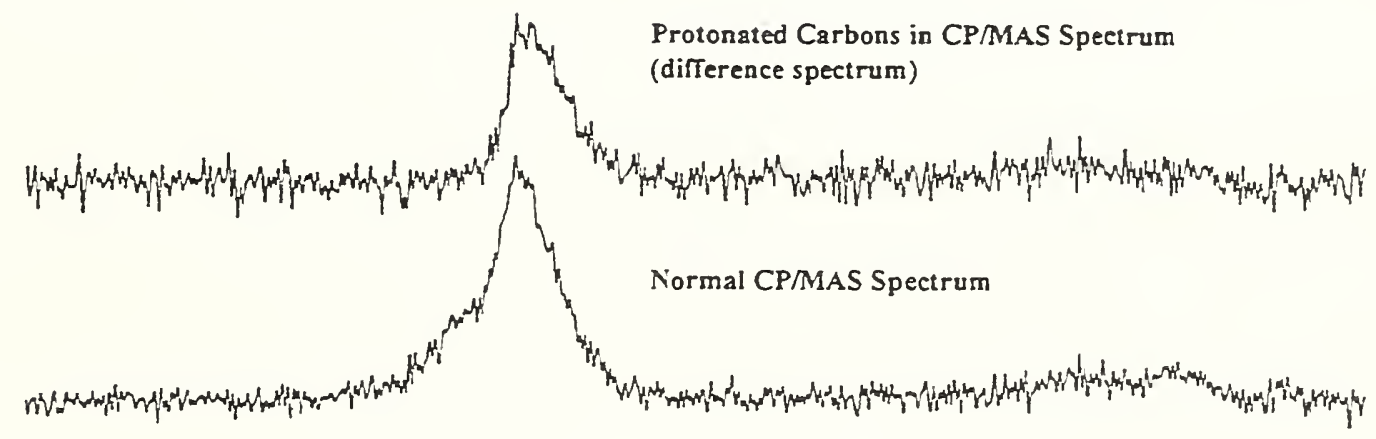

Intertupted Decoupling Spectrum
Non - protonated Carbons in CPIMAS Spectrum

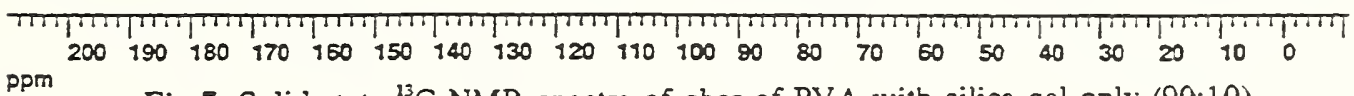

Fig.7. Solid state ${ }^{13} \mathrm{C}$ NMR spectra of char of PVA with silica gel only $(90: 10)$.

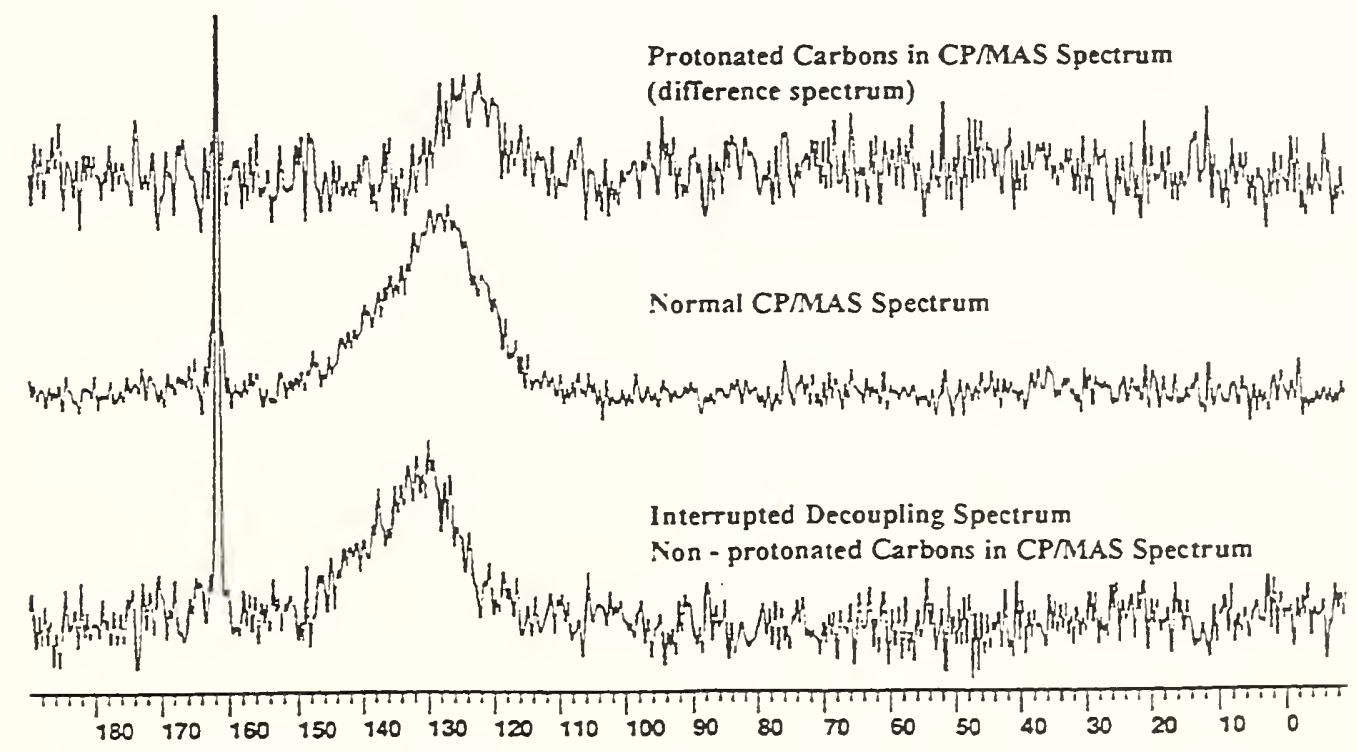

Fig. 8. Solid state ${ }^{23} \mathrm{C}$ NMR spectra of char of PVA with silica gel /. $\mathrm{K}_{2} \mathrm{CO}_{3} \quad$ (90:6:4). 


\section{Discussion}

Henri Mitler: I noticed that you used $6 \%$ and $4 \%$ additions. If there a reason?

Jeffrey Gilman: We haven't explore optimizing that ratio.

Richard Lyon: Did you plan on trying it on systems that would really benefit from something like this like epoxies that have terminal pendant groups that would really benefit from that boost of charge.

Jeffrey Gilman: We are in the process of doing that now.

Richard Lyon: What about moisture sensitivity?

Jeffrey Gilman: We are going to look at other sources of bases and other types of bases and other sources of potassium which seems to be very important.

END: March 19, 1996 
FIRES AFTER EARTHQUAKES 
2 I 2 


\section{PROGRESS REPORT ON \\ FIRES FOLLOWING THE NORTHRIDGE EARTHQUAKE}

\author{
David D. Evans and William D. Walton \\ Building and Fire Research Laboratory \\ National Institute of Standards and Technology \\ U.S. Department of Commerce \\ Gaithersburg, Maryland 20899
}

\author{
Frederick W. Mowrer \\ Fire Protection Engineering Dept. \\ University of Maryland \\ College Park, MD 20742
}

\section{INTRODUCTION}

Fires following earthquakes can add substantially to the community wide destruction initiated by strong earthquakes and the associated after shocks. The loss of life and property caused by fire occurs in a different time frame than the structural and property damage caused directly by the earthquake. While most of the loss caused by shaking occurs during the time of ground movement, there is usually no fire loss during that time. Fire loss directly attributable to the earthquake begins immediately following the earthquake and can continue for days afterward. The destruction potential associated with postearthquake fires is strongly dependent on the damages to the community lifeline systems and to the weather conditions, especially the wind speed.

The January 17, 1994, Northridge earthquake resulted in fires which challenged the resources of the fire service due to the number of fires, disruption of the water supply, and damage to fire protection systems within buildings [1]. The Los Angles City Fire Department responds to over 900 fire, medical, and other emergences on a typical day. This number increased to over 2200 on the day of the earthquake and remain at txice the normal level in the following days. The majority of the estimated 30 to 50 significant post-earthquake fires were located in the San Fernando Valley and were confined to the building of fire origin either by separation or by fire department action. Fortunately there was no loss of life from fire. A principal cause of the fires involved natural gas leaks. A small number of fires were caused by hazardous chemical interactions. A small number of wildland fires occurred that were attributed to earthquake related causes, most likely arcing in overhead power lines. Since the wind was light and the vegetation was not excessively dry, these fires were easily extinguished.

Fires in most of the buildings were confined to the building of fire origin due to a combination of factors including light winds, building construction, building separation, and the actions of the fire department. Experience and predictions of the impact of fire following earthquake indicate that at wind speeds above $9 \mathrm{~m} / \mathrm{s}(20 \mathrm{mi} / \mathrm{hr})$ the fire spread and associated loss increase dramatically [2].

Building-to-building fire spread was limited to three manufactured housing developments (mobile home parks). Figure 1 shows the typical spacing between units in one of these developments. Figure 2 shows an area of the development destroyed by fire. The Building and Fire Research Laboratory staff investigated these areas in detail during the week following the earthquake. Particular attention was paid to areas where the building to building spread of fire was prevented.

Observations made at one of the manufactured housing developments after the fires indicates the method of unit-to-umit fire spread was primarily through windows. Once a unit became completely involved in fire, the thermal radiation was sufficient to cause either the breakage of windows in an adjacent unit or ignite combustibles within the unit directly through window openings. The fire department reported low water pressure in the area, which combined with multiple independent fires and limited resources 
hindered fire fighting operations. The fire spread was stopped either by separation such as roads and open areas or by fire department operations.

A unique aspect of the construction of these manufactured units played a role in limiting the spread and assisting fire department actions. As the units burned from the inside, the carport roofs collapsed from the building side, coming to rest on the outside supporting columns. In this way the carport roofs formed fire breaks as shown in figure 3. Although these roofs would not necessarily have survived the fire by themselves, they reduced the fire exposure on adjacent units and thus enhanced the resistance to community wide fire spread.

This investigation showed the importance of flame spread between building based on ignition of interiors through windows. Research was initiated at BFRL to quantify the effectiveness of different window systems and selected resident self-help strategies in resisting penetration of fire into building interiors through glazed and protected window openings. Preliminary results from this study are presented below.

\section{FIRE RESISTANCE MEASUREMENTS OF WINDOW GLAZING SYSTEMS}

In theory, fire penetration of a structure could occur as a result of radiant transmission through the window glazing, but glazing materials of practical interest are virtually opaque in the infrared wavelengths associated with natural fires, so this mode of fire penetration is not believed to be significant. Alternatively, the incident heat flux from an external fire can heat the glazing, causing thermally-induced stresses in the glazing, particularly at the frame where the glazing is shielded from the incident heat flux. When these stresses exceed the strength of the glazing material, the material breaks, typically with bifurcating cracks propagating from the shielded edge of the glazing material. Breaking of the window glazing material is suspected to be one of the principal failure mechanism associated with post-earthquake fire spread between residential structures.

An experimental study has been conducted to evaluate the performance of window glazing systems and potential protective treatments under simulated exterior fire exposure conditions. In these experiments, different glazing systems and treatments have been exposed to various uniform heat fluxes from electric and gas-fire radiant panels. Both small- and large-scale experiments have been conducted. In the smallscale experiments, $23 \mathrm{~cm}$ wide by $33 \mathrm{~cm}$ high glazing materials were installed in wooden frames and exposed to a gas-fire radiant panel. In the large-scale experiments, commercially available double hung windows, nominally $60 \mathrm{~cm}$ wide by $90 \mathrm{~cm}$ high, were installed in a $1.2 \mathrm{~m}$ wide by $2.4 \mathrm{~m}$ tall wood frame wall and subjected to the heat flux from a large-scale electrically powered radiant panel. Both single-pane and double-pane window assemblies have been tested in wood, vinyl clad wood and vinyl frames.

Several potential protective treatments also have been evaluated. These treatments have included: the use of insect screens and aluminum foil on the exposed side (outside) of the windows, the use of aluminum foil and a vinyl solar film on the inside of the windows, the use of double-pane windows, and the use of special glazing materials, such as high temperature glass, in place of ordinary plate glass.

Results of the study are still being analyzed, but some preliminary observations can be made. Figure 4 shows that for heat fluxes below about $0.5 \mathrm{~W} / \mathrm{cm}^{2}$, single pane glazing remains intact indefinitely. Insect screens placed between the heat source and the glazing seem to increase the imposed heat load (product of the imposed heat flux and the time to breakage) required for failure by nominally about $25-30 \%$. Such an increase is consistent with the shading factor provided by insect screens. A vinyl solar film bubbled and smoked when heated; it did not seem to offer any benefit. Aluminum foil applied to the unexposed side (inside) of the glazing also did not offer any benefit. If anything, this treatment causes earlier failure 
of the glass due to reflection of heat transmitted through the glass back into the glass.

Perhaps the most promising treatment for self-help by building owners and occupants is the application of aluminum foil on the outside (exposed) of a window. Glazing systems with this treatment survived indefinitely under test conditions, indicated by data points on the abscissa of the graph in figure 4 . Even with the introduction of a hole in the center of the aluminum foil covering to simulate a tear, this treatment provides approximately a three- to four-fold increase in the failure time when compared to exposed glass. Other treatments that offer some benefit, albeit at greater cost, include the use of alternative glazing materials, such as high temperature glass, or glazing systems with multiple pane assemblies.

One assembly that performed particularly poorly was the vinyl-framed window. Upon heating, this window assembly lost its strength and drooped, permitting openings through the window to develop even while the glazing itself remained intact.

As a final note, in most of the experiments where the glass broke, it remained in place after breaking.

\section{1995 POST-EARTHQUAKE FIRE AND LIFELINES WORKSHOP}

In addition to the on-site investigation, a post-earthquake fire and lifeline workshop was held by the Building and Fire Research Laboratory, National Institute of Standards and Technology on January 30 31, 1995, in Long Beach, California [3]. The objective of the workshop was to assess technology development and research needs and make recommendations that could reduce the number and severity of future post-earthquake fires. The workshop brought together leaders in the fire service; fire protection engineering; codes and standards; insurance; transportation; and water, gas, power distribution, and telecommunication utilities with experience in dealing with consequences of earthquakes. The workshop participants developed a list of priority project areas where further research, technology development, or information collection and dissemination would serve as a vital step in reducing the losses from future post-earthquake fires.

The research and development needs generated by the participants are separated into two broad categories; ignition and fire spread, and fire control. Under the category of ignition and fire spread all the research needs related to either the direct source of ignition or the first fuel ignited, as well as factors that contribute to fire spread. The category of fire control includes research needs related to systems and personnel whose functions include the control and extinguishment of fires. The following summarizes the findings of the panels by topic areas.

\section{Ignition and Fire Spread}

Although investigations are conducted following most major earthquakes, there remains a lack of knowledge conceming the causes of fires and how fires spread from building to building. A process for collecting and a clearinghouse for storing post-earthquake fire incident data need to be established. Further, a methodology should be developed specifically designed to evaluate the impact of actions intended to reduce the number of fires and control their spread. In order to reduce the potential for a post-earthquake conflagration, the potential pathways for building-to-building fire spread need to be identified and practical measures to control the spread need to be developed.

Failures in power and gas distribution systems have been identified as factors contributing to the initiation of fires following past earthquakes. The technical feasibility of seismically operated shutoffs 
and control mechanisms should be assessed along with a cost/benefit analysis for the use of these systems. Further, guidelines for their installation and use of these devices should be developed. As a part of this analysis the susceptibility of gas leaks to ignition should be examined.

The movement of manufactured housing units during past earthquakes has caused damage to utility lines which resulted in fires. The types of support and anchoring which have successfully maintained these manufactured housing units during previous earthquakes should be examined and guidelines for new and retrofit installations developed.

\section{Fire Control}

Adequate and reliable water supplies are required for both manual firefighting and automatic fire sprinkler systems. Disruptions to the primary municipal water supplies have been common in past earthquakes. Seismic design standards for water supply systems should be evaluated as well as techniques for the rapid assessment and restoration of damage to systems. Further, guidelines for coordination between fire departments and water agencies need to be developed. Since municipal water supplies are often disrupted, altemative water supply sources and distribution systems should be considered. Experience gained in using rural water supplies may be beneficial.

Water based fire protection systems such as automatic sprinklers are an important feature in the fire protection design for many buildings. A database of water based system performance during past earthquakes should be established to assist in identifying causes of past failures. The adequacy of design, installation, and maintenance practices should be evaluated and recommendations for new and retrofit systems be developed. Guidelines also are required to assess the condition of systems following an earthquake to determine if they have retained their design effectiveness.

Passive fire protection systems such as fire resistant assemblies may be damaged during an earthquake even though their condition may not be readily apparent. Guidelines for evaluating the condition of passive fire protection features need to be developed.

Lifeline systems play an important role in controlling fires and handling emergencies following an earthquake. The guidelines for the installation and retrofit of lifeline systems should be reviewed and recommendations for codes and standards developed. In addition, guidelines and procedures for the rapid restoration of lifeline systems should be examined.

Emergency service personnel are unable to respond to all emergencies following a large earthquake; therefore, it is important that the public have adequate information and training to reduce the likelihood of fires starting and take actions to control their spread. Although public information material does exist, the material should be examined basti on the experience gained in recent earthquakes. Citizen volunteer response teams are being trained in some areas and the experience gained should be made widely available.

Experience has shown that following earthquakes, there is frequently inadequate communication between lifeline providers and emergency service organizations. Guidelines for cooperation should be developed based on the successful plans that exist in some communities. Further, since industrial facilities have specialized requirements to handle large emergencies, methods to evaluate their resource requirements and mutual aid plans should be developed. 
Water supplies may be limited following an earthquake and control of large spreading fires may be difficult. The new water additives intended to enhance the firefighting capabilities of water should be examined as a means to assist in the control of building-to-building fire spread with limited resources.

Models presently available to predict fire growth within buildings do not generally have the capability to predict fire spread between buildings. Methods to predict accurately the spread of fires between buildings damaged by earthquakes should be developed to assist in developing post-earthquake fire protection strategies.

\section{FIRE AND LIFELINE STUDIES FUNDED BY NIST}

In response to the workshop recommendations, NIST funded eleven research grants to address some of the major issues with regard to fire safety and lifelines. Most of these studies are still underway, with final reports due late in 1996 or early in 1997. Those that have issued final reports are indicated with the reference. The research grant projects and objectives are:

\section{Northridge Post-earthquake Monograph on Lifelines Performance}

Objectives: to conduct a follow-up lifeline investigation of the Northridge earthquake and publish a monograph of the observations and lessons leamed. The monograph includes the information collected in the initial investigation conducted immediately following the earthquake [4].

\section{Protection of building envelope from external fire sources}

Objectives: to evaluate the fure exposure conditions that cause glass to fail, examine the protection afforded by strategies that could easily be retrofitted, and address the protection of soffit vents from external fire penetration in single family homes.

\section{Fire-related aspects of the Northridge earthquake}

Objectives: to investigate and fully document fires, fire spread and fire department operations, provide analysis of this data in support of future estimation of fires following earthquakes. Summarize lessons learned and insights resulting from this earthquake, in support of loss reduction practices and mitigation of potential conflagrations and large loss fires following earthquakes.

\section{Analysis of fire sprinkler svstem performance in the Northridge earthquake}

Objectives: to analyze the performance of fire sprinkler systems in the Northridge earthquake in relation to the specific earthquake protection measures employed in their design and installation and develop proposed changes to the national installation standard, NFPA 13, which should improve future system performance by bringing brace fasteners up to current levels of technology.

Fire hazards and mitigation measures associated with seismic damage of water-heaters and related components

Objectives: to assess damage of nonstructural elements in buildings which may lead to fire hazards; review current codes and provisions related to seismic design of water heaters and related components; develop, through analysis and experiments, mitigation measures which can be effective in minimizing their fire hazards; and recommend specific seismic code provisions and design guidelines for this class of nonstructural components.

\section{Evaluation of passive fire protection systems following earthquakes}

Objectives: to conduct a post-earthquake safety evaluation of the passive fire prevention features of buildings and add such evaluation to the ATC-20 document, "Procedures for Post-Earthquake Safety 
Evaluation Of Buildings, which in its current form lacks procedures for fire protection system evaluation.

Reliability and restoration of water supplv svstems following earthquakes

Objectives: to assess post-earthquake system reliability, and make recommendations to enhance postearthquake operability of domestic water supply and/or alternate water supply systems, and enable quick restoration of service following an earthquake.

Seismic risk assessment of liquid fuel svstems

Objectives: to review and integrate available methods and procedures of seismic risk assessment and loss estimation, develop a framework for risk assessment that can logically accommodate the state-of the-art results of research and development effort on the physical and functional performance of the liquid fuel transmission systems subjected to earthquakes, identify and highlight the design issues that must reflect the risk concept in the process of the development of design guides, and develop and draft an outline of design guides.

Seismic performance of liquid fuel tanks

Objectives: to document and evaluate the performance of liquid fuel tanks during past major earthquakes, particularly the Northridge earthquake, assess their performance with respect to the current design and construction practices, and develop recommendations for improving their future performance.

The assessment and mitigation of earthquake hazard to electric power

Objectives: to develop 1) guides for selection, installation, and operation of emergency power, 2) guides for seismic evaluation and design of high voltage substation, and 3) fragility curves for substation equipment.

Method to evaluate building fire protection features following earthquakes

Objectives: to develop a methodology which can be used by fire inspectors to evaluate the condition of building fire protection features following earthquakes. The methodology shall address systems such as: automatic fire sprinklers; fire standpipes; dry chemical, liquid and gaseous fire suppression systems; fire pumps; fire alarms; fire detectors; automatic door closers; emergency egress lighting; and smoke control systems.

\section{CONCLUSIONS}

Investigations of post-earthquake fire events have suggested that flame penetration though broken windows is a significant means of fire spread in residential communities. Measurements have shown that covering windows with readily available aluminum foil can substantially increase the resistance of window glazing to breakage under simulated radiant exposures from external fires.

Research is underway to increase the understanding of the performance of fire safety systems and lifelines systems subjected to a strong earthquake and the aftermath. Results of this research will provide guidance to national consensus standards efforts and regulatory authorities. 


\section{REFERENCES}

1. D. Todd, N. Carino, R.M. Chung, H.S. Lew, A.W. Taylor, W.D. Walton, J.D. Cooper, and R. Nimis, 1994 Northridge Earthquake Performance of Structures, Lifelines, and Fire Protection Systems, NIST Special Publication 862, National Institute of Standards and Technology, Gaithersburg MD, 20899, May, 1994.

2. Fire Following Earthquake -- Estimates of the Conflagration Risk to Insured Property in Greater Los Angles and San Francisco, All-Industry Research Advisory Council, Oak Brook, IL, 1987.

3. RM. Chung, N. H. Jason, B. Mohraz, F. W. Mowrer, and W. D. Walton, editors, PostEarthquake Fire and Lifelines Workshop: Long Beach California January 30-31, 1995, NIST Special Publication 889, National institute of Standards and Technology, Gaithersburg, MD 20899, August, 1995.

4. Northridge Earthquake Lifeline Performance and Post-Earthquake Response, Technical Council for Lifeline Earthquake Engineering, Washington, DC, August, 1996. 


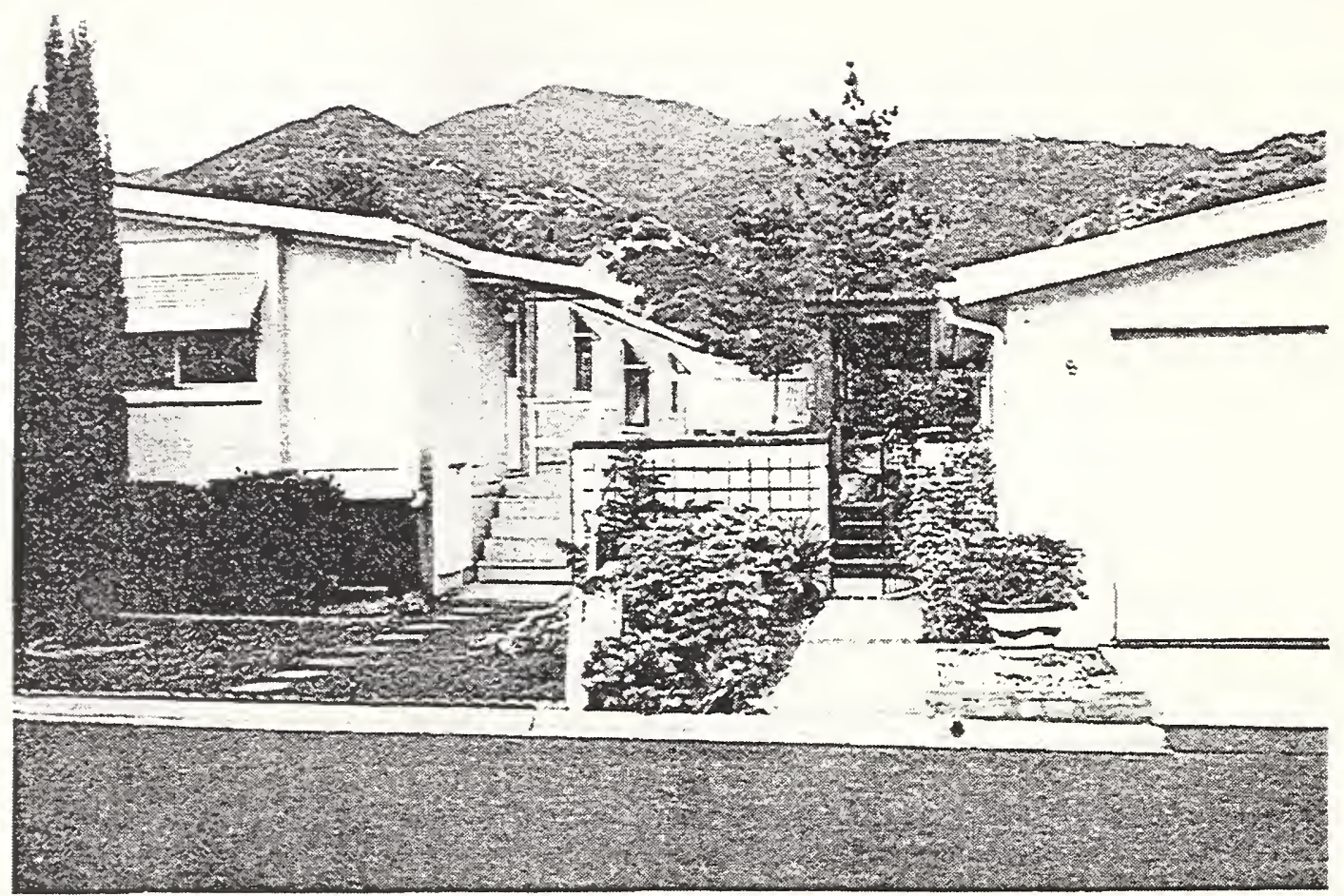

Figure 1. Typical unit spacing in a manufactured housing development.

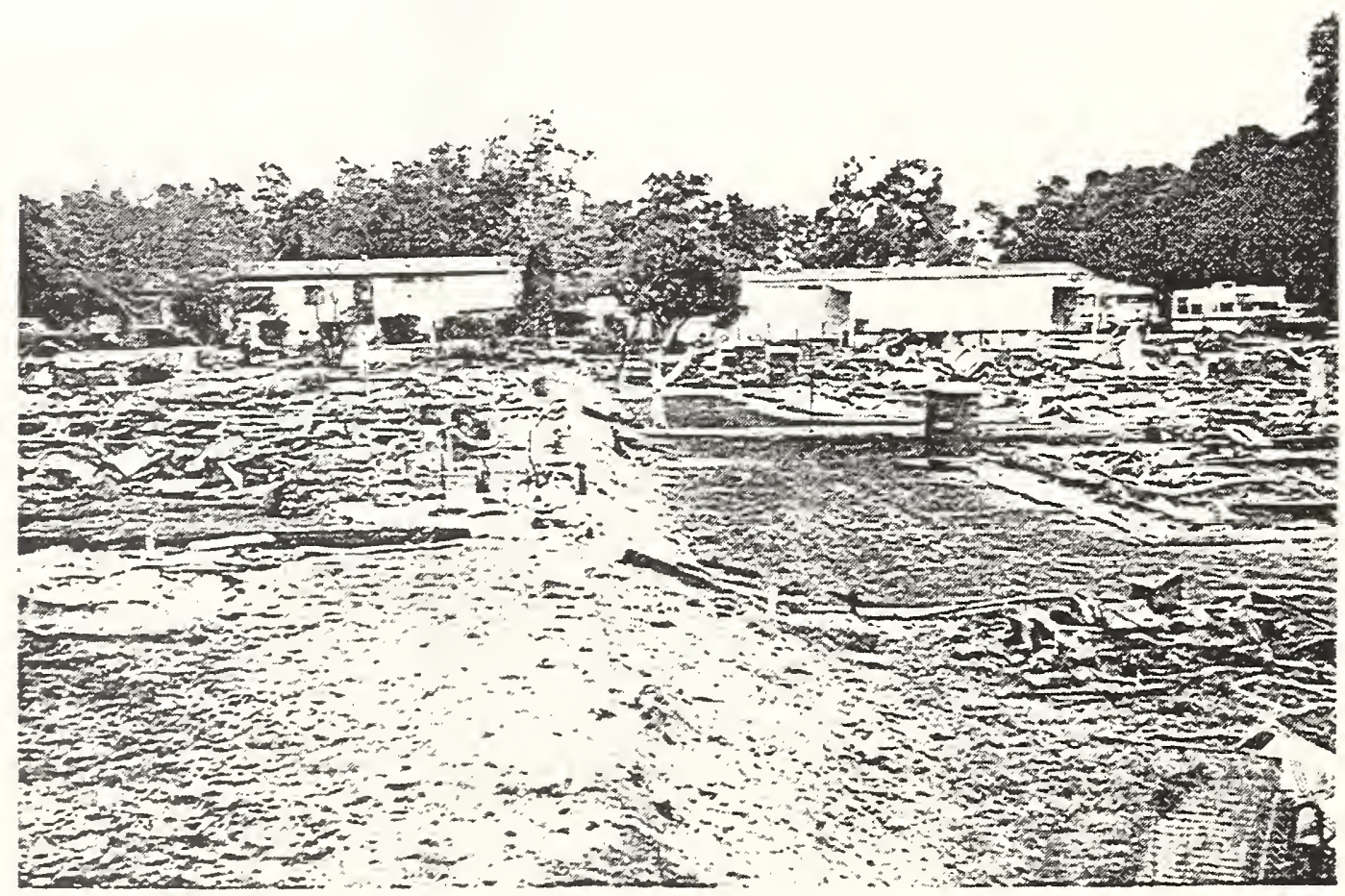

Figure 2. Impact of a multiple unit fire in manufactured housing development. 


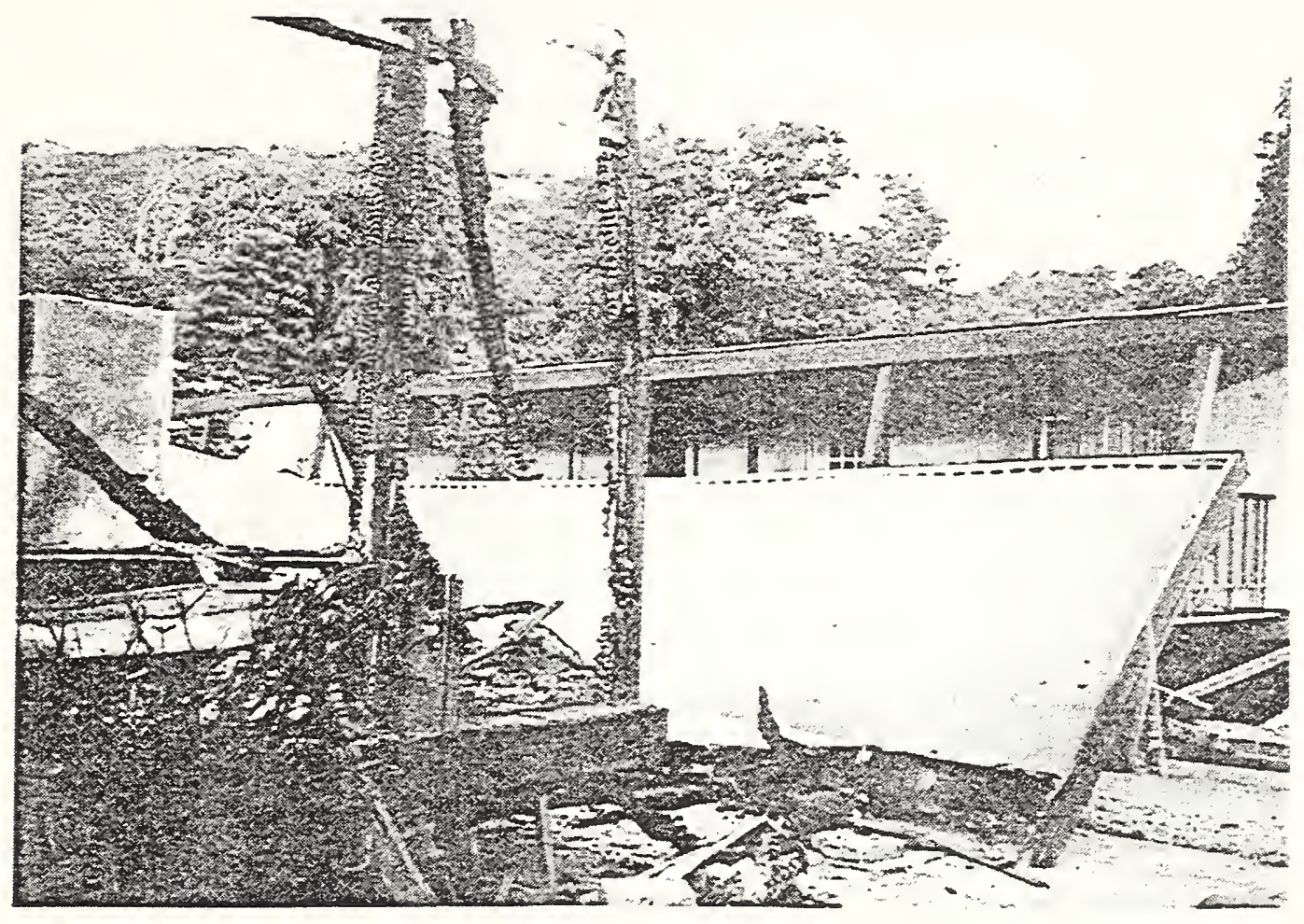

Figure 3. Collapsed roof which acted as a fire break in a manufactured housing development.

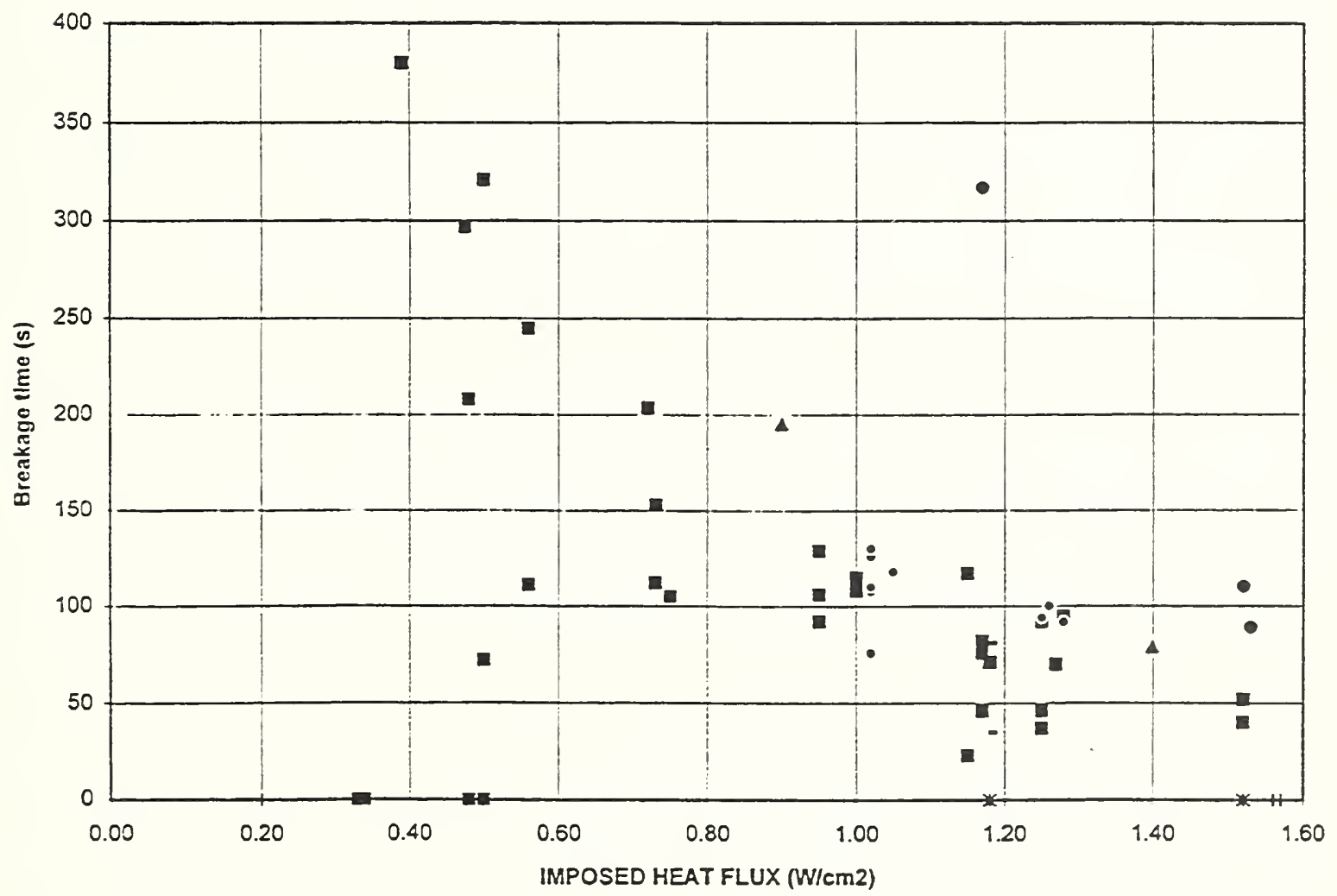

\begin{tabular}{|l|}
\hline - SS \\
DS \\
- Screen \\
$x$ Foil \\
- Foil w/hole \\
+ High temp \\
- Shade
\end{tabular}

Figure 4. Data on resistance of glazing to breakage from an imposed heat flux. 
2 82 


\title{
PROGRESS REPORT ON FIRES FOLLOWING \\ THE 1995 GREAT HANSHIN-AWAJ EARTHQUAKE
}

\author{
Akihiko Hokugo \\ Building Research Institute \\ Ministry of Construction \\ 1 Tatehara, Tsukuba, 305, Japan
}

\section{INTRODUCTION}

At 5:46 a.m., on January 17, 1995, an earthquake, with an epicenter only $14 \mathrm{~km}$ under the ground and hypocentral region just beneath Kobe and other cities between Kobe and Osaka, registered 7.2 on the Richter scale. As Kobe city is Japan's sixth largest city with a population of 1.4 million, the damage caused by this massive earthquake was the worst in postwar Japan. The Great Hanshin Earthquake destroyed more than 200,000 buildings killing more than 5,500 people, and injuring and burying over 40,000 others. More than 200 fires broke out after the earthquake and burnt down more than 7,000 houses or areas covering over 65 hectares, killing more than 350 people who had been buried under the damaged structures.

Investigations were conducted by fire departments and several researchers at universities and research institutes. The following summarizes the findings of the investigations following this earthquake.

\section{IGNITIONS AND CAUSES OF FIRES}

During the first 3 days following the earthquake, more than 200 fires occurred in the Hyogo and Osaka prefectures. Of those, 138 fires took place in Kobe city. Table 1 lists the number of post-earthquake fires by date in 7 cities where the damage of structures was very severe. In the 13 minutes after the earthquake, 60 fires broke out in Kobe city. Fire Research Institute collected data on fire incidents which occurred in 10 days after the earthquake from fire agencies in these areas. $[1,2]$

Kobe Fire Department investigated 175 fires which occurred in 10 days after the earthquake. 81 ignition sources and scenarios have been identified. 44 fires were caused by electric appliances and electrical system malfunctions, 5 fires by gas heaters and stoves, 5 fires by kerosene heaters and 6 fires were associated with gas leaks. Causes of 94 cases have not been identified because the fire were too severe to find any evidence. [1] Because fire suppression and emergency response were the primary priorities following the earthquake, their investigations were not conducted in a timely manner.

Researchers at the Kobe University headed by Professor Murosaki Yoshiteru investigated the causes of the fires by hearing about 400 people who observed the fire. They conducted this investigation from 3 days after the earthquake until February 5. Their investigation covers 181 fires which occurred in 3 days after the earthquake in Kobe, Amagasaki, Nishinomiya, Ashiya, Takarazuka, Itami and Kawanishi city. They pointed out that more than half of the causes were associated with gas and electricity and this tendency is similar to the fire causes of the Northridge earthquake. $[3,4]$ 
Fire Investigation Section at National Research Institute of Police Science conducted a study on causes of electrical fires following the Great Hanshin Earthquake. Data on the ignition time of fires and the recovery time of electricity were collected and it was concluded that some fires occurred as the result of electricity recovery. [5]

Table 1. Number of Post-earthquake Fires by Date in Kobe and adjacent areas in Hyogo and Osaka Prefectures. $\left.{ }^{1)}, 2\right)$

\begin{tabular}{l|cccc:c:c:c:c}
\hline Name of & Jan. 17 Jan. 17 Jan. 17 Jan. 17 & Jan. 17 & Jan. 18 Ian. 19 & \\
Ward and City & $-6: 00$ & $-9: 00$ & $-12: 00$ & $-24: 00$ & Total & Total & Total & Total \\
\hline Higashi-Nada & 10 & 4 & 0 & 3 & 17 & 2 & 4 & 23 \\
Nada Ward & 13 & 2 & 1 & 1 & 17 & 2 & 0 & 19 \\
Chuo Ward & 8 & 7 & 2 & 3 & 20 & 3 & 3 & 26 \\
Hyogo Ward & 11 & 3 & 2 & 1 & 17 & 4 & 3 & 24 \\
Nagata Ward & 12 & 2 & 2 & 1 & 17 & 1 & 4 & 22 \\
Suma Ward & 4 & 7 & 2 & 0 & 13 & 2 & 1 & 16 \\
Tarumi Ward & 0 & 4 & 1 & 1 & 6 & 0 & 0 & 6 \\
Kita Ward & 0 & 0 & 0 & 1 & 1 & 0 & 0 & 1 \\
Nishi Ward & 1 & 0 & 0 & 0 & 1 & 0 & 0 & 1 \\
\hline Kobe City & 59 & 29 & 10 & 11 & 109 & 14 & 15 & 138 \\
\hdashline Ashiya & 4 & 5 & 0 & 0 & 9 & 2 & 2 & 13 \\
Nishinomiya & 11 & 13 & 2 & 4 & 30 & 2 & 3 & 35 \\
Takarazuka & 2 & 0 & 1 & 1 & 4 & 0 & 0 & 4 \\
Itami & 2 & 5 & 0 & 0 & 7 & 0 & 0 & 7 \\
Kawanishi & 1 & 2 & 0 & 0 & 3 & 1 & 0 & 4 \\
Amagasaki & 3 & 3 & 1 & 1 & 8 & 0 & 1 & 9 \\
Akashi & 0 & 1 & 4 & 1 & 6 & 1 & 0 & 7 \\
Awaji & 1 & 1 & 0 & 0 & 2 & 1 & 0 & 3 \\
\hline Hyogo Pref. & 83 & 59 & 18 & 18 & 178 & 21 & 21 & 220 \\
Osaka City & 7 & 6 & 1 & 2 & 16 & 2 & 2 & 20 \\
Toyonaka & 2 & 3 & 0 & 1 & 6 & 1 & 2 & 9 \\
Suita & 1 & 1 & 0 & 0 & 2 & 1 & 0 & 3 \\
Takatsuki & 1 & 0 & 0 & 0 & 1 & 0 & 0 & 1 \\
Sakai- & 1 & 0 & 0 & 0 & 1 & 1 & 1 & 3 \\
Takaishi & & & & & & & \\
Osaka Pref. & 12 & 10 & 1 & 3 & 26 & 5 & 5 & 36 \\
Total & 95 & 69 & 19 & 21 & 204 & 26 & 26 & 256 \\
\hline
\end{tabular}

\section{AREA OF CONFLAGRATIONS AND FIRE STOP FACTORS}

Building Research Institute investigated the buildings surrounding the 150 fires. Maps indicating types of buildings surrounding the fires were made and areas were measured. Table 2 lists the number of large fires ( burned area, $3,300 \mathrm{~m}^{2}$-), medium-sized fires $\left(1,000-3,300 \mathrm{~m}^{2}\right)$, small fires $\left(-1,000 \mathrm{~m}^{2}\right)$, confined fires and fire loss. 7 fires spread over $33,000 \mathrm{~m}^{2}$. [6] 
Fire Research Institute investigated fire stop factors for the 22 large fires following the Great Hanshin Earthquake in Kobe city by observing the buildings surrounding the fires and hearing the people who observed the fires. Table 3 indicates the fire stop factors for the major large fire sites whose area exceeds 33,000 square meters. [7]

Table 2. Number of Post-earthquake Fires by Area and Fire Loss 1), 6)

\begin{tabular}{|c|c|c|c|c|c|c|c|}
\hline $\begin{array}{l}\text { Name of } \\
\text { Ward and City }\end{array}$ & $\begin{array}{l}\text { No. of } \\
\text { Large } \\
\text { Fires }\end{array}$ & $\begin{array}{l}\text { No. of } \\
\text { Middle } \\
\text { Fires }\end{array}$ & $\begin{array}{l}\text { No. of } \\
\text { Small } \\
\text { Fires }\end{array}$ & $\begin{array}{l}\text { No. of } \\
\text { Confined } \\
\text { Fires }\end{array}$ & $\begin{array}{l}\text { Total } \\
\text { No. of } \\
\text { Fires }\end{array}$ & $\begin{array}{l}\text { Total } \\
\text { Number of } \\
\text { burned } \\
\text { Buildings }\end{array}$ & $\begin{array}{l}\text { Total area } \\
\text { of fire } \\
\text { sites } \\
\mathrm{m}^{2}\end{array}$ \\
\hline Nishi & 0 & 0 & 0 & 1 & 1 & 1 & 77 \\
\hline Tarumi & 0 & 0 & 0 & 5 & 5 & 5 & 173 \\
\hline Suma & 1 & 2 & 4 & 3 & 10 & 351 & 98,552 \\
\hline Nagata-Suma & 2 & - & - & - & 2 & 1583 & - \\
\hline Nagata & 14 & 3 & 2 & 3 & 22 & 2926 & 303,558 \\
\hline Hyogo & 4 & 4 & 5 & 1 & 14 & 972 & 127,055 \\
\hline Kita & 0 & 0 & 0 & 1 & 1 & 1 & 54 \\
\hline Chuo & 0 & 3 & 3 & 16 & 22 & 88 & 14,542 \\
\hline Nada & 3 & 4 & 6 & 5 & 18 & 561 & 65,318 \\
\hline Higashi-Nada & 3 & 5 & 3 & 9 & 20 & 333 & 32,886 \\
\hline Kobe & 27 & 21 & 23 & 44 & 115 & 6814 & 642,215 \\
\hline Ashiya & 0 & 0 & 3 & 11 & 14 & 22 & 2,925 \\
\hline Nishinomiya & 0 & 1 & 7 & 22 & 30 & 66 & 8,259 \\
\hline Takarazuka & 0 & 0 & 3 & 11 & 14 & - & - \\
\hline Itami & 0 & 0 & 0 & 7 & 7 & 7 & - \\
\hline Kawanishi & 0 & 0 & 0 & 3 & 3 & 3 & - \\
\hline Amagasaki & 0 & 2 & 0 & 5 & 7 & 10 & 2,090 \\
\hline Total & 27 & 24 & 34 & 95 & 180 & 6922 & 655,489 \\
\hline
\end{tabular}

Table 3. Fire Stop Factors at the Major Fire Sites ${ }^{7)}$

\begin{tabular}{l|cccccccc}
\hline & $\begin{array}{l}\text { Main } \\
\text { Street, } \\
\text { Railroad }\end{array}$ & $\begin{array}{l}\text { Open } \\
\text { Space }\end{array}$ & $\begin{array}{l}\text { Fire } \\
\text { Resistive } \\
\text { building }\end{array}$ & Suppression & Total & Area ${ }^{6)}$ & \multicolumn{2}{l}{$\begin{array}{l}\text { Ignition } \\
\text { Time }\end{array}$} \\
\hline Mizukasa Nishi Park & $42 \%$ & $23 \%$ & $27 \%$ & $8 \%$ & 2,345 & 121,783 & $7-9$ a.m. \\
Sugawara Market & 40 & 33 & 27 & 0 & 1,320 & 77,297 & instant \\
Takahashi Hospital & 65 & 24 & 10 & 2 & 1,258 & 68,850 & instant \\
Eigeyama South & 23 & 34 & 25 & 17 & 2,745 & 61,337 & instant \\
Shin-Nagata Sta. S. & 59 & 11 & 25 & 5 & 1,058 & 39,570 & instant \\
Kobe Department S. & 24 & 16 & 34 & 26 & 1,080 & 35,100 & 7 a.m. \\
Nishidai Market & 23 & 25 & 28 & 24 & 1,195 & 34,407 & instant \\
\hline Total & 38 & 25 & 25 & 12 & 11,001 & 438,344 & - \\
\hline
\end{tabular}

Ignition time is of 17 January 1995. 
Researchers at the Tokyo University headed by Professor Sugawara Shinichi and aluminum framed windows manufacturers conducted an investigation on the openings of houses surrounding the 27 fire sites. They pointed out that most of the windows that prevented the fire from spreading was aluminum framed windows with reinforced glass and concluded that the aluminum fire resistant doors at the openings of houses are essential for the prevention of the fire spreading because the fire spread is caused through the openings when the building itself is not damaged by the earthquake. [8]

\section{FIRE SPREAD MECHANISMS AND VELOCITY}

Tokyo Fire Department investigated the sites of the 11 large fires following the Great Hanshin Earthquake in Kobe city. Fire spread was investigated by hearing the people who observed the fires and fire fighters. Photographs taken from helicopters were also used to know the front lines of fire spread. Velocity of the fire spread was obtained by measuring the distance between front lines at different times. [9]

The Japanese Association for Fire Science and Engineering (JAFSE) conducted a survey to ask the people whose houses were burnt down or about to be burnt down. About 12,000 questionnaires were sent to the 47 urban fire sites and 32 apartment buildings where the fires were confined. About 3,600 people returned the questionnaires. In addition to this survey, JAFSE has been collecting the photographs which were taken during the fires to find out fire spread mechanisms. Over 1,000 photographs has been collected from newspapers, fire departments and the people who responded to the questionnaires. Questionnaires survey and collection of photographs has been supported mainly by FRI and BRI. The work is on the way but a complete result has not yet been obtained.

\section{CIVIL ACTIVITIES}

Researchers at the Kobe University headed by Professor Murosaki Yoshiteru investigated civil activities by hearing about 1,260 people at refuge sites. They asked the people's actions during and after the earthquake motion. Only 9 percent took action to prevent the ignition of fires because the shock was so intense to do so. In one to two hours after the shock, 36 percent of people went to nearby refuge sites because their houses were demolished. This early refuge made it impossible to take appropriate actions to prevent the ignition of fires and in fighting incipient fires. [10]. Kobe Fire Department conducted a similar survey by hearing about 860 people at refuge sites. [11]

According to the fire sites investigation by the Kobe University, civil fire fighting took place at 77 fire sites out of 94.21 out of 24 confined fires were extinguished by the civil fire fighting. $[3,4]$

Civil fire fighting and other aspects of activities during the fires were also asked in detail in the questionnaires by the JAFSE. 33 percent out of people who used kerosene heaters at the time of earthquake turned off kerosene heaters, 34 percent turned off gas heaters and 51 percent turned off gas cooking stoves. As mentioned above, the work is on the way but a complete result has not yet been obtained.

\section{HIGH-RISE FIRES}

BRI investigated some fires in high-rise apartment buildings and combined the data with Kobe University's data to analyze the tendency of high-rise fires following the earthquake. $[12,13]$ 


\section{DAMAGES TO THE FIRE PROTECTION EQUIPMENT AND COMPARTMENTS}

Researchers at Nagoya University headed by Tujimoto Makoto investigated the damages to the fire protection equipment and compartments. Fire Prevention Committee at Architecture Institute of Japan, Dr. Morita Masahiro at Science University of Tokyo, Construction companies and related companies, fire departments of Kobe City and Osaka City conducted similar investigations at some high-rise buildings. These investigations were conducted not for the occurred fires but for the future fires in high rise buildings because this time the earthquake took place very early in the morning and there were no major fires in high rise office buildings. [1416]

\section{REFERENCES}

1. Fire Department of Kobe City ; Fires following the 1995 Southern Hyogo Prefecture Earthquake, 14 April 1995.

2. Fire Research Institute ; Data base of the fires following the 1995 Southern Hyogo Prefecture Earthquake.

3. Murosaki, Y. et al at Kobe Univ.; Fire Causes following the 1995 Great Hanshin and Awaji Earthquake, 10 June 1995.

4. Murosaki, Y. et al at Kobe Univ. and Institute of Environment and Safety Planning; Fire Spread following the 1995 Great Hanshin and Awaji Earthquake, 28 March 1995.

5. National Research Institute of Police Science, National Police Agency; Study on causes of electrical fires simultaneously broke out in earthquake, 18 May 1995.

6. Building Research Institute ; The first stage Report of the Investigation on the 1995 Southern Hyogo Prefecture Earthquake, February 1995.

7. Fire Research Institute ; The Investigation Report on the Fires in Kobe City following the Southern Hyogo Prefecture Earthquake, March 1995.

8. Sugawara Shinichi; Damages at the openings of Houses by the fires following the Southern Hyogo Prefecture Earthquake, December 1995.

9. Tokyo Fire Department; The Investigation Report on the Fires in Kobe City following the Southern Hyogo Prefecture Earthquake, December 1995.

10. Murosaki, Y. et al at Kobe Univ.; Civil fire fighting during the Fires following the 1995 Great Hanshin and Awaji Earthquake, May 1995.

11. Fire Department of Kobe City ;Civil activities during the 1995 Great Hanshin and Awaji Earthquake, 12 April 1995.

12. Building Research Institute; The Intermediate Report of Investigation on the 1995 Southern Hyogo Prefecture Earthquake, August 1995.

13. Building Research Institute ; The Final Report of Investigation on the 1995 Southern Hyogo Prefecture Earthquake, March 1996.

14. Tsujimoto Makoto ; Damages to the Fire Protection Equipment and Fire Resistant Doors, Proceedings of the Symposium on the fires following the 1995 Southern Hyogo Prefecture Earthquake, Architecture Institute of Japan ,19 August 1995.

15. Fire Prevention Committee at Architecture Institute of Japan; Damages to the Fire Protection System by the 1995 Southern Hyogo Prefecture Earthquake, 19 August 1995.

16. Morita Masahiro; Damages to the Fire Protection Equipment, Proceedings of the Symposium on the fires following the 1995 Southern Hyogo Prefecture Earthquake, Japan Association for Fire Science and Engineering, 26 May 1995. 
2 88 
in The 1995 Great Hanshin Earthquake

\section{Ai SEKIZAWA}

Fire Research Institute

Fire Defense Agency

3-14-1, Nakahara, Mitaka, Tokyo 181

\section{ABSTRACT}

A number of concurrent post-earthquake fires occurred in the Hanshin Earthquake. In terms of fire incidence in the regions heavily struck by the earthquake, fires occurred almost uniformly in proportion to the degree of structural damage even other than Nagata-ward in Kobe City where large fires were concentrated. This fact indicates that most of the post-earthquake fires were suppressed in their early stage by fire brigades. The reason for concentration of large fires around Nagata-ward is not only high incidence of fires, but also higher risks of fire spread attributed to city configuration, i.e. ratio of wooden structures, building coverage in the area, and the width of streets, etc. in those regions. In addition, the performance of firefighting activities in the early stage is another key factor to the difference of fire damage by region.

The pattern of ignitions in time order and causes of post-earthquake fires are very similar to those observed in the Northridge Earthquake. These similarities in fire ignitions on both the two earthquakes should be remarked and studied further.

Keywords : post-earthquake fire, conflagration, city configuration, firefighting activity.

\section{INTRODUCTION}

Several crucial lessons were learned from the Northridge Earthquake in Los Angeles in 1994. One year later, the Great Hanshin Earthquake ( officially referred to as "the Off-South Hyogo Prefecture Earthquake" by the Japan Meteorological Agency : JMA ) happened revealing them to us again on January 17, 1995. It can be said the worst scenario expected to which we did not have enough time to apply the lessons from the Northridge Earthquake to mitigate potential risk in Japan.

Even though the degrees of damage were different, these two earthquakes under the large modern cities have quite a few similarities in terms of structural damages, disruption of lifelines, pattern of fire outbreaks, some barriers to firefighting activities such as damaged fire hydrants, and so on. However, it is also important to point out a significant difference in the damages of the two earthquakes, that is, occurrence of conflagrations in city areas. In the Northridge Earthquake, no conflagration took place except in special occasions like mobile home parks. On the other hand, there were such fires found in Kobe. For this difference in risks of fire spread, the conditions in the city areas, such as building coverage and width of streets, are considered as ones of the most significant reasons. Because, if a number of fires occur simultaneously beyond the firefighting capability to control in zones having high dense wooden structures, fires would inevitably spread.

The Hanshin Earthquake left us a significant task: how to improve means of fire spread prevention in city areas with zones having very packed wooden structures in Japan. In this paper, the post-earthquake fires and fire stop factors in the Hanshin Earthquake are discussed first, and then the outline of firefighting activities in the early stage is discussed. 


\section{POST-EARTHQUAKE FIRES}

\subsection{Post-Earthquake Fires in Time Order}

Table 1 and Table 2 show the number of fires in time order following the Hanshin Earthquake by city in suffered regions. As seen in Table 1 which shows the number of fires following the earthquake in Kobe City, 109 fires started on the day of the earthquake, and in the following two days, 14 and 15 fires started. The number of the fires gradually decreased, and during 10 days after the quake, 175 fires occurred. However, all the twelve large fires, more than $10,000 \mathrm{~m}^{2}$ in bumed area, occurred on the day of the earthquake, January 17 . Also, 60 fires started shortly after the earthquake, thus, the situation can be called "a number of concurrent fires just after an earthquake".

Figure $1^{1)}$ provides the data on the number of fires by hours in Kobe City on January 17. The time period " 5 " on the horizontal line in the figure stands for 14 minutes after 5:46 a.m. when the earthquake struck.

As it is clear from Figure 1, about half of the fires on 17th occurred in 14 minutes just after the main shock. On the other hand, during the day of 17 th, about the other half occurred after 6:00 a.m. and some started one by one hours later after the earthquake. Actually, this pattern of occurrence of fires is very similar to the one observed in the Northridge Earthquake.

Figure $2^{2)}$ shows fires by hours on the day of the Northridge Earthquake in the jurisdiction of the Los Angeles City Fire Department. The profile of the fire occurrence in time order is very similar to that of Kobe City. For this pattern of fire ignitions, the Los Angeles City Fire Department explains as follows. The first wave was formed by a number of fires starting simultaneously just after the main shock. These fires were mainly caused by gas leakage. Also, the second wave was formed by sporadic fires which were mostly electrical fires derived from the recovery of the electricity supply in collapsed structures. The same situation of fire ignitions were observed again in the Hanshin Earthquake. The similarities in the patterns and causes of fire ignitions on both the two earthquakes should be remarked.

\subsection{Regional Distribution of Fires}

Figure $3^{1)}$ shows the regional distribution of fires from January 17 to January 19 . In terms of fire incidence in regions heavily struck by the earthquake, fires occurred almost uniformly even other than Nagata-ward in Kobe City where large fires were concentrated. The incidence of fires following the earthquake seems to be in proportion to the degree of structural damage. This fact indicates that most of the post-earthquake fires were suppressed in their early stage by fire brigades as mentioned later. The concentrated occurrence of large fires happened around Nagata-ward not simply because of high incidence of fires, but because of the higher risks of fire spread, i.e. ratio of wooden structures, building coverage in the area, and width of streets, etc. in those regions. (See Figure $4^{3)}$ and Figure 5 )

\subsection{Fires by Size by Region}

Table 3 and Figure 6 show numbers and percentages of post-earthquake structure fires from January 17 to January 19 by size and by region. The total number of fires by January 19 is 231, 132 of which are in Kobe, but other 99 are out of Kobe. However, twelve very large fires having burned area over $10,000 \mathrm{~m}^{2}$ were concentrated around Nagata-ward in Kobe and, among 48 large fires having bumed area over $1000 \mathrm{~m}^{2}$ in the table, 47 fires occurred in Kobe City except the one in Nishinomiya City. In terms of average burned structures per fire as an index of average fie size by region, the cities other than Kobe have less than 2.0 (only Nishinomiya has 2.4, but still very small ). Also, less damaged regions in Kobe like Tarumiward, Kita-ward, and Nishi-ward have less than 2.0. Only south east part of Kobe City had many large fires. This is also explained by the same reason as mentioned above. 


\section{THE FACTORS CONTRIBUTING TO FIRE TERMINATION}

\subsection{The Investigation on Large Fires in Kobe}

In 6 days from January 26 to 31 in 1995, the Fire Research Institute organized an 11 -member team including the author and investigated 21 large fire sites around Nagata-ward shown in Table 4. The purpose of this investigation was to obtain and maintain important information to clarify the areas burned by large fires and the factors of fire spread and its termination.

The results gained from the investigation on fire spread boundaries and factors of fire terminations were recorded on 1/2500-scaled maps. For example, Figure 7 demonstrates the fire site near Mizukasa-nishi Park. The paper space does not allow the explanation of the other fire sites, which are described in the Report ${ }^{\text {g }}$ of the Fire Research Institute.

\subsection{Proportion of Each Fire Stop Factor}

Table 4 shows total extension distances along the fire spread boundaries of each large fire and the proportion of extension distances by fire stop factor which were estimated from the investigation.

At any fire spread boundary, fire brigades must have worked to extinguish fires finally. However, the fire stop factors treated here stand for factors that mainly functioned for fire termination. For instance, in the case that fire termination would have been difficult only by firefighting activity without factors as roads, open spaces, and fire-resistive buildings, the physical factors are considered as main factors. Also, in the case that fire fighting activity was done and essential or that it would not have been possible to put out fires without firefighting activity, suppression is chosen as a main factor, even if there are some other factors. Therefore, the proportion of factors was estimated after one factor was chosen as the main one, although several factors may have worked synthetically in some cases. Also, the effects of change of wind velocity and/or direction need to be considered to have our final results.

The tendency over the 21 fire sites is shown in Table 5: "Roads and railways" (40\% of the total ), "open spaces" (about 23\%), and "fire-resistive buildings and walls" (also about 23\%). These physical factors are up to $86 \%$ of the total. This result seems quite natural, since each fire site investigated was large enough to be beyond firefighting capability to control and needed natural fire stop factors on most part of fire spread boundaries to go off. On the other hand, "suppression" which was done mainly by firefighting activities of fire brigades and partly by activities of citizen volunteer teams, occupies about 14\%. However, considering the definition of a main factor here and total extension distances on fire spread boundaries stopped by "suppression" in the 21 fire sites, $2,316 \mathrm{~m}$, the figure $14 \%$ is not small.

\subsection{Comparison with Large Fires in The Past}

The results described above were compared with the results of the 1923 Great Kanto Earthquake's proportion of fire stop factors. In the result ${ }^{\text {}) ~}$ of the Kanto Earthquake whose total extension distances of fire spread boundaries of all large fires is $59.6 \mathrm{~km}$, the firefighting activities as a fire stop factor score $15.1 \%$, and the figure is close to $14 \%$ of that of the case in Kobe. In 1923, there was no category of fire-resistive buildings, maybe since there were not so many fire-resistive buildings and fire-proof wooden buildings. Open spaces, including roads (27\%), occupied $72.5 \%$ of the total figure.

As one of very few recent large fires, the Sakata City Conflagration occurred in 1976. In this fire, the proportion of fire-resistive buildings in the total extension distances of fire spread boundaries was $16.7 \%$.

From these facts, the proportion of "fire-resistive buildings and walls" (24\%) observed in this investigation is not a small figure, that is, the role of fire-resistive buildings as a fire stop 
factor cannot be neglected. Also, "roads and railroads" ( $40 \%$ of the total ) plays an important role as a fire stop factor even though some are still narrow.

\section{FIRE FIGHTING ACTIVITIES IN THE EARLY STAGE}

\subsection{Recognition of Fire Ignitions by The Fire Departments in The Early Stage}

From just after the earthquake, all of the 118 emergency telephone lines became busy at the Headquarters of Kobe City Fire Department. Incident report calls to the Headquarters counted 441 by 7:00 a.m. and more than 6,000 by the end of January 17 . However, most of the calls by 6:00 a.m. did not reply to the receivers. This kind of troubles, caused by malfunction of exchangers or transmittance system of Nippon Telegraph and Telephone Corporation ( NTT ), decreased as time passed. However, about $40 \%$ of the 6,872 calls received on the day did not transmit sounds.

However, even if they had received normal report calls, most of the calls could have been not emergency but to ask for information, as seen in Table $5^{\text {ग }}$. Thus, it is doubtful whether incident reports on fires could be obtained effectively through the emergency telephone line, 119 , in the case. It is very important to grasp disaster information actively and voluntarily as the experience of Nishinomiya City Fire Department.

The Headquarters of Nishinomiya City Fire Department recognized 16 fires on January 17. The professional fire brigades and volunteer fire corps found 11 out of the 16 by themselves, while working or supervising from some higher places. For 3 of the fires, citizen directly came to fire stations to report. Then, they had only one fire report call through 119 as the earliest notice. Actually, this has not been unexpected at all. The same thing happened in past earthquakes such as the 1993 Kushiro-oki Earthquake, and they will probably be observed again in future earthquakes as well.

The telephone plays a significant role in the modem society. The emergency call ( to 119) system and the automatic dispatch control system, based on the calling system, naturally work in the general time. However, it should be considered that they may not be so reliable that they work always at the time of severe disasters like earthquakes. For example, there was considerable delay in recognizing occurrence of post-earthquake fires by the fire departments in the early stage after the Hanshin Earthquake as shown in Figure 8.

Therefore, it is absolutely needed to think of the system of collecting and communicating disaster information by alternative channels. Although it might not sound highly sophisticated, the author believes that "the system of collecting information by people" is necessary. In other words, we should consolidate the system of receiving direct reports from citizen, and also recognize the significance of collecting disaster information by fire personnel on their way to summon or patrolling fire engines in districts of jurisdiction. And, actually, the effectiveness of patrolling fire engines to collect information was already demonstrated by the experience of Los Angeles City Fire Department in the Northridge Earthquake.

\subsection{The Performance of Fire Brigades Activities in the Early Stage}

\section{- In Kobe City, Nishinomiya City, and Ashiya City -.}

Table 6 provides the data of post-earthquake structure fires in Kobe City, Nishinomiya City, and Ashiya City by 7:00 a.m. on January 17 and the initial conditions of fire brigades toward the fires in the initial stage.

In Kobe City, by 7:00 a.m., there were 63 simultaneous fires. Actually, the number of fires was more than that of fire engines, 40. Regarding three wards, Tarumi, Kita, and Nishi in Kobe, there was only one fire so that the fire stations there had much capacity left in the early stage to cope with fires in their jurisdictions, because firefighting operation of each fire station in the initial stage at an earthquake is usually concentrated upon districts within its own 
jurisdiction. Therefore, the three wards had better be excluded when the firefighting capability in the early stage for Kobe City is considered. Then, they had only 28 fire engines, which were ready, against 62 simultaneous fires. In other words, as simply calculated, they could not cope with 34 fires at least. In Table 6 , the number of large fires which burned more than $1,000 \mathrm{~m}^{2}$ is roughly equal to the number, the difference between the number of simultaneous fires and that of fire engines ready to go in each city or ward. This fact indicates that the extent of fire damage at an earthquake depends basically upon the balance of number of simultaneous fires and the fire fighting capability such as number of fire engines ready for use, if water supply like cisterns at least is secured.

The contents of firefighting activities in the cities of Nishinomiya and Ashiya are worth close studies. Nishinomiya City is one forth as large as Kobe City in population, and also its number of fires is about one forth as well by chance, as seen in the table. The Nishinomiya Fire Department possessed 14 fire engines to take care of 16 fires, and besides, they could have cooperation of the volunteer fire corps that possess more fire engines than the professional fire brigades have. They also had as many cisterns as Kobe City did. These factors effectively worked to hold fire damage the least, even though they could not use fire hydrants. The two fires "burning more than $1,000 \mathrm{~m}^{2}$ " were suppressed before they burned more than $2,000 \mathrm{~m}^{2}$.

The city development standard of Nishinomiya City demands at least one cistern per three of water supplies for firefighting. This is the reason the city has a lot of water reservoirs including private ones. In addition to that, they learned a lesson from a serious shortage of water in the summer of 1994, and made the "Manual of Firefighting Operation for Special Occasions with Shortage of Water". Before the earthquake, they already had practical training to use rivers and swimming pools, supposing a suspension of water supply, and this preparedness significantly helped to distinguish fires in the earthquake.

On the other hand, Ashiya City is populated about 87,000 and had simultaneous eight fires following the earthquake that is as high incidence as that of Nagata-ward in Kobe. Although fire hydrants were not available, the fire brigades put out fires so successfully that six fires burned only one building for each and that none burned more than $1,000 \mathrm{~m}^{2}$. They distinguished all the simultaneous fires in the early stage, which number was more than that of fire engines they had. Many fires, fortunately, started in fire-resistive structures. In addition, there are two other reasons pointed out: the cooperation of the volunteer fire corps who possess fire engines and the adequate use of two rivers running through the city.

\subsection{The Dilemma How To Put The Priority on Firefighting or Rescue}

The firefighting activities in the early stage, especially in Kobe City, were surely influenced by a dilemma how they should put the priority on firefighting or rescue of the buried alive in a number of collapsed buildings. However, the essence of this issue is, not the question of decision by fire personnel on site to distinguish fire or to rescue the buried, but exactly the fact itself that too many structures were destroyed as ever seen before and too many people were buried alive there. The basic purpose of the fire brigades' activity in the early stage at an earthquake is, obviously, firefighting to prevent conflagration, because fire service personnel are unable to respond all emergencies following a large earthquake.

Although a number of incidents to need rescue work happened at the same time in the Hanshin Earthquake, the author think that these happenings should be discussed as one of the crucial issues other than firefighting activities in the early stage. In other words, the fundamental countermeasures to be taken are to build earthquake-proof structures and also to reinforce existing old structures to be safe for preventing the same tragic situation. Moreover, a national emergency rescue system should be considered after the experiences of the USAR in the United States for incidents which need rescue work beyond the capability of a few local fire departments. 


\section{CONCLUDING REMARKS}

As observed in Kobe City at the time of the Hanshin Earthquake, it might possibly happen again in future earthquakes that some fires are not suppressed in the early stage and grow up to conflagrations.

The point is how we prevent fire spread to minimize fire damage, but the solution should not be looked for only in firefighting capability, which has limitation. A fire that has grow'n to a conflagration can not be easily stopped only by firefighting activity. For example, the 1976 Sakata City Conflagration did not stop until it went to a big river, the Niida River, that is a natural fire stop boundary, even though 217 fire engines in total for there days were on duty. In order to prevent a conflagration, fire-proof city planning such as construction of fire stop boundaries along roads by widening and/or construction of fire-resistive structures, arrangement of location of parks, green zones, promenades, and open water or water reservoirs etc. is significantly necessary besides reinforcement of the firefighting capability.

\section{REFERENCES}

1) Sekizawa, A : "Post-Earthquake fires and Firefighting Activities in the Hanshin Earthquake", Science Journal KAGAKU, Iwanami-Shoten, 1996.2.

2) Scawthorn, C., et al : "Fire-Related Aspects of the January 17, 1994 Northridge Earthquake", Chapter 8, Spectra, Earthquake Engineering Research Institute, Oakland CA.

3) Kajima Corporation: The Second Report on the Damage in the 1995 Southern Hyogo Prefecture Earthquake, 1995.3.

4) Building Research Institute : The Interim Investigation Report on the Damage in the 1995 Southem Hyogo Prefecture Earthquake, 1995.8.

5) Fire Research Institute : The Investigation Report on the Fires and Fire Stop Factors in Kobe City in the 1995 Southern Hyogo Prefecture Earthquake, 1995.3.

6) Tokyo Fire Department : The Research Report on Firefighting against District Fires, 1976.3.

7) Murozaki, Y. : "Overview of the fire damage", Proceedings of the Symposium on Lessons from Fire Damage in the 1995 Southem Hyogo Prefecture Earthquake, Fire Safety Division of Architectural Institute of Japan, 1995.8. 


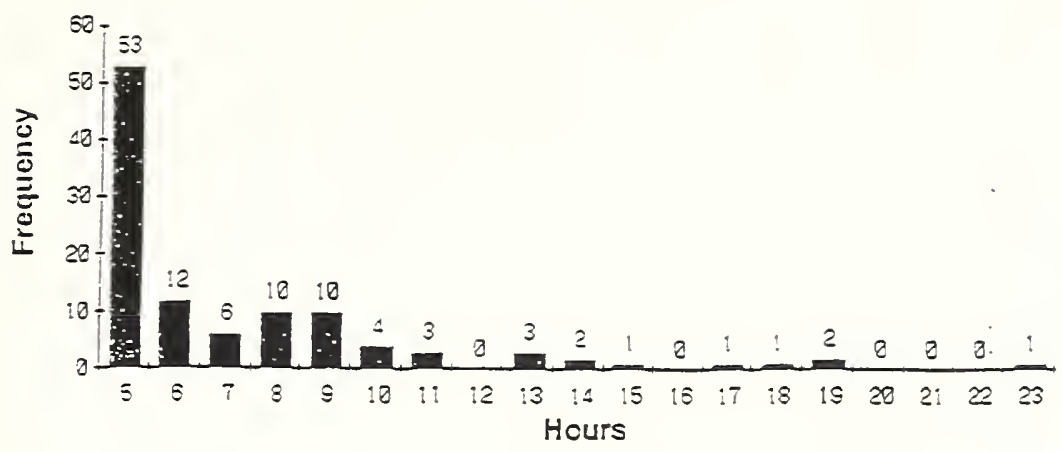

Figure 1. Frequency of fires by hours within January 17 in Kobe City for the 1995 Hanshin Earthquake."

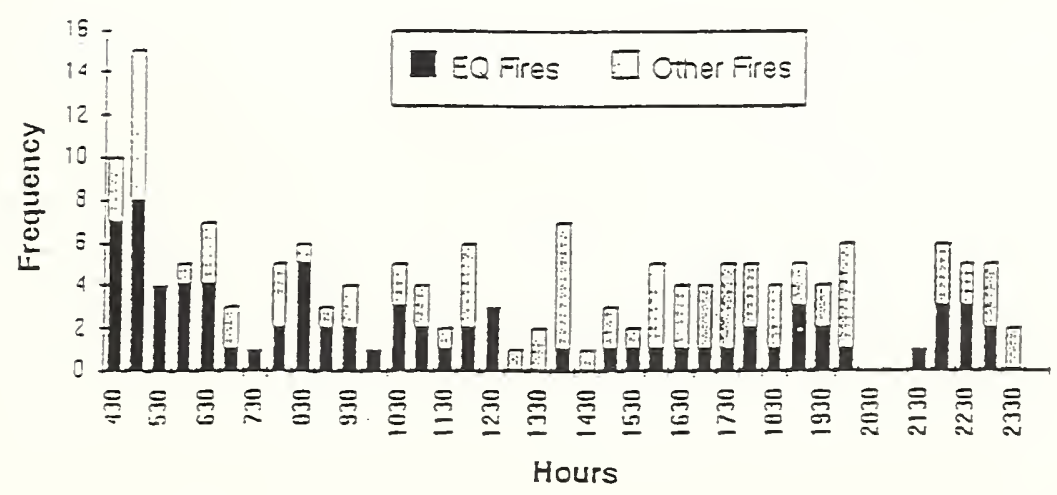

Figure 2. Frequency of fires by hours within January 17 in Los Angeles for the 1994 Northridge Earthquake. ${ }^{21}$

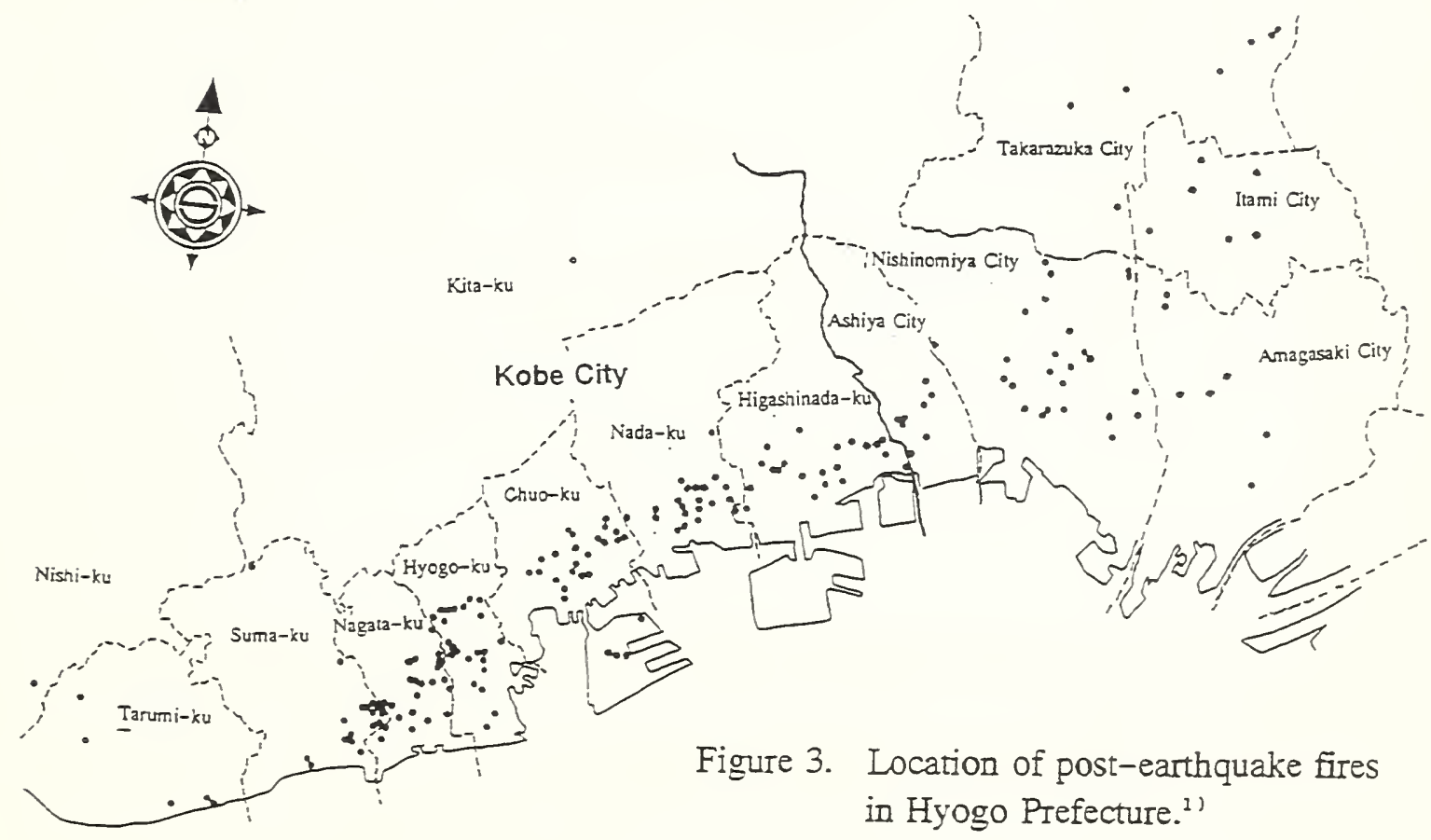




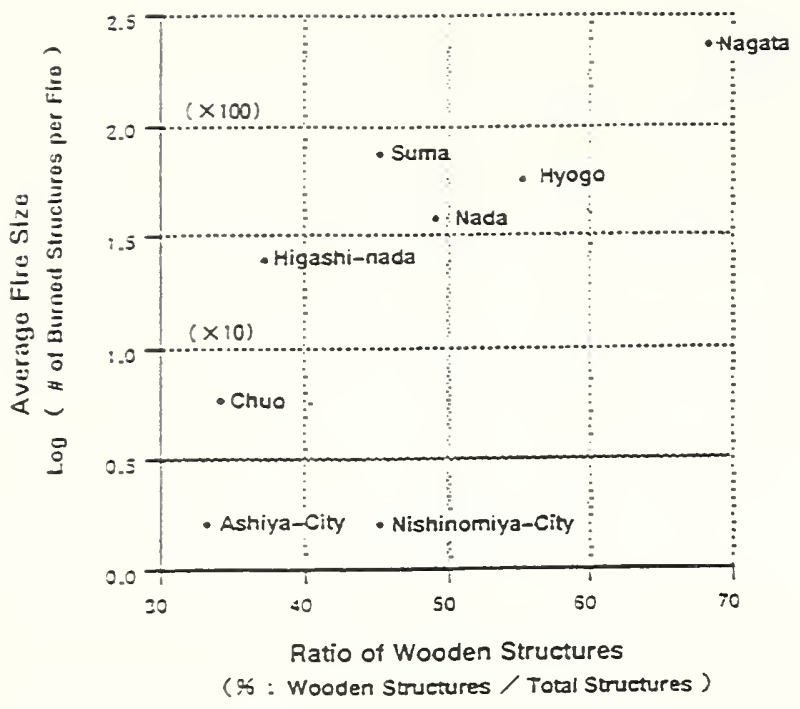

Figure 4. Relation between average fire size and ratio of wooden structures by region. ${ }^{31}$

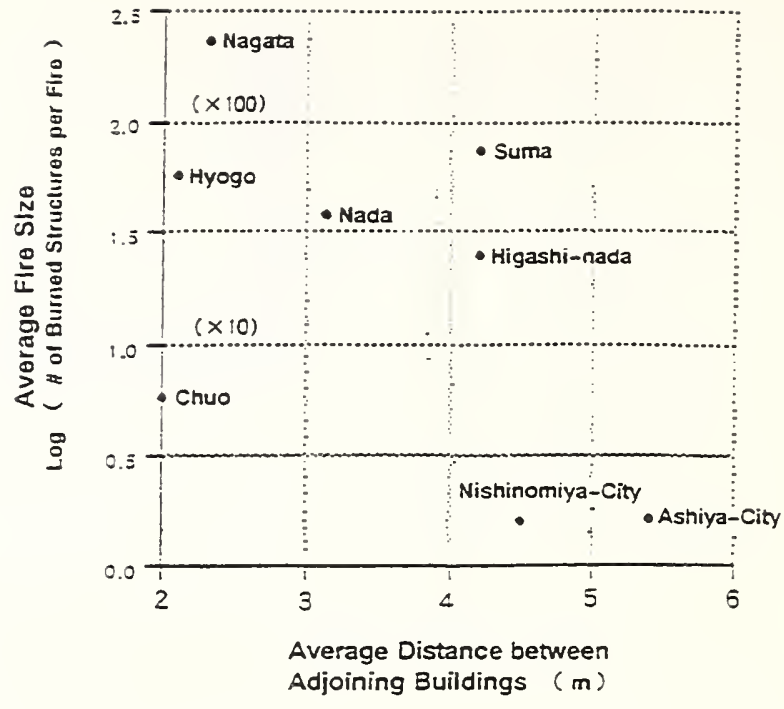

Figure 5. Relation between average fire size and average distance $^{4)}$ between adjoining buildings by region.

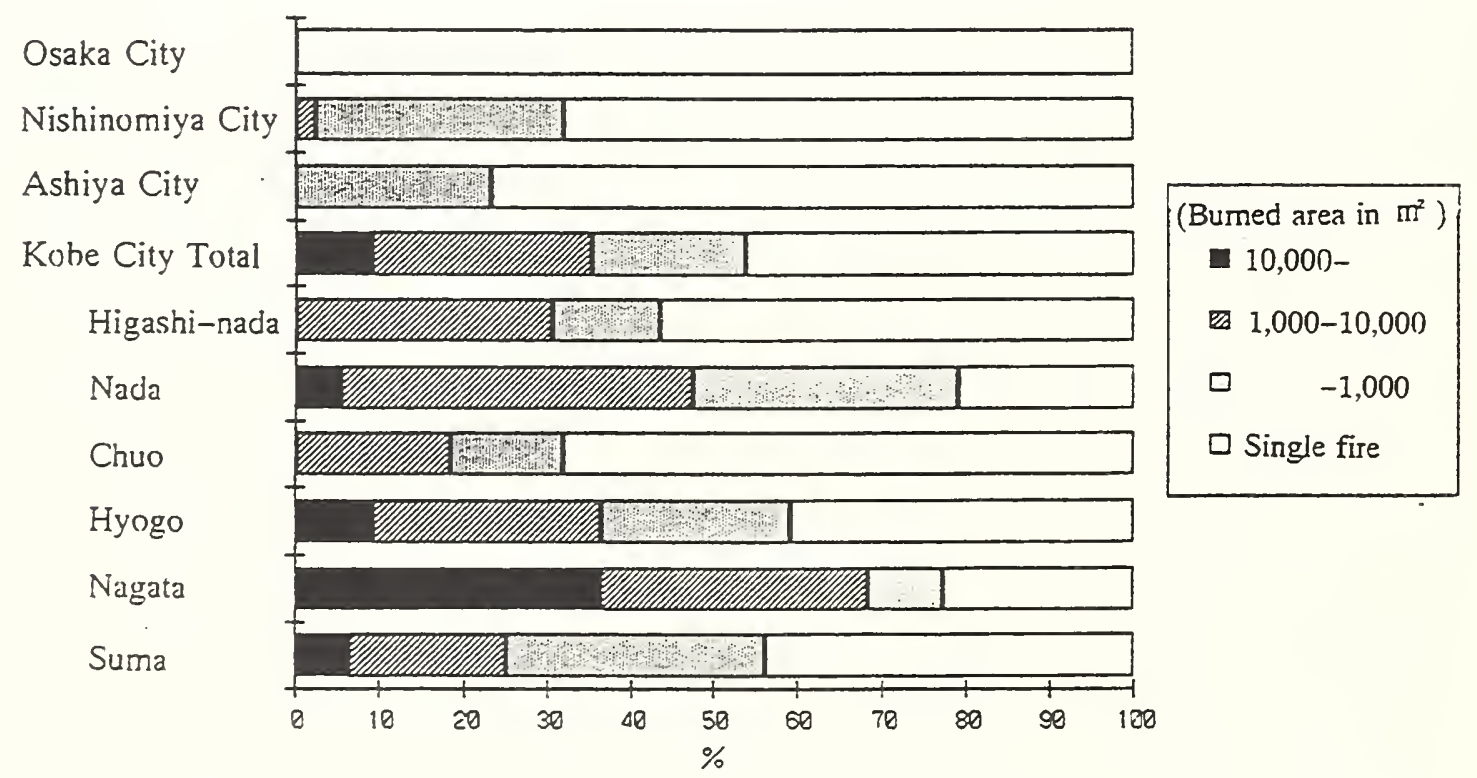

Figure 6. Proportions of post-earthquake structure fires by size by region.

(Fires for January 17 th-19th) 


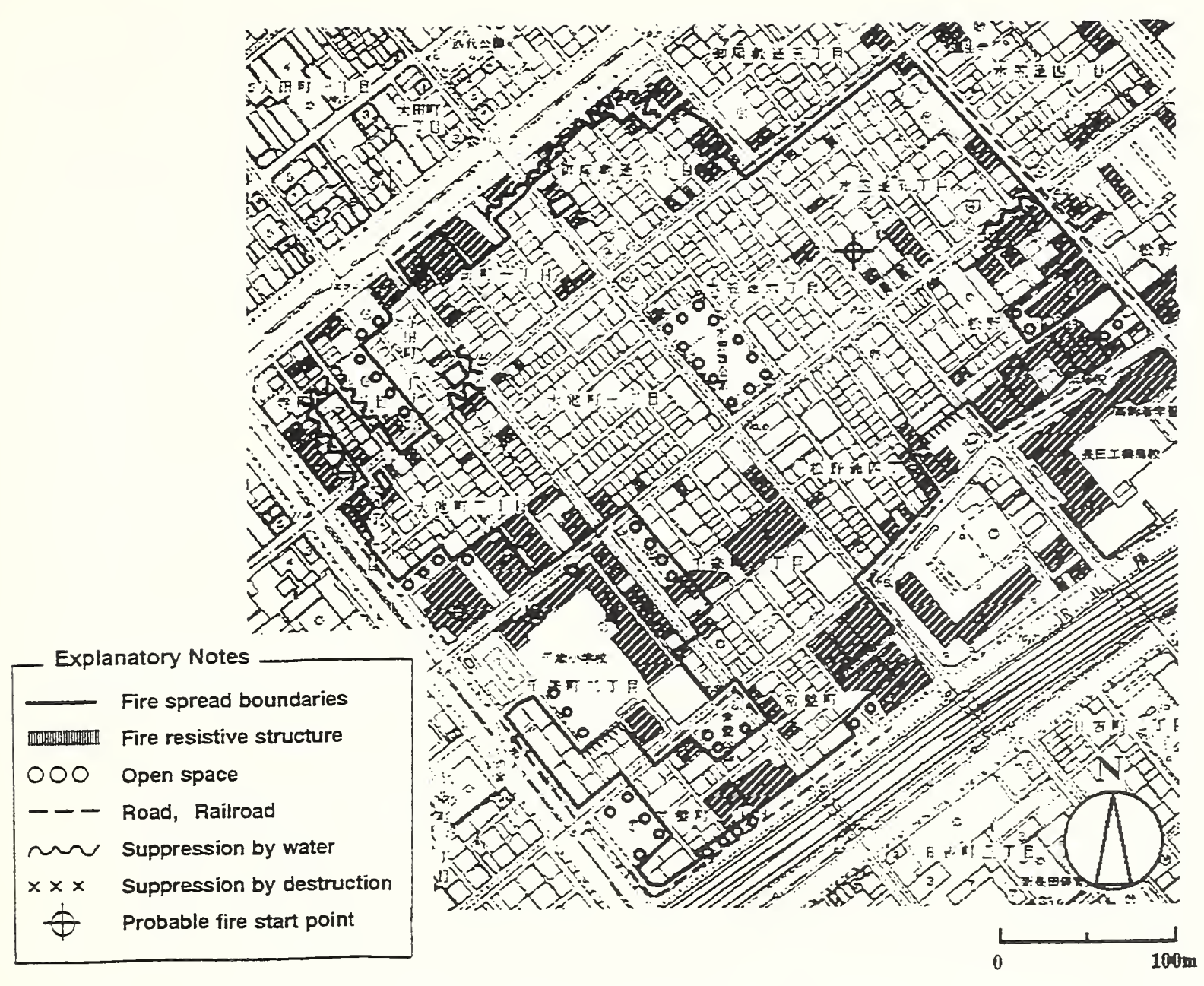

Figure 7. Fire spread boundaries and fire stop factors of the fire site near Mizukasa-nishi Park. ${ }^{5)}$

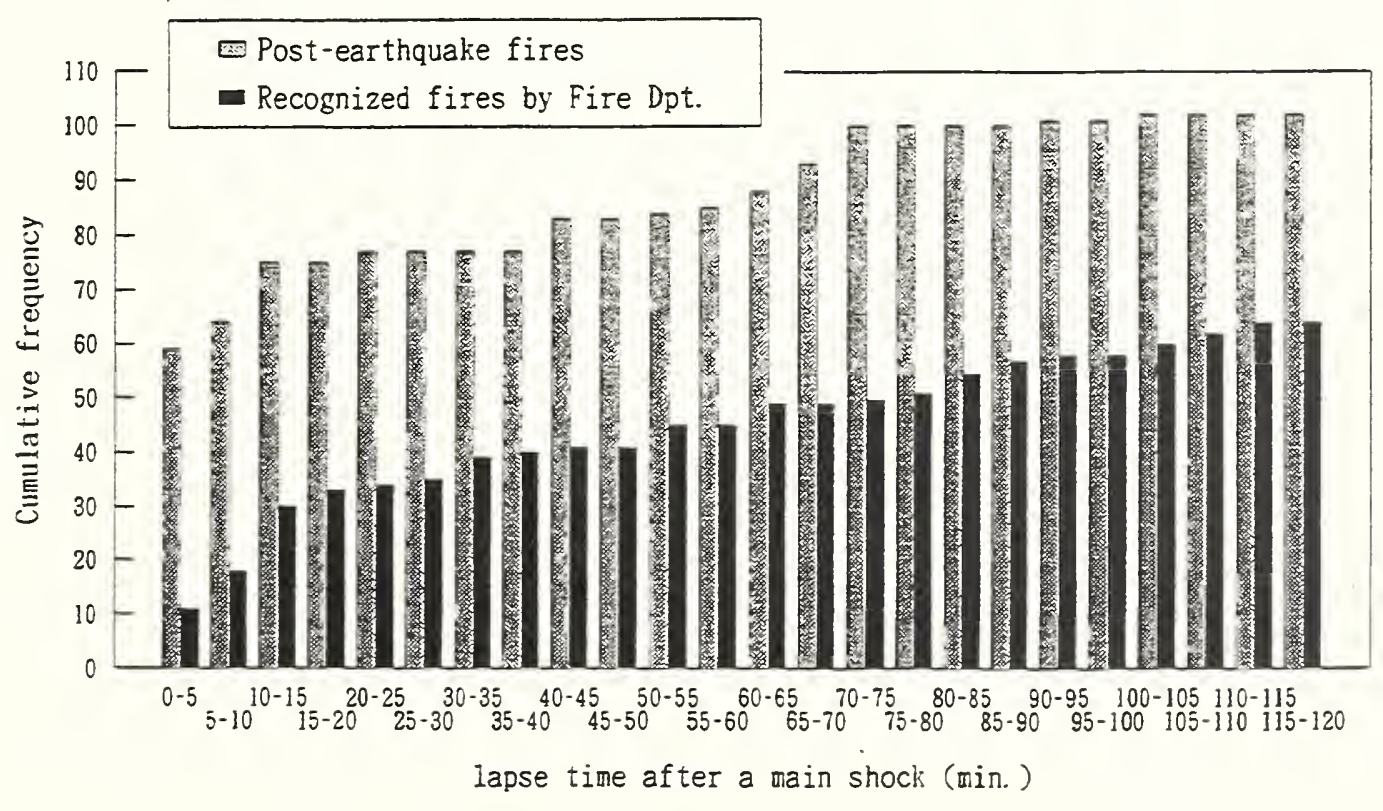

Figure 8. Cumulative frequency of post-earthquake fires and recognized ones by fire departments by lapse time after a main shock. 


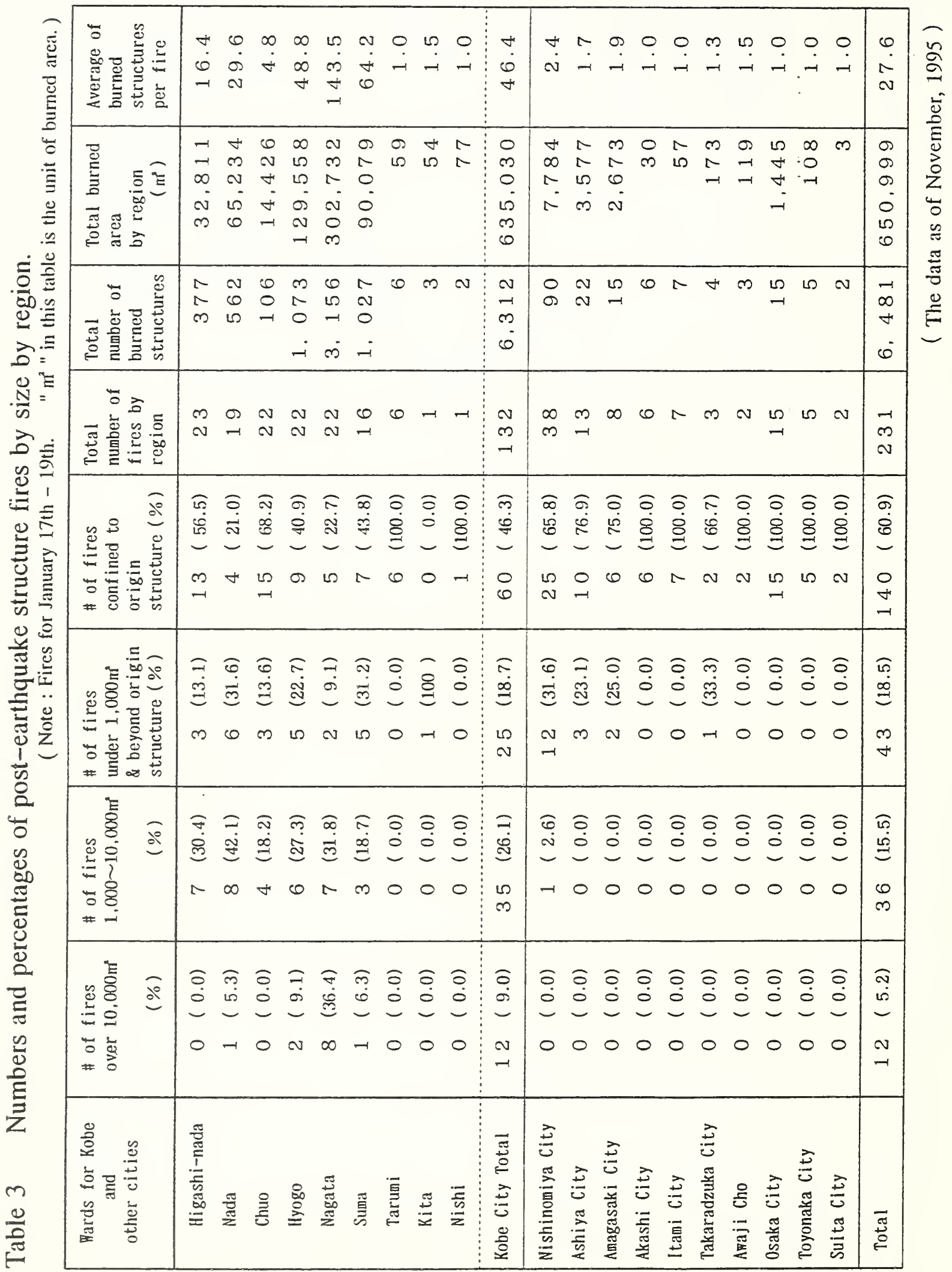


Table 1 Post-earthquake fires in time order for January 17-19 in Kobe City.

\begin{tabular}{|l|rrrrr|c|c|c|c|}
\hline $\begin{array}{l}\text { Kobe City } \\
\text { by ward }\end{array}$ & $\begin{array}{l}\text { 1/17 } \\
\sim 6: 00\end{array}$ & $\sim 7: 00$ & $\sim 8: 00$ & $\sim 9: 00$ & $\sim 24: 00$ & $\begin{array}{c}1 / 17 \\
\text { Total }\end{array}$ & $\begin{array}{c}1 / 18 \\
\text { Total }\end{array}$ & $\begin{array}{c}1 / 19 \\
\text { Total }\end{array}$ & $\begin{array}{c}1 / 17-19 \\
\text { Total }\end{array}$ \\
\hline Higashi-nada & 10 & 1 & 2 & 1 & 3 & 17 & 2 & 4 & 23 \\
Nada & 13 & 0 & 1 & 1 & 2 & 17 & 2 & 0 & 19 \\
Chuo & 8 & 4 & 2 & 1 & 5 & 20 & 3 & 3 & 26 \\
Hyogo & 11 & 0 & 2 & 2 & 2 & 17 & 4 & 3 & 24 \\
Nagata & 13 & 1 & 0 & 0 & 3 & 17 & 1 & 4 & 22 \\
Suma & 4 & 4 & 0 & 4 & 1 & 13 & 2 & 1 & 16 \\
Tarumi & 0 & 0 & 0 & 0 & 6 & 6 & 0 & 0 & 6 \\
Kita & 0 & 0 & 0 & 0 & 1 & 1 & 0 & 0 & 1 \\
Nishi & 1 & 0 & 0 & 0 & 0 & 1 & 0 & 0 & 1 \\
\hline Kobe City Total & 60 & 10 & 7 & 9 & 23 & 109 & 14 & 15 & 138 \\
\hline
\end{tabular}

( The data as of November, 1995 )

Table 2 Post-earthquake fires in time order for January 17-19 in cities other than Kobe.

\begin{tabular}{|c|c|c|c|c|c|c|c|c|c|}
\hline $\begin{array}{l}\text { Cities } \\
\text { other than Kobe }\end{array}$ & $\begin{array}{l}1 / 17 \\
\sim 6: 00\end{array}$ & $\sim 7: 00$ & $-8: 00$ & $-9: 0$ & $24: 00$ & $\begin{array}{l}1 / 17 \\
\text { Total }\end{array}$ & $\begin{array}{l}1 / 18 \\
\text { Total }\end{array}$ & $\begin{array}{l}1 / 19 \\
\text { Total }\end{array}$ & $\begin{array}{l}1 / 17-19 \\
\text { Total }\end{array}$ \\
\hline Ashiya & 4 & 4 & 1 & 0 & 0 & 9 & 2 & 2 & 13 \\
\hline Nishinomiya & 11 & 11 & 1 & 1 & 10 & 34 & 4 & 3 & 41 \\
\hline Takaradzuka & 2 & 0 & 0 & 0 & 2 & 4 & - & - & 4 \\
\hline Itami & 2 & 2 & 2 & 1 & 0 & 7 & - & - & 7 \\
\hline Kamanishi & 1 & 1 & 0 & 0 & 0 & 2 & - & - & 2 \\
\hline Amagasaki & 3 & 2 & 1 & 0 & 2 & 8 & - & - & 8 \\
\hline Awa j icho & 1 & 1 & 0 & 0 & 0 & 2 & - & - & 2 \\
\hline Osaka & 7 & 4 & 1 & 1 & 2 & 15 & - & - & 15 \\
\hline Toyonaka & 3 & 1 & 0 & 1 & 0 & 5 & - & - & 5 \\
\hline Suita & 1 & 1 & 0 & 0 & 0 & 2 & - & - & 2 \\
\hline Other Cities Total & 37 & 26 & 5 & 4 & 16 & 88 & 6 & 5 & 99 \\
\hline
\end{tabular}

Note: " - " meens no post-earthquake fire reported.

( The data as of November, 1995 ) 


\begin{tabular}{|c|c|c|c|}
\hline $\begin{array}{l}\stackrel{0}{0} \\
\stackrel{0}{0} \\
\stackrel{0}{0}\end{array}$ & 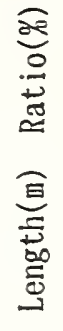 & 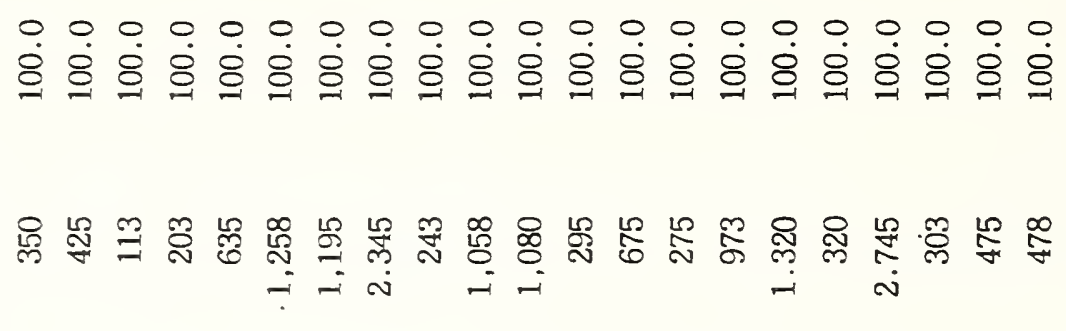 & 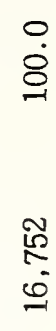 \\
\hline 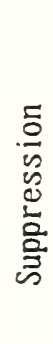 & 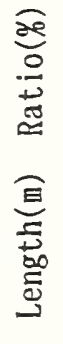 & 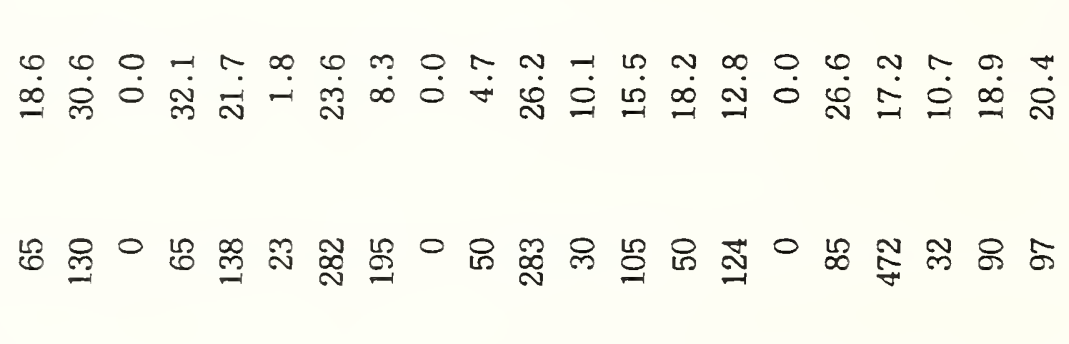 & $\frac{\frac{6}{m}}{\sim}$ \\
\hline 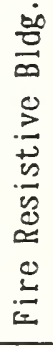 & 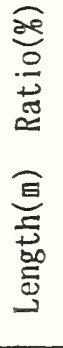 & 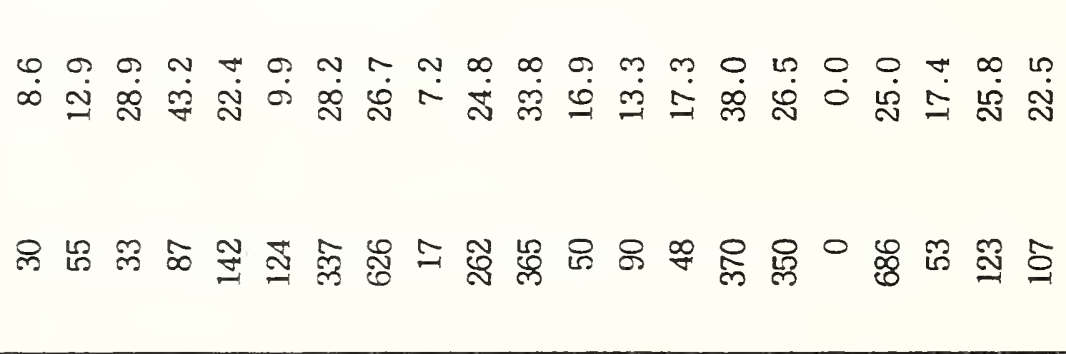 & $\begin{array}{l}\text { 乌ొ } \\
\text { న్ }\end{array}$ \\
\hline 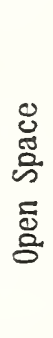 & 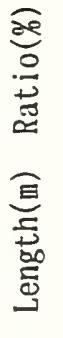 & 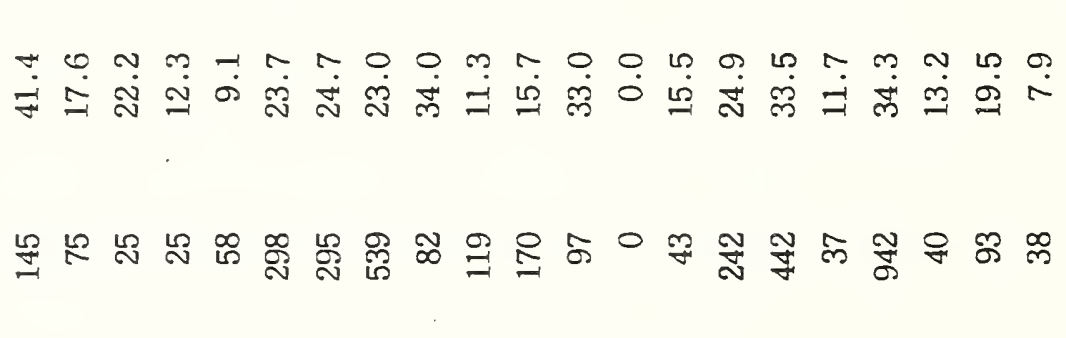 & $\begin{array}{l}\text { D } \\
\infty \\
\text { n' }\end{array}$ \\
\hline 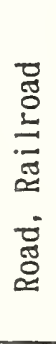 & 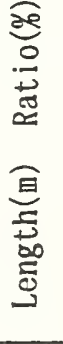 & 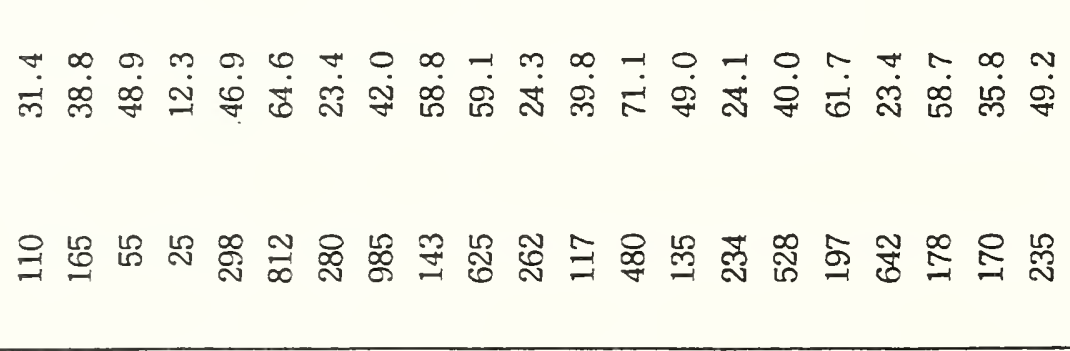 & $\begin{array}{l}0 \\
60 \\
0 \\
0\end{array}$ \\
\hline 0 & 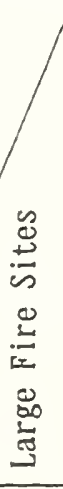 & 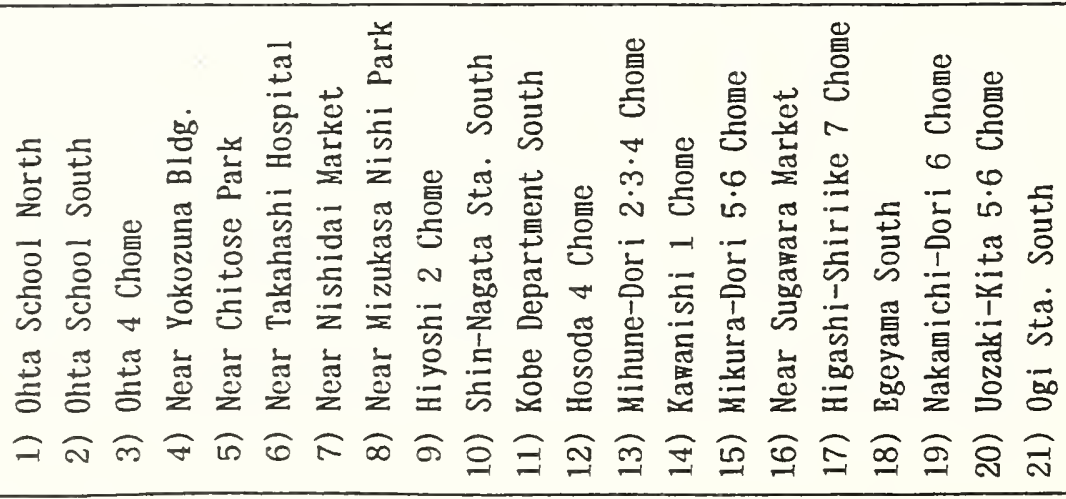 & 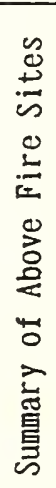 \\
\hline
\end{tabular}


Table 5 The state of emergency call through 119 on January 17 at the Headquarters of Kobe Fire Department. ${ }^{7}$

\begin{tabular}{|c|c|c|c|c|c|c|c|c|}
\hline & \multicolumn{3}{|c|}{ Emergency call } & \multirow{2}{*}{$\begin{array}{l}\text { Communication } \\
\text { within Fire } \\
\text { Department }\end{array}$} & \multicolumn{3}{|c|}{ Not emergency call } & \multirow{2}{*}{ Total } \\
\hline & Fire & Ambulance & Others & & Inquiry* & No response & Others & \\
\hline $5: 46 \sim 6: 00$ & 0 & 0 & 2 & 0 & 10 & 24 & 0 & 36 \\
\hline $6: 00 \sim 7: 00$ & 11 & 17 & 45 & 7 & 74 & 243 & 8 & 405 \\
\hline $7: 00 \sim 8: 00$ & 19 & 19 & 96 & 15 & 43 & 234 & 20 & 446 \\
\hline $5: 46 \sim 24: 00$ & 237 & 297 & 981 & 258 & 2130 & 2762 & 207 & 6872 \\
\hline
\end{tabular}

* To ask information on the earthquake.

Table 6 Performance of fire brigades activity in the early stage."

- in Kobe City, Nishinomiya City, and Ashiya City

\begin{tabular}{|c|c|c|c|c|c|c|c|}
\hline \multirow{2}{*}{ City or Ward } & \multirow{2}{*}{$\begin{array}{l}\text { Number } \\
\text { of } \\
\text { burned } \\
\text { structures }\end{array}$} & \multicolumn{3}{|c|}{ Structure fires until 7:00 am Jan.17 } & \multirow{2}{*}{$\begin{array}{l}\text { Nuaber of fire } \\
\text { engines ready at } \\
\text { the earthquake }\end{array}$} & \multirow{2}{*}{$\begin{array}{l}\text { Damaged to } \\
\text { fire hydrants }\end{array}$} & \multirow{2}{*}{$\begin{array}{l}\text { Number of } \\
\text { cisterns }\end{array}$} \\
\hline & & $\begin{array}{l}\text { Total } \\
\text { number }\end{array}$ & $\begin{array}{l}\text { Fires spread } \\
\text { over } 1000 \mathrm{~m}^{\prime \prime} \\
\text { burned area }\end{array}$ & $\begin{array}{l}\text { Single fires } \\
\text { (\# of } R C \\
\text { buildings) }\end{array}$ & & & \\
\hline Nishinomiya City & 90 & 16 & $2(13 \%)$ & $7(3)$ & 14 (+Volunteer's) & Danaged & 927 \\
\hline Ashiya City & 22 & 8 & $0(0 \%)$ & $6(4)$ & 5 (+Volunteer's) & Damaged & 60 \\
\hline Kobe City Total & 7,453 & 63 & $37(59 \%)$ & $17(10)$ & 40 & mostly Damaged & 1.303 \\
\hline Higashinada & 379 & 9 & $5(56 \%)$ & $3(3)$ & 5 & used up to 2 hours & 38 \\
\hline Nada & 625 & 13 & $7(54 \%)$ & $2(2)$ & 4 & Damaged & 100 \\
\hline Chuo & 115 & 7 & $2(29 \%)$ & $4(2)$ & 5 & Partially damaged & 147 \\
\hline Byogo & 1.097 & 11 & $5(45 \%)$ & $3(0)$ & 5 & Damaged & 104 \\
\hline Nagata & 4,073 & 14 & $11(79 \%)$ & $2(1)$ & 5 & Damaged & 93 \\
\hline Suma & 1,149 & 8 & $5(63 \%)$ & $2(1)$ & 4 & Damaged & 129 \\
\hline Tarug i & 9 & 0 & 0 & 0 & 4 & Damaged & 77 \\
\hline Kita & 3 & 0 & 0 & 0 & 5 & unknom & 259 \\
\hline Nishi & 2 & 1 & $0(0 \%)$ & $1(1)$ & 3 & Damaged & 356 \\
\hline
\end{tabular}

(The data as of November,1995) 


\section{Discussion}

Charles Scawthorn: Thank you for an excellent talk. Embers, fire brands, did they play any role in Kobe?

Ai Sekizawa: According to the testimony of the fire fighters of Kobe City, even if they believed that they could successfully stop the fire at some point, they saw the fire brands behind them, and another fire started.

Howard Baum: Is there any record of the natural winds that were present during the period of the fire?

Ai Sekizawa: Are you talking about the official record?

Howard Baum: Either that or any information that any other observers recorded. Basically, it would be very interesting to know what the wind patterns were in the affected area during the fire.

Ai Sekizawa: When it comes to the local wind speed and direction, I do not believe any official record has been prepared. We'll be able to come up with some information from testimony or some photographic evidence.

Donald Bathurst: I've got three questions. The fire department response seemed to be very good, but what impact did the attempt to rescue people have on their ability to respond. You showed the correlation between the number of fires and the number of engines available, but how many of those were diverted toward rescue? And was there an impact from the damage to the infrastructure in preventing the fire department from setting up in a timely manner.

Ai Sekizawa: I do not believe there is any specific data showing the impact correlation between rescue operation and extent of fires in other obstructions. In reality, as I mention in my paper, there is a huge dilemma faced by the people who are involved in these operations because they have to engage in rescue operations at the same time as fire fighting operations, and there is no definite division between those operations. They have to engage in whatever they have to do. I do not believe that there is any data which clearly shows the impact on the part of rescue operation.

Donald Bathurst: And the last point, there was significant relay operation with all the pumps. Were there any problem with fuel? Getting fuel for those long term operations?

Ai Sekizawa: Starting from the afternoon of the 11th, since we obtained assistance from neighboring towns and cities, we had pretty much established logistic conditions. We had help in that manner, including fuel, so we didn't experience any particular problems. The most difficult problem we faced was the severe congestion of traffic. Therefore, even if they wanted to rush to help us, they couldn't arrive at the affected area as soon as they wanted. 
THE 1994 NORTHRIDGE EARTHQUAKE

AND THE FIRES THAT FOLLOWED

\author{
Frank W. Borden \\ Assistant Chief \\ Los Angeles City Fire Department \\ 1700 Stadium Way \\ Los Angeles, California 90012
}

\begin{abstract}
The purpose of this paper is to provide an overview of the fire problem that occurred as a result of the January 17, 1994, Northridge Earthquake. A brief review of the factors involved in ignition, spread, response and suppression operations will lead into discussions concerning the fires that followed the 6.8M earthquake and analysis of what was learned.
\end{abstract}

\title{
I. INTRODUCTION - FIRE FOLLOWING EARTHQUAKE
}

Fires that occur as a result of the damage caused by earthquakes are but one of many consequences of the shaking. Iarge fires following an earthquake in urban areas are relatively rare but have occasionally been of catastrophic proportions. The two largest peace time urban fires from earthquakes were the 1906 San Francisco and 1923 Tokyo disasters.

The January 17, 1995 Kobe Japan Earthquake resulted in major losses as a result of the fires that swept many residential and commercial neighborhoods. It is estimated that over 6,500 buildings were burned totalling nearly 700,000 square meters of area.

There are many factors involved in contributing to the magnitude of the fire problem following an earthquake.

Ignition

Post earthquake ignitions occur from many forces caused by the shaking intensity such as breakage, open flame, chemical reaction, electrical wiring, friction and other factors. The number of ignitions can be related to shaking intensity and building density, construction and occupancy type.

Multiple ignitions will overwhelm the ability of the Fire Department to respond and potentially lead to conflagrations.

\section{Fire Report and Response}

Locating and reporting fires following the earthquake is problematic due to the damage created and concurrent loss of normal communications systems. Delays in response result in fire spread and as these fires grow it will eventually take more suppression resources to control them. Mutual Aid fire companies were requested from outside Los Angeles County. 
Fire Growth and Spread

Fire growth and spread may be related to building density, open spaces, construction, contributing materials such as natural gas, flammable chemicals, other combustible fuel, and of course weather conditions. Wind speed is the most critical factor in fires considered of conflagration proportions.

\section{Fire Suppression}

Rapid response and access to the fire is critical to controlling the growth of fire. Response may be made by convergent or trained volunteers, and professional firefighters. Building sprinkler systems may also be part of the suppression equation if they are not damaged and if water is available. of primary concern is the availability of water for firefighting since this is a system commonly damaged in large earthquakes. Initial firefighting operations will be impacted by other response priorities such as structural collapse rescue and immediate evacuation of areas threatened by fast moving fires or hazardous chemical spills. The priority for emergency responders is saving Iives with the protection of property as a secondary priority.

\section{The Northridge Earthquake - An Overview}

At 0431 hours on January 17, 1994, the Los Angeles area was rocked by an earthquake whose damage in recent history was precedented only by the San Francisco Earthquake in 1906. The Northridge Earthquake registered $6.8 \mathrm{MW}$ on a thrust fault 12 miles beneath the San Fernando Valley. The earthquake resulted in 57 deaths, over 9,000 injuries and losses exceeding $\$ 20$ billion.

Peak ground accelerations were measured at $1.8 \mathrm{~g}$ and over 58,000 buildings were damaged. The City's infrastructure was badly damaged including the loss of electrical power and thousands of breaks in natural gas lines, and water mains.

The city was in total darkness from the loss of power and initial damage assessment was difficult for emergency response personnel. Telephone communications were compromised from damage and overuse resulting in delays to many emergency incidents.

\section{Fires}

Department resources, confronted with significant obstacles such as total darkness, inadequate water pressure, electrical hazards, and roadways blocked with debris, were effectively utilized in rapidly controlling fires, using quick known down tactics, preventing extension to exposed structures, and moving on to the next prioritized incident. 
Due to water shortages, 29 water tank trucks were requested through the Statewide Fire Department Mutual Aid system and were strategically located with fire companies to respond as an added resource. Swimming pools were also effectively utilized as a water supply for firefighting. Fire-Department water dropping helicopters were even used in dropping over 15,000 gallons of water on structure fires which is not a standard operational procedure.

During the first 27 hours and 29 minutes ( 0431 hours on January 17, 1994, to 0800 hours on January 18, 1994) following the earthquake, 2,332 incidents were created in the Fire Department dispatch center. The average number of incidents in a 24-hour period is about 900. There were actually more incidents handled by LAFD resources during the peak incident time period but not reported.

The summary of incidents for this $27-1 / 2$ hours is as follows:

776 incidents or $33.3 \%$ were fire incidents

1,111 incidents or $47.6 \%$ were EMS incidents

445 incidents or $19.1 \%$ were other incidents

Due to the unusually high number of incidents reported immediately after the earthquake, the Fire Department dispatch center (OCD) reduced the number and types of resources dispatched to each. Eight minutes after the earthquake, dispatch assignments of Department resources were degraded to the earthquake mode. Three minutes later, assignments were further degraded to the disaster mode. In the disaster mode there is a major assignment reduction with 13 no send fire categories. The Department remained in the disaster mode until 2119 hours on January 18 , 1994.

Many of the structure fires that occurred during this time frame resulted in the utilization of minimal Department resources. During normal operations, many of these structure fires would have utilized many more resources, and been classified as major emergencies. Fire Department Command established strategy to prevent incidents from reaching catastrophic proportions. Incidents were prioritized by field officers to mitigate the potential for conflagrations. For those incidents that exceeded available resource capability, emphasis was placed on exposure protection.

The most common types of incidents created on January 17 and 18, 1994, falling within the fire category are listed below: 
Incident Type

Reported Structure Fires

Brush/Grass/Trees

Rubbish Fires

Pole Fires

Automobile Fires

Automatic Alarms

Illegal Burning
Number of

Incidents Created

660

38

17

17

14

12

12
Percent of Total

Fire Incidents

other incidents in this category bring the total to $100 \%$.

As a result of investigations conducted by the LAFD Arson section after the earthquake, it was determined that 158 actual structure fires occurred during the 27-1/2 hour period following the initial shock. This number accounts for fires occurring at a single address and does not include the multiple fires that occurred within a mobile home park. The fires were scattered throughout the city of Los Angeles, with the majority of the incidents occurring closest to the epicenter of the earthquake. Further complicating fire suppression efforts were damage to water mains, inadequate water pressure, and collapsed structures. A brief synopsis of some of the structure fires follows:

\section{Blucher Avenue - January 17, 1994, 0431 hours}

The collapse of a two-story apartment complex over carport resulted in the structure crushing numerous automobiles. A fire started in the carport at the onset of the earthquake. Due to the condition of the structure and the potential for further collapse, the fire extended into the apartments. Loss estimated at $\$ 1,500,000$.

18110 Andrea Circle North - January 17, 1994, 0431 hours A fire, determined to originate at the gas meter, extended to 17 units in three separate buildings in a multiple unit, apartment complex. Loss estimated at $\$ 500,000$.

\section{W. Nordoff street, Building 2, Cal state Northridge -} January 17,1994

Two separate fires and chemical spills were discovered on the second and third floors of the three-story, $75 \times 200$ foot science building of the cal state Northridge Campus. The fire rooms contained unknown types and amounts of chemicals. The cause of the fire is undetermined at this time.

16700 Chatsworth Street - January 17, 1994, 0432 hours A fire resulting from combustibles placed too close to a heat source resulted in the destruction of 14 units in three buildings of an apartment/condominium complex. No loss was estimated. 
19155 Victory Boulevard - January 17, 1994

A fire originating from a natural gas leak resulted in the total burnout of a 50 × 150 foot apartment/condominium complex. No loss was estimated.

15455 Glenoaks Boulevard - January 17, 1994, 0434 hours Earthquake cased a break or leak in natural gas lines resulting in the destruction of 58 mobile homes. Loss estimated at $\$ 5,000,000$.

13320 Riverside Drive - January 17, 1994, 0435 hours A fire in a machinery room on the roof of a two-story medical building caused $\$ 250,000$ damage. The investigation determined that the fire was a result of a broken natural gas line to a water heater that was knocked over by the shock of the earthquake.

15831 Olden Street - January 17, 1994, 0435 hours

A fire determined to be the result of leaking natural gas destroyed 22 mobile homes at a mobile home park. No loss was estimated.

19240 Dearborn Street - January 17, 1994, 0440 hours A fire originating from a broken natural gas line at the water heater resulted in a complete burnout of a single-family dwelling. Loss estimated at $\$ 400,000$.

21001 Plummer Street, \#61 - January 17, 1994, 0440 hours A fire, of undetermined origin, occurred in a mobile home resulting in the fatality of a 90-year old female.

2741 S. Palm Grove Avenue - January 17, 1994, 0440 hours A fire originating immediately after the earthquake at the natural gas meter resulted in a $\$ 250,000$ loss to a multiple unit residence.

365 West 47th Place - January 17, 1994, 0453 hours Shock of the earthquake caused the natural gas flex line to a clothes dryer located on a service porch of a single-family dwelling to rupture causing natural gas to leak into dwelling. Gas was ignited by a pilot light in the kitchen. Loss estimated at $\$ 140,000$.

15445 Cobalt Way - January 17, 1994, 0500 hours Several fires, originating in separate mobile homes, eventually destroyed 54 mobile homes in a mobile home park. The fires originated as a result of natural gas leaks following the earthquake. Loss estimate at $\$ 1,500,000$. 
17221 West Willard Street - January $17,1994,0500$ hours

A fire involving a single-family dwelling originated when a block wall fell, as a result of the earthquake, onto the gas meter shearing the riser at ground level. The natural gas was ignited from unknown sources entering the structure. Loss. estimated at $\$ 50,000$.

5622 N. El Canon Avenue - January 17, 1994, 0501 hours

Fire in a single-family dwelling occurred when a shelf, located above a water heater, broke away from the wall and dropped material. The material apparently broke the gas line leading to the water heater regulator which eventually ignited the material. Loss estimated at $\$ 250,000$.

$11700 \mathrm{~N}$. Balboa Boulevard - January 17, 1994, 0527 hours A break in a 12-inch underground natural gas main resulted in destroying five single-family dwellings and damaging one other. Loss estimated at $\$ 1,500,000$.

2324 S. Chariton Street - January 17, 1994, 0530 hours Fire originating from a broken natural gas connection to a floor furnace in a single-family dwelling extended vertically through the walls and into the attic. Loss was estimated at $\$ 200,000$.

14011 Ventura Boulevard - January 17, 1994, 0530 hours

A fire of undetermined origin caused an estimated $\$ 500,000$ worth of damage to a two-story, multi-unit commercial structure. Fire appeared to originate on the second floor.

3845 N. Bobstone Drive - January 17, 1994, 0533 hours structure fire in a single-family dwelling. Probable cause was determined to be a natural gas leak due to the earthquake. Loss estimated at $\$ 400,000$.

634 O'Melveny Avenue - January 17, 1994, 0613 hours

Earthquake caused a rupture in a crude oil pipeline causing oil to flow and burn in the street. One single-family dwelling and 15 vehicles destroyed, and two single-family dwellings damaged. Loss estimated at $\$ 2,500,000$.

$4360 \mathrm{~N}$. Ventura Canyon Avenue - January 17, 1994, 0643 hours structure fire in a collapsed, two-story, 14-unit apartment with underground parking, 40 × 150 feet in dimension. Fire involved several units in the rear of the complex. Unable to determine cause due to the building collapse. No estimated loss.

$10630 \mathrm{~N}$. Louise Avenue - January 17, 1994, 0652 hours A fire, of undetermined origin, occurred in a single-family dwelling. Fire exposed a second single-family dwelling located at $10636 \mathrm{~N}$. Louise Avenue resulting in the total loss of that structure. Loss estimated at $\$ 400,000$. 
13320 W. Riverside Drive - January 17, 1994, 0740 hours Fire originating in the machinery room of a three-story medical office building resulted in an estimated $\$ 500,000$ loss. Probable cause was a natural gas leak.

$7225 \mathrm{~W}$. Hollywood Boulevard - January 17, 1994, 0813 hours Incendiary fire in one unit of a 74-unit apartment hours. No estimated loss.

$9250 \mathrm{~W}$. Owensmouth Avenue - January 17, 1994, 0843 hours Undetermined fire in a 50 x 250 foot metal clad commercial occupancy. Loss estimated at $\$ 500,000$.

1818 S. Stoner Avenue - January 17, 1994, 0844 hours Fire in one unit of a 16-unit apartment house. Cause was determined to be an electrical lamp that was knocked over during the earthquake onto clothing. Heat from lamp ignited clothing when power restored.

$18111 \mathrm{~W}$. Nordoff street, Building 3, Cal state Northridge January 17,1994

A second fire and chemical spill resulting from broken/spilled chemical containers co-mingling as a result of the earthquake occurred on the third floor of a three-story, $75 \mathrm{x} 200$ foot science building on the Cal state Northridge Campus. The loss was estimated at $\$ 50,000$.

\section{$18111 \mathrm{~W}$. Nordoff street, Building 1, Cal state Northridge -} January 17,1994

A third fire and chemical spill on the Cal state Northridge Campus occurred on the third floor of science Building 1. The fire, similar to the fire that occurred in Building 3, was a result of a chemical reaction with broken/spilled chemicals. The loss was estimated at $\$ 50,000$.

1118 W. 3rd street - January 17, 1994, 0931 hours Structure fire in a single-family dwelling caused by a broken flex natural gas line at the water heater. Fire extended to two, four-unit apartment houses. Loss estimated at $\$ 500,000$.

11275 Westminster Avenue - January 17, 1994, 1102 hours Fire occurred in a multi-unit apartment complex due to natural gas leak in line leading from meters to apartments. Break in line due to earthquake. Natural gas found source of ignition in apartment complex. Loss estimated at $\$ 125,000$.

19360 Rinaldi street - January 17, 1994, 1216 hours The earthquake caused an air conditioning unit on the roof of a single-story commercial occupancy to knock over causing the motor to eventually burnout and short. Air conditioning unit caught fire and spread to the structure. No loss estimated. 
17515 W. Enadia Street - January 17, 1994, 1230 hours

A fire in the garage of a single-family dwelling occurred shortly after the electricity was restored to the area. Loss estimated at $\$ 105,000$.

12021 Riverside Drive - January 17, 1994, 1400 hours

A fire causing $\$ 400,000$ in damage occurred in a two-story dental office. An investigation determined the fire was caused by an electrical short in the attic area originating when the power was restored to the area.

14025 Ventura Boulevard - January 17, 1994, 1842 hours

A fire of undetermined cause, resulted in the destruction of the entire second floor of a multi-occupancy commercial. The fire originated on the second floor and resulted in a $\$ 1,500,000$ loss.

"Other" type incidents created on January 17 and 18, 1994:

Incident Type

Leaking Natural Gas

Electrical/wires Down

Hazardous Conditions

HazMat/Chem Investigations

water Problems

Elevators

Invalid Assists
Number of Incidents Created

126

99

99

45

44

13

8
Percent of Total Fire Incidents

$28.3 \%$

$22.2 \%$

$22.2 \%$

$10.1 \%$

$9.8 \%$

$2.9 \%$

$1.8 \%$

other incidents in this category bring the total to $100 \%$

There were 445 incidents created within this category. Although not reported as actual fire incidents, these incidents required the dispatch of fire suppression resources.

The largest number of incidents created in this group was due to reported natural gas leaks (approximately 28.3\% of the total within this group). The figures for actual natural gas services shut down by LAFD personnel are not available.

Many buildings, throughout the entire city of Los Angeles, sustained total or partial collapse from the initial January 17 , 1994, earthquake and from the subsequent aftershocks. Structural damage, although related to the distance from the epicenter, was scattered indiscriminately throughout the southern California region. Given the intensity of the earthquake, the time of day, and the number of buildings that collapsed, the number of deaths resulting from the collapse of buildings was remarkably small. 


\section{Analysis}

Fire following earthquake will continue to be a factor for emergency responders and disaster planners. Much needs to be done in researching areas such as ignition sources., spread factors, and fire modelling in order to mitigate against their destructive impact.

Scientists predict that southern California faces an $86 \%$ chance of a magnitude 7 or greater earthquake by the year 2024. As a result of the Northridge Earthquake, several actions were taken by the City of Los Angeles to assist in the control of future fires following earthquakes. A city ordinance was enacted calling for the installation of automatic gas shut off valves on structures. Studies are underway on pipeline safety, mobile home anchoring systems, auxiliary water supply, and controlling reenergizing the electrical systems in damaged buildings/areas.

The potential for major post earthquake fires in the United states clearly exists in the New Madrid (Central United states) and Puget sound (Pacific Northwest) seismic zones, as well as in California. Research into prevention and mitigation of these fires is crucial if future catastrophes are to be avoided.

\section{REFERENCES}

LAFD Northridge Earthquake Report 1994

EQE International Northridge Earthquake Summary Report, March 1994

Fire Following Earthquake, AIRAC, Dr. Charles Scawthorn, March 1987

EQE International, Review, Fall 1993

Fire Engineering Magazine, August 1994, The Northridge Earthquake

Fire Engineering Magazine, May 1995, The Kobe Earthquake, Frank Borden 


\section{Discussion}

Edward Zukoski: I'd like to address a question to you and the previous speaker. Would the use of the kind of cisterns in Japan be of any help to us in Los Angeles?

Frank Borden: We have no water cisterns in Los Angeles, and I personally think along with many other people in my field that they are a very important part of our water supply system and should be actually installed along with other things. And a matter of fact, one of the lessons learned from Northridge and Kobe was for our water and power department and the fire department to work together to research the idea of implementing those tanks within our city.

Carole Womeldorf: When the fire trucks are on patrol right after the earthquake, what types of detection methods do you have on the fire trucks to detect fires?

Frank Borden: That may be a good area for research, because we have none other than our eyes and our ears. Some people call it a windshield survey. Many of the Los Angeles police department helicopters and some fire helicopters have infrared capability where they can see on a screen where fires are in total darkness. I think that's something that we need to pursue, at least infrared from aerial operation.

John Rockett: Back in the 1970s, Mission Research did a lot of work with the Los Angeles Fire Department and with the California Division of Forestry primarily directed at the urban brush fire. I wondered if there were any lessons to be learned from these earthquakes which would lead to improvements of those systems.

Frank Borden: Very much so. Thank you for that question. That system that was developed that long ago has evolved tremendously and today, it is a system that is used for all types of incidents. As a matter of fact, the National Fire Academy teaches what we call the incident command system and multiagency coordination, and this is actually occurring all over the country. For the earthquake, this system was applied on a very large scale and worked very well. The state of California has mandated the use of this system because of the Oakland Hills fire and the problems that occurred up there between fire departments and the fact that they weren't using the same systems. So all response agencies in the state of California by the end of this year will be using the incident command system (ICS) and multiagency coordination.

Question: I found in your documents that there is a very precise, very well documented record of individual fire incidence. I was wondering if this was a normal practice that you keep such a record for every fire? And is that record made public?

Frank Borden: Yes. We are hoping that in the very near future a full report on the earthquake including locations and fire problems will be published. I know the department has been working on that. People do have access to fire report records through our department. It costs a person coming in a little bit of money for administrative processing. 


\title{
THE PERFORMANCE OF FIRE PROTECTION OF BUILDINGS AGAINST THE FIRES FOLLOWING THE GREAT HANSHIN-AWAJI EARTHQUAKE
}

\author{
Akihiko Hokugo \\ Building Research Institute \\ Ministry of Construction \\ 1 Tatehara, Tsukuba, 305, Japan
}

\begin{abstract}
High levels of amplitude acceleration ( $300 \mathrm{gal}$ to $800 \mathrm{gal}$ ) and velocity ( $80 \mathrm{kine}$ ) were observed over a wide area at the time of the 1995 Great Hanshin-Awaji Earthquake. The locations where the fires started are almost uniformly distributed in areas where building suffered severe structural damage, mainly areas where the seismic intensity reached seven in JMA scale.
\end{abstract}

Thanks to the weak winds at the time of the earthquake, the speed of the fire spread was far below that of past urban fires. Seven large fires that destroyed an area of more than 33,000 sq. meters each, broke out in areas with large concentrations of wooden houses. Most of the fire preventive wooden buildings that caught fire did so mainly because fire spread from the building's openings, not from their walls. Most of the cease burning lines in urban areas were wide streets, railways, parks and similar large spaces, rows of buildings of fireproof construction, and fire proof buildings constructed on large building lots. This confirms that it is extremely important to provide more space between buildings and improve buildings' fire proof performance in order to prevent the spread of urban fires.

\section{LARGE NUMBER OF SIMULTANEOUS IGNITIONS}

During the first 3 days following the earthquake, more than 200 fires occurred in the Hyogo and Osaka prefectures ${ }^{1)}$. Of those, 138 fires took place in Kobe city ${ }^{2)}$. Figure 1 shows 150 fire sites where investigations by Building Research Institute were conducted.

The fires started in areas where building suffered severe structural damage. Table 1 shows the relationship between the structural damage of the buildings in which the fires started and the causes of fires. As the structural damages severer, the number of fires increases. In this table causes of fires are categorized into three groups, gas related fires, electricity related fires and other fires. Gas related fires has strong relation to the damage of buildings. Electricity related fires occurred evenly to the structural damage. This indicates that gas related fire most likely occurred when the building was collapsed or severely damaged and as the result the gas pipes were cut or unconnected.

\section{LARGE CONFLAGRATIONS}

Of 150 fire sites investigated by BRI, 73 fires involved single building and 77 involved multiple buildings. The number of fire sites less than 1,000 square meters is 33. Seven fires spread over 33,000 square meters. Other 37 fire sites are between 1,000 and 33,000 square meters. Figure 2 shows large conflagrations whose area is over 3,300 square meters in Nagata, Suma, Hyogo, Nada and Higashi-Nada Ward. 


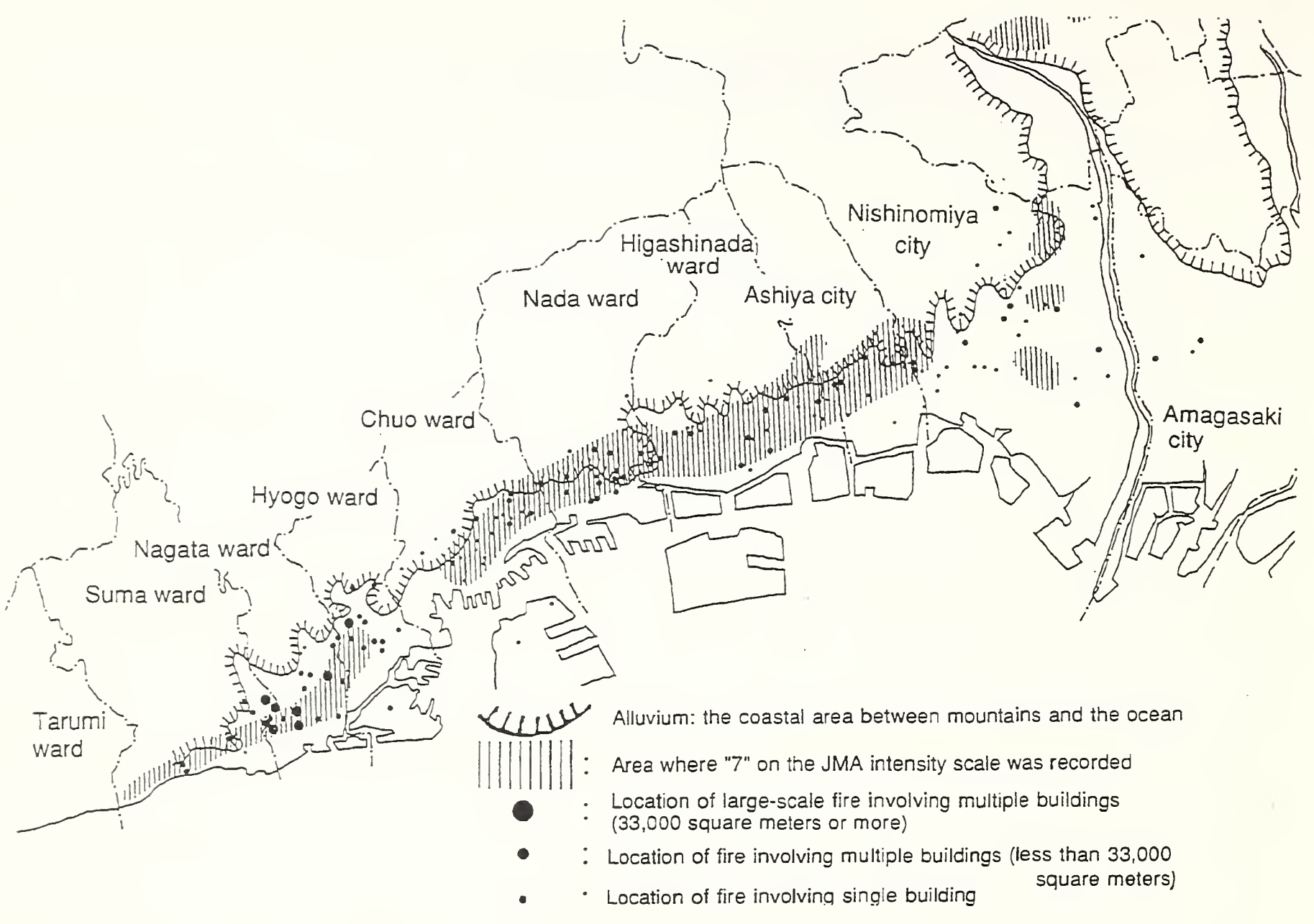

Figure 1. Location of buildings surveyed for fire damage (also shows the alluvium extending from Kobe to Amagasaki and the area where " 7 " on the JMA intensity scale was recorded )

Table 1. The relationship between the structural damage of the buildings and the causes of fires ${ }^{3)}$.

\begin{tabular}{|c|c|c|c|c|c|c|}
\hline $\begin{array}{l}\text { Degree of } \\
\text { Structural } \\
\text { Damage }\end{array}$ & $\begin{array}{l}\text { Number of } \\
\text { Buildings }\end{array}$ & $\begin{array}{l}\text { Gas Related } \\
\text { Fires* }\end{array}$ & $\begin{array}{l}\text { Electricity } \\
\text { Related } \\
\text { Fires * }\end{array}$ & $\begin{array}{l}\text { Other } \\
\text { Fires * }\end{array}$ & Unknown * & Total * \\
\hline $\begin{array}{l}\text { Collapsed or } \\
\text { Destroyed }\end{array}$ & 93,567 & $10(10.7)$ & $4(4.3)$ & $11(11.8)$ & $32(34.2)$ & $57(60.9)$ \\
\hline $\begin{array}{l}\text { Moderate } \\
\text { Damage }\end{array}$ & 90,908 & $5(5.5)$ & $4(4.4)$ & $1(1.1)$ & $14(15.4)$ & $24(26.4)$ \\
\hline $\begin{array}{l}\text { Slight damage } \\
\text { or undamaged }\end{array}$ & 358,525 & $8(2.3)$ & $18(5.0)$ & $11(3.1)$ & $33(9.2)$ & $70(19.5)$ \\
\hline Unknown & & 1 & 6 & 5 & 18 & 30 \\
\hline Total & 543,000 & $24(4.4)$ & $32(5.9)$ & $28(5.2)$ & $97(17.7)$ & $181(33.3)$ \\
\hline
\end{tabular}




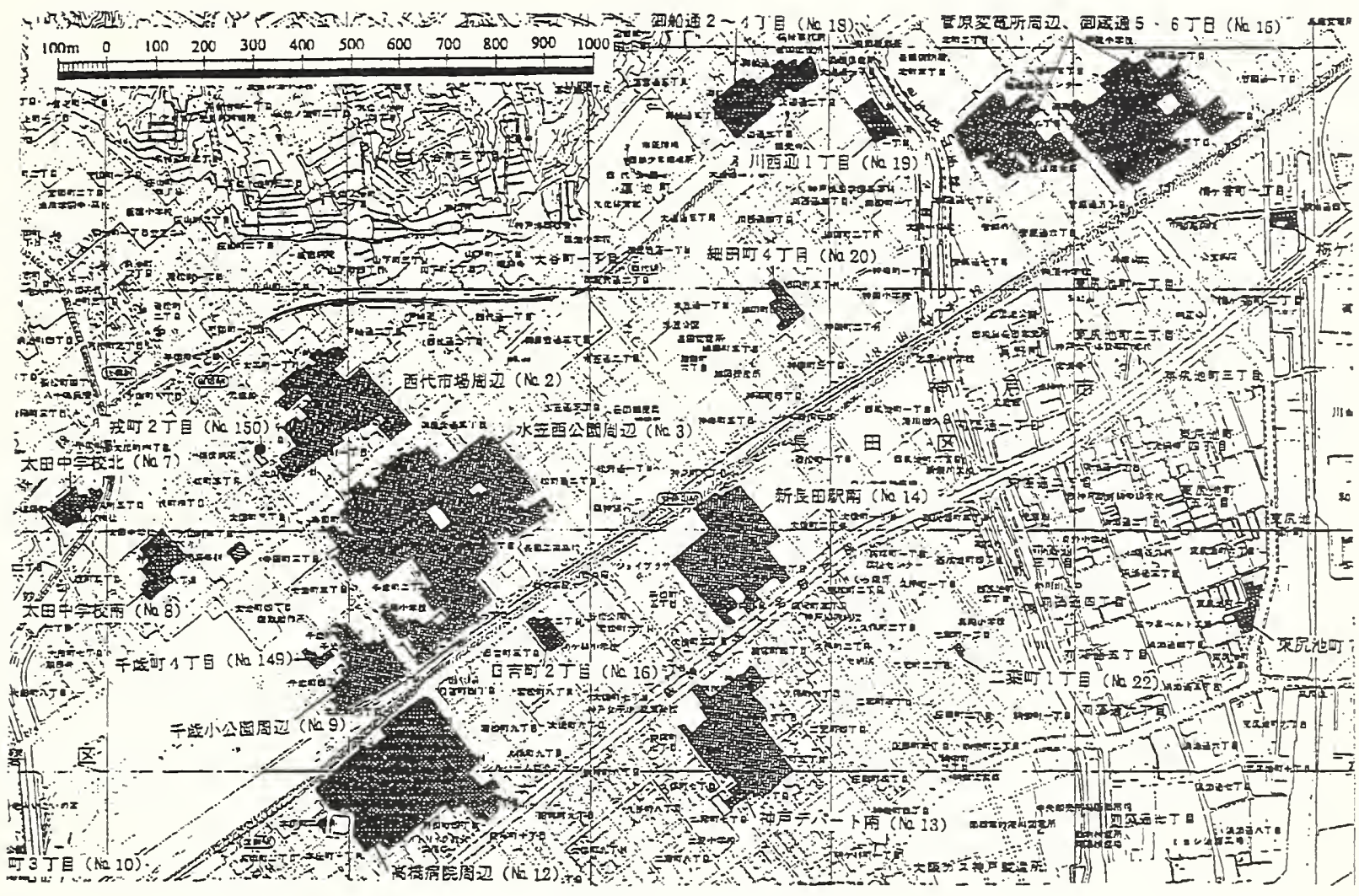

\section{Nagata and Suma wards}

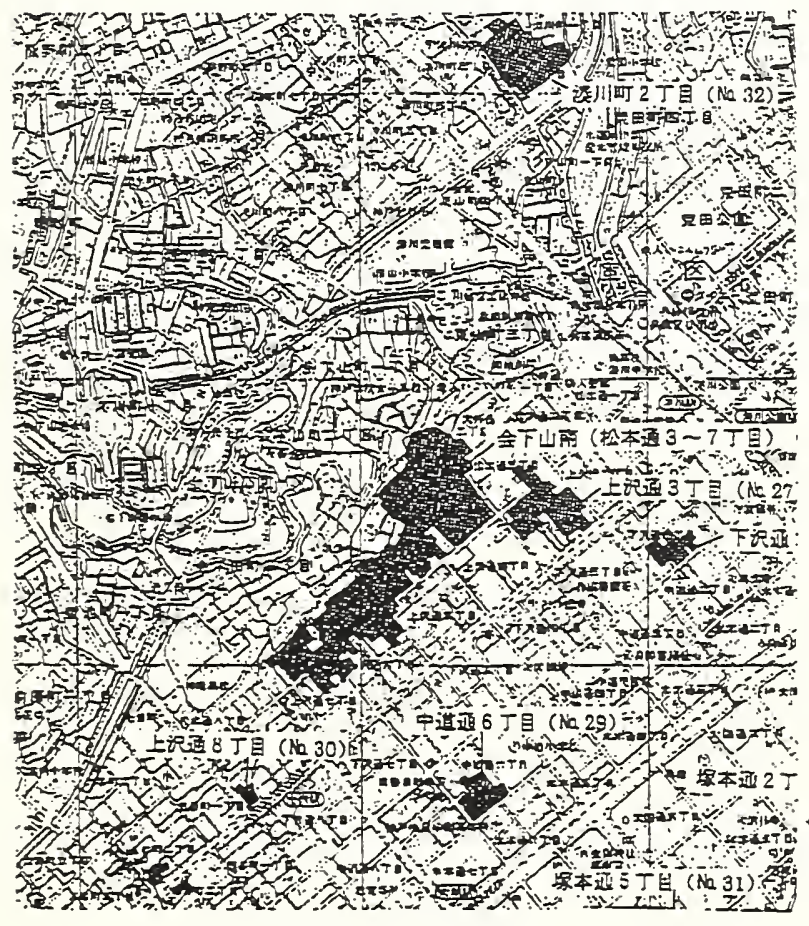

Hyogo Ward

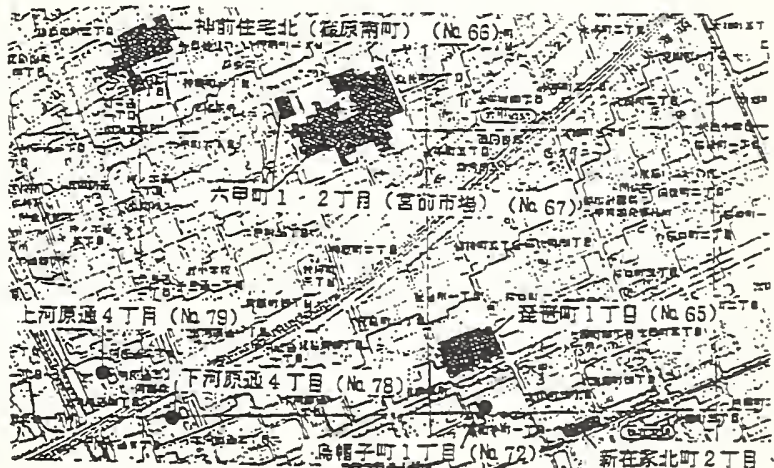

\section{Nada Ward}

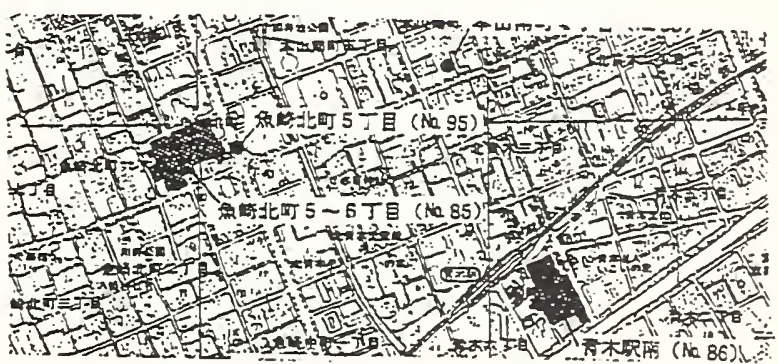

Higashi-Nada Ward

Figure 2. Large Conflagrations in Kobe City 


\section{VELOCITY OF FIRE SPREAD AND WIND}

Figure 3 shows an example of fire spread recorded by news videos. Fire spread was relatively slow, averaging about 20 to 30 meters per hour. The main reason for this slow speed was that there was a slow wind and sometimes no wind on the day of the earthquake as shown in Figure 4. The average wind velocity in Kobe was 2.6 meters per second.

Figure 5 indicates the relation between the wind velocity and the velocity of fire spread. When the wind velocity is higher than 3 meters per second, the velocity of leeward fire spread increases obviously.

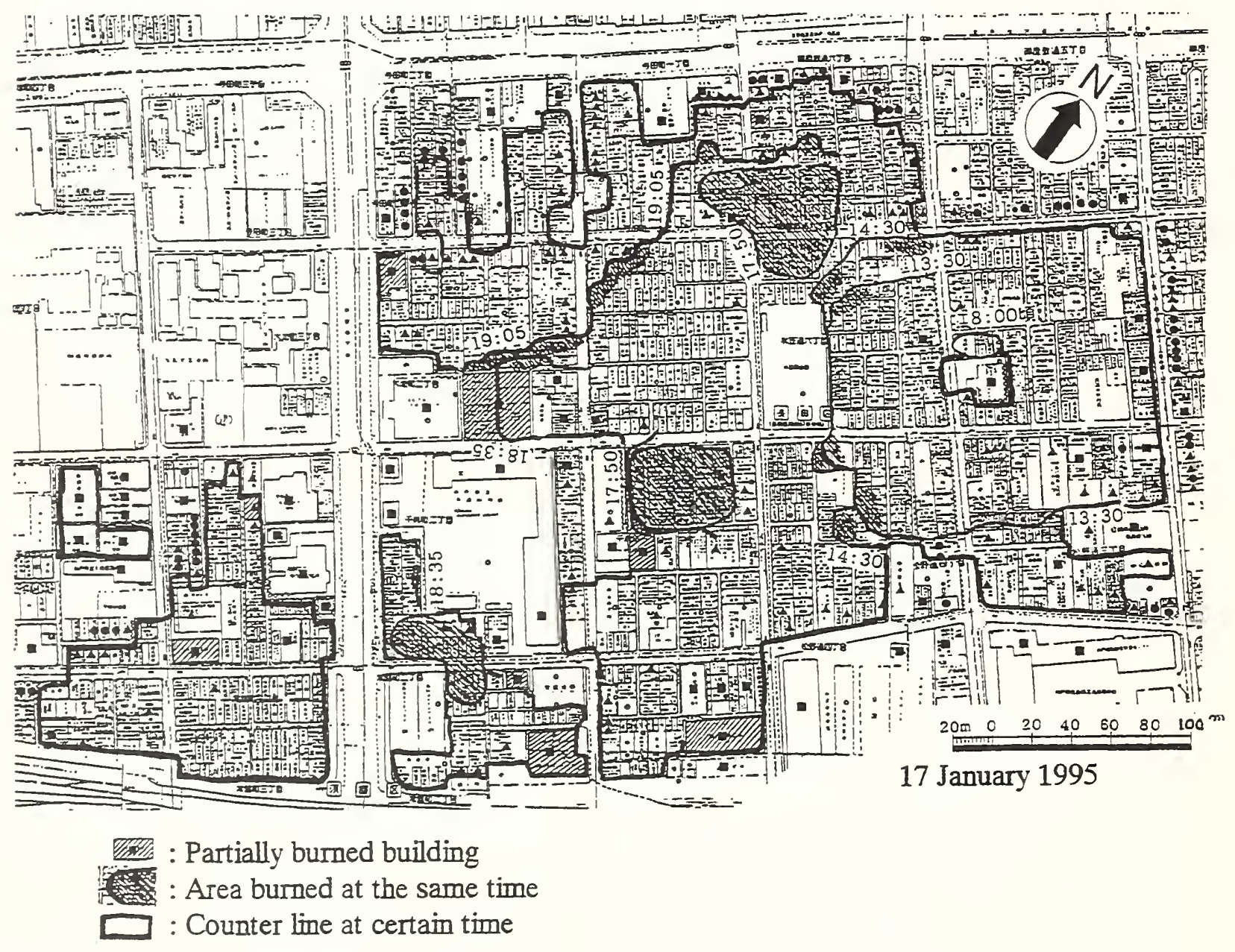

Index for building structure

- : Fire resistive building

$\Delta$ : Semi-fire resistance building

- : Wooden building with non-combustible materials on the exterior wall

$\circ$ : Wooden building without non-combustible materials on the exterior wall 


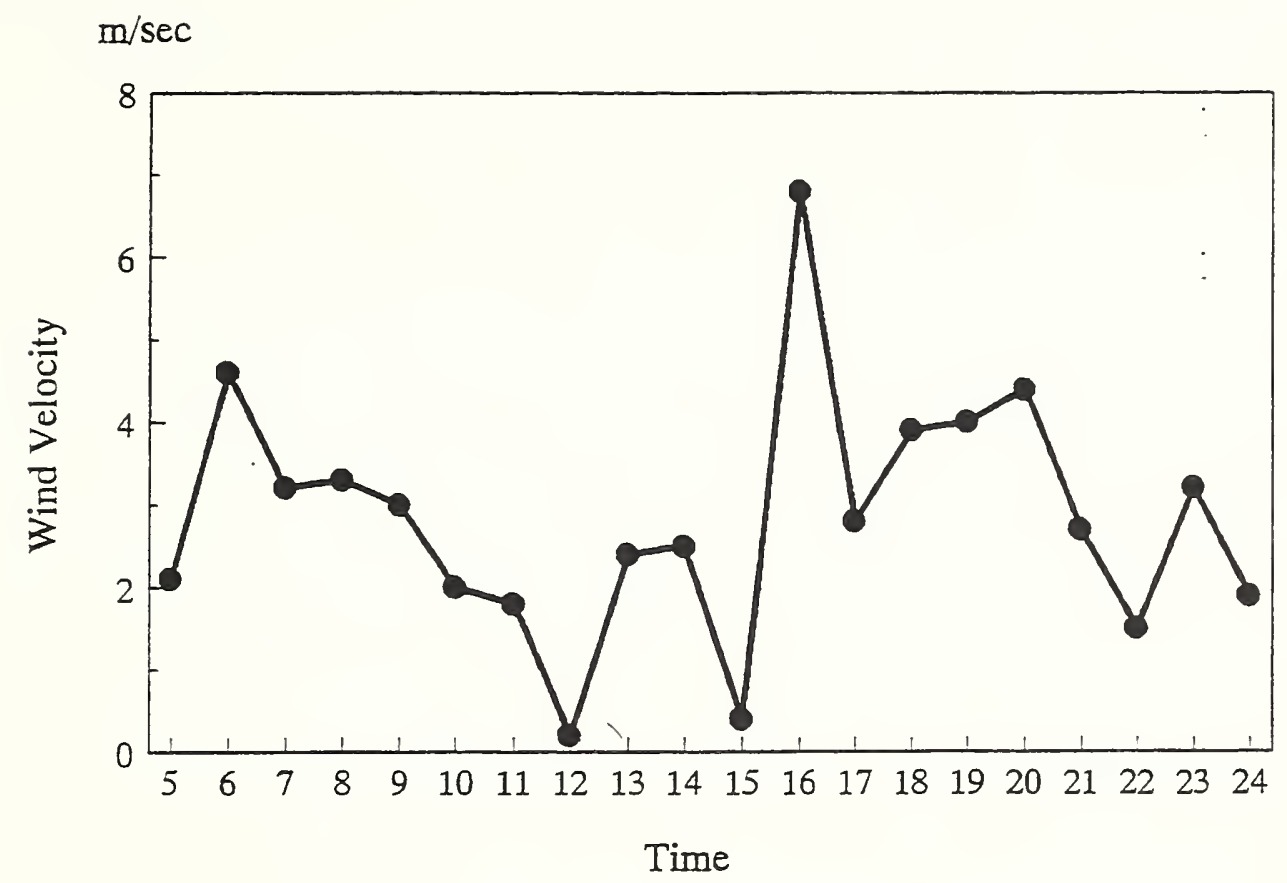

Figure 4. Wind Velocity at Kobe Meteorological Observatory in Chuo Ward on the Day of the Earthquake

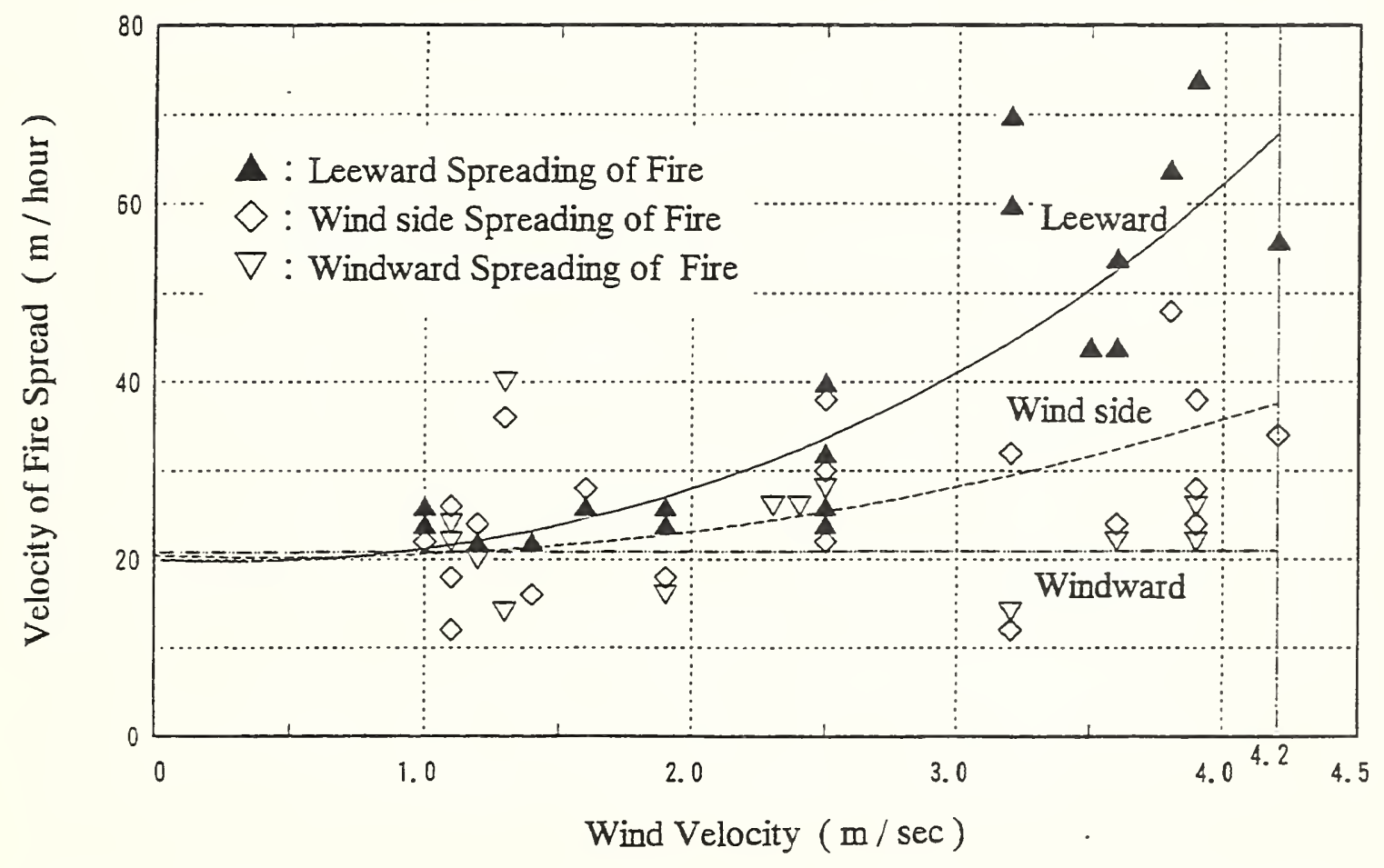

Figure 5. Relation between the wind velocity and the velocity of fire spread ${ }^{4)}$ 
There is some evidence that the fire spread faster in the blocks of wooden buildings without non-combustible materials on the exterior wall than in the blocks of wooden buildings without non-combustible materials and in the blocks which have some fire resistive buildings ${ }^{5 \text { ) }}$.

\section{FIRE SPREAD MECHANISMS}

The fires spread widely because the buildings were so close together especially in Nagata and Hyogo wards as shown in Figures 6,7 and 8. In those fire sites in Nagata and Hyogo wards, there were a lot of wooden dwellings whose exterior walls were not covered with non-combustible materials. Although many of the conventional post \& beam construction dwellings had been covered with non-combustible materials, they were severely damaged by the earthquake. In the case when the fire preventive wooden buildings constructed appropriately in accordance with the present aseismatic standards and suffered a little damage, most of them that caught fire did so mainly because fire spread from the building's openings, not from their walls ${ }^{6}$.

The combustible contents of the houses, synthetic rubber in small shoes factories especially in Nagata ward and collapsed buildings on the street contributed to the fire spread as shown in Figure 10.

There are at least 20 cases where new ignitions were caused by burning wood and embers flying through the air ${ }^{7)}$. Because fireproof roofing was shaken down by the earthquake, it was easy to ignite with burning wood and embers in those wooden house areas, although people out there extinguished most of the small ignitions.

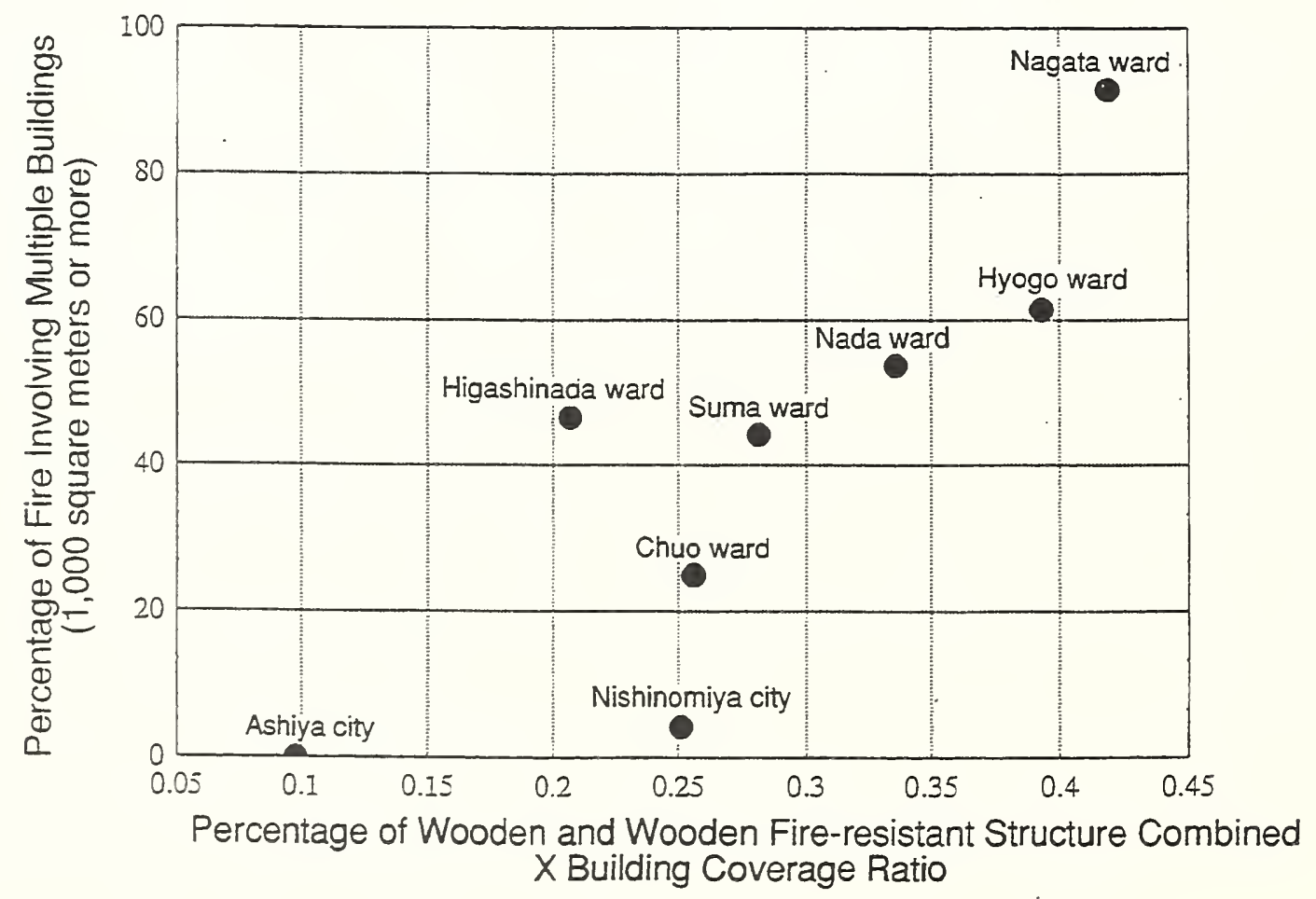

Figure 6. Relation between Percentage of Fire Involving Multiple Buildings and Wooden Building Coverage Ratio (Ward and City ) 


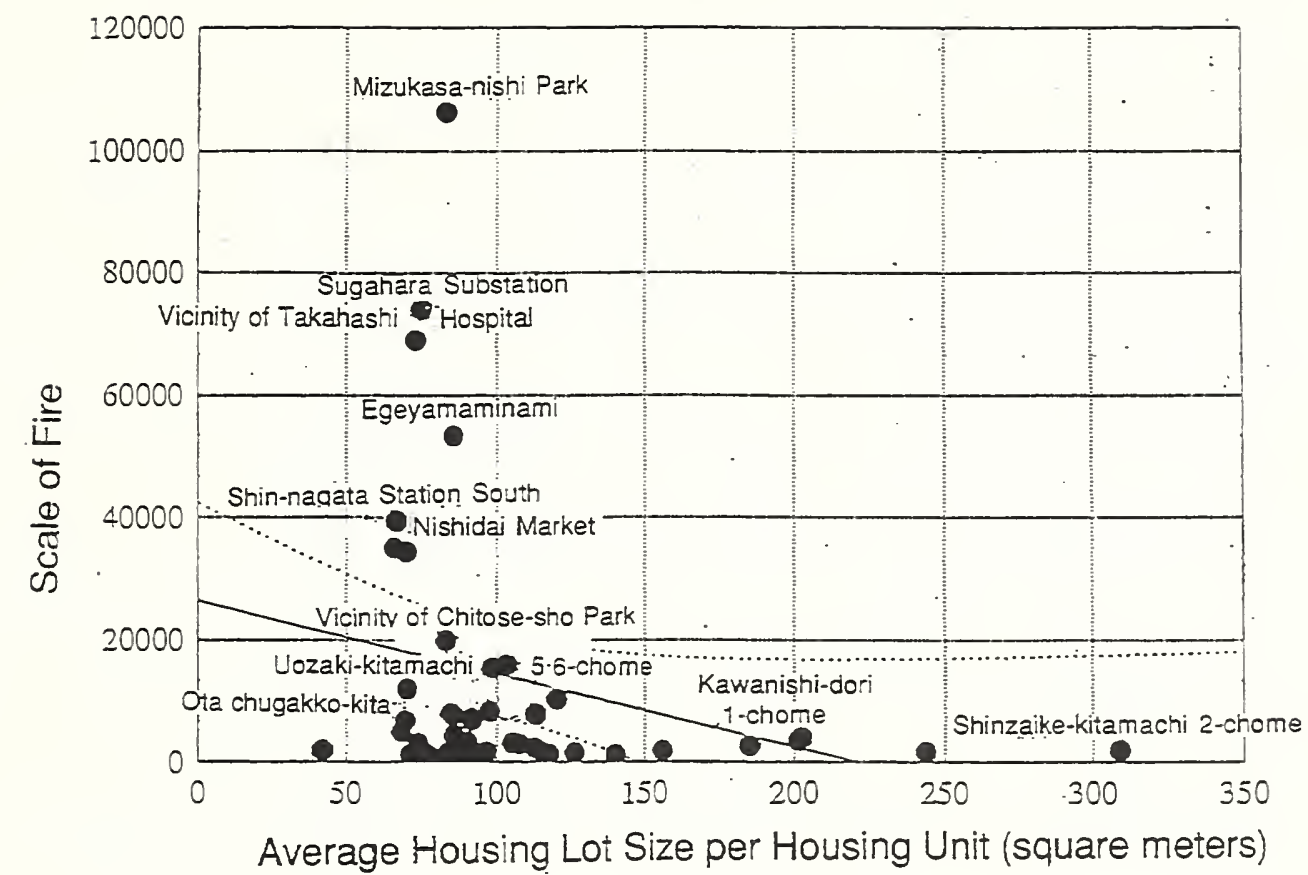

Figure 7. Relation between Scale of Fire and Average Housing Lot Size per Housing Unit ( Fire Site)

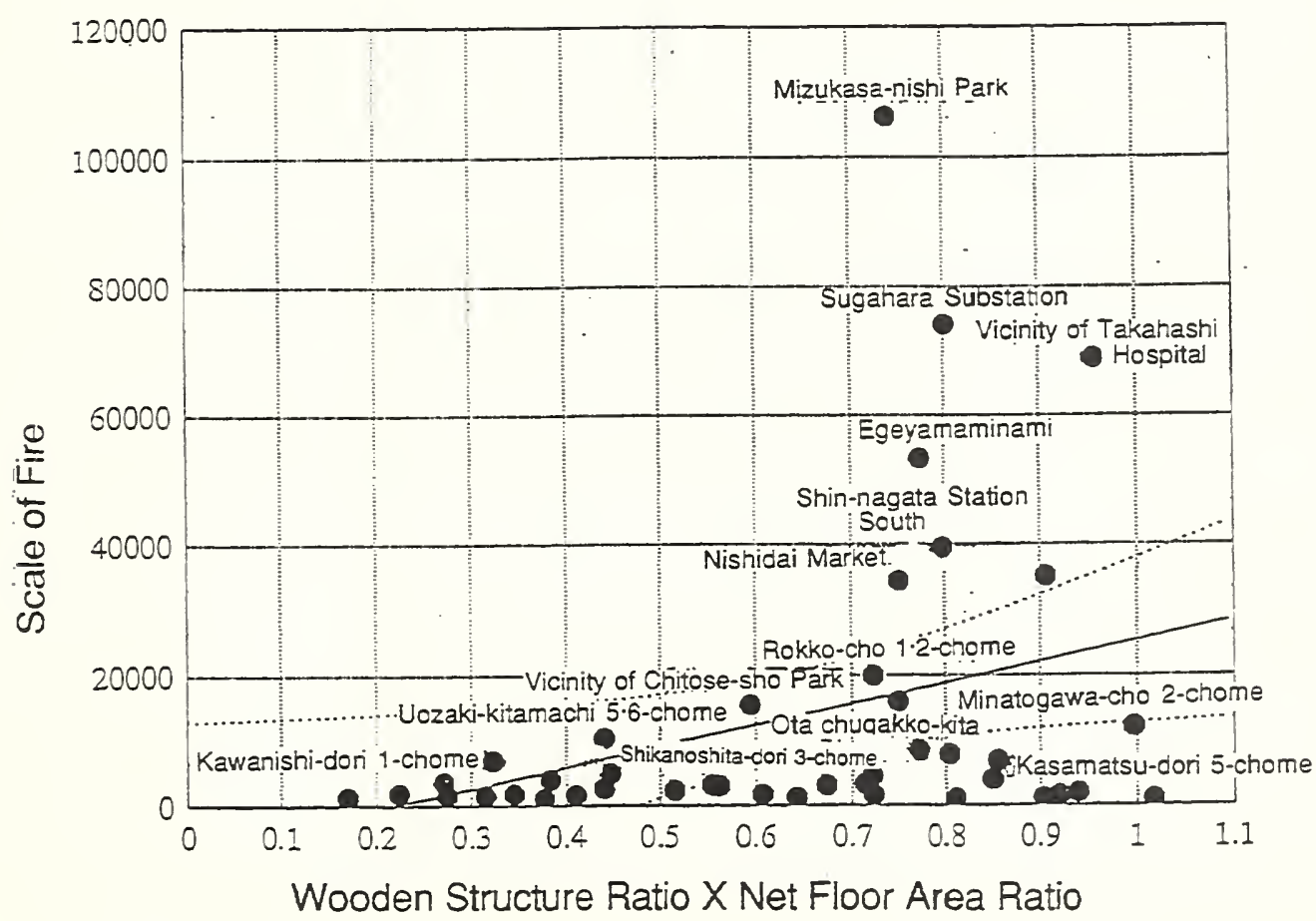

Figure 8. Relation between Scale of Fire and Wooden Structure Floor Area Ratio ( Fire Site ) 


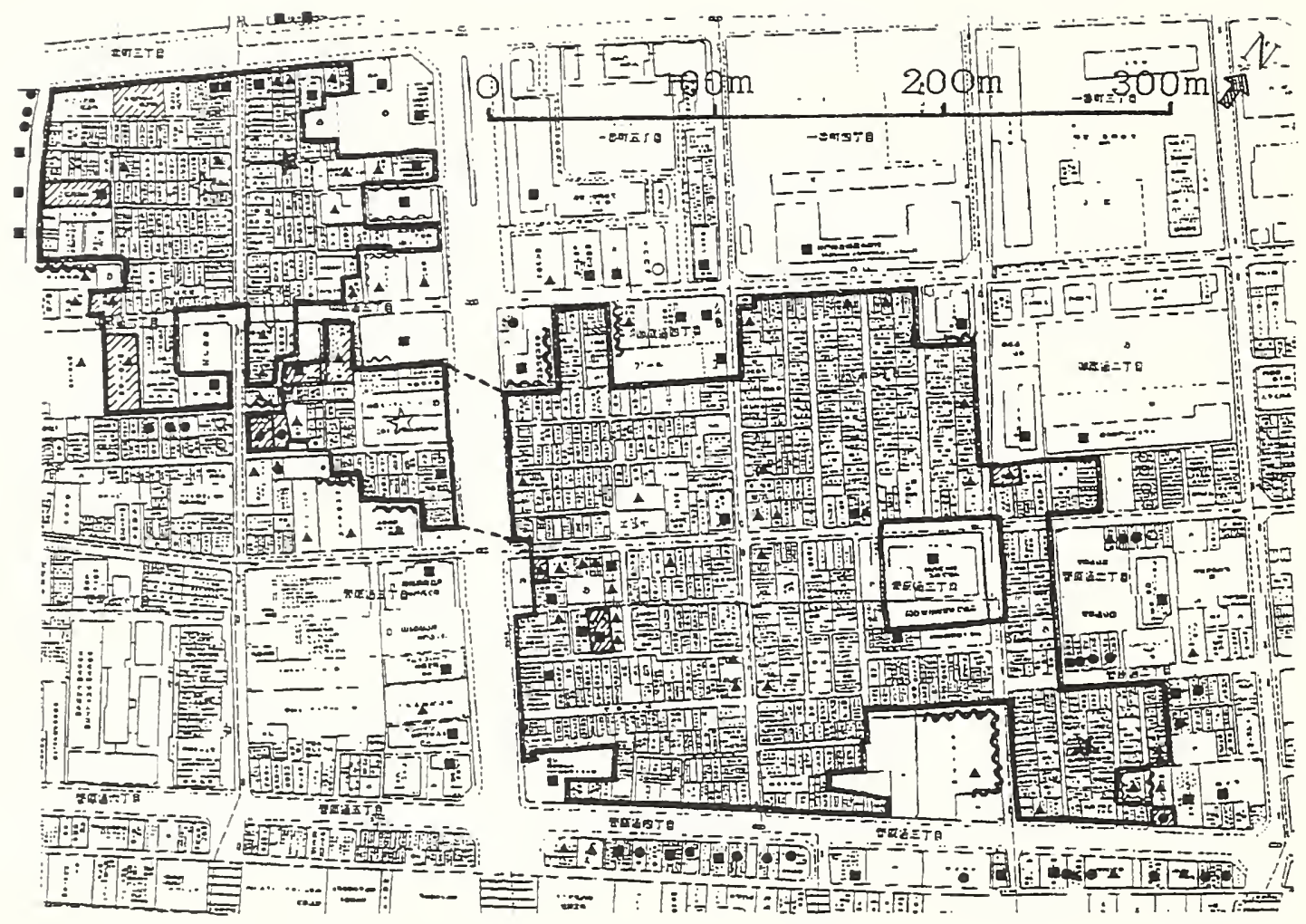

Index for building structure

a : Fire resistive building

$\Delta$ : Semi-fire resistance building

- : Wooden building with non-combustible materials on the exterior wall

$\circ$ : Wooden building without non-combustible materials on the exterior wall

Index for Ignition

* : Fire origin ( an eye-witness )

Index for Fire Spread

is : Ignition by burning wood and embers flying through the air

( Estimated by the evidence of sight )

$c:$ Fire Spread through the collapsed building on the street by fire

Index for Fire-Arresting Line

_ Fire spread boundaries

चII : Partially burned building

m: Fire damage only on the exterior wall 


\section{FIRE STOP FACTORS}

Most of the cease burning lines in urban areas were wide streets, railways, parks and similar large spaces, rows of buildings of fireproof construction, and fire proof buildings constructed on large building lots.

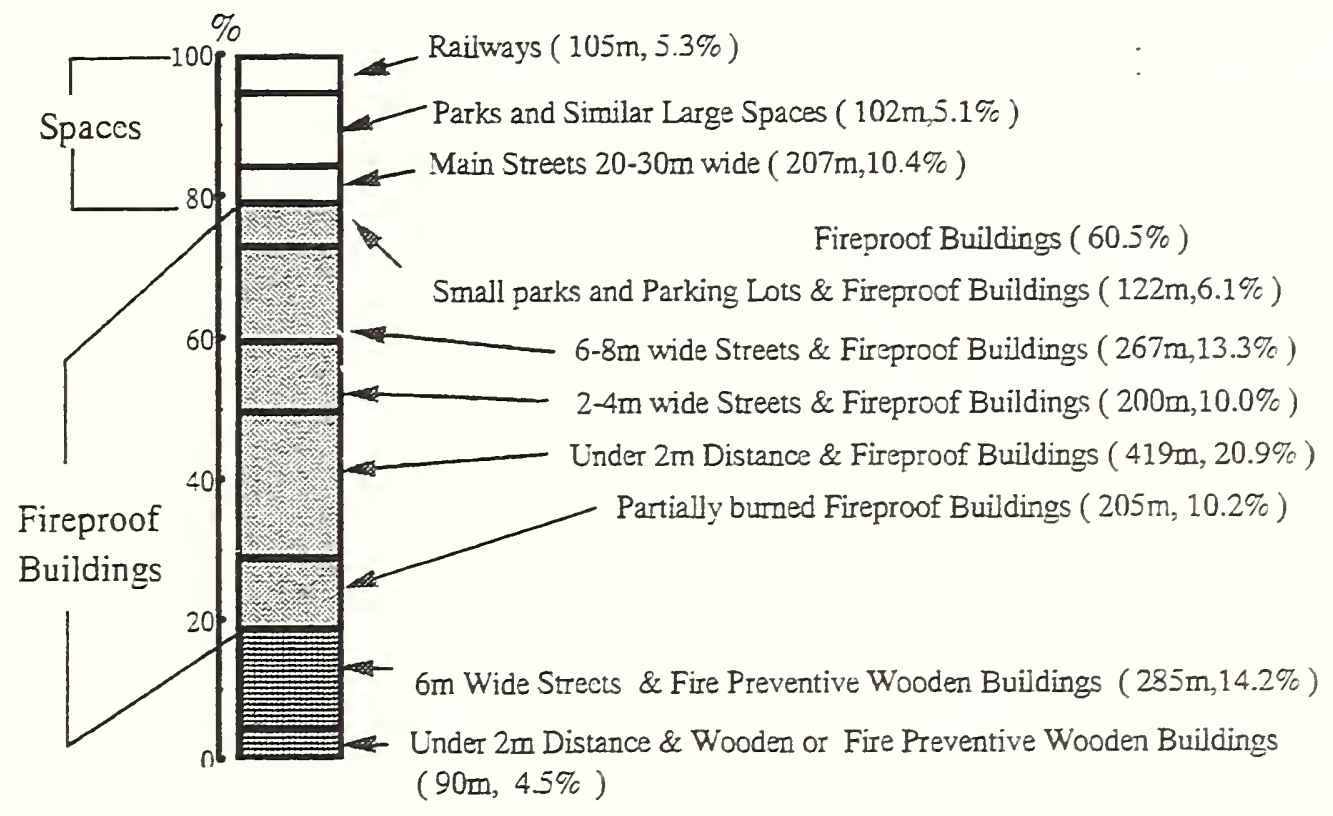

Figure 10. Fire Stop Elements at the Fire Site around the Mizukasa Nishi Park (BRI)

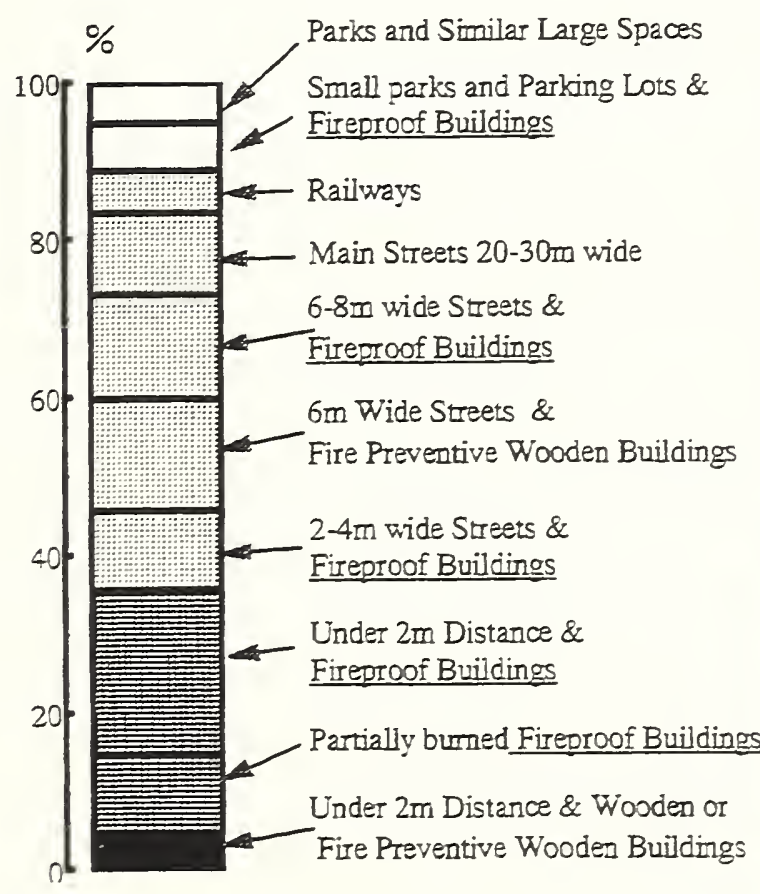

(BRI Investigation)

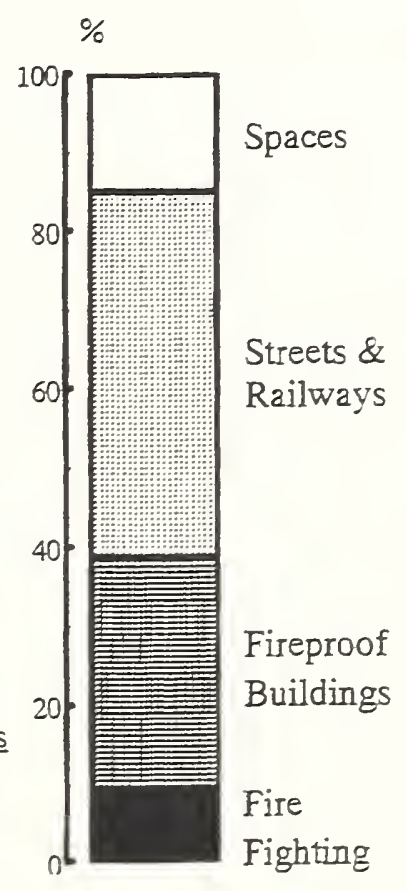

(FRI Investigation)

Figure 11. Fire Stop Factors Comparison between the result of BRI investigation and FRI investigation at the Fire Site around the Mizukasa Nishi Park 
Figure 10 shows fire stop elements at the fire site around the Mizukasa Nishi Park. This fire site was a typical residential area with large concentrations of wooden houses. About 60 percent of the cease burning lines consisted of fireproof buildings.

Figure 11 shows a comparison between the result of BRI investigation and FRI investigation at the Mizukasa Nishi Park. To make it the same definition, spaces factor around the park was omitted from the result of FRI investigation.

The streets \& railways factor is the main fire stop factor at the FRI investigation. The fireproof buildings factor is the main fire stop factor at BRI investigation. Had the buildings along the $6-8 \mathrm{~m}$ streets been wooden buildings, those wooden buildings at the cease buming lines would have been easily ignited. From this point of view, improvement of fire protection of buildings is more important than streets widening.

\section{CONCLUSION}

Fortunately the wind was weak during the first hours after the earthquake and catastrophic fires such as the 1923 Great Kanto Earthquake Fire were avoided. Further studies including detailed analysis of fire spread mechanisms and experimental studies for the conditions with strong winds are necessary for the disaster prevention planning. It is necessary to promote the improvement of urban residential areas by, for example, carrying out the systematic reconstruction of buildings in areas with high concentrations of decrepit wooden dwellings.

\section{REFERENCES}

1. Fire Research Institute; Data base of the fires following the 1995 Southern Hyogo Prefecture Earthquake.

2. Fire Department of Kobe City ; Fires following the 1995 Southern Hyogo Prefecture Earthquake, 14 April 1995.

3. Building Research Institute ; The Final Report of Investigation on the 1995 Southern Hyogo Prefecture Earthquake, March 1996.

4. Tokyo Fire Department ; The Investigation Report on the Fires in Kobe City following the Southern Hyogo Prefecture Earthquake, December 1995.

5. Murosaki, Y. et al at Kobe Univ. and Institute of Environment \& Safety Planning; Fire Spread in the 1995 Great Hanshin and Awaji Earthquake, 28 March 1995.

6. Sugawara Shinichi; Damages at the openings of Houses by the fires following the Southern Hyogo Prefecture Earthquake, December 1995.

7. Murosaki, Y.; "Problems around the fires following the 1995 Southern Hyogo Prefecture Earthquake", Proceedings of the Symposium on Earthquake and Fires, Japan Association for Fire Science and Engineering, 26 May 1995.

8. Fire Research Institute; The Investigation Report on the Fires in Kobe City following the Southern Hyogo Prefecture Earthquake, March 1995. 


\section{Discussion}

William Pitts: I know there has been some work in Japan on modeling the spread of urban fires, and it seems like you have enough data to do some sort of comparisons. Have you made any. comparisons of model with fire spread in the urban environment?

Akihiko Hokugo: This is the very topic that Dr. Tanaka is supposed to conduct some research from now on.

Takeyoshi Tanaka: We haven't started the construction of modeling of fire spread yet. We just started to collect some basic data.

Henri Mitler: Were you able to establish any statistics on how many of those fires that were induced in fire-proof buildings penetrated through broken windows?

Akihiko Hokugo: Since we had to deal with so many buildings, it was quite difficult for us to understand what kind of actual structure those buildings had. Therefore, we feel that it will be difficult to collect some meaningful statistics concerning that specific point.

Edward Zukoski: Is there any good data on the rate of fire propagation through buildings that were very severely damaged and sort of flattened by the earthquake as compared to buildings that were not very severely damaged?

Akihiko Hokugo: According to the statistics collected by the University of Kobe, when they compared the external damage of the buildings as a result of fire, in terms of fire resistant buildings, the more destruction those buildings experienced, the higher the extent of fire spread to upper floors. So if you compare individual buildings, the destroyed and severely damaged buildings tend to allow the fire to spread to the upper floors. 
3 24 


\title{
FIRES FOLLOWING THE NORTHRIDGE AND KOBE EARTHQUAKES
}

\author{
Charles Scawthorn \\ Senior Vice President, EQE Intemational \\ San Francisco CA 94104 USA and Tokpo 107 Japan
}

\begin{abstract}
Large fires following an earthquake in an urban region are potentially of catastrophic proportions - recent earthquakes such as the 1994 Northridge and 1995 Kobe earthquakes, as well as several rece nt large non-earthquake conflagrations, including the 1991 East Bay Hills and 1993 Southern California wild fires demonstrate that this potential is still quite real. The Northridge and Hanshin events are strikingly similar in magnitude, ground motions, affected population, time of day and season and other factors (eg, for both events, total earthquake-related fires were about 110). However, Northridge resulted in relatively small posteearthquake fire losses, while Hanshin resulted in the loss of 5,500 buildings. The factors that contributed to this quite different outcome were primarily the performance of the water system, the traffic congestion, and the building density in Kobe versus Los Angeles. Mitigation of the fire following problem is complex, but involves a combination of increasing public and fire service awareness, improving water supply and other lifeline seismic resistance, and structurally upgrading the building stock. Several cities in north America, including San Francisco, Berkeley and Vancouver (B.C., Canada) have active programs to mitigate the fire potential following earthquakes.
\end{abstract}

\section{THE 1994 NORTHRIDGE, CALIFORIIA EARTHQUAKE}

The Northridge earthquake was the largest earthquake to occur within a US city in more than 20 years. Because the earthquake primarily affected only a limited area, this paper focuses on the experiences of two heavily effected fire departments, the Los Angeles City Fire Department and the Santa Monica Fire Department.

Affected Area and Fire Protection. The 4:31 AM january 17, $1994 \mathrm{M}_{\mathrm{w}} 6.7$ earthquake was centered under the Northridge section of the San Fernando Valley area of the Los Angeles region. The event resulted in Modified Mercalli Intensity (MMI) shaking intensities greater than MMI VIII over approximately 700 square miles of the northern Los Argeles area, Figure 1. The population most heavily affected was in the Sar Fernando Valley, which is primarily protected by the Los Angeles City Fire 
Department. Table 1 lists fire departments significantly affected by the earthquake, and their summary statistics (see Scawthorn et al 1994 for additional detail).

Response and Related Aspects in the Northridge Earthquake. Approximately 110 fires were reported as earthquake-related on January 17, as shown in Table 2. For Los Angeles City, Santa Monica, Pasadena, Burbank, and Glendale, the number of fires is shown as reported by each fire department's incident reports. These reports reflect initial assessment of the probable cause, and may or may not include all earthquakerelated fires. Furthermore, what is counted as a single incident report may involve multiple structures. Lastly, in the case of mobile home parks, a single incident in some cases even involves multiple ignitions, since several mobile home parks had several fires, but were only listed as one incident, at one address. The major damage caused by the Northridge earthquake and the largest number of earthquake-related fires was concentrated in the San Fernando Valley. This area is served primarily by the Los Angeles City Fire Department (LAFD).

Los Angeles City Fire Department. The Northridge earthquake reportedly caused or was a contributing factor in 77 fires in the LAFD service area. These earthquake-related fires are located on the map in Figure 2. The 77 fires were among a total of 161 fires that occurred on the day of the earthquake. Earthquake-related fires predominate the calls for the first three hours. During the remainder of the day, the earthquake is a factor in almost one half of the fires. The presence of aftershocks, the shifting of damaged structures, and the turning off and on of utilities as a result of the initial shock had apparently caused new fires to occur. Structure fires predominate (86\%) the earthquake-related fires. As noted above, each report of a mobile home fire may actually involve multiple structures, since a single report may have been written for an entire mobile home park consisting of multiple burn sites. Fires classified as "outside" include the incident on Balboa Boulevard in Granada Hills where ground movement broke gas and water mains beneath the street, igniting the escaping gas and causing fires in five surrounding homes. More than $70 \%$ (66) of the earthquake-related fires occurred in single- or multiple-family residences, as might be expected from the building stock that is typical in the San Fernando Valley. The major cause of ignition was electric arcing as the result of a short circuit, although gas flame from an appliance is also a recurring source of ignition.

The large number of dispatches at mid morning were mostly hazardous condition calls due to the reported leakage of natural gas. Hazardous condition dispatches and other public service assistance account for over $75 \%$ of the dispatches.

Water Supply. The Northridge earthquake effected the water supply for portions of the San Fernando Valley. Breaks occurred in at least six trunk lines and a large number of leaks occurred at other locations. The Department of Water and Power estimated that approximately 3,000 leaks were caused by the earthquake, including two lines of the Los Angeles Aqueduct. Pump stations and storage tanks also sustained damage. The damage to the system resulted in a water shortage that had to be made up by water tenders. LAFD reported lack of water pressure at hydrants in much of the west and north portions of the San Fernando Valley. Due to this lack of water pressure, LAFD 
resorted to drafting from alternative sources, including the large number of backyard. swimming pools in the area. SMFD reports the water supply system for Santa Monica suffered no significant impairment as a result of the earthquake.

\section{THE 1995 HANSHNN (KOBE), JAPAN EARTHQUAKE}

Affected Area and Fire Protection. The 5:46 AM January 17, $1995 \mathrm{M}_{\mathrm{W}} 6.9$ OMA M7.2) Hanshin (official name: Hyogo-ken Nambu) earthquake was centered under the northern tip of Awaji island near Kobe, in the Kansai region of Japan. The event resulted in Modified Mercalli Intensity (MMI) shaking intensities greater than MMI VII over approximately 400 square $\mathrm{km}$ of the Kobe-Ashiya-Nishinomiya area, Figure 3.

The Kobe Fire Department (KFD) is a modern, well-trained fire response agency, organized into Prevention, Suppression, and General Affairs sections, and a Fire Academy. The city is divided into 11 wards for fire protection purposes. KFD maintains 11 fire stations and 15 branch stations, served by 1,298 uniformed personnel. Equipment includes two helicopters, two fireboats, and 196 vehicles. Other equipment includes 72 portable pumps. Fire engines carry predominantly 50- and 65-millimeter hose; larger hose is not available except for drafting purposes. KFD has a civil disaster prevention program as well as a cadre of volunteer fire corps with about 4,000 members. This corps provides the first on-scene engagement of the fire, performing functions such as giving directions to arriving emergency vehicles and helping to guide people to safety

Response and Related Aspects in the Hanshin Earthquake. KFD had mirimal staffing on duty at the time of the earthquake, possibly because the previous day had been a holiday. Initial actions included recalling off-duty personnel and responding to fire calls. Approximately 100 fires broke out within minutes, primarily in densely built-up, low-rise areas of the central city, which comprise mixed residential-commencial occupancies, predominantly of wood construction. Within 1 to 2 hours, several large conflagrations had developed. There were a total of 108 fires reported in Kobe on January 17 (Kobe FD, 1995), the majority being in the wards of Higashi Nada, Nada, Hyogo, Nagata, and Suma, Figure 4 (after National Fire Research Institute, 1995). Mades of fire reporting were unclear as of this writing, and fire response was hampered by extreme traffic congestion, and collapsed houses, buildings, and rubble in the streets. Because of the numerous collapses, many areas were inaccessible to vehicles.

Fire spread was via radiant heat and flame impingement, building to building in the densely built-up areas. The wind was calm, and fire advance was relatively slow. In a number of cases, fires were observed to have stopped at relatively narrow fire breaks (e.g., 10 meters) or, in at least one case, at a high-rise apartment building, probably as a result of active fire fighting. The final bumed area in Kobe was estimated at 1 million square meters, with $50 \%$ of this in the Nagata Ward.

The Ashiya Fire Department reported 11 fires on January 17; nine of them were before 7:30 a.m. Distribution of the fires was along an east-west line about 1 kilometer wide centered on National Route 2 . The total bumed area for the 11 fires was about 4,400 square meters. 
Water Supply. Fire water is primarily from the city water system, served by gravity from 30 reservoirs. Of these, 22 have dual tanks, with one tank having a seismic shutoff valve so that, in the event of an earthquake, one tark's contents is conserved for fire fighting. In this event, all 22 valves functioned properly, conserving 30,000 cubic meters of water, which, however, could not be delivered because of approximately 2,000 breaks in the underground piping. Kobe has approximately 23,500 fire hydrants, typically flush-mounted (i.e., under a steel plate in the sidewalk or street) with one 150 millimeter-diameter hose connection. The city has provided underground storage of water for disaster fire fighting in 968 cisterns, generally of 40,000-liter capacity, sufficient for about a 10-minute supply of a pumper. All engines carry hard suction, so that additional water can be drafted from Osaka Bay or the several streams running through Kobe.

Kobe sustained approximately 1,750 breaks in its underground distribution system. Water for fire-fighting purposes was available for 2 to 3 hours, including the use of underground cisterns. Subsequently, water was available only from tanker trucks. KFD attempted to supply water with a fireboat and relay system, but this was unsuccessful due to the relatively small hose used by KFD. The first author overflew the area at about 5:00 p.m. on January 17 and was able to observe all of the larger fires (about eight in all) from an altitude of less than 300 meters. No fire streams were observed, and all fires were burning freely - several with flames 6 meters or more in height. No fire apparatus were observed in the vicinity of the large fires, although fire apparatus could be seen at other locations (their activities were unclear from the air). Some residents formed bucket brigades (with sewer water) to try to control the flames.

\section{Northridge/Kobe - Comparative Analysis}

Several observations emerge from these two earthquakes, which are summarized in Table 3. Specifically:

Ignitions: There were a significant number of earthquake-related fires in both events. In fact, the total number of ignitions is comparable - 110 for the Northridge event, versus 108 for Kobe City. Considering that the great majority of the 77 ignitions within the City of Los Angeles occurred within the MMI VII isoseismal, and that this area contains about one third of the total Los Angeles City population of 3.4 million, this equates to about 1 ignition per 14,719 population for the Northridge event. In the case of Kobe (population 1.5 million), the 108 ignitions equates to 1 ignition per 13,676 population. Thus, ignition rate is also comparable.

Response: In the case of Northridge, ignitions were all brought under control within several hours of the earthquake. Furthermore, the resources of the Los Angeles region were sufficient to deal with all fire ignitions, as well as other emergencies, such as search and rescue, hazardous materials releases, etc. This is an excellent response, and is due to the large well-equipped fire service in the Los Angeles region, which has dealt with a large number of fires and other emergencies in the 
last several years. The fire service in Los Angeles equates to approximately 1 firefighter per 1,338 population, while in Kobe this ratio is about 1/1,138, or quite similar. However, Los Angeles region is significantly larger than Kobe, so that Los Angeles had more than four times the total resources of Kobe, a significant difference.

Weather Conditions: Wind, humidity and other conditions were favorable in both cases and not a major problem.

Water Supply: In Los Angeles, while firefighting water supply failed in the heavily affected portions of Northridge, firefighters were able to avail themselves of alternative sources (e.g., backyard swimming pools). in Kobe, due to the more than 2,000 breaks in the underground water distribution system, the fire department was without water within several hours, and found it difficult to relay or otherwise obtain water for firefighting purposes. As a result, fire spread in Kobe was significantly greater than in Northridge, resulting in the destruction of perhaps 5,000 buildings in Kobe.

The 1994 Northridge and 1995 Hanshin earthquakes and associated fires reinforce the following points:

- Earthquakes in urban areas continue to cause multiple simultaneous ignitions, and degrade emergency response due to impaired commurications, transportation and water supply

- These events are replicable, as shown by comparison of the 1971 San Fernando and 1994 Northridge events (Scawthom, et al, 1995), and by comparison of the ignition rates and other factors in the Northridge and Hanshin events, providing some validation for simulation modeling and projections for larger events

- Under adverse conditions, large conflagrations are possible in modern cities, as shown by events in Califormia (i.e., the 1991 East Bay Hills Fire, and the 1993 Southern California wildfires), and by the Hanshin earthquake in Japan.

- Projections for larger earthquakes in Los Angeles indicate perhaps 500 ignitions within several hours - the situation is worse in Tokyo.

This accumulation of experience leads to the conclusion that the potential exists for large conflagrations following a major earthquake in an urban area. Under adverse meteorological and other conditions, these conflagrations may burn for several days, replicating the events of 1906 in San Francisco, and 1923 in Tokyo. Extensive, welldrilled mutual aid systems are required, in order to mobilize large resources in response, but the deployment of these resources will be hampered by transportation 
Fires Following Northridge and Kobe Earthquakes

UJNR Panel on Fire, Gaithersburg, March 1996

C Scawthom, Page 6

difficulties and, perhaps most tellingly, failure of firefighting water supplies. Improvements in planning and infrastructure are absolutely essential to forestall this potential.

Table 1.

Fire Departments Affected by the January 17, 1994 Northridge Earthquake

\begin{tabular}{|l|c|c|c|c|c|}
\hline Fire Department & $\begin{array}{c}\text { Estimated } \\
\text { Population } \\
\text { (thousands) }\end{array}$ & $\begin{array}{c}\text { Area } \\
\text { (Sq } \\
\text { Miles) }\end{array}$ & $\begin{array}{c}\text { Number } \\
\text { of } \\
\text { Stations }\end{array}$ & $\begin{array}{c}\text { Fire } \\
\text { Fighting } \\
\text { Personnel }\end{array}$ & $\begin{array}{c}\text { Number } \\
\text { of } \\
\text { Engines }\end{array}$ \\
\hline Los Angeles City & 3,400 & 469 & 104 & 2,865 & 104 \\
\hline $\begin{array}{l}\text { Los Angeles } \\
\text { County }\end{array}$ & 2,896 & 2,234 & 127 & 1,842 & 144 \\
\hline Ventura County & 700 & 126 & 30 & 327 & $40+/-$ \\
\hline Santa Monica & 97 & 8 & 4 & 100 & 5 \\
\hline Burbank & 94 & 17 & 6 & 120 & 6 \\
\hline Pasadena & 132 & 23 & 8 & 150 & 8 \\
\hline Glendale & 166 & 30 & 9 & 167 & 9 \\
\hline South Pasadena & 25 & 3 & 1 & 27 & 2 \\
\hline Beverly Hills & 34 & 6 & 3 & 81 & 7 \\
\hline Culver City & 41 & 5 & 3 & 66 & 5 \\
\hline Fillmore & 12 & 2 & 1 & 9 & 1 \\
\hline
\end{tabular}

Table 2.

Fire Following the January 17, 1994 Northridge Earthquake

\begin{tabular}{|l|r|}
\hline \multicolumn{1}{|c|}{ Community } & \multicolumn{1}{|c|}{$\begin{array}{c}\text { Number of } \\
\text { Earthquake- } \\
\text { Related Fires }\end{array}$} \\
\hline Los Angeles City & 77 \\
\hline $\begin{array}{l}\text { Los Angeles } \\
\text { County }\end{array}$ & -15 \\
\hline Ventura County & -10 \\
\hline Santa Morica & 4 \\
\hline Burbank & 0 \\
\hline Pasadena & 1 \\
\hline Glendale & 0 \\
\hline South Pasadena & 0 \\
\hline Beverly Hills & 1 \\
\hline Culver City & 0 \\
\hline Fillmore & 2 \\
\hline \multicolumn{1}{|c|}{ TOTAL } & -110 \\
\hline
\end{tabular}


Fires Following Northridge and Kobe Earthquakes

UJNR Panel on Fire, Gaithersburg, March 1996

C. Scawthom, Page 7

Table 3

Hanshin and Northridge Earthqquakes: Comparative Analysis.

\begin{tabular}{|c|c|c|c|}
\hline ASPECT & FACIOR & NORTHRIDGE & HANSHEN \\
\hline \multirow[t]{2}{*}{ Event } & Magnitude $\left(M_{w}\right)$ & 6.7 & 6.9 \\
\hline & Date (winter) & $\operatorname{Jan} 17$ & $\operatorname{Jan} 17$ \\
\hline \multirow{4}{*}{$\begin{array}{l}\text { Region } \\
\text { Ignitions }\end{array}$} & $\begin{array}{c}\text { Time } \\
\text { Population (MMI 8) }\end{array}$ & $\begin{array}{c}0431 \\
1 \sim 1.5 \text { million }\end{array}$ & $\begin{array}{c}0546 \\
2 \text { million }\end{array}$ \\
\hline & $\begin{array}{c}\text { Density (pop/sq km) } \\
\text { Number (total) }\end{array}$ & $\begin{array}{c}1,000 \sim 1,500 \\
110\end{array}$ & $\begin{array}{c}4,000 \\
108 \\
\end{array}$ \\
\hline & Structural Fires & $86 \%$ & $97 \%$ \\
\hline & Rate (MMI 7) Ign/pop: & 14,719 & 13,676 \\
\hline \multirow[t]{5}{*}{ Response } & FD Communications & manual dispatch & \\
\hline & Resources (ff/popul): & 1,338 & 1,138 \\
\hline & Stations & 104 & 26 (Kobe) \\
\hline & Traffic Congestion & Minor & Major \\
\hline & Mutual Aid & Available - not needed & after $10 \mathrm{hrs}$ \\
\hline \multirow[t]{2}{*}{ Water } & Water System Damage & Some & Total? \\
\hline & Cisterns & $\begin{array}{c}\text { Swimming Pools } \\
\text { Calm }\end{array}$ & $\begin{array}{c}\text { 946, mostly } 40 \text { tons (10 } \\
\text { mins) } \\
\text { Minor }\end{array}$ \\
\hline Gas & Automatic Shut-offs & ? few $\%$ & $\begin{array}{l}70 \% \text { - ineffective due to } \\
\text { structl collapse }\end{array}$ \\
\hline Spread & & Minor & Major. 5,000 bldgs \\
\hline
\end{tabular}

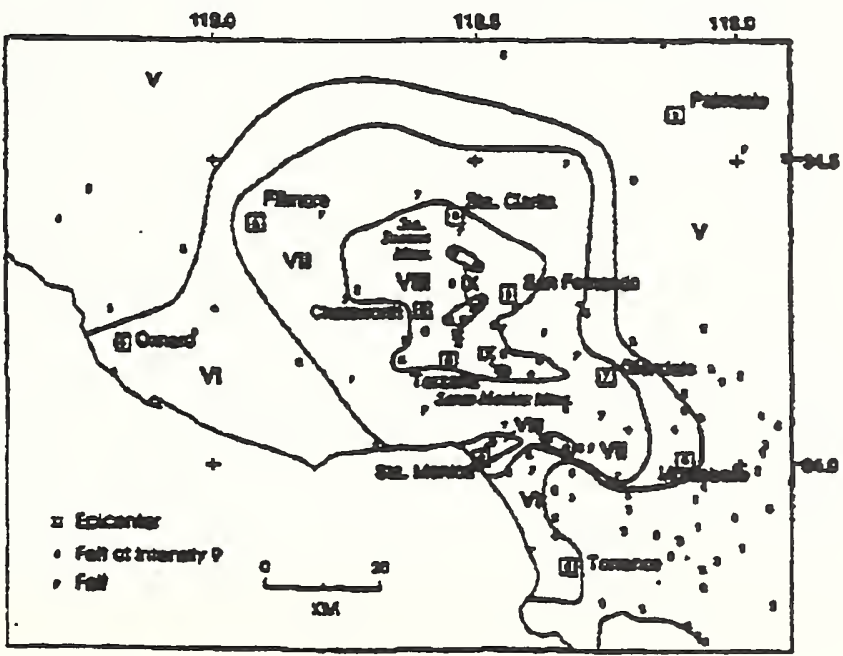

Figure 1. MMI Map for the January 17, 1994 Northridge Earthquake

(from J. Dewey, USGS) 
Fires Following Northridge and Kobe Earthquakes

UTNR Panel on Fire, Gaithersburg, March 1996

C. Scawthom, Page 8

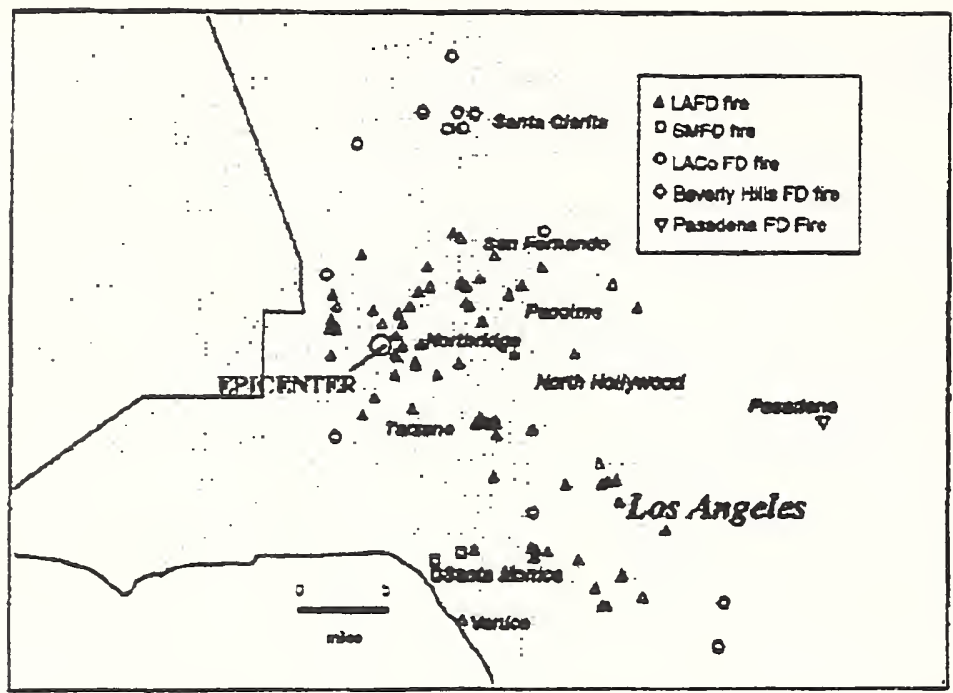

Figure 2. Earthquake-Related Fires, January 17, 1994, LA County Fires Only.

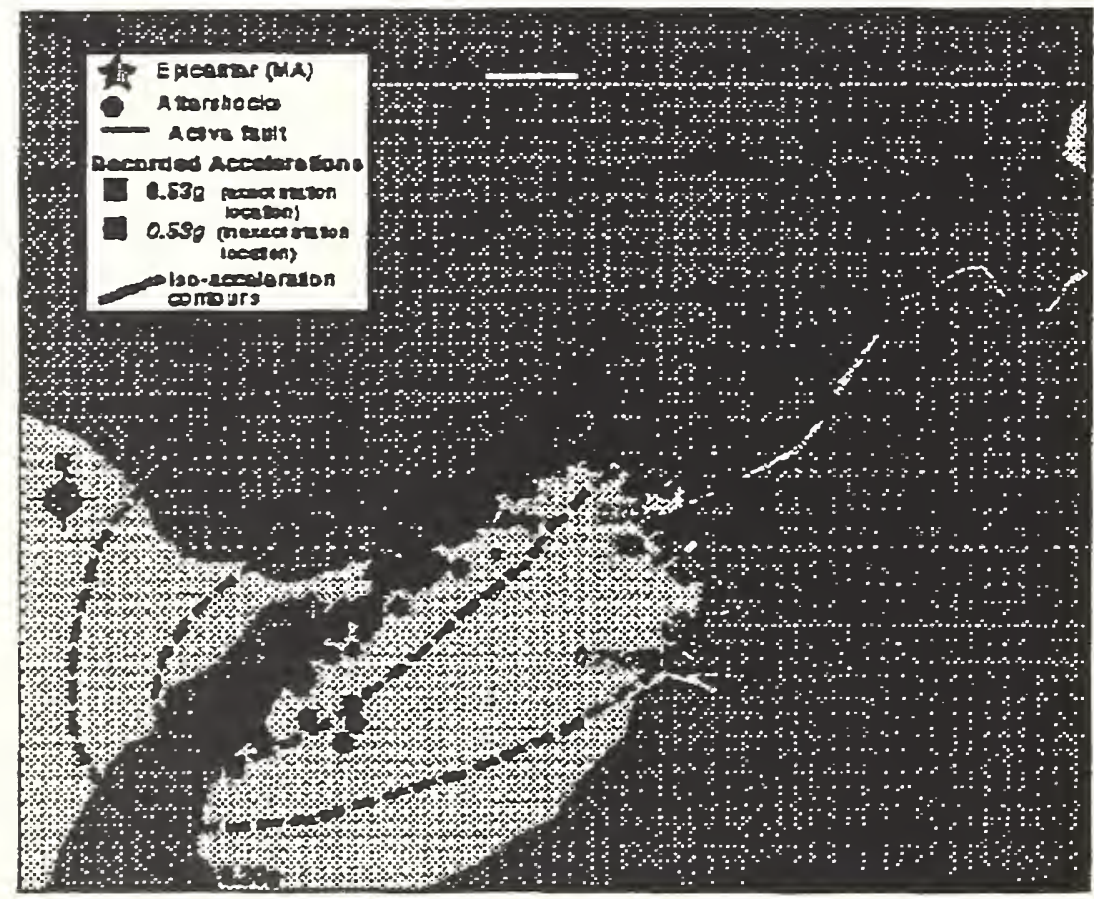

Figure 3. Shaking Intensity, Hanshin Earthquake (after EQE, 1995) 
Fires Following Northridge and Kobe Earthquakes

UJNR Panel on Fire, Gaithersturg, March 2996

C. Scawthorn, Page 9

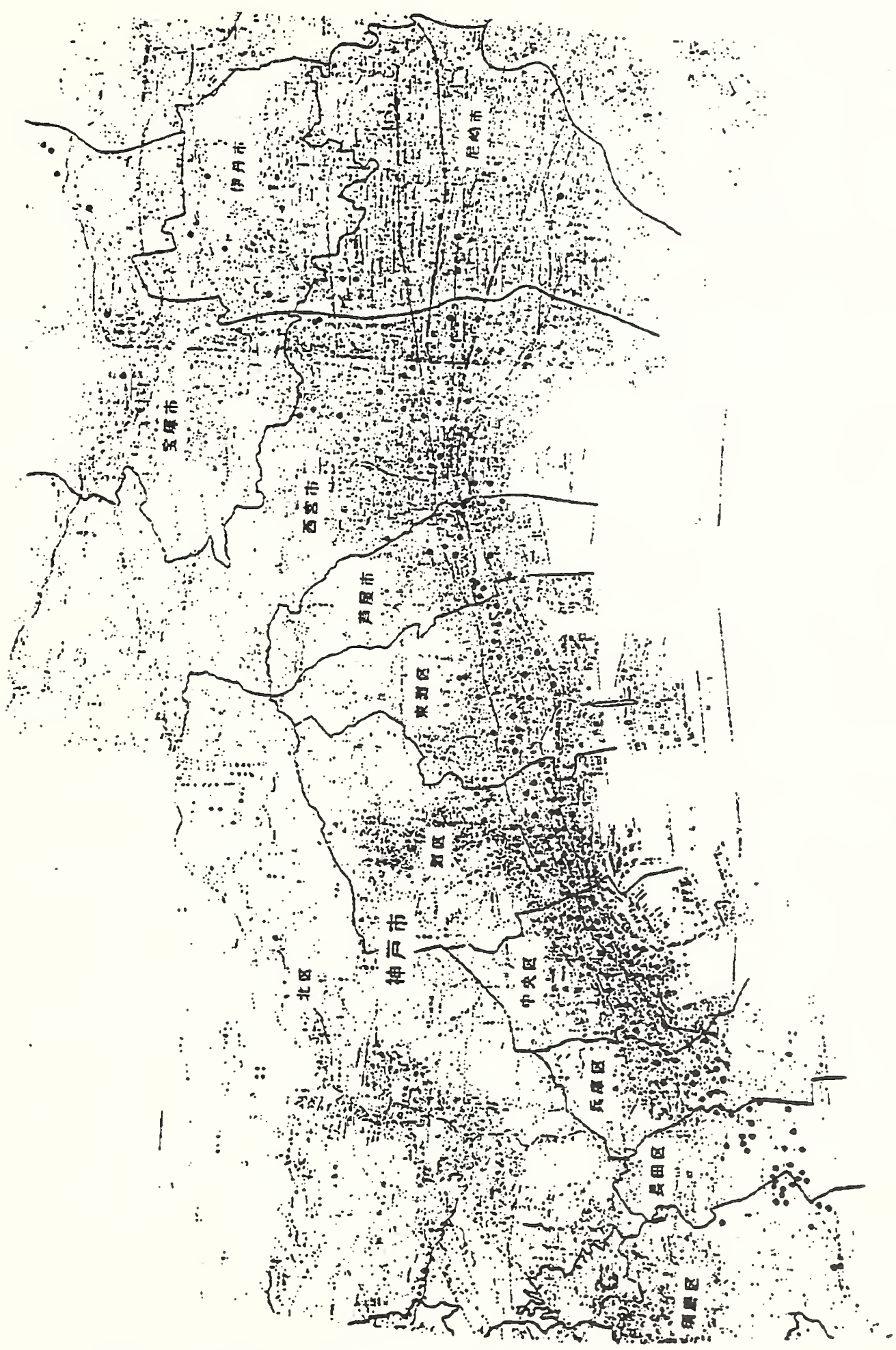

Figure 4. Ignition Distribution, Hanshin Earthquake

(after National Fire Research Institute, 1995) 


\section{REFERENCES}

Anthony, D. F., Managing the Disaster, Fire Engineering, Pennwell Publishing Company, August 1994.

Dewey, J.W., B.G. Reagor, and L. Dengler (1994) Isoseismal map of the Northridge California earthquake of January 17, 1994: Poster talk presented at the 89th Annual Meeting of the Seismological Society of American, Pasadena CA.

Earthquake Engineering Research Institute (1995) The Hyogo-Ken Nanbu Earthquake, January 17, 1995, Preliminary Reconnaissance Report, Earthquake Engineering Research Institute, Oakland CA.

EQE International (1995) The January 17, 1995 Kobe Earthquake, An EQE Summary Report, EQE International, San Francisco CA.

Kobe Fire Department (1995) Heisei 7 Hyogo Ken Nanbu Earthquake - Regarding Kobe City Damage and Fire Department Activities, Kobe City.(in Japanese)

Los Angeles City Fire Department (1987), Earthquake Emergency Operational Plan, 1987.

Los Angeles City Fire Department (1994), '94 Earthquake After Action Report, March 9, 1994.

Los Angeles County Fire Department (1994) Northridge Earthquake Fact Sheet.

Manning, D. O., Message from LAFD Chief Engineer and General Manager, Fire Engineering, Pennwell Publishing company, August 1994.

National Fire Research Institute (1995) Report on the Hyogo Ken Nanbu Earthquake and Urban Fires in Kobe City.

Scawthorn, C. And Khater, M. (1992) Fire Following Earthquake - Conflagration Potential in the Greater Los Angeles, San Francisco, Seattle and Memphis Areas, prepared for the Natural Disaster Coalition by EQE International, San Francisco, CA. [EQE's study for the Natural Disaster Coalition, Fire Following Earthquake in the Greater Los Angeles, San Francisco, Seattle and Memphis Areas, is based on methodologies originated by Dr. Scawthorn and is available from the National Committee on Property Insurance, 75 Tremont Street, Suite 510, Boston, MA 02108-3910, (617) 722-0200.]

Scawthorn, C. and Khater, M. (1994) Fires Caused by Earthquakes: A Greater Threat Than Many Realize, NFPA Journal, May/June, p. 82-86.

Scawthorn, C., Cowell, A. et al (1995) Fire-related Aspects of the January 17, 1994 Northridge Earthquake, Ch. 8, Spectra, Earthquake Engg. Research Inst., Oakland CA. (in press). 


\section{Discussion}

John Rockett: I have essentially the same question for you and the other speakers. There has been a great deal of interest in recent years in the extension of sprinkler systems to a wider variety of buildings. If one goes through the streets of Chicago, you see the remnants of old elevated tanks or the frames that they were on. It has become the philosophy that municipal water systems are so reliable that we don't need back-up systems. Do we need back-up systems for sprinklers in earthquake-prone areas?

Charles Scawthorn: I think the answer is case-by-case. You can't make a blanket statement. The Uniform Building Code presently requires for highrise and larger buildings in a seismic zone 3 and 4, which is basically the western United States, a minimum back-up water supply of about 15,000 gallons. That is precisely because of earthquakes. The lowrise building, say a warehouse, the building code would not require a back-up water supply within the building. It just takes it off the mains. When you lose pressure, the sprinkler system is going to be largely ineffective. In that situation, I think you have to look at the building density, the occupancy, and many other factors to decide whether a back-up is appropriate or you just want to rely on insurance and evacuation of people. I think that this kind of concept I just outlined falls under performance-based codes.

\section{Ai Sekizawa:}

Let me talk about the situations in Japan. As Mr. Scawthorn mentioned, there is a building code requirement that when buildings exceed a certain size, a sprinkler system or back-up water supply system is required. However, we experienced a problem at the time of great earthquake of Kobe. Many of those buildings that were equipped with back-up water supply systems had those tanks situated on the top of the building. Because of the earthquake shaking, the water tank was damaged, and the water in that tank escaped. Therefore, the actual system was not usable when needed. So we now need to work on ensuring the integrity of the tanks so that they themselves are seismic. Therefore, it is desirable to install those tanks underground rather than on the top of the buildings. In the case of Michiomia City, they had under ground tanks for the additional water supply, and they could successfully use that water when an earthquake happened. Therefore, their response activities were quite effective. That water supply is used not only for the fire of buildings, but also to extinguish fire in the streets or in the city or town.

Charles Scawthorn: Thank you for pointing that out Dr. Sekizawa. In the U.S., typically the pattern in a highrise building is that the water tank is in the basement and you have a back-up fire pump and back-up fuel supply in the basement. When the sprinklers trigger, then the pressure drops and the pumps kick in and utilize the back-up water supply. I think the entire system of back up motors, fire pump, piping and so on, can be improved a whole lot. 
3 $3 \underline{6}$ 
Causes of the Seismic Fires following the Great Hanshin-Awaji Earthquake-Survey

Kazuyoshi OHNISHI

\author{
Department of Architecture and Civil Enginerring \\ KOBE University \\ 1-1 Rokko-dai cho, Nada-ku, Kobe 657 JAPAN
}

\begin{abstract}
This study will pay particular attention to the causes of the fires that occurred simultaneously and in large numbers following the earthquake. In order that such a massive disaster never repeats itself, we must accurately determine what events took place during this earthquake and what measures were taken. The seven cities targeted for in Hyogo Prefecture and the seismic fires considered here are those that started over the three days immediately following the earthquake between January 17, 18 and 19 . Interview surveys with residents in areas that were judged to be the source of fires, concerned whether the fires actually occurred, determining the spots of fire outbreaks, and studying the causes of the fires, the spreading of fires, and fire-fighting activities. . The number of seismic fires that started immediately after the earthquake up to January 19 totaled 181 in surveyed cities, then 96 were single fires and 85 were spread fires. Out of the 84 fires whose causes could be conjectured, a total of 56 cases (66.7\%) were related to electricity and gas; the major characteristic of the Great Hanshin-Awaji Earthquake. In the North Ridge Earthquake of 1994, it is also conjectured that many fires were caused by a similar phenomenon.
\end{abstract}

\title{
1. Introduction
}

On January 17, 1995 at 5:46 a.m., the Hanshin area was hit by a violent earthquake, measuring 7 on the Japanese seismic scale. The epicenter was under the northern part of Awaji Island. The earthquake registered 7.2 on the Richter scale and the shallow depth of the epicenter was only about $20 \mathrm{~km}$; unusual for earthquakes. Having occurred immediately beneath large cities such as Kobe, the temblor produced enormous damage. The life-support services of electricity, water, and gas were cut and transportation networks of roads and railways were paralyzed. Amidst these circumstances, fires broke out simultaneously and in large numbers, sending up raging flames in numerous places across town and spewing up black columns of smoke. Indeed even several minutes after the earthquake 60 fires broke out simultaneously in Kobe city area, but only eighty pomper teams with 292 firemen, who belong to Kobe city Fire Department, could arrest the spread of fires just after the earthquake. This event happened before the lessons from the North Ridge Earthquake that occurred in the United States in 1994 could be fully digested. The Great Hanshin-Awaji Earthquake again clearly brought to fore the characteristics and problems of seismic fires. 


\section{Method of Study}

\subsection{Scope of investigation}

The seven cities targeted for survey were Kobe, Amagasaki, Nishinomiya, Ashiya, Takarazuka, Itami and Kawanishi in Hyogo Prefecture. The seismic fires considered here are those that started over the three days immediately following the earthquake on January 17.

\subsection{Method of interview survey}

Using the fire list of the fire departments of the above seven cities of between January 17 , 18 and 19, interview surveys with residents in areas that were judged to be the source of fires, concerned whether the fires actually occurred, determining the spots of fire outbreaks, and studying the causes of the fires, the spreading of fires, and fire-fighting activities.

\subsection{Compilation of fire list}

In areas where many fires started at the same time, the work of sorting through the lists of all reported fires is in itself a large task. Therefore, surveys were undertaken as lists became available.

2.3.1. With regard to the cities of Kobe and Nishinomiya, the fire report lists were obtained from the municipal fire departments and interviews on residents were duly conducted. Later, supplementation and correction of exact location of fire outbreaks were conducted, based on the new report lists obtained afterwards from the municipal fire authorities and from the research findings from fire sites as issued by the Ministry of Construction's Building Research Institute.

2.3.2. With regard to the cities of Amagasaki and Ashiya, the fire survey findings gathered by the cities' fire departments were obtained, and based on these findings, the number of fires were determined and interviews with residents were held.

2.3.3. With regard to the cities of Itami, Takarazuka and Kawanishi, the causes of fires were determined based on the investigation results of the fire departments. Interviews with residents were not conducted.

\section{General Facts about the Fires}

\subsection{Number of fires}

The number of fires that started immediately after the earthquake up to January 19 totaled 181 in the seven cities surveyed. Table 1 gives a breakdown according to the size of fires. Ninty-six were single fires (fire was limited to one building, or in the case of an apartment block, even if the fire spread from one apartment to another, the fire was contained within the same block) and 85 were fires where flames spread to affect more than one building.

3.1.1. Regarding the size of the fires that spread, small-scale fires (less than $1,000 \mathrm{~m}^{2}$ of fire spread) numbered 34 , medium-scale fires $\left(1,000 \mathrm{~m}^{2}\right.$ or more but under $\left.3,300 \mathrm{~m}^{2}\right)$ numbered 24 , and large-scale fires $\left(3,300 \mathrm{~m}^{2}\right.$ or more) numbered 27 . Fires of $33,000 \mathrm{~m}^{2}$ or more 
numbered 10.

Note 1) The figures for the amount of areas burnt by fire were taken from data given by the Ministry of Construction, Building Research Institute. In general, areas burnt in fires are determined according to the area of the building, but here, the land area was used for its measure.

Note 2) Because there are cases when more than one exact location of fire breaks combined to form a large-scale fire, the number of large-scale fires became 22 .

Fig. 1 examines the relation between the number of fires starting and the time elapsed after the earthquake. Out of the 181 incidents of fire, 91 (50.3\%) started within one hour of the earthquake. There were 137 fires (75.7\%) that started within 6 hours, and $157(86.7 \%)$ within 24 hours. After 24 hours, 23 fires (12.7\%) broke out. Thus, the striking feature of this earthquake is that many fires started long after the earthquake's occurrence.

Table 1 Number of fires according to the size

\begin{tabular}{l|rcccc}
\hline & single bldg. & small-scale & medium-scale & large-scale & Total \\
\hline Kobe city & 44 & 23 & 21 & 17 & 115 \\
Higashinada-ku & 9 & 3 & 5 & 3 & 20 \\
Nada-ku & 5 & 6 & 4 & 3 & 18 \\
Chuo-ku & 16 & 3 & 3 & 0 & 22 \\
Hyogo-ku & 1 & 5 & 4 & 4 & 14 \\
Nagata-ku & 3 & 2 & 3 & 14 & 22 \\
Suma-ku & 3 & 4 & 2 & 3 & 12 \\
Tarumi-ku & 5 & 0 & 0 & 0 & 5 \\
Kita-ku & 1 & 0 & 0 & 0 & 1 \\
Nishi-ku & 1 & 0 & 0 & 0 & 1 \\
Amagasaki city & 5 & 0 & 2 & 0 & 7 \\
Nishinomiya city & 23 & 7 & 1 & 0 & 14 \\
Ashiya city & 11 & 3 & 0 & 0 & 4 \\
Takarazuka city & 3 & 1 & 0 & 0 & 7 \\
Itami city & 7 & 0 & 0 & 0 & 3 \\
Kawanishi city & 3 & 0 & 24 & 27 & 1 \\
\hline Total & 9 & 3 & & 7 & 1 \\
\hline
\end{tabular}

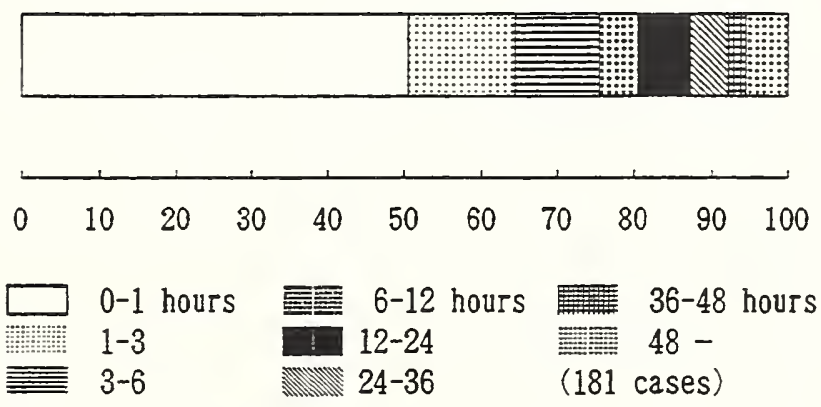

Fig 1 When fires occured after the Earthquake? 


\subsection{Incidence of fire}

Table 2 gives the incidence of fire broken down by cities and wards (the number of fires starting per 10,000 households). Taking areas in the seven cities surveyed as a whole, the rate was 1.68; however, in the Chuo and Nagata wards of Kobe City, and in Ashiya City, the incidence was high at over 4.00 .

Table 3 gives the incidence of fires in major earthquakes of the past. The only earthquake that had previously registered 7 on the Japanese scale was the Fukui Earthquake of 1948. The incidence of fire in Fukui City at that time was as high as 15.5. Comparing this with the Great Hanshin-Awaji Earthquake, the incidence is far lower than that of the Fukui

Table 2 Incidence of fire broken down by cities and wards

\begin{tabular}{l|rcc}
\hline & N of fires & N of family & Incidence of fire \\
\hline Kobe city & 115 & 530063 & 2.17 \\
Higashinada-ku & 20 & 72625 & 2.75 \\
Nada-ku & 18 & 53530 & 3.36 \\
Chuo-ku & 22 & 50146 & 4.39 \\
Hyogo-ku & 14 & 51726 & 2.71 \\
Nagata-ku & 22 & 52308 & 4.21 \\
Suma-ku & 12 & 61664 & 1.95 \\
Tarumi-ku & 5 & 80664 & 0.62 \\
Kita-ku & 1 & 46267 & 0.22 \\
Nishi-ku & 1 & 61133 & 0.16 \\
Ashiya city & 14 & 32186 & 4.35 \\
Nishinomiya city & 31 & 156671 & 1.98 \\
Itami city & 7 & 62556 & 1.12 \\
Kawanishi city & 3 & 43906 & 0.68 \\
Amagasaki city & 7 & 183842 & 0.38 \\
Takatazuka city & 4 & 67357 & 0.59 \\
\hline \multicolumn{1}{c|}{ Total } & 181 & 1076581 & 1.68 \\
\hline
\end{tabular}

Table 3 Incidence of fires in major earthquakes of the past in Japan

\begin{tabular}{|c|c|c|c|c|c|}
\hline $\begin{array}{l}\text { Name of Earthquake in } \\
\text { the past }\end{array}$ & $\begin{array}{l}\text { Kanto } \\
\mathrm{EQ} \text {. }\end{array}$ & $\begin{array}{l}\text { Fukui } \\
\mathrm{EQ} \text {. }\end{array}$ & $\begin{array}{l}\text { Niigata } \\
\mathrm{EQ} \text {. }\end{array}$ & $\begin{array}{l}\text { Tokachi-oki } \\
\text { EQ. }\end{array}$ & $\begin{array}{l}\text { i Miyagi-oki } \\
\text { EQ. }\end{array}$ \\
\hline $\begin{array}{l}\text { year } \\
\text { date }\end{array}$ & $\begin{array}{r}1923 \\
\text { Sep. } 1\end{array}$ & $\begin{array}{r}1948 \\
\text { Jun.28 }\end{array}$ & $\begin{array}{r}1964 \\
\text { Jun.16 }\end{array}$ & $\begin{array}{r}1968 \\
\text { May.16 }\end{array}$ & $\begin{array}{r}1978 \\
\text { Jun.12 }\end{array}$ \\
\hline $\begin{array}{l}\text { season } \\
\text { time }\end{array}$ & $\begin{array}{l}\text { summer } \\
\text { noon } \\
11: 58\end{array}$ & $\begin{array}{c}\text { summer } \\
\text { evening } \\
16: 13\end{array}$ & $\begin{array}{l}\text { summer } \\
\text { afternoon } \\
13: 01\end{array}$ & $\begin{array}{cc}\text { spring } & \mathrm{s} \\
\text { morning } & \mathrm{e} \\
9: 49 & 1\end{array}$ & $\begin{array}{l}\text { summer } \\
\text { evening } \\
17: 14\end{array}$ \\
\hline Japanese seismic scale & 6 & 7 & 5 & 5 & 5 \\
\hline $\begin{array}{l}\text { Incidence of fire per } \\
10,000 \text { family }\end{array}$ & 2.02 & 15.5 & 1.26 & 1.78 & 0.373 \\
\hline $\begin{array}{l}\text { Incidence of civil } \\
\text { fire-fighting }\end{array}$ & $41.4 \%$ & $0.0 \%$ & $44.4 \%$ & $57.1 \%$ & $50.0 \%$ \\
\hline
\end{tabular}


Table 4 Causes of fire which could be conjectured

\begin{tabular}{|c|c|c|c|c|c|c|c|}
\hline time elasped after $E Q$ & $\begin{array}{l}\text { hrs } \\
0-1\end{array}$ & $1-6$ & $6-12$ & $12-24$ & $24 \cdot-48$ & $48-$ & Total \\
\hline leak of gas + catching fire & 4 & 3 & 1 & 1 & 1 & 0 & 10 \\
\hline $\begin{array}{l}\text { other reasons with gas appliance } \\
\text { (appliance for boiling water etc.) }\end{array}$ & 4 & 1 & 0 & 0 & 0 & 0 & 5 \\
\hline leak of gas + electrical leak & 4 & 4 & 0 & 1 & 0 & 0 & 9 \\
\hline electrical leak short & 4 & 3 & 0 & 0 & 1 & 2 & 10 \\
\hline $\begin{array}{l}\text { other kind of electric appliance } \\
\text { (electrical stove etc.) }\end{array}$ & 2 & 5 & 0 & 3 & 0 & 2 & 12 \\
\hline $\begin{array}{c}\text { other reasons with gas appliance } \\
\text { A kind of electric }\end{array}$ & 2 & 5 & 0 & 1 & 2 & 0 & 10 \\
\hline kind of kerosene stove & 7 & 0 & 1 & 1 & 0 & 0 & 9 \\
\hline others & 12 & 1 & 1 & 2 & 1 & 2 & 19 \\
\hline$T \circ t a 1$ & 39 & 22 & 3 & 9 & 5 & 6 & 84 \\
\hline
\end{tabular}


Table 5 Causes of fire in past major earthquakes in Japan

\begin{tabular}{|c|c|c|c|c|c|}
\hline Causes of fires & $\begin{array}{l}\text { Kanto } \\
\text { EQ. } \\
(1923)\end{array}$ & $\begin{array}{l}\text { Fukui } \\
\text { EQ. } \\
\text { (1948) }\end{array}$ & $\begin{array}{l}\text { Niigata } \\
\text { EQ. } \\
(1964)\end{array}$ & $\begin{array}{l}\text { Tokachi-oki } \\
\text { EQ. } \\
(1968)\end{array}$ & $\begin{array}{l}\text { Miyagi-oki } \\
\text { EQ. } \\
(1978)\end{array}$ \\
\hline medicine (or gunpowder) & 47 & 11 & 3 & 4 & 7 \\
\hline gas & 15 & - & - & - & 2 \\
\hline $\begin{array}{l}\text { portable cooking stove } \\
\text { by charcoal fire, brazie }\end{array}$ & 181 & 10 & 1 & - & - \\
\hline kitchen, cookroom & 43 & 17 & - & - & - \\
\hline kerosene cooking stove & 3 & - & - & 6 & - \\
\hline kerosene stove & 0 & - & - & 13 & - \\
\hline $\begin{array}{l}\text { others (electric leak, } \\
\text { candle, chacoal fire) }\end{array}$ & 38 & 5 & 5 & 19 & 6 \\
\hline unknown & 18 & - & 3 & 3 & - \\
\hline a leap of the flames & 68 & - & - & - & - \\
\hline Total & $413(345)$ & 48 & 12 & 45 & 15 \\
\hline
\end{tabular}

Note) The numbers of fires given for the Great Kanto Earthquake (1923) are those that occurred in the then Tokyo and Kanagawa Prefectures.

The numbers of fires given for the Miyagi Offshore Earthquake (1978) are those that occurred in Miyagi Prefecture./E 
by kerosene stoves.

After this, the anti-seismic extinguishing device for kerosene stoves became mandatory. Nonetheless, in the Great Hanshin-Awaji Earthquake, 9 fires (10.7\%) broke out because of kerosene stoves.

\section{Causes of Fire Analyzed by Time}

\subsection{Causes of fire starting within one hour of the earthquake}

5.1.1. The number of fires that started due to electrical reasons was 8 in the first hour. This is probably because electricity supply was cut off after the earthquake but returned immediately because of the automatic power restoration system.

5.1.2. Of all the 15 fires directly caused by gas, 8 occurred immediately after the earthquake. This is thought to be because the computerized shut-off devices which are supposed to cut the gas supply did not operate effectively and as a result, gas filled the damaged buildings or rubble, and was ignited.

5.1.3. Out of the 9 fires caused by kerosene stoves, 7 started immediately after the earthquake. These are believed to have started because the anti-seismic devices of kerosene stoves did not work effectively due to the sudden shock of the earthquake or because old types or broken stoves were being used.

5.1.4. Other causes of fire starting immediately after the earthquake include: automobile (4 cases), candle (1), charcoal (\%\%rentan\%\%) (1), cigarette (1), chemicals (3), and propane gas (2), making a total of 12 fires. Fires caused by naked flames created by the burning of charcoal, etc. decreased dramatically in comparison with past earthquakes.

\subsection{Causes of fires starting more than one hour after the earthquake}

5.2.1. Twenty four out of the 32 fires due to electrical causes started between one hour and 3 days after the earthquake. More fires broke out during this time sequence than immediately after the earthquake. The reason for this is thought to be caused by the power restoration system after the electricity was initially cut. It is conjectured that fires started because power supply was resumed, without due consideration given to the state of damage of the buildings in the area or the state of the electrical facilities and equipment remaining the buildings.

5.2.2. Seven out of the 15 fires caused by gas started more than one hour after the earthquake. The reason for this is thought to be the delay in the emergency shut-off of supply. In the interviews, eye-witnesses accounts state that gas flames rose up for several hours after the earthquake.

5.2.3. Other causes of fire include: kerosene stove (2), candle (2), and portable cooking stove (shichirin) (1), making for a total of 5 fires. These are thought to be accidental fires because fire was used to obtain heat and light, in conditions where there was no gas, water, or electricity, by people who were not staying in emergency shelters. 
Besides these, other cases of fire broke out as time passed following the earthquake, including cases of rescue workers using oxyacetylene equipment causing 2 fires, as well as 2 cases of arson.

\section{Summary}

The research on the recent Great Hanshin-Awaji Earthquake was conducted through interviews with residents for the purpose of determining the numbers of fires and causes of fires. However, in many places such as Nagata ward, where the fire was of a massive scale, it was difficult to find people who were actually present at the time the fire started, making it difficult to specify the cause of fire. Work will be continued on determining the causes of these fires.

The facts to be noted are that the fires occurred in many places at once, that there was difficulty in water availability because of burst water mains, that there were delays in firefighting equipment arriving at the site because of traffic chaos, and that there was difficulty in systematic organization because of the confusion in communication networks. Owing to these and other factors, if fires break out at the same time and at many different locations, it becomes impossible for public fire-fighting forces to respond to the situation.

In the case of scale 7 earthquakes, the firefighters have an inordinately difficult situation to contend with. The lesson for the future is that we must plan countermeasures in a step-bystep manner, as in preventing fires from occurring, in preventing fires from spreading, and in protecting the lives of residents. What is most important among these is to establish measures for preventing fires from breaking out in the first place. To prevent fires, we must scientifically reveal every single one of the causes of such fires and find the means to overcome them.

In addition, we need to reexamine the method for the restoration of electricity and the system for the cutting off of the gas supply.

\section{Acknowledgement}

This study is based on valuable research results that could not have been obtained without the cooperation of the many survey volunteers who participated in the interviews which were conducted under difficult circumstances soon after the earthquake disaster. Our most sincere gratitude goes to the disaster victims who responded to our interviews, Professor Murozaki of the Department of Architecture \& Civil Engineering, Faculty of Engineering, Kobe University, who was the author's academic supervisor and who was in overall charge of the field survey, the students of Professor Murozaki's Laboratory of Regional Safety \& Security Planning, the Institute of Regional Environment \& Disaster Prevention Co., Ltd., Kyoto University, Tohoku Art and Technology College, Hosei University, Keiou University, Science University of Tokyo, Nihon University, and many other voluntary helpers. 


\title{
DURABLE AGENTS FOR EXPOSURE PROTECTION IN WILDLAND/URBAN INTERFACE CONFLAGRATIONS
}

\author{
Daniel Madrzykowski \\ Gary L. Roadarmel \\ Laurean A. DeLauter \\ National Institute of Standards and Technology \\ Building and Fire Research Laboratory \\ Gaithersburg, Maryland 20899
}

\begin{abstract}
The objective of this study was to investigate the capability of "durable agents" to protect building exterior materials from ignition. The "durable agents" used in this study included: a protein-based, compressed air foam, and two different water thickeners, also known as gelling agents. Two exterior wall sections forming a corner were pre-treated with a "durable agent" and exposed to a $50 \mathrm{~kW}$ fire for 10 minutes. Flame extension on the exterior siding was recorded during the tests. This study demonstrated that durable agents applied to combustible exterior siding reduce the likelihood of ignition and flame spread.
\end{abstract}

\section{BACKGROUND}

Wildland/urban interface conflagrations, such as the Oakland Hills fire in 1991 which destroyed 2889 homes [1], overwhelm the resources of the fire department. Some homes which were located in the path of the fire were saved, with little or no damage, due to the intervention of the fire department or the homeowners. The homes were saved by applying water to surfaces of the house throughout the time the home was exposed to a fire threat. Unfortunately, this method of protection requires fire fighters or homeowners to remain at the house, placing them in a dangerous situation and in the case of the fire fighters limiting the number of homes that they can protect. During large fire incidents the water supply may be diminished or depleted during the course of the fire.

A promising concept for protecting buildings in the path of an advancing wildland fire is to have the homeowner or fire department cover structures with a water based, compressed air foam or gelling agent prior to evacuating the area. Building and Fire Research Laboratory (BFRL) conducted a small scale study in 1988 on the ignition inhibiting properties of one type of Class A foam. Class A foam is intended for use as a suppression agent on "combustibles such as vegetation, wood, cloth, paper, rubber and many plastics"[2]. Class A foam may also be used for exposure protection. The results of the BFRL study indicated that compressed air, Class A foam exhibited an ignition-inhibiting capability twice that of an equal mass of water for untreated T1-11 textured plywood siding and the compressed air foam had a retention efficiency approximately 20 times that of water on the test samples [3].

The Class A foams are based on synthetic hydrocarbon surfactants which makes them good wetting agents. As a result, Class $\mathrm{A}$ foams tend to drain and break down rapidly relative to 
protein based foams or water thickeners [4]. Therefore the protein based foams and water thickeners are referred to as "durable agents" to distinguish them from the Class A foams.

Experience from previous wildland/urban interface fires [5] shows that the fires which are spread by burning brands start small in areas where the brands can collect, such:as under eaves or in corners. More data are needed to quantify the range of conditions which a wildland/urban interface fire can impose on a structure. However, if the structure can be protected from the thermal insult of a small fire for a short period of time, the chances of the structure igniting during a large fire incident may decrease dramatically.

\section{EXPERIMENTAL APPROACH}

The objective of this study was to investigate the capability of durable agents to protect building exterior materials from ignition. The durable agents used in this study included: a protein-based, compressed air foam and two different water thickeners, also known as gelling agents.

The experimental structure used in this investigation was composed of two $1.2 \mathrm{~m} \times 2.4 \mathrm{~m}$ high ( $4 \mathrm{ft} \times 8 \mathrm{ft}$ ) walls forming a comer, as shown in Figure 1. A partial attic assembly was positioned over the walls. The technique and the materials used in the construction of the corner are representative of construction techniques used in many parts of the United States. The overhang or eave of the structure is important because it traps heat, provides a path for flames into the attic, and allows ability of the agent to cling to the inverted surface to be examined. Each wall was built using $38 \mathrm{~mm} \times 89 \mathrm{~mm}$ (nominally 2 in. $\times 4$ in.) wood structural members. The vertical members were spaced $406 \mathrm{~mm}$ (16 in) on center. The void between the vertical members was filled with $89 \mathrm{~mm}$ ( $3.5 \mathrm{in}$ ) thick blankets of fiberglass insulation. The "interior" wall surface was constructed of $13 \mathrm{~mm}(0.5 \mathrm{in})$ thick sheets of gypsum board. In this study, three different "exterior" siding materials were used: $0.64 \mathrm{~mm}$ ( $0.025 \mathrm{in})$ thick aluminum, $16 \mathrm{~mm}(0.625 \mathrm{in})$ thick T1-11 textured plywood and $1.1 \mathrm{~mm}(0.045 \mathrm{in})$ thick vinyl. The design of the aluminum, and the vinyl sidings simulated $102 \mathrm{~mm}$ (4 in) wide clapboards. The soffit materials were consistent with the siding materials used for a given experiment.

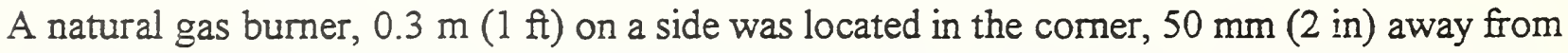
the face of the walls and $0.3(1 \mathrm{ft})$ above the floor. The $50 \mathrm{~mm}(2 \mathrm{in})$ gap between the wall and the burner was needed to reduce the flow of the durable agents onto the burner and to minimize any change in the characteristics of the fire. The gap was filled with ceramic fiber to minimize air entrainment on the wall sides of the burner. The burner was calibrated to provide a steady state $50 \mathrm{~kW}$ fire for the duration of each experiment.

Baseline experiments were performed with each exterior siding material (i.e., no agent application). The experiments were repeated with the durable agents applied to the combustible exterior siding materials. The agents were applied with commercially available equipment to the entire surface of the siding and underside of the eaves. The applications were made at 15 minutes prior to ignition. If the agent was successful in limiting flame spread with a pre-treatment time of 15 minutes, tests were conducted with a pre-treatment time of one hour. The experiments were conducted in a laboratory at temperatures between $21^{\circ} \mathrm{C}$ and $27^{\circ} \mathrm{C}$ and with relative humidities in the range of $45 \%$ to $70 \%$. 
The compressed air foam applications were made with protein-based foam concentrate batch mixed with water in a $3 \%$ solution (i.e., 97 parts water to 3 parts foam concentrate by volume). Although compressed air foam units can produce a wide range of foam expansions, for exposure protection, a stable foam is desired. A stable foam will drain water slowly, be composed of many tiny bubbles, and have the consistency of shaving cream. Foams with low: expansions of 3 to 5 , are wet and unstable. Foams with expansions of 40 to 50, are very stable, but very dry. Foams with expansions between 22 and 30 were used for the fire experiments in this study.

The water thickeners were proportioned at $3 \%$ with an in line eductor in a $38 \mathrm{~mm}$ (1.5 in) hose Two types of nozzles were used during the experiments, a $13 \mathrm{~mm}(0.5 \mathrm{in}$.) smooth bore tip, and a fog nozzle.

\section{EXPERIMENTAL RESULTS AND DISCUSSION}

The results of the baseline experiments (i.e., no agent application) are given in Table 1. The time to initial flame extension and time to flame extension to the eave are the average times from three experiments. Initial flame extension was defined for the purposes of this study as. combustion of the siding material and a measurable change in flame height relative to the flame height from the burner. Flame extension to the eave was recorded when the flames had extended to the top of the wall.

After 10 minutes of exposure to the $50 \mathrm{~kW}$ burner, aluminum siding had melted in the areas exposed to direct flame impingement. A smoldering fire had started on the structural members inside the wall, but there was no flame extension. On average, flames attached to the T1-11 siding and began spreading at approximately 200 seconds after ignition. The flames extended to the underside of the eave approximately 80 seconds later. The fire was limited to surface burning until the flames penetrated the vents in the eave and spread fire into the attic space.

The vinyl siding softened, deformed and charred prior to igniting. Small areas in the center of the flame impingement region charred and opened up which exposed the paperboard substrate within 60 seconds of ignition. Less than 90 seconds after ignition, the flames began to spread upward and within another 50 seconds the flames were into the attic space. A vinyl soffit was used with the vinyl siding. The soffit soften and dropped out leaving an open path for flame spread into the attic area.

The protein-based compressed air foam (CAF) when applied formed a 75 to $150 \mathrm{~mm}$ ( 3 to 6 in.) thick layer on the siding materials. The time to initial flame extension was almost twice as long with the foam applied to the T1-11 siding as compared to the untreated siding. There was no flame spread up to the eave throughout the duration of the test, as shown in Table 2. After the burner was shut off, the flames which had attached to the siding self extinguished. The foam proved even more effective on the vinyl siding only allowing flame extension in one out of the three cases. In that experiment initial flame extension occurred at 555 seconds after ignition and the flames extinguished within a minute after the bumer was shut off.

The water thickener, referred to as Gel A in the Table 2, when applied to the siding left a coating approximately 6 to $12 \mathrm{~mm}(0.25$ to 0.5 in.) thick. Immediately after application the gel began dripping and running down the surface of the siding. At the time of ignition, 15 minutes after application, the thickness of the agent on the siding had been reduced, with the run off pooled 
in front of the walls on the floor. Mass retention was not characterized during these fire tests. The time to initial flame extension with Gel A was almost double the time for the untreated T1-11 siding. In two out of three cases the flame grew and extended to the eave, whereas in the foam cases, the flame never extended to the eave. For the tests with Gel A applied to vinyl siding flame extension to the eave and flame penetration into the attic occurred in all of the tests. There was no noticeable difference in the coating or the performance with the use of different nozzles.

Since the vinyl siding proved to be the most challenging scenario in this study, Gel B was only used with vinyl siding. Gel B when applied to the vinyl siding left a coating of approximately 6 to $12 \mathrm{~mm}(0.25$ to $0.5 \mathrm{in}$.). Gel B appeared to flow down the siding at a slower rate than $\mathrm{Gel}$ $\mathrm{A}$, therefore more agent remained on the siding at the time of ignition. In the three tests performed with a fifteen minute pre-treatment, Gel B prohibited flame extension. Again in the area of flame impingement, the vinyl deformed and charred however the fire never attached to the surface to extend the flame. Tests with a 60 minute pretreat time were also conducted with Gel B. Again no flame extension was observed.

\section{CONCLUSIONS}

This study demonstrates that durable agents applied to combustible exterior siding reduced the likelihood of ignition and flame spread, under laboratory conditions, i.e., no wind, no exposure to the sun and moderate relative humidity. Not all of these agents performed equally, and differences in equipment requirements, environmental concerns, and usability need to be accounted for in a full evaluation of these agents. Further research needs to be conducted on mass retention and fire testing under more realistic weather conditions.

\section{REFERENCES}

1. Steckler, K.D., Evans, D.D., and Snell, J.E. Preliminary Study of the 1991 Oakland Hills Fire and Its Relevance to Wood-Frame, Multi-Family Building Construction, NISTIR 4724 Nat. Inst. Stand \& Tech. Gaithersburg, MD, Nov. 1991.

2. NFP.A 298, Standard on Fire Fighting Foam Chemicals for Class A Fuels in Rural, Suburban, and Vegetated Areas, 1994 ed. National Fire Protection Association, Quincy, MA. 1995.

3. Madrzykowski, D. Study of the Ignition Inhibiting Properties of Compress Air Foam, NISTIR 88-3880 Nat. Instit. of Stand \& Tech. Gaithersburg, MD, Oct. 1988.

4. Rochna, R.R. Foam on the Range. Fire Chief Magazine, Vol. 38, No. 6, June1994.

5. Tran, H.C., Cohen, J.D., and Chase, R.A. Modeling Ignition of Structures in Wildland/Urban Interface Fires. Proceedings, 1st International Fire and Materials Conference, Arlington, VA Sept 24-25, 1992. 
Table 1. Flame extension times for untreated siding materials

\begin{tabular}{|c|c|c|}
\hline Siding Material & $\begin{array}{c}\text { Time to Initial Flame } \\
\text { Extension } \\
(\mathrm{s})\end{array}$ & $\begin{array}{c}\text { Time of Flame Extension to Eave } \\
(\mathrm{s})\end{array}$ \\
\hline Aluminum & no extension & no extension \\
\hline T1-11 Plywood & 203 & 288 \\
\hline Vinyl & 82 & 130 \\
\hline
\end{tabular}

Table 2. Flame extension times for siding materials treated with "durable agents," 15 minute pretreatment times.

\begin{tabular}{|c|c|c|}
\hline $\begin{array}{c}\text { Siding Material } \\
\text { w/agent }\end{array}$ & $\begin{array}{c}\text { Time to Initial Flame } \\
\text { Extension } \\
\text { (s) }\end{array}$ & $\begin{array}{c}\text { Time of Flame Extension to Eave } \\
\text { (s) }\end{array}$ \\
\hline $\begin{array}{c}\text { T1-11 Plywood } \\
\text { w/CAF.A }\end{array}$ & 392 & no extension to eave \\
\hline $\begin{array}{c}\text { T1-11 Plywood } \\
\text { w/Gel A }\end{array}$ & 387 & no extension to eave \\
\hline $\begin{array}{c}\text { Vinyl } \\
\text { w/CAF A }\end{array}$ & $555+$ & 427 \\
\hline $\begin{array}{c}\text { Vinyl } \\
\text { w/Gel A }\end{array}$ & 255 & no extension \\
\hline $\begin{array}{c}\text { Vinyl } \\
\text { w/Gel B }\end{array}$ & no extension & \\
\hline
\end{tabular}

* Flame extended to the eave in 2 out of 3 experiments.

+ Flame extension was observed in 1 out 3 experiments. 


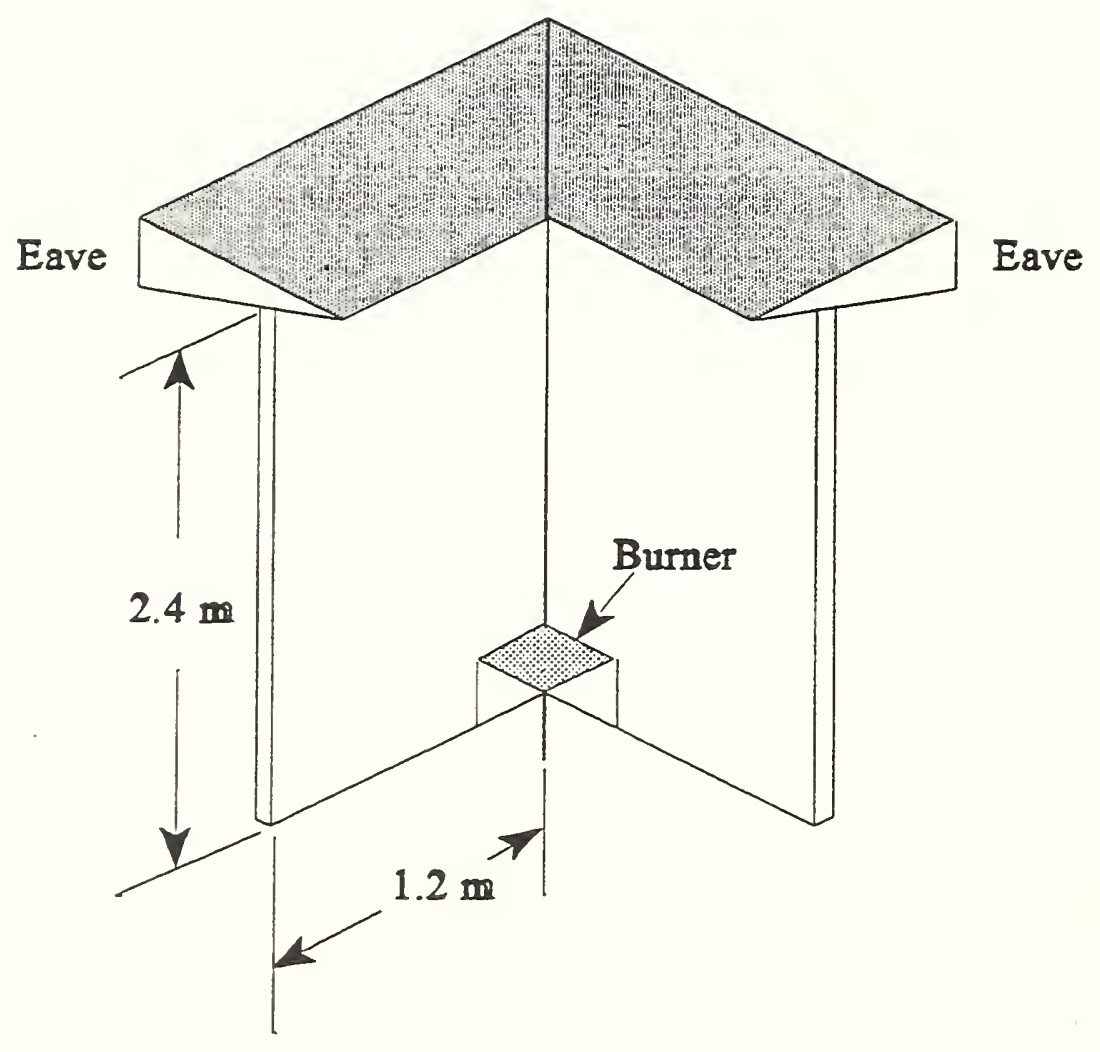

Figure 1 Schematic of Fire Test Arrangement 


\title{
Smoke Plumes from Large Fires
}

\author{
Kevin B. McGrattan, Howard R. Baum and R.G. Rehm \\ National Institute of Standards and Technology \\ Gaithersburg, Maryland 20899 USA
}

\begin{abstract}
A large eddy simulation (LES) model of smoke plumes generated by large outdoor fires is presented. The plume is described in terms of steady-state convective transport by a uniform ambient wind of heated gases and particulate matter introduced into a stably stratified atmosphere by a continuously burning fire. The Navier-Stokes equations in the Boussinesq approximation are solved numerically with a constant eddy viscosity representing dissipation on length scales below the resolution limits of the calculation. The effective Reynolds number is high enough to permit direct simulation of the large scale mixing over two to three orders of magnitude in length scale. Particulate matter, or any non-reacting combustion product, is represented by Lagrangian particles which are advected by the fire-induced flow field. Results of the model are compared with a series of field experiments.
\end{abstract}

\section{INTRODUCTION}

There is growing interest in the environmental consequences of large fires, since the transport of combustion products by a windblown fire plume can distribute potentially hazardous materials over a wide area. Buoyant windblown plumes have been studied since the early 1960's. A summary of the early work together with a useful bibliography is given by Turner (1973). For summaries of more recent work see Turner (1985) and Wilson (1993). Virtually all the models described in these works are integral models, where the profiles of physical quantities in cross-sectional planes perpendicular to the wind direction are assumed, together with simple laws relating entrainment into the plume to macroscopic features used to describe its evolution. A great many of the models in use for air quality assessment simply use Gaussian profiles of pollutant density. Unfortunately, the plume structures actually observed are too complex to be described in terms of a few simple parameters.

Most of the assumptions required by integral models can be removed by taking advantage of the enormous advances in computational fluid dynamics that have occurred since most of these models were developed. This is especially true if it is assumed that the component of the fluid velocity in the direction of the ambient wind is literally the wind speed. The neglect of streamwise perturbations to the ambient wind is an old idea in aerodynamics, where it has been used to study aircraft wake dynamics since the 1930's (Batchelor, 1967). Once this approximation is made, the plume (or wake) can be studied as a two-dimensional time dependent entity. The large scale structure of the plume can then be determined in detail at moderate computational cost. The small scale "sub-grid" mixing and dissipation is represented with a constant eddy viscosity. This permits the mathematical structure of the Navier-Stokes equations to be retained. The effective Reynolds number, defined by the buoyancy induced velocity, plume height and eddy viscosity, is chosen to be above $10^{4}$. Thus, at least two orders of magnitude of dynamically active length scales in all coordinate directions are permitted, leaving open the possibility of comparison, with laboratory scale 
experiments (where the Reynolds numbers are low enough to be simulated directly). It is in this sense that the model described here is a large eddy simulation.

This approach was first used to study the settling of a smoke plume in an unstratified atmosphere by Ghoniem et al. (1993). That study was performed using Lagrangian vortex dynamics techniques. More recently, Zhang and Ghoniem have studied plume rise in a linearly stratified atmosphere using this methodology (Zhang, 1994). In these studies the main emphasis was on the mixing process as it affected the plume structure. The present work uses finite-difference methods to determine the large scale mixing combined with Lagrangian transport of the smoke or other pollutants. The effect of sub-grid scale velocity fluctuations on the dispersion of the smoke is accounted for explicitly, and the ambient temperature profile is subject only to the constraint that it is stable over the altitudes occupied by the plume.

\section{MATHEMATICAL MODEL}

The plume is described in terms of steady-state convective transport by a uniform ambient wind of heated gases and particulate matter introduced into a stably stratified atmosphere by a continuously burning fire. As described above, the most important assumption is that the component of the velocity in the direction of the wind is the ambient wind and thus is known. Since the firebed itself is not the object under study, only the overall heat release rate and the fraction of the fuel converted to particulate matter need be specified. The simulation begins several fire diameters downwind of the fire, where the plume is characterized by relatively small temperature perturbations, and minimal radiation effects. In this region the plume gases ascend to an altitude of neutral buoyancy, and then gradually disperse. The trajectory of the plume is governed by the ambient wind, the atmospheric stratification and the buoyancy induced convection. As it is not our objective to calculate the local meteorology, it is assumed that the ambient temperature profile as a function of height is available. The problem can now be reduced to the study of an equivalent two-dimensional unsteady problem in the cross-wind plane moving downstream at the ambient wind speed.

Given these assumptions, the mathematical model of a smoke plume consists of the conservation equations of mass, momentum and energy which govern the temperature $T$, pressure $p$, density $\rho$, and crosswind velocity components $(v, w)$ in a plane $(y, z)$ normal to the direction $(x)$ of the uniform ambient wind. It is convenient to divide the temperature and pressure fields into mean background values $T_{0}(z)$ and $p_{0}(z)$ plus perturbations induced by the fire, $\tilde{T}$ and $\tilde{p}$. Similarly, the density $\rho$ is decomposed into an ambient density $\rho_{0}$ and a small thermally induced perturbation $\tilde{\rho}$, which is related to the temperature perturbation through the equation of state taken in the small disturbance, low Mach number form appropriate to this problem

$$
\frac{\rho-\rho_{0}}{\rho_{0}}=-\frac{T-T_{0}}{T_{0}}
$$

The ambient density is related to the background pressure through the hydrostatic balance

$$
\frac{d p_{0}}{d z}=-\rho_{0} g
$$

Assuming that the perturbations to the background temperature and density are small beyond a few diameters of the fire downwind of the firebed, we can write the conservation 
equations describing the steady-state plume in the Boussinesq approximation as follows

Conservation of mass

$$
\frac{\partial v}{\partial y}+\frac{\partial w}{\partial z}=0
$$

Conservation of lateral and vertical momentum

$$
\begin{gathered}
\rho_{0}\left(U \frac{\partial v}{\partial x}+v \frac{\partial v}{\partial y}+w \frac{\partial v}{\partial z}\right)+\frac{\partial \tilde{p}}{\partial y}=\mu\left(\frac{\partial^{2} v}{\partial y^{2}}+\frac{\partial^{2} v}{\partial z^{2}}\right) \\
\rho_{0}\left(U \frac{\partial w}{\partial x}+v \frac{\partial w}{\partial y}+w \frac{\partial w}{\partial z}\right)+\frac{\partial \tilde{p}}{\partial z}+\tilde{\rho} g=\mu\left(\frac{\partial^{2} w}{\partial y^{2}}+\frac{\partial^{2} w}{\partial z^{2}}\right)
\end{gathered}
$$

Conservation of energy

$$
\rho_{0} c_{p}\left(U \frac{\partial \tilde{T}}{\partial x}+v \frac{\partial \tilde{T}}{\partial y}+w \frac{\partial \tilde{T}}{\partial z}\right)-\left(\frac{d p_{0}}{d z}-\rho_{0} c_{p} \frac{d T_{0}}{d z}\right) w=k\left(\frac{\partial^{2} \tilde{T}}{\partial y^{2}}+\frac{\partial^{2} \tilde{T}}{\partial z^{2}}\right)
$$

where $c_{p}$ is the specific heat of air, $k$ the thermal conductivity, and $\mu$ the dynamic viscosity. The viscosity and thermal conductivity are to be regarded as "eddy" coefficients whose primary role is to provide sinks of kinetic and thermal energy that are actually the result of sub-grid scale dissipative processes. This will be discussed in more detail below. The uniform ambient wind speed $U$ is taken to be constant. For mathematical consistency, $U$ is much larger than the buoyancy induced crosswind velocity components, and the rates of change of physical quantities in the windward direction are much slower than those in the crosswind plane. These assumptions are quite realistic several flame lengths downwind of the firebed. Since $U$ does not change, there is no need for a windward component of the momentum equations. The details of the firebed are not being simulated, so the only information about the fire required is the overall convective heat release rate $Q$ and the particulate mass flux. The initial temperature distribution in the plume cross section is assumed to be Gaussian and satisfy the following integral

$$
\int_{-\infty}^{\infty} \int_{0}^{\infty} \rho_{0} c_{p} U \tilde{T} d z d y=Q
$$

The particulate matter (or any non-reacting combustion product) is tracked through the use of Lagrangian particles which are advected with the overall flow. The initial particulate distribution mimics the initial temperature distribution. If either more detailed experimental data or the results of a local simulation of the fire bed dynamics is available, then these could be used in lieu of the Gaussian profile.

Details of the numerical methodology used to solve the above equations are given in Baum (1994). Briefly, the steady-state, three-dimensional equations are written as a twodimensional initial value problem where the windward spatial coordinate $x$ is replaced by a pseudo-temporal coordinate. Figure 1 shows the results of a sample computation, illustrating the position of the initial slice and the extent of the computational domain. The plume is visualized by interpolating the particle locations onto the computational grid, and then 


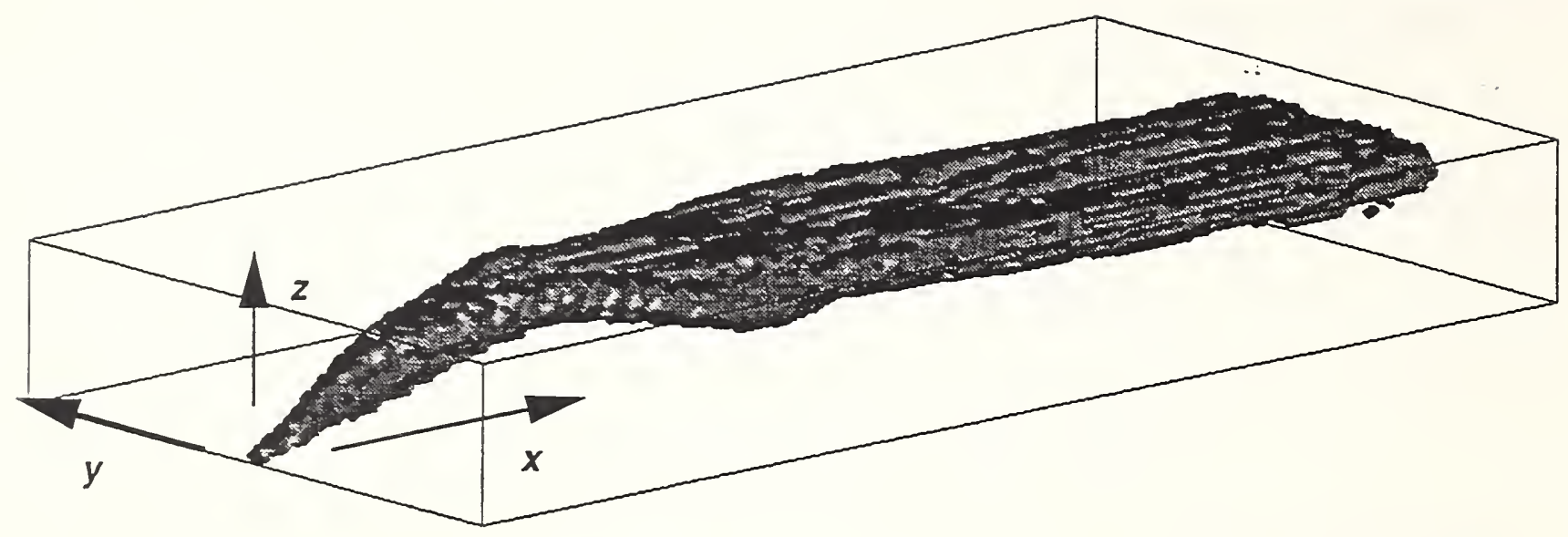

Figure 1: Results of a sample calculation showing the visible extent of the smoke particulate from a large fire. The length of the viewbox is 8 kilometers, the height is $1 \mathrm{~km}$, and the lateral extent $4 \mathrm{~km}$. The wind speed for this example is about $6 \mathrm{~m} \mathrm{~s}^{-1}$, the stratification height is roughly half of a kilometer, and the heat release rate for the fire is about 200 megawatts (MW).

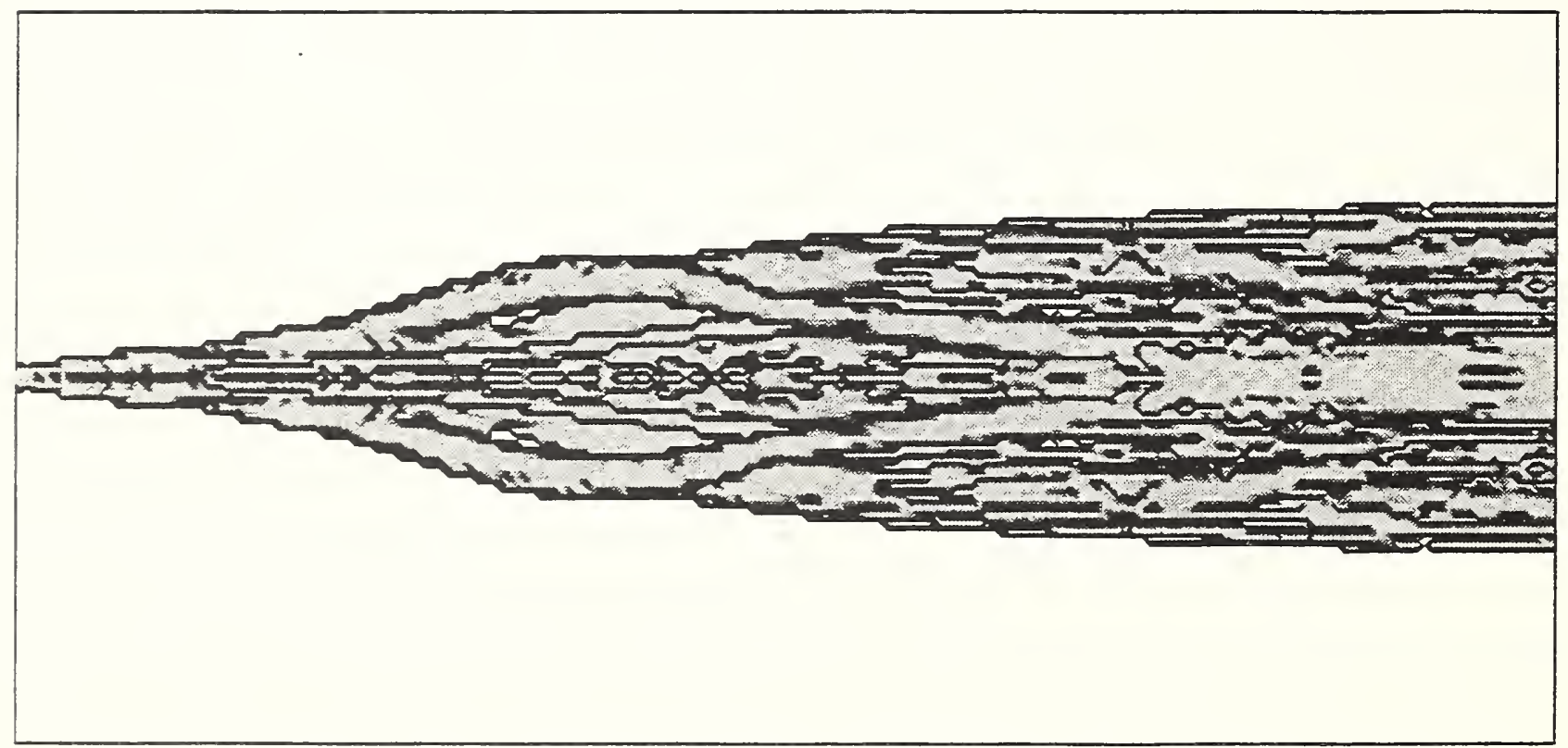

Figure 2: The plume shown in Fig. 1, shown from below. Note the two counterrotating vortices that entrain most of the fresh air into the smoke plume. 


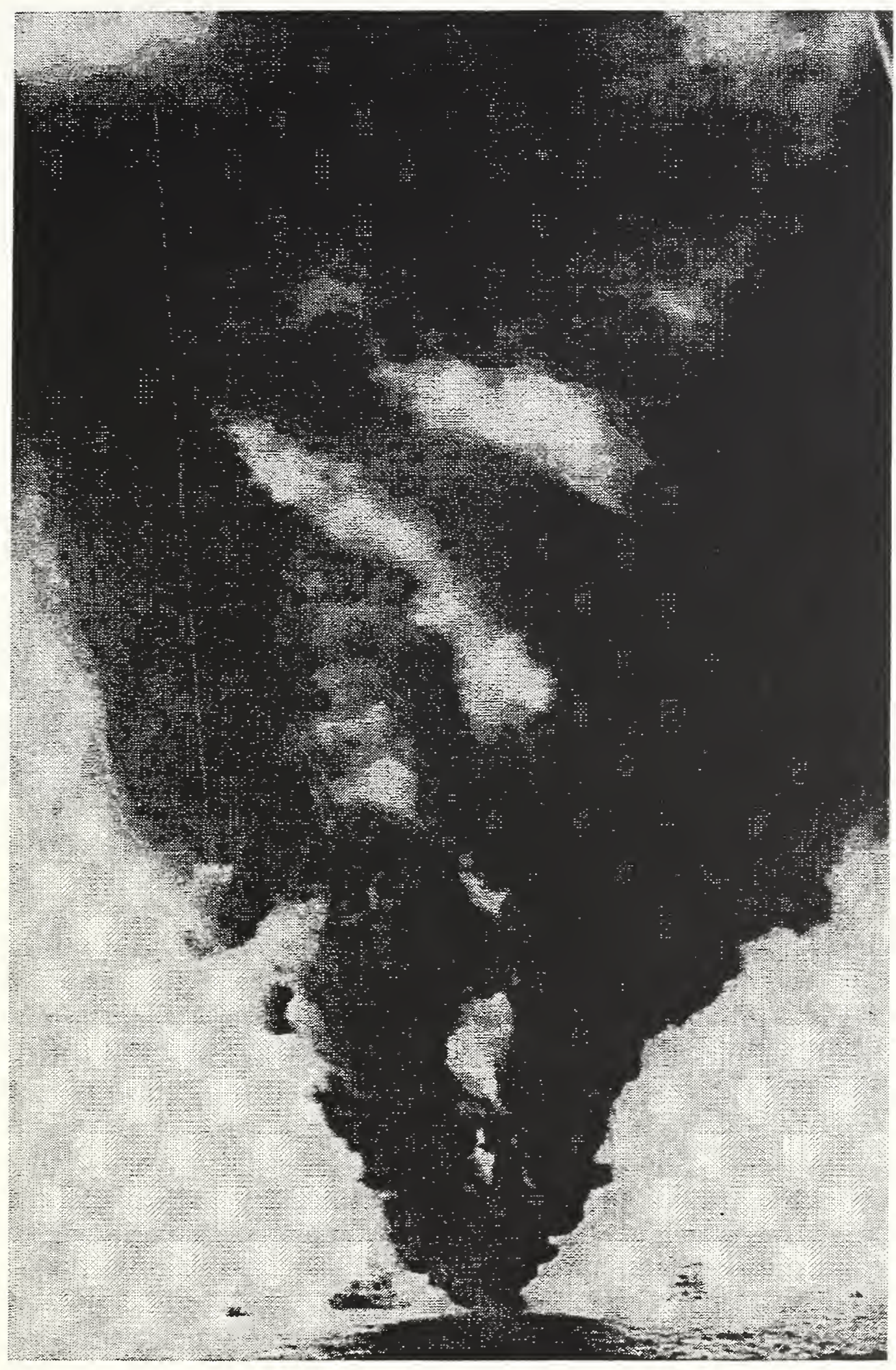

Figure 3: Photograph taken from about $200 \mathrm{~m}$ downwind of the Newfoundland Offshore Burn Experiment (NOBE) showing the two large counter-rotating vortices which characterize the structure of the rising smoke plume. 


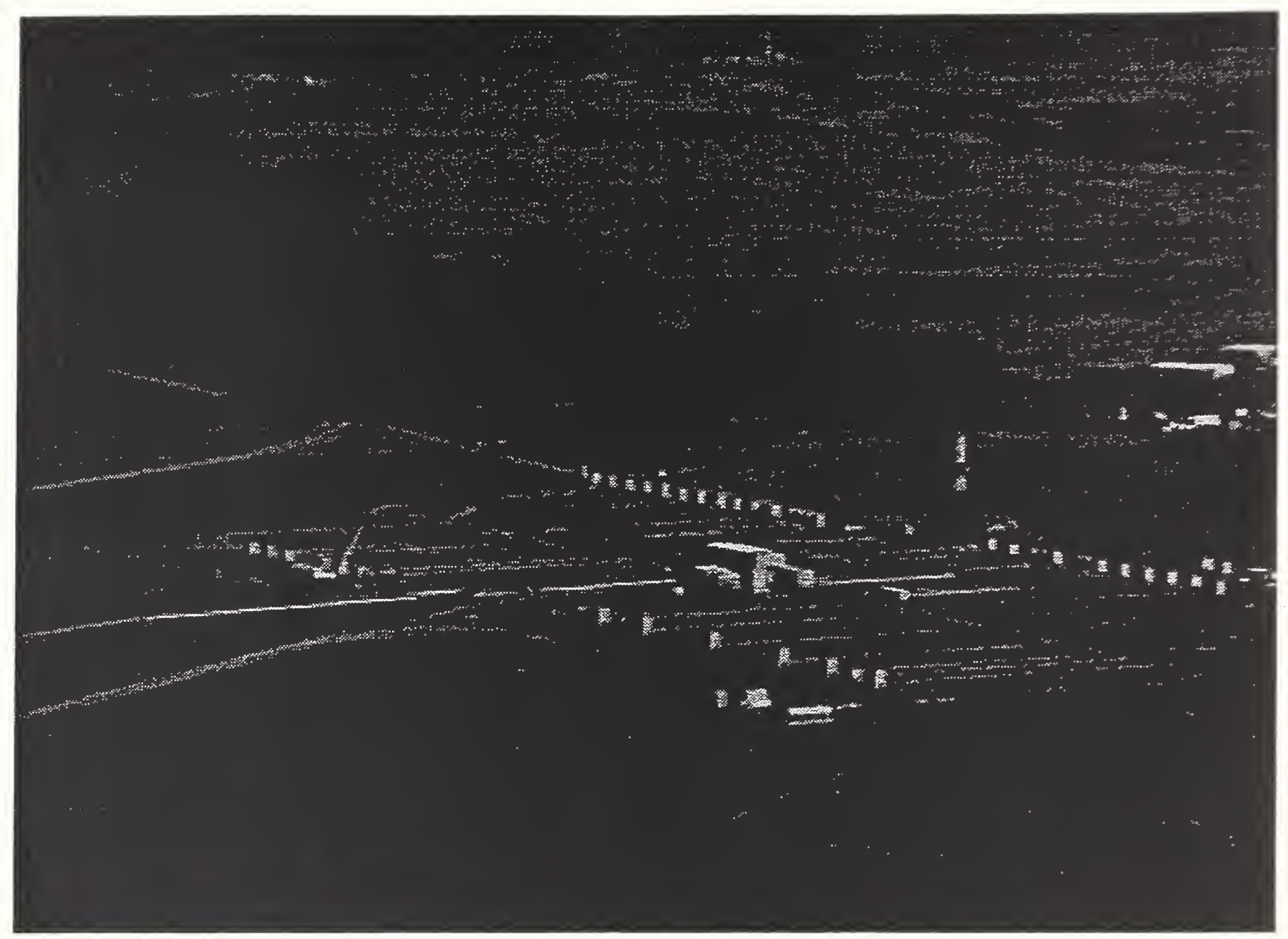

Figure 4: Aerial view of second Alaska Clean Seas emulsion burn experiment, Prudhoe Bay, Alaska, September 1994.

plotting the isosurface on which the particulate density is a chosen value. An interesting view of the plume shown in Fig. 1 from underneath is shown in Fig. 2. From this perspective, one can observe the two large counter-rotating vortices which entrain much of the fresh air; mixing with and cooling the combustion gases. These vortices are readily observed in actual large scale experiments (See Fig. 3).

\section{RESULTS}

In early September 1994, Alaska Clean Seas conducted at their Fire Training Ground in Prudhoe Bay, Alaska, three mesoscale burns to determine the feasibility of burning emulsified oil. An aerial photograph of one of the burns is shown in Fig. 4. Each burn consisted of burning an oil mixture within the confines of a fire-resistant circular boom which floated in a pit filled with water. The boom diameter was roughly $9 \mathrm{~m}$, and the rectangular pit was roughly $20 \mathrm{~m}$ by $30 \mathrm{~m}$. The first and third burns consumed emulsions of salt water and $17.4 \%$ evaporated Alaskan North Slope (ANS) crude. Emulsion breakers were applied to these mixtures. The second burn consumed fresh ANS crude. Heat release rates for the three burns were estimated to be 55,186 and $98 \mathrm{MW}$, respectively. The mass flux of 
particulate was based on a smoke yield for ANS crude of $11.6 \%$.

At the request of the Alaska office of the US Environmental Protection Agency, the EPA's Emergency Response Team (EPA/ERT) came to Prudhoe Bay with 12 MIE real-time aerosol monitors (RAM-1). These instruments employ a sensing principle that is based on the detection of near-forward electromagnetic radiation in the near infrared. The amount of scattered radiation detected quantifies particulate and aerosol concentrations. The twelve instruments were set out on tripods, spread out in rows of three or four, at distances ranging from 1 to $5 \mathrm{~km}$ from the burn site. The deployment strategy varied from burn to burn, depending on the weather conditions and the terrain over which the plume was expected to loft. The instruments were set to sample every second, and then log the 5 second average. Global positioning instruments recorded the locations of the individual devices. Atmospheric temperatures, wind speeds and wind directions were measured with a weather station suspended from a small tethered blimp, deployed just after the burns were completed.

Figure 5 summarizes the results of the experiments, showing the model prediction of ground level particulate concentration versus the actual measurements made in the field. The field measurements were averaged over the time of the burn. Neither the model predictions nor the RAM data were uniform in space or in time, due in part to random fluctuations in wind direction, convective cells which are not accounted for in the model, small terrain effects, and unsteady burning of the fuel. Nevertheless, the agreement between the time-averaged model predictions and field measurements are quite good, showing particulate concentrations ranging from 0 to $80 \mu \mathrm{g} \mathrm{m}^{-3}$ along the narrow path over which the plume is lofted. In addition to ground level instruments, a small airplane was hired to fly in the vicinity of the plume and record plume positions at various times, as well as to photograph the burn site and the plume. According to flight track data, the plume from the first burn rose to a height of about $550 \mathrm{~m}$ and the plume from the second burn rose to about $400 \mathrm{~m}$. These measurements are in very good agreement with model predictions, based on atmospheric profiles obtained with a helium blimp and a helicopter. The visibility on the day of the third burn was very limited, and all aircraft were grounded.

\section{CONCLUSION}

The model of plume dispersion presented above is best described as a large eddy simulation valid in an intermediate region downstream from the fire. This region begins a few tens of meters from the fire and extends a few tens of kilometers downwind. Nearer to the fire the temperatures are high, the radiation field intense, and the Boussinesq approximation is not valid. Also, the approximations which allow replacement of a three-dimensional steady plume by a two-dimensional time dependent one are not valid near the fire because the buoyant velocity of the plume is comparable to the ambient wind speed. Further than a few tens of kilometers downwind of the fire, larger scale meteorological effects begin to dominate, and flat terrain, steady wind assumptions break down. However, within the limits of applicability, the present model offers a high resolution representation of a smoke plume that is consistent with both the current understanding of plume dynamics and with experimental data. Moreover, the computations reported here are well within the range of current generation workstations (where in fact they have been performed). It is hoped that future work will improve the current capabilities of the model. 

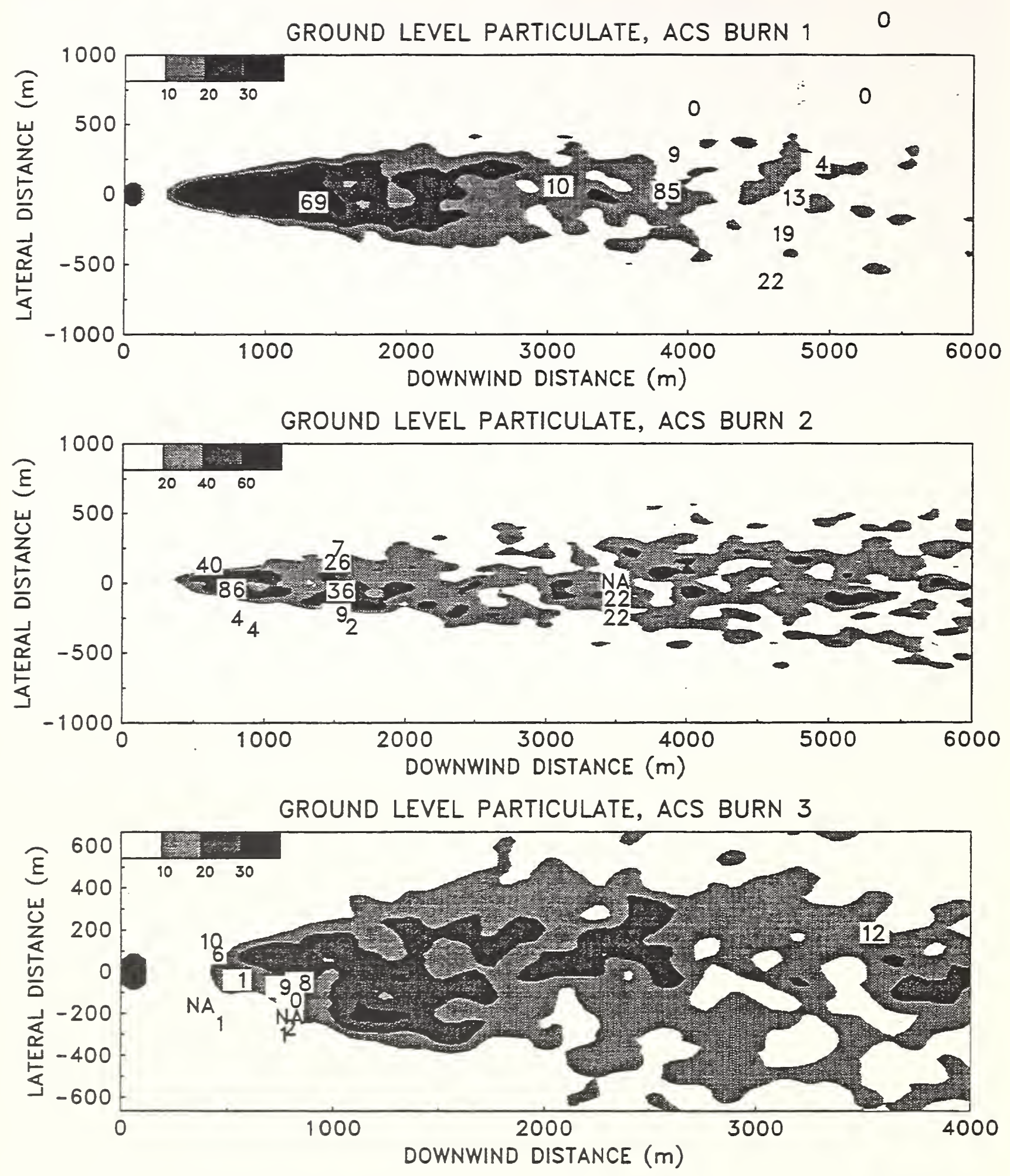

Figure 5: Predicted ground level particulate concentrations from the LES model (shaded contours) along with actual time-averaged RAM data for the three ACS Emulsion Burns (numerical labels). All concentrations are given in units of $\mu \mathrm{g}$ $\mathrm{m}^{-3}$. 
The comparisons between the field experiments and the predictions of the LES model show good agreement given the uncertainty of the input parameters. This favorable comparison suggests that the uncertainty of the model prediction is commensurate with the uncertainty of the input parameters, and that the limitations resulting from the physical assumptions of the model are outweighed by the uncertainty of the input parameters.

Acknowledgement - This work was funded by the Technology Assessment and Research Program for Offshore Minerals Operations, Minerals Management Service, US Department of the Interior. Additional funding was provided by the Alaska Department of Environmental Conservation (ADEC).

\section{REFERENCES}

Batchelor, G.K. (1967) An Introduction to Fluid Dynamics, pp. 580-593, Cambridge University Press, Cambridge.

Baum, H.R., McGrattan, K.B. and Rehm, R.G. (1994) Simulation of Smoke Plumes from Large Pool Fires, Twenty-fifth Symposium (International) on Combustion, The Combustion Institute, Pittsburgh, Pennsylvania, pp. 1463-1469.

Ghoniem, A.F., Zhang, X., Knio, O.M., Baum, H.H., and Rehm, R.G. (1993) Journal of Hazardous Materials 33, 275-293.

McGrattan, K.B., Putorti, A.D., Twilley, W.H., and Evans, D.D. (1993) Smoke Plume Trajectory from In Situ burning of Crude Oil in Alaska, NISTIR 5273, National Institute of Standards and Technology, Gaithersburg, Maryland, 20899.

McGrattan, K,B., Rehm, R.G. and Baum, H.R. (1994) Fire-Driven Flows in Enclosures, J. Comput. Phys. 110, 285-291.

Turner, J.S. (1973) Buoyancy Effects in Fluids, pp. 165-206, Cambridge University Press, Cambridge.

Turner, J.S. (1985) Atmospheric Environment 19, 1215-1218.

Wilson, R.B. (1993) Atmospheric Environment, 27B, 41-57.

Zhang, X. and Ghoniem, A.F. (1994) A computational model for the rise and dispersion of wind-blown, buoyancy driven plumes II - Linearly stratified Atmospheres, Atmospheric Environment, 28, 3005-3018. 


\section{Discussion}

Yoshihiko Hayashi: Thank you. In connection with the very last slide, you said that you have conducted rather extensive calculations with regard to mesh intervals. I'm sure there is some limitation in terms of the capacity of the calculated errors and so forth. But how did you go about it in terms of measuring the first mesh intervals of the smoke plume?

Kevin McGrattan: That's a good question. Without getting into too much detail that people may not appreciate: If you look closely at this slide, you can see the mesh buried underneath the terrain. The spatial resolution of this calculation is on the order of 100-150 meters. The reason why we chose this resolution is that for Alaska, the terrain data that is available via the United States Geological Survey is roughly on the order of 200 meter resolution. So there is no point in doing a more finely resolved calculation if we don't know the terrain better to 200 meters.

Yoshihiko Hayashi: In this calculation, you took into consideration of the complexity of the terrain of various areas. Is it common practice that when you make this kind of calculation, terrain factors are always taken into consideration?

Kevin McGrattan: No. In fact, most atmospheric dispersion model available today are based on much simpler principles. The current models which are known as Gaussian models, maintained by the Environmental Protection Agency, are basically empirical correlations based on the notion that plumes spread in a Gaussian factor. These models, because they are very simplistic, do not usually account for terrain. So much of what we are doing here is relatively new because of the fact that the computers that we have available to us are powerful enough to resolve the plume at resolutions that we think are important.

Hiroshi Koseki: First of all, I would like to express my appreciation that I had a very precious opportunity of participating in some of the research activities in this area, as Mr. Mulholland mentioned in his presentation two days ago. I understand that you have been examining the spread of smoke plumes produced by various fires. And I also understand that those smoke plumes tend to have very extensive distribution. When you examine the movement of smoke plumes, do you also look at the different sizes of the smoke or the magnitude of smoke?

Kevin McGrattan: Yes we do. In fact, a lot of the measurements that Doug Walton has made and that you have made, involve the distribution of particulate size from these fires. We generally assume, based on measurements made with cascade impactors, that the smoke particles are on the order of ten micrometers or less. This is what in this country is call PM10 particulate, and this is the form of the particulate that gets into your lungs and is most.harmful. Because the particulate is relatively small, its deposition is not important in relation to the overall convective motion of the air. In other words, the time scales that we're looking at show very little deposition of the PM10 particulate. So to answer your question very simply, once we know the smoke yield, we assume that that smoke is all being convected with the plume, and the calculation does not discern the various sizes of the particles. As long as we're assured that it's roughly on the order of ten microns or less, we treat it as a passive scalar. I've mainly talked about smoke because for these crude oil fires, it is the smoke that is considered most harmful. However, there's nothing that prevents us from considering any other passive quantity produced by the fire. These calculations will work equally well for benzene, $\mathrm{SO}_{2}$, or whatever else you could think of. 


\section{DETECTION}


3 6 는 


\title{
PROGRESS REPORT ON FIRE DETECTION RESEARCH IN THE UNITED STATES
}

\author{
William L. Grosshandler \\ Building and Fire Research Laboratory \\ National Institute of Standards and Technology \\ Technology Administration \\ U.S. Department of Commerce \\ Gaithersburg, MD 20899
}

\section{Introduction}

Opportunities exist for new concepts in automatic fire detection that do a better job of discriminating a threatening fire from a non-threatening condition across the spectrum of applications. These opportunities arise because of a number of factors, including an increased need to protect more complex and variable structures, the need to replace an aging generation of smoke detectors, the need for detection systems to respond more quickly in tandem with less efficient halon replacement fire suppression systems, and the desire to better safeguard the public and meet evolving regulations. A technological push is being provided by new sensor technologies, by more sophisticated signal processing software, and by a greater understanding of fire physics and improved prediction capability for smoke movement. Competition from new technologies introduced internationally provides additional prodding for the U.S. industry to become fully aware of opportunities to enter new market areas and exploit advances in related technologies and scientific disciplines.

An assessment of advanced fire detection technologies, up to the time of the previous UJNR meeting, was made by Grosshandler [1]. That review sets the stage for what has transpired since. The results of much of the recent research from U.S. organizations can be found in the Proceedings of the Fourth International Symposium on Fire Safety Science, and in the proceedings of Automatische Brandentdeckung (AUBE) '95. In the case of the former publication, only seven of the more than 100 papers deal directly with the issue of fire detection. The latter publication is devoted entirely to the topic, but fewer than $10 \%$ of the articles were contributed by U.S. researchers. This relatively small output motivated BFRL to organize a workshop [2] on the subject of fire detector research in hopes of stimulating more U.S. activities.

\section{NIST Workshop}

The objective of the workshop was to identify the needs of users and specifiers of fire detection systems which are not currently being met by the U.S. fire protection industry; to highlight future needs which may result from new developments in the construction, transportation, and manufacturing sectors, or from regulatory changes; to identify generic, technological barriers which may limit the U.S fire protection industry from fully meeting the users' needs; and to develop a research agenda and recommend priorities to enable U.S. industry to overcome these technological barriers. A series of experts from industry, government, certifying organizations and academia were invited to review the various applications for fire detection systems and to discuss recent developments that could impact the future of the industry. The speakers were divided into focused panels of users and specifiers, systems and components manufacturers, regulators and certifiers, and researchers. Small working groups were convened after the panel discussions to identify critical research issues, concentrating on sensors, signal processing, systems integration and regulations.

The ultimate goals of a comprehensive and integrated research program were identified and include a lower ratio of false-positive-to-actual-fire indications, pre-fire warning for protection of high value operations, more fool-proof installation and maintenance methods, component compatibility for 
system upgrade, a wider range of fires detectable, reliable detection of noxious fire precursors, faster and more precise response of fire detection systems customized to particular processes, earlier warning in connection with halon-alternative suppression systems, situation monitoring following automatic suppression, means to evaluate system trade-offs with the advent of performance-based standards, combination gas sensors for fire/environmental monitoring, and the capability for partial integration of fire detection with other building control functions. Technological barriers which might inhibit attainment of these goals and a research plan to enable the barriers to be breached were discussed. The proceedings of the workshop are available from NIST [2].

\section{Fire Signatures}

Measurements of the chemical and physical emissions from small fires are being taken at BFRL to develop a database of standard fire signatures. The intent is to eventually catalogue the major gases, particulates, and electromagnetic radiation emitted very early in a fire as means to develop new fire sensing strategies and certification procedures. In addition, the time varying temperature and velocity fields above these small fires are being probed. The initial study has been limited to smoldering cotton and pyrolyzing wood [3]. Future work will move on to flaming liquids and solids. Additional details are provided in the paper by Grosshandler presented elsewhere in these proceedings.

The Consumer Product Safety Commission and the U.S. Fire Administration sponsored research at BFRL to identify the conditions which exist on and above a residential kitchen cooking surface just prior to ignition of different foods [4]. The objective was to determine the feasibility of installing a sensor (or multiple sensors) on the stove which could interrupt power before ignition occurred, while being mindful of the need not to interrupt normal cooking operations. Temperature, velocity, gas concentration and smoke attenuation were recorded as bacon, soy bean oil, and sugar were heated to flaming conditions. No definitive conclusions have yet been drawn, and the work is continuing.

Milke [5] conducted bench-top and full-scale room experiments to identify major constituents formed early in small fires, and the response of existing detectors to them. He examined both flaming and non-flaming conditions, and also recorded the response of sensors to non-fire stimuli. Included in the study were over a dozen possible environmental sources likely to confound a fire detector. A paper describing this research can be found in these proceedings.

Goedeke and Gross [6] were concerned with sources of false alarms due to non-fire electromagnetic radiation present in aircraft hangars. They measured the spectral radiation from small pools of jet fuel and noted the fluctuation frequencies. Their investigation identified numerous potential sources of ultraviolet and infrared radiation (other than a fire) that, if modulated and in close enough proximity, could fool optical detectors into an alarm mode.

\section{Fire Sensors}

Thermal: There have been a number of different studies on new ways to sense the heat from a fire which could lead to detection systems that are more responsive and discriminatory. Latimer et al. [7] evaluated the ability of a thin-film heat flux gauge to sense a fire. By placing two micro-sensors side by side, each with a different absorptivity, they attempted to separate the radiative and convective contributions of heat flux, and to use this information to increase system selectivity. Sivathanu and Gore [8] proposed to make use of the radiation reflected from the surroundings to detect a fire located out of the direct line of sight of the sensor. Two silicon diode photo-detectors were used, one employing a narrow-band filter at $900 \mathrm{~nm}$ and the other a narrow-band filter at $1000 \mathrm{~nm}$. By ratioing the voltages and analyzing the time varying fluctuations, they are able to measure the approximate temperature of the infrared source and to discriminate an out-of-direct-sight fire from a warm background object. Bakkom et al. [9] placed cards painted with thermochromic liquid crystals close to the ceiling of a half-scale room to demonstrate that a thermal plume indicative of a fire could be located quickly and remotely with a low resolution video camera.

Gas: There is a great deal of research currently underway in the U.S. on new gas sensing 
technologies. In a workshop held at NIST [10], a number of techniques were mentioned that might be adaptable to early fire detection. Conductometric microsensor arrays are particularly attractive because a large number can be etched into a single silicon wafer, with each sensor doped differently. The substrate temperature can be programmed to minimize power consumption, to increase selectivity, or even to bake off surface contaminants [10 and 2, pp. 13-17].

Aerodyne Research, Inc. [11] has recently completed an assessment of a smart infrared fire detection system which focused on $\mathrm{CO}$ emission. By comparing performance trade-offs in optical component size and system sensitivity, they hoped to show that a practical and economical remote $\mathrm{CO}$ detection system was feasible. They concluded that the current state-of-technology is insufficient to render such a device competitive in the marketplace. On the other hand, Advanced Fuel Research [12 and 13] is continuing their efforts to devise a Fourier-transform infrared spectrometer for use in detecting a range of combustion gases and particulates in building fires. They plan to overcome some of the price and operational issues through clever software development and the selection of appropriate but less expensive components.

Smoke: The Consumer Product Safety Commission surveyed home owners with smoke detectors to ascertain the state of detector operability among the general U.S. public [14]. In a second CPSC study [15], interviews were held with fire investigators to determine how well smoke detectors performed in situations where an actual fire had occurred. The remains of the detectors were examined in an attempt to pin-point causes in cases where no detector-initiated alarm was sounded.

Aggarwal and Motevalli [16] measured the response of smoke detectors located on the ceiling of a $1.8 \mathrm{~m}$ high enclosure in an attempt to discriminate the smoke produced among different fuel sources. Scattered light, ionization, and obscuration methods were used to sense the smoke, while wood material, natural fabrics, different plastics, cooking oil and bread were used as fuels. An analysis based upon the Mie theory suggested that the response of the detectors is in fact fuel dependent, but that more precise measurements are necessary to reduce ambiguity.

Hughes Associates, Inc. [17], added a CO sensor to conventional smoke detectors to increase sensitivity and reduce the number of false alarms. Their evaluation program included measuring the response of the combination detector to a range of fire and non-fire sources of gases and particulates. They were encouraged by their ability to establish correlations between $\mathrm{CO}$ levels and ionization response, and to discriminate fire from non-fire events.

Field models have proven useful in guiding the selection of smoke detector sites on ceilings that are not flat. Forney et al. [18] demonstrated (numerically) how beams of various depth and spacing influence the movement of smoke, and the likely consequence of this altered flow on the time required for a smoke detector to alarm. Davis et al. [19] extended this work to include sloped ceilings and other geometrical complexities. Notarianni and Davis [20] examined the impact of high ceiling heights and cross-flows on smoke movement to a detector. They compared predictions of time-to-alarm using a field model to zone models and other less rigorous methods of analysis.

\section{Detection Algorithms}

Advances also have taken place in the U.S. in the development of signal processing and decisionmaking algorithms. Smith [21] examined the principle function of a fire detection system, and suggested that an economical basis for installing multiple sensors must lie in the quantity and quality (i.e., value) of the information provided. Knowledge Industries [22] received an SBIR grant from NIST to explore decision-theoretic methods for minimizing false alarms in residential fire detection. This method had been used successfully in the past for integrating complex, multiple sensory information such as that associated with real-time space vehicle control, and medical condition diagnosis. A belief network relating the levels of measured $\mathrm{CO}, \mathrm{CO}_{2}$, tin oxide sensor voltage, and room temperature to the probability of a fire was constructed, and the functionality of the method was demonstrated. An alternative approach to sensor data fusion for fire detection is currently being conducted by Opto-Knowledge Systems, Inc. [23]

Neural networks were utilized to train gas and smoke sensors in experiments by Milke [5] to 
increase the rate of successfully classifying flaming fires, smoldering fires, and nuisance signals. This work is summarized in the current UJNR proceedings.

An algorithm was developed by Richards et al. [24] to solve an inverse heat transfer problem. The time-varying temperature distribution was known from measurements at several positions within a room, and the location and source of the heat (i.e. the fire) was sought. The model was able to predict within one to three minutes (depending upon the fire size) the location of the fire within one meter, but the heat release rate of the fire could only be predicted within a factor of five.

A computer simulation of photons emanating from a small fire and interacting with walls and participating media is being undertaken by Sivathanu and Gore [8]. The objective of their research is to determine the feasibility of determining explicitly the spectral radiative intensity on a near-infrared temperature sensor. This theoretical work is complimented by experiments described above.

\section{Fire Detection in Aircraft Hangars}

The U.S. Navy sponsored a series of tests to evaluate the performance of heat, smoke and radiation detectors used to protect aircraft hangars. Other branches of the Defense Department, NIST and five U.S. industry groups participated. Measurements of plume temperature, fuel mass loss, and wind speed were taken in actual aircraft hangars fires. Pools of jet fuel, $0.3 \mathrm{~m}$ to $4.6 \mathrm{~m}$ across, released heat at rates estimated to be between $100 \mathrm{~kW}$ and $40 \mathrm{MW}$. In addition to pool size, the researchers varied fuel type, ventilation conditions, and ambient temperature. Of particular interest were the effects of hangar ceiling height $(15 \mathrm{~m}$ and $22 \mathrm{~m}$ ) and geometry (e.g., draft curtains, cross-beams, and slope). Extensive data were compiled and are available in the final report [25]. A summary of the results with key findings regarding detector spacing, threshold fire size and the impact of draft curtains on time to activation are given in the article by Notarianni et al. [26]

\section{Fire-emulator/detector-evaluator}

The concept of a universal fire-emulator/detector-evaluator (FE/DE) was presented by Grosshandler [27]. The emulator portion of the FE/DE would produce a well controlled environment to simulate the atmosphere anywhere in or adjacent to a room that contains a growing fire. The signatures identified from standard fire tests would act as input to a computer simulation of flow within the room. Detector evaluation would be accomplished by inserting the sensing device into the FE/DE, which would be programmed to reproduce the environment at the detector as predicted from the flow model. The detector evaluator will have the capability to assess the performance of multi-sensor and line detectors. Progress on the construction of the FE/DE at NIST is included in another paper by the author compiled in these proceedings.

\section{References}

1. Grosshandler, W.L., "An Assessment of Technologies for Advanced Fire Detection," Heat and Mass Transfer in Fire and Combustion Systems, HTD-vol. 223, pp. 1-10, ASME, December 1992.

2. Grosshandler, W.L., "Proceedings of the 1995 Workshop on Fire Detector Workshop," NISTIR 5700, Nat. Inst. of Stand. and Tech., Gaithersburg, MD, June 1995.

3. Cleary, T., Grosshandler, W., Nyden, M., and Rinkinen, W., "Signatures of Smoldering/Pyrolyzing Fires for Multi-element Detector Evaluation," Interflam '96, Cambridge, England, March 26-28, 1996.

4. Johnsson, E.L., "Study of Technology for Detecting Pre-ignition Conditions of Cooking-related 
Fires Associated with Electric and Gas Ranges and Cooktops," NISTIR 5729, Nat. Inst. of Stand. and Tech., Gaithersburg, MD, October 1995.

5. Milke, J.A., "Application of Neural Networks for Discriminating Fire Detectors, "Proceedings, Automatische Brandentdeckung, AUBE '95, Duisburg, Germany, pp. 212-222, April 1995.

6. Goedeke, A.D., and Gross, H.G., "Characteristics of Optical Fire Detector False Alarm Sources and Qualifications Test Procedures to Improve Immunity," Final Report, U.S. Air Force Civil Engineering Support Agency, Tyndall AFB, Contract No. F08635-91-C-0129, Donmar Limited, Newport Beach, CA, October 8, 1992.

7. Lattimer, B., Terrell, J.P., and Langley, L.W., "Evaluate Heat Flux Microsensor for Automatic Fire Detection," SBIR phase I final report, DoC contract number 50-DKNA-3-00118, Vatell Corporation, Christiansburg, VA, January 1993.

8. Sivathanu, Y.R., and Gore, J.P., "Fire Detection Using Reflected Near Infrared Radiation and Source Temperature Discrimination," U.S. Dept. of Commerce Research Grant No. 60NANB5D0113, Purdue University, Sept. 1, 1995.

9. Bakkom, A.W., Richards, R.F., and Plumb, O.A., "Design of a Prototype Video-based Fire Detection System," Annual Conference on Fire Research: Book of Abstracts, October 17-20, 1994, NISTIR 5499, S. Smith (editor), Nat. Inst. of Stand. and Tech., p.141, September 1994.

10. Semancik, S., and Whetstone, J.R., "NIST Programs and Facilities in Gas Sensing and Related Areas," NIST Workshop on Gas Sensors: Strategies for Future Technologies, NIST Special Publication 865, S. Semancik, ed., December 1994, and references therein.

11. Niple, E., "A Smart IR Fire Detection System," Progress Report \#1\&2, Dept. of Commerce SBIR Contract No. 50-DKNB-5-00144, Aerodyne Research, Inc., Billerican MA, December 1995.

12. Serio, M.A., Bonanno, A.S., Knight, K.S., and Newman, J., "An FT-IR Based System for Fire Detection," Annual Conference on Fire Research, NISTIR 5499, S. Smith, ed., p. 139, September 1994.

13. Serio, M.A., Bonanno, A.S., Knight, K.S., Wojtowicz, M.A., and Solomon, P.R., "Advanced Infrared Systems for Detection of Building Fires," Phase I Final Report, Dept. of Commerce SBIR Contract No. 50-DKNA-4-00096, Advanced Fuel Research, East Hartford CT, February $1,1995$.

14. Smith, C.L., "Smoke Detection Operability Survey Report and Findings," Directorate for Economic Analysis, U.S. Consumer Product Safety Commission, Bethesda, MD, October 1994.

15. Smith, L.E., "Fire Incident Study National Smoke Detector Project," Directorate for Epidemiology, U.S. Consumer Product Safety Commission, Bethesda, MD, January 1995.

16. Aggarwal, S., and Motevalli, V., "Study of Smoke Generated by non-Flaming Sources for Fuel Identification," Proceedings, Automatische Brandentdeckung, AUBE '95, Duisburg, Germany, pp. 180-192, April 1995. 
17. Gottuk, D.T., Roby, R.J., and Beyler, C.L., "Advanced Fire Detection Based upon Combined Conventional Smoke and CO Sensors," SBIR final report, DoC contract number 50-DKNA-30016, Hughes Associates, Inc., Columbia, MD, February 1994.

18. Forney, G.P., Bukowski, R.W., and Davis, W.D., International Fire Detection Research Project - Field Modeling: Effects of Flat Beamed Ceilings on Detector and Sprinkler Response, Technical Report Year 1, National Fire Protection Research Foundation, Quincy, MA, October 1993.

19. Davis, W.D., Forney, G.P., and Bukowski, R.W., "Developing Detector Siting Rules for Computational Experiments in Spaces with Complex Geometries," Proceedings, Automatische Brandentdeckung, AUBE '95, Duisburg, Germany, pp. 419-428, April 1995.

20. Notarianni, K., and Davis, W., The Use of Computer Models to Predict Temperature and Smoke Movement in High Bay Spaces, NISTIR 5304, December 1993.

21. Smith, R.L., "Performance Parameters of Fire Detection Systems," NISTIR 5439, Nat. Inst. of Stand. and Tech., Gaithersburg MD, June 1994.

22. Shwe, M., Peot, M., and Provan, G., "Decision-theoretic Control of Centralized Fire Alarm Systems," SBIR phase I final report, NOAA contract number 50-DKNA-4-00109, Knowledge Industries, Palo Alto, CA, January 1995.

23. Gat, N., "Sensor Data Fusion for an Intelligent Fire Detection and Control System," Progress Report, Dept. of Commerce SBIR Contract No. 50-DKNB-5-00143, Opto-Knowledge Systems, Inc., Manhattan Beach, CA, December 1995.

24. Richards, R.F., Munk, B.N., Plumb, O.A., and Grosshandler, W.L., "Fire Detection and Solution through Inverse Problem Solution," Proceedings, Automatische Brandentdeckung, AUBE '95, Duisburg, Germany, pp. 170-179, April 1995.

25. Notarianni, K.A., Gott, J.E., Davis, W., Lowe, D., and Laramee, S., "Final Report on High Bay Hangar Facilities for Detector Sensitivity and Placement," NIST Special Publication, in press, 1996.

26. Notarianni, K., Gott, J.E., Davis, W., Lowe, D., and Laramee, S., "Analysis of High Bay Hangar Facilities for Detector Sensitivity and Placement," Interflam '96, Cambridge, England, March 26-28, 1996.

27. Grosshandler, W.L., "Towards the Development of a Universal Fire Emulator/Detector Evaluator," Proceedings, Automatische Brandentdeckung, AUBE '95, Duisburg, Germany, pp. 368-380, April 1995. 


\section{Discussion}

James Bold (??): Point of clarification, in the European Wood Fire Test, they have one test for flaming, one for smoldering. How do they control that fire in order to reproducibly produce smoldering without converting to flaming?

William Grosshandler: The smoldering fire is identical to the one we were testing, and we were very pleased to find that it is highly reproducible based upon their description of the machine.

James Bold: However, I would expect a significant variation from one laboratory to another because they only specify a temperature on the hot plate. So it's possible that there will be variation from laboratory to laboratory. 
$\underline{3} \geq \underline{0}$ 


\title{
Progress Report on Detection Research in Japan
}

\author{
Hiroyuki TAMURA and Daisuke KOUZEKI
}

\author{
Alarm and Communication Section \\ Fire Research Institute \\ Fire Defense Agency \\ 3-14-1 Nakahara, Mitaka-shi, Tokyo 181,Japan
}

Reviewed is progress in the fire detection technology in J apan after the 12th UJNR Meeting in 1992.

1. Study on the fire detection technology with the plural sensor's signal.

Takahashi et al. have developed a system to evaluate the probability of fire based on profile curve of temperature and smoke density.[1,11,23]

Hosokawa et al. have developed a system to distinguish real fire from false fire $w$ ith fuzzyreasoning based on data of temperature, smoke density, $\mathrm{CO}$ concentration and infrared radiation from human-body.[2]

Tamura et al. have proposed a technology to detect fire based on fuzzy expert system.[3]

Ito et al. have reduced the number of false alarms by making neural-network learn s ensors' signal. This system is tuned not to respond to pulse-input.[4]

Hosokawa et al. have applied neural-network technique to tuning the membership function for their fuzzy expert system.[12,21,22]

The SECOM Co., Ltd. has developed the flame detector that works when both the strength of infrared and the strength of ultraviolet are over a definite level. The detector can reduce the number of false alarms without losing its' sensitivity.

Many researches have been made to develop a system which detect real fire using the data of temperature, smoke density, and/or the concentration of $\mathrm{CO}$ gas. Even though u sing these data, it left being difficult to discern smoke from fire and s moke from cigarette, or other source due to human activities. It is expected that development of technology to detect the existence of human within the area watched can solve the issue, if problems of privacy and costs are worked out.

2. Study on the fire detection technology using odor sensor, sound sensor and ray sensor.

Okayama et al. have tried to detect fire in the its' early stage using odor sensor. $[5,6,7,8,14,15,16,17]$

Waki et al. have investigated the burning sound and developed a technology to detect fire by sound. The equipment is set to be activated when value of square integration of sound spectrum $(0.05-3 \mathrm{~Hz})$ from fire is over a threshold.[25]

Takahashi et al. have investigated the frequency and the amplitude of infrared signals from fire, and proposed a system which works only when the light input scatters.[20]

Fujiwara et al. have developed a technology to evaluate the heat release rate of fire using the output signals from a color video camera.[10]

In these researches, a lot of experiments under environmental noise have been conducted, and it is shown that countermeasures for the environmental noise are required in order to fire detector with odor sensor, sound sensor and optical sensor.

3. Study on the fire detection technology toward specific fires. 
Tamura et al. have investigated the behavior of fire detection under the condition of a rooffire of a cultural building thatched with the dry cogongrasses, the Japanese cypress and the like. This study showed that a suitable detector varied with the kind of roof material.[19]

Nakai et al. have applied a piezoelectric $\mathrm{HCl}$ sensor to detecting a PVC electric cable fire. This sensor responds to $\mathrm{HCl}$ gas $(5 \mathrm{ppm})$ in usual surroundings, and it becomes possible to detect a small PVC cable fire using this sensor.[18]

Noda et al. have investigated to detect an electric cable fire in a room which is well ventilated. The sensors used here are highly sensitive smoke sensor, $\mathrm{HCl}$ sensor and a general smoke detector.[24]

Ishii et al. have proposed a system which can detect a fire in the cable tunnel with optical fiber temperature sensor. They also tried to estimate the fire dimension using the sensor outputs.[29]

It is possible to adopt suitable sensors in addition to heat and smoke detector, in order to construct a fire detection system from specific subject. In future, it is anticipated that the application of this type fire detection system will be magnified.

4. Study on fire modeling for the fire detection.

$\mathrm{S}$ atoh has simulated the fire gas flow in air-conditioned rooms with computer-fluid-dynamics model and investigated the effective setting up of fire detectors.[9,13]

\section{REFERENCE}

1) Takahashi, N., Katayama, K., Imagawa, K. and Takizawa, K. :"Distinction of Trends in Sensor Outputs for Thermal and Smoke Sensor Integration and Fusion", (a).

2) Hosokawa, M., Satoh, K., Kouzeki, D., Tamura, H., Kusanagi, S., Kurio, T., Fujioka, T., Watabe, Y., and Nakanishi, S. :"Study of Fuzzy-reasoning Intelligent Fire Detector", (a).

3) Tamura, H., Satoh, K., Kouzeki, D., Hosokawa, M., Kusanagi, S., Fujioka, T., Watabe, Y., Kurio, T. and Nakanishi, S. :"Study on Fuzzy-expert Fire Detection System using Two kinds of Sensors", (a).

4) Ito, M., Ono, T. and Ishii, H. :"Detection of Fire by using Multi-layered Neural Network Addition to Temporal Factor-, (a).

5) Okayama, Y., Nohmi, T. and Fenn, j. :"Odor Analysis during Early Smoldering", (a).

6) Maekawa, M., Okayama, Y. and Nohmi, T. :"Odor Analysis and it's Identification Limit", (a).

7) Tanaka, K., Fujii, K. and Okayama, Y. :"Diffusion Experiment of Odor and Smoke During Early Smoldering", (a).

8) Koide, A., Kobayashi, M. and Okayama, Y. :"Development of Odor Sensor to Detect Fires

in Their Early Stage", (a).

9) Satoh, K. :"Study of Behavior of Fire Gas Flow in Air-conditional Flow", (a).

10) Fujiwara, Y., Ono, T. and Ishii, H. :"Analysis Heat of Fire by Color TV Camera", (b).

11) Takahashi, N., Katayama, K., Imagawa, K. and Takizawa, K. :"Applications of Sensor Fusion to Spot Type Combination Heat and Smoke Detectors", (b).

12) Hosokawa, M. :"Study of Fire Judging Rule Using Fuzzy Reasoning", (b).

13) Satoh, K. :"Study of Fire Detection in Air-conditioned Rooms", (b).

14) Ito, T., Ueno, H. and Okayama, Y. :"System to Detect Abnormal Environment by Odor Sensor and Neural Net", (b).

15) Ueno, H., Yokoo, A., Kobayashi, M. and Okayama, Y. :"Discrimination of Odor in the Early Stage of a Fire by Two Different Odor Sensors", (b). 
16) Maekawa, M. and Nohmi, T. :"Odor Analysis and it's Identification Limit(2)", (b).

17) Maekawa, M. Yamauchi, K. and Nohmi, T. :"Odor Analysis and it's Identification Limit (3)": (b).

18) Nakai, T., Nemoto, M, Watanabe, K., Yuchi, S., Yamano, S. and Yamauchi, Y. :"Detection of PVC Electric Cable Fire by a Piezoelectric HCL(Hydrogen Chloride) Sensor", (c).

19) Tamura, H., Takahashi, S., Yamashita, K. and Aizawa, M."Study on the Fire Protection Method of Cultural Property Buildings which are thatched with the Cogongrasses and others (Part 2 Fire detection)", (c).

20) Takahashi, E. and Yamaguchi, J. :"Fire Detection Method Using Dynamic Spectrum and Amplitude Distribution", (c).

21) Nakanishi, S., Kurio, T., Nomura, J. and Tanaka, O. :"Development of Intelligent Fire Alarm System with Neuro-fuzzy Method", (c).

22) Hosokawa, M. :"Study of Fire Judging Rule Using Fuzzy Reasoning -Generation of Fuzzy Membership Function Using Genetic Algorithm-", (c).

23) Takahashi, N., Katayama, K., S himizu, T. and Nakayama, T. :"A F ire Prediction applied Fuzzy Measures of DS Theory to Analogue Type Detectors", (c).

24) Noda, S., Abe, T. and Ichimura, M. :"An Experimental Study of Early Warning Fire Detection System in Telecommunications Equipment Rooms", (c).

25) Waki, T., Ohta, F. and Nakanishi, M. :"Burning Sounds Frequency Analysis", Report of Fire Science Laboratory (1993).

26) Waki, T., Nakanishi, M. and Sasaki, K. :"Burning Sounds Frequency Analysis (Series 2)", Report of Fire Science Laboratory (1994).

27) Waki, T., Nakanishi, M. and Sasaki, K. :"Burning Sounds Frequency Analysis (Series 3)". Report of Fire Science Laboratory (1995).

28) SECOM Product Catalog "Flame Checker", SECOM Co.,Ltd.

29) Ishii, H., Kawamura, K., Ono, T., Yamamoto, H., Noda, S. and Kikkawa, A :"Fire Detection in a Cable Tunnel by using Optical Fiber Temperature S ensor", National Convention Record of the Institute of Electrical Engineers Japan (1995).

(a) : P roceeding of Annual Meeting of the Japan Association for Fire Science and Engineering, (1993)

(b) : P roceeding of Annual Meeting of the J apan Association for Fire Science and Engineering, (1994)

(c) : Proceeding of Annual Meeting of the Japan Association for Fire Science and Engineering, (1995) 
3 74 


\title{
TEST FIRE SIGNATURES AND THE FIRE-EMULATOR/DETECTOR-EVALUATOR
}

\author{
William L. Grosshandler and Thomas Cleary \\ Building and Fire Research Laboratory \\ National Institute of Standards and Technology \\ Technology Administration \\ U.S. Department of Commerce \\ Gaithersburg, MD 20899
}

\begin{abstract}
Existing methods for evaluating the performance of smoke and thermal detectors are reviewed. The levels of combustion gases, smoke, temperature and velocity likely to be produced in the European standard detector test fires are discussed, and this information is used to establish the operating conditions. for a fire-emulator/detector-evaluator (FE/DE). The first-generation FE/DE being developed by BFRL will handle multi-criteria spot-type detectors over the anticipated range of realistic operating conditions. Measurements in non-flaming test fires (TF 2 and TF 3) are described and example signatures are proposed for training the $\mathrm{FE} / \mathrm{DE}$ to evaluate combination smoke/heat/gas detection systems.
\end{abstract}

\section{Background}

Accepted test methods exist for the current generation of smoke and thermal detectors. The methods can be categorized as either synthetic or actual full-scale simulations. Full-scale simulations involve the placement of the detector in a large room [about $11 \mathrm{~m}$ long by $7 \mathrm{~m}$ wide and 3 to $4 \mathrm{~m}$ high in the Underwriters Laboratory ${ }^{1}$ and the European ${ }^{2}$ tests] and measuring the detector's response to a variety of actual small fires. These include flammable liquids, flaming wood cribs, burning plastics, and smoldering wood, cotton and newspaper. The UL test has the detector located near the ceiling and 5.4 $\mathrm{m}$ from the fire centerline. The CEN 54 test places the detector $3 \mathrm{~m}$ from the centerline of the fire.

The synthetic simulations take place in smaller chambers designed to duplicate either the smoke or heat produced during a fire. One of these, the Underwriters Laboratory smoke box ${ }^{1,3}$, is about 1.7 $\mathrm{m}$ long, $0.5 \mathrm{~m}$ wide and $0.5 \mathrm{~m}$ high. A circulating fan with a straightening section is used to provide a uniform, laminar flow with air speeds between 0.16 and $0.76 \mathrm{~m} / \mathrm{s}$. A second UL chamber $(0.30 \mathrm{~m} \times$ $0.34 \mathrm{~m} \mathrm{x} 0.61 \mathrm{~m}$ ) is used to evaluate the sensitivity of the smoke detector to changes in velocity. In this test, a smoldering cotton wick is placed at different locations below the detector with a counterflow of air in the range of $0.16 \mathrm{~m} / \mathrm{s}$ to $1.52 \mathrm{~m} / \mathrm{s}$.

European standard CEN 54, part $7^{4}$ describes a procedure for synthesizing a controlled smoke environment to test ionization and optical detectors. A paraffin oil aerosol is introduced to a closed circuit wind tunnel with a $0.38 \mathrm{~m}$ square cross-section and a $0.75 \mathrm{~m}$ to $1.75 \mathrm{~m}$ long measuring zone. Air speeds are maintained between $0.1 \mathrm{~m} / \mathrm{s}$ and $1 \mathrm{~m} / \mathrm{s}$, and the aerosol is polydisperse with the dominant area-weighted diameter being between $0.5 \mu \mathrm{m}$ and $1.0 \mu \mathrm{m}$. Different designs exist for aerosol generators (e.g., refs. 5 and 6). Provision is made to test the detector at ambient temperatures, and for temperatures increasing from $20{ }^{\circ} \mathrm{C}$ to $50{ }^{\circ} \mathrm{C}$ at a rate of $1{ }^{\circ} \mathrm{C} / \mathrm{min}$. Air speeds are up to $10 \mathrm{~m} / \mathrm{s}$.

The performance of heat detectors is evaluated according to UL $521^{7}$ and UL $539^{8}$ in synthetic environments consisting of a temperature controlled oven and a $0.8 \mathrm{~m} \mathrm{x} 0.25 \mathrm{~m} \mathrm{x} 0.4 \mathrm{~m}$ chamber with $1.2 \mathrm{~m} / \mathrm{s}$ air flow. Temperature gradients can be set to greater than $7^{\circ} \mathrm{C} / \mathrm{min}$ to test rate-of-rise detectors. Sprinkler-type thermal sensors are also evaluated full-scale, in a large room $18 \mathrm{~m}$ on a side and $4.8 \mathrm{~m}$ high. A burning $1.7 \mathrm{~m}^{2}$ pool of ethanol provides the source of heat.

Open or closed circuit wind tunnels are specified in CEN 54, parts 5, 6, and 8, for testing the 
different types of thermal detectors. ${ }^{9-11}$ The key parameters synthesized are the air velocity $(0.8 \mathrm{~m} / \mathrm{s} \pm$ $\left.0.1 \mathrm{~m} / \mathrm{s} @ 25^{\circ} \mathrm{C}\right)$, the maximum heating rate $\left(30^{\circ} \mathrm{C} / \mathrm{min}\right)$, and the maximum temperature $\left(170{ }^{\circ} \mathrm{C}\right)$. The accuracy on control of temperature measured $0.23 \mathrm{~m}$ upstream of the detector is specified to be within $2{ }^{\circ} \mathrm{C}$.

Test fires such as those used in the UL and CEN standards have only been partially characterized. Pfister ${ }^{12}$ measured the $\mathrm{H}_{2}, \mathrm{CO}_{2}, \mathrm{CO}$ and total hydrocarbon levels at the ceiling above the center of the CEN flaming wood fire (TF 1), the pyrolyzing wood fire (TF 2), the smoldering cotton fire (TF 3), the UL 217 smoldering paper fire, and an overheated transformer. The response of radiation, ionization, light scattering and thermal detectors to these fires was also recorded. Jackson and Robins ${ }^{13}$ sampled the plume gases $3 \mathrm{~m}$ off the centerline near the ceiling in all six CEN test fires. They quantified the temperature, $\mathrm{CO}$ and $\mathrm{H}_{2}$ mole fractions, relative humidity, and oxygen depletion. Smoke levels were monitored with optical and ionization detectors. Cleary and co-workers ${ }^{14,}$ is focused on the pyrolyzing wood fire (TF 2) and the smoldering cotton fire (TF 3). Their measurements are summarized later in this paper.

\section{Attributes of a Fire-emulator/detector-evaluator}

Existing test fixtures emulate a number of important aspects of actual fires, but each is limited to single-element detectors that respond either to smoke or heat. Ideally, detector evaluation should be independent of the detector operating principle, and many characteristics of an early fire should be duplicated simultaneously during the evaluation. The concept for a more universal fire emulator was introduced by Grosshandler ${ }^{16,17}$ for the purpose of providing a well-controlled environment into which fire sensors could be exposed to highly reproducible, time-varying concentrations of combustion products at predetermined temperatures and flow velocities. A properly designed fire-emulator/detector-evaluator (FE/DE) would have distinct advantages over existing detector test methods. These include the following:

- Test-to-test variations would be minimized.

- Single element gas, smoke, and thermal sensors, as well as multi-criteria detectors, could be evaluated under identical conditions.

- Line-type detectors, new combinations of existing sensors, and novel devices operating on new principles all could be accommodated.

- The emission of noxious combustion gases and the precautions necessary for live-fire tests could be minimized.

Table 1 lists the different types of detectors that one may want to evaluate in a FE/DE. Included are point (or spot) detectors that sense heat or the composition of combustion products (with single or multiple sensing elements); detectors that sense the state of the environment along a line (e.g., contiguous temperature sensitive wires) or through an open path (e.g., laser light attenuation across a room); and volume detectors that respond to portions of the electromagnetic or acoustic field (e.g., uv flame detectors). Across the top of the table are different fire conditions that need to be emulated. These include various fuels and ignition sources, smoldering combustion, deflagrations and detonations. A "0" indicates that a particular combination of emulator/detector already exists. For example, methods have been established to evaluate single-element temperature detectors in constant pressure flames (i.e., deflagrations) for burning liquids and solids, or for smoke detectors exposed to smoldering solids.

Number "1" symbolizes a detector/fire scenario that will be emulated in the first generation FE/DE. This encompasses single-element point detectors that sense combustion gases, combination multielement detectors, and line detectors exposed to smoldering and flaming combustion. A second generation emulator (FE/DE II) will be required to properly assess volume-detectors and some line-type detectors. These and their applications are noted with a "2". Version III ("3" in Table 1) is proposed to be aimed at ignition and pre-detonation events, sensed by fast response volume- and line-detectors, and must have the capability to withstand explosions. Some combinations of sensing systems will require 
Table 1. Fire-emulator/detector-evaluator fire applications matrix

\begin{tabular}{|c|c|c|c|c|c|c|c|}
\hline \multirow{2}{*}{ Detector Evaluated } & \multicolumn{3}{|c|}{ Ignition } & \multirow{2}{*}{$\begin{array}{l}\text { Smolder Wave } \\
\text { (heat source or } \\
\text { self-sustained) }\end{array}$} & \multirow{2}{*}{$\begin{array}{c}\text { Deflagration } \\
\text { (gas, liq.; sol.) }\end{array}$} & \multicolumn{2}{|c|}{ Detonation } \\
\hline & spark & hot surf. & flame & & & gas & aerosol \\
\hline \multicolumn{8}{|l|}{ Point single-elem., gas: } \\
\hline $\mathrm{CO}, \mathrm{CO}_{2}, \mathrm{H}_{2} \mathrm{O}, \mathrm{O}_{2}$ & - & - & - & 1 & 1 & 0,3 & - \\
\hline $\mathrm{NO}_{x}, \mathrm{HCs}, \mathrm{CH}_{4}, \mathrm{H}_{2}$ & - & - & - & 1 & 1 & 0,3 & - \\
\hline $\mathrm{HCl}, \mathrm{HF}, \mathrm{HCN}, \mathrm{SO}_{x}$ & - & - & - & 1 & 1 & - & - \\
\hline \multicolumn{8}{|l|}{ Point single-elem., particle: } \\
\hline smoke $<100 \mathrm{~nm}$ & -- & - & - & 0,1 & 0,1 & - & - \\
\hline smoke $>100 \mathrm{~nm}$ & - & - & - & 0,1 & 0,1 & - & - \\
\hline fuel aerosols & -- & -- & - & 1 & - & - & 3 \\
\hline \multicolumn{8}{|l|}{ Point single-elem., therm.: } \\
\hline temperature & - & -- & - & 0,1 & 0,1 & - & - \\
\hline heat flux & - & - & - & 0,1 & 0,1 & - & - \\
\hline Point multi-elem., combi. & - & - & - & 1 & 1 & 3 & 3 \\
\hline \multicolumn{8}{|l|}{ Line-detector, contiguous: } \\
\hline gas & - & - & - & 1,2 & 1,2 & 3 & - \\
\hline temperature & - & - & - & 1,2 & 1,2 & - & - \\
\hline \multicolumn{8}{|l|}{ Line-detector, open-path: } \\
\hline gas & - & - & 3 & 1,2 & 1,2 & - & - \\
\hline aerosol & - & - & 3 & 1,2 & 1,2 & 3 & 3 \\
\hline temperature & - & - & 3 & 1,2 & 1,2 & - & - \\
\hline pressure & 3 & - & 3 & 1,2 & 1,2 & 3 & 3 \\
\hline radiation & 3 & 3 & 3 & 1,2 & 1,2 & 3 & 3 \\
\hline \multicolumn{8}{|l|}{ Volume-detector: } \\
\hline narrow-band uv/vis/ir & $0,2,3$ & $0,2,3$ & $0,2,3$ & 0,2 & 0,2 & 0,3 & 0,3 \\
\hline broadband & $0,2,3$ & $0,2,3$ & $0,2,3$ & 0,2 & 0,2 & 0,3 & 0,3 \\
\hline sonic pressure & 2,3 & - & 2,3 & 2,4 & 2,4 & 0,3 & 0,3 \\
\hline ultra-sonic & - & 4 & - & 4 & 4 & 3 & 3 \\
\hline Combination (pt./line/vol.) & $2,3,4$ & $2,3,4$ & $2,3,4$ & 2,4 & 2,4 & 3,4 & 3,4 \\
\hline
\end{tabular}

Notes: 0 . emulation and/or evaluation procedure already exists

1. version I of $F E / D E$

2. version II of FE/DE (open plume, vol. detectors)
3. version $I I$ of FE/DE (explosion proof, fast response det.)

4. customized evaluation required

- does not apply 
customized testing/certification facilities, as indicated by a "4". No number ("-") in the table signifies that a given detector is inappropriate for a given fire threat (e.g., a point smoke detector is unable to sense a spark quickly enough to warn of a pending detonation event, and a sonic pressure detector is unsuited for sensing a hot surface just prior to ignition).

A similar matrix, shown in Table 2, can be generated for nuisance signals. The detectors are identical to the ones listed in Table 1. Different sources of non-fire signals include the gases, aerosols, and heat which are produced during cooking or from operating combustion engines; natural fogs and dust suspensions; consumer products such as cleaning fluids and solvents (prevaporized and in aerosol form); radiation across the entire electromagnetic spectrum; and mechanical disturbances (e.g., vibrations, dirt, insects, pressure fluctuations). Currently, there are no generally accepted procedures for evaluating the susceptibility of a fire detector to non-fire signals (hence, no "0" entries in Table 2). The boxes marked with a "1" have been targeted for emulation in FE/DE I. The numbers 2, 3 and 4 have the same meaning as in Table 1. Note that stray high energy and stray very long wavelength electromagnetic radiation and mechanical disturbances require custom fixtures (marked "4" in table) and will not be emulated; however, the influence of uv, visible and ir radiation on triggering false alarms in open-path fire detectors could be attempted.

\section{Operating Parameters for FE/DE}

A range of some of the conditions to which a fire detector is likely to be exposed can be gleaned from previous measurements in test fires and from the sensitivity levels specified in the current UL and CEN standards. The velocity, temperature, gas composition, smoke levels, and radiation are discussed in the following paragraphs.

Velocity Field: The normal air velocities in the vicinity of a point detector are small, often below $0.5 \mathrm{~m} / \mathrm{s}$. Directly over a thermal plume the velocity can exceed $2 \mathrm{~m} / \mathrm{s}$, and near a ventilation duct or an open window wind speeds over $5 \mathrm{~m} / \mathrm{s}$ would not be uncommon. The FE/DE should, therefore, be able to control the mean air flow between $0.5 \mathrm{~m} / \mathrm{s}$ and $5 \mathrm{~m} / \mathrm{s}$.

The transport of combustion products into a detector and the response of a line-type sensor may be influenced by the scale and intensity of the turbulence as well as the average free-stream velocity. These parameters are small in near-laminar flows. Turbulence intensities between $3 \%$ and $20 \%$ may be encountered in turbulent buoyant jets and duct flows. The turbulence scale for a given flow is distributed between small, isotropic fluctuations (less than a millimeter) and scales of the order of the size of dominant objects within the structure (tens of centimeters). No data exist on the actual turbulence parameters likely to be encountered by detectors in the field, in either a fire or non-fire situation. Different size wire meshes can be used to generate small scale turbulence; and large eddies can be generated by placing bluff bodies in the free stream within the FE/DE ahead of the detector location.

Temperature Field: From the measurements of Jackson and Robins ${ }^{13}$, one knows the peak temperatures at the detector location in the CEN 54 fires. These vary between $1{ }^{\circ} \mathrm{C}$ above the ambient for the smoldering wood and cotton fires (TF2 and TF3) and $58^{\circ} \mathrm{C}$ above the ambient for TF5, the flaming heptane pool. The maximum rates of temperature change measured were between $0.1^{\circ} \mathrm{C} / \mathrm{min}$ and $30^{\circ} \mathrm{C} / \mathrm{min}$. In order to accommodate the current generation of thermally released sprinkler heads, the ideal FE/DE needs to produce air temperatures up to $170{ }^{\circ} \mathrm{C}$.

Data have not been reported on the temperature fluctuations adjacent to the detector in either the CEN 54 or UL test procedures. For conventional, slow-reacting heat sensors the value of the fluctuation is not important. For miniature heat sensors with a short response time, however, the temperature spectrum could provide useful information. It is unlikely that frequencies faster than $0.5 \mathrm{~Hz}$ will be emulated in the first generation FE/DE.

Gas Composition: The concentrations of some of the gaseous products formed in small test fires have been reported in the literature ${ }^{12,13}$. Maximum deviations from ambient conditions attained at the detector site were in the range of thousands of parts per million by volume of $\mathrm{CO}_{2}, \mathrm{H}_{2} \mathrm{O}$ and $\mathrm{O}_{2}$; hundreds of ppmv for $\mathrm{CO}$; and tens of ppmv for $\mathrm{H}_{2}$. Non-zero values of hydrocarbon species, acid 
Table 2. Fire-emulator/detector-evaluator nuisance applications matrix

\begin{tabular}{|c|c|c|c|c|c|c|c|c|c|c|}
\hline \multirow{2}{*}{ Detector Evaluated } & \multicolumn{3}{|c|}{$\begin{array}{c}\text { Cooking, } \\
\text { Engine Exhaust }\end{array}$} & \multicolumn{2}{|c|}{$\begin{array}{r}\text { Natural } \\
\text { Aerosols } \\
\end{array}$} & \multicolumn{2}{|c|}{$\begin{array}{l}\text { Consumer } \\
\text { Products }\end{array}$} & \multicolumn{2}{|c|}{$\begin{array}{c}\text { Electromagnetic } \\
\text { Radiation }\end{array}$} & \multirow{2}{*}{$\begin{array}{c}\begin{array}{c}\text { Mechanical } \\
\text { Disturb. }\end{array} \\
\text { obstruct., } \\
\text { vib., press. }\end{array}$} \\
\hline & gas & part. & heat & dust & water & gas & part. & $\begin{array}{c}x \text {-ray, } \\
\gamma \text {-ray, RF }\end{array}$ & $\begin{array}{l}\text { uv, ir } \\
\text { vis. }\end{array}$ & \\
\hline \multicolumn{11}{|l|}{ Point single-elem., gas: } \\
\hline $\mathrm{CO}, \mathrm{CO}_{2}, \mathrm{H}_{2} \mathrm{O}, \mathrm{O}_{2}$ & 1 & -- & - & - & 1 & 1 & - & 4 & - & 4 \\
\hline $\mathrm{NO}_{x}, \mathrm{HCs}, \mathrm{CH}_{4}, \mathrm{H}_{2}$ & 1 & -- & - & - & - & 1 & - & 4 & - & 4 \\
\hline $\mathrm{HCl}, \mathrm{HF}, \mathrm{HCN}, \mathrm{SO}_{x}$ & 1 & - & - & - & - & 1 & - & 4 & - & 4 \\
\hline \multicolumn{11}{|l|}{ Point single-elem., part.: } \\
\hline smoke $<100 \mathrm{~nm}$ & - & 1 & - & 1 & 1 & - & 1 & 4 & -- & 4 \\
\hline smoke $>100 \mathrm{~nm}$ & - & 1 & -- & 1 & 1 & -- & 1 & 4 & -- & 4 \\
\hline fuel aerosols & - & 1 & - & 1 & 1 & - & 1 & 4 & -- & 4 \\
\hline \multicolumn{11}{|l|}{ Pt. single-elem., thermal: } \\
\hline temperature & - & -- & 1 & -- & - & - & -- & 4 & - & 4 \\
\hline heat flux & - & - & 1 & - & - & - & - & 4 & - & 4 \\
\hline $\begin{array}{l}\text { Point multi-elem. } \\
\text { combinations }\end{array}$ & 1 & 1 & 1 & 1 & 1 & 1 & 1 & 4 & 4 & 4 \\
\hline \multicolumn{11}{|l|}{ Line-det., contiguous: } \\
\hline gas & 1,2 & -- & - & - & 1,2 & 1,2 & - & 4 & 4 & 4 \\
\hline temperature & - & - & 1,2 & - & - & - & - & 4 & - & 4 \\
\hline \multicolumn{11}{|l|}{ Line-det., open-path: } \\
\hline gas & 1,2 & - & 1,2 & 1,2 & 1,2 & 1,2 & 1,2 & 4 & 1,2 & 4 \\
\hline aerosol & - & 1,2 & - & 1,2 & 1,2 & - & 1,2 & 4 & 1,2 & 4 \\
\hline temperature & - & - & 1,2 & - & - & - & - & 4 & 1,2 & 4 \\
\hline pressure & 1,2 & - & 1,2 & - & - & - & - & 4 & - & 4 \\
\hline radiation & - & - & 1,2 & 1,2 & 1,2 & - & 1,2 & 4 & 1,2 & 4 \\
\hline \multicolumn{11}{|l|}{ Volume-detector: } \\
\hline narrow-band, uv/vis/ir & 2 & 2 & 2 & 2 & 2 & 2 & 2 & 4 & 2 & 4 \\
\hline broadband & 2 & 2 & 2 & 2 & 2 & 2 & 2 & 4 & 2 & 4 \\
\hline sonic pressure & - & - & 2 & - & - & - & - & 4 & - & 4 \\
\hline ultra-sonic & - & - & - & - & - & - & - & 4 & - & 4 \\
\hline $\begin{array}{l}\text { Combination } \\
\text { (pt./line/vol.) }\end{array}$ & 2,4 & 2,4 & 2,4 & 2,4 & 2,4 & 2,4 & 2,4 & 4 & 2,4 & 4 \\
\hline
\end{tabular}

Note: See table 1 for explanation of numbers. 
gases, and NO are undoubtedly present and are just now being quantified ${ }^{14,15}$ in standard fires used to evaluate detector response.

As a minimum, the first generation $\mathrm{FE} / \mathrm{DE}$ should be capable of emulating the build-up of $\mathrm{CO}_{2}$, $\mathrm{CO}$, and $\mathrm{H}_{2} \mathrm{O}$ as measured. The magnitude of the turbulent concentration fluctuations is unknown and would be difficult to simulate. Minor species like $\mathrm{H}_{2}, \mathrm{CH}_{4}$, and higher/oxygenated hydrocarbons could be added in a straight forward manner once enough data have been accumulated from actual test fires. Acid gases and noxious species such as $\mathrm{NO}, \mathrm{HBr}, \mathrm{HCl}, \mathrm{HF}, \mathrm{HCN}$, and $\mathrm{SO}_{2}$ could cause operational problems if present in too high a concentration, but at low levels diluted in $\mathrm{N}_{2}$ they could also be injected into the FE/DE in a controlled manner. This would be necessary if the choice of fuels is broadened to include substances like PVC, nylon and fire retarded materials.

Particulate Matter: Many studies have been undertaken on the properties of condensed matter formed in fires (i.e, smoke). To properly describe particulate matter requires knowledge of its chemical composition, size distribution, number density, and morphology. Because soot invariably is not chemically equilibrated with its gaseous surroundings, satisfactory means to predict the quantity or attributes of solid material produced in real fires do not exist.

Current methods for evaluating fire detectors recognize two qualitatively different kinds of smoke: soot formed during short residence times in flaming fires, and aerosols formed during pyrolysis and in smoldering fires. The maximum values of extinction coefficient measured by Jackson and Robins ${ }^{13}$ range from $0.03 \mathrm{~m}^{-1}$ for the flaming ethanol fire (TF 6) to $0.69 \mathrm{~m}^{-1}$ for the smoldering cotton. The precise character of these smokes has not been measured in test fire settings. Instead, the industry has relied on the response of standard smoke detectors to characterize the particulate emissions, operating on either the ionizing radiation or scattered light principle. In contrast, the detailed composition, concentration and morphology of soot formed in a number of simple laboratory burners have been well documented (e.g., refs. 18-20). Similar detailed measurements are required in the plume above the test fires to verify that the concept of two types of smoke ("gray" and "black") is sufficient to evaluate detectors that may operate on different principles.

Smoke is emulated by misting paraffin oil in the CEN 54 fire simulation apparatus. Methods to control droplet size and number density have been developed for some of these tests. The index of refraction and morphology of particles formed during pyrolysis and smoldering fires may be duplicated reasonably by this approach; however, the imaginary part of the index of refraction is much higher for particles formed in flames, rendering the paraffin less than satisfactory for emulating flaming fire smoke.

The ideal FE/DE would produce on demand a fire aerosol with specified composition, size distribution, number density and morphology. A match of these properties would guarantee the appropriate index of refraction. Control of all these attributes of smoke in the FE/DE is well beyond the current state-of-technology. A combination of smoke generators operating on different principles may be required.

Electromagnetic Radiation: Smoldering fires emit thermal radiation associated with hot smoke particles and pyrolysis gases. Small flaming fires emit radiation from the ultraviolet through the infrared. A large number of commercial flame and spark detectors are on the market that operate on the basis of sensing one or more portions of the electromagnetic spectrum. Radiation travels in all directions from the source and reflects from surfaces in the volume being protected, making the performance of a flame detector highly dependent upon the geometry of the room. Thus, a fire emulator that is satisfactory for detectors of gas, heat or smoke at one point may be unsuited for detectors of radiation which monitor the entire room volume.

A heated, black surface would yield a continuous source of visible and infrared radiation, and a small methane flame would reproduce the UV due to chemiluminescence and the infrared bands associated with hot water vapor and $\mathrm{CO}_{2}$. It would be necessary to vary in a tightly controlled manner the temperature and solid angle of the radiation sources as viewed from the detector location. The fluctuating radiation signals, which characterize flickering flames, would also need to be controlled.

Nuisance electromagnetic radiation can result from any portion of the spectrum. Properly 
emulating the viewing angle would also apply here. False sources of UV can be traced to solar radiation, lightning, fluorescent lights, welding operations, and sparks from electrical machinery. Interior lighting and solar radiation are large sources of visible radiation, and hot surfaces such as exhaust manifolds or space heaters emit strongly in the infrared. Although it is conceivable that a universal facility could be designed to accommodate each of these nuisance sources, the first generation FE/DE will not include this capability. False electrical signals can be generated if the detector or processing electronics are exposed to RF, $\mathrm{x}$-rays or $\gamma$-rays of sufficient intensity. A separate chamber specifically designed to control these extra long and extra short wavelengths is necessary and will not be a part of the FE/DE.

Nuisance Aerosols: The procedures developed to emulate the velocity, temperature, and concentration fields of actual test fires in the FE/DE could be adapted easily to yield the environment produced by non-fire sources of false alarms such as extreme temperatures, wind, excess $\mathrm{CO}$ from automobile exhausts, volatile liquids, or space heaters. The aerosols generated by non-fire sources, however, require special attention. A powder generator similar to that used to seed LDA gas flows could prove useful for emulating nonorganic dusts or pollen. Household chemicals and cooking aerosols could be dispensed in the same manner as the paraffin oil soot emulator. The charring of food could be simulated by placing specimens on a small electric hot plate or under a radiant "broiler". The concentration levels of the nuisance aerosol around the fire detector and the rate at which they build must bracket the conditions most likely to be encountered by the detector in the field. Too little is known about these conditions to specify them for the first generation FE/DE.

Table 3 is a summary of the conditions to be emulated in the first version FE/DE. The maximum design velocity is limited to $2.5 \mathrm{~m} / \mathrm{s}$ even though higher flows are conceivable. However, this value most likely exceeds the velocities attained at the detector location in current test fires and is more than twice the value specified in existing UL and CEN simulators. The blower will be selected large enough to allow higher velocities by limiting the temperature increase or by reducing the test section area. The maximum temperature observed in the field and shown in the table $\left(170^{\circ} \mathrm{C}\right)$ refers to the current limit imposed for sprinkler activation testing. By limiting the $\mathrm{FE} / \mathrm{DE}$ to a maximum of $100{ }^{\circ} \mathrm{C}$ one loses the capability to reproduce Parts 5,6 and 8 of CEN 54. This is not thought to be critical, however, since $100{ }^{\circ} \mathrm{C}$ is well above the temperature where a fire detector should respond, and is a warmer environment than most would expect to be placed. A number of conditions are listed in Table 3 as "tbd" (to be determined) because measurements of these quantities have not been made with the precision necessary (if made at all) to bracket the dynamic range.

\section{Components of the FE/DE}

Figure 1 is a block diagram showing the major components of the proposed FE/DE-I facility. The detector will be located in the test section, which is designed to handle single-point, multi-point, and line-type detection systems. Upstream of the test section is the smoke and particle injection system. Care will be taken to minimize the loss of aerosol mass to the walls of the emulator. The gases $\left(\mathrm{N}_{2}, \mathrm{CO}_{2}, \mathrm{CO}\right.$, $\mathrm{H}_{2}$ and $\mathrm{CH}_{4}$ ) will mix uniformly into the airstream prior to the addition of the particulate matter. A separate gas generator will be used to produce water vapor. The heater and blower are located, in order, upstream of the mixing section. Both will use programmable closed-loop feedback control from precision temperature and velocity sensors located in the test section.

The detector/evaluator test section will be $0.9 \mathrm{~m}$ wide by $0.3 \mathrm{~m}$ high. A length of $4 \mathrm{~m}$ has been chosen to allow enough room for a ceiling boundary layer to develop and also to provide space for the evaluation of different types of line detectors. There will be provision for point detectors to be tested in vertical and horizontal orientations. Access ports will allow measurement of the composition, temperature, and velocity to ensure that the fire signature is properly emulated at the location of the detector.

The major combustion gases will be stored in two cylinders pressurized with nitrogen. Programmable mass flow controllers will meter the appropriate amount of gas to a tubular ring injector. It is proposed that water vapor be generated in a methane burner and routed to the center of the duct. 


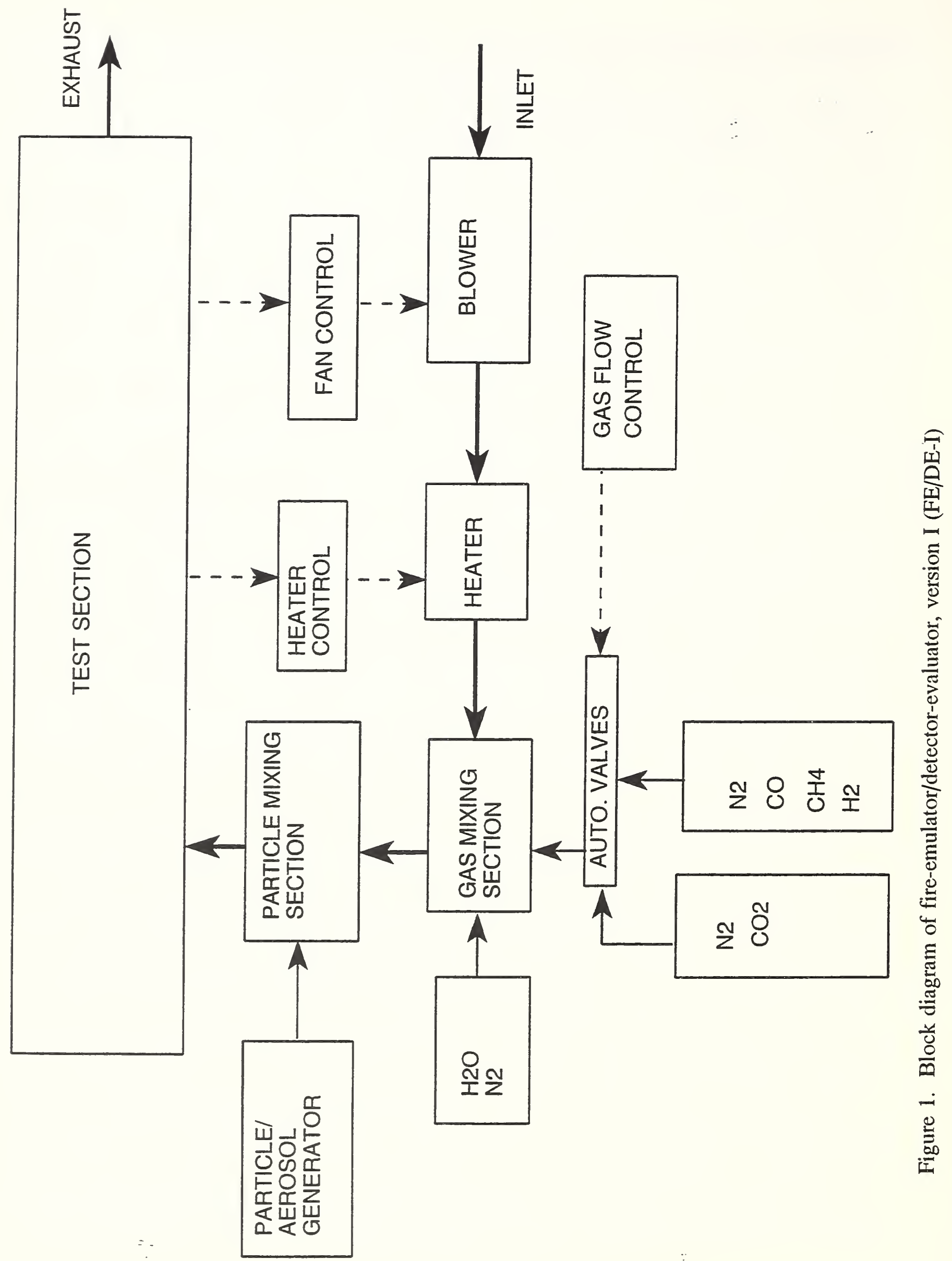


Table 3. Dynamic range of fire characteristics emulated in FE/DE-I

\begin{tabular}{c|c|c|c|c|c}
\hline Parameter & $\begin{array}{c}\text { Observed in } \\
\text { Field }\end{array}$ & Range in FE/DE & Max Gradient & RMS Deviation & $\begin{array}{c}\text { Fluctuation } \\
\text { Frequency }\end{array}$ \\
\hline Velocity & 0.5 to $5 \mathrm{~m} / \mathrm{s}$ & 0.5 to $2.5 \mathrm{~m} / \mathrm{s}$ & $0.1 \mathrm{~m} / \mathrm{s}^{2}$ & 2 to $10 \%$ & 0.1 to $1000 \mathrm{~Hz}$ \\
\hline $\begin{array}{c}\text { Temperature } \\
\begin{array}{c}\Delta \mathrm{CO}_{2} \\
\text { vol. fraction }\end{array}\end{array}$ & -30 to $170^{\circ} \mathrm{C}$ & 25 to $100{ }^{\circ} \mathrm{C}$ & $30{ }^{\circ} \mathrm{C} / \mathrm{min}$ & uncontrolled & $<0.5 \mathrm{~Hz}$ \\
\hline $\begin{array}{c}\Delta \mathrm{H}_{2} \mathrm{O} \\
10^{-6} \text { vol. fraction }\end{array}$ & 0 to 12,000 & 0 to 12,000 & $120 / \mathrm{s}$ & uncontrolled & $<0.1 \mathrm{~Hz}$ \\
\hline $\begin{array}{c}\Delta \mathrm{O}_{2} \\
10^{-6} \text { vol. fraction }\end{array}$ & 0 to $-12,000$ & 0 to $-12,000$ & $-120 / \mathrm{s}$ & uncontrolled & $<0.1 \mathrm{~Hz}$ \\
\hline $\begin{array}{c}\mathrm{CO} \\
10^{-6} \text { vol. fraction }\end{array}$ & 0 to 400 & 0 to 400 & $10 / \mathrm{s}$ & uncontrolled & uncontrolled \\
\hline $\begin{array}{c}\mathrm{H}_{2} \\
10^{-6} \text { vol. fraction }\end{array}$ & 0 to 70 & 0 to 70 & $10 / \mathrm{s}$ & uncontrolled & uncontrolled \\
\hline $\begin{array}{c}\mathrm{CH} \\
10^{-6} \text { vol. fraction }\end{array}$ & unknown & 0 to 200 & $10 / \mathrm{s}$ & uncontrolled & uncontrolled \\
\hline $\begin{array}{c}\text { oxygenated or } \\
\text { higher hydrocarbons }\end{array}$ & unknown & tbd* & tbd & uncontrolled & uncontrolled \\
\hline $\begin{array}{c}\text { HCl, NO } \\
\text { particulate } \\
\text { extinction coeff. }\end{array}$ & 0 to $0.7 \mathrm{~m}{ }^{-1}$ & 0 to $0.7 \mathrm{~m}^{-1}$ & $.01 \mathrm{~m} / \mathrm{s}$ & uncontrolled & uncontrolled \\
\hline $\begin{array}{c}\text { IR radiation } \\
\text { unknown }\end{array}$ & unknown & tbd & tbd & tbd & 0 to 20 Hzz \\
\hline
\end{tabular}

*to be determined

Liquid water could be sprayed through a hollow-cone pressure jet nozzle into methane combustion products, producing a maximum ratio of $\mathrm{H}_{2} \mathrm{O}$ to $\mathrm{CO}_{2}$ estimated to be 17:1.

Nine annular-shaped resistance heaters, $5 \mathrm{~kW}$ each, will be placed in series to control the air temperature. The outer diameter and total length of the heater will be $0.5 \mathrm{~m}$ and $0.69 \mathrm{~m}$, respectively. Power to the heater will be provided by a programmable, $160 \mathrm{~A}$ control panel. The blower will be a direct drive tubeaxial fan with a $1 \mathrm{~kW}$ motor, capable of moving over $1100 \mathrm{~L} / \mathrm{s}$ through a pressure drop of $350 \mathrm{~Pa}$.

Details of the smoke generator have not yet been developed. One concept is to use a rich, premixed propene/air burner to generate smoke in an attempt to emulate a plastic or liquid fuel fire. Nitrogen gas would be used to transport the soot to the $0.3 \mathrm{~m}$ by $0.3 \mathrm{~m}$ square duct. The concentration of soot in the nitrogen would be varied by adjusting the flame equivalence ratio and fuel flow rate. The total flow to the emulator duct would be controlled by regulating the bypass valve. Smoldering and pyrolyzing smoke would be formed in a radiant heated chamber into which a controlled amount of nitrogen and air would flow. A bypass system similar to the one used with the propene/air burner would be used to adjust the total flow to the duct. A yet-to-be-designed fluidized bed system would be used to deliver non-flame solid particulate matter. Each of the three aerosol producing systems would be characterized before installation to ascertain the exact nature of the particles and how it changes with operating conditions. 


\section{Signatures from Smoldering/Pyrolyzing Fires for "Training" Emulator}

Two of the test fires described in CEN 54, TF 2 and TF 3 , involve smoldering materials. An electric heater is used to char wood in TF 2. Power is supplied continuously up to the point of flaming, which typically occurs more than 10 minutes into the test. A self-sustained smoldering cotton wick is used in TF 3. The data presented here are from measurements in the plume close to the fuel source. A schematic of the test room is shown in Fig. 2; Fig. 3 is a sketch of the gas sampling system. Details of the experimental facility and instrumentation are described elsewhere ${ }^{14}$. The primary objective of these measurements was to quantify the source of important chemical species and particulate matter, the plume momentum, and the thermal energy provided by the fire. This information can be used to specify the source terms of hydrodynamic models of the actual space to be protected, which in turn dictate the conditions to be emulated in the FE/DE for evaluating a detecting system sited in the room.

Smoldering Wood (TF 2): Beechwood blocks with a total mass between $121 \mathrm{~g}$ to $127 \mathrm{~g}$ were placed uniformly around two concentric circles on top of a $0.22 \mathrm{~m}$ diameter electrical hot plate. The test began by initially applying $1.9 \mathrm{~kW}$ of power to the hot plate. The temperature on the surface of the hot plate reached $600{ }^{\circ} \mathrm{C}$ in $490 \mathrm{~s} \pm 15 \mathrm{~s}$ for the five different runs, which was well within the time limit specified in CEN 54. Mass loss became perceptible about $20 \mathrm{~s}$ into the test. The peak pyrolysis rate was $0.26 \mathrm{~g} / \mathrm{s} \pm 0.01 \mathrm{~g} / \mathrm{s}$, occurring $620 \mathrm{~s} \pm 10 \mathrm{~s}$ after heating began. Figure 4 is a plot of the mass loss rate for each test. The repeatability for the first $720 \mathrm{~s}$ is remarkable considering that the loss rate is computed from the gradient of the instantaneous mass of fuel measured by the load cell. The wood eventually burst into flames (creating the spikes in Fig. 4), but always after at least $70 \%$ of the mass had been lost.

The plume temperatures, averaged over multiple runs, $0.88 \mathrm{~m}$ above the hot plate, are plotted in Fig. 5. The centerline temperature reaches $45^{\circ} \mathrm{C}$ in $660 \mathrm{~s}$, and decreases with increasing radius in all directions. Velocities at the same axial position peaked at slightly over $1.0 \mathrm{~m} / \mathrm{s}$ on the centerline. The volume fractions of $\mathrm{CO}_{2}, \mathrm{CO}$ and $\mathrm{H}_{2} \mathrm{O}$ measured on the hot plate centerline were reported by Cleary ${ }^{14,15}$. The ratio of $\mathrm{CO}$ to $\mathrm{CO}_{2}$ was approximately constant at $1 / 3$ throughout the heating period. The water was released from the wood much sooner, but reached its maximum volume fraction $(0.45 \%)$ at about the same point in time. The total hydrocarbon levels were measured with a flame ionization detector, while methane, acetaldehyde, and acetic acid were found from line-of-sight FTIR analysis. Laser extinction through the plume, $0.22 \mathrm{~m}$ above the smoldering wood, was measured and showed good repeatability. Assuming Beer's law held, the extinction coefficient at $633 \mathrm{~nm}$ averaged across the plume diameter was found to be about $10 \mathrm{~m}^{-1}$.

Smoldering Cotton (TF 3): Six independent experiments were run using the smoldering cotton fires. Figure 6 shows the cumulative mass loss, normalized by the initial mass of fuel, for the different runs. The large variation in burning rates was attributable to the state of the wick material. A higher burning rate occurred if the wicks remained straight and in close contact with each other. If the individual wicks separated from one another and curled outward, more heat was lost to the environment and the chimney-like structure of the initial wick sample was destroyed, affecting the entrainment of oxygen into the smoldering zone. It is unclear which initial conditions led to the outward curling of the wicks.

The radial variations in temperature were examined $0.05 \mathrm{~m}$ above the top of the wick. The centermost temperatures were within $20^{\circ} \mathrm{C}$ of the those measured half way to the edge of the fuel source. Temperatures at the edge rarely exceeded $40{ }^{\circ} \mathrm{C}$, and were as much as $100{ }^{\circ} \mathrm{C}$ below the centerline values. This suggested a top-hat profile near the fuel surface with a high temperature uniform core increasing in time to about $120{ }^{\circ} \mathrm{C}$ and a close-to-ambient value at the edge of the fuel. The average temperatures measured with time on the fuel centerline $0.16 \mathrm{~m}$ above the top of the wicks (see Fig. 7) increased steadily for $650 \mathrm{~s}$ to about $70^{\circ} \mathrm{C}$, and thereafter increased at a slower rate to a maximum of $88^{\circ} \mathrm{C}$ at $1500 \mathrm{~s}$. Temperature excursions exceeding $100^{\circ} \mathrm{C}$ can be seen in the run-to-run deviations.

The attenuation of laser intensity through the plume $0.01 \mathrm{~m}$ above the smoldering cotton wicks led to an estimated extinction coefficient (at $633 \mathrm{~nm}$ ) $600 \mathrm{~s}$ into the test of $16 \mathrm{~m}^{-1} \pm 6 \mathrm{~m}^{-1}$. Measured values of the $\mathrm{CO}, \mathrm{CO}_{2}$ and $\mathrm{H}_{2} \mathrm{O}$ are presented in the report by Cleary ${ }^{15}$. 


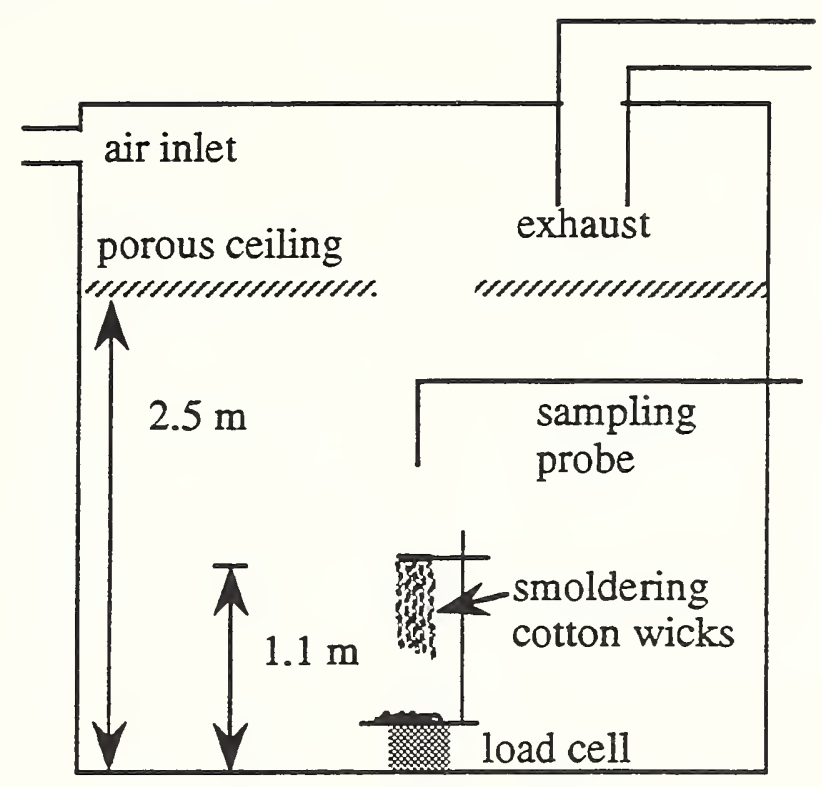

Figure 2. Schematic of detector fire test room (set up for TF 3).

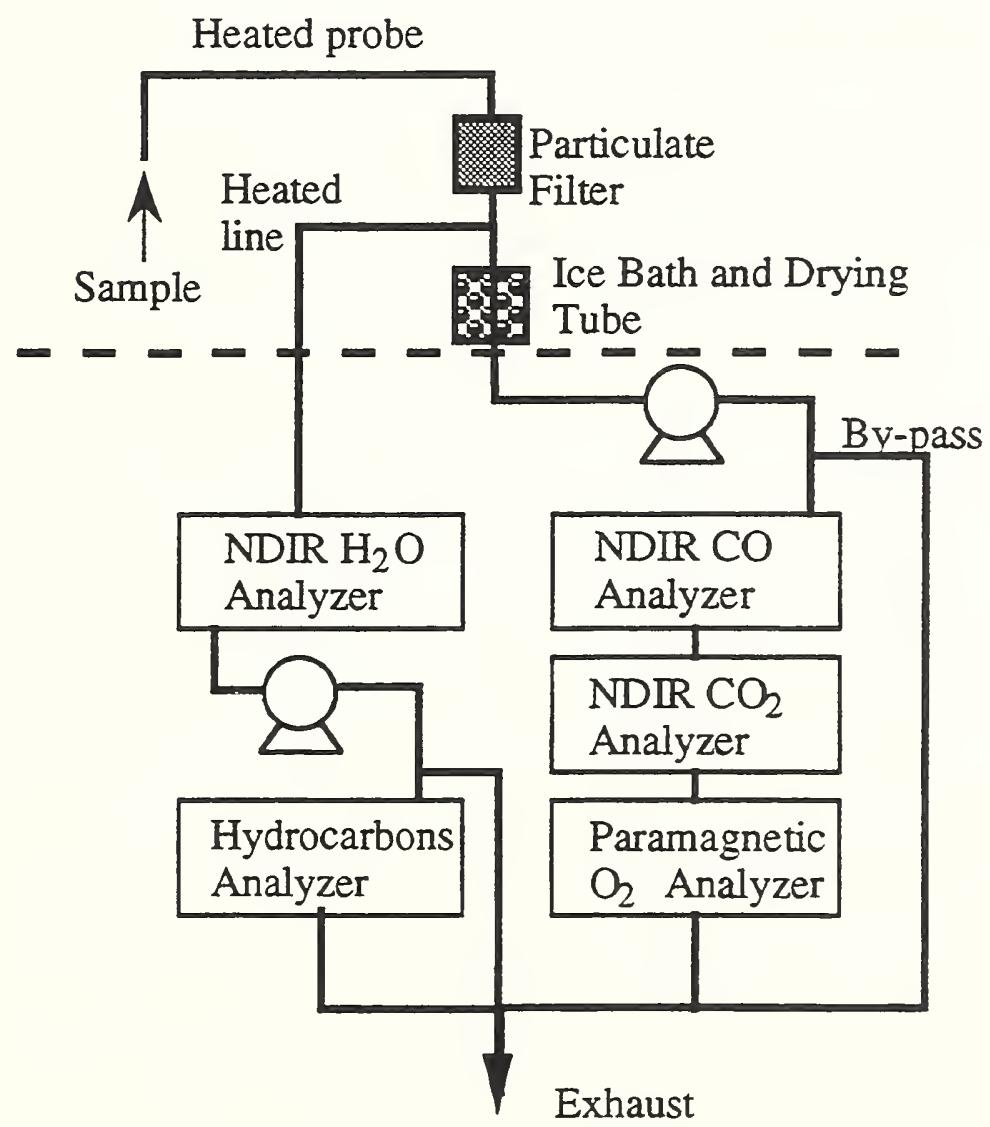

Figure 3. Block diagram of gas sampling system for test fire plume measurements. 


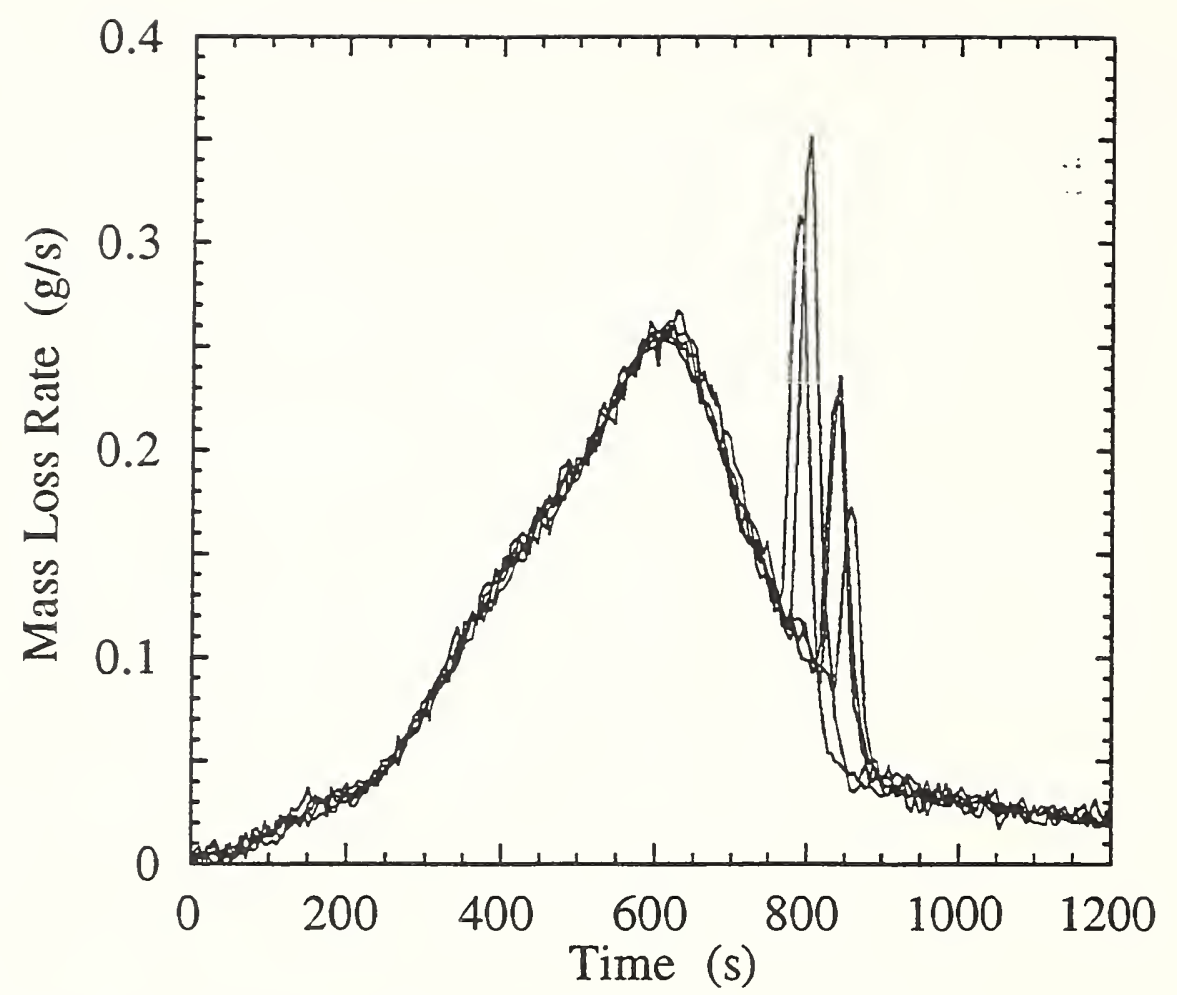

Figure 4. Composite mass loss rate of five charring wood tests (TF 2).

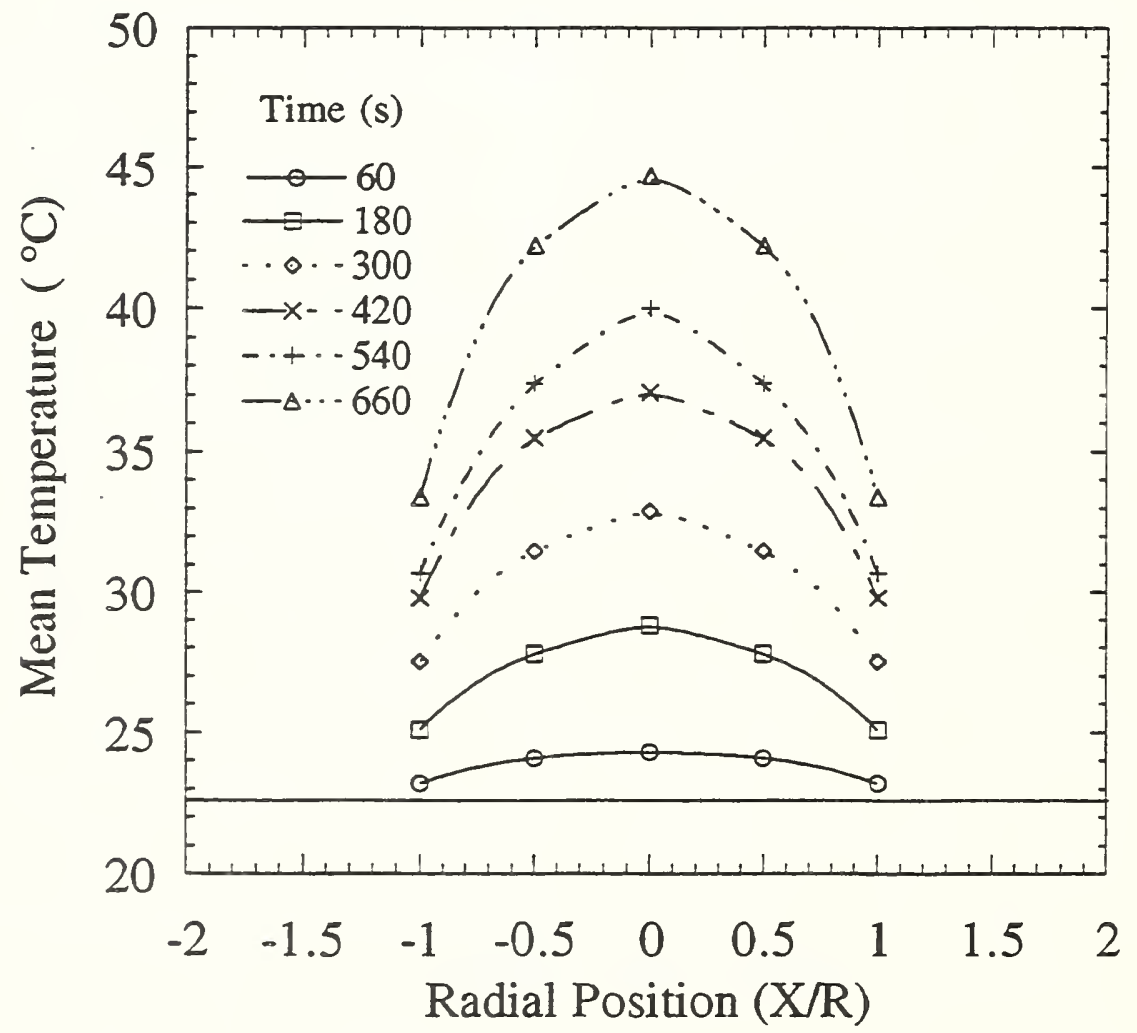

Figure 5. Radial temperature distribution as a function of time in plume $0.88 \mathrm{~m}$ above charring wood test fire. 


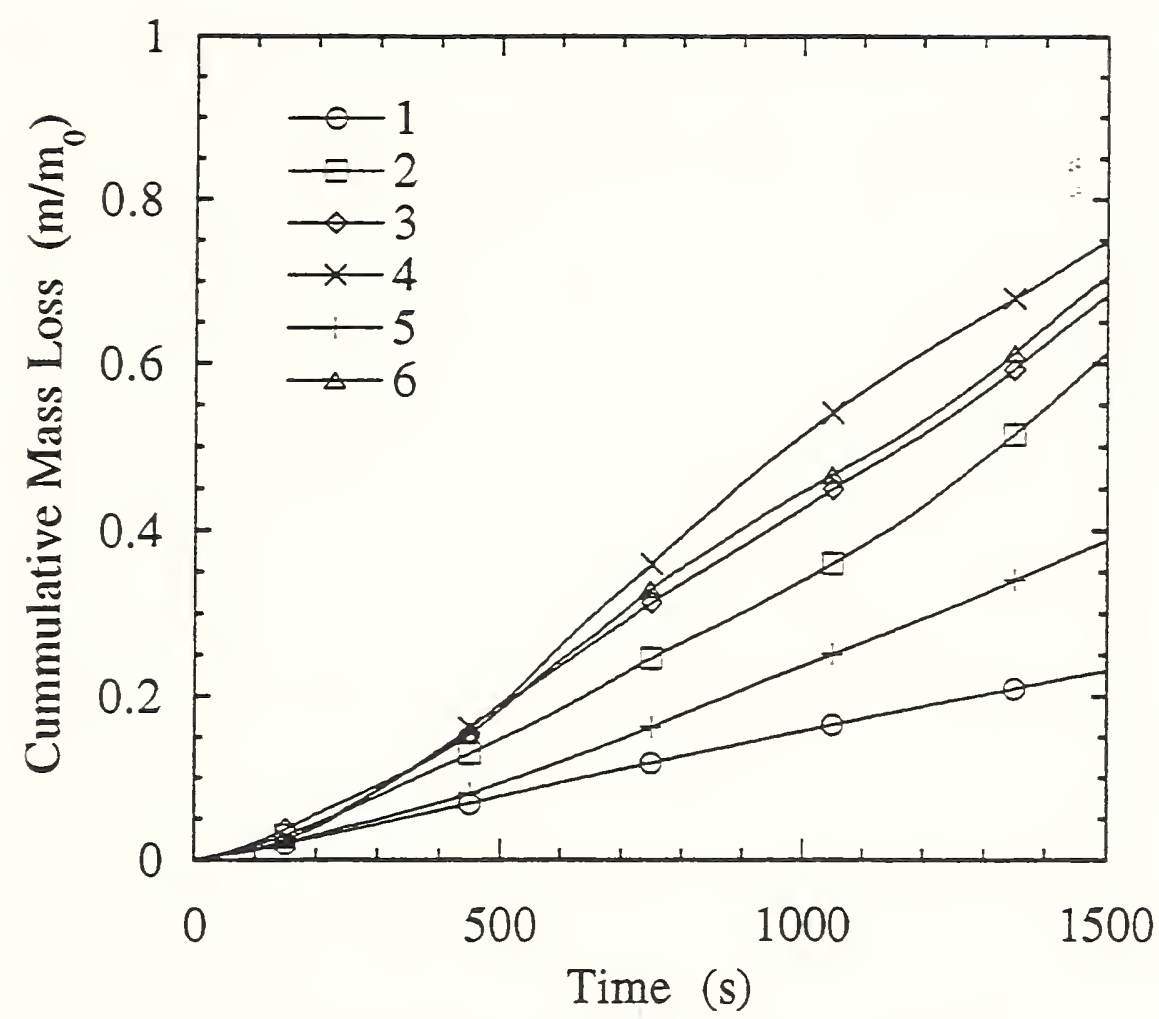

Figure 6. Mass loss from six smoldering cotton wick test fires (TF 3).

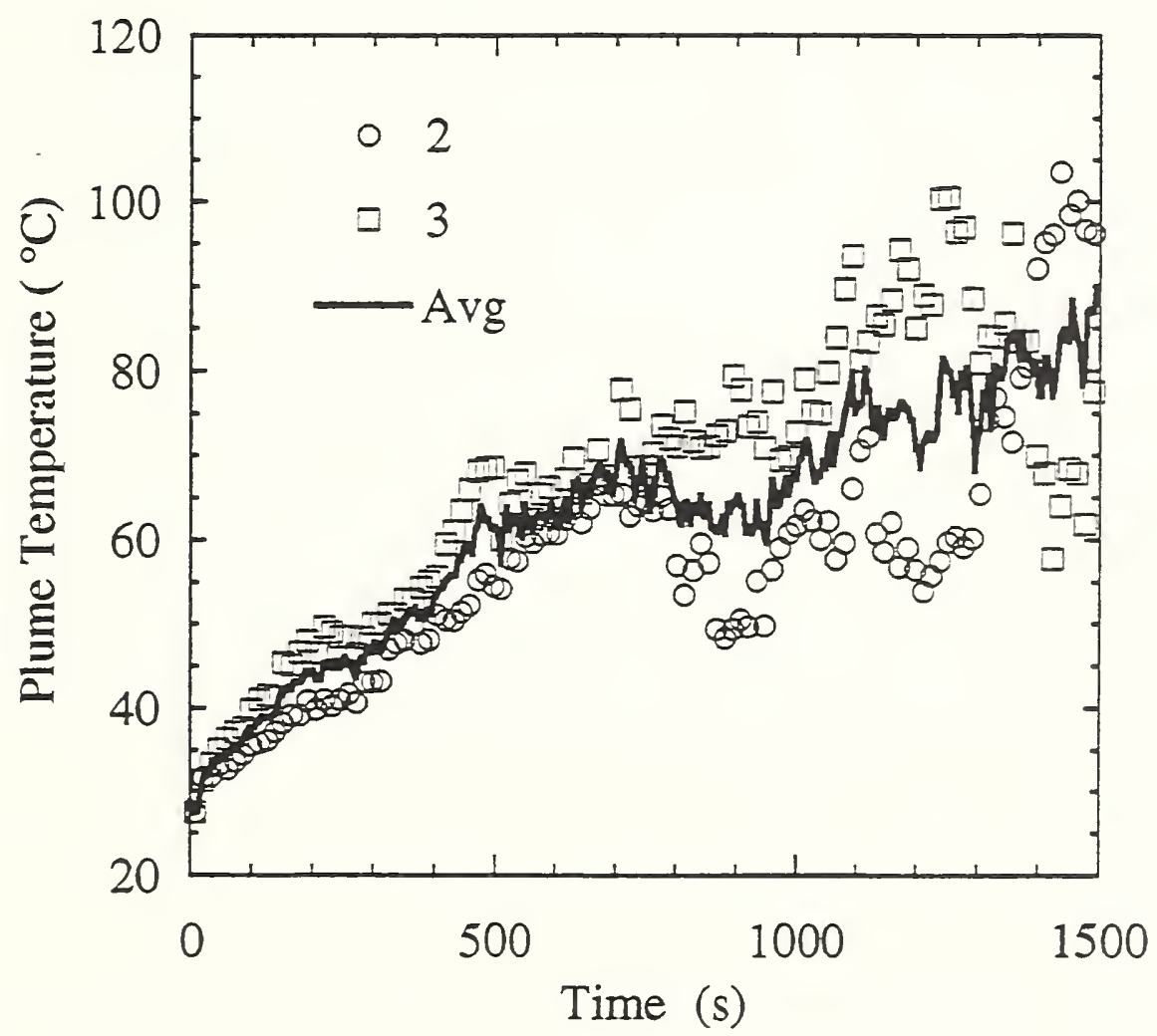

Figure 7. = Plume centerline temperature $0.16 \mathrm{~m}$ above cotton wicks (TF 3). 
Generic Signatures for TF 2 and TF 3: Pfister $^{12}$ and Jackson and Robins ${ }^{13}$ measured the ceiling temperatures and combustion products in the TF 2 and TF 3 fires burning in a standard CEN 54 room. Their reported temperature increases and volume fractions of $\mathrm{CO}$ and $\mathrm{CO}_{2}$ are 6 to 8 times lower than those measured here in the smoldering wood fire, $0.88 \mathrm{~m}$ above the hot plate. The hydrocarbon levels measured previously ${ }^{12}$ are a factor of 60 times lower than those measured here, an inconsistency suggesting a possible sampling problem in the older study or a difference in operating procedure. Comparing the previous measurements taken near the ceiling in TF 3 , the values of $\mathrm{CO}_{2}, \mathrm{CO}$ and temperature increase measured $0.16 \mathrm{~m}$ above the cotton wick in the current study are greater by over an order of magnitude. Modeling of plume entrainment and the ceiling jet are required to ascertain if the different studies are in general agreement with each other.

Preliminary time signatures of temperature, velocity, $\mathrm{H}_{2} \mathrm{O}, \mathrm{CO}_{2}$ and $\mathrm{CO}$ suggested for these two smoldering fires are plotted in Figs. 8 and 9. Note that they refer to predicted centerline values $0.88 \mathrm{~m}$ and $0.16 \mathrm{~m}$ above the TF 2 and TF 3 fires, respectively. As more testing is completed, additional species volume fractions (e.g., $\mathrm{H}_{2}, \mathrm{CH}_{4}, \mathrm{NO}$ ) and electromagnetic spectra will be added to these signatures. The standard deviation and characteristic fluctuating frequencies will also be accumulated. With this information, computational fluid dynamics can be used to model the transport of heat and species throughout the CEN 54 test room or any other volume in need of protection. This modeling approach has been used in a number of situations to determine the impact of room geometry ${ }^{21}$, distance from the fire $^{22}$, and room ventilation ${ }^{23}$ on the likely response of a fire detector. The response of a multi-criteria detector to a smoldering fire can also be evaluated by exposing the sensors to the temperatures and concentrations predicted by the fluid mechanical models. The fire-emulator/detector-evaluator will be constructed expressly for this purpose. The data summarized in Figs. 6 and 7 are, thus, key to the successful implementation of the FE/DE concept.

\section{References}

1. UL 217: Standard for Single and Multiple Station Smoke Detectors, Underwriters Laboratories, Inc., Northbrook IL, 1993.

2. CEN 54: Components of Automatic Fire Detection Systems; Part 9. Fire sensitivity test, European Committee for Standardization, July 1982.

3. UL 268: Standard for Smoke Detectors for Fire Protective Signaling Systems, Underwriters Laboratories, Inc., Northbrook IL, 1989.

4. CEN 54: Components of Automatic Fire Detection Systems; Part 7. Point-type smoke detectors; Detectors using scattered light, transmitted light or ionization, European Committee for Standardization, April 1988.

5. Ahonen, A.I., and Sysiö, P.A., A Wind Tunnel System for Testing Smoke Detectors, Research Report 221, Technical Research Centre of Finland, Espoo, Finland, 1983.

6. Voigt, H., and Avlund, M., Standardized Smoke: Problems Related to Generation of Smoke with Properties as Specified in New European Draft Standards for Automatic Smoke Detectors, prEN54 Part 7 and 9, Report \#ECR-94, Danish Research Center for Applied Electronics, Hoersholm, June 1980.

7. UL 521: Heat Detection for Fire Protective Signaling Systems, Underwriters Laboratories, Inc., Northbrook IL, 1993.

8. UL 539: Single and Multiple Station Heat Detectors, Underwriters Laboratories, Inc., Northbrook IL, 1991.

9. CEN 54: Components of Automatic Fire Detection Systems; Part 5. Heat sensitive detectors point detectors containing a static element, European Committee for Standardization, 1977.

10. CEN 54: Components of Automatic Fire Detection Systems; Part 6. Heat sensitive detectors; rate of rise point detectors without a static element, European Committee for Standardization, July 


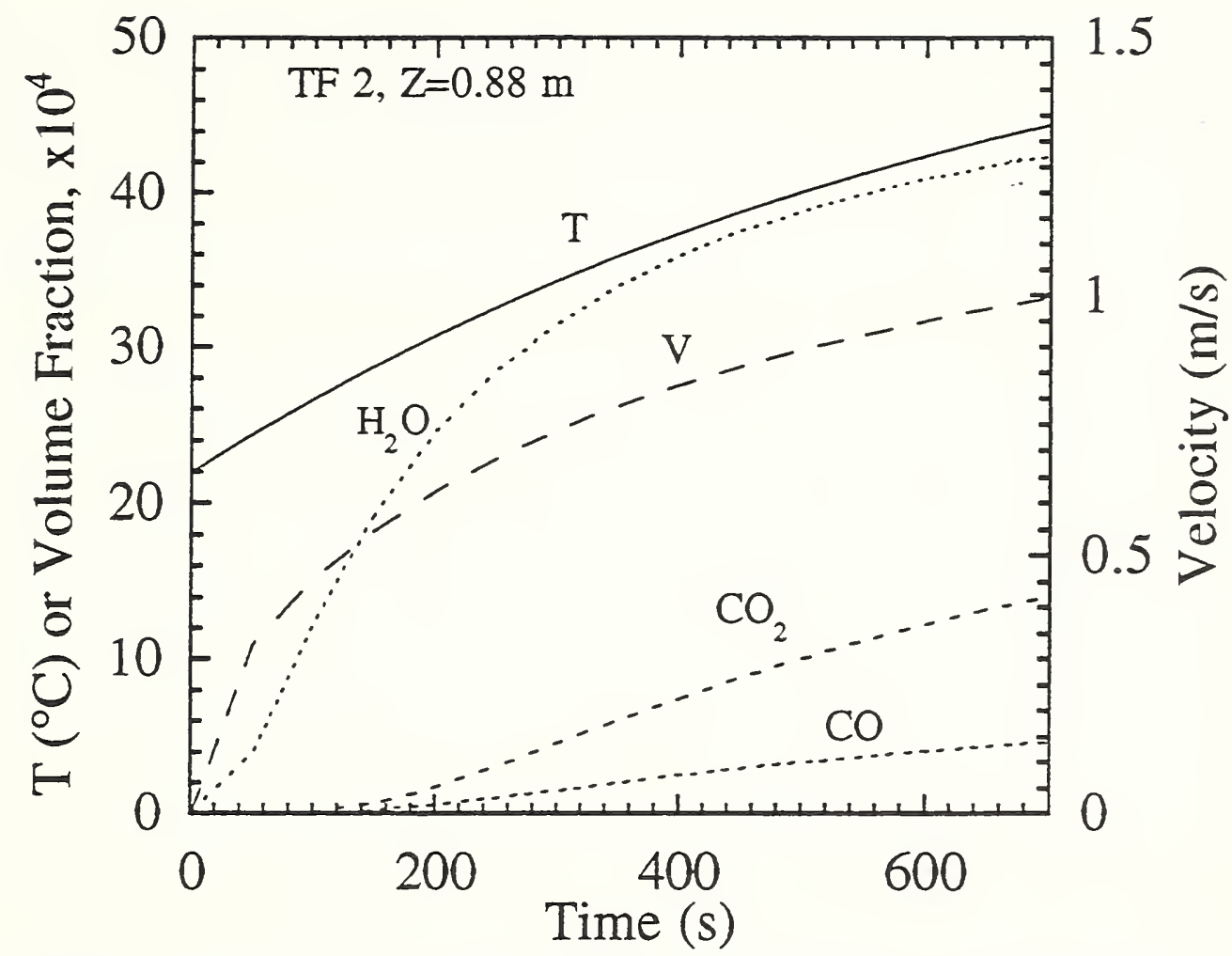

Figure 8. Generic signature for temperature, velocity and species on centerline $0.88 \mathrm{~m}$ above charring wood fire (TF 2).

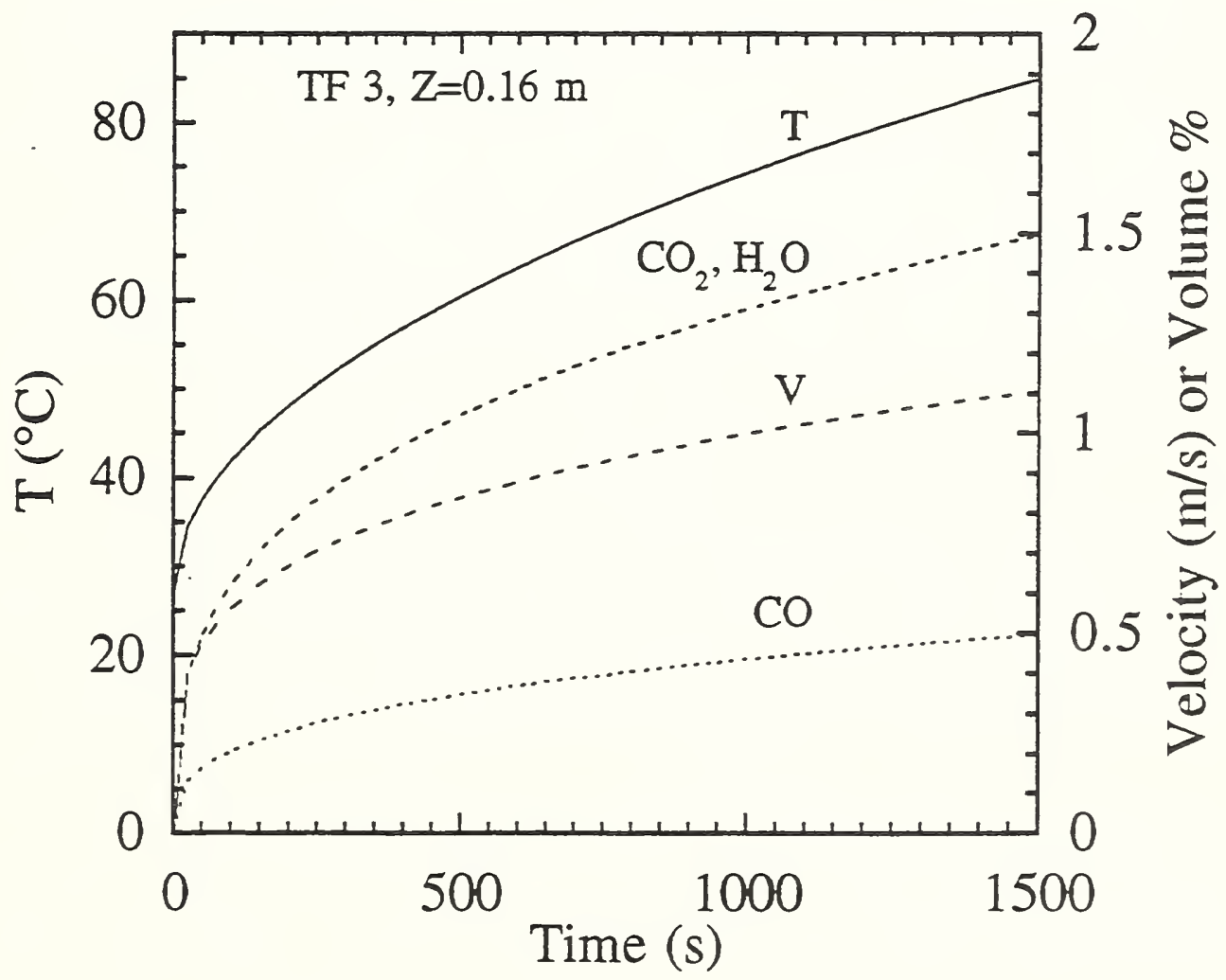

Figure 9. Generic signature for temperature, velocity and species on centerline $0.16 \mathrm{~m}$ above smoldering cotton wicks (TF 3). 
1982.

11. CEN 54: Components of Automatic Fire Detection Systems; Part 8. High temperature heat detectors, European Committee for Standardization, April 1988.

12. Pfister, G., "Detection of Smoke Gases by Solid State Sensors - A Focus on Research Activities," Fire Safety Journal 6, 165-174 (1983).

13. Jackson, M.A., and Robins, I., "Gas Sensing for Fire Detection: Measurements of $\mathrm{CO}, \mathrm{CO}_{2}, \mathrm{H}_{2}$, $\mathrm{O}_{2}$, and Smoke Density in European Standard Fire Tests," Fire Safety Journal 22, 181-205 (1994).

14. Cleary, T., Grosshandler, W., Nyden, M., and Rinkinen, W., "Signatures of Smoldering/Pyrolyzing Fires for Multi-element Detector Evaluation," Interflam '96, Cambridge, England, March 1996.

15. Cleary, T., and Grosshandler, W., Signatures of Smoldering Fires for Use in a Fire-emulator/ Detector-evaluator, NISTIR, National Institute of Standards and Technology, in press, 1996.

16. Grosshandler, W.L., "Towards the Development of a Universal Fire Emulator/Detector Evaluator," Proceedings, Automatische Brandentdeckung, AUBE '95, Duisburg, Germany, p. 363, April, 1995.

17. Grosshandler, W.L. (editor), Proceedings of the 1995 Workshop on Fire Detector Research, NISTIR 5700, August, 1995.

18. Lee, T.G.K., and Mulholland, G., Physical Properties of Smokes Pertinent to Smoke Detector Technology, NBSIR 77-1312, November 1977.

19. Mulholland, G., and Ohlemiller, T.J., "Aerosol Characterization of a Smoldering Source," Aerosol Science and Technology 1, 59-71 (1982).

20. Sivathanu, Y.R., and Faeth, G.M., "Soot Volume Fractions in the Overfire Region of Turbulent Diffusion Flames," Combst. Flame 30, 227-284 (1990).

21. Forney, G.P., Bukowski, R.W., and Davis, W.D., International Fire Detection Research Project - Field Modeling: Effects of Flat Beamed Ceilings on Detector and Sprinkler Response, Technical Report Year 1, National Fire Protection Research Foundation, Quincy, MA, October 1993.

22. Baum, H.R., McGrattan, K.B., and Rehm, R.E., "Mathematical Modeling and Computer Simulation of Fire Phenomena," Fire Safety Science - Proceedings of Fourth International Symposium, T. Kashiwagi, ed., IAFSS, USA, pp. 185, 1994.

23. Satoh, K., "Study of Early and Reliable Fire Detection in Air-conditioned Rooms, "Fire Safety Science-Proceedings of the Fourth Int'l Symposium, T. Kashiwagi, ed., IAFSS, USA, p. 173, 1994. 


\title{
INDUSTRY ADVANCES IN FIRE DETECTION TECHNOLOGY
}

\author{
Ronald K. Mengel \\ Pittway Systems Technology Group \\ 3825 Ohio Avenue \\ St. Charles, Illinois 60174
}

\section{ABSTRACT \\ Fire Characterization - Research \& Application}

The fire detection industry, independently and also in concert with research organizations, has long studied the concept of the characterization of fires; searching for scenarios enabling electromechanical sensors to mimic the ability of human senses to determine when, and when not, to initiate an alarm condition. We, in fact, are receiving progress reports of continuing work in this area at this conference. The use of these techniques in the field, however, has not been extensively realized to this point in time.

Industry advances in the recent past have been concentrated in several other areas encompassing all aspects of the fire detection system, including its role as a sub-system in total building control.. Many of these advances concern themselves with the same goal as that pursued by the application of fuzzy logic or neural networks - differentiation of the fire and non-fire environments.

The industry or "applied" technological advances the industry has made attack the problem at several levels, from the basic sensors used, through the communication paths within the system, to the integration of these systems within the building environment. At each level, the ability of the system to more precisely make the fire/non-fire determination in the face of real world environments was a key design goal.

\section{Sensor Performance}

Little change has occurred in the past several years in regard to basic sensing techniques in fire detection systems. In the research associated with fire characterization, other sensors, e.g., gas, chemical, etc. have been investigated. At this point in time, however, the ionization and photoelectronic smoke sensors remain the choice in reliable early detection in today's detection systems.

Therefore, recent advances within industry related to detectors have concentrated in applying signal processing techniques to remove or reduce unwanted sources of distortion of the desired information. These unwanted distortions include electromagnetic interference as well as non-fire environmental influences such as air borne dust, air gusts and moisture. 
Sensor electromechanical design advances have made major contributions to the reduction of unwanted alarms, and must be given attention in any discussion of this topic. Recent advances in this area have concentrated in improved EMI rejection as well as significant improvement in the ability of smoke detectors to exclude insects and, in the case of the photoelectronic detectors, ambient or stray light. It is important to note that these improvements have been accomplished in the face of significant market pressure to decrease the profile dimension of these sensors.

While these electromechanical advances are no doubt of equal significance in improving fire alarm system performance, the spotlight has been captured in the past one or two years by advances in multisensor and signal processing developments. The downward migration of microprocessor power and technology to include sensors and other distributed modules has provided impetus for these developments. We have come, in a sense, "full circle" in fire detection decision making. We started with all of the decision making in the detector, moved toward a significant portion (if not all) of the alarm decision being made in the control unit, to today's systems which utilize "distributed intelligence". This has created much debate within the fire alarm system design community as to where and how the signal processing should be done.

\section{Multisensor Technology}

A discussion of multisensor technology requires that we first define the term. For the purposes of this discussion, we will define it as: a combination of fire sensors plus the associated signal processing and algorithms, regardless of the physical location of each of these elements within the fire alarm system. This definition is necessary if we are to include all points of view and design approaches currently in use in the United States, e.g. it includes the processing of information from spatially separated sensors. In general, however, the term is used to describe multiple sensors within a single spot-type detector housing.

Obviously the category does not attempt to restrict the type of sensors which can be combined, but as stated earlier, the combination of smoke and heat sensors is by far the most prevalent. The working group currently developing the ISO product testing standard for multisensor detectors, of which I am a member, spent a great deal of time on the question of scope. While the standard will allow for other combinations in the future, no specific requirements for other combinations will be included in the first version.

The combinations in use today generally include either one or two smoke sensors, a heat sensor used in either a supplemental or full voting fashion, plus the software containing the signal processing and combinational algorithms. The physical arrangement of the sensors in single housing units is also an important element in determining overall performance, as it can bias the relative signal strength from the individual sensors. If we examine the historical development of multisensor technology, we find first a combination of an ionization sensor and a photoelectronic sensor in a non-concentric arrangement coupled with an "or" logic circuit. While in the strictest interpretation of our 
working definition this can be considered a "multisensor" detector, it is truly only two independent sensors within one housing. The same is, of course true of similar units using a smoke sensor and heat sensor so combined.

Today's units combine the individual sensors physically to provide, as nearly as practical, simultaneous sampling of the same sample volume, and utilize sophisticated signal processing techniques to improve signal to noise, long term drift compensation, and rejection of environmental short term effects (see Figures $4 \& 5$ ). In addition, they utilize algorithms which provide increased selectivity by the ability to "tune" the system to the individual application. One example is shown the attached Figures $1,2, \& 3$. In this example of a multisensor unit having an ionization sensor, a photoelectronic sensor, and a thermal sensor, five level of sensitivity are included, all within the U.L. listing for the combined unit. The three signals can then be examined in both amplitude and time against an algorithm to ultimately arrive at the alarm decision.

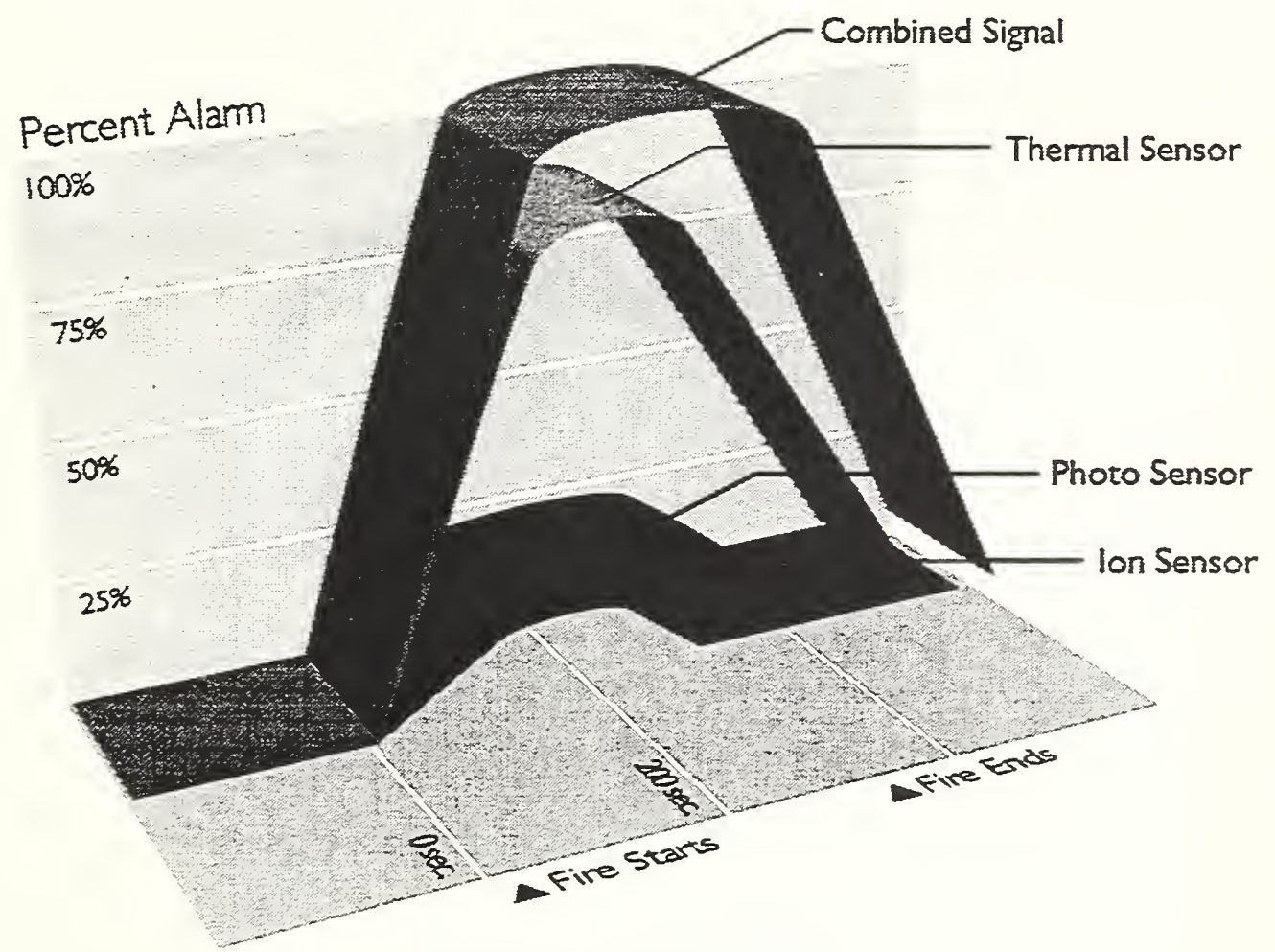

Figure 1 - Alcohol Fire 


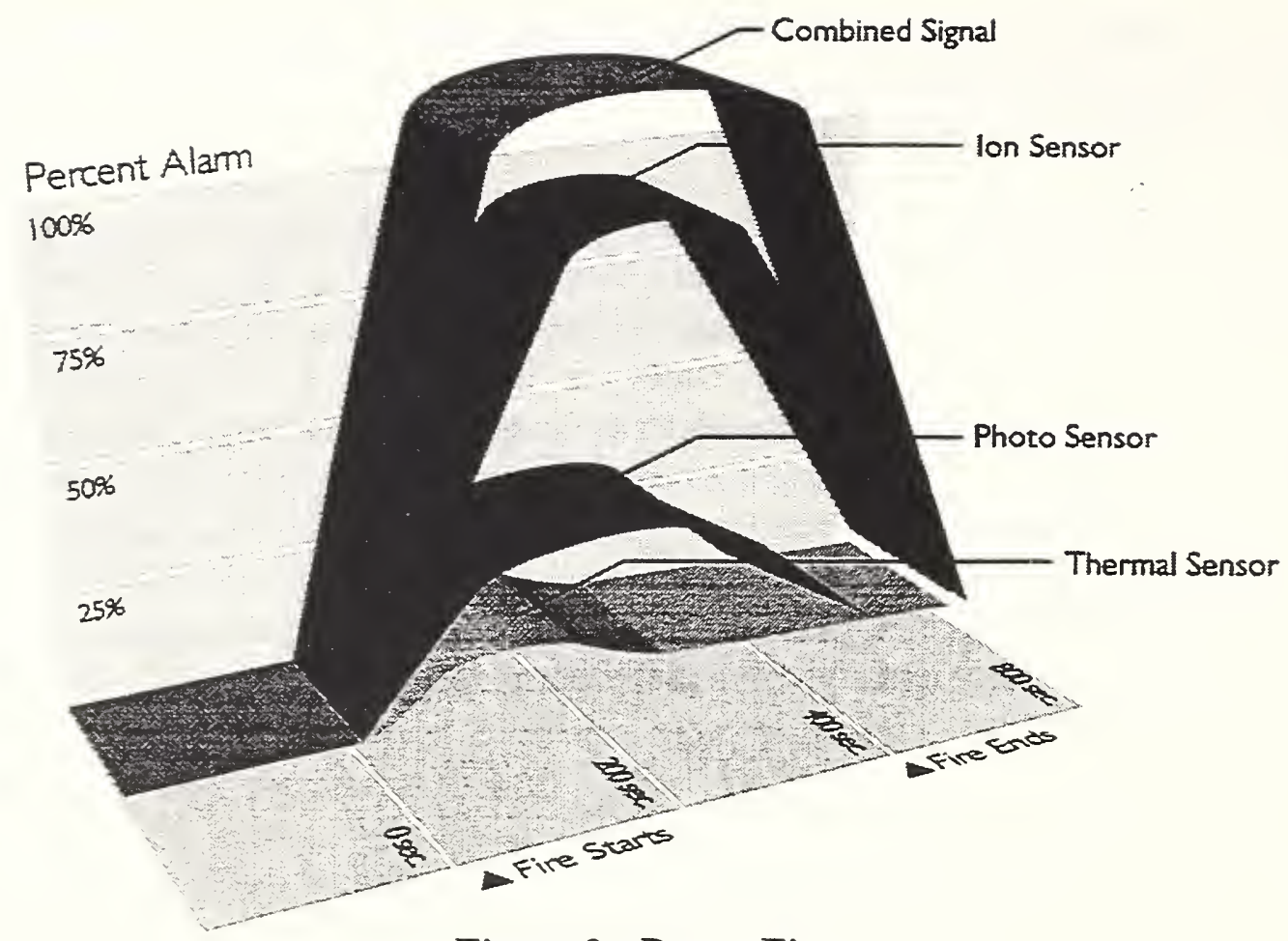

Figure 2 - Paper Fire

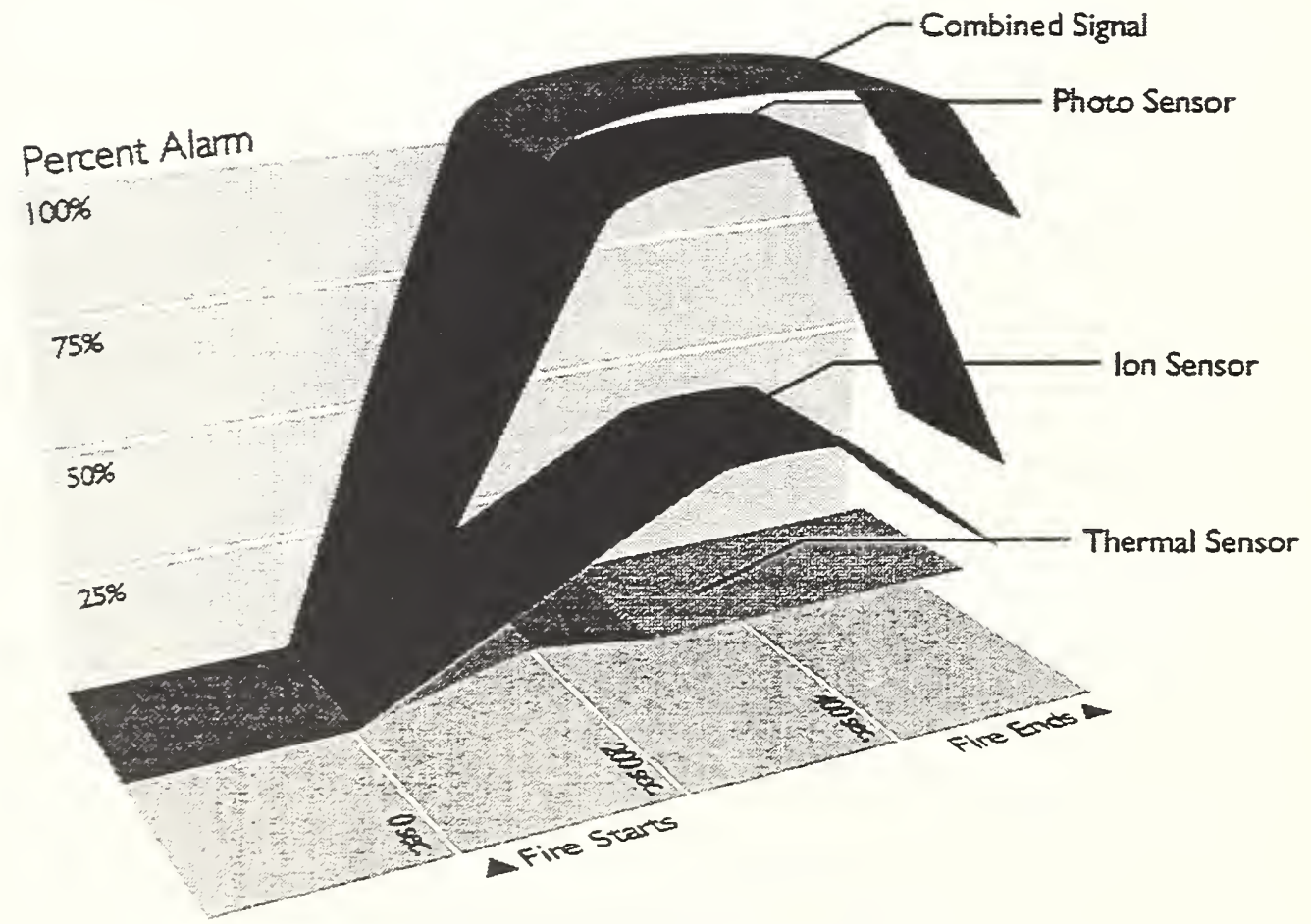

Figure 3 - Smoldering Fire 


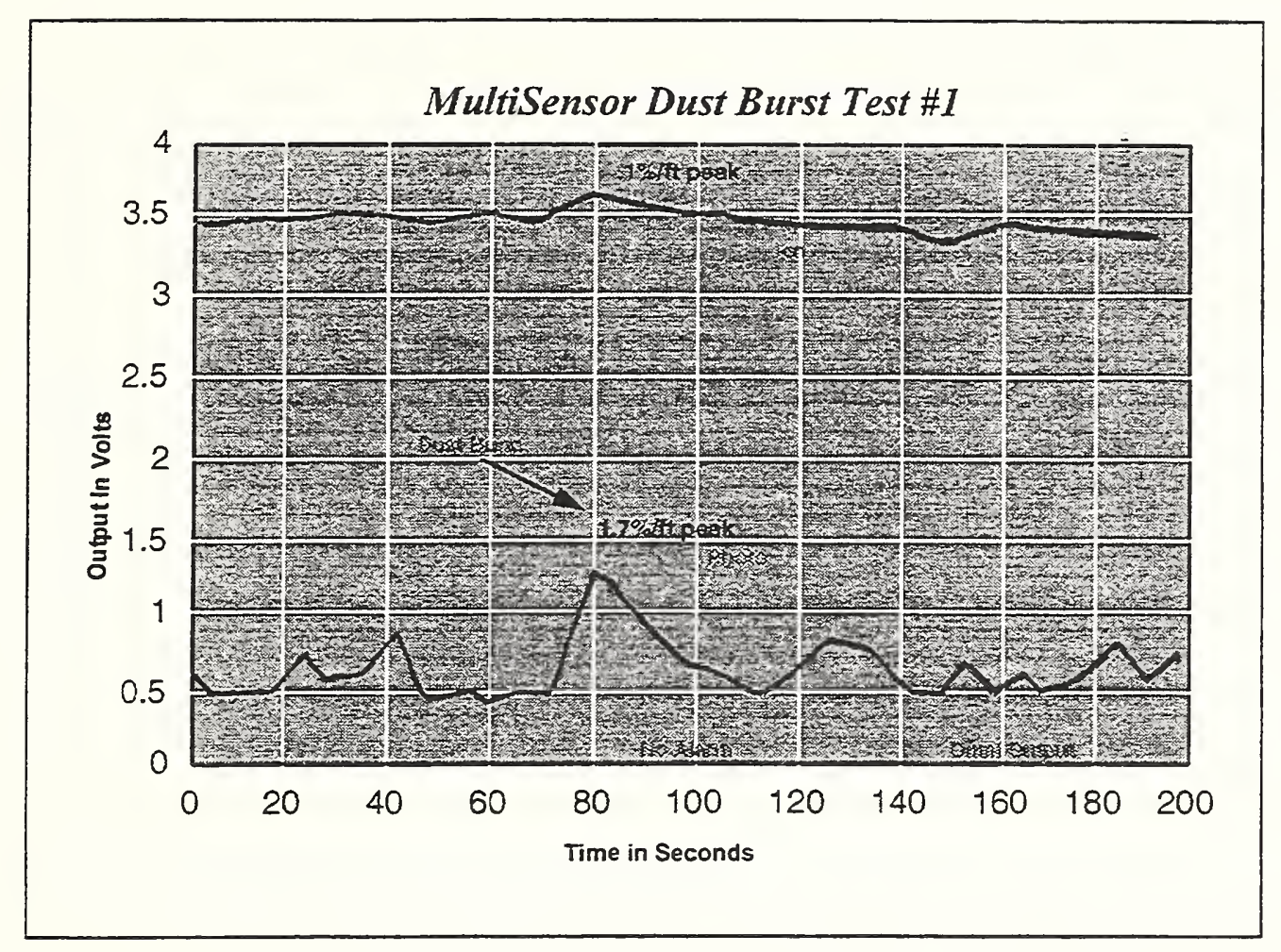

Figure 4

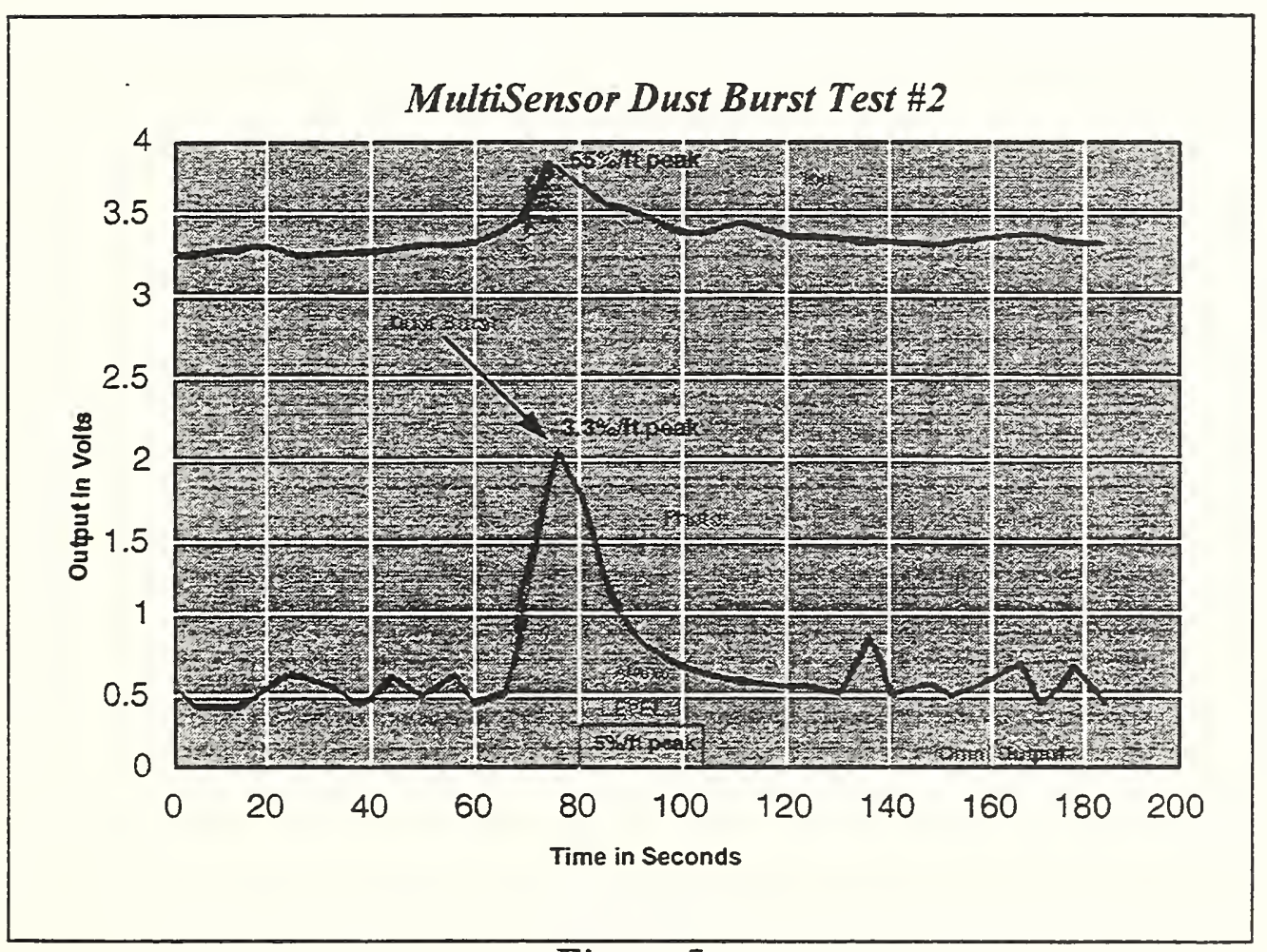

Figure 5 


\section{Discussion}

Henri Mitler: My thought, along the lines of your suggestion, is that we want to approximate what a human sensor does. What a human sensor does is it smells something or hears something or sees something. It then investigates and therefore, it seems to me that if it were not cost inhibited, the detector which upon noticing something, should begin a search pattern.

Ronald Mengel: I couldn't agree more, and I think that's some of the work that Dr. Grosshandler mentioned in his paper. This is more or less the pre-fire condition sensor that I think we need to get into if we are going to realize that sort of capability.

Question: It seems to be that you've pointed out a weakness in the development in the detector technology and that is that the certification process only requires that you detect fires and that there's really nothing in either of the U.S. or the European standards that require that you cannot detect non-fires. Do you see any prospects for bringing another test of a similar nature in a CL or CDN type standard and are you prepared to recommend what those tests will be?

Ronald Mengel: Industry has spent a good deal of time and effort attempting to find repeatable non-fire equipment. To date, we haven't had a great deal of success, and this is why we see the reluctance of the European fire community to accept anything at this point. 


\title{
FIRE DETECTION IN ATRIUM BUILDINGS
}

\author{
JUZO UNOKI \\ George KAWADA
}

NOHMI BOSAI LTD.

7-3, Kudan Minami 4-chome, Chiyoda-ku, Tokyo 102

\section{ABSTRACT}

With advance of building-construction techniques and for more effective use of land, new forms of buildings such as ones with extended height, deeper underground floor levels or larger space have been or are being built in Japan in recent years. This also applies to atrium buildings having large well space, and besides the comfort and convenience they offer to occupants and visitors in the premises, their use tends to be more diversified as seen in similar structures such as large-scale exhibtion hall and dome stadium.

While admitting merits and advantages of the atrium space, one should be aware on the other hand that there is a danger that it could become a big weak point in fire protection for the building premises. If a fire breaks out in the atrium, smoke rapidly spreads into upper floors through the atrium space, causing a serious hindrance in the way of occupants' escape from the premises. Therefore, it is necessary to detect a fire in its early stage and to facilitate fire suppression, for which an effective system configuration with fire detection and extinguishing systems must be planned for the atrium. This paper introduces the fire detection systems installed in the atrium buildings and typical examples of their installations in Japan.

\section{Fire detection systems in atria}

As compared with occupied spaces in ordinary buildings, the atrium has a much higher ceiling and far greater space volume. Therefore, even if conventional spot type smoke detectors or heat detectors are installed on the atrium ceiling, we can hardly expect their effect because smoke and heat generated from fire in its incipient stage are diluted to undetectable smoke density and airflow temperature levels before they reach the detectors. Furthermore, in the case of an atrium with its ceiling constructed with a transparent or semi-transparent material to admit natural light, the temperature in the space close to the atrium ceiling is high due to solar radiation and influence of the air conditioner and creates a hot zone, which might possibly prevent the updraft from the source of fire from reaching the ceiling. In view of these considerations including their maintenance and inspection the following fire detection systems have been installed for fire protection of the atrium buildings.

\section{(1) Projected beam type smoke detector}

This detector monitors a space over a long distance and detects smoke in a wide range. Therefore, it can detect a fire in its early stage even if smoke spreads in the entire space. 


\section{1) Sensitivity and installation standard}

The projected beam type smoke detector operates on obscuration of the beam by smoke. This obscuration is related to the beam length as per the Lambert-Beer Law, and accordingly the longer the distance is, the higher obscuration becomes in the same smoke density. Therefore, it is the considered necessary to adapt the sensitivity of the projected beam type smoke detector to a monitoring distance (beam length) and to review the installation standard which determines horizontal spacing between the detectors for effective and early detection of fire occurring in one supervisory zone. A number of fire tests have been conducted by fire research bodies to measure smoke distribution and densities near the ceiling surfaces. Fig. 1 shows distribution of smoke from a smouldering wood fire generating little heat measured below the ceiling surface. Fig.2 shows results of the test indicating the relationship between monitoring distances and obscurations. From these test data it has been found that a fire can be well detected if the distance between the fire point and the detector beam is less than 7m. Accordingly, it has been decided that the space between the detectors shall not exceed $14 \mathrm{~m}$. The test data also indicated detector sensitivities of $30 \sim 60 \%$ as being appropriate for the monitoring distance of 29m, and 15 30\% for the monitoring distance of $5 \mathrm{~m}$.

Based on these findings, the monitoring distances related to the sensitivities of the detectors have been determined as shown in Table 1.

As for siting of the detectors in the atria, it has been recommended to install two detectors, i.e. one above the other in spaces with a height of 20 60m, and three detectors, i.e. one each at the upper, intermediate and lower levels in spaces exceeding 60m in height.

\section{2) Design}

The detector comprises a light transmitter and a light receiver which are installed at a distance between $5 \mathrm{~m}$ and $100 \mathrm{~m}$ from each other. It detects reduction in the light reaching the receiver due to obscuration of the beam by smoke and transmits a fire signal to a control panel. Fig.3 is a block diagram of the projected beam type smoke detector.

\section{3) Functions}

\section{a. Sensitivity setting}

The smoke sensitivity of the detector can be set to a predetermined value by setting the sensitivity switch to the corresponding sensitivity depending upon the monitoring distance between 5m and 100m. (Table 1)

\section{b. Sensitivity compensation}

The detector has a compensation function to avoid any change in the smoke sensitivity until the light reaching the receiver is reduced to about 50\% of the initial light intensity due to contamination of the lenses of the light transmitter and the light receiver or disalignment of the beam caused over an extend period.

\section{c. Trouble alarm}

A trouble alarm is initiated to a control panel at the time the received light reaches $50 \%$ of the initial light intensity due to open in the dete- 
ctor power supply line, momentary interruption of the beam or contamination of the lenses of the light transmitter and the light receiver.

d. Remote operation test

The operation test of the detector can be carried out by means of the test switch provided on the control panel or close to the detector.

\section{e. Indications}

The detector gives a fire alarm indication, a trouble indication, a power-on indication and a remote operation test indication by means of LEDs.

\section{(2) Flame detectors}

With adoption of technical standards for flame detectors responding to radiant energy from flame in the Ministerial Ordinance of Fire Service Law of Japan, there has been increasing use of this type of detector for fire monitoring in the atrium space in recent years. The flame detectors are suited for early detection of a small flame occurring close to the floor surface of the atrium. They are roughly classified into the ultraviolet type flame detector and the infrared type flame detector which respectively detect ultraviolet and infrared radiation from flames. A flame detector which combines these two detecting principles and makes a fire judgement is also available. In this paper, the authors describe the infrared type flame detector.

\section{1) Design}

Flames developing in the course of combustion of substances contain many infrared rays of resonance radiation from carbon dioxide with peak wavelength of $4.3 \mu$ m and fluctuation of frequency within a range of $1 \sim 15 \mathrm{~Hz}$. The detector receives this wavelength through special optical filters, detects the frequency typical of flames by means of AC selective converter circuit comprising electric bandpass filters and sends a fire signal to a control panel. Fig.2 shows spectral distribution of different lights from flames. Fig. 3 is a block diagram of the detector.

2) Eunctions

\section{a. Sensitivity}

The detector detects an n-heptane fire in a vessel of 1 m ${ }^{2}$ within 30 seconds.

\section{b. Angle of view and monitoring distance}

The monitoring ditance is determined for every $5^{\circ}$ angle of view. As shown in Fig. 4 the detector has monitoring distances of $20 \mathrm{~m}$ in the front, 13m in the direction of $50^{\circ}$ on both sides of the front, and a conical monitoring range with maximum $100^{\circ}$ angle of view.

\section{c. Indication}

On operation of the detector, the response lamp lights up. 
(3) Fire scanning device

This device has come to be used recently for fire monitoring in large spaces such as a dome stadium. By converting the heat radiation energy from a fire to an electrical signal and determining its level, the device pinpoints the fire source and provides a fire extinguishing system such as a water cannon with information on the fire point location. The detector employs a method of locating a fire point by two-dimensional thermal image and is capable of monitoring a wide plane at a time.

\section{1) Design}

This device comprises an infrared image type fire detector and a control panel. The infrared image type fire detector uses an infrared camera and is equipped with an optical scanning means for two-dimensional detection of a fire. The detector is installed on a motor-driven swivel base, which, repeatedly rotates $180^{\circ}$ on both sides in about 25 seconds in normal monitoring state. Fig.7 is a block diagram of the fire scanning device. Fig. 8 shows the design of the infrared image type fire detector.

2) Functions

a. Sensitivities

The detector can be set to the following sensitivity levels. High sensitivity level $\cdots$ a level at which n-heptane fire in a vessel of $0.5 \mathrm{~m}^{2}$ is to be detected at a distance of $200 \mathrm{~m}$.

Low sensitivity lebel $\cdots$ a level at which n-heptane fire in a vessel of $1 \mathrm{~m}^{2}$ is to be detected at a distance of 200 m.

b. Monitoring for plural fires

The device is capable of continuing its monitoring for other fire sources after it has initiated a fire alarm.

c. Trouble alarm

A trouble alarm is initiated to a control panel on the part of the fire extinguishing system in case of troubles such as malfunction of any of the equipment and transmission error.

d. Remote operation test

The operation test of the fire scanning device can be carried out by means of the test switch on the control panel for the fire extinguishing system.

2. Reference of installations

\section{(i) Edo Tokyo Museum}

Edo Tokyo museum is a 7-storey building with 1 basement floor, and a total floor area of $45,000 \mathrm{~m}^{2}$. It has a unique design like a sky museum with an exhibition hall, storerooms, and a restaurant in the upper floors; con- 
ference rooms, multi-purpose halls and an administration office in the lower floors; and a various event space open to the air in the intermediate level. The exhibition hall situated on the upper part is supported by four pillars at a height exceeding 30m from the ground and forms a large atrium type space with a floor space of $12,000 \mathrm{~m}^{2}$ and a maximum ceiling height of 26m so that full scale large items can be exhibited.

For early fire detection the projected beam type smoke detector with a monitoring destance of 50m has been installed in the large space of the exhibition hall, and flame detectors whose sensitivity is not influenced by air current have been installed in the event space open to the air.

\section{(2) Fakuoka dome}

Eukuoka dome is a giant 7-storey building with a total floor area of $176,000 \mathrm{~m}^{2}$. It has an appearance of a cylindrical building which closely resembles the colosseum in the ancient Roman times and is covered with a hemispherical roof. It also has an outside diameter of 222m, an inside diameter of 200m and a height of $70 \mathrm{~m}$ from the arena floor surface to the top of the roof, which form a large scale space. A typical feature of Fukuoka dome is that it can flexibly be adapted to seasons and weather because the roof can be opened and closed. Therefore, besides its use as a baseball stadium, it can be used for multiple purposes such as concert hall having a maximum seating capacity of 50,000 audiences, exhibitions, assemblies etc. This arena space is monitored by means of the fire scanning device. The infrared image type fire detector has been installed at the highest position of the cylindrical part of the dome, i.e. at a height of $31 \mathrm{~m}$ from the ground and monitors the entire area of the arena of 200m in diameter by horizontally scanning the area at all times. For monitoring the non-supervised area directly below this fire detector, the flame de tectors have been installed. The fire scanning device is linked with a water cannon extinguishing system which automatically directs the cannon toward the fire point and controls the direction and pattern of water discharge to effectively extinguish the fire with the fire alarm and information on location of the fire received from the fire scanning device.

\section{Conclusion}

The authors have described the actual state of fire detection in atrium buildings in Japan. With advance of building-construction techniques, it is assumed that the forms of atria will be more diversified in the future. Under the circumstances we must promote development of new fire protection technologies for atrium buidings which take account of not only fire detection but also its effective combination with fire extinguishing means. Since the atrium space would have more different construction from the ordinary building space, it is also important, when discussing the fire protection for atrium buildings, to establish an organic cooperative system with ventilation system and other associated building management systems in each individual case rather than attempting to deal with this matter by fire protection systems alone. Therefore, it will be an important theme to be discussed in the future in an endeavor to find a way to collectively use all these systems on the basis of prompt and appropriate judgement of the situations in the premises. 


\section{PEFERENCES}

1) T. Yamada, Fire Safety Measures in Atrium, Fire Science Seminar, October 1993

2) Report on Investigation concerning Fire Prevention Measures for LargeScale Buildings, Special Buildings etc., Tokyo Fire Department, March 1992

3) Report on Fire Tests of Projected Beam Type Detector, LS Research Team, December 1981

4) J. Unoki, Fire Safety Plans for Museums in Japan, Japan-China Museum Property Preservation Symposium, August 1995

5) Fukuoka Dome, AYUMI No. 138, June 1993, internal periodical publication 


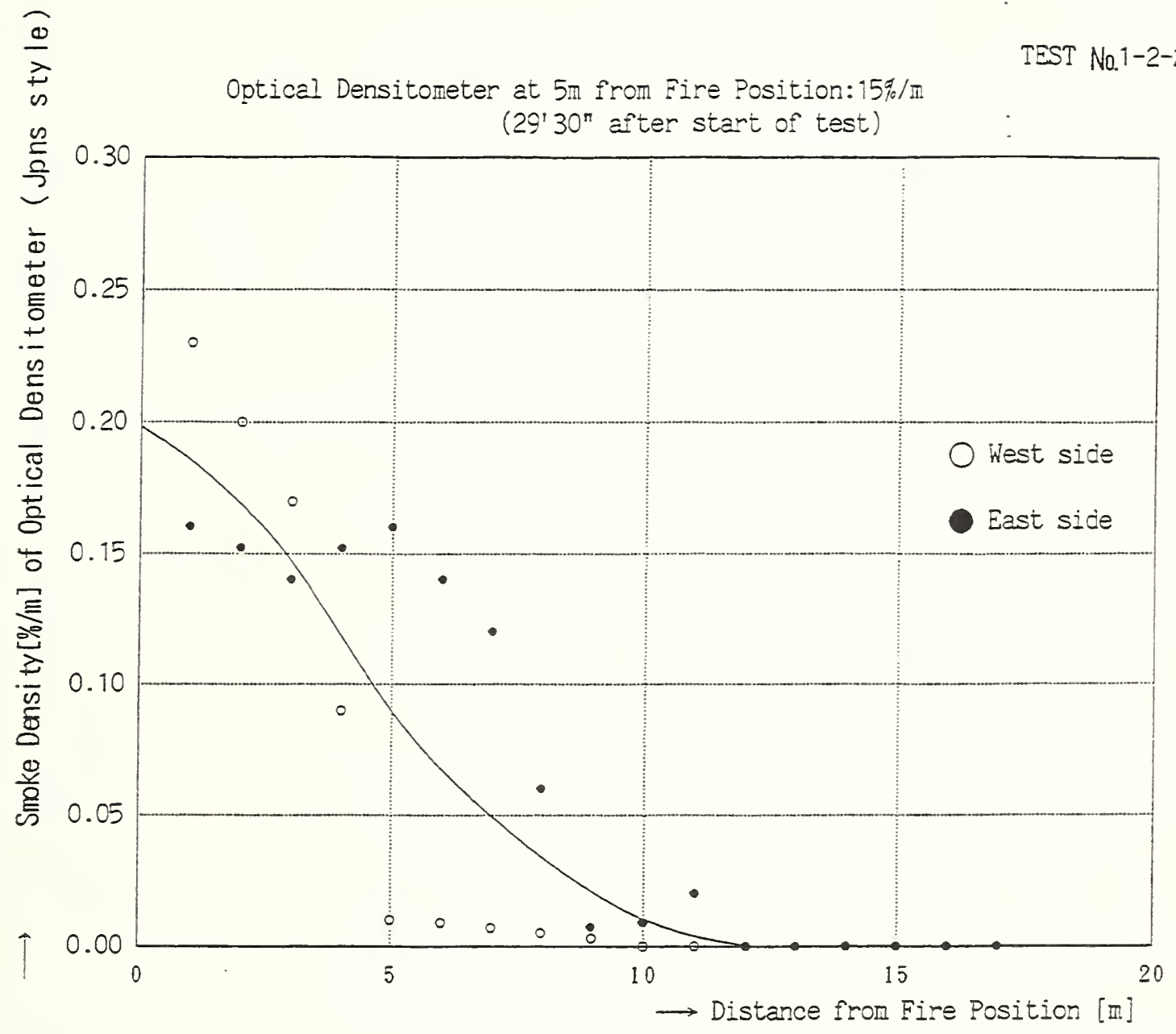

Fig. 1 Smoke distribution on ceiling surface during smouldering of wood 
Optical Densitometer at $5 \mathrm{~m}$ from Fire Position:15\%/m

(29' $30^{\prime \prime}$ after start of test)

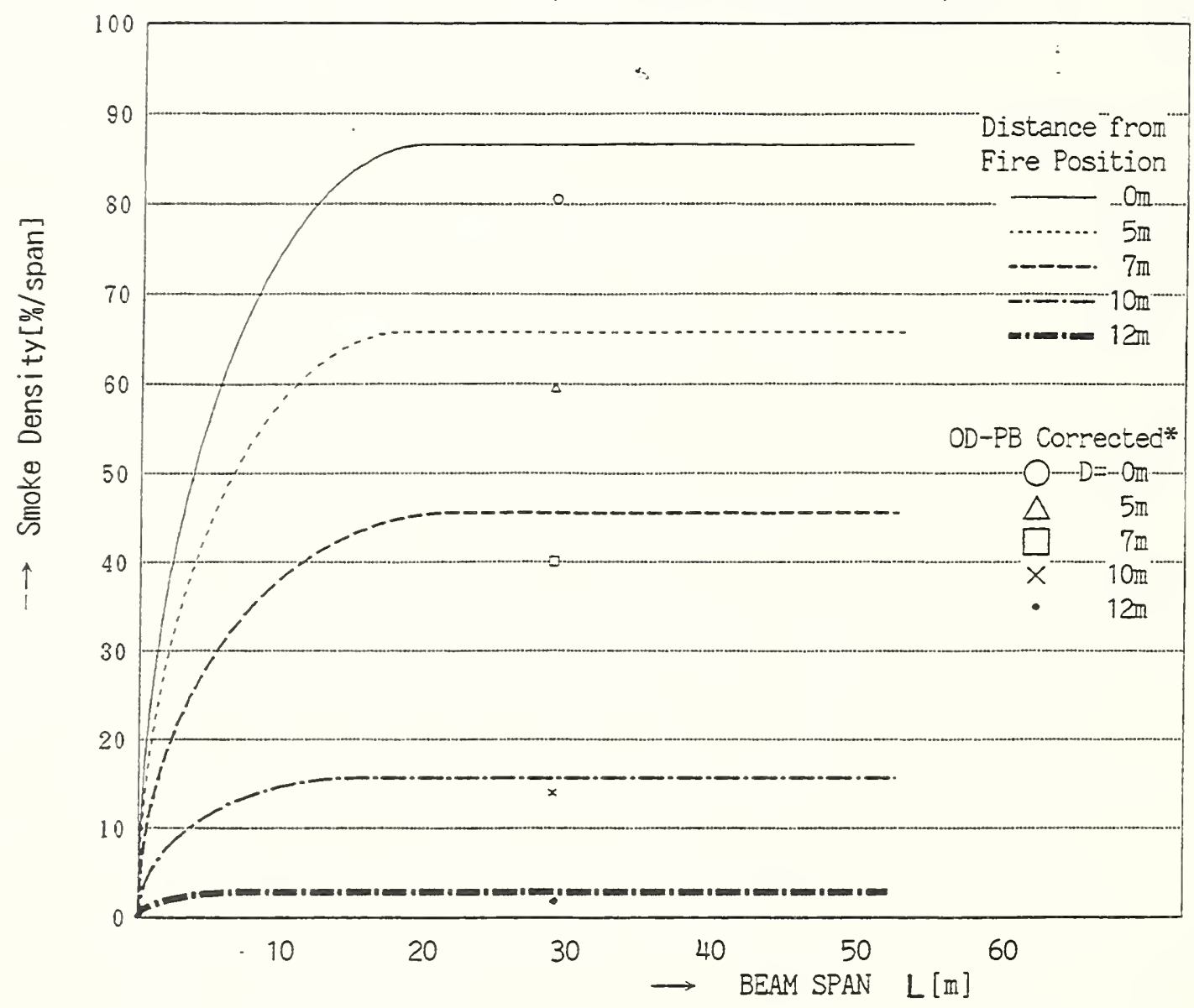

Fig.2 Monitoring distance v.s. Smoke Density of Projected Bean Smoke Detector Expected

*OD-PB Corrected : Opeical Densitometve - Projected Beam Smoke Detector Correction

\begin{tabular}{|c|r|r|}
\hline Type & Ordinary type, Class 1 & Ordinary type, Class 2 \\
\hline Monitoring & $5 \sim 20 \mathrm{~m}: 20 \%$ & $5 \sim 20 \mathrm{~m}: 30 \%$ \\
distance & $20 \sim 40 \mathrm{~m}: 30 \%$ & $20 \sim 40 \mathrm{~m}: 50 \%$ \\
sensitivity & $40 \sim 100 \mathrm{~m}: 50 \%$ & $40 \sim 100 \mathrm{~m}: 70 \%$ \\
\hline
\end{tabular}

Table 1 Monitoring distances and sensitivities of projected beam type smoke detectors 


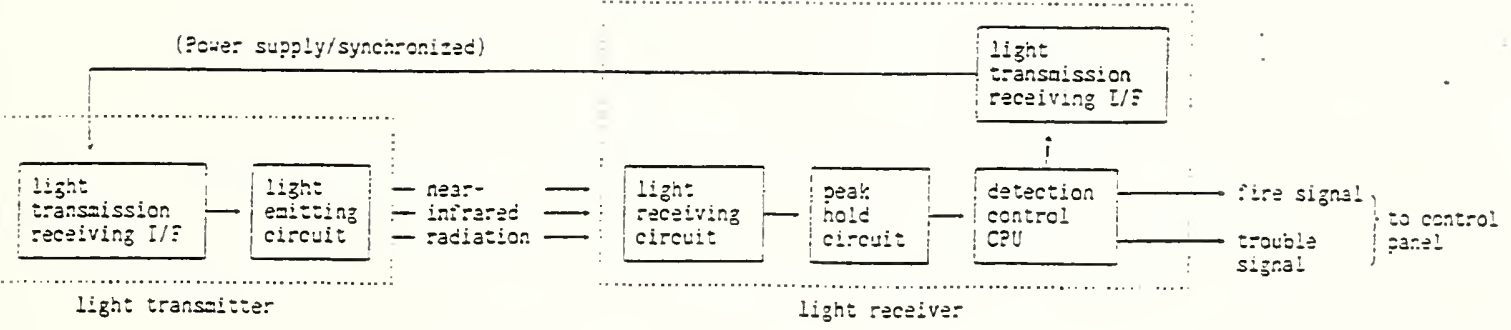

Fig.3 Block diagram of projected beam type smoke detector
(a) candle flame
(b) gasoline fire
(c) town gas fire
(d) sunlight
(e) incandescent lamp
(d) Hot substance

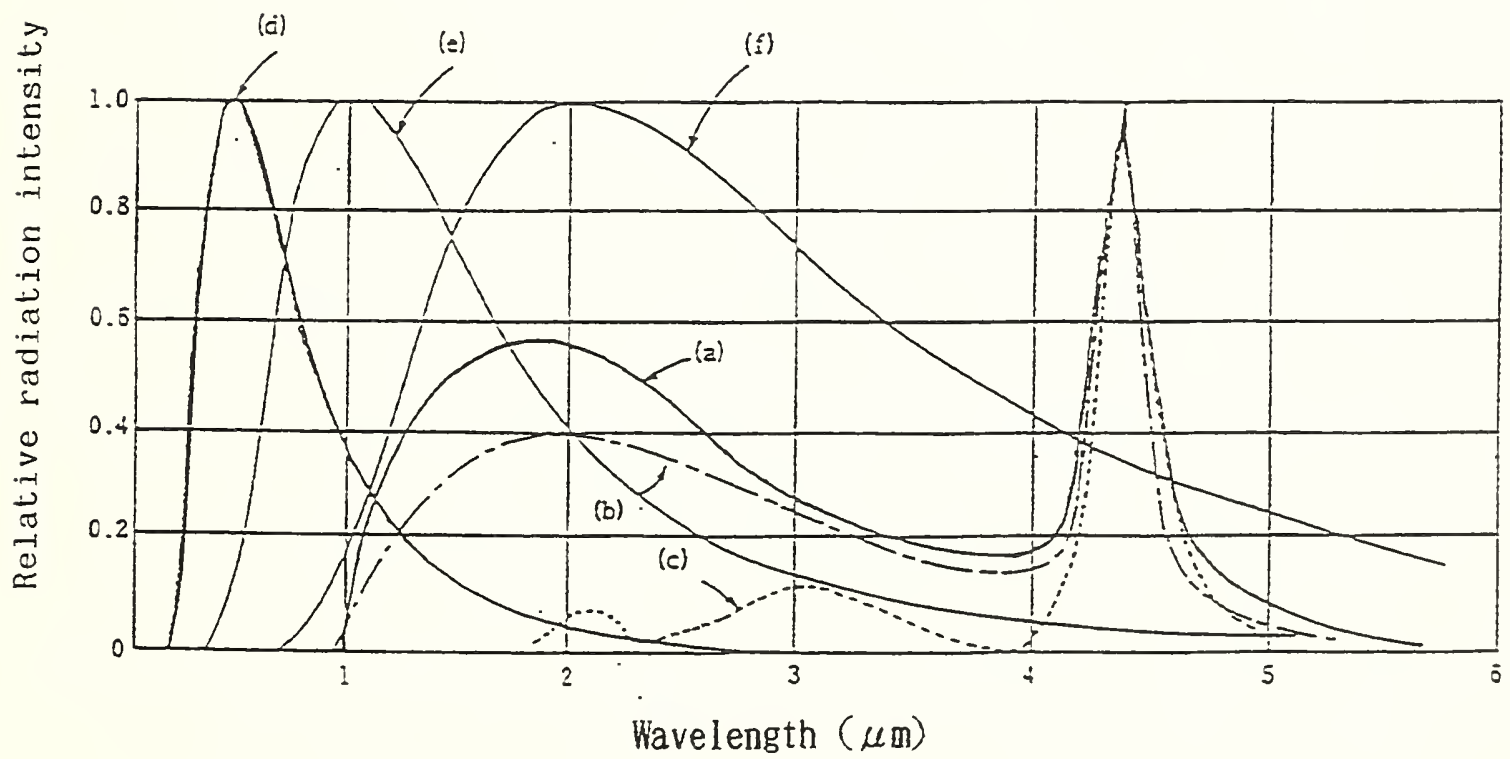

Fig. 4 Spectral distribution characteristics 


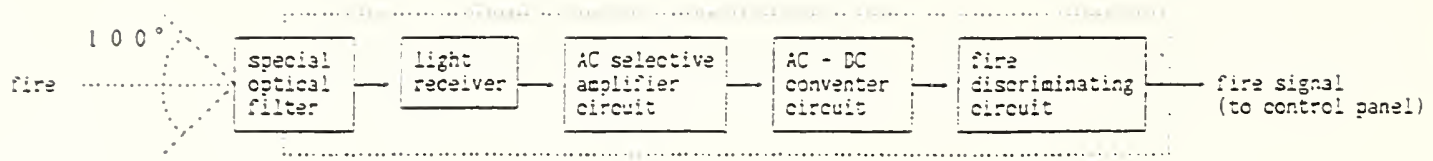

Eig.5 Block diagram of flame detector

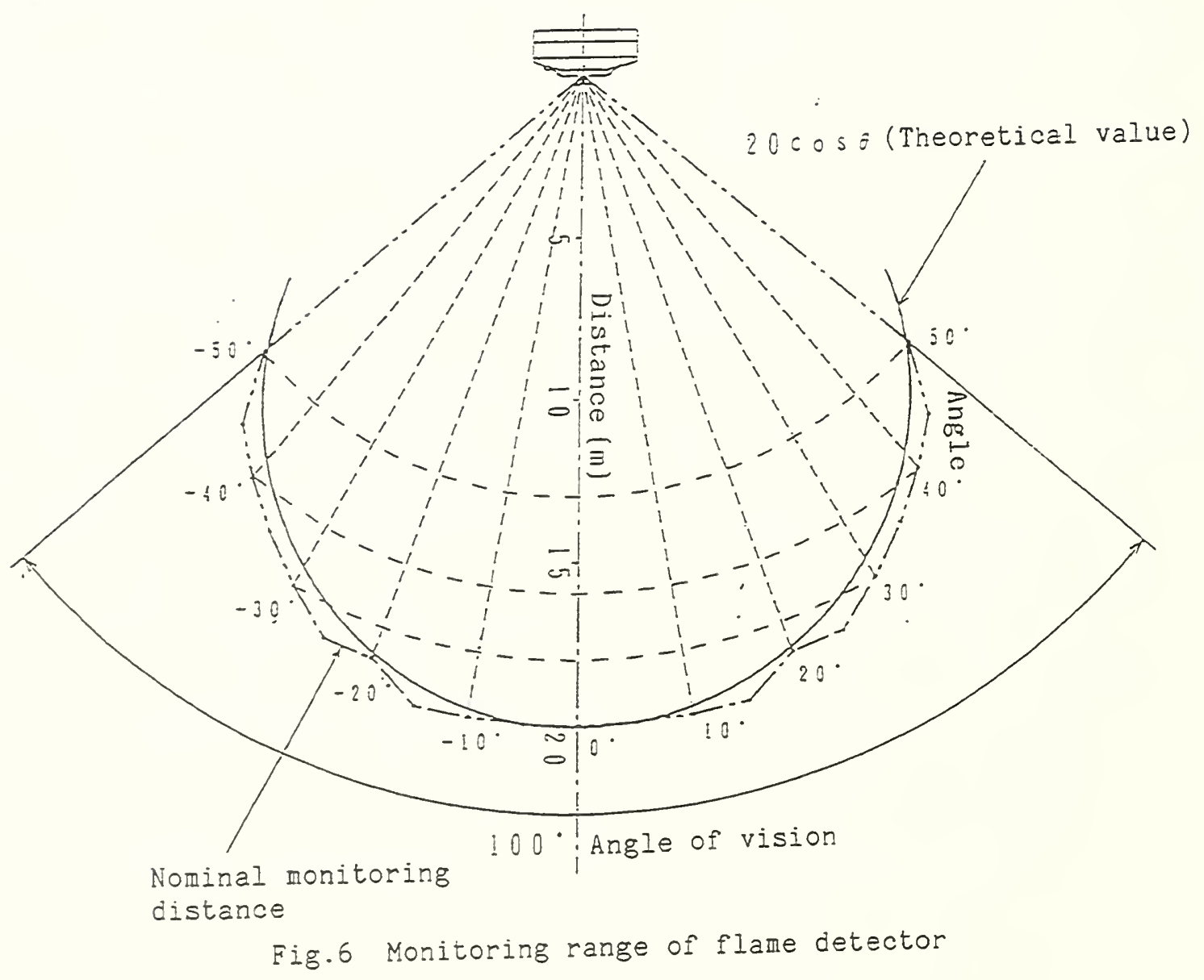




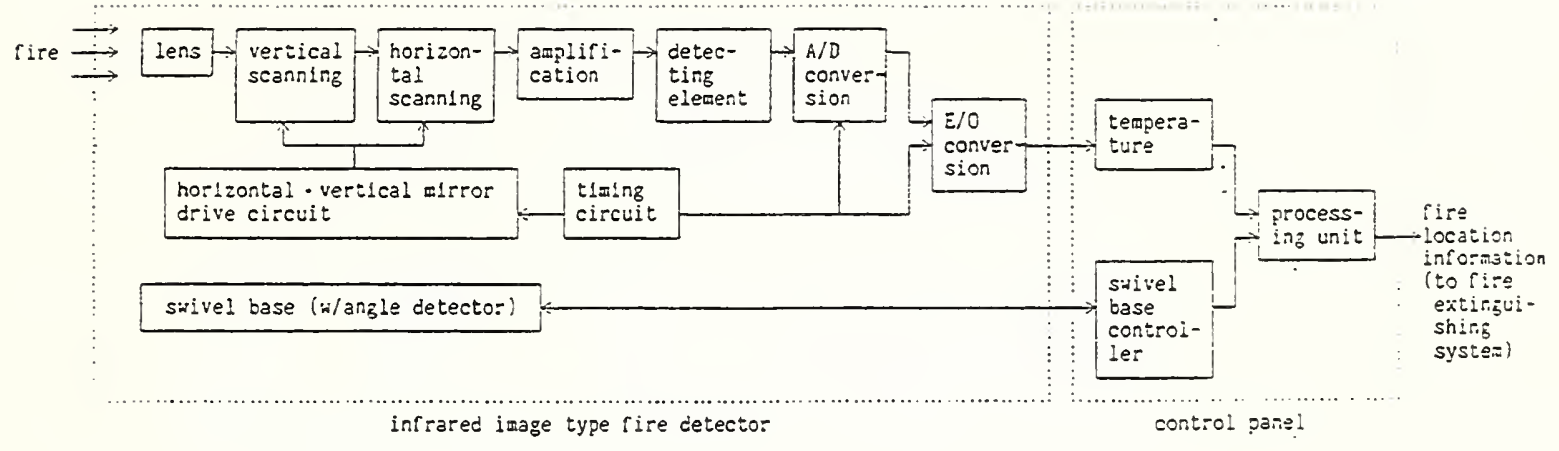

Fig.7 Block diagram of fire scanning device

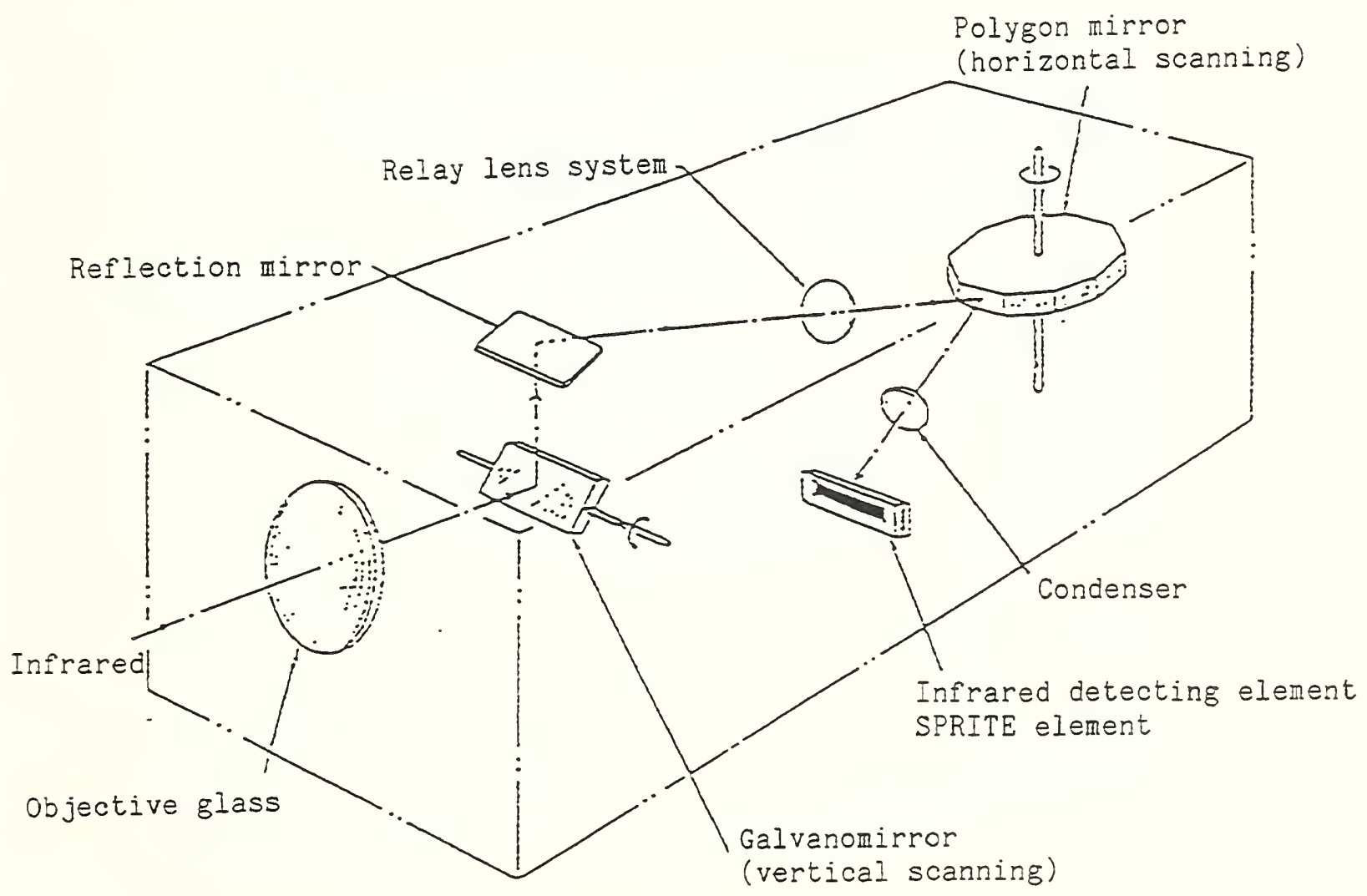

Fig.8 Design of infrared image type fire detector 


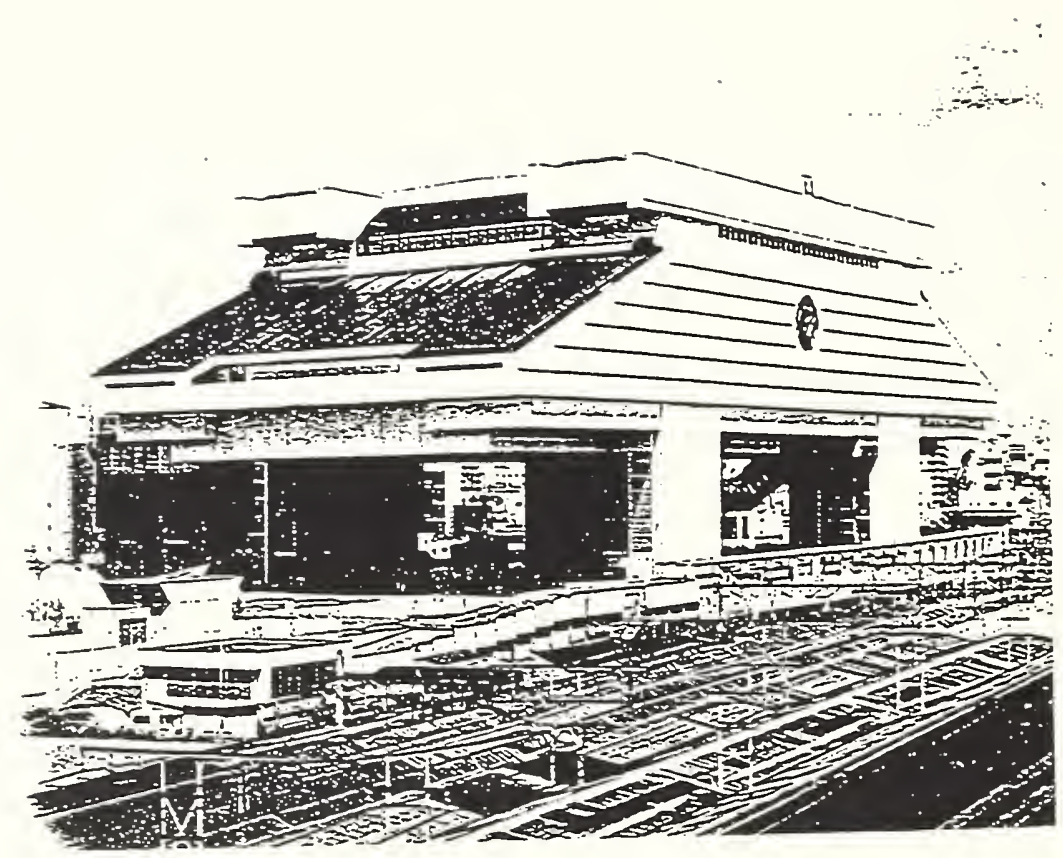

Photo 1 Edo Tokyo Museum

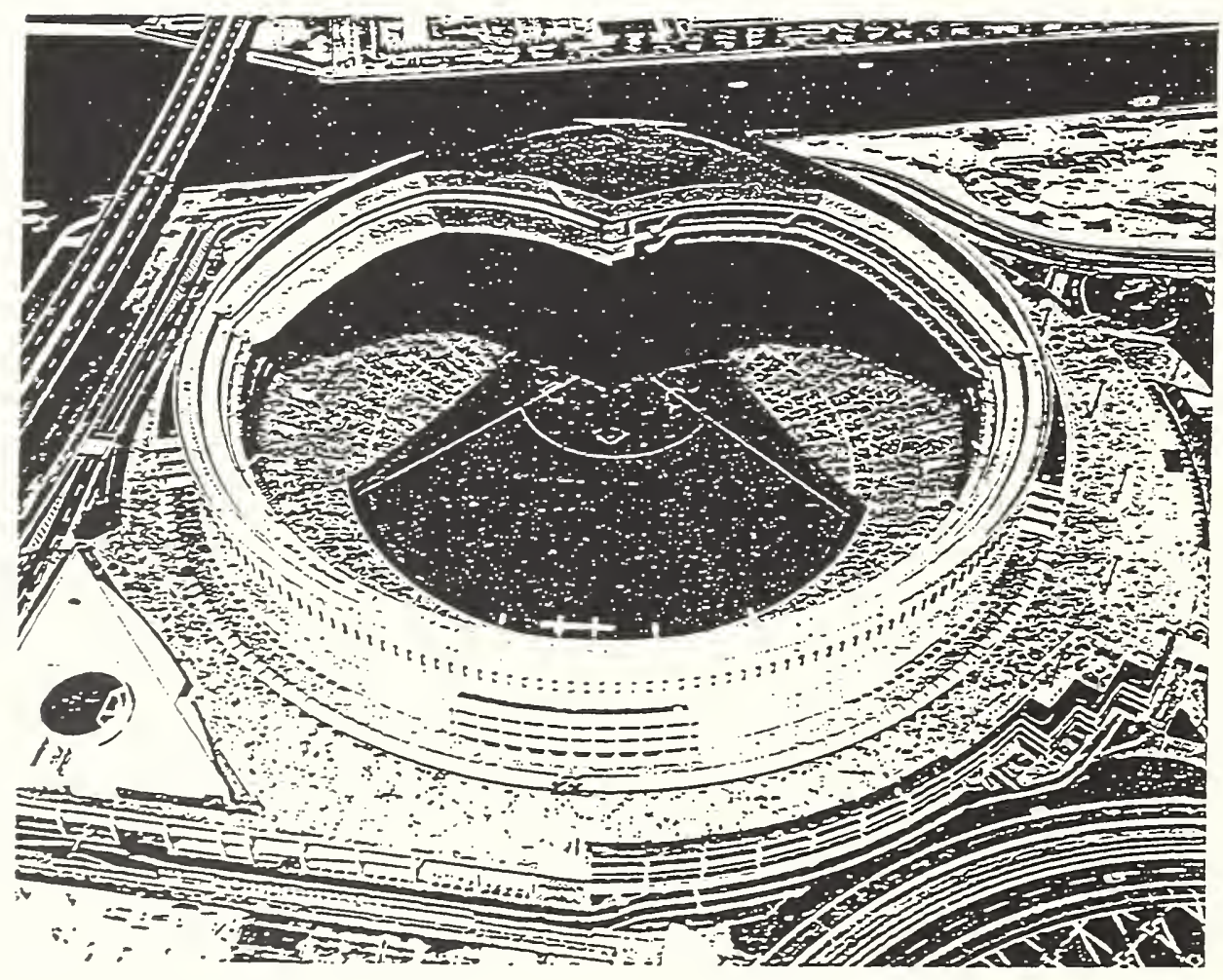

Photo 2 Fukuoka dome 


\section{Discussion}

Walter Jones: Do I understand that this moving camera is currently in use in such buildings?

Joji Kawada: This is the actual camera which is installed in an atrium. 
410 


\author{
Multivariate Methods for Fire Detection \\ James A. Milke and Thomas J. McAvoy \\ University of Maryland, College Park, MD
}

\begin{abstract}
Research is being conducted to describe the characteristics of an improved fire detector which promptly reacts to smoke while discriminating between smoke and odors from fire and nonfire sources. Discrimination is accomplished by comparing signature response patterns from fire and environmental sources collected in small- and large-scale tests. Smoke and odors are produced in the tests from a variety of conditions: flaming, pyrolyzing and heated samples, and nuisance sources, such as aerosols, household products and cooked food. Measurements include light obscuration, temperature, mass loss, $\mathrm{CO}, \mathrm{CO}_{2}, \mathrm{O}_{2}$ and oxidizable gas concentrations. The feasibility of an elementary expert system to classify the source of the signatures from small-scale experiments was demonstrated in the first phase. In the second and third phases, an expert system was developed by Multivariate statistical methods to distinguish between fire and non-fire sources. In addition, the presence of a fire could be identified despite the interjection of signatures from nuisance sources which could mask the fire signatures.
\end{abstract}

\title{
Introduction
}

Prompt fire detection is the primary objective of automatic fire detection. The time to detection can be increase by increasing the sensitivity of the detector. However, a highly sensitive detector may be prone to provide unnecessary alarms because contemporary smoke detectors cannot discriminate between smoke and odor sources. Data from U.S. fire incidents during the 1980's indicates that $95 \%$ of all alarms from smoke detectors were unnecessary [1]. One solution proposed for minimizing unnecessary alarms without sacrificing prompt activation involves using intelligence along with combinations of current sensor technology.

Research is being conducted via an interdisciplinary team at the University of Maryland to determine the characteristics of a sensitive, discriminating detector. The research is being conducted by teams in the Departments of Fire Protection Engineering and Chemical Engineering. The fire protection engineering team is concentrating on identifying signatures from fire and non-fire sources. The chemical engineering team is applying Multivariate statistical methods to investigate the sensor response patterns and provide the discrimination capability between fire and non-fire sources. This effort has been conducted in three oneyear phases.

\section{Small-scale Experimental Program}

Initially, small-scale tests were conducted to characterize the signatures from fire and non-fire sources [2]. The experiments were designed to be conceptually similar to those by Okayama 
[3], with modifications incorporated to provide a greater range of measurements for describing the signature.

The small-scale experimental apparatus was a simplified tunnel which included measurement equipment (light obscuration, temperature, gas species concentrations $\left(\mathrm{CO}, \mathrm{CO}_{2}\right.$ and $\left.\mathrm{O}_{2}\right)$ and presence of any oxidizable gas) and a means for generating odors. The presence of oxidizable gases was measured by a Taguchi metal oxide sensor. Sources of the smoke or odor were placed under a hood at the inlet end of the apparatus. A variety of fuels and environmental sources were selected to be representative of a residential environment. Smoke and odors were produced from a wide range of conditions: samples with flaming and pyrolyzing combustion, heated samples and aerosols.

An elementary expert system successfully classified 28 of 31 sources. The rules of the expert system were:

- $\mathrm{CO}_{2}$ concentration exceeds $1500 \mathrm{ppm}$ only for flaming fires

- Peak CO concentration exceeds 28 ppm and Taguchi detector response less than $6 \mathrm{~V}$ is acquired only for pyrolyzing solids.

- All other combinations are acquired from nuisance sources.

An ellipsoidal neural network was applied to the small-scale data, using two-thirds of the data for training and the remainder for testing [4]. An improved classification rate was obtained, accurately classifying all sources except one smoldering source (which was classified as a flaming source).

The level of success attained from the small-scale experimental program confirmed the feasibility of the concept presented by Okayama. However, the success of the expert system and neural network only related to the limited range of fuel sources investigated and the small-scale test apparatus.

\section{Large-Scale Experimental Program}

In the second and third phases, large-scale experiments were conducted to determine whether the trends identified in the small-scale experimental effort were also applicable in large-scale environments. The large-scale experiments were conceptually similar to the small-scale experiments where signatures from a wide variety of fires and environmental sources were monitored and sensor response patterns were explored. In the second phase, either fire or non-fire sources were introduced alone. In contrast, in the third phase mixed sources including both fire and non-fire sources were provided simultaneously.

The large-scale experiments were conducted in a $3.6 \times 3.6 \mathrm{~m}$ room with a height of $2.4 \mathrm{~m} \mathrm{[5]}$. Measurements included temperature, mass loss of the fire sources, $\mathrm{CO}, \mathrm{CO}_{2}$ and $\mathrm{O}_{2}$ concentrations, light obscuration and the voltage output from two metal oxide sensors 
(Taguchi model 822 and 880 ). In addition, two commercial smoke detectors (one photoelectric and one ionization) were located on the ceiling, at the center of the room. A diagram of the room, including the relative locations of the sensors is provided as Figure 1.

The metal oxide sensors responded to the presence of oxidizable gases and environmental odors respectively. Mass loss measurements were used to estimate the yield fractions of the signatures from the fire sources. Because the tests were conducted in an unconditioned space, data was collected for at least two minutes prior to introducing any source in order to document ambient conditions.

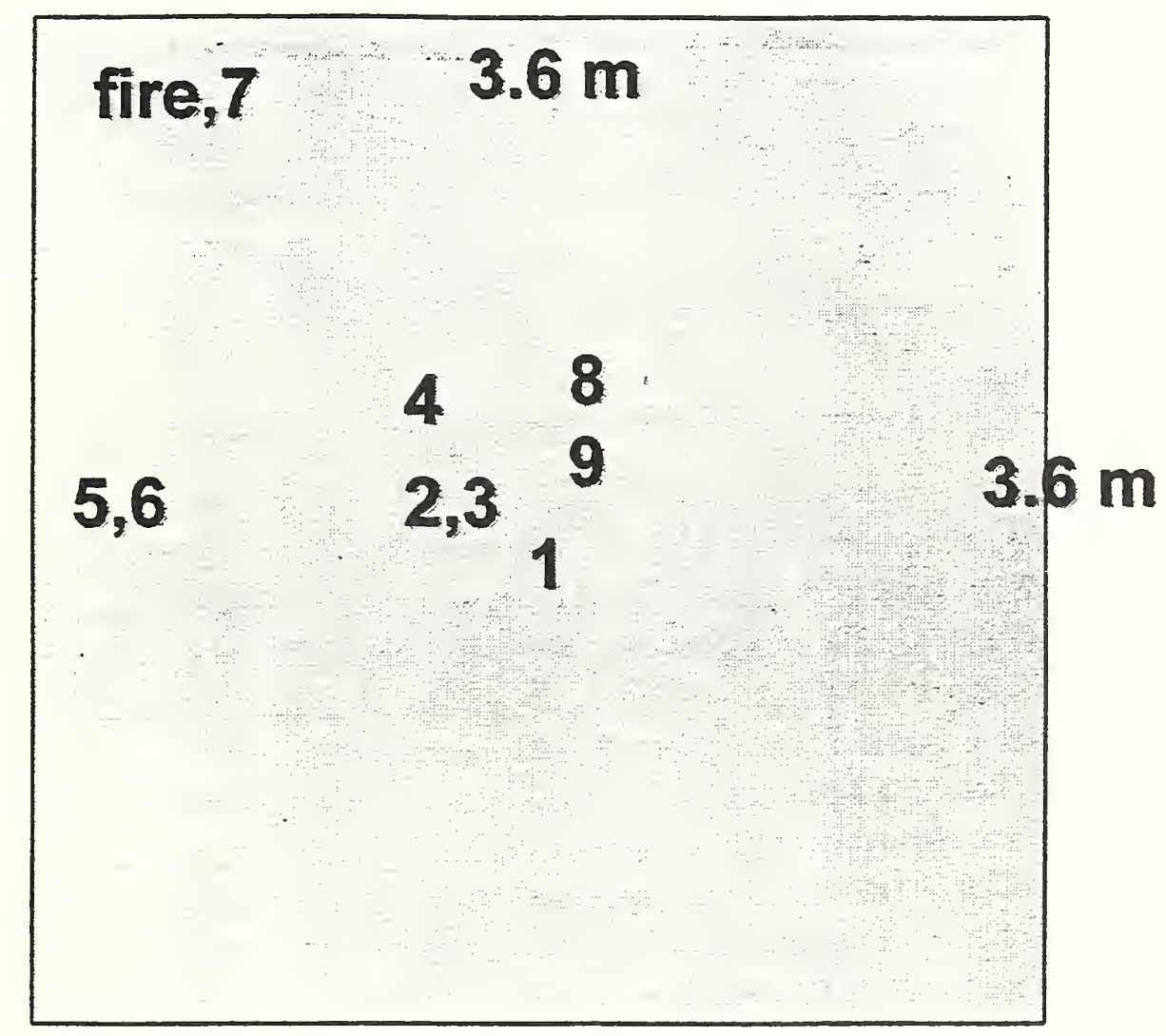

1. Thermocouple tree

3. Taguchi 880

5. Photocell

7. Load cell

9. Photoelectric smoke detector
2. Taguchi 822

4. $4.75 \mathrm{~mm}$ copper sampling tube

6. Helium-neon laser

8. Ionization smoke detector

Figure 1. Diagram of Test Room 


\section{Single Source Experiments}

The variety of sources used in the second phase to generate conditions within the room are summarized in Table 1. The sources were intended to be representative of fire and nuisance sources in residences. The 87 tests involved introducing 34 flaming sources, 16 smoldering sources and 37 nuisance sources.

Table 1. Test Sources

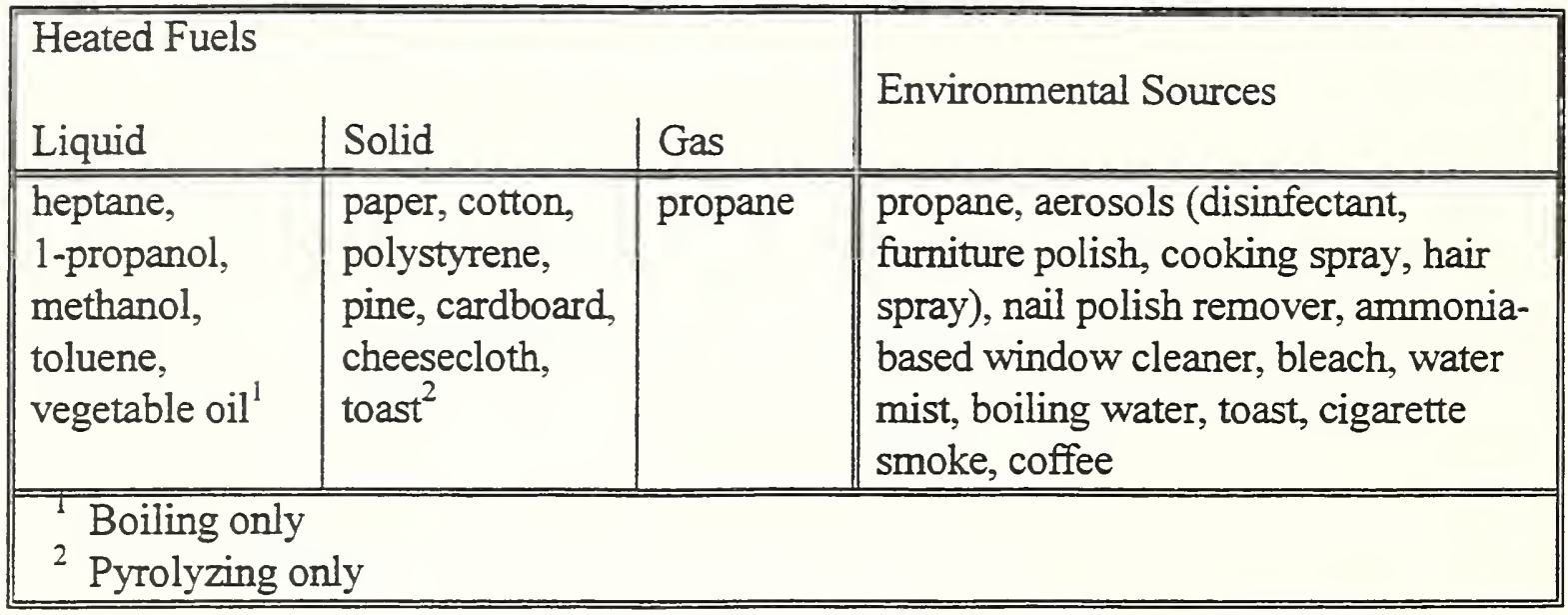

Data from the sensors is reviewed for the purpose of identifying patterns associated with the categories of sources. General trends are noted from a manual review of the maximum values recorded for each sensor. An expert system similar to that developed for the data from the small-scale tests is developed. In addition, a Multivariate statistical analysis is applied to the maximum values recorded for each sensor during each test to identify the nature of the source. The type of statistical analysis, a principal component analysis (PCA) makes use of the experimental maxima, arranged in a data matrix, $\mathbf{X}$ [6-8]. Each row of $\mathbf{X}$ consists of one set of readings for all $m$ sensors of the $x_{i}$ variables under consideration. The number of rows in $\mathrm{X}$ equals the number of experiments. PCA determines the linear combinations of the maxima that are capable of explaining most of the variations in the measurements. The linear combination are called scores, $t_{i}$, and the number of $t_{i}$ 's used is typically much smaller than the number of sensors. These scores are used to reconstruct the raw sensor measurements. The squared difference between the raw sensor values and the reconstructed values is called the squared prediction error (SPE). The SPE is used to detect abnormal situations [7]. Both the scores and the SPE reflect all of the sensor measurements because both the scores and the $\mathrm{SPE}$ involve data compression as well as synthesis.

Measurements from the following six sensors used in the tests are applied to develop the PCA model: $\mathrm{CO}, \mathrm{CO}_{2}$, two Taguchi sensors (T880 and T822), temperature and smoke obscuration. The data for each sensor is scaled to zero mean and unit variance. The data collected from each sensor prior to the introduction of the source is used to establish normal, 
background conditions for that test. Three PCA components explain approximately $76 \%$ of the variability in the normal data. Consequently, three components are used to classify the sources.

The SPE is used to flag abnormal situations, with its confidence limit set at $99.5 \%$. The SPE is successful in identifying all 87 tests as differing from normal conditions. The scores $\left(t_{i}\right)$ are used to distinguish the type of source, using the following rules:

- if $t_{3}>5$, then the source is a flaming fire

- if $-8<t_{2}<0$, then the source is a smoldering fire

- otherwise the source is a nuisance source.

The results of applying the above rules are summarized in Table 2. All of the flaming sources are properly classified, with smoldering sources classified properly in $88 \%$ of the tests and nuisance and ambient sources classified properly in $73 \%$ of the tests. $27 \%$ of the nuisance source cases are misidentified as smoldering sources and hence represent false alarms. Improved classification is expected by increasing the number of sensors to respond to additional attributes of the signatures.

Table 2. Classification of Test Sources

\begin{tabular}{|c|c|c|c|c|c|}
\hline & \multicolumn{3}{|c|}{ Classification } & \multicolumn{2}{|c|}{ Summary } \\
\hline & Flaming & Smoldering & $\begin{array}{l}\text { Nuisance/ } \\
\text { Ambient }\end{array}$ & Total & $\%$ Correct \\
\hline Flaming Fire & 34 & & & 34 & 100 \\
\hline $\begin{array}{l}\text { Smoldering } \\
\text { Fire } \\
\end{array}$ & & 14 & 2 & 16 & 88 \\
\hline $\begin{array}{l}\text { Nuisance/ } \\
\text { Ambient }\end{array}$ & & 10 & 27 & 37 & 73 \\
\hline Total & & & & 87 & 86 \\
\hline
\end{tabular}

In addition to the improved classification rate, the time for detection of the sensors with the PCA-based intelligence (the "prototype detector") is significantly less than that for the commercial detector. The time required for detection is reduced an average of $109 \mathrm{~s}$, with the detection time for the prototype detector being 18 to $259 \mathrm{~s}$ less than that for the first responding commercial detector.

Mixed Source Experiments 
The variety of sources used in the third phase to generate conditions from combinations of sources within the room are summarized in Table 3. A set of baseline tests are conducted using each of the sources alone.

Table 3. Combinations of Fire and Non-Fire Sources

\begin{tabular}{|l|c|c|c|c|c|}
\hline & \multicolumn{5}{|c|}{ Non-fire Sources } \\
\hline Fire Sources & None & $\begin{array}{c}\text { Aerosol } \\
\text { Disinfectant }\end{array}$ & $\begin{array}{c}\text { Window } \\
\text { Cleaner }\end{array}$ & $\begin{array}{c}\text { Aerosol } \\
\text { Hairspray }\end{array}$ & $\begin{array}{c}\text { Boiling } \\
\text { Water }\end{array}$ \\
\hline None & - & $\mathrm{x}$ & $\mathrm{x}$ & $\mathrm{x}$ & $\mathrm{x}$ \\
\hline Heptane & $\mathrm{x}$ & $\mathrm{x}$ & $\mathrm{x}$ & $\mathrm{x}$ & $\mathrm{x}$ \\
\hline Paper & $\mathrm{x}$ & $\mathrm{x}$ & $\mathrm{x}$ & $\mathrm{x}$ & $\mathrm{x}$ \\
\hline Cloth & $\mathrm{x}$ & $\mathrm{x}$ & $\mathrm{x}$ & $\mathrm{x}$ & $\mathrm{x}$ \\
\hline Hamburger & $\mathrm{x}$ & $\mathrm{x}$ & $\mathrm{x}$ & $\mathrm{x}$ & $\mathrm{x}$ \\
\hline
\end{tabular}

A manual review of the data from the mixed sources indicates that the expert system from the previous phase can be amended to properly classify many of the sources. Application of the PCA approach to the data from the mixed sources is in progress.

\section{Summary}

As a result of the experimental effort, an early fire detector consisting of an array of sensors appears feasible, with discrimination provided by a Multivariate statistical analysis of the sensor responses. Additional research is necessary to compare the performance and cost of a detector using a limited number of sensors, including sensor types currently used in commercial detectors with a detector containing an array of sensors such as incorporated in this experimental investigation.

\section{Acknowledgments}

This project is supported by the Building and Fire Research Laboratory of the National Institute of Standards and Technology (NIST). Dr. William Grosshandler is the technical monitor.

\section{Selected References}

[1] Hall, J.R., "The Latest Statistics on U.S. Home Smoke Detectors," Fire J., 83, 1, 1989, $39-41$. 
[2] Denny, Samuel, "Development of a Discriminating Fire Detector for Use in Residential Occupancies," Report FP 93-07, M.S. Thesis, College Park, Fire Protection Engineering, University of Maryland, December 1993.

[3] Okayama, Y., "Approach to Detection of Fires in Their Very Early Stage by Odor Sensors and Neural Net", Proceedings of the 3rd International Symposium of Fire Safety Science, 1991, p. 955-964.

[4] Pan, D., "Applications of Pattern Recognition Using Neural Networks," M.S. Thesis, College Park, Chemical Engineering, University of Maryland, 1994.

[5] Hagen, B.C., "Evaluation of Gaseous Signatures in Large-Scale Test," Report FP 94-05, M.S. Thesis, College Park, Fire Protection Engineering, University of Maryland, December 1994.

[6] Dong, D. and McAvoy, T.J., 'Nonlinear Principal Component Analysis - Based on Principal Curves and Neural Networks," Computers and Chemical Engineering, in press.

[7] Kresta, J., MacGregor, J., and Marlin, T., "Multivariate Statistical Monitoring of Process Operating Performance," Canadian J. Chemical Engineering, 69, 35-47, 1991.

[8] Nomikos, P., and MacGregor, J., "Monitoring Batch Processes Using Multi-Way PCA," AIChE J, 1994. 


\section{Discussion}

Walter Jones: In your early tables on what you could attempt either correctly or incorrectly, there were a total of twelve at $80 \mathrm{~cm}$. Either you detected fire where there was not fire, or you did not detect a fire where there was one. Which is about a $15 \%$ false alarm rate. It appears to me it is considerably better than what exists now. However, the only downside to this would appear to be that you need multiple detectors. Given the earlier point by Mr. Mengel that price is the driving factor, is there any hope for multiple detectors again on this improvement?

James Milke: I agree that I think we showed the key advantages to multisensors being incorporated into the detectors. We have not been involved in any multiple sensors, however, and I would suspect at this point in time, such would be fairly expensive. However, there is an economy of scale that it is well recognized, so it may become economically more feasible later.

Question: I have to comment on your including toast as both a fire source and a false alarm. This raises a very interesting issue. In our testing, we also had toast as both a fire source and a false alarm source. The issue really is when the toast is burning in the toaster, when it's charcoaling in the toaster, you would probably consider it a nuisance, but when it transitions to flaming, which ours did in several tests, now it's a real fire source. Where's the line? And I think toast is not the only case where that happens. Another example that we saw is a pilot lamp. If you're just heating it up, it creates aerosols that are a nuisance, but if it heats too long it turns into a fire. I think this raises a very interesting point about nuisance alarms sources versus real alarm sources.

James Milke: I'd like to respond to your comment in two ways. One, is that our own classification of what's a nuisance, what's a non-fire source can be ambiguous by itself so that some of the false alarm rates we get are high. The other point that you make is that there is a possibility for the nuisance source to transition to something else and that is very difficult balance to try and obtain. 


\author{
William D. Davis, and Kathy A. Notarianni \\ Building and Fire Research Laboratory \\ National Institute of Standards and Technology
}

\title{
I. Introduction/Background
}

The National Aeronautics and Space Administration, together with the National Institute of Standards and Technology are in the third year of a five year project designed to set guidelines for fire protection in high bay facilities. There is a special need to address fire protection issues for high ceiling height (high bay) spaces. NASA has numerous high bay spaces that are used to perform a variety of functions. Many of these functions are critical to meeting the goals of the NASA strategic plan. Examples of high bay spaces at NASA include those used for clean rooms, shuttle simulators, assembly/storage, vacuum and vibration chambers, vehicle assembly, and/or testing facilities with payloads. These spaces represent some of the most difficult fire protection challenges in that detection of a fire in a large space may be delayed due to the distance smoke and products of combustion must travel to reach the detector, the large amount of ambient air for smoke dilution, the high dollar value of these spaces, and the low damage threshold of a clean room. Some of these spaces also involve forced air flow.

Accurate detector predictions are important in these large spaces, as timely detection of a fire is more difficult due to the distance heat and products of combustion must travel to reach sprinklers and detectors. Since fires frequently grow at an exponential rate, an increased time to detection results in larger fires to be suppressed by, e.g., an automatic sprinkler system. Even a modest uncertainty in the prediction of the activation time may lead to a large uncertainty in the fire size used to predict the hazard, a central element of many fire protection analyses. The issue of prediction of activation time was addressed during the first year of this project. Experimental measurements taken during a fire in a $30 \mathrm{~m}$ (100 ft) high aircraft hangar were compared to the predictions of the fire models DETACT-QS, LAVENT, and FPETOOL, and also to the predictions of a computational fluid dynamics (CFD) model, HARWELL FLOW3D. The CFD calculations, using modified $\mathrm{k}-\varepsilon$ parameters, provided the best agreement with experimental measurements. The CFD model was then used to model the hot gas movement in a NASA Goddard Space Flight Center high bay clean room. Information regarding detector placement and sensitivity was obtained as a result of this effort. A final report, "The Use of Computer Models to Predict Temperature and Smoke Movement in High Bay Spaces", NISTIR 5304, Dec 1993, was delivered to NASA. This report fully documents the modeling procedure, inputs and results.

In the second year of this study, NASA locations were surveyed to determine the number and size of existing high bay facilities, their usage, and criticality to the NASA mission. The survey identified special hazards, target fire sizes, existing detection and suppression systems, and the presence of forced air flows. Site visits were conducted to view high bay spaces at selected locations. Results of the survey were correlated to produce a matrix of NASA high bay spaces. 
CFD modeling was used during the third year of the study to determine the appropriateness of available detection and suppression systems for each type of NASA space. Based on the modeling results, guidelines were drawn up to indicate which detection and suppression strategies would be successful as a function of fire size, forced ventilation, and ceiling height. The activation of a suppression system was modeled, not the capability of the system to extinguish the fire.

\section{Survey Results}

A survey form was developed that identified the occupancy/use of the space, the primary concern with fire protection in the space, and any information on any special geometry, temperature, or activity concerns in this space.

Data were also collected to be used in the modeling of a space. Variables such as the ceiling height(s), whether or not the ceiling is flat, the depth and thickness of various beams below the ceiling, and the presence of ceiling vents and/or draft curtains affecting the smoke flow in the space and thus the detection of a fire. The rate of air flow, and the uniformity of the flow from floor to ceiling were used to calculate the effect of forced air flow on the plume.

Information on the type and amount of any materials contained in this space was collected so that special consideration could be given to, e.g., explosive or radioactive materials. An estimate of the maximum acceptable fire size, defined as the largest fire size tolerable in the space considering the dollar value loss potential and criticality to the NASA mission was collected so that suitable detection strategies could be determined. On the survey form, NASA engineers were asked to select a maximum acceptable fire size from one of three choices. Choices were a $50 \mathrm{~kW}$ fire (ex. a wastebasket fire), up to a $1 \mathrm{MW}$ fire (ex. a $1.5 \mathrm{~m}$ ( $5 \mathrm{ft}$.) floor area of $4.6 \mathrm{~m}$ (15 ft.) high storage of ordinary combustibles), or a greater than $1 \mathrm{MW}$ fire (ex. $>0.8 \mathrm{~m}$ diameter JP-4 fire).

Information on automatic smoke detectors (e.g., photoelectric, ionization, beam, and/or continuous sampling), heat detectors (e.g. fixed temperature, rate of rise, or combination), and flame detection (infrared or ultraviolet) is used to determine the adequacy of existing fire protection in the space. Information on whether the space contains an automatic extinguishing system, and what type, was also obtained for each space.

A plan view including the length and width of the space, the approximate location of ceiling vents and/or draft curtains, and the approximate location of ceiling detectors was drawn for each space. Also drawn for each space was an elevation which includes the ceiling height(s), the geometrical configuration of the ceiling, the direction of forced air flow, and the approximate location of wall detectors. This information was also needed for modelling of the space.

\section{Analysis}

Data from the surveys were collected and analyzed. A total of 70 high bay spaces were identified at 13 NASA locations. These are all NASA owned buildings and do not include 
buildings which NASA contractors own.

Most locations reporting radiation detectors had a combination UV/IR detector. The term thermal/smoke was used to refer to smoke detectors, heat detectors, and automatic fire sprinklers.

The high bay spaces were grouped by height into three categories: 18 to $26 \mathrm{~m}$ ( 60 to $85 \mathrm{ft}$ ), 27 to $37 \mathrm{~m}$ (90 to $120 \mathrm{ft}$ ), and over $37 \mathrm{~m}$ (120 ft). Most of the spaces in the inventory had flat ceilings.

There were a total of 36 spaces (51\%) between 18 and $26 \mathrm{~m} \mathrm{(60} \mathrm{and} 85 \mathrm{ft})$ high; $25 \%$ of these had no detectors, $39 \%$ had thermal/smoke detectors with no forced air flow, $22 \%$ had thermal/smoke detectors with forced air flow, and $14 \%$ had radiation detectors.

There were a total of 25 spaces (36\%) between 27 and $37 \mathrm{~m}$ (90 and $120 \mathrm{ft}$ ) high; $32 \%$ of these spaces had no detectors, $32 \%$ had thermal/smoke detectors with no forced air flow, $4 \%$ had thermal/smoke with forced air flow, and $32 \%$ had radiation detectors.

There were a total of 9 spaces (13\%) with ceiling heights greater than $37 \mathrm{~m}(120 \mathrm{ft}) ; 45 \%$ of these spaces had no detectors, $22 \%$ had thermal/smoke detectors with forced air flow, and $33 \%$ had radiation detectors.

Thirty nine percent of the structures had a maximum acceptable fire size of less than $50 \mathrm{~kW}$. Some interesting observations were that 7 structures having a maximum acceptable fire size of less than $50 \mathrm{~kW}$ had no detectors. Only 6 structures (9\%) reported a maximum acceptable fire size of greater than $1 \mathrm{MW}$. Nine structures (13\%) had forced air flow, $89 \%$ of which had ceiling heights between 18 and $27 \mathrm{~m}$ (60 and $85 \mathrm{ft}$ ).

\section{Site Selection for Modeling}

From the survey, it was decided that the bulk of the mission critical spaces were located at Kennedy Space Center. These spaces presented some of the most demanding fire detection problems due to the presence of hypergols and the associated hazards posed by the spill of these fuels. Based on the importance of the high bay structures to the NASA mission and the potential for useful information to be gained from the modeling, it was decided to run computer fire model simulations for an orbiter processing facility, and a payload processing facility. The loss of either of these facilities due to a fire would have a significant impact on the NASA shuttle schedule. Both of these structures are clean rooms with approximately $30 \mathrm{~m}$ (100 ft) high ceilings.

A third high bay to be modelled is a $26 \mathrm{~m}$ (95 ft) high hangar at Langley Research Center. This space has a sloping ceiling and includes draft curtains. While the maximum ceiling height is 26 $\mathrm{m}$, the sloping ceiling provides locations in the building where the ceiling height is as low as 18 $\mathrm{m}(60 \mathrm{ft})$.

\section{Computer Modeling}


Several fire scenarios were chosen to be analyzed for each high bay category. For clean rooms or in situations where hypergolic fuels were present, survey results suggested that fires be detected by the time their heat release rate had reached $50 \mathrm{~kW}$. In less hazardous situations, heat release rates of $1 \mathrm{MW}$ or more might be tolerated before detection. This talk focuses on the detection of t-squared fires reaching $50 \mathrm{~kW}, 1 \mathrm{MW}$, and $4 \mathrm{MW}$. It is assumed that a time of 100 $\mathrm{s}$ would be required for the fires to reach their maximum size. In addition, a rapidly growing fire to $50 \mathrm{MW}$ is analyzed. The $50 \mathrm{MW}$ fire would simulate a significant spill of hypergolic fuels from an orbiter payload.

The response of smoke, fusible link, heat, UV/IR, and obscuration detectors were modelled using algorithms developed for the CFD fire model using the fire scenarios given above. In the first two categories where ceiling heights range from $18 \mathrm{~m}$ to $37 \mathrm{~m}$ ( $60 \mathrm{ft}$ to $120 \mathrm{ft}$ ), the modelling was done for both forced and unforced air flows. The forced air flows were chosen to represent specific flows observed in NASA clean rooms. These flows typically originate from ceiling mounted supplies and exit at wall mounted returns near the floor. There were no clean rooms in the NASA inventory for the third category where ceiling heights exceeded $37 \mathrm{~m} \mathrm{(120} \mathrm{ft)} \mathrm{and}$ so forced air flows were not included in the analysis for this category.

This presentation will focus on the results of the modelling of heat, fusible links, smoke and obscuration detectors in high bay spaces. Expected ceiling temperatures and activation times are presented in the analysis of fusible link activation and heat detection at the ceiling of these spaces for the fire scenarios under consideration. An estimate of activation time for smoke and obscuration detectors is presented based on the predictions of the smoke movement models used in the calculations. In particular, the effect on smoke movement of forced air flow from ceiling mounted supplies in the orbiter processing facility and the payload processing facility will be discussed. In each of these facilities, workstands or the presence of the orbiter coupled with forced air flow complicate smoke movement and make detection strategies more challenging.

\section{Discussion}

James Quintiere: To come back to the point of scale models, it would seem that these facets of this problem could ideally be complimented by using the scale models. Have you thought of that?

William Davis: Well, you always have to have scale models. The analysis that will be done this year at NIST will probably dictate the experimental areas that need to be looked at. But certainly, scale models with their ability to be run many times are sometimes very preferable to a large-scale experiment which you can only conduct once or twice or three times with not as much control.

James Quintiere: I think the scale model could serve to validate your computer results better than the full-scale tests.

William Davis: Having the opportunity to use these full-scale tests, which weren't run for this particular project, is very useful. We need small-scale tests, and we certainly can't afford to buy a million dollars of full-scale tests. 
RESOLUTIONS

$42 \underline{3}$ 
$4 \underline{2} 4$ 


\section{RESOLUTIONS}

The members of the United States-Japan Conference on Development and Utilization of Natural Resources' Panel on Fire Research and Safety are quite pleased with the results of the $13^{\text {th }}$ Joint panel, held in Gaithersburg, MD, March 12 - 20. This panel continues to facilitate the exchange of important research ideas between two of the world's leading fire research communities. We greatly appreciate the support from Factory Mutual Research Corporation, Hughes associates, National Fire Protection Association, and Underwriters Laboratories. The Japanese Delegation generously supported the Kawagoe Symposium. We wish to thank the Building and Fire Research Laboratory, National Institute of Standards and Technology for its hospitality. We note that each time we meet, the quality of our technical communication improves. The MiniSymposia for Professor Kawagoe and Professor Zukoski were very informative and memorable. The following resolutions summarize the consensus reached.

It is hereby resolved that:

1. The objectives of the meetings of this panel are to:

a. exchange particularly interesting technical information regarding our latest research.

b. promote cooperative research on focused areas within fire safety science, such as: fires after earthquakes, performance based material development and testing, performance based fire codes, detection and fire suppression, fluid mechanics of fires, computer based fire modeling, burning behavior of real objects, and risk and hazard analyzes.

2. The next $\left(14^{\text {th }}\right)$ meeting of UJNR Panel on Fire Research and Safety will be held in May 1998 in Tokyo or Tsukuba. The panel members will utilize the International Association for Fire Safety Science meetings and other international meetings to informally exchange technical information in the interim.

3. Within 3 months, the Chairmen will come to consensus on the selection of specific focused area topics of strong mutual interest and support and appoint coordinators from each country for each topic. The coordinators will facilitate joint research efforts by identifying panel members who are particularly interested in their focused areas. The structure of the technical sessions at the next meeting will reflect the focused area topics and include pertinent research papers and in-depth discussion periods.

4. Our formal presentations will include expanded progress reports covering developments in broad areas of fire safety science research between meetings by both Japan and the United States. 
5. Recent United State-Japan joint research has achieved great success. We are pleased with the collaborations among Drs. Tanaka and Pitts; Drs. Hasemi, Zukoski and Quintiere; Drs. Yamada and Cooper; Drs. Sekizawa and Hall; Drs. Koseki, Evans, Madrzykowski and Mulholland, Drs. Baum and Yamada (FRI); and Dr. Saito (FRI) and Ms. Womeldorf (NIST). The panel proposes to continue such highly productive research.

6. Panel members are encouraged to exchange information regarding prospective experimental and database research with the purpose of soliciting useful comments. New technical information and new research reports issued in each country should be sent to all panel members as soon as they are available. We encourage informal communications among panel members by electronic media. It is important that all the relevant reports for the next meeting be sent in time to be received in the other country at least two months before the $14^{\text {th }}$ meeting. 


\section{AUTHOR INDEX}

VOLUME 2

B

Baum, H., 351

Borden, F., 303

C

Cleary, T., 375

D

Davis, W., 419

DeLauter, L., 345

E

Evans, D., 273

F

Fukuda, T., 91

G

Gann, R., 111

Gilman, J., 261

Grosshandler, W., 363,

375

H

Hamins, A., 119

Hasemi, Y., 161, 173, 225

Hayashi, Y., 3

Hirschler, M., 167

Hokugo, A., 283, 313

I
Iwata, Y., 235

J

Jones, W., 7, 61, 81, 105

K

Kaplan, C., 15

Kashiwagi, T., 167, 261

Kawada, G., 397

Kikuchi, R., 225

Koseki, H., 41
Kouzeki, D., 371

Kozeki, D., 145

Kurioka, H., 31

L

Lomakin, S., 261

Lyon, R., 249

\section{M}

Madrzykowski, D., 345

Marchal, A., 173

Mawhinney, J., 137

McAvoy, T., 411

McGrattan, K., 351

Mengel, R. 391

Milke, J., 411

Mochida, A., 3

Mowrer, F., 273

Murakami, S., 3

\section{N}

Notarianni, K., 419

O

Ohlemiller, T., 215

Ohmiya, Y., 69

Ohnishi, K., 337

$\mathbf{P}$

Peacock, R., 81, 105

Pitts, W., 51

Portier, R., 105

R

Rehm, R., 351

Reneke, P., 81

Richardson, J., 137

Ritchie, S., 261

Roadarmel, G., 345

$\mathrm{S}$

Saito, N., 115, 129
Satoh, H., 31

Scawthorn, C., 325

Sekizawa, A., 145, 289

Shaddix, C., 15

Smyth, K., 15

Sugawa, O., 31

Suzuki, K., 145

$T$

Takaike, R., 225

Takemoto, A., 145

Tamura, H., 371

Tanaka, T., 69, 91

U

Unoki, J., 397

W

Wakamatsu, T., 69, 91

Walton, W., 273

Y

Yamada, T., 21

Yamamoto, E., 225

Yanai, E., 145, 235

Yoshida, M., 173, 225 



\section{MANUSCRIPT REVIEW AND APPROVAL}

\section{INSTRUCTIONS: ATTACH ORIGINAL OF THIS FORM TO ONE (1) COPY OF MANUSCRIPT AND SEND TO THE} SECRETARY, APPROPRIATE EDITORIAL REVIEW BOARD.

\section{TITLE AND SUBTITLE (CITE IN FULL)}

Thirteenth Meeting of the UJNR Panel on fire Research and Safety, March $13-20,1996$

\begin{tabular}{l|l}
\hline OONTRACT OR GRANT NUMBER & TYPE OF REPORT AND/OR PERIOD COVERED
\end{tabular}

AUTHOR(S) (LAST NAME, FIRST INITIAL, SECOND INITIAL)

Beall, K.A., Editor
PERFORMING ORGANIZATION (CHECK $(X)$ ONE BLOCK) 叉 NIST/GAITHERSBURG

NIST/BOULDER

Z JILAVBOULDER

ABORATORY AND DIVISION NAMES (FIRST NIST AUTHOR ONLY)

Building and Fire Research Laboratory, Fire Science Division (865)

PONSORING ORGANIZATION NAME AND COMPLETE ADDRESS (STREET, CITY, STATE, ZIP)

ROPOSED FOR NIST PUBLICATION

JOURNAL OF RESEARCH (NIST JRES)

J. PHYS. \& CHEM. REF. DATA (JPCRD)

HANDBOOK (NIST HB)

SPECIAL PUBLICATION (NIST SP)

TECHNICAL NOTE (NIST TN)

MONOGRAPH (NIST MN)
NATL. STD. REF. DATA SERIES (NIST NSRDS)
$\square$ FEDERAL INF. PROCESS. STDS. (NIST FIPS)
LIST OF PUBLICATIONS (NIST LP)
NIST INTERAGENCYINTERNAL REPORT (NISTIR)

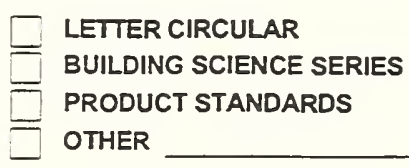

LETTER CIRCULAR

PRODUCT STANDARDS

- OTHER

ROPOSED FOR NON-NIST PUBLICATION (CITE FULLY

ZU.S. — FOREIGN

PUBLISHING MEDIUM
$\square$ PAPER
$\square$ DISKETTE (SPECIFY)
$\square$ OTHER (SPECIFY)

UPPLEMENTARY NOTES

BSTRACT (A 2000-CHARACTER OR LESS FACTUAL SUMMARY OF MOST SIGNIFICANT INFORMATION. IF DOCUMENT INCLUDES A SIGNIFICANT BIBLIOGRAPHY OR LTTERATURE SURVEY, CITE IT HERE. SPELL OUT ACRONYMS ON FIRST REFERENCE.) (CONTINUE ON SEPARATE PAGE, IF NECESSARY.)

the 13th meeting of the U.S.-Japan Panel on Fire Research and Safety was held at the National Institute of Standards and Technology March 13-20, 1996. The core of the meeting consisted of technical sessions on design/risk/hazard/performance standards, burning of real objects, xperimental refinement and validation of fire models, suppression, materials testing, Letection, and fires after earthquakes. The last of these topics took on special meaning in the wake of two disasters since the l2th meeting: a major earthquake in Northridge,

falifornia and the Great Hanshin-Awaji Earthquake on the largest Japanese island of Honshu. In addition, the meeting hosted two one-day symposia honoring two long-time principals of fire research in general and this UJNR Panel in particular. The first was in honor of Professor Edward Zukoski on the occasion of his retirement from the California Institute of echnology. The second was in memory of Professor Kunio Kawagoe of the Building Research institute and Tokyo Science University.

\section{KEY WORDS (MAXIMUM OF 9; 28 CHARACTERS AND SPACES EACH; SEPARATE WITH SEMICOLONS; ALPHABETIC ORDER; CAPITALIZE ONLY PROPER NAMES)}

bulding fires, building materials, combustion toxicology; compartment fires; computers; halons; hazard assessment 

